Florida International University FIU Digital Commons

FIU Electronic Theses and Dissertations

University Graduate School

5-11-2018

\title{
Exposure to Estrogenic Endocrine Disrupting Chemicals and Brain Health
}

\author{
Mark Preciados \\ Department of Environmental \& Occupational Health, Florida International University, mprec001@fiu.edu
}

DOI: $10.25148 /$ etd.FIDC006573

Follow this and additional works at: https://digitalcommons.fiu.edu/etd

Part of the Bioinformatics Commons, Biostatistics Commons, Environmental Public Health Commons, Epidemiology Commons, Genetics Commons, and the Molecular Genetics Commons

\section{Recommended Citation}

Preciados, Mark, "Exposure to Estrogenic Endocrine Disrupting Chemicals and Brain Health" (2018). FIU Electronic Theses and Dissertations. 3741.

https://digitalcommons.fiu.edu/etd/3741

This work is brought to you for free and open access by the University Graduate School at FIU Digital Commons. It has been accepted for inclusion in FIU Electronic Theses and Dissertations by an authorized administrator of FIU Digital Commons. For more information, please contact dcc@fiu.edu. 


\title{
FLORIDA INTERNATIONAL UNIVERSITY
}

Miami, Florida

\section{EXPOSURE TO ESTROGENIC ENDOCRINE DISRUPTING CHEMICALS AND \\ BRAIN HEALTH}

\author{
A dissertation submitted in partial fulfillment of \\ the requirements for the degree of \\ DOCTOR OF PHILOSOPHY \\ in
}

PUBLIC HEALTH

by

Mark Vicera Preciados 
To: $\quad$ Dean Tomas R. Guilarte

R.Stempel College of Public Health and Social Work

This dissertation, written by Mark Vicera Preciados, and entitled Exposure to Estrogenic Endocrine Disrupting Chemicals and Brain Health, having been approved in respect to style and intellectual content, is referred to you for judgment.

We have read this dissertation and recommend that it be approved.

$\begin{array}{r}\hline \text { Alok Deoraj } \\ \hline \text { Vijaya Narayanan } \\ \hline \text { Quentin Felty } \\ \hline \text { Changwon Yoo } \\ \hline \text { Deodutta Roy, Major Professor }\end{array}$

Date of Defense: May 11, 2018

The dissertation of Mark Vicera Preciados is approved.

Dean Tomas R. Guilarte R.Stempel College of Public Health and Social Work

Andrés G. Gil

Vice President for Research and Economic Development and Dean of the University Graduate School

Florida International University, 2018 
C Copyright 2018 by Mark Vicera Preciados

All rights reserved. 


\section{DEDICATION}

To my Mom and Dad, for always sacrificing and providing me with all the resources needed to succeed. As I have become older and wiser, I have learned to value your example and advice.

To my wife, Jaemy, your confidence, never give up attitude, and support is all I could ask for in a spouse. You are one of the great foundations in my life. 


\section{ACKNOWLEDGMENTS}

I would like to acknowledge my committee members, Dr. Alok Deoraj, Dr. Vijaya Narayanan, Dr. Quentin Felty, and Dr. Changwon Yoo, thank you for your expertise, advice, and time in my $\mathrm{PhD}$ journey.

I would also like to acknowledge my major professor, Dr. Deodutta Roy, you are truly a great professor, who always looks out for the well-being of your students. Thank you for helping me gain the confidence I needed to finish my dissertation and to see the magic in the process. 


\title{
ABSTRACT OF THE DISSERTATION \\ EXPOSURE TO ESTROGENIC ENDOCRINE DISRUPTING CHEMICALS AND \\ BRAIN HEALTH
}

by

\author{
Mark Vicera Preciados
}

Florida International University, 2018

Miami, Florida

\section{Professor Deodutta Roy, Major Professor}

The overall objective of this dissertation was to examine exposures to the estrogenic endocrine disrupting chemicals (EEDCs), phthalates, bisphenol-A (BPA), and the metalloestrogens cadmium (Cd), arsenic (As), and manganese (Mn) in an older geriatric aged-population and examine associations with brain health. Given the evidence that EEDCs affect brain health and play a role in the development of cognitive dysfunction and neurodegenerative disease, and the constant environmental exposure through foods and everyday products has led this to becoming a great public health concern. Using a bioinformatic approach to find nuclear respiratory factor 1 (NRF1) gene targets involved in mitochondrial dysfunction, that are both estrogen and EEDC-sensitive, we found several genes involved in the gene pathways of Alzheimer's disease (AD): APBB2, EIF2S1, ENO1, MAPT, and PAXIP1. Using the Center for Disease Control and Prevention (CDC), National Health and Nutrition Examination Survey (NHANES) 20112014 datasets to assess EEDC bioburden and associations with surrogate indicators of brain health, which include cognitive scores, memory questions, and taste and smell data, we found phthalate bioburden to be significantly higher in those with adverse brain health 
and significantly higher in females. In our logistic regression model when controlling for all known and suspected covariates in $\mathrm{AD}$, in females, the phthalates in females ECP, MBP, MOH, MZP, and MIB in males and the phthalates COP, ECP, MBP, MC1, MEP, $\mathrm{MHH}, \mathrm{MOH}$, and MIB were significantly associated with poor cognitive test scores, poor memory, and taste and smell dysfunction. Among the metalloestrogens, Cd bioburden was higher in those with poor cognitive performance, poor memory, and taste and smell dysfunction, with the trend more significant in males. Among oral contraceptive (OC) and HRT (hormone replacement therapy) use, in our logistic regression model when controlling for all known and suspected covariates in AD, past OC and HRT use was associated with better cognitive test scores. The study provides further evidence of the complex role EEDCs play in overall brain health through other biological mechanisms and fills a gap in knowledge that demonstrates EEDCs effects on brain health in a geriatric age population. 


\section{TABLE OF CONTENTS}

\section{CHAPTER}

PAGE

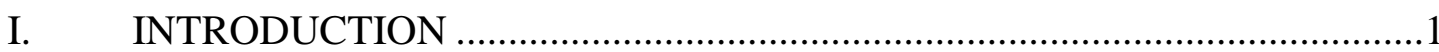

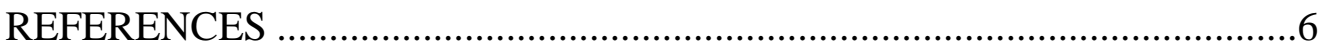

II. MANUSCRIPT 1.............................................................................12

EXPOSURE TO ESTROGENIC ENDOCRINE DISRUPTING

CHEMICALS AND THEIR ROLE BRAIN HEALTH

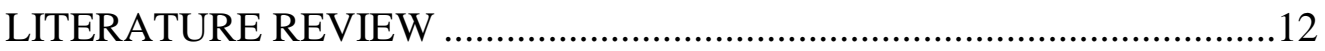

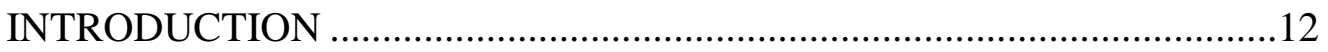

Estrogens and Brain Health .........................................................................13

Oral Contraceptives (OC) and Brain Health ..................................................15

Hormonal Replacement Therapy (HRT) and Brain Health ..............................15

Bisphenol - A (BPA) and Brain Health.....................................................16

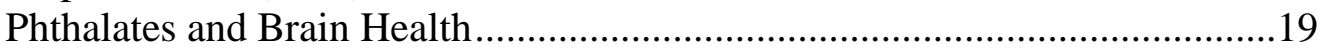

Polychlorinated Biphenyls (PCBs) and Brain Health ....................................20

Cadmium (Cd) and Brain Health ..............................................................22

Arsenic (As) and Brain Health..................................................................23

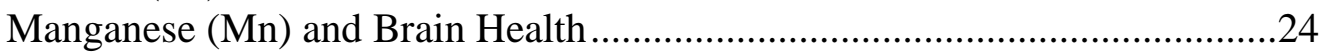

Mechanisms of Action of EEDCs in Brain Health ........................................26

Nuclear Respiratory Factor 1 (NRF1) Genes, EEDCs, and Brain Health .......27

Bioinformatic Method: Estrogen and NRF1 responsive Genes and EEDCs ..28

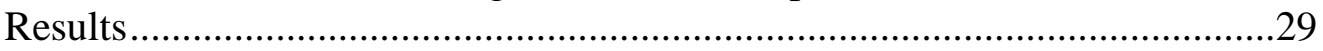

NRF1, Mitochondrial Dysfunction, and Neurodegenerative Disease ..............34

NRF1-mediated Regulation of Neurogenesis and Synaptogenesis ...................35

Gender Bias and NRF1 Regulated Genes-EEDC Interactions in AD ..............37

NRF1 and Estrogen Responsive Genes in the AD Pathway.............................37

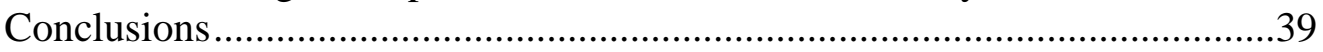

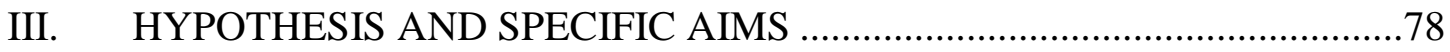

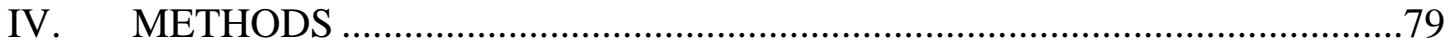

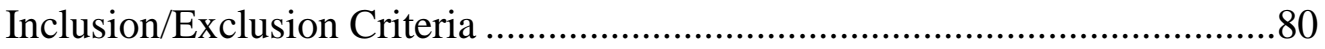

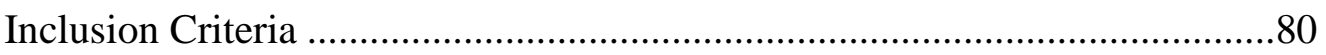

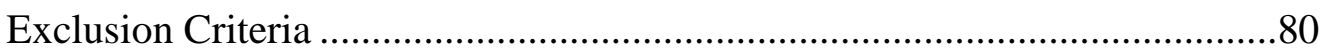

Phthalate Exposure Assessment and Measurements...............................80

BPA Exposure Assessment and Measurements....................................81

Cadmium Exposure Assessment and Measurements..............................82 
Manganese Exposure Assessment and Measurements ................................8 82

Arsenic Exposure Assessment and Measurements .......................................83

Oral Contraceptive Use Assessment and Measurements ...............................84

Hormonal Replacement Therapy Use Assessment and Measurements ...........84

Assessment of Surrogate Brain Health Indicators - Cognitive Scores............84

CERAD Word Learning Subtest - Immediate Recall and Delayed Recall .....84

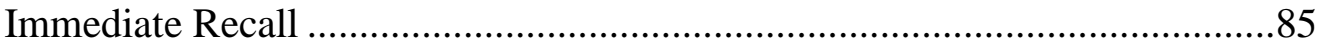

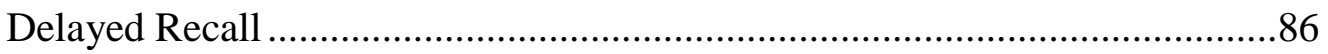

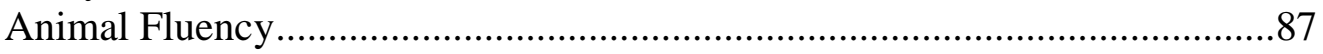

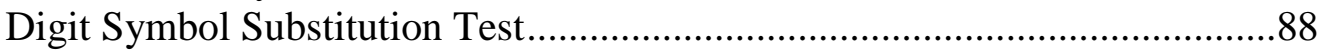

Assessment of Surrogate Brain Health Indicators - Memory Function ..........90

During the past 12 months, have you experienced confusion or memory loss that is happening more often or getting worse? ...................................90

During the past 7 days, how often have you had trouble remembering

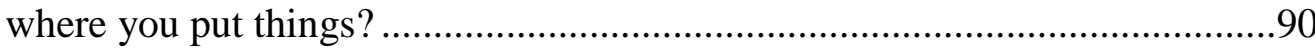

Are you limited in any way because of difficulty remembering or because you experience periods of confusion?.

Assessment of Surrogate Brain Health Indicators - Taste and Smell

Function

Do you sometimes smell and unpleasant, bad, or burning odor when nothing is there?....

During the past 12 months have you had a taste or other sensation in your mouth that does not go away?

Covariates and Confounding Variables ....................................................95

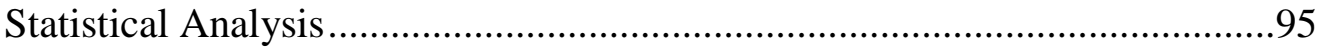

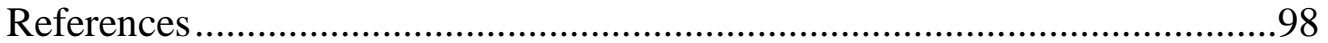

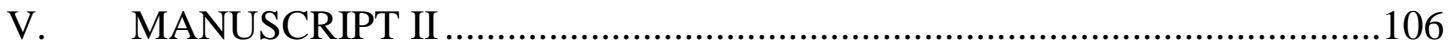

EXPOSURE TO PHTHALATES AND BISPHENOL-A AND

ASSOCIATIONS WITH BRAIN HEALTH: NHANES 2011-2014 ............106

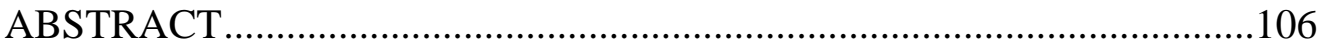

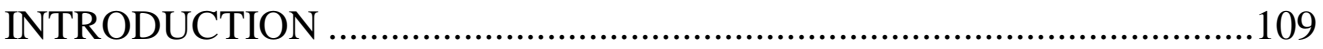

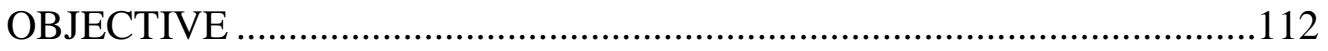

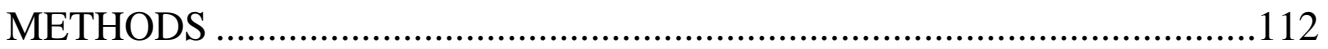

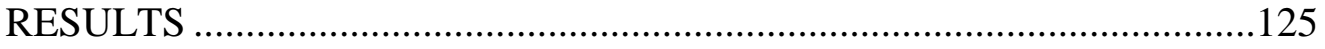

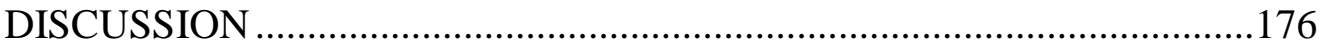

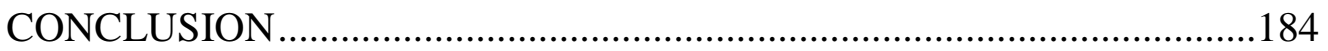

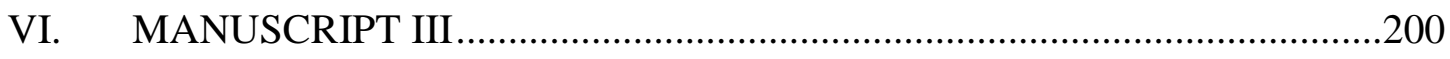

EXPOSURE TO METALLOESTROGENS AND ASSOCIATIONS

WITH BRAIN HEALTH: NHANES 2011-2014 ......................................200

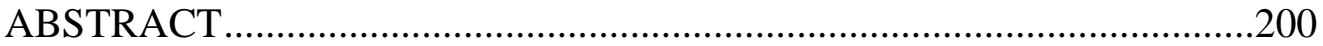

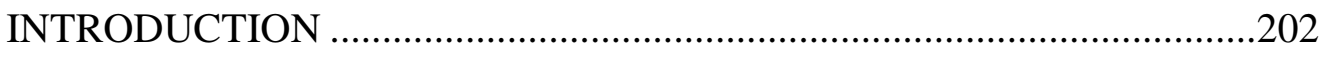




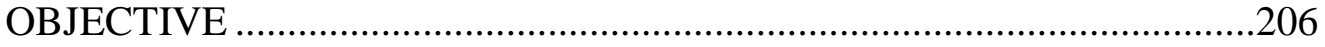

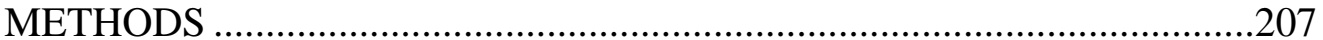

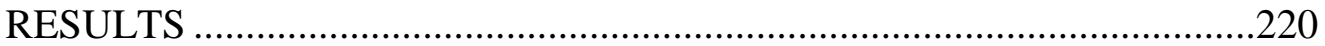

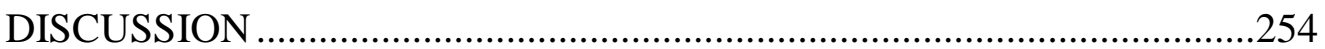

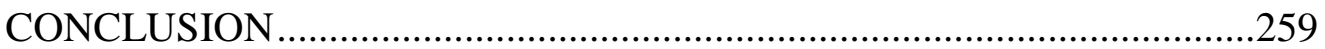

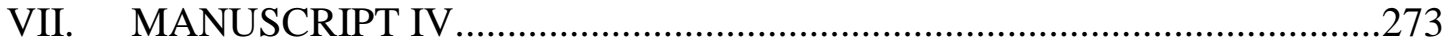

EXPOSURE TO ORAL CONTRACEPTIVES AND HORMONE REPLACEMENT THERAPY ASSOCIATIONS WITH BRAIN

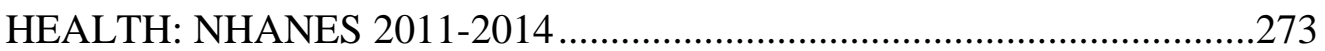

ABSTRACT ......................................................................................2.

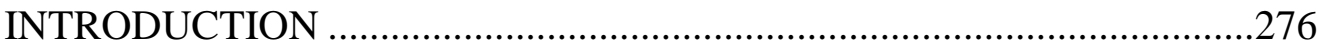

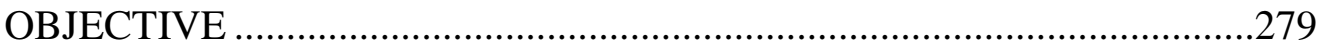

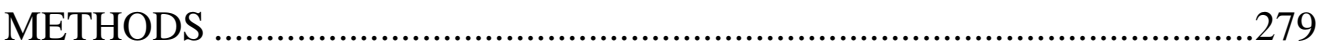

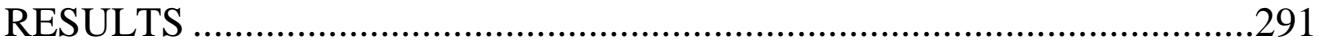

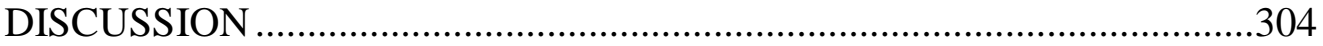

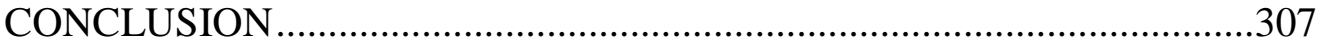

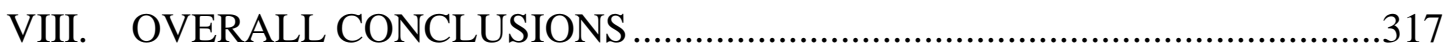

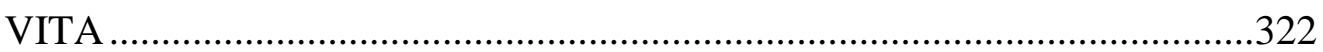




\section{LIST OF TABLES}

TABLE

PAGE

\section{Manuscript I}

Table 1: Top 10 enriched pathways for E2 and NRF1-Target Genes Associated with Estrogenic Endocrine Disruptors - BPA, PCB, Phthalates, As, Cd, and Mn.

Table 2: Interaction of Estrogenic Endocrine Disrupting Chemicals Modified Genes with Estrogen Signaling and NRF1 network genes in the Individual Neurodegenerative Disease

\section{Manuscript II}

Table 1.1 - Descriptive Statistics -- Surrogate brain health indicators and covariates for Phthalates and BPA.

Table 1.2 Geometric Mean Urinary Phthalate and BPA Levels by Immediate Recall Cut-Off Score for Subjects >= 60 years of age, NHANES 2011-2014, over the LOD ...130

Table 1.3 Age-Specific Geometric Mean Urinary Phthalate and BPA Levels by Immediate Recall Cut-Off Scores for Subjects $>=60$ years of age, NHANES 2011-2014, over the LOD

Table 1.4 Gender-specific Geometric Mean Urinary Phthalate and BPA Levels by Immediate Recall Cut-Off Scores for Subjects $>=60$ years of age, NHANES 2011-2014, over the LOD.

Table 1.5 Race-specific Geometric Mean Urinary Phthalate and BPA Levels by Immediate Recall Cut-Off Score for Subjects $>=60$ years of age, NHANES 2011-2014, over the LOD.

Table 1.6 Estimated ORs (95\% CI) of Urinary Phthalate and BPA Levels by Immediate Recall Cut-Off Score for Subjects $>=60$ years of age, NHANES 2011-2014 
Table 1.7 Geometric Mean Urinary Phthalate and BPA Levels by Delayed Recall Cut-Off Score for Subjects >= 60 years of age, NHANES 2011-2014, over the LOD ...135

Table 1.8 Age-Specific Geometric Mean Urinary Phthalate and BPA Levels by Delayed Recall Cut-Off Score for Subjects $>=60$ years of age, NHANES 2011-2014, over the LOD

Table 1.9 Gender-specific Geometric Mean Urinary Phthalate and BPA Levels by Delayed Recall Cut-Off Score for Subjects $>=60$ years of age, NHANES 2011-2014, over the LOD

Table 1.10 Race-specific Geometric Mean Urinary Phthalate and BPA Levels by Delayed Recall Cut-Off Scores for Subjects $>=60$ years of age, NHANES 2011-2014, over the LOD. .136

Table 1.11 Estimated ORs (95\% CI) of Urinary Phthalate and BPA Levels by Delayed Recall Cut-Off Score for Subjects $>=60$ years of age,

NHANES 2011-2014

Table 1.12 Geometric Mean Urinary Phthalate and BPA Levels by Animal Fluency Cut-Off Scores for Subjects >= 60 years of age, NHANES 2011-2014, over the LOD .140

Table 1.13 Age-Specific Geometric Mean Urinary Phthalate and BPA Levels by Animal Fluency Cut-Off Score for Subjects $>=60$ years of age, NHANES 2011-2014, over the LOD

Table 1.14 Gender-specific Geometric Mean Urinary Phthalate and BPA Levels by Animal Fluency Cut-Off Score for Subjects $>=60$ years of age, NHANES 2011-2014, over the LOD

Table 1.15 Race-specific Geometric Mean Urinary Phthalate and BPA Levels by Animal Fluency Cut-Off Score for Subjects $>=60$ years of age, NHANES 2011-2014, over the LOD 141 
Table 1.16 Estimated ORs (95\% CI) of Urinary Phthalate and BPA Levels by

Verbal Fluency Score for Subjects >= 60 years of age, NHANES 2011-2014.

Table 1.17 Geometric Mean Urinary Phthalate and BPA Levels by Digit Symbol Substitution Test (DSST) Cut-Off Score for Subjects $>=60$ years of age, NHANES 2011-2014, over the LOD

Table 1.18 Age-Specific Geometric Mean Urinary Phthalate and BPA Levels by Digit Symbol Substitution (DSST) Cut-Off Scores for Subjects $>=60$ years of age, NHANES 2011-2014, over the LOD.

Table 1.19 Geometric Mean Urinary Phthalate and BPA Levels by Digit Symbol Substitution (DSST) Cut-Off Score for Subjects $>=60$ years of age, NHANES 2011-2014, over the LOD. 146

Table 1.20 Race-specific Geometric Mean Urinary Phthalate and BPA Levels by Digit Symbol Substitution (DSST) Cut-Off Score for Subjects $>=60$ years of age, NHANES 2011-2014, over the LOD.

Table 1.21 Estimated ORs (95\% CI) of Urinary Phthalate and BPA Levels by Digit Symbol Substitution Test (DSST) Score for Subjects $>=60$ years of age, NHANES 2011-2014

Table 1.22 Geometric Mean Urinary Phthalate and BPA Levels by responses, "During the past 12 months, experienced confusion or memory loss that is happening more often or is getting worse?" for Subjects $>=60$ years of age, NHANES 2011-2014, over the LOD.

Table 1.23 Age-Specific Geometric Mean Urinary Phthalate and BPA Levels by responses, "During the past 12 months, experienced confusion or memory loss that is happening more often or is getting worse?" for Subjects $>=60$ years of age, NHANES 2011-2014, over the LOD.

Table 1.24 Geometric Mean Urinary Phthalate and BPA Levels by responses, "During the past 12 months, experienced confusion or memory loss that is happening more often or is getting worse?" for Subjects $>=60$ years of age, NHANES 2011-2014, over the LOD 
Table 1.25 Race-specific Geometric Mean Urinary Phthalate and BPA Levels by responses, "During the past 12 months, experienced confusion or memory loss that is happening more often or is getting worse?" for Subjects $>=60$ years of age,

NHANES 2011-2014, over the LOD.

Table 1.26 Estimated ORs (95\% CI) of Urinary Phthalate and BPA Levels by responses, "During the past 12 months, you experienced confusion or memory loss that is happening more often or is getting worse?" for Subjects $>=60$ years of age, NHANES 2011-2014

Table 1.27 Geometric Mean Urinary Phthalate and BPA Levels by Responses, "During the past 7 days, how often have you had trouble remembering where you put things, like your keys or your wallet?" for Subjects $>=60$ years of age, NHANES 2011-2014, over the LOD. 156

Table 1.28 Age-Specific Geometric Mean Urinary Phthalate and BPA Levels by Responses, "During the past 7 days, how often have you had trouble remembering where you put things, like your keys or your wallet?" for Subjects $>=60$ years of age, NHANES 2011-2014, over the LOD.

Table 1.29 Gender-Specific Geometric Mean Urinary Phthalate and BPA Levels by Responses, "During the past 7 days, how often have you had trouble remembering where you put things, like your keys or your wallet?" for Subjects $>=60$ years of age, NHANES 2011-2014, over the LOD.

Table 1.30 Race-specific Geometric Mean Urinary Phthalate and BPA Levels by Responses, "During the past 7 days, how often have you had trouble remembering where you put things, like your keys or your wallet?" for Subjects $>=60$ years of age, NHANES 2011-2014, over the LOD.

Table 1.31 Estimated ORs (95\% CI) of Urinary Phthalate and BPA by Responses, "During the past 7 days, how often have you had trouble remembering where you put things, like your keys or your wallet?" for Subjects $>=60$ years of age, NHANES 2011-2014

Table 1.32 Geometric Mean Urinary Phthalate and BPA Levels by Responses, "Are you limited in any way because of difficulty remembering or because you experience periods of confusion?" for Subjects $>=60$ years of age, 
Table 1.33 Age-Specific Geometric Mean Urinary Phthalate and BPA Levels by Responses "Are you limited in any way because of difficulty remembering or because you experience periods of confusion?" for subjects $>=60$ years of age, NHANES 2011-2014, over the LOD.

Table 1.34 Geometric Mean Urinary Phthalate and BPA Levels by Responses, "Are you limited in any way because of difficulty remembering or because you experience periods of confusion?" for subjects $>=60$ years of age,

NHANES 2011-2014, over the LOD.

Table 1.35 Race-specific Geometric Mean Urinary Phthalate and BPA Levels by Responses, "Are you limited in any way because of difficulty remembering or because you experience periods of confusion?" for Subjects $>=60$ years of age, NHANES 2011-2014, over the LOD.

Table 1.36 Estimated ORs (95\% CI) of Urinary Phthalate and BPA Levels by Responses, "Are you limited in any way because of difficulty remembering or because you experience periods of confusion?" for Subjects $>=60$ years of age, NHANES 2011-2014

Table 1.37 Geometric Mean Urinary Phthalate and BPA Levels by Responses, "Do you sometimes smell an unpleasant, bad or burning odor when nothing is there?" for Subjects $>=60$ years of age, NHANES 2011-2014, over the LOD.

Table 1.38 Age-specific Geometric Mean Urinary Phthalate and BPA Levels by Responses, "Do you sometimes smell an unpleasant, bad or burning odor when nothing is there?" for Subjects >= 60 years of age, NHANES 2011-2014, over the LOD 168

Table 1.39 Gender-specific Geometric Mean Urinary Phthalate and BPA Levels by Responses, "Do you sometimes smell an unpleasant, bad or burning odor when nothing is there?" for Subjects >= 60 years of age, NHANES 2011-2014, over the LOD

Table 1.40 Race-specific Geometric Mean Urinary Phthalate and BPA Levels by Responses, "Do you sometimes smell an unpleasant, bad or burning odor when nothing is there?" for Subjects >= 60 years of age, NHANES 2011-2014, over the LOD . 
Table 1.41 Estimated ORs (95\% CI) of Urinary Phthalate and BPA Levels by Responses, "Do you sometimes smell an unpleasant, bad or burning odor when nothing is there?" for Subjects >= 60 years of age, NHANES 2011-2014

Table 1.42 Geometric Mean Urinary Phthalate and BPA Levels by Responses, "During the past 12 months have you had a taste or other sensation in your mouth that does not go away?" for Subjects >= 60 years of age, NHANES 2011-2014, over the LOD

Table 1.43 Age-Specific Geometric Urinary Mean Phthalate and BPA Levels by Responses, "During the past 12 months have you had a taste or other sensation in your mouth that does not go away?" for Subjects $>=60$ years of age,

NHANES 2011-2014, over the LOD.....

Table 1.44 Geometric Mean Phthalate and BPA Levels by Responses, "During the past 12 months have you had a taste or other sensation in your mouth that does not go away?" for Subjects >= 60 years of age, NHANES 2011-2014, over the LOD

Table 1.45 Race-specific Geometric Mean Phthalate and BPA Levels by Responses, "During the past 12 months have you had a taste or other sensation in your mouth that does not go away?" for Subjects >= 60 years of age, NHANES 2011-2014, over the LOD

Table 1.46 Estimated ORs (95\% CI) of Urinary Phthalate and BPA Levels by Responses, "During the past 12 months have you had a taste or other sensation in your mouth that does not go away?" for Subjects $>=60$ years of age,

NHANES 2011-2014

Table 1.47 - Summary Table of EEDCs overall significance of GMs by Surrogate of Brain Health Indicators $(* \mathrm{p}<0.05, * * \mathrm{p}<0.01, * * * \mathrm{p}<0.001)$

Table 1.48 - Summary Table of EEDCs overall significance of GMs by Surrogate of Brain Health Indicators in males $(* \mathrm{p}<0.05, * * \mathrm{p}<0.01, * * * \mathrm{p}<0.001) \ldots$

Table 1.49 - Summary Table of EEDCs overall significance of GMs by Surrogate of Brain Health Indicators in females $(* \mathrm{p}<0.05, * * \mathrm{p}<0.01, * * * \mathrm{p}<0.001)$. 


\section{MANUSCRIPT III}

Table 1.1 - Descriptive Statistics -- Surrogate brain health indicators and covariates for $\mathrm{Cd}$ and $\mathrm{Mn}$

Table 1.1a - Descriptive Statistics -- Surrogate brain health indicators and covariates for As

Table 1.2 Geometric Mean Urinary Metalloestrogen Levels by Immediate Recall Cut -Off Score for Subjects >= 60 years of age, NHANES 2011-2014, over the LOD .........224

Table 1.3 Age-Specific Geometric Mean Urinary Metalloestrogen Levels by Immediate Recall Cut-Off Score for Subjects >= 60 years of age, NHANES 2011-2014, over the LOD

Table 1.4 Gender-Specific Geometric Mean Urinary Metalloestrogen Levels by Immediate Recall Cut-Off Score for Subjects >= 60 years of age, NHANES 2011-2014, over the LOD

Table 1.5 Race-specific Geometric Mean Urinary Metalloestrogen Levels by Immediate Recall Cut-Off Score for Subjects $>=60$ years of age, NHANES 2011-2014, over the LOD.

Table 1.6 ORs and 95\% CI for Urinary Metalloestrogen Levels by Immediate Recall Cut-Off Scores for Subjects >= 60 years of age, NHANES 2011-2014

Table 1.7 Geometric Mean Urinary Metalloestrogen Levels by Delayed Recall Cut-Off Score for Subjects >= 60 years of age, NHANES 2011-2014, over the LOD ...227

Table 1.8 Age-Specific Geometric Mean Urinary Metalloestrogen Levels by Delayed Recall Cut-Off Score for Subjects $>=60$ years of age, NHANES 2011-2014, over the LOD

Table 1.9 Gender-specific Geometric Mean Urinary Metalloestrogen Levels by Delayed Recall Cut-Off Score for Subjects $>=60$ years of age, NHANES 
Table 1.10 Race-specific Geometric Mean Urinary Metalloestrogen Levels by Delayed Recall Cut-Off Score for Subjects $>=60$ years of age, NHANES 2011-2014, over the LOD.

Table 1.11 ORs and 95\% CI for Urinary Metalloestrogen Levels by Delayed Recall Cut-Off Score for Subjects >= 60 years of age, NHANES 2011-2014

Table 1.12 Geometric Mean Urinary Metalloestrogen Levels by Animal Fluency Cut-Off Score for Subjects >= 60 years of age, NHANES 2011-2014, over the LOD ...230

Table 1.13 Age-Specific Geometric Mean Urinary Metalloestrogen Levels by Animal Fluency Cut-Off Score for Subjects >= 60 years of age, NHANES 2011-2014, over the LOD

Table 1.14 Gender-specific Geometric Mean Urinary Metalloestrogen Levels by Animal Fluency Cut-Off Score for Subjects $>=60$ years of age, NHANES 2011-2014, over the LOD

Table 1.15 Race-specific Geometric Mean Urinary Metalloestrogen Levels by Animal Fluency Cut-Off Score for Subjects $>=60$ years of age, NHANES 2011-2014, over the LOD.

Table 1.16 ORs and 95\% CI for Urinary Metalloestrogen Levels by Animal Fluency Scores for Subjects $>=60$ years of age, NHANES 2011-2014

Table 1.17 Geometric Mean Urinary Metalloestrogen Levels by Digit Symbol Substitution (DSST) Cut-Off Scores for Subjects $>=60$ years of age, NHANES 2011-2014, over the LOD

Table 1.18 Age-Specific Geometric Mean Urinary Metalloestrogen Levels by Digit Symbol Substitution (DSST) Cut-Off Scores for Subjects $>=60$ years of age, NHANES 2011-2014, over the LOD.

Table 1.19 Gender-Specific Geometric Mean Urinary Metalloestrogen Levels by Digit Symbol Substitution (DSST) Cut-Off Score for Subjects $>=60$ years of age, NHANES 2011-2014, over the LOD. 
Table 1.20 Race-specific Geometric Mean Urinary Metalloestrogen Levels by Digit Symbol Substitution (DSST) Cut-Off Scores for Subjects $>=60$ years of age,

NHANES 2011-2014, over the LOD. .235

Table 1.21 ORs and 95\% CI for Urinary Metalloestrogen Levels by Digit Symbol Substitution (DSST) Score for Subjects $>=60$ years of age, NHANES 2011-2014 .235

Table 1.22 Geometric Mean Urinary Metalloestrogen Levels by Y/N, "During the past 12 months, you experienced confusion or memory loss that is happening more often or is getting worse?" for Subjects $>=60$ years of age,

NHANES 2011-2014, over the LOD. .238

Table 1.23 Age-Specific Geometric Mean Urinary Metalloestrogen Levels by $\mathrm{Y} / \mathrm{N}$, "During the past 12 months, you experienced confusion or memory loss that is happening more often or is getting worse?" for Subjects $>=60$ years of age, NHANES 2011-2014, over the LOD. .238

Table 1.24 Gender-Specific Geometric Mean Urinary Metalloestrogen Levels by $\mathrm{Y} / \mathrm{N}$, "During the past 12 months, you experienced confusion or memory loss that is happening more often or is getting worse?" for Subjects $>=60$ years of age, NHANES 2011-2014, over the LOD.

Table 1.25 Race-specific Geometric Mean Urinary Metalloestrogen Levels by Y/N, "During the past 12 months, you experienced confusion or memory loss that is happening more often or is getting worse?" for Subjects $>=60$ years of age,

NHANES 2011-2014, over the LOD.

Table 1.26 ORs and 95\% CI for Urinary Metalloestrogen Levels by Y/N, "During the past 12 months, you experienced confusion or memory loss that is happening more often or is getting worse?" for Subjects >= 60 years of age, NHANES 2011-2014

Table 1.27 Geometric Mean Urinary Metalloestrogen Levels by Y/N, "During the past 7 days, how often have you had trouble remembering where you put things, like your keys or your wallet?" for Subjects $>=60$ years of age, NHANES 2011-2014, over the LOD. 
Table 1.28 Age-Specific Geometric Mean Urinary Metalloestrogen Levels by Y/N, "During the past 7 days, how often have you had trouble remembering where you put things, like your keys or your wallet?" for Subjects $>=60$ years of age, NHANES 2011-2014, over the LOD

Table 1.29 Gender-Specific Geometric Mean Urinary Metalloestrogen Levels by Y/N, "During the past 7 days, how often have you had trouble remembering where you put things, like your keys or your wallet?" for Subjects $>=60$ years of age, NHANES 2011-2014, over the LOD

Table 1.30 Race-specific Geometric Mean Urinary Metalloestrogen Levels by $\mathrm{Y} / \mathrm{N}$, "During the past 7 days, how often have you had trouble remembering where you put things, like your keys or your wallet?" for Subjects $>=60$ years of age, NHANES 2011-2014, over the LOD

Table 1.31 ORs and 95\% CI for Urinary Metalloestrogen by $\mathrm{Y} / \mathrm{N}$, "During the past 7 days, how often have you had trouble remembering where you put things, like your keys or your wallet?" for Subjects $>=60$ years of age, NHANES 2011-2014

Table 1.32 Geometric Mean Urinary Metalloestrogen Levels by $\mathrm{Y} / \mathrm{N}$, "Are you limited in any way because of difficulty remembering or because you experience periods of confusion?" for Subjects $>=60$ years of age, NHANES 2011-2014, over the LOD

Table 1.33 Age-Specific Geometric Mean Urinary Metalloestrogen Levels by Y/N "Are you limited in any way because of difficulty remembering or because you experience periods of confusion?" for subjects $>=60$ years of age, NHANES 2011-2014, over the LOD

Table 1.34 Gender-Specific Geometric Mean Urinary Metalloestrogen Levels by Y/N, "Are you limited in any way because of difficulty remembering or because you experience periods of confusion?" for subjects $>=60$ years of age, NHANES 2011-2014, over the LOD

Table 1.35 Race-specific Geometric Mean Urinary Metalloestrogen Levels by $\mathrm{Y} / \mathrm{N}$, "Are you limited in any way because of difficulty remembering or because you experience periods of confusion?" for Subjects $>=60$ years of age, NHANES 2011-2014, over the LOD. .246 
Table 1.36 ORs and 95\% CI for Urinary Metalloestrogen Levels by $\mathrm{Y} / \mathrm{N}$, "Are you limited in any way because of difficulty remembering or because you experience periods of confusion?" for Subjects $>=60$ years of age, NHANES 2011-2014

Table 1.37 Geometric Mean Urinary Metalloestrogen Levels by Y/N, "Do you sometimes smell an unpleasant, bad or burning odor when nothing is there?" for Subjects $>=60$ years of age, NHANES 2011-2014, over the LOD. .248

Table 1.38 Age-Specific Geometric Mean Urinary Metalloestrogen Levels by $\mathrm{Y} / \mathrm{N}$, "Do you sometimes smell an unpleasant, bad or burning odor when nothing is there?" for Subjects >= 60 years of age, NHANES 2011-2014, over the LOD

Table 1.39 Gender-Specific Geometric Mean Urinary Metalloestrogen Levels by Y/N, "Do you sometimes smell an unpleasant, bad or burning odor when nothing is there?" for Subjects >= 60 years of age, NHANES 2011-2014, over the LOD

Table 1.40 Race-specific Geometric Mean Urinary Metalloestrogen Levels by Y/N, "Do you sometimes smell an unpleasant, bad or burning odor when nothing is there?" for Subjects >= 60 years of age, NHANES 2011-2014, over the LOD

Table 1.41 ORs and 95\% CI for Urinary Metalloestrogen Levels by Responses, "Do you sometimes smell an unpleasant, bad or burning odor when nothing is there?" for Subjects >= 60 years of age, NHANES 2011-2014 .250

Table 1.42 Geometric Mean Urinary Metalloestrogen Levels by Y/N, "During the past 12 months have you had a taste or other sensation in your mouth that does not go away?" for Subjects >= 60 years of age, NHANES 2011-2014, over the LOD...252

Table 1.43 Age-Specific Geometric Urinary Mean Metalloestrogen Levels by $\mathrm{Y} / \mathrm{N}$, "During the past 12 months have you had a taste or other sensation in your mouth that does not go away?" for Subjects $>=60$ years of age, NHANES 2011-2014, over the LOD 
Table 1.44 Geometric Mean Metalloestrogen Levels by Y/N, "During the past 12 months have you had a taste or other sensation in your mouth that does not go away?" for Subjects >= 60 years of age, NHANES 2011-2014, over the LOD.

Table 1.45 Race-specific Geometric Mean Metalloestrogen Levels by Y/N, "During the past 12 months have you had a taste or other sensation in your mouth that does not go away?" for Subjects >= 60 years of age, NHANES 2011-2014, over the LOD

Table 1.46 ORs and 95\% CI for Urinary Metalloestrogen Levels by Y/N, "During the past 12 months have you had a taste or other sensation in your mouth that does not go away?" for Subjects >= 60 years of age, NHANES 2011-2014

Table 1.47 - Summary Table of EEDCs overall significance of GMs by Surrogate of Brain Health Indicators $(* \mathrm{p}<0.05, * * \mathrm{p}<0.01, * * * \mathrm{p}<0.001)$

Table 1.48 - Summary Table of EEDCs overall significance of GMs by Surrogate of Brain Health Indicators in males $(* \mathrm{p}<0.05, * * \mathrm{p}<0.01, * * * \mathrm{p}<0.001)$ .256

Table 1.49 - Summary Table of EEDCs overall significance of GMs by Surrogate of Brain Health Indicators in females $(* \mathrm{p}<0.05, * * \mathrm{p}<0.01, * * * \mathrm{p}<0.001)$

\section{MANUSCRIPT IV}

Table 1.1 - Descriptive Statistics -- Surrogate brain health indicators and covariates for OC use

Table 1.1a - Descriptive Statistics -- Surrogate brain health indicators and covariates for HRT use.

Table 1.2 Immediate Recall Cut-off Scores Chi-Square Results

Table 1.3 Immediate Recall Cut-off Scores Chi-Square Results

Table 1.4 - ORs and 95\% CI for OC and HRT Use Levels by Immediate Recall

Cut-off Scores for Female Subjects >= 60 years of age, NHANES 2011-2014. 
Table 1.5 Delayed Recall Cut-off Scores Chi-Square Results

Table 1.6 Delayed Recall Cut-off Scores Chi-Square Results .295

Table 1.7 - ORs and 95\% CI for OC and HRT Use Levels by Delayed Recall Cut-off Scores for Female Subjects >= 60 years of age, NHANES 2011-2014 .296

Table 1.8 Animal Fluency Cut-off Scores Chi-Square Results .296

Table 1.9 Animal Fluency Cut-off Scores Chi-Square Results .296

Table 1.10 - ORs and 95\% CI for OC and HRT Use Levels by Animal Fluency Cut-off Scores for Female Subjects >= 60 years of age, NHANES 2011-2014.....

Table 1.11 DSST Cut-off Scores Chi-Square Results .297

Table 1.12 DSST Cut-off Scores Chi-Square Results

Table 1.13 - ORs and 95\% CI for OC and HRT Use Levels by DSST Cut-off Scores for Female Subjects >= 60 years of age, NHANES 2011-2014 .298

Table 1.14 During the past 12 months, have you experienced confusion or memory loss that is happening more often or getting worse? Chi-Square Results

Table 1.15 During the past 12 months, have you experienced confusion or memory loss that is happening more often or getting worse? Chi-Square Results

Table 1.16 - ORs and 95\% CI for OC and HRT Use Levels by Y/N "During the past 12 months, you experienced confusion or memory loss that is happening more often or is getting worse?" for Female Subjects >= 60 years of age, NHANES 2011-2014 ....299

Table 1.17 During the past 7 days, how often have you had trouble remembering where you put things? Chi-Square Results .300

Table 1.18 During the past 7 days, how often have you had trouble remembering where you put things? Chi-Square Results .300 
Table 1.19 - ORs and 95\% CI for OC and HRT Use Levels by Y/N "During the past 7 days, how often have you had trouble remembering where you put things, like your keys or your wallet?" for Female Subjects >= 60 years of age, NHANES 2011-2014 ...300

Table 1.20 Are you limited in any way because of difficulty remembering or because you experience periods of confusion? Chi-Square Results

Table 1.21 Are you limited in any way because of difficulty remembering or because you experience periods of confusion? Chi-Square Results

Table 1.22 - ORs and 95\% CI for OC and HRT Use Levels by Y/N "Are you limited in any way because of difficulty remembering or because you experience periods of confusion?" for Female Subjects >= 60 years of age, NHANES 2011-2014

Table 1.23 Do you sometimes smell and unpleasant, bad, or burning odor when nothing is there? Chi-Square Results

Table 1.24 Do you sometimes smell and unpleasant, bad, or burning odor when nothing is there? Chi-Square Results

Table 1.25 - ORs and 95\% CI for OC and HRT Use Levels by Y/N "Do you sometimes smell an unpleasant, bad or burning odor when nothing is there?" for Female Subjects $>=60$ years of age, NHANES 2011-2014 .303

Table 1.26 During the past 12 months have you had a taste or other sensation in your mouth that does not go away? Chi-Square Results 303

Table 1.27 During the past 12 months have you had a taste or other sensation in your mouth that does not go away? Chi-Square Results

Table 1.28 - ORs and 95\% CI for OC and HRT Use Levels by Y/N "During the past 12 months have you had a taste or other sensation in your mouth that does not go away?" for Female Subjects >= 60 years of age, NHANES 2011-2014 


\section{LIST OF FIGURES}

FIGURE

PAGE

\section{Manuscript I}

Figure 1. Venn diagram showing the individual pharmacological estrogen or estrogenic Endocrine Disruptor (EED) modified genes common to both NRF1 and/or estrogen signaling pathways. NRF1 target genes were mapped between 17-beta estradiol (E2) and pharmacological estrogen ethinyl estradiol or PCBs or BPA or phthalates or $\mathrm{Cd}$ or As or Mn.

Figure 2. Gene set pathway enrichment analysis of 17-beta estradiol (E2) responsive NRF1 target genes associated with Alzheimer Disease (AD). E2 responsive NRF1 genes are in black. The network includes the top 30 related genes. Right panel show GO annotations of NRF1 enrichment network genes of E2 associated with biological process pathways involving multiple E2 and NRF1 genes and Q-value as a probability showing number of genes out of the total genes annotated to each GO term.

Figure 3. Gene set pathway enrichment analysis of ethinyl estradiol responsive E2 and NRF1 common target genes associated with Alzheimer Disease (AD). Ethinyl estradiol responsive NRF1 genes are in black. Genes denoted by a diamond-shaped node are E2 and NRF1. The network includes the top 30 related genes. Right panel show GO annotations of E2 and NRF1 enrichment network genes of ethinyl estradiol associated with biological process pathways involving multiple E2 and NRF1 genes and Q-value as a probability showing number of genes out of the total genes annotated to each GO term

Figure 4. Gene set pathway enrichment analysis of bisphenol-A (BPA) responsive R2 and NRF1common target genes associated with Alzheimer Disease (AD). Bisphenol-A responsive NRF1 genes are in black. Genes denoted by a diamond-shaped node are E2 and NRF1 responsive. The network includes the top 30 related genes. Right panel show GO annotations of E2 and NRF1 enrichment network genes of BPA associated with biological process pathways involving multiple E2 and NRF1 genes and Q-value as a probability showing number of genes out of the total genes annotated to each GO term. 
Figure 5. Gene set pathway enrichment analysis of dibutyl phthalate modified E2 and NRF1 common target genes associated with Alzheimer Disease (AD). Dibutyl phthalate responsive NRF1 genes are in black. Genes denoted by a diamond-shaped node are E2 and NRF1 responsive. The network includes the top 30 related genes. Right panel show GO annotations of E2 and NRF1 enrichment network genes of dibutyl phthalate associated with biological process pathways involving multiple E2 and NRF1 genes and Q-value as a probability showing number of genes out of the total genes annotated to each GO term.

Figure 6. Gene set pathway enrichment analysis of diethylhexyl phthalate responsive E2 and NRF1 common target genes associated with Alzheimer Disease (AD).

Diethylhexyl phthalate responsive NRF1 genes are in black. Genes denoted by a diamond-shaped node are E2 and NRF1 responsive. The network includes the top 30 related genes. Right panel show GO annotations of E2 and NRF1 enrichment network genes of diethylhexyl phthalate associated with biological process pathways involving multiple E2 and NRF1 genes and Q-value as a probability showing number of genes out of the total genes annotated to each GO term.

Figure 7. Gene set pathway enrichment analysis of cadmium responsive E2 and NRF1 common target genes associated with Alzheimer Disease (AD). Cadmium responsive NRF1 genes are in black. Genes denoted by a diamond-shaped node are E2-and NRF1 responsive. The network includes the top 30 related genes. Right panel show GO annotations of E2 and NRF1 enrichment network genes of cadmium associated with biological process pathways involving multiple E2 and NRF1 genes and Q-value as a probability showing number of genes out of the total genes annotated to each GO term

Figure 8. Gender differences in the NRF1 network from brain microarray data of 79 male and female AD subjects and controls from the GEO database 


\section{ABBREVIATIONS AND ACRONYMS}

\begin{tabular}{|c|c|}
\hline $\mathrm{AD}$ & Alzheimer's Disease \\
\hline ADHD & Attention Deficit Hyperactive Disorder \\
\hline ALS & Amyotrophic Lateral Sclerosis \\
\hline APOE & Apolipoprotein E \\
\hline APP & Amyloid Precursor Protein \\
\hline AS & Arsenic \\
\hline BPA & Bisphenol-A \\
\hline $\mathrm{CD}$ & Cadmium \\
\hline CDC & Center for Disease Control and Prevention \\
\hline CERAD & Consortium to Establish a Registry for Alzheimer's Disease \\
\hline $\mathrm{CI}$ & Cognitive Impairment \\
\hline $\mathrm{CNP}$ & Mono (carboxynonyl) Phthalate \\
\hline $\mathrm{COP}$ & Mono (carboxyoctyl) Phthalate \\
\hline DHNES & Division of Health and Nutritional Examination Surveys \\
\hline DSST & Digit Symbol Substitution Test \\
\hline $\mathrm{ECP}$ & Mono-(2-ethyl-5-carboxypentyl) Phthalate \\
\hline EDC & Endocrine Disrupting Chemical \\
\hline $\mathrm{EE}$ & Ethinyl Estradiol \\
\hline EEDC & Estrogenic Endocrine Disrupting Chemical \\
\hline EPA & Environmental Protection Agency \\
\hline ER & Estrogen Receptors \\
\hline
\end{tabular}




\begin{tabular}{|c|c|}
\hline HRT & Hormone Replacement Therapy \\
\hline HT & Huntington's Disease \\
\hline LOD & Limit of Detection \\
\hline MBP & Mono-n-butyl Phthalate \\
\hline MBzp & Mono-benzyl Phthalate \\
\hline MC1 & Mono-(3-carboxypropyl) Phthalate \\
\hline MCI & Mild Cognitive Impairment \\
\hline MEC & Mobile Evaluation Clinic \\
\hline MEP & Mono-ethyl phthalate \\
\hline $\mathrm{MHH}$ & Mono (2-ethyl-5-hydroxy-hexyl) Phthalate \\
\hline MIB & Mono-isobutyl Phthalate \\
\hline $\mathrm{MN}$ & Manganese \\
\hline $\mathrm{MOH}$ & Mono-(2-ethyl-5-oxohexyl) Phthalate \\
\hline MZP & Mono-benzyl Phthalate \\
\hline NCHS & National Center for Health Statistics \\
\hline NHANES & National Health and Nutritional Examination Survey \\
\hline NMDA & N-Methyl-D-aspartate \\
\hline NRF1 & Nuclear Respiratory Factor 1 \\
\hline $\mathrm{OC}$ & Oral Contraceptive \\
\hline PCB & Polychlorinated Biphenyl \\
\hline PD & Parkinson's Disease \\
\hline PSU & Primary Sampling Units \\
\hline
\end{tabular}


RR

UPDRS

WHIMS

WHISCA
Relative Risk

Unified Parkinson's Disease Rating Scale

Women's Health Initiative Memory Study

Women's Health Initiative Study of Cognitive Aging 


\section{CHAPTER I}

\section{INTRODUCTION}

Endocrine disrupting chemicals (EDCs) are of important public health concern and are linked to diseases of all human systems ${ }^{1}$. They affect all the sensitive periods of human life: gestation, childhood, puberty, reproductive life, and old age ${ }^{1}$. EDCs include numerous chemicals found in our environment, both natural and man-made. These include industrial chemicals, plasticizers, pesticides, pharmaceutical drugs, and phytoestrogens ${ }^{2}$. EDCs affect the human body by imitating, blocking, and/or altering hormonal function ${ }^{1}$. EDCs exert their effects by being able to enter the body by dermal, ingestion, and inhalation routes ${ }^{2}$. There widespread distribution in our environment is due to their use in most manufacturing processes ${ }^{1}$ and their production from e-wastes ${ }^{3}$. Estrogenic endocrine disrupting chemicals (EEDCs) are a specific type of EDC, which affect bodily process that are influenced and modulated by estrogen hormones ${ }^{4}$.

EEDCs as a Brain Health Concern: EEDCs have been implicated as one of the causes of neurodegenerative disease and adverse brain health ${ }^{1}$. Adverse brain health can be defined by neurodegeneration that encompasses any pathological condition affecting neurons ${ }^{5}$. Neurodegenerative diseases, a product of adverse brain health, have varied symptoms which affect neurons from different parts of the brain, with the most publicized diseases being Alzheimer's disease (AD), Parkinson's disease (PD), amyotrophic lateral sclerosis, (ALS), and Huntington's disease (HD) ${ }^{5}$. The ability of various EEDCs to mimic estrogen and affect its role in brain health, and their widespread dissemination in the environment makes this an important public health concern. 
Estrogen's Role in Brain Health: Estrogen role in the brain is extensive. Estrogen has been shown to influence sexual differentiation ${ }^{6}$, plays a role as a neuroprotectant and promote anti-inflammatory effects on the brain ${ }^{7-12}$, promotes neuroplasticity and synaptogenesis ${ }^{13-17}$, and regulates and protects mitochondrial function in the brain ${ }^{18-20}$. A majority of studies have focused on EEDCs effects on early human development and the brain, and there are few studies that look at an older geriatric aged population. Due to EEDCs ability to interact with estrogen and disrupt its role in brain development and protection, merits further study on its effects on the aging brain. Of the hundreds of EEDCs that human beings are exposed to, this dissertation focuses on a selection of the most prevalent EEDCs in our environment with proven estrogenic activity: Phthalates, bisphenol-A (BPA), cadmium (Cd), manganese (Mn), and arsenic (As).

Phthalates: Phthalates are class of estrogenic endocrine disrupting chemicals that have a role in the development of neurodegenerative disease. Phthalates, also known as plasticizers, are chemicals used in plastics to make them flexible and strong ${ }^{21-23}$. Exposure to phthalates comes through ingestion, inhalation, and to a lesser extent, dermal contact with phthalate-containing products ${ }^{24}$. Phthalates are found in most consumer products. These include the following: wall coverings, tablecloths, floor tiles, furniture, upholstery, shower curtains, garden hoses, baby products, toys, shoes, packing materials, medical devices, paints, glue, nail polish, hair spray, insect repellents, food packaging materials, cosmetics, insecticides, and drug products ${ }^{21-23}$. Phthalates have short biological half-lives and do not accumulate, with urine as the primary route of excretion 25. Specific phthalates are also found in high levels among the US population. One study, found the body burden of the phthalates mono-ethyl phthalate (MEP), mono-n-butyl 
phthalate (MBP) and and mono-benzyl phthalate (MBzp) in 97\% of samples tested from the CDC NHANES 1999-2000 datasets ${ }^{26}$. As such, most of the US population has some measurable levels of phthalates in their bodies ${ }^{25}$.

Phthalates have also been demonstrated to have estrogenic activity and affinity for estrogen receptors (ER) ${ }^{27,28}$ and have been shown to interact with estrogen-responsive genes implicated in various neurodegenerative disorders ${ }^{29}$.

Bisphenol-A (BPA): BPA is a synthetically created chemical that is used to create polycarbonate plastics and resins ${ }^{30}$. BPA is found in many consumer products and plastics. These products include the following: baby bottles, compact discs, impactresistant safety equipment, medical devices, food cans and tops, water supply pipes, ATM receipts and dental sealants and composites ${ }^{30}$. Food storage containers that have BPA can cause it to leech into foods by the use of high heat ${ }^{30}$. It is of note that during the 2003-2004 NHANES data cycle, BPA was detected in 93\% of urine samples collected from subjects 6 years of age and older ${ }^{31}$.

BPA has been shown to be weakly estrogenic and have a low affinity for binding to ER receptors, but is speculated to exert its effects through other non-classical pathways 32. BPA has also been shown to interact with estrogen-responsive genes that are implicated in neurodegenerative disease pathways ${ }^{29}$.

Cadmium (Cd): $\mathrm{Cd}$ is a naturally occurring metal in the earth's crust and a natural part of water in the ocean ${ }^{33}$. People are exposed through eating food, cigarette smoke, drinking water, and air ${ }^{33}$. Cd is introduced into the food chain through soil and food contact surfaces, and through foods such as green vegetables, grains, legumes, and meats ${ }^{33}$. Occupation exposures are highest in occupations involving the manufacture of $\mathrm{Cd}$ - 
containing products ${ }^{33}$. Inhalation and oral routes of exposure are predominant, followed by dermal exposure ${ }^{33}$. Current evidence supports the toxicity of $\mathrm{Cd}$ and its effects on the developing organism, reproductive toxicity, hepatic effects, hematological effects, and immunological effects ${ }^{33}$. Cd has also been demonstrated to have estrogenic properties ${ }^{34}$ and has been shown to interact with estrogen-responsive genes implicated in various neurodegenerative disorders ${ }^{29}$.

Arsenic (As): As is an element in the environment, found in the earth's crust, and is considered a metalloid ${ }^{35}$. Populations are usually exposed through the air, drinking water, and food, with food being the main source of As in a population, with some areas having naturally high levels of As ${ }^{35}$. Occupational exposure occurs through individuals working in metal working, wood treatment, and those in working in the production and application of pesticides ${ }^{35}$. As is also used in the animal and poultry feed as an antimicrobial compound ${ }^{35}$. The main routes of exposure are inhalation and oral, with dermal exposure being considered a minor route ${ }^{35}$. As has been associated with various health conditions and affects every organ system ${ }^{35}$. Genetic polymorphisms are suspected of causing some individuals to be more sensitive to $\mathrm{As}^{35}$. As has also been demonstrated to have estrogenic properties ${ }^{34}$ and has been shown to interact with estrogen-responsive genes implicated in various neurodegenerative disorders ${ }^{29}$. Manganese (Mn): $\mathrm{Mn}$ is a metal that is an essential nutrient required as a cofactor for various enzymatic processes and is found as a naturally occurring element in grains and fruit ${ }^{36} . \mathrm{Mn}$ is used in industrial processes and products and exposure can occur through inhalation, oral, dermal, and occupational routes ${ }^{36}$. Mn has the potential to accumulate in organisms at the bottom tier of the food chain and has been linked to various health issues 
that include neurological dysfunction ${ }^{36}$. Mn has also been demonstrated to have estrogenic properties ${ }^{34}$ and has been shown to interact with estrogen-responsive genes implicated in various neurodegenerative disorders ${ }^{29}$.

Oral Contraceptives (OC): Oral contraceptives containing specifically containing synthetic estrogens such as ethinyl estradiol are considered EEDCs due to its ability to mimic estrogen and influence the reproductive cycle. $\mathrm{OC}$ use has been shown to reduce the amount of available endogenous estrogen in the body ${ }^{37}$. OCs has varying effects brain structure, function, and cognition ${ }^{38}$. Most recent studies have shown varying effects; however, the studies do not differentiate between the various types of contraceptives, making it difficult to find out if the contraceptives used contained ethinyl estradiol and inconsistencies are found in the reporting of the type of OC used ${ }^{39}$. Hormonal Replacement Therapy (HRT): Hormone replacement therapy, or HRT, has been shown to be associated with the start of neurodegenerative disease, although this has been related to a time dependent response, which depends on when the HRT was initiated. Observation studies and analyses with women indicated the use of HRT's containing estrogen to be associated with a reduced risk of Alzheimer's disease. Studies suggest that the timing of HRT use during parts of the menopausal stage may dictate whether beneficial adverse effects are observed ${ }^{40,41}$.

\section{National Health and Nutrition Examination Survey (NHANES): NHANES is a} continuous cross-sectional data collection carried out by the Center for Disease Control and Prevention (CDC) utilizing a complex multi-stage sampling design that creates a survey representative of the non-institutionalized population of the United States ${ }^{42,43}$. The survey has been conducted since 1999 and consists of an at-home questionnaires 
followed by a standardized physical examination and specimen collection conducted in mobile examination centers (MEC) ${ }^{42,43}$. Eligibility is determined using preset selection probabilities for the desired demographic subdomains ${ }^{43}$. A household screener is performed before to determine if any household members are eligible for the interview and examination ${ }^{43}$. The interview collects demographic, health, nutrition, and household information, while the physical examination includes physical measurements, dental examination, and the collection of blood and urine specimens for laboratory testing ${ }^{43}$. Prior to any to interviews and examinations, informed consent was obtained and all procedures were approved by the CDC Institutional Review Board ${ }^{44}$.

The goal of this research was to find out if exposure to EEDCs (phthalates, BPA, $\mathrm{Cd}, \mathrm{As}$, and Mn) are associated with adverse brain health. Using the CDC's NHANES 2011 to 2014 datasets, statistical analyses were performed on surrogates of brain health indicators, urinary EEDC levels, and associated variables.

\section{REFERENCES}

1. Bergman A, Heindel JJ, Jobling S, Kidd KA, Zoeller TR. State of the science of endocrine disrupting chemicals. World Heal Organ United Nations Environ Program. 2012:1-38. http://apps.who.int/iris/handle/10665/78102.

2. Diamanti-Kandarakis E, Bourguignon J-P, Giudice LC, et al. Endocrine-disrupting chemicals: an Endocrine Society scientific statement. Endocr Rev. 2009;30(4):293342. doi:10.1210/er.2009-0002.

3. Chen A, Dietrich KN, Huo X, Ho SM. Developmental neurotoxicants in e-waste: An emerging health concern. Environ Health Perspect. 2011. doi:10.1289/ehp.1002452. 
4. Kiyama R, Wada-Kiyama Y. Estrogenic endocrine disruptors: Molecular mechanisms of action. Environ Int. 2015. doi:10.1016/j.envint.2015.05.012.

5. Przedborski S, Vila M, Jackson-Lewis V. Neurodegeneration: What is it and where are we? J Clin Invest. 2003. doi:10.1172/JCI200317522.

6. Gillies GE, McArthur S. Estrogen Actions in the Brain and the Basis for Differential Action in Men and Women: A Case for Sex-Specific Medicines. Pharmacol Rev. 2010;62(2):155-198. doi:10.1124/pr.109.002071.

7. Chakrabarti M, Haque A, Banik NL, Nagarkatti P, Nagarkatti M, Ray SK. Estrogen receptor agonists for attenuation of neuroinflammation and neurodegeneration. Brain Res Bull. 2014;109:22-31. doi:10.1016/j.brainresbull.2014.09.004.

8. Hristian C, Ruber JG, Alter W, et al. Production and actions of estrogens. $N$ Engl J Med. 2002;346(5). doi:10.1016/S0960-0760(01)00184-4.

9. Green PS, Simpkins JW. Neuroprotective effects of estrogens: Potential mechanisms of action. Int J Dev Neurosci. 2000;18(4-5):347-358. doi:10.1016/S0736-5748(00)00017-4.

10. Wise PM, Dubal DB, Wilson ME, Rau SW, Liu Y. Estrogens: trophic and protective factors in the adult brain. Front Neuroendocrinol. 2001;22(1):33-66. doi:10.1006/frne.2000.0207.

11. Pozzi S, Benedusi V, Maggi A, Vegeto E. Estrogen action in neuroprotection and brain inflammation. Ann N Y Acad Sci. 2006;1089(2006):302-323. doi:10.1196/annals.1386.035.

12. Arevalo M-A, Azcoitia I, Garcia-Segura LM. The neuroprotective actions of oestradiol and oestrogen receptors. Neuroscience. 2015;16(1):17-29. doi:10.1038/nrn3856.

13. Leranth C, Petnehazy O, MacLusky NJ. Gonadal hormones affect spine synaptic density in the CA1 hippocampal subfield of male rats. $J$ Neurosci. 2003;23(5):1588-1592. 
14. MacLusky NJ, Hajszan T, Prange-Kiel J, Leranth C. Androgen modulation of hippocampal synaptic plasticity. Neuroscience. 2006;138(3):957-965. doi:10.1016/j.neuroscience.2005.12.054.

15. Parducz a., Hajszan T, MacLusky NJ, et al. Synaptic remodeling induced by gonadal hormones: Neuronal plasticity as a mediator of neuroendocrine and behavioral responses to steroids. Neuroscience. 2006;138(3):977-985. doi:10.1016/j.neuroscience.2005.07.008.

16. Ogiue-Ikeda M, Tanabe N, Mukai H, et al. Rapid modulation of synaptic plasticity by estrogens as well as endocrine disrupters in hippocampal neurons. Brain Res Rev. 2008;57(2):363-375. doi:10.1016/j.brainresrev.2007.06.010.

17. Hojo Y, Murakami G, Mukai H, et al. Estrogen synthesis in the brain-Role in synaptic plasticity and memory. Mol Cell Endocrinol. 2008;290(1-2):31-43. doi:10.1016/j.mce.2008.04.017.

18. Simpkins JW, Yi KD, Yang S, Dykens J a. Mitochondrial mechanisms of estrogen neuroprotection. Biochim Biophys Acta. 2011;1800(10):1113-1120. doi:10.1016/j.bbagen.2009.11.013.Mitochondrial.

19. Arnold S, Beyer C. Neuroprotection by estrogen in the brain: The mitochondrial compartment as presumed therapeutic target. J Neurochem. 2009;110(1):1-11. doi:10.1111/j.1471-4159.2009.06133.x.

20. Grimm A, Schmitt K, Lang UE, Mensah-Nyagan AG, Eckert A. Improvement of neuronal bioenergetics by neurosteroids: Implications for age-related neurodegenerative disorders. Biochim Biophys Acta - Mol Basis Dis. 2014;1842(12):2427-2438. doi:10.1016/j.bbadis.2014.09.013.

21. Agency for Toxic Substances and Disease Registry (ATSDR). Toxicological Profile for Diethyl Phthalate. Agency Toxic Subst Dis Regist. 1995;(June):1-158.

22. Agency for Toxic Substances and Disease Registry (ATSDR). TOXICOLOGICAL PROFILE FOR DI-n-BUTYL PHTHALATE. Agency Toxic Subst Dis Regist. 2001;(September):225. 
23. Agency for Toxic Substances and Disease Registry (ATSDR). Toxicological profile: di(2-ethylhexyl)phthalate (DEHP). Agency Toxic Subst Dis Regist. 2002;(September). http://www.atsdr.cdc.gov/toxprofiles/tp.asp?id=684\&tid=65.

24. Heudorf U, Mersch-Sundermann V, Angerer J. Phthalates: Toxicology and exposure. Int J Hyg Environ Health. 2007;210(5):623-634. doi:10.1016/j.ijheh.2007.07.011.

25. Miodovnik A, Edwards A, Bellinger DC, Hauser R. Developmental neurotoxicity of ortho-phthalate diesters: Review of human and experimental evidence. Neurotoxicology. 2014;41:112-122. doi:10.1016/j.neuro.2014.01.007.

26. Silva MJ, Barr DB, Reidy JA, et al. Urinary levels of seven phthalate metabolites in the U.S. population from the National Health and Nutrition Examination Survey (NHANES) 1999-2000. Environ Health Perspect. 2004;112(3):331-338. doi:10.1289/ehp.6723.

27. Blair RM, Fang H, Branham WS, et al. The estrogen receptor relative binding affinities of 188 natural and xenochemicals: structural diversity of ligands. Toxicol Sci. 2000;54(1):138-153. doi:10.1093/toxsci/54.1.138.

28. Jobling S, Reynolds T, White R, Parker MG, Sumpter JP. A variety of environmentally persistent chemicals, including some phthalate plasticizers, are weakly estrogenic. Environ Health Perspect. 1995;103(6):582-587. doi:10.1289/ehp.95103582.

29. Preciados M, Yoo C, Roy D. Estrogenic Endocrine Disrupting Chemicals Influencing NRF1 Regulated Gene Networks in the Development of Complex Human Brain Diseases. Int J Mol Sci. 2016;17(12):2086. doi:10.3390/ijms17122086.

30. ATSDR. Toxicological Profile for Phenol. Agency Toxic Subst Dis Regist. 2008:1269. doi:10.3109/15569529909037564.

31. Calafat AM, Ye X, Wong LY, Reidy JA, Needham LL. Exposure of the U.S. population to Bisphenol A and 4-tertiary-octylphenol: 2003-2004. Environ Health Perspect. 2008;116(1):39-44. doi:10.1289/ehp.10753. 
32. Alonso-Magdalena P, Ropero AB, Soriano S, et al. Bisphenol-A acts as a potent estrogen via non-classical estrogen triggered pathways. Mol Cell Endocrinol. 2012;355(2):201-207. doi:10.1016/j.mce.2011.12.012.

33. Agency for Toxic Substances and Disease Registry (ATSDR). Toxicological Profile for Cadmium. Agency Toxic Subst Dis Regist. 2012;(September). http://www.atsdr.cdc.gov/toxprofiles/tp5.pdf\%5Cnhttp://www.ncbi.nlm.nih.gov/bo oks/NBK158845/.

34. Choe SY, Kim SJ, Kim HG, et al. Evaluation of estrogenicity of major heavy metals. Sci Total Environ. 2003;312(1-3):15-21. doi:10.1016/S00489697(03)00190-6.

35. Agency for Toxic Substances and Disease Registry (ATSDR). TOXICOLOGICAL PROFILE FOR ARSENIC. Health Effects. Agency Toxic Subst Dis Regist. 2007;(August).

36. Agency for Toxic Substances and Disease Registry (ATSDR). Toxicological Profile for Manganese. ATSDR - Toxicol Profiles. 2000;(September):504.

37. Griksiene R, Ruksenas O. Effects of hormonal contraceptives on mental rotation and verbal fluency. Psychoneuroendocrinology. 2011;36(8):1239-1248. doi:10.1016/j.psyneuen.2011.03.001.

38. Pletzer BA, Kerschbaum HH. 50 years of hormonal contraception - time to find out, what it does to our brain. Front Neurosci. 2014. doi:10.3389/fnins.2014.00256.

39. Beltz AM, Hampson E, Berenbaum SA. Oral Contraceptives and Cognition: A Role for Ethinyl Estradiol. Horm Behav. 2015. doi:10.1016/j.yhbeh.2015.06.012.

40. Maki PM, Henderson VW. Hormone therapy, dementia, and cognition: the Women's Health Initiative ten years on. Climacteric. 2012;15(3):256-262. doi:10.1016/j.biotechadv.2011.08.021.Secreted.

41. Xiao M, Cao GL, Marshall C, Hu G. Hypothesis: Multiple factors are associated with the lack of any beneficial effects of oestrogen-replacement therapy in the late postmenopausal stage. Clin Exp Pharmacol Physiol. 2010;37(8):873-876. doi:10.1111/j.1440-1681.2010.05400.x. 
42. Johnson CL, Paulose-Ram R, Ogden CL, et al. National health and nutrition examination survey: analytic guidelines, 1999-2010. Vital Health Stat 2. 2013;(161):1-24. http://www.ncbi.nlm.nih.gov/pubmed/25090154.

43. Johnson CL, Dohrmann SM, Burt VL, Mohadjer LK. National Health and Nutrition Examination Survey: Sample Design , 2011 - 2014. Vital Heal Stat. 2014;2(162):1-33. http://www.cdc.gov/nchs/data/series/sr_02/sr02_162.pdf.

44. Centers for Disease Control and Prevention (CDC). National Center for Health Statistics (NCHS). National Health and Nutrition Examination Survey NCHS Research Ethics Review Board (ERB) Approval. Hyattsville, MD: U.S. Department of Health and Human Services, Centers for Disease Control and Prevention. https://www.cdc.gov/nchs/nhanes/irba98.htm. Published 2016. 


\section{CHAPTER II}

MANUSCRIPT I

\section{EXPOSURE TO ESTROGENIC ENDOCRINE DISRUPTING CHEMICALS AND THEIR ROLE IN BRAIN HEALTH}

\section{LITERATURE REVIW}

\section{INTRODUCTION}

Estrogenic endocrine disrupting chemicals (EEDCs) are endocrine disruptors that mimic and affect the function of estrogen ${ }^{1}$. They can affect the human body through every route of exposure and are found in almost all manufactured products ${ }^{2}$. The global increase in manufacturing and e-waste production have increased exposures ${ }^{3}$. The effects of these chemicals are far reaching since they affect every part of the human timeline, from birth to old age, and have even been demonstrated to produce adverse health effects in later generations ${ }^{4}$.

These chemicals are of great concern to public health since they are linked to almost every disease of every human system including neurodegenerative diseases ${ }^{4,5}$. EEDCs have also been associated with other neurological disorders such as attention deficit and hyperactive disorder (ADHD) as well as learning disabilities and aggressiveness ${ }^{6}$. EEDCs have also been demonstrated to affect the young through maternal exposure, producing neurological issues such as abnormal rearing behavior, locomotion issues, anxiety disorder, learning difficulties, memory issues, and abnormal neuronal development ${ }^{7,8}$. 


\section{Estrogens and Brain Health}

Estrogens have many roles in the human brain. Estrogen plays a role in sexual differentiation in early brain development ${ }^{9}$. Estrogen has been found to potent neuroprotectant and decreases inflammation in the brain ${ }^{10-15}$. Estrogen has been demonstrated to stimulate the grown of neurons and synapses ${ }^{16-20}$, and protect the function of mitochondria in the brain ${ }^{21-23}$.

Epidemiological studies have shown the effects of estrogen on brain health. In the review of epidemiological studies pertaining to estrogen and brain health, exposure was assessed through serum or blood measures, or through a surrogate of exposure. The surrogate of exposure to estrogen was quantified as the start of menarche to the end of menopause. Studies were also only used if exogenous estrogen exposure was either controlled for, or excluded, as exogenous estrogen can affect the natural levels of estrogen in the body.

Cross-sectional studies have differing results. In a study of 393 postmenopausal geriatric-age women, no significant association was found between sex hormone levels and neuropsychological test performance ${ }^{24}$. Another study consisting of 760 post-menopausal women looking at life-long endogenous estrogen exposure and using a surrogate of estrogen exposure did not find any associations with cognitive tests ${ }^{25}$. A study of 11094 postmenopausal women found significant associations between surrogates of estrogen exposure and delayed recall scores ( $\mathrm{p}$-value $=0.001,95 \% \mathrm{CI}, 0.008-0.02)$ and mini-mental state exam scores ${ }^{26}$. In a study of 181 geriatric-aged men, higher estrogen serum levels were associated with better verbal memory assessment scores (Beta $=0.17$, $p$-value $<0.02)$ 
${ }^{27}$. In a cross-sectional study of 579 females, the age of Parkinson's disease (PD) onset was positively associated with surrogates of estrogen exposure $\left(7.10,[3.31], \mathrm{P}\right.$-value=0.032) ${ }^{28}$.

Several case-control studies were identified that assessed the effects of endogenous estrogen exposure on brain health. In a case-control study of 50 Alzheimer's disease (AD) cases and 93 controls, women with the lowest endogenous estradiol levels were 4 times as likely to have $\mathrm{AD}$ compared to women with the highest estradiol levels $(\mathrm{OR}=4.2,95 \% \mathrm{CI}$ $1.1-15.6)^{29}$. In a case-control study of 131 amyotrophic lateral sclerosis (ALS) cases and 430 age-matched controls a longer reproductive time-span decrease ALS risk $(\mathrm{OR}=0.95$, 95\% CI 0.91-0.98) and longer endogenous estrogen exposure decreases ALS risk $(\mathrm{OR}=0.95,95 \% \text { CI } 0.89-1.01)^{30}$.

Several cohort studies were identified that assessed the effects of endogenous estrogen exposure on brain health. In a cohort of 3601 postmenopausal women, it was found after adjusting for covariates, women with longer reproductive spans had an increased risk of dementia compared to women with the shortest reproductive spans $(\mathrm{RR}=1.78,95 \% \mathrm{CI} 1.12-2.84)$ and an increased risk of $\mathrm{AD}(\mathrm{RR}=1.51,95 \% \mathrm{CI} 0.91-2.50)$ with the risk was more pronounced in carriers of the APOE allele ${ }^{31}$. In a cohort study of 119 women with Down's syndrome, women with low bioavailable estrogen were more likely to develop $\mathrm{AD}\left(\mathrm{HR}=4.1,95 \%\right.$ CI 1.2-13.9) ${ }^{32}$. A study of 133 postmenopausal females found longer duration of months with endogenous estrogen exposure had a protective effect against $\mathrm{AD}$ risk (p-value $=0.0235,96 \% \mathrm{CI} 0.9907-0.9993$ ) with the effect becoming stronger when subjects were over the total median number of months (pvalue $=0.00754,96 \%$ CI $0.004118-0.005289)^{33}$. 


\section{Oral Contraceptives (OC) and Brain Health}

OCs containing synthetic estrogens, such as ethinyl estradiol (EE), are considered EEDCs since they imitate estrogen and cause changes to the human reproductive cycle. OC use has been demonstrated to reduce endogenous estrogen in the body ${ }^{34}$. Most studies do not differentiate if contraceptives do, or do not contain ethinyl estradiol and inconsistencies are found in the reporting of the type of OC used ${ }^{35}$

A cross-sectional study examined OC effects on spatial and verbal abilities found OC users to perform better on spatial ability and verbal tests ${ }^{35}$. Another cross-sectional study found OC users to have better cognitive scores compared to non-users ${ }^{36}$. Another

study found OC use to negatively affect cognition and verbal fluency ${ }^{34} \mathrm{~A}$ review by Warren et al. suggest an overall positive effect with OC use and verbal memory ${ }^{37}$.

\section{Hormonal Replacement Therapy (HRT) and Brain Health}

Initial observation studies indicate the use of hormone replacement therapy (HRT) containing synthetic estrogen to be associated with a reduced risk of $\mathrm{AD}^{38}$. Studies suggest that the timing of HRT use during specific times during menopause may dictate whether beneficial effects are observed ${ }^{38,39}$. In the Women's Health Initiative Memory Study (WHIMS), a randomized, double-blind, placebo-controlled clinical trial, HRT treatment of estrogen with progestin increased the risk for dementia in postmenopausal women and did not prevent cognitive impairment ${ }^{40}$. In the same trial, it was found that estrogen-only HRT increased the risk for both dementia and cognitive impairment ${ }^{41}$. The Women's Health Initiative Study of Cognitive Aging (WHISCA) further supports HRT use and its' association with neurodegenerative disease, with mixed findings. One finding from the WHISCA study found conjugated equine estrogen with 
medroxyprogesterone acetate (estrogen + progestin) appeared to negatively impact verbal memory, but positively affect figural memory among postmenopausal women, free of probable dementia compared to controls ${ }^{42}$ Another study found estrogen alone, as conjugated equine estrogen, did not improve cognitive functioning and lowered certain cognitive functions in women with prior hysterectomy ${ }^{43}$. Other studies have indicated that no improvement in using estrogen only treatments for cognitive function ${ }^{44-47}$.

Newer studies give mixed findings. One cohort study showed increased AD risk amongst women who used HRT more than five years after menopause but observed a decreased risk of $\mathrm{AD}$ if used within five years of menopause ${ }^{48}$. Another recent metaanalysis showed no association between postmenopausal HRT use and AD and dementia 49. While another study found an increase in Parkinson's disease (PD) risk dependent on the type of HRT treatment ${ }^{50}$.

\section{Bisphenol-A (BPA) and Brain Health}

BPA is a synthetically created chemical that is used to create polycarbonate plastics and resins ${ }^{51}$. BPA is found in many consumer products and plastics. These products include the following: baby bottles, compact discs, impact-resistant safety equipment, medical devices, food cans and tops, water supply pipes, ATM receipts and dental sealants and composites ${ }^{51}$. Food storage containers that have BPA can cause BPA to leech into foods by the use of high heat ${ }^{51}$. It is of note that during the 2003-2004 NHANES data cycle, BPA was detected in $93 \%$ of urine samples collected from subjects 6 years of age and older ${ }^{52}$.

BPA has been shown to be weakly estrogenic and have a low affinity for binding to ER receptors, but is speculated to exert its effects through other non-classical pathways 
53. BPA has also been shown to interact with estrogen-responsive genes that are implicated in neurodegenerative disease pathways ${ }^{54}$. BPA has been demonstrated to be able to cross the blood brain barrier ${ }^{55,56}$.

Animal studies have demonstrated BPA to have negative effects on memory ${ }^{57-72}$, negatively affect neurogenesis ${ }^{62,72}$, negatively affect structure of dendritic spines and synaptogenesis $57,61,66,69,71,73$, and negatively affect cellular processes, protein expression, and gene expression ${ }^{60,64,66-68,70}$. Other studies show BPA produces no negative effects on spatial and working memory ${ }^{74-76}$.

BPA was found to cause hyperactivity in rats ${ }^{77}$. A single dose of BPA administered to neonatal mice was observed to affect cognitive function and alter adult spontaneous behavior, mid and high dosed mice ${ }^{78}$. In utero exposure to BPA in mice was shown to reduce dendritic spine densities in the hippocampal CA1 region and was not dose dependent ${ }^{79}$. BPA was shown to effect neural ectoderm specification and neural progenitor cells in mouse embryonic stem cells ${ }^{80}$. In an animal model using C.elegans, early embryogenesis exposure to BPA and BPS was shown to cause changes in behavior and learning into adulthood ${ }^{81}$. BPA was shown to decrease the proliferation of multipotent neural progenitor cells and produce cytotoxicity in F1 mice, and in low-doses stimulated neuronal differentiation which might disrupt brain development ${ }^{82}$.

Animal studies have indicated bisphenol-A to affect various aspects of memory at lower than the US Environmental Protection Agency's (EPA) reference safe daily limit of $50 \mathrm{ug} / \mathrm{kg} /$ day ${ }^{83}$. The types of memory affected include spatial memory, visual memory, object recognition, working memory, reference memory and navigational memory 57,58,62,63,66,69,71-73,84. Animal studies have also indicate affects to locomotor function 58,66 . 
In humans, prenatal BPA exposure has been demonstrated to cause aggressive and hyperactive behavior in offspring from mothers with higher BPA levels when compared to offspring with mothers that have lower BPA levels ${ }^{85}$. This human epidemiological study is consistent with animal studies that also show the same effect ${ }^{86-93}$.

Prenatal urinary BPA concentrations in the human mother and child were associated with anxiety, depression, and hyperactivity ${ }^{94}$. In autistic children, plasma levels of BPA and phthalates were significantly higher compared to controls ${ }^{95}$. One study using cross-sectional data from the Canadian Health Measures Survey found children taking psychotropic medications was associated with urinary BPA (OR 1.59; 95\% CI 1.05-2.40) ${ }^{96}$. Another study assessing prenatal exposure to bisphenol A and phthalates and infant neurobehavior at five weeks found no associations with bisphenol A and some associations with phthalate exposure and improved neurobehavior ${ }^{97}$. In a prospective cohort study following African-American and Dominican-American women from pregnancy to children's age of 7-9, it was found that among boys, high prenatal BPA concentrations was associated with increased internalizing and externalizing behaviors in boys, with a decrease in internalizing behavior in girls, and high postnatal BPA concentrations was associated with increased internalizing and externalizing behaviors in girls more than in boys ${ }^{98}$. Other studies have found were related to a decrease in hyperactivity symptoms in boys and an increase in anxiety, depression, and externalizing behavior in young girls ${ }^{85,99}$. Other studies have found no associations between maternal BPA levels and autism ${ }^{100}$. 


\section{Phthalates and Brain Health}

Phthalates are class of estrogenic endocrine disrupting chemicals that have a role in the development of neurodegenerative disease. Phthalates, also known as plasticizers, are chemicals used in plastics to make them flexible and strong ${ }^{101-103}$. Exposure to phthalates comes through ingestion, inhalation, and to a lesser extent, dermal contact with phthalate-containing products ${ }^{104}$. Phthalates are found in most consumer products. These include the following: wall coverings, tablecloths, floor tiles, furniture, upholstery, shower curtains, garden hoses, baby products, toys, shoes, packing materials, medical devices, paints, glue, nail polish, hair spray, insect repellents, food packaging materials, cosmetics, insecticides, and drug products ${ }^{101-103}$. Phthalates have short biological halflives and do not accumulate, with urine as the primary route of excretion ${ }^{105}$. Specific phthalates are also found in high levels among the US population. One study, found the body burden of the phthalates mono-ethyl phthalate (MEP), mono-n-butyl phthalate (MBP) and mono-benzyl phthalate (MBzp) in 97\% of samples tested from the CDC NHANES 1999-2000 datasets ${ }^{106}$. As such, most of the US population has some measurable levels of phthalates in their bodies ${ }^{105}$.

Phthalates have also been demonstrated to have estrogenic activity and affinity for estrogen receptors ${ }^{107,108}$ and have been shown to interact with estrogen-responsive genes implicated in various neurodegenerative disorders ${ }^{54}$. Phthalates have also been demonstrated to cross the blood brain barrier ${ }^{109,110}$

Animal studies have shown phthalates to have adverse effects on brain health. Negative effects include adversely affecting learning ${ }^{111,112}$, adversely affecting memory ${ }^{111,113}$, interfering with locomotion ${ }^{113}$; negatively affecting social behavior ${ }^{112}$, and 
producing cellular effects such as cell death, synaptic loss, and synaptic dysfunction ${ }^{114}$. Some animal studies indicate improvement in memory ${ }^{115}$ and possible dose-dependent effects, where memory improves with one dose, but degrades with another ${ }^{116}$. A recent review of phthalates and neuroplasticity suggests phthalates negatively affect neurogenesis and plasticity in animal models ${ }^{114,117}$.

There are a group of studies that have found associations between phthalates and brain behavioral disorders such as autism spectrum disorder (ASD) and attention deficit hyperactive disorder (ADHD). One study examining exposure to EEDCs and autism and ADHD found intracranial exposure to several classes of EEDCs, which include phthalates, caused significant hyperactivity in neonatal rats with observable gene alteration ${ }^{118}$. A retrospective study followed children 1-6 years of age with a follow-up at 6-8 years of age and found the children with exposure to phthalates from PVC pipe dust were more likely to develop ASD ${ }^{119}$. Phthalates are suspected in the development of neurodegenerative diseases ${ }^{110}$. In rat model study, phthalate exposure was shown to impair cognition and increase the levels of phospho-Tau, a precursor of AD development, in exposed rat offspring ${ }^{120}$. Currently no definitive epidemiological studies have been conducted with phthalates and neurodegenerative diseases.

\section{Polychlorinated Biphenyls (PCBs) and Brain Health}

PCBs are synthetic organic chemicals composed in different combinations of 209 different chlorinated compounds, or congeners ${ }^{121}$. PCBs have been used as coolant and lubricants in transformers, and its manufacture was banned in 1977 because of harmful health effects, however, old products may still contain them ${ }^{121}$. PCBs have been found to cause cancer in animals ${ }^{121}$. Routes of exposure included occupational exposure, 
breathing air near contaminated areas, using older products manufactured before and around 1977, and eating contaminated food ${ }^{121}$. PCBs bio accumulate in the food chain and remain active in the environment for extended periods of time ${ }^{121}$.

Recent animal and cell studies have shown PCBs to adversely affect brain health. Animal studies have shown exposure to PCBs affect brain health by affecting social behavior $^{122}$, increased hyperactivity ${ }^{123}$, reduction in learning ability ${ }^{124}$, and physiological effects on the brain such as neuronal degradation ${ }^{123,125}$, neuronal loss and damage $^{126}$, and susceptibility to amyloid stress and reduced expression of synaptic proteins ${ }^{125}$

Recent cell studies have shown PCBs to cause cellular death ${ }^{127}$ and interfere with estrogen's neuroprotective effects on neurons and brain cells ${ }^{128}$.

Cross-sectional studies have shown the effects of PCBs on brain health. In children, PCB exposure is associated with lower IQ ${ }^{129}$ and lower visual memory function ${ }^{130}$. In older populations, PCB exposure is associated with lower verbal learning and memory ${ }^{131}$, lower verbal memory and depressive symptoms ${ }^{132}$, lower score on memory and learning measures ${ }^{133}$, and attenuation of emotional wellbeing and attentional functioning ${ }^{134}$.

In a nested case-control study in Finland, serum PCBs were not associated with PD development ${ }^{135}$. In a retrospective mortality study examining PCB exposure amongst workers in capacitor plants, sex-specific analyses found higher deaths from PD amongst exposed women ${ }^{136}$. In a case-control study examining post-mortem brain tissue from PD, $\mathrm{AD}$, and control patients, PCB levels were higher in PD groups, and when stratified by age, were higher amongst women ${ }^{137}$. 


\section{Cadmium (Cd) and Brain Health}

$\mathrm{Cd}$ is a naturally occurring metal in the earth's crust and a natural part of water in the ocean ${ }^{138}$. People are exposed through eating food, cigarette smoke, drinking water, and air ${ }^{138}$. Cd is introduced into the food chain through soil and food contact surfaces, and through foods such as green vegetables, grains, legumes, and meats ${ }^{138}$. Occupation exposures are highest in occupations involving the manufacture of Cd-containing products ${ }^{138}$. Inhalation and oral routes of exposure are predominant, followed by dermal exposure ${ }^{138}$. Current evidence supports the toxicity of $\mathrm{Cd}$ and its effects on the developing organism, reproductive toxicity, hepatic effects, hematological effects, and immunological effects ${ }^{138}$. Cd has also been demonstrated to have estrogenic properties 139 and has been shown to interact with estrogen-responsive genes implicated in various neurodegenerative disorders 54 .

In a study using hippocampal CA1 neurons, cadmium was shown to negatively affect synaptic transmission and neural plasticity ${ }^{140}$. In a study using neural PC12 and SH-SY5Y cells, cadmium induced apoptosis in the neural cells ${ }^{141,142}$. In a zebrafish animal model, cadmium has been shown to inhibit neurogenesis in embryonic development ${ }^{143}$. Cadmium has been shown to cause cell death in rat cerebellum cortical neurons by affecting calcium homeostasis ${ }^{144}$ and also cause cell death by damaging mitochondria in rat oligodendrocytes ${ }^{145}$. Cadmium has been shown to interact with beta amyloid peptides which is involved in the development of $\mathrm{AD}^{146}$.

A study that examined the cerebrospinal fluid of ALS patients observed higher levels of various metals, including cadmium ${ }^{147}$. A case-control study examining heavy metal levels in hair samples from a group of Mongolian people found elevated cadmium, 
as well as other heavy metals in those with Parkinson-like symptoms ${ }^{148}$. In a study of boiler workers and occupational exposure, exposure to various heavy metals, including cadmium was associated with conditions similar to PD and $\mathrm{AD}^{149}$.

\section{Arsenic (As) and Brain Health}

As is an element in the environment, found in the earth's crust, and is considered a metalloid ${ }^{150}$. Populations are usually exposed through the air, drinking water, and food, with food being the main source of As in a population, with some areas having naturally high levels of As ${ }^{150}$. Occupational exposure occurs through individuals working in metal working, wood treatment, and those in working in the production and application of pesticides ${ }^{150}$. As is also used in the animal and poultry feed as an antimicrobial compound ${ }^{150}$. The main routes of exposure are inhalation and oral, with dermal exposure being considered a minor route ${ }^{150}$. As has been associated with various health conditions and affects every organ system ${ }^{150}$. Genetic polymorphisms are suspected of causing some individuals to be more sensitive to $\mathrm{As}^{150}$. As has also been demonstrated to have estrogenic properties ${ }^{139}$ and has been shown to interact with estrogen-responsive genes implicated in various neurodegenerative disorders ${ }^{54}$.

In animal studies using rats, arsenic exposure through ingest water has been shown to impair neurogenesis, worsened spatial memory, and promote abnormal neural synapses growth. ${ }^{151}$. Animal studies in rats have demonstrated arsenic exposure to affect synaptic plasticity, by affecting the expression of NDMA receptors ${ }^{152,153}$ and downregulating the PTEN-Akt-Creb signaling pathway and damaging cerebral neurons 154. Arsenic was demonstrated to cause oxidative stress and cell death in cultured neuronal cells, when administered with dopamine ${ }^{155}$. In a study using a cholinergic 
neuronal cell line overexpressing amyloid precursor protein (APP) and exposing it to sodium arsenite and its metabolite, dimethyl arsenic acid, increased APP production was observed ${ }^{156}$.

In a cohort study consisting of 133 men and 201 women, long-term low level exposure to arsenic from groundwater was found to be associated with poorer scores in language, visuospatial skills, and executive functioning, global cognition, processing speed, and immediate memory ${ }^{157}$. In another study, consisting of 526 subjects genotyped according to the AS3MT gene, exposure to higher low level arsenic in groundwater reduced cognitive functioning, but the results differed with amongst the different SNPs 158. In a case-control study measuring heavy metal serum levels in 89 Ad patients and 188 cognitively normal controls, there was no difference in serum arsenic levels between the AD group and controls ${ }^{159}$. Another study with a cohort consisting of $733 \mathrm{AD}$ patients, 127 individuals with mild cognitive impairment (CI), and 530 individuals of normal cognition, found that exposure to low level arsenic exposure from groundwater was found to be associated with poorer neuropsychological performance ${ }^{160}$.

\section{Manganese (Mn) and Brain Health}

$\mathrm{Mn}$ is a metal that is an essential nutrient required as a cofactor for various enzymatic processes and is found as a naturally occurring element in grains and fruit ${ }^{161}$. $\mathrm{Mn}$ is used in industrial processes and products and exposure can occur through inhalation, oral, dermal, and occupational routes ${ }^{161}$. Mn has the potential to accumulate in organisms at the bottom tier of the food chain and has been linked to various health issues that include neurological dysfunction ${ }^{161}$. Mn has also been demonstrated to have 
estrogenic properties ${ }^{139}$ and has been shown to interact with estrogen-responsive genes implicated in various neurodegenerative disorders ${ }^{54}$.

Recent epidemiological studies have assessed the effects of manganese on brain health. A cross-sectional study by Hozumi et al. ${ }^{162}$, analyzed the cerebrospinal fluid of various neurodegenerative disease patients and found a higher level of manganese among PD patients ( $\mathrm{p}$-value $<0.05$ ). In another cross-sectional study assessing children's intellectual functioning and arsenic and manganese exposure, blood manganese levels were negatively associated with full scale IQ test scores (p-value $<0.05$ ), working memory (p-value $<0.05$ ), and perceptual memory (p-value $<0.05){ }^{163}$. A cross-sectional study by Kim et al. ${ }^{164}$ examining low-level manganese exposure in adults of a Ohio community found subtle subclinical effects in Unified Parkinson's Disease Rating Scale (UPDRS) and postural sway test for PD. A cross-sectional study of school-aged children in Brazil found inverse scores on executive function and attention tests with manganese levels ${ }^{165}$. A study amongst school-children in Canada found low-level manganese exposure in drinking water was associated with poorer neurobehavioral functions ${ }^{166}$. Koc et al. ${ }^{167}$ found higher levels of metal, including manganese, in hair samples of AD patients compared to controls.

A case-control study Miyake et al. ${ }^{168}$, assessing dietary intake of heavy metals amongst PD patients found no association with manganese intake. A case-control study by Roos et al. ${ }^{147}$ found elevated manganese levels in cerebrospinal fluid of ALS patients. A study by Kumudini et al. ${ }^{169}$ found not correlation between manganese blood levels in PD patients compared to controls. A case control study by Garzillo et al. ${ }^{170}$ found no association between manganese levels in ALS patients vs controls. A study by Kihira et 
al. ${ }^{171}$ found elevated manganese levels in ALS patients vs controls from hair samples. Another study by Arain et al. ${ }^{172}$ found higher levels of manganese and aluminum in hair samples of patients suffering from neurodegenerative disease.

A small cohort study that followed 26 welders exposed to manganese found after a 3.5-year follow-up found worsened olfactory, extrapyramidal, and mood disturbances 173. A cohort study following asymptomatic welder trainees with no previous manganese exposure found low-level exposure to cause sub-clinical brain changes in subjects before any measurable learning deficits may occur ${ }^{174}$.

Evidence suggests manganese having a role in neurotoxicity. Chronic manganese exposure has been shown to promote the build-up of the metal in the basal ganglia, white matter, and cortical structures of the brain ${ }^{175}$. manganese has also been shown to cause an inhibitory effect on NMDA receptors ${ }^{176}$ Although manganese is an essential nutrient and has beneficial uses in the human body, increased levels of manganese in the body can lead to PD-like symptoms and developmental exposure has been shown to negatively affect neurological development ${ }^{177}$. Manganese appears to interfere with dopaminergic synaptic transmission, by possibly impairing presynaptic dopamine release ${ }^{177}$. A study using a monkey model showed manganese exposure caused neurotoxicity by inhibiting dopamine neurotransmission ${ }^{178}$.

\section{Mechanisms of Action of EEDCs and Brain Health}

From our review, it is demonstrated that physiologic pharmacologic, and chemical forms of estrogen and EEDCs affect brain health. The exact molecular mechanisms are not clear. Estrogens have a clear gender difference as they regulate the both men and women's sexual development. In females, they are produced mainly by the ovaries, in the 
testis in males, but are produced in both men and women from the adrenal gland, brain, and fat cells. In the brain, estrogen is produced by both neurons and glial cells through the aromatization of testosterone ${ }^{20,179}$. Early animal studies in rhesus monkeys found circulating estrogen in the brains of in the fetal and postnatal stages of development ${ }^{180}$. Circulating estrogen produced in the hypothalamus may also play a role in the release of gonadotropin releasing hormone $(\mathrm{GnRH})$, further adding to the complexity of estrogen's role in the brain. There is evidence to suggest that phthalates, BPA, PCBs, Cd, As, and Mn influence GnRH regulation through the hypothalamic-pituitary-ovarian axis since they also bind to estrogen receptors ${ }^{4,181-183}$ and have been demonstrated to cross the blood brain barrier ${ }^{110,117,184-186}$ and have shown estrogenic activity $53,107,108,139$. The most widely research idea regarding estrogen and the brain estrogens and EEDCs effects are mediated through estrogen receptors which are all expressed in the brain ${ }^{8}$. There is also evidence that suggests EEDC's adverse effects in the brain area results of reactive oxygen species (ROS) production and oxidative stress in the brain. Studies suggest phthalate metabolites, BPA, and metalloestrogns are associated oxidative stress and ROS production ${ }^{141,148,155,184,187,188}$.

\section{Nuclear Respiratory Factor 1 (NRF1) Genes, EEDCs, and Brain Health}

Nuclear respiratory factor 1 is a transcription factor that acts on genes encoding for mitochondrial respiratory subunits, heme biosynthetic enzymes, and regulatory factors involved in the creation and transcription of mitochondrial DNA ${ }^{189}$. Other possible extra-mitochondrial processes that are affected by NRF-1 include RNA metabolism, splicing, cell cycle, DNA damage repair, protein translation initiation, and ubiquitin-mediated protein degradation ${ }^{190}$. Since mitochondrial dysfunction has been 
implicated as a possible pathway for neurodegenerative diseases ${ }^{191}$, gene targets of NRF1 directly affect brain health and may provide insight into mechanisms of these diseases. NRF-1 mediate oxidative stress responses by regulating the expression of genes involved in cell cycle, DNA repair, cell apoptosis and mitochondrial biogenesis. NRF1 is highly expressed in human fetal brain ${ }^{192}$.

A recent study based on data from SK-N-SH human neuroblastoma cells showed that NRF1 DNA motif(s) are present in the promoters of 2470 genes ${ }^{190}$. The study speculates that NRF1 targets may be involved in the development of adverse brain health and neurodegenerative disease ${ }^{190}$. The study stated that seven out of 2470 NRF1 target genes have significant relationships with several neurodegenerative conditions. The NRF1 target genes- PARK2, PARK6 (PINK1), PARK7, PAELR (GPR37) are associated with Parkinson's disease. NRF1 target genes -PSENEN AND MAPT are involved in Alzheimer's disease. TAF4, a NRF1 target gene, is associated with Huntington's disease 190.

\section{Bioinformatic Method: Estrogen and NRF1 responsive Genes and EEDCs}

We used the Comparative Toxicogenomics Database (CTD), which contains gene information based on curated information about chemical-gene/protein interactions, chemical-disease and gene-disease relationships. Using a bioinformatics approach, we examined the gene-EDC interactions associated with both estrogen and NRF1 signaling pathways, and neurodegenerative diseases. Findings of EDCs-modified estrogen signaling and NRF1 signaling genes with exposure to natural estrogen, pharmacological estrogen, PCBs, phthalate, BPA, As, Cd, or Mn are summarized in Tables 1 and 2. 


\section{$\underline{\text { Results }}$}

\section{7-Beta Estradiol interacting genes which are also NRF1 target genes: The}

CTD search results showed that there were 6695 genes related to 17-beta estradiol (E2) (Figure 1). The 724 genes out of the 6695 E2 modified genes are NRF1 target genes (Figure 1). Three of the common E2 and NRF1 target genes, GPR37, MAPT, and PSENEN are associated with neurodegenerative disease ${ }^{190}$. Table 1 showing enriched pathway analysis revealed the top pathways associated with E2 responsive NRF1 target genes, that included:1) Disease (86 genes), 2) Metabolism (86 genes),3) Gene Expression (77 genes), 4) Signal Transduction (72 genes), 5) Immune System (67 genes), 6) Cell Cycle (62 genes), 7) Metabolic pathways (62 genes), 8) Metabolism of proteins (48 genes), 9) Developmental Biology (43 genes), and 10) Mitotic M-M/G1 phases (35 genes).

\section{Ethinyl Estradiol Interacting genes common to both E2 and NRF1 target}

genes: The CTD search revealed that there were 6049 genes related to the active estrogenic chemical in oral contraceptives, ethinyl estradiol (EE) (Figure 1). Out of the 6049 EE modified genes, 800 genes are NRF1 target genes and 331 genes are target genes of both E2 and NRF1 (Figure 1). Two of the genes, MAPT and PSENEN, observed interacting with all three EE, E2 and NRF1 are associated with neurodegenerative disease

190. Table 1 showing enriched pathway analysis revealed the top pathways associated with the number of common E2, E2 and NRF1 target genes, that included: 1) Metabolism (49 genes), 2) Disease (48 genes), 3) Gene Expression (43 genes), 4) Immune System (36 genes), 5) Cell Cycle (35 genes), 6) Metabolic pathways (33 genes), 7) Signal 
Transduction (33 genes), 8) Metabolism of Proteins (28 genes), 9) Developmental Biology (25 genes), and 10) Cancer Pathways (22 genes).

Bisphenol A and Interactions with NRF1 Target Genes: The CTD search revealed there are 19113 genes related to BPA. Out of the 19113 interacting genes, 2113 are NRF1 target genes, and 673 are target genes of both BPA and E2 (Figure 1). Three of the genes, GPR37, MAPT and PSENEN observed interacting with all three BPA, E2, and NRF1 are associated with neurodegenerative disease ${ }^{190}$. Enriched pathway analysis revealed the top pathways associated with number of common BPA/E2/NRF1 target genes, that included: 1) Metabolism (85 genes), 2) Disease (84 genes), 3) Gene Expression (71 genes), 4) Signal Transduction (68 genes), 5) Immune System (66 genes), 6) Cell Cycle (61 genes), 7) Metabolic Pathways (58 genes), 8) Metabolism of Proteins (47 genes), 9) Developmental Biology (41 genes), and Cancer Pathways (34 genes) (Table 1).

Phthalates and Interactions with NRF1 Target Genes: The CTD search revealed there were 4816 genes interacting with dibutyl phthalate (DBP) and 1344 genes with diethylhexyl phthalate (DEHP). The 756 DBP interacting genes are NRF1 target genes, whereas 149 DEHP interacting genes are NRF1 target genes. Among DBP responsive genes, there are 309 genes common E2 and NRF1 target genes and 86 DEHP associated genes are common E2 and NRF1 target genes (Figure 1). MAPT, a common DEHP, E2 and NRF1 target genes are associated with neurodegenerative disease ${ }^{190}$. Enriched pathway analysis revealed the top pathways associated with number of common DBP/E2/NRF1 target genes, that included: 1) Metabolism (56 genes), 2) Disease (45 genes), 3) Gene Expression (39 genes), 4) Metabolic Pathways (38 genes), 5) 
Cell Cycle (34 genes), 6) Signal Transduction (32 genes), 7) Immune System (31 genes), 8) Metabolism of Proteins (25 genes), 9) Mitotic M-M/G1 phases (23 genes), and Developmental Biology (21 genes). Enriched pathway analysis of E2/NRF1/DEHPresponsive genes revealed the top pathways for gene involvement that includes: 1) Metabolism (21 genes), 2) Metabolic pathways (15 genes), 3) Disease (15 genes), 4) Immune System (12 genes), 5) Metabolism of proteins (11 genes), 6) Cell Cycle (9 genes), 7) Cellular response to stress (8 genes), 8) Cancer Pathways (8 genes), 9) Developmental Biology (8 genes), and 10) Cell cycle (5 genes) (Table 1).

\section{Polychlorinated Biphenyls (PCBs) and Interactions with NRF1 Target}

Genes: There were 648 genes interacting with polychlorinated biphenyls (PCBs). The 433 PCBs genes are NRF1 target genes, and the 53 PCBs genes are common E2 and NRF1 target genes (Figure 1). Enriched pathway analysis of common PCBs/E2/NRF1associated genes revealed the top pathways for gene involvement that included: 1) Cell Cycle (13 genes), 2) Mitotic M-M/G1 phases (9 genes), 3) DNA replication (4 genes), 4) Cell Cycle (4 genes), 5) DNA replication and repair (3 genes), and 6) Pancreatic cancer (3 genes) (Table 1).

Cadmium and Interactions with NRF1 Target Genes: Using CTD search we found 2458 genes interacting with cadmium (Cd). The $263 \mathrm{Cd}$ interacting genes are NRF1 target genes and 143 interacting genes are common E2 and NRF1 target genes (Figure 1). GPR37 gene, a common Cd/E2/NRF1 gene, is associated with neurodegenerative disease ${ }^{190}$. Enriched pathway analysis of $\mathrm{Cd} / \mathrm{E} 2 / \mathrm{NRF} 1$ common genes revealed the top pathways that included: 1) Metabolism (26 genes), 2) Gene Expression (25 genes), 3) Disease (24 genes), 4) Signal Transduction (21 genes), 5) Metabolic 
pathways (20 genes), 6) Immune System (18 genes), 7) Cell Cycle (17 genes), 8) Cancer Pathways (15 genes), 9) Developmental Biology (15 genes), 10) Metabolism of proteins (12 genes) (Table 1).

Arsenic and Interactions with NRF1 Target Genes: The CTD search revealed there were 4037 genes related to arsenic (As). The 520 As interacting genes are NRF1 target genes and the 190 As interacting genes are common E2 and NRF1 target genes (Figure 1). Enriched pathway analysis of As/E2/NRF1 common genes revealed the top pathways that included: 1) Metabolism (33 genes), 2) Immune System (30 genes), 3) Disease (27 genes), 4) Signal Transduction (24 genes), 5) Developmental Biology (22 genes), 6) Metabolic pathways (20 genes), 7) Cell Cycle (19 genes), 8) Gene Expression (19 genes), 9) Cancer Pathways (17 genes), and 10) Cellular responses to stress (16 genes) (Table 1).

Manganese and Interactions with NRF1 Target Genes: Using CTD search we found 462 genes interacting with manganese (Mn). The $50 \mathrm{Mn}$ interacting genes are NRF1 target genes and the $30 \mathrm{Mn}$ interacting genes are common E2 and NRF1 target genes (Figure 1). Enriched pathway analysis of common Mn/E2/NRF1 genes revealed the top pathways that included: 1) Cellular responses to stress (6 genes), 2) Developmental Biology (5 genes), 3) Tuberculosis (4 genes), 4) p53 signaling pathway (3 genes), Small cell lung cancer ( 3 genes), Apoptosis ( 3 genes), Cell cycle (3 genes) (Table 1).

Association of EDC interacting genes common to both E2- and NRF1 targets with Neurodegenerative Diseases: Table 2 summarizes EEDs modified genes, which are common E2 and NRF1 target genes and their involvement with the specific type of brain disease, such as AD, PD, HD, ALS, Autism Spectrum Disorder, and Brain 
Neoplasms. Out of the 6643 E2 interacting genes, there were 1413 genes associated with nervous system disease.

The CTD search of E2 interacting genes in AD revealed 61 genes: ACE, ACHE, AMFR, APBB2, APOE, APP, ARC, ATP5A1, BAX, BCHE, BCL2, BDNF, BIN1,CALM1,CASP3,CHRNA7,CHRNB2,CLU,CRH,CYP46A1,DHCR24,DPYSL2,EI F2S1,ENO1,EPHA1,ESR1,F2,GAPDHS,GSK3B,HFE,HMOX1,IGF1,IGF1R,IGF2,IGF 2R,IL1B,INS,INSR,LEP,MAOB,MAPT,MIR146A,MPO,NOS3,NPY,PAXIP1,PICAL M,PLAU,PPARG,PRNP,PSEN2,SLC2A4,SOD2,SORL1,TF,TFAM,TNF,TPI1,TREM2 ,VEGFA, VSNL.The bold indicates E2 interacting genes which are NRF1 target genes. With AD, the six E2-responsive genes are NRF1 target genes: APBB2, DPYSL2, EIF2S1, ENO1, MAPT, AND PAXIP1. These genes are also responsive to the following EEDCs: ethinyl estradiol (APBB2, DPYSL2, EIF2S1, ENO1, MAPT, and PAXIP1), bisphenol-A (APBB2, EIF2S1, ENO1, MAPT, and PAXIP1), dibutyl phthalate (DPYSL2, EIF2S1, ENO1), diethylhexyl phthalate (DPYSL2 and MAPT), dibutyl phthalate (DPYSL2, EIF2S1, ENO1), cadmium (ENO1), arsenic (ENO1 and MAPT), and manganese (MAPT) (Table 5).

With PD, the eight E2-responsive NRF1 target genes are: GAK, HSPA9, MAPT, PARK2, PARK7, PINK1, RPL14, AND VPS35. These genes are also responsive to the following EEDCs: ethinyl estradiol (HSPA9, MAPT), bisphenol-A (HSPA9, MAPT, RPL14), dibutyl phthalate (HSPA9, RPL14), diethylhexyl phthalate (HSPA9, MAPT), cadmium (RPL14), and arsenic (HSPA9 and MAPT) (Table 5). The CTD search revealed that PCBs-interacting nine genes: HMOX1, IL6, NQO1, RPS8, SLC18A2, SNCA, 
SOD1, SOD2, TNF are associated with Parkinson Disease. Both SOD and TNF E2 interacting genes are NRF1 target genes.

With HD, the two E2-responsive NRF1 target genes are: AIFMI and IP6K2. These genes are also responsive to the following EEDCs: ethinyl estradiol (AIFM1), bisphenol-A (AIFM1 and IP6K2), dibutyl phthalate (IP6K2), cadmium (AIFM1), and arsenic (IP6K2) (Table 2).

With ALS, the E2-responsive NRF1 target genes are CHMP2B and GSR. These genes are also responsive to the following EEDCs: ethinyl estradiol (GSR), bisphenol-A (GSR and $C H M P 2 B)$, dibutyl phthalate $(G S R)$, diethylhexyl phthalate $(G S R)$, cadmium $(G S R)$, and arsenic $(G S R)$ (Table 2).

With ASD, the E2-responsive NRF1 target genes are CIRBP, PCDH9, and GTF2I. These genes are also responsive to the following EEDCs: ethinyl estradiol (CIRBP and GTF2I), bisphenol-A (CIRBP, GTF2I, and PCDH9), polychlorinated biphenyls $(C I R B P)$, cadmium $(C I R B P)$, and arsenic (PCDH9) (Table 2).

With brain neoplasms, the E2-responsive NRF1 target genes are PCNA, PTCH1, and RELA. These genes are also responsive to the following EEDCs: ethinyl estradiol (PCNA and PTCH1), bisphenol-A (PCNA and RELA), dibutyl phthalate (PCNA and PTCH1), cadmium (RELA), arsenic (PCNA and PTCH1), and manganese (RELA) (Table 2).

\section{NRF1, Mitochondrial Dysfunction, and Neurodegenerative Disease}

Mitochondria are known as the powerhouses of cells. They are important for cell viability and function ${ }^{193}$. They control cell process such as energy production, calcium signaling, and apoptosis ${ }^{193}$. Research has suggested that mitochondrial dysfunction is a 
cause and not a result of neurodegenerative diseases ${ }^{191}$ It is suggested mitochondrial dysfunction plays a major role, in $\mathrm{AD}, \mathrm{PD}, \mathrm{HD}$, and ALS through the oxidative phosphorylation dysfunction ${ }^{191}$. Mitochondrial dysfunction may also increase ROS generation, cause abnormal protein-protein interactions, and may lead to loss of cellular integrity and cell death ${ }^{194}$. There is also evidence to suggest mitochondrial dysfunction may play a role in neuronal plasticity and maintenance ${ }^{194}$. Sex steroid have been observed to regulate mitochondrial function ${ }^{195}$, and provide targets for estrogenic endocrine disruptors such as bisphenol-A, phthalates, polychlorinated biphenyls, metals, oral contraceptives, and hormonal replacement therapies ${ }^{1}$. NRF1 regulates the expression of nuclear genes that encode that encode mitochondrial proteins that function in metabolic pathways such as the trichloroacetic acid cycle (TCA), oxidative phosphorylation, heme synthesis, and in mitochondrial DNA replication and transcription (eg, mitochondrial transcription factor A [Tfam] ${ }^{189,190}$. Since mitochondrial dysfunction has been implicated as a possible pathway for neurodegenerative diseases ${ }^{191}$, gene targets of NRF-1 directly affect brain health and may provide insight into mechanisms of these diseases.

\section{NRF1-mediated Regulation of Neurogenesis and Synaptogenesis}

Estrogen has been demonstrated to have a significant role in controlling neural progenitors in the developing embryonic brain. Estrogen has been shown to induce proliferation and differentiation of neural progenitor ${ }^{196-198}$. Neural progenitors have also been demonstrated to express estrogen receptors ${ }^{197,198}$, which provide a site in EEDCs to exert their effects. EEDCs have been shown to cross the placental and blood brain barrier

${ }^{6}$, but the mechanisms regarding the effects on the early stages of neural development are 
largely not known. Studies suggest neural development is affected by exposures to EEDCs. Low-dose exposure to bisphenol-A and $\mathrm{S}$ has been shown to induce hypothalamic neurogenesis in an embryonic zebrafish at a point in time analogous to the second trimester of human development ${ }^{199}$. Several classes of phthalates were shown to prevent neural stem cell proliferation in a rat mesencephalic stem cell model ${ }^{200}$. Polychlorinated biphenyls have also been demonstrated to interfere with neuronal cell differentiation in a rat embryonic neural stem cell model ${ }^{201}$. Arsenic was shown to substantially inhibited neuronal differentiation in human embryonic neural stem cells ${ }^{202}$. Manganese has also been demonstrated to cause cell death in a rat neural stem cell model through a mitochondrial-mediated pathway ${ }^{203}$. The generation of new neurons from neural progenitor stem cells, the growth of axons and dendrites and the formation and reorganization of synapses are examples of neuroplasticity. All these processes seem to be regulated by nuclear respiratory factor 1 (NRF1) target network genes. For example, recently, it has been shown that NRF1 regulates neurite outgrowth - a critical process in neuronal development in neuroblastoma cells and hippocampal neurons by regulating its target gene, Synapsin $1^{204}$. Another fifteen genes involved in different biological processes of neurons, cell cycle-related genes- MAPRE3, NPDC1, SUV39H2, SKA3, transport-related genes- RAB3IP, TRAPPC3, signal transduction-related genes- SMAD5, PIP5K1A, USP10, SPRY4, transcription-related genes- GTF2F2, NR1D1, and regulation of GTPase activity-related genes- RHOA, RAPGEF6, SMAP1, have been reported to contain NRF1 binding motif(s) in their promoters and mRNA levels of 12 of these genes are regulated by NRF1 ${ }^{205}$. Overexpression or knockdown of MAPRE3, NPDC1, SMAD5, USP10, SPRY4, GTF2F2, SKA3, RAPGEF6 positively regulates, where as 
RHOA and SMAP1 negatively regulates neurite outgrowth. Three hypothetical genes FAM134C, C3orf10, and ENOX1 involved in neurite outgrowth are regulated by NRF1. FAM134C positively regulates and C3orf10 negatively regulates neurite outgrowth ${ }^{206}$ In summary, it appears that NRF1 regulates neurite outgrowth through cell cycle-, transport, signal transduction-, transcription-, regulation of GTPase activity-related genes and hypothetical genes. This suggests that NRF1 regulates neuronal differentiation through a variety of biological processes.

\section{Gender Bias and NRF1 Regulated Genes-EEDC Interactions in AD}

Sex differences in nervous system diseases, such as multiple sclerosis, Parkinson's disease, and Alzheimer's disease, exist in prevalence, severity, and progression of pathologies and consequently has become a major obstacle for the treatment. Cognitive disturbances are frequent in brain health deficit-related disease. Men show greater cognitive impairment in schizophrenia, whereas women show more severe dementia and cognitive decline with Alzheimer's disease. Alzheimer's disease (AD) disproportionally affects women $(\mathrm{F}: \mathrm{M} \approx 2: 1)$ and the total number of Americans aged 65 years and older with $\mathrm{AD}$ is projected to increase from 5.1 million to 13.8 million by $2050{ }^{207}$ Despite tremendous progress in understanding the pathogenesis of $\mathrm{AD}$, the molecular basis underlying the sex-dependent differences in AD remains largely unknown. and consequently, has become a major obstacle for the development of new sex-based molecular targets of AD.

\section{NRF1 and Estrogen Responsive Genes in the AD Pathway}

Many genes modified by EEDs are common targets of both (E2) and NRF1 and some of these genes are involved with the specified brain diseases. Therefore, using 
NRF1-EDC interacting genes identified from CTD (Table 4), we focused our efforts on $\mathrm{AD}$ and conducted enrichment pathway analysis, which revealed that many of the NRF1 target genes interacting with each specific EEDC and they are part of pathway of AD (Figures 2-7). Enrichment pathway analysis was performed using Cytoscape and Genemania. The search of enriched pathways showed that top 10 E2 interacting genes in AD: APOE, APP, ATP5A1, CALM1, CASP3, GSK3B, IL1B, MAPT, PSEN2 and TNF underlie the enrichment of the Kyoto Encyclopedia of Genes and Genomes (KEGG) Alzheimer's disease pathway. With AD, the six E2-responsive genes are NRF1 target genes: APBB2, DPYSL2, EIF2S1, ENO1, MAPT, AND PAXIP1. These genes are also responsive to the following EEDs: ethinyl estradiol (APBB2, DPYSL2, EIF2S1, ENO1, $M A P T$, and PAXIP1) BPA (APBB2, EIF2S1, ENO1, MAPT, and PAXIP1), dibutyl phthalate (DPYSL2, EIF2S1, ENO1), diethylhexyl phthalate (DPYSL2 and MAPT), dibutyl phthalate (DPYSL2, EIF2S1, ENO1) (Figures 2-7, Table 2). GO annotations of E2 and NRF1 enrichment network genes of EEDs revealed multiple common E2 and NRF1 genes associated with carbohydrate metabolic pathways, which was common among all EEDCs (Figures 2-7). Other two biological process pathways showing association with multiple common E2 and NRF1 genes interacting with E2, EE, BPA or phthalates were translation (translation initiation, translation initiation factory activity and translation factory activity, nucleic acid binding) and glial/oligodendrocytes growth and differentiation (Figures 2-7). To validate our CTD findings, we used Bayesian network (BN) analysis ${ }^{208}$ of microarray data of 79 subjects from the Gene Expression Omnibus (GEO) database ${ }^{209}$, which showed the female NRF1 gene network is different from the male network. It was also observed that both NRF1 expression and gender were 
associated with AD (Figure 8). Genes associated with AD - APLP1, APP, GRIN1, GRIN2B, MAPT, PSEN2, PEN2, and IDE are also NRF1-regulated and E2-responsive and may contribute to NRF1 gender differences and may play a role in the prevention of AD by E2.

\section{$\underline{\text { Conclusions }}$}

Estrogenic endocrine disrupting chemicals go further than the known novel mechanisms of endocrine disruption. Gene-gene and gene-environment provide alternative paths for endocrine disruption. Bioinformatics analysis of gene-EEDCs interactions and brain disease associations identified numerous NRF1 regulated genes that were altered by exposure to estrogen, phthalate, BPA, and metalloestrogens. EEDCmodified genes in brain health deficits are part of estrogen and nuclear respiratory factor 1 signaling pathways. Our findings suggest that in addition to estrogen signaling, these chemicals influence NRF1 factor regulated communities of genes across genomic and epigenomic multiple networks may contribute in the development of complex chronic human brain health deficits. 

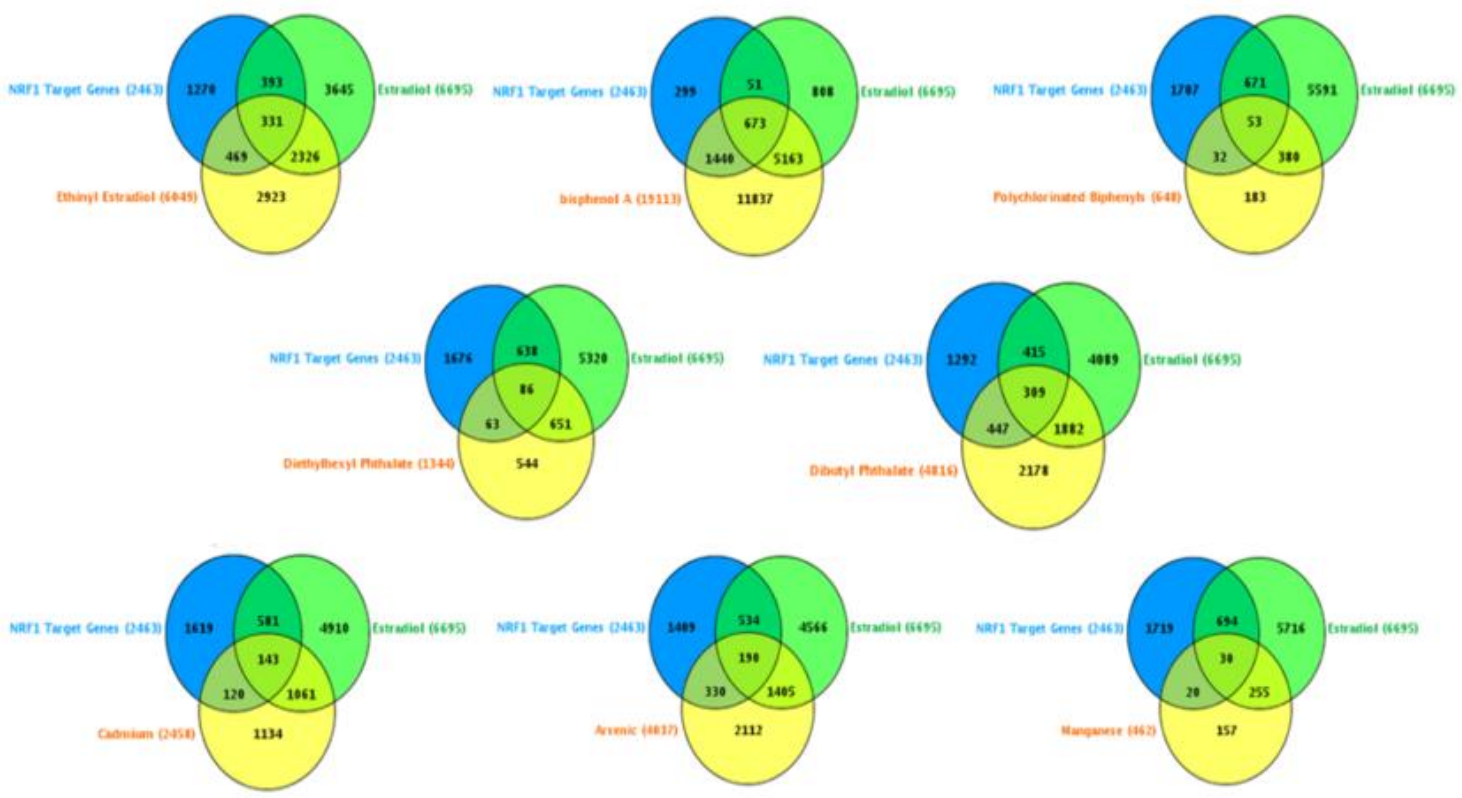

Figure 1: A Venn diagram showing the individual pharmacological estrogen or estrogenic Endocrine Disruptor (EED) modified genes common to both NRF1 and/or estrogen signaling pathways. NRF1 target genes were mapped between 17-beta estradiol (E2) and pharmacological estrogen lethinyl estradiol or PCBs or BPA or phthalates or Cd or As or Mn.

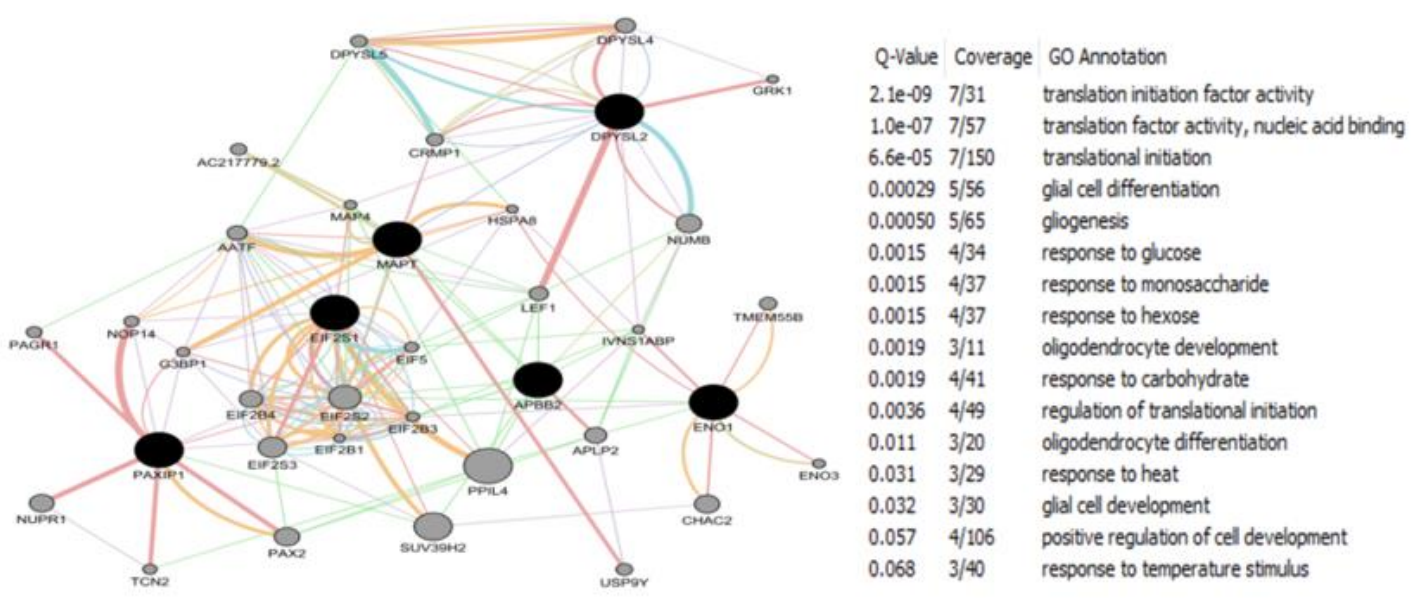

Figure 2. Gene set pathway enrichment analysis of 17-beta estradiol (E2) responsive NRF1 target genes associated with Alzheimer Disease (AD). E2 responsive NRF1 genes are in black. The network includes the top 30 related genes. Right panel show GO annotations of NRF1 enrichment network genes of E2 associated with biological process pathways involving multiple E2 and NRF1 genes and Q-value as a probability showing number of genes out of the total genes annotated to each GO term. 


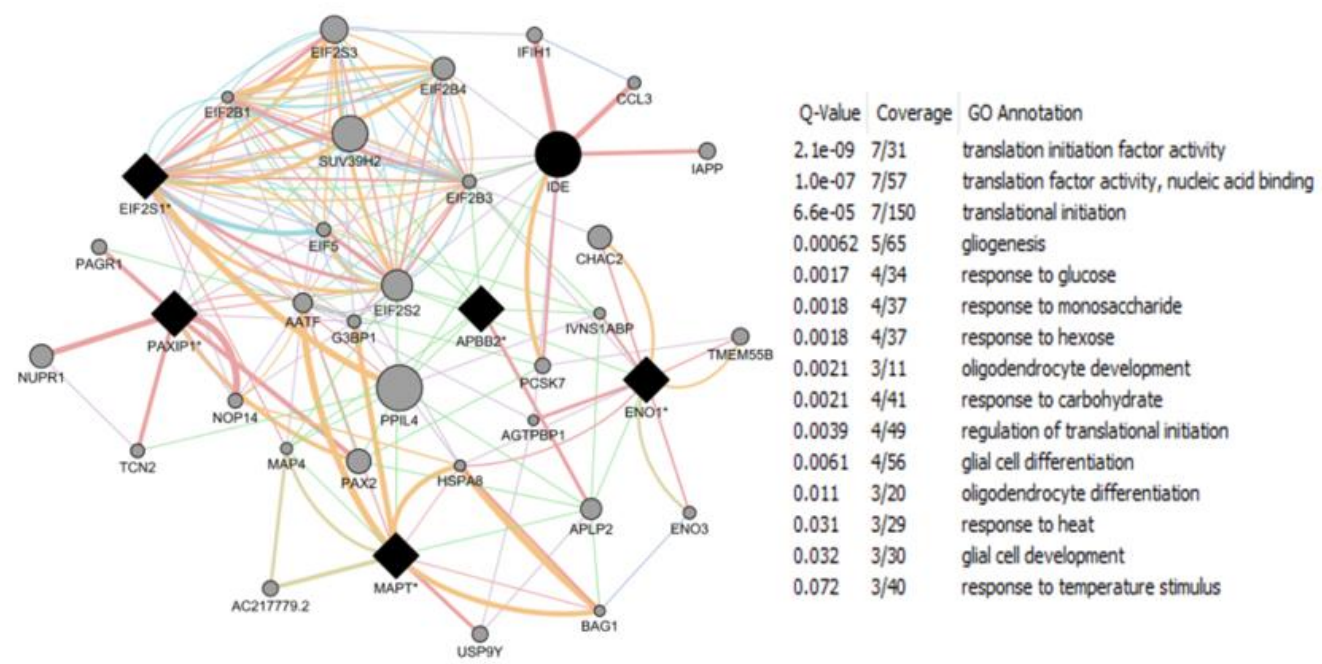

Figure 3. Gene set pathway enrichment analysis of ethinyl estradiol responsive E2 and NRF1 common target genes associated with Alzheimer Disease (AD). Ethinyl estradiol responsive NRF1 genes are in black. Genes denoted by a diamond-shaped node are E2 and NRF1. The network includes the top 30 related genes. Right panel show GO annotations of E2 and NRF1 enrichment network genes of ethinyl estradiol associated with biological process pathways involving multiple E2 and NRF1 genes and Qvalue as a probability showing number of genes out of the total genes annotated to each GO term.

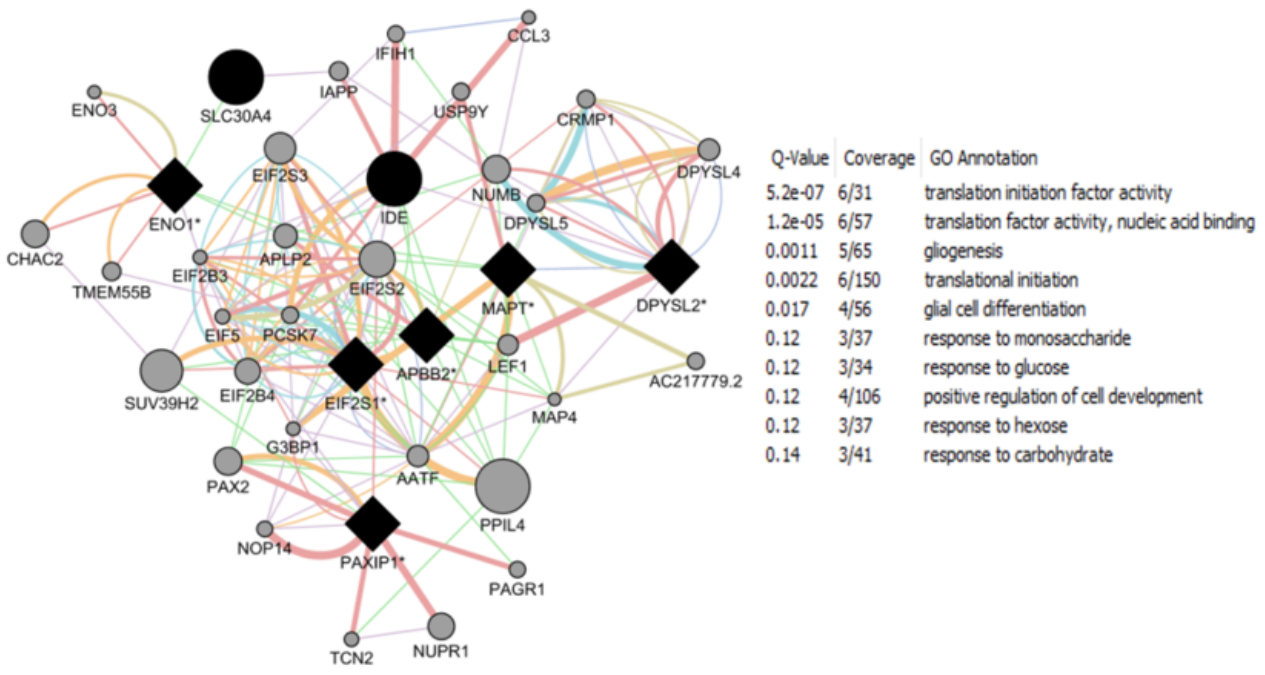

Figure 4. Gene set pathway enrichment analysis of bisphenol-A (BPA) responsive R2 and NRF1common target genes associated with Alzheimer Disease (AD). Bisphenol-A responsive NRF1 genes are in black. Genes denoted by a diamond-shaped node are E2 and NRF1 responsive. The network includes the top 30 related genes. Right panel show GO annotations of E2 and NRF1 enrichment network genes of BPA associated with biological process pathways involving multiple E2 and NRF1 genes and Q-value as a probability showing number of genes out of the total genes annotated to each GO term. 


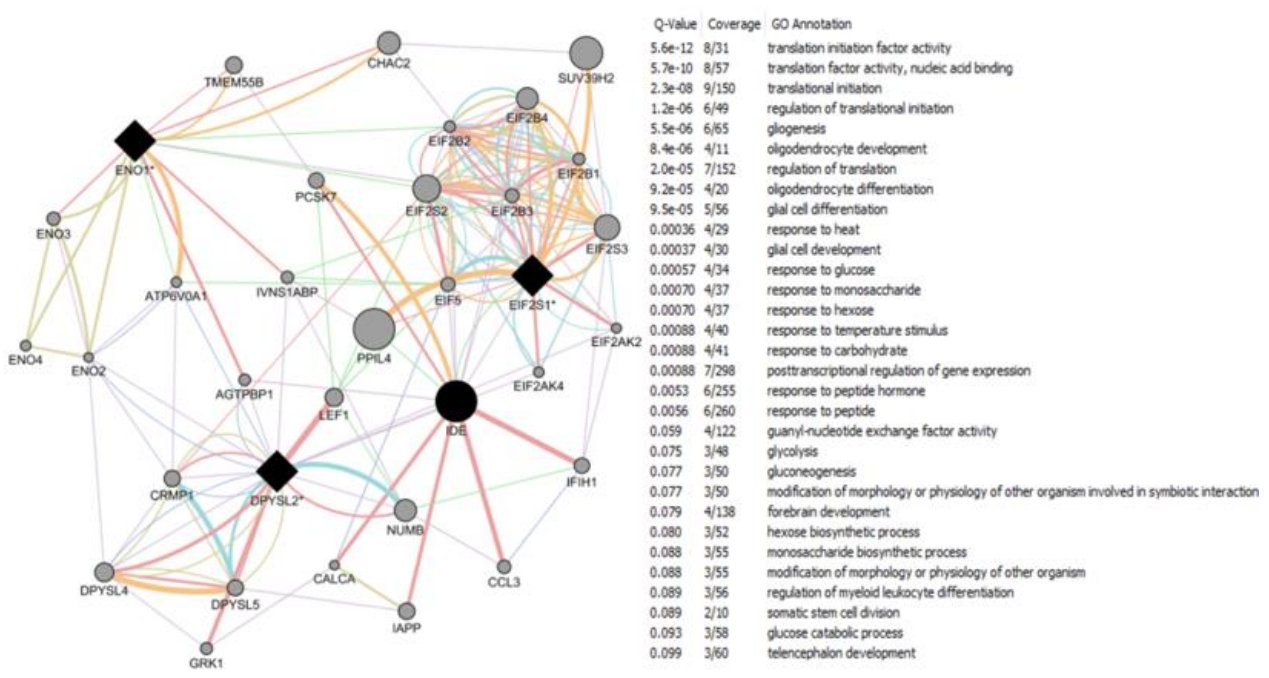

Figure 5. Gene set pathway enrichment analysis of dibutyl phthalate modified E2 and NRF1 common target genes associated with Alzheimer Disease (AD). Dibutyl phthalate responsive NRF1 genes are in black. Genes denoted by a diamond-shaped node are E2 and NRF1 responsive. The network includes the top 30 related genes. Right panel show GO annotations of E2 and NRF1 enrichment network genes of dibutyl phthalate associated with biological process pathways involving multiple E2 and NRF1 genes and Q-value as a probability showing number of genes out of the total genes annotated to each $\mathrm{GO}$ term.

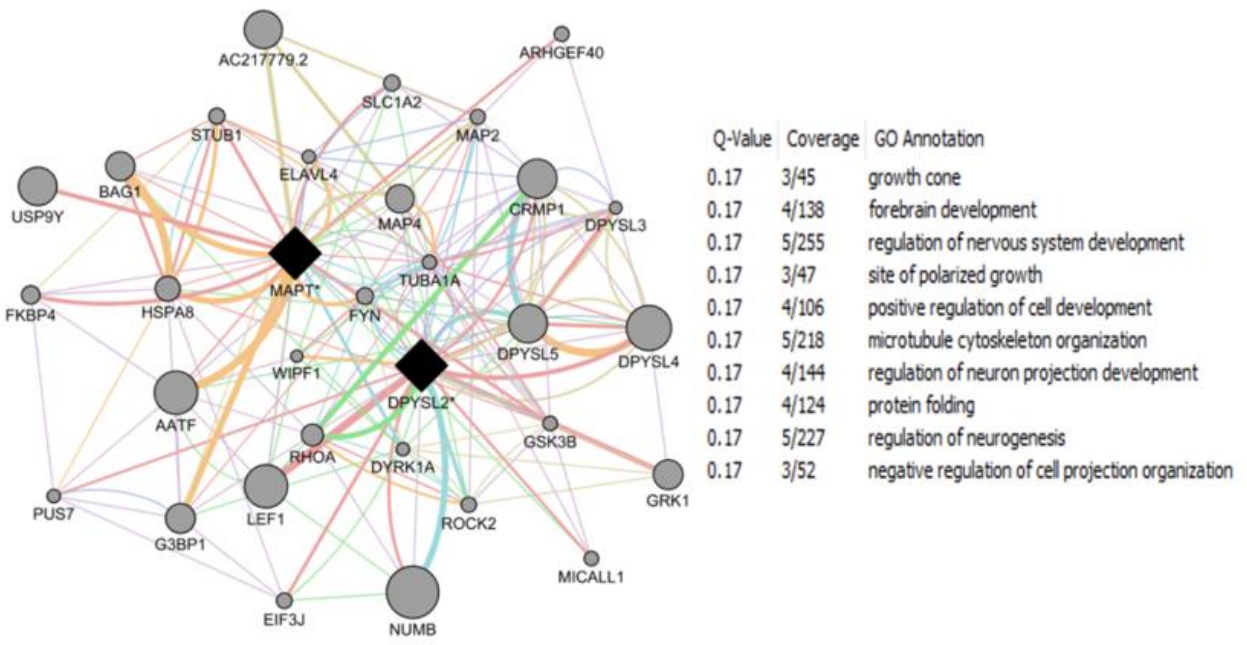

Figure 6. Gene set pathway enrichment analysis of diethylhexyl phthalate responsive E2 and NRF1 common target genes associated with Alzheimer Disease (AD). Diethylhexyl phthalate responsive NRF1 genes are in black. Genes denoted by a diamond-shaped node are E2 and NRF1 responsive. The network includes the top 30 related genes. Right panel show GO annotations of E2 and NRF1 enrichment network genes of diethylhexyl phthalate associated with biological process pathways involving multiple E2 and NRF1 genes and Q-value as a probability showing number of genes out of the total genes annotated to each GO term. 


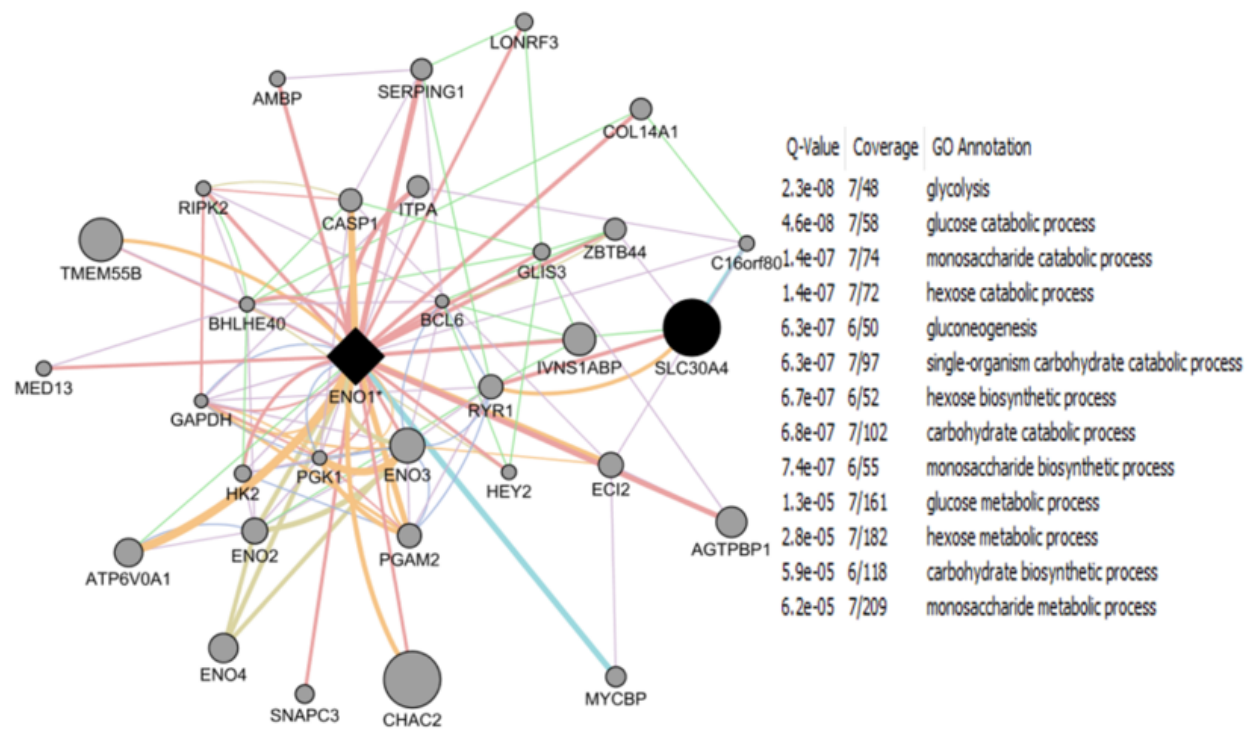

Figure 7. Gene set pathway enrichment analysis of cadmium responsive E2 and NRF1 common target genes associated with Alzheimer Disease (AD). Cadmium responsive NRF1 genes are in black. Genes denoted by a diamond-shaped node are E2-and NRF1 responsive. The network includes the top 30 related genes. Right panel show GO annotations of E2 and NRF1 enrichment network genes of cadmium associated with biological process pathways involving multiple E2 and NRF1 genes and Qvalue as a probability showing number of genes out of the total genes annotated to each GO term.

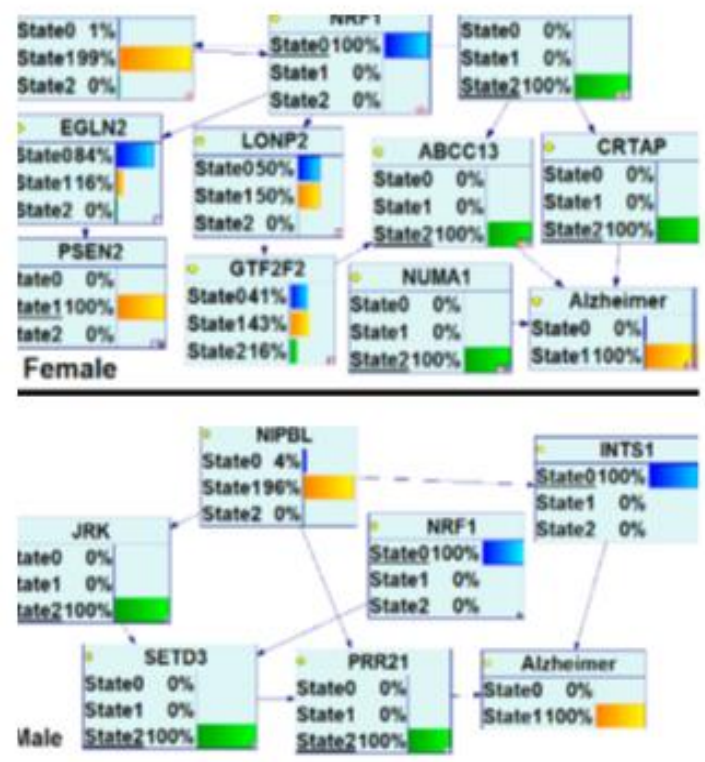

Figure 8. Gender differences in the NRF1 network from brain microarray data of 79 male and female AD subjects and controls from the GEO database. 

and $\mathrm{Mn}$

\section{Beta-estradiol (E2) interacting NRF1 target genes}

\begin{tabular}{|c|c|c|}
\hline KEGG Pathway & $\begin{array}{l}\text { Number of } \\
\text { genes: }\end{array}$ & Annotated Genes \\
\hline Disease & 86 & $\begin{array}{l}\text { ADCY9|AKT2|ALDOA|AP1S1|CCNT2|CDC42|CDK1|CDKN2B|CHMP2A|CHMP2B|CHMP5|CREB1|CSK|CSNK1A1| } \\
\text { CTBP1|CYB5A|ENO1|EPS15L1|ERCC2|EXT2|FASN|FZD4|GLB1|GTF2E2|HDAC2|HDAC3|HDAC4|HMMR|HRAS|H } \\
\text { SP90AA1|IRS1|MAP2K7|MKNK1|MMADHC|MTHFD1|NAMPT|NPM1|NUP107|NUP50|OS9|P4HB|PAPSS1|PFKFB4| } \\
\text { PIP5K1B|PLCG1|POLR2A|POM121|PPIA|PRKAR2A|PSENEN|PYGL|RAC1|RAE1|RAF1|RAN|RBX1|RHOA|RPL10| } \\
\text { RPL13A|RPL14|RPL36|RPS13|RPS16|RPS21|RPS29|RPS9|SHC1|SLC25A10|SLC37A4|SMARCA4|SNW1|SOS1|SRC|S } \\
\text { TUB1|STX1A|TAF12|TAF5|TCEB2|TGIF1|TNKS2|TPR|UBA52|WNT5A|XRCC5|XRCC6|YWHAZ }\end{array}$ \\
\hline Metabolism & 86 & $\begin{array}{l}\text { ACADM|ADCY9|ADI||AGPAT5|AKR7A2|ALAS1|ALDOA|AMACR|ATP5O|AUH|BCAT1|BCAT2|BSG|CBR1|COX5 } \\
\text { B|COX6C|CTPS1|CYB5A|CYCS|DTYMK|ELOVL4|ENO1|ERCC2|ETFB|ETFDH|EXT2|FASN|FDPS|GCLC|GLB1|GN } \\
\text { G5|GPD1L|GSR|GSS|GSTM3|HDAC3|HMMR|HSD17B4|HSP90AA1|INPP5A|INPP5K|IP6K2|LDHA|LPCAT3|LSS|M } \\
\text { GST3|MMADHC|MPC2|MTHFD1|NAMPT|NDUFA3|NDUFB4|NUBP1|NUP107|NUP50|OAZ2|ODC1|P4HB|PAICS|P } \\
\text { APSS1|PDHB|PFKFB4|PIP5K1B|PLA2G12A|PLCG1|PLD1|POM121|PPAT|PRKAR2A|PSAP|PYGL|RAE1|RAN|SDH } \\
\text { A|SGMS1|SIN3B|SLC16A1|SLC25A10|SLC37A4|SLC44A2|STX1A|SUCLA2|TPMT|TPR|UBA52|VAPB }\end{array}$ \\
\hline $\begin{array}{c}\text { Gene } \\
\text { Expression }\end{array}$ & 77 & 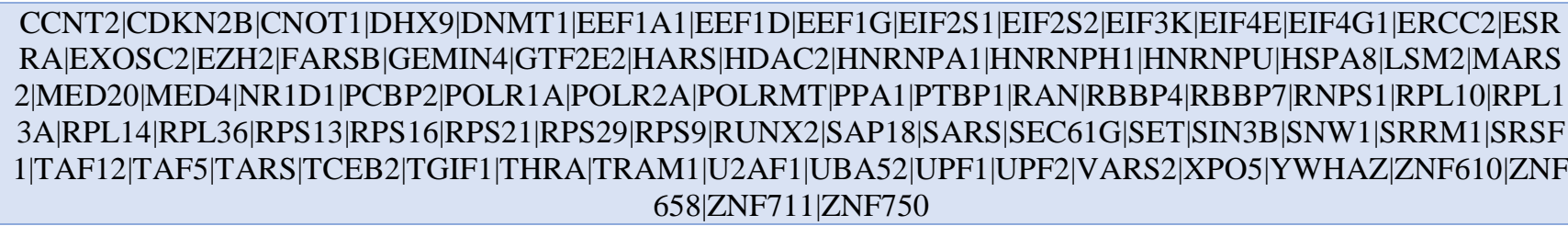 \\
\hline $\begin{array}{c}\text { Signal } \\
\text { Transduction }\end{array}$ & 72 & $\begin{array}{l}\text { ABI1|ADCY9|AKT2|ARHGDIA|BRK1|CASP2|CCNT2|CDC42|CDK1|CDKN2B|CREB1|CRK|CRKL|CSK|CSNK1A1| } \\
\text { CTBP1|DAAM1|DGKH|E2F1|EIF4E|EIF4G1|EPS15L1|FSTL3|FZD4|GNG5|GPR37|HDAC2|HDAC3|HDAC4|HRAS|H } \\
\text { SP90AA1|IRS1|JAK2|LFNG|MAPK7|MEF2A|MKNK1|OS9|P4HB|PFN1|PIP5K1B|PLCG1|PRDM4|PRKAR2A|PSAP|P } \\
\text { SENEN|PTCH1|PTK2|RAC1|RAF1|RBX1|RELA|RHOA|RIPK2|ROCK1|RPS6KA5|RPS6KB1|SHC1|SMARCA4|SNW1 } \\
\text { |SOCS1|SOS1|SRC|STARD13|STUB1|TGIF1|THBS2|TNKS2|TRIO|UBA52|WNT5A|YWHAZ }\end{array}$ \\
\hline Immune System & 67 & $\begin{array}{l}\text { ABI1|ADCY9|AKT2|ANAPC1|ANAPC11|AP1S1|ARPC2|ARPC5|BRK1|CASP2|CDC34|CDC42|CDK1|CREB1|CRK|C } \\
\text { RKL|CSK|CTSD|DHX9|DYNLL1|EIF4E|EIF4G1|FADD|FZR1|HRAS|HSP90AA1|IP6K2|IRS1|JAK2|KIF18A|KIF4A|M } \\
\text { AP2K7|MAPK7|MEF2A|NUP107|NUP50|PCBP2|PELI1|PLCG1|PLD1|POM121|PRKAR2A|PRKDC|PTK2|PVR|RAC1| } \\
\text { RAE1|RAF1|RBX1|RELA|RIPK2|RNF19B|RPS6KA5|SEC61G|SHC1|SOCS1|SOS1|SRC|STUB1|TCEB2|TPR|TUBB4B } \\
\text { |UBA52|UBE2D4|XRCC5|XRCC6|YWHAZ }\end{array}$ \\
\hline Cell Cycle & 62 & $\begin{array}{l}\text { ANAPC1|ANAPC11|APITD1|ATM|AURKA|AURKB|BUB1|CCNB1|CCNE1|CDC7|CDK1|CDKN2B|CDKN2D|CDT1| } \\
\text { CENPN|CKS1B|CLASP2|DKC1|DSN1|DYNLL1|E2F1|ERCC6L|FBXO5|FZR1|GOLGA2|HSP90AA1|KIF18A|LIN37|M } \\
\text { AD2L1|MCM2|MCM4|MCM6|MLH3|NEK2|NPM1|NUP107|NUP50|OIP5|ORC6|PCNA|PLK4|POLA1|POM121|PPP1R }\end{array}$ \\
\hline
\end{tabular}




\begin{tabular}{|c|c|c|}
\hline & & $\begin{array}{c}\text { 12A|RAB1B|RAD50|RAE1|RBBP4|RBBP7|RBBP8|RBL2|RSF1|SDCCAG8|SET|SMC4|SPC24|SPDL1|TPR|TUBB|TUB } \\
\text { B4B|UBA52|ZWINT }\end{array}$ \\
\hline $\begin{array}{l}\text { Metabolic } \\
\text { pathways }\end{array}$ & 62 & $\begin{array}{c}\text { ACADM|ADI1|ALAS1|ALDOA|AMACR|ATP5O|AUH|BCAT1|BCAT2|CBR1|COX5B|COX6A2|COX6C|CTPS1|HAD } \\
\text { 1|DGKH|DNMT1|DTYMK|ENO1|EXT2|FASN|FBL|FDPS|FUT8|GAA|GCLC|GFPT1|GLB1|GSS|HSD17B4|INPP5A|IN } \\
\text { PP5K|LAP3|LDHA|LSS|MECR|MTHFD1|NDUFA3|NDUFB4|ODC1|PAICS|PAPSS1|PDHB|PGAM5|PGAP1|PIGA|PIP } \\
\text { 5K1B|PLA2G12A|PLCG1|PLD1|POLA1|POLE4|POLG2|POLR1A|POLR2A|PPAT|SDHA|SGMS1|SHMT2|SUCLA2|S } \\
\text { UFU|TGDS }\end{array}$ \\
\hline $\begin{array}{l}\text { Metabolism of } \\
\text { proteins }\end{array}$ & 48 & $\begin{array}{c}\text { B3GNTL1|CCT3|CCT6A|CCT8|CTSD|HAD1|DDIT3|DNAJB9|DNAJC3|DOHH|EEF1A1|EEF1D|EEF1G|EIF2S1|EIF2S } \\
\text { 2|EIF3K|EIF4E|EIF4G1|EIF5A|FBXW5|FUT8|GFPT1|GLB1|GRPEL2|HSPA9|HSPD1|IGFBP3|MANEA|PFDN1|PGAP1 } \\
\text { |PIGA|RPL10|RPL13A|RPL14|RPL36|RPS13|RPS16|RPS21|RPS29|RPS9|SEC61G|SHC1|STX1A|THBS2|TRAM1|TSP } \\
\text { YL2|TUBB4B|UBA52 }\end{array}$ \\
\hline $\begin{array}{l}\text { Developmental } \\
\text { Biology }\end{array}$ & 42 & $\begin{array}{l}\text { ABLIM2|AKT2|ARPC2|ARPC5|CDC42|CDK1|CLASP2|CREB1|CRMP1|CTNNA2|DPYSL2|HDAC3|HRAS|HSP90AA } \\
\text { 1|HSPA8|ITGA1|KIF4A|MED11|MED18|MED20|MED4|MEF2A|MYH10|MYH14|NRTN|PFN1|PLCG1|PSENEN|PTK2 } \\
\text { |RAC1|RAF1|RHOA|ROCK1|RPS6KA5|RPS6KA6|SCN3B|SDCBP|SIAH2|SOS1|SPTAN1|SRC|TRIO|TUBB4B }\end{array}$ \\
\hline $\begin{array}{l}\text { Mitotic M- } \\
\text { M/G1 phases }\end{array}$ & 35 & $\begin{array}{l}\text { ANAPC1|ANAPC11|APITD1|AURKB|BUB1|CCNB1|CDC7|CDK1|CDT1|CENPN|CLASP2|DSN1|ERCC6L|FBXO5|G } \\
\text { OLGA2|KIF18A|MAD2L1|MCM2|MCM4|MCM6|NUP107|NUP50|ORC6|POLA1|POM121|RAB1B|RAE1|SET|SMC4|S } \\
\text { PC24|SPDL1|TPR|TUBB4B|UBA52|ZWINT }\end{array}$ \\
\hline KEGG Pathway & $\begin{array}{l}\text { Number of } \\
\text { Genes: }\end{array}$ & Ethinyl estradiol interacting common E2- and NRF1-target genes \\
\hline Metabolism & 49 & $\begin{array}{c}\text { ACADM|ADCY9|AGPAT5|ALAS1|ALDOA|ATP5O|BCAT1|BSG|CBR1|COX5B|COX6C|CTPS1|CYB5A|CYCS|ENO1 } \\
\text { |ERCC2|ETFDH|EXT2|FASN|FDPS|GCLC|GLB1|GSR|GSS|GSTM3|HDAC3|HSD17B4|HSP90AA1|INPP5A|LDHA|LS } \\
\text { S|MGST3|MTHFD1|NAMPT|NUP50|ODC1|P4HB|PAPSS1|PDHB|PPAT|PSAP|PYGL|RAE1|RAN|SGMS1|SIN3B|SLC } \\
\text { 16A1|SLC25A10|SLC37A4 }\end{array}$ \\
\hline Disease & 48 & $\begin{array}{c}\text { :ADCY9|AKT2|ALDOA|AP1S1|CDC42|CDK1|CHMP2A|CREB1|CSK|CSNK1A1|CYB5A|ENO1|ERCC2|EXT2|FASN| } \\
\text { GLB1|GTF2E2|HDAC2|HDAC3|HRAS|HSP90AA1|MAP2K7|MTHFD1|NAMPT|NPM1|NUP50|OS9|P4HB|PAPSS1|PS } \\
\text { ENEN|PYGL|RAE1|RAN|RBX1|RHOA|RPL36|RPS16|RPS9|SLC25A10|SLC37A4|SMARCA4|STUB1|TAF12|TAF5|T } \\
\text { CEB2|WNT5A|XRCC5|YWHAZ }\end{array}$ \\
\hline $\begin{array}{c}\text { Gene } \\
\text { Expression }\end{array}$ & 43 & $\begin{array}{c}\text { DNMT1|EEF1A1|EEF1G|EIF2S1|EIF2S2|EIF3K|EIF4E|ERCC2|EZH2|FARSB|GTF2E2|HARS|HDAC2|HNRNPA1|HN } \\
\text { RNPH1|HNRNPU|HSPA8|MED4|NR1D1|PCBP2|POLR1A|POLRMT|RAN|RBBP4|RBBP7|RNPS1|RPL36|RPS16|RPS9 } \\
\text { |SAP18|SARS|SEC61G|SET|SIN3B|SRSF1|TAF12|TAF5|TARS|TCEB2|THRA|UPF1|VARS2|YWHAZ }\end{array}$ \\
\hline Immune System & 36 & $\begin{array}{c}\text { ABI1|ADCY9|AKT2|ANAPC1|ANAPC11|AP1S1|ARPC2|ARPC5|CASP2|CDC42|CDK1|CREB1|CRK|CSK|CTSD|DY } \\
\text { NLL1|EIF4E|FADD|FZR1|HRAS|HSP90AA1|MAP2K7|MAPK7|NUP50|PCBP2|PTK2|PVR|RAE1|RBX1|SEC61G|SOC } \\
\text { S1|STUB1|TCEB2|TUBB4B|XRCC5|YWHAZ }\end{array}$ \\
\hline Cell Cycle & 35 & \\
\hline
\end{tabular}




\begin{tabular}{|c|c|c|}
\hline & & $\begin{array}{l}\text { ANAPC1|ANAPC11|AURKB|BUB1|CCNB1|CCNE1|CDC7|CDK1|CDT1|CKS1B|DYNLL1|E2F1|FZR1|GOLGA2|HSP } \\
\text { 90AA1|LIN37|MAD2L1|MCM2|MCM4|MCM6|NPM1|NUP50|ORC6|PCNA|PLK4|PPP1R12A|RAE1|RBBP4|RBBP7|R } \\
\text { BBP8|RSF1|SET|SPC24|SPDL1|TUBB4B }\end{array}$ \\
\hline $\begin{array}{l}\text { Metabolic } \\
\text { pathways }\end{array}$ & 33 & $\begin{array}{l}\text { ACADM|ALAS1|ALDOA|ATP5O|BCAT1|CBR1|COX5B|COX6C|CTPS1|DNMT1|ENO1|EXT2|FASN|FDPS|GCLC|G } \\
\text { LB1|GSS|HSD17B4|INPP5A|LDHA|LSS|MECR|MTHFD1|ODC1|PAPSS1|PDHB|PGAM5|PIGA|POLE4|POLG2|POLR } \\
\text { 1A|PPAT|SGMS1 }\end{array}$ \\
\hline $\begin{array}{c}\text { Signal } \\
\text { Transduction }\end{array}$ & 33 & $\begin{array}{l}\text { ABI1|ADCY9|AKT2|CASP2|CDC42|CDK1|CREB1|CRK|CSK|CSNK1A1|E2F1|EIF4E|HDAC2|HDAC3|HRAS|HSP90 } \\
\text { AA1|MAPK7|OS9|P4HB|PFN1|PRDM4|PSAP|PSENEN|PTCH1|PTK2|RBX1|RHOA|SMARCA4|SOCS1|STUB1|TRIO| } \\
\text { WNT5A|YWHAZ }\end{array}$ \\
\hline $\begin{array}{l}\text { Metabolism of } \\
\text { proteins }\end{array}$ & 28 & $\begin{array}{l}\text { B3GNTL1|CCT3|CCT6A|CCT8|CTSD|DDIT3|DNAJC3|EEF1A1|EEF1G|EIF2S1|EIF2S2|EIF3K|EIF4E|EIF5A|FBXW5| } \\
\text { GLB1|GRPEL2|HSPA9|HSPD1|IGFBP3|PFDN1|PIGA|RPL36|RPS16|RPS9|SEC61G|TSPYL2|TUBB4B }\end{array}$ \\
\hline $\begin{array}{l}\text { Developmental } \\
\text { Biology }\end{array}$ & 25 & $\begin{array}{c}\text { AKT2|ARPC2|ARPC5|CDC42|CDK1|CREB1|CTNNA2|HDAC3|HRAS|HSP90AA1|HSPA8||ITGA1|MED11|MED4|MY } \\
\text { H10|NRTN|PFN1|PSENEN|PTK2|RHOA|RPS6KA6|SDCBP|SIAH2|TRIO|TUBB4B }\end{array}$ \\
\hline & & Bisphenol A interacting common E2- and NRF1-target genes \\
\hline KEGG Pathway & $\begin{array}{l}\text { Number of } \\
\text { genes: }\end{array}$ & Annotated Genes \\
\hline Metabolism & 85 & 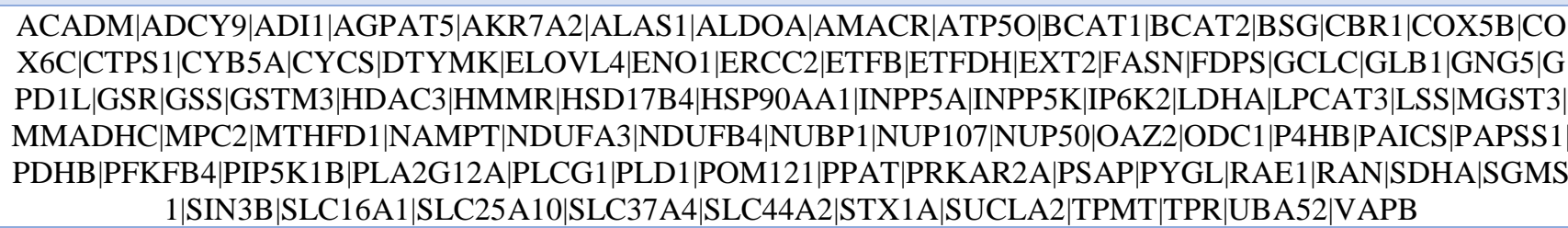 \\
\hline Disease & 84 & $\begin{array}{l}\text { ADCY9|AKT2|ALDOA|AP1S1|CCNT2|CDC42|CDK1|CDKN2B|CHMP2A|CHMP2B|CHMP5|CREB1|CSK|CSNK1A1| } \\
\text { CTBP1|CYB5A|ENO1|ERCC2|EXT2|FASN|FZD4|GLB1|GTF2E2|HDAC2|HDAC3|HDAC4|HMMR|HRAS|HSP90AA1 } \\
\text { |IRS1|MAP2K7|MKNK1|MMADHC|MTHFD1|NAMPT|NPM1|NUP107|NUP50|OS9|P4HB|PAPSS1|PFKFB4|PIP5K1B| } \\
\text { PLCG1|POLR2A|POM121|PPIA|PRKAR2A|PSENEN|PYGL|RAC1|RAE1|RAF1|RAN|RBX1|RHOA|RPL13A|RPL14|R } \\
\text { PL36|RPS13|RPS16|RPS21|RPS29|RPS9|SHC1|SLC25A10|SLC37A4|SMARCA4|SNW1|SOS1|SRC|STUB1|STX1A|TA } \\
\text { F12|TAF5|TCEB2|TGIF1|TNKS2|TPR|UBA52|WNT5A|XRCC5|XRCC6|YWHAZ }\end{array}$ \\
\hline $\begin{array}{c}\text { Gene } \\
\text { Expression }\end{array}$ & 71 & $\begin{array}{c}\text { CCNT2|CDKN2B|CNOT1|DHX9|DNMT1|EEF1A1|EEF1D|EEF1G|EIF2S1|EIF2S2|EIF3K|EIF4E|EIF4G1|ERCC2|ESR } \\
\text { RA|EXOSC2|EZH2|FARSB|GEMIN4|GTF2E2|HARS|HDAC2|HNRNPA1|HNRNPH1|HNRNPU|HSPA8|LSM2|MARS } \\
\text { 2|MED20|MED4|NR1D1|PCBP2|POLR1A|POLR2A|PPA1|PTBP1|RAN|RBBP4|RBBP7|RNPS1|RPL13A|RPL14|RPL36 } \\
\text { |RPS13|RPS16|RPS21|RPS29|RPS9|RUNX2|SAP18|SARS|SEC61G|SET|SIN3B|SNW1|SRRM1|SRSF1|TAF12|TAF5|T } \\
\text { ARS|TCEB2|TGIF1|THRA|TRAM1|U2AF1|UBA52|UPF1|UPF2|XPO5|YWHAZ|ZNF711 }\end{array}$ \\
\hline $\begin{array}{c}\text { Signal } \\
\text { Transduction }\end{array}$ & 68 & $\begin{array}{l}\text { ABI1|ADCY9|AKT2|ARHGDIA|BRK1|CASP2|CCNT2|CDC42|CDK1|CDKN2B|CREB1|CRK|CRKL|CSK|CSNK1A1| } \\
\text { CTBP1|DAAM1|DGKH|E2F1|EIF4E|EIF4G1|FSTL3|FZD4|GNG5|GPR37|HDAC2|HDAC3|HDAC4|HRAS|HSP90AA1| } \\
\text { IRS1|JAK2|LFNG|MAPK7|MKNK1|OS9|P4HB|PFN1|PIP51B|PLCG1|PRKAR2A|PSAP|PSENEN|PTCH1|PTK2|RAC } \\
\text { 1|RAF1|RBX1|RELA|RHOA|RIPK2|ROCK1|RPS6KA5|RPS6KB1|SHC1|SMARCA4|SNW1|SOCS1|SOS1|SRC|STARD } \\
\text { 13|STUB1|TGIF1|THBS2|TNKS2|UBA52|WNT5A|YWHAZ }\end{array}$ \\
\hline
\end{tabular}




\begin{tabular}{|c|c|c|}
\hline Immune System & 66 & $\begin{array}{c}\text { ABI1|ADCY9|AKT2|ANAPC1|ANAPC11|AP1S1|ARPC2|ARPC5|BRK1|CASP2|CDC34|CDC42|CDK1|CREB1|CRK|C } \\
\text { RKL|CSK|CTSD|DHX9|DYNLL1|EIF4E|EIF4G1|FADD|FZR1|HRAS|HSP90AA1|IP6K2|IRS1|JAK2|KIF18A|KIF4A|M } \\
\text { AP2K7|MAPK7|NUP107|NUP50|PCBP2|PELI1|PLCG1|PLD1|POM121|PRKAR2A|PRKDC|PTK2|PVR|RAC1|RAE1|R } \\
\text { AF1|RBX1|RELA|RIPK2|RNF19B|RPS6KA5|SEC61G|SHC1|SOCS1|SOS1|SRC|STUB1|TCEB2|TPR|TUBB4B|UBA52| } \\
\text { UBE2D4|XRCC5|XRCC6|YWHAZ }\end{array}$ \\
\hline Cell Cycle & 61 & $\begin{array}{c}\text { ANAPC1|ANAPC11|ATM|AURKA|AURKB|BUB1|CCNB1|CCNE1|CDC7|CDK1|CDKN2B|CDKN2D|CDT1|CENPN| } \\
\text { CKS1B|CLASP2|DKC1|DSN1|DYNLL1|E2F1|ERCC6L|FBXO5|FZR1|GOLGA2|HSP90AA1|KIF18A|LIN37|MAD2L1| } \\
\text { MCM2|MCM4|MCM6|MLH3|NEK2|NPM1|NUP107|NUP50|OIP5|ORC6|PCNA|PLK4|POLA1|POM121|PPP1R12A|RA } \\
\text { B1B|RAD50|RAE1|RBBP4|RBBP7|RBBP8|RBL2|RSF1|SDCCAG8|SET|SMC4|SPC24|SPDL1|TPR|TUBB|TUBB4B|U } \\
\text { BA52|ZWINT }\end{array}$ \\
\hline $\begin{array}{l}\text { Metabolic } \\
\text { pathways }\end{array}$ & 58 & $\begin{array}{l}\text { ACADM|ADI1|ALAS1|ALDOA|AMACR|ATP5O|BCAT1|BCAT2|CBR1|COX5B|COX6C|CTPS1|HAD1|DGKH|DNM } \\
\text { T1|DTYMK|ENO1|EXT2|FASN|FBL|FDPS|FUT8|GAA|GCLC|GFPT1|GLB1|GSS|HSD17B4|INPP5A|INPP5K|LDHA|L } \\
\text { SS|MECR|MTHFD1|NDUFA3|NDUFB4|ODC1|PAICS|PAPSS1|PDHB|PGAM5|PGAP1|PIGA|PIP5K1B|PLA2G12A|PL } \\
\text { CG1|PLD1|POLA1|POLG2|POLR1A|POLR2A|PPAT|SDHA|SGMS1|SHMT2|SUCLA2|SUFU|TGDS }\end{array}$ \\
\hline $\begin{array}{c}\text { Metabolism of } \\
\text { proteins }\end{array}$ & 47 & $\begin{array}{c}\text { B3GNTL1|CCT3|CCT6A|CCT8|CTSD|HAD1|DDIT3|DNAJB9|DNAJC3|DOHH|EEF1A1|EEF1D|EEF1G|EIF2S1|EIF2S } \\
\text { 2|EIF3K|EIF4E|EIF4G1|EIF5A|FBXW5|FUT8|GFPT1|GLB1|GRPEL2|HSPA9|HSPD1|IGFBP3|MANEA|PFDN1|PGAP1 } \\
\text { |PIGA|RPL13A|RPL14|RPL36|RPS13|RPS16|RPS21|RPS29|RPS9|SEC61G|SHC1|STX1A|THBS2|TRAM1|TSPYL2|TU } \\
\text { BB4B|UBA52 }\end{array}$ \\
\hline $\begin{array}{c}\text { Developmental } \\
\text { Biology }\end{array}$ & 41 & $\begin{array}{c}\text { ABLIM2|AKT2|ARPC2|ARPC5|CDC42|CDK1|CLASP2|CREB1|CRMP1|CTNNA2|DPYSL2|HDAC3|HRAS|HSP90AA } \\
\text { 1|HSPA8|ITG1|KIF4A|MED11|MED18|MED20|MED4|MYH10|MYH14|NRTN|PFN1|PLCG1|PSENEN|PTK2|RAC1| } \\
\text { RAF1|RHOA|ROCK1|RPS6KA5|RPS6KA6|SCN3B|SDCBP|SIAH2|SOS1|SPTAN1|SRC|TUBB4B }\end{array}$ \\
\hline $\begin{array}{l}\text { Pathways in } \\
\text { cancer }\end{array}$ & 34 & $\begin{array}{l}\text { AKT2|ARAF|CCNE1|CDC42|CDKN2B|CKS1B|CKS2|CRK|CRKL|CTBP1|CTNNA2|CYCS|E2F1|FADD|FZD4|HDAC2 } \\
\text { |HRAS|HSP90AA1|MSH2|PLCG1|PLD1|PTCH1|PTK2|RAC1|RAF1|RASSF1|RBX1|RELA|RHOA|SOS1|SUFU|TCEB2| } \\
\text { TPR|WNT5A }\end{array}$ \\
\hline \multicolumn{3}{|r|}{ Dibutyl Phthalate interacting common E2- and NRF1-target genes } \\
\hline KEGG Pathway & $\begin{array}{l}\text { Number of } \\
\text { genes: }\end{array}$ & Annotated Genes \\
\hline Metabolism & 56 & $\begin{array}{l}\text { ACADM|ADCY9|ADI1|AKR7A2|ALAS1|ALDOA|AMACR|ATP5O|BCAT1|BCAT2|BSG|CBR1|CYB5A|CYCS|DTY } \\
\text { MK|ENO1|ETFB|ETFDH|EXT2|FASN|FDPS|GCLC|GLB1|GNG5|GSR|GSS|HDAC3|HMMR|HSD17B4|HSP90AA1|IN } \\
\text { PP5A|IP6K2|LDHA|LPCAT3|LSS|MGST3|MTHFD1|NAMPT|NDUFA3|NDUFB4|NUBP1|NUP50|OAZ2|ODC1|PAPSS } \\
\text { 1|PLA2G12A|PLD1|POM121|PRKAR2A|PYGL|RAE1|RAN|SDHA|SGMS1|SLC25A10|UBA52 }\end{array}$ \\
\hline Disease & 45 & $\begin{array}{c}\text { ADCY9|ALDOA|CCNT2|CDK1|CREB1|CSNK1A1|CTBP1|CYB5A|ENO1|EXT2|FASN|GLB1|HDAC3|HMMR|HSP90 } \\
\text { AA1|MTHFD1|NAMPT|NPM1|NUP50|PAPSS1|POLR2A|POM121|PRKAR2A|PYGL|RAE1|RAF1|RAN|RHOA|RPL13 } \\
\text { A|RPL14|RPL36|RPS13|RPS21|RPS29|SLC25A10|SMARCA4|SNW1|SRC|STUB1|TCEB2|TGIF1|TNKS2|UBA52|WN } \\
\text { T5A|XRCC5 }\end{array}$ \\
\hline
\end{tabular}




\begin{tabular}{|c|c|c|}
\hline $\begin{array}{c}\text { Gene } \\
\text { Expression }\end{array}$ & 39 & $\begin{array}{c}\text { CCNT2|DNMT1|EEF1A1|EEF1D|EIF2S1|EIF2S2|EIF4E|EIF4G1|ESRRA|EXOSC2|EZH2|GEMIN4|HNRNPA1|HNRNP } \\
\text { U|HSPA8|LSM2|MED20|POLR1A|POLR2A|PTBP1|RAN|RBBP4|RNPS1|RPL13A|RPL14|RPL36|RPS13|RPS21|RPS29 } \\
\text { |SET|SNW1|SRSF1|TARS|TCEB2|TGIF1|THRA|U2AF1|UBA52|XPO5 }\end{array}$ \\
\hline $\begin{array}{l}\text { Metabolic } \\
\text { pathways }\end{array}$ & 38 & $\begin{array}{c}\text { ACADM|ADI1|ALAS1|ALDOA|AMACR|ATP5O|BCAT1|BCAT2|CBR1|COX6A2|DNMT1|DTYMK|ENO1|EXT2|FAS } \\
\text { N|FBL|FDPS|GCLC|GFPT1|GLB1|GSS|HSD17B4|INPP5A|LDHA|LSS|MTHFD1|NDUFA3|NDUFB4|ODC1|PAPSS1|P } \\
\text { GAM5|PLA2G12A|PLD1|POLE4|POLR1A|POLR2A|SDHA|SGMS1 }\end{array}$ \\
\hline Cell Cycle & 34 & $\begin{array}{l}\text { ANAPC1|ANAPC11|APITD1|ATM|AURKB|CCNB1|CCNE1|CDC7|CDK1|CDKN2D|CDT1|CENPN|CKS1B|CLASP2|F } \\
\text { BXO5|FZR1|HSP90AA1|MCM2|MCM4|MCM6|NPM1|NUP50|OIP5|ORC6|PCNA|POM121|PPP1R12A|RAE1|RBBP4|S } \\
\text { ET|SPC24|SPDL1|UBA52|ZWINT }\end{array}$ \\
\hline $\begin{array}{c}\text { Signal } \\
\text { Transduction }\end{array}$ & 32 & $\begin{array}{l}\text { ADCY9|ARHGDIA|CCNT2|CDK1|CREB1|CSNK1A1|CTBP1|DAAM1|EIF4E|EIF4G1|GNG5|HDAC3|HSP90AA1|JAK } \\
\text { 2|LFNG|MEF2A|PRDM4|PRKAR2A|PTCH1|RAF1|RHOA|SMARCA4|SNW1|SOCS1|SRC|STUB1|TGIF1|THBS2|TNK } \\
\text { S2|TRIO|UBA52|WNT5A }\end{array}$ \\
\hline Immune System & 31 & $\begin{array}{l}\text { ADCY9|ANAPC1|ANAPC11|ARPC2|ARPC5|CDK1|CREB1|CTSD|EIF4E|EIF4G1|FADD|FZR1|HSP90AA1|IP6K2|JA } \\
\text { K2|MEF2A|NUP50|PLD1|POM121|PRKAR2A|PRKDC|PVR|RAE1|RAF1|RNF19B|SOCS1|SRC|STUB1|TCEB2|UBA5 } \\
\text { 2|XRCC5 }\end{array}$ \\
\hline $\begin{array}{l}\text { Metabolism of } \\
\text { proteins }\end{array}$ & 25 & $\begin{array}{c}\text { CCT3|CTSD|DDIT3|DNAJB9|DNAJC3|EEF1A1|EEF1D|EIF2S1|EIF2S2|EIF4E|EIF4G1|FBXW5|GFPT1|GLB1|HSPA9| } \\
\text { HSPD1|PFDN1|RPL13A|RPL14|RPL36|RPS13|RPS21|RPS29|THBS2|UBA52 }\end{array}$ \\
\hline $\begin{array}{l}\text { Mitotic M- } \\
\text { M/G1 phases }\end{array}$ & 23 & $\begin{array}{l}\text { ANAPC1|ANAPC11|APITD1|AURKB|CCNB1|CDC7|CDK1|CDT1|CENPN|CLASP2|FBXO5|MCM2|MCM4|MCM6|N } \\
\text { UP50|ORC6|POM121|RAE1|SET|SPC24|SPDL1|UBA52|ZWINT }\end{array}$ \\
\hline $\begin{array}{l}\text { Developmental } \\
\text { Biology }\end{array}$ & 21 & $\begin{array}{c}\text { ARPC2|ARPC5|CDK1|CLASP2|CREB1|CTNNA2|DPYSL2|HDAC3|HSP90AA1|HSPA8|ITGA1|MED20|MEF2A|MYH } \\
\text { 10|NRTN|RAF1|RHOA|SCN3B|SDCBP|SRC|TRIO }\end{array}$ \\
\hline & & Diethylhexyl Phthalate interacting common E2- and NRF1-target genes \\
\hline KEGG Pathway & $\begin{array}{l}\text { Number of } \\
\text { Genes: }\end{array}$ & Annotated Genes \\
\hline Metabolism & 21 & $\begin{array}{l}\text { ACADM|ALAS1|ALDOA|BCAT2|BSG|CYCS|FASN|FDPS|GSR|GSTM3|HSD17B4|HSP90AA1|LDHA|LSS|MMADH } \\
\text { C|NDUFA3|ODC1|PAPSS1|PLD1|PYGL|SLC37A4 }\end{array}$ \\
\hline $\begin{array}{l}\text { Metabolic } \\
\text { pathways }\end{array}$ & 15 & ACADM|ALAS1|ALDOA|BCAT2|DNMT1|FASN|FDPS|HSD17B4|LDHA|LSS|NDUFA3|ODC1|PAPSS1|PLD1|POLE4 \\
\hline Disease & 15 & $\begin{array}{c}\text { AKT2|ALDOA|FASN|HDAC2|HDAC4|HSP90AA1|IRS1|MMADHC|NPM1|PAPSS1|PYGL|RBX1|RPL36|RPS13|SLC3 } \\
\text { 7A4 }\end{array}$ \\
\hline Immune System & 12 & AKT2|ANAPC11|CDC34|DYNLL1|EIF4G1|HSP90AA1|IRS1|KIF18A|PLD1|RBX1|SEC61G|TUBB4B \\
\hline $\begin{array}{l}\text { Metabolism of } \\
\text { proteins }\end{array}$ & 11 & СCT3|CCT6A|DNAJB9|EIF4G1|GRPEL2|HSPA9|HSPD1|RPL36|RPS13|SEC61G|TUBB4B \\
\hline Cell Cycle & 9 & ANAPC11|CCNB1|CCNE1|DYNLL1|HSP90AA1|KIF18A|NPM1|TUBB4B|ZWINT \\
\hline $\begin{array}{l}\text { Cellular } \\
\text { responses to } \\
\text { stress }\end{array}$ & 8 & ANAPC11|CBX2|CCNE1|CYCS|GSR|HSP90AA1|HSPA8|RBX1 \\
\hline
\end{tabular}




\begin{tabular}{|c|c|c|}
\hline $\begin{array}{l}\text { Pathways in } \\
\text { cancer }\end{array}$ & 8 & AKT2|CCNE1|CYCS|HDAC2|HSP90AA1|MSH2|PLD1|RBX1 \\
\hline $\begin{array}{l}\text { Developmental } \\
\text { Biology }\end{array}$ & 8 & AKT2|DPYSL2|HSP90AA1|HSPA8|ITGA1|SCN3B|SIAH2|TUBB4B \\
\hline Cell cycle & 5 & ANAPC11|CCNB1|CCNE1|HDAC2|RBX1 \\
\hline \multicolumn{3}{|r|}{ Polychlorinated Biphenyls interacting common E2- and NRF1-target genes } \\
\hline KEGG Pathway & $\begin{array}{l}\text { Number of } \\
\text { Genes: }\end{array}$ & Annotated Genes \\
\hline Cell Cycle & 13 & AURKA|AURKB|CDK1|CDT1|CENPN|CKS1B|FBXO5|MCM2|MCM6|PCNA|PLK4|SMC4|ZWINT \\
\hline $\begin{array}{l}\text { Mitotic M- } \\
\text { M/G1 phases }\end{array}$ & 9 & AURKB|CDK1|CDT1|CENPN|FBXO5|MCM2|MCM6|SMC4|ZWINT \\
\hline $\begin{array}{l}\text { DNA } \\
\text { Replication }\end{array}$ & 4 & CDT1|MCM2|MCM6|PCNA \\
\hline Cell cycle & 4 & CDK1|MCM2|MCM6|PCNA \\
\hline $\begin{array}{l}\text { DNA } \\
\text { replication }\end{array}$ & 3 & MCM2|MCM6|PCNA \\
\hline $\begin{array}{c}\text { DNA } \\
\text { replication and } \\
\text { repair }\end{array}$ & 3 & CDK1|PCNA|UNG \\
\hline $\begin{array}{l}\text { Pancreatic } \\
\text { cancer }\end{array}$ & 3 & ARAF|CDC42|RAF1 \\
\hline \multicolumn{3}{|r|}{ Cadmium interacting common E2- and NRF1-target genes } \\
\hline KEGG Pathway & $\begin{array}{l}\text { Number of } \\
\text { Genes: }\end{array}$ & Annotated Genes \\
\hline Metabolism & 26 & $\begin{array}{l}\text { ACADM|ALDOA|AUH|BCAT1|COX5B|COX6C|CYCS|DTYMK|ENO1|FASN|GCLC|GSR|GSS|GSTM3|HSD17B4|HS } \\
\text { P90AA1|INPP5K|LDHA|MGST3|OAZ2|PAICS|RAN|SDHA|SGMS1|SLC16A1|SLC37A4 }\end{array}$ \\
\hline $\begin{array}{l}\text { Gene } \\
\text { Expression }\end{array}$ & 25 & $\begin{array}{l}\text { CCNT2|CDKN2B|DNMT1|EEF1A1|EEF1D|EEF1G|EXOSC2|HARS|HNRNPA1|HNRNPH1|HSPA8|LSM2|MARS2|ME } \\
\text { D20|RAN|RPL10|RPL13A|RPL14|RUNX2|SRRM1|TAF12|TCEB2|THRA|XPO5|YWHAZ }\end{array}$ \\
\hline Disease & 24 & $\begin{array}{c}\text { ALDOA|CCNT2|CDC42|CDK1|CDKN2B|CREB1|CTBP1|ENO1|FASN|HSP90AA1|IRS1|OS9|PPIA|RAC1|RAF1|RAN| } \\
\text { RPL10|RPL13A|RPL14|SHC1|SLC37A4|TAF12|TCEB2|YWHAZ }\end{array}$ \\
\hline $\begin{array}{c}\text { Signal } \\
\text { Transduction }\end{array}$ & 21 & $\begin{array}{c}\text { ARHGDIA|CCNT2|CDC42|CDK1|CDKN2B|CREB1|CTBP1|E2F1|FSTL3|GPR37|HSP90AA1|IRS1|OS9|PTK2|RAC1|R } \\
\text { AF1|RELA|ROCK1|RPS6KB1|SHC1|YWHAZ }\end{array}$ \\
\hline $\begin{array}{l}\text { Metabolic } \\
\text { pathways }\end{array}$ & 20 & $\begin{array}{c}\text { ACADM|ALDOA|AUH|BCAT1|COX5B|COX6C|DNMT1|DTYMK|ENO1|FASN|GAA|GCLC|GSS|HSD17B4|INPP5K| } \\
\text { LDHA|PAICS|SDHA|SGMS1|SHMT2 }\end{array}$ \\
\hline
\end{tabular}




\begin{tabular}{|c|c|c|}
\hline Immune System & 18 & $\begin{array}{c}\text { CDC42|CDK1|CREB1|CTSD|HSP90AA1|IRS1|KIF4A|PELI1|PTK2|PVR|RAC1|RAF1|RELA|RNF19B|SHC1|TCEB2|U } \\
\text { BE2D4|YWHAZ }\end{array}$ \\
\hline Cell Cycle & 17 & $\begin{array}{c}\text { AURKA|BUB1|CCNB1|CCNE1|CDK1|CDKN2B|CKS1B|E2F1|FBXO5|HSP90AA1|MAD2L1|MCM2|MCM4|MCM6|N } \\
\text { EK2|OIP5|SMC4 }\end{array}$ \\
\hline $\begin{array}{l}\text { Pathways in } \\
\text { cancer }\end{array}$ & 15 & CCNE1|CDC42|CDKN2B|CKS1B|CTBP1|CYCS|E2F1|HSP90AA1|MSH2|PTK2|RAC1|RAF1|RASSF1|RELA|TCEB2 \\
\hline $\begin{array}{l}\text { Developmental } \\
\text { Biology }\end{array}$ & 15 & CDC42|CDK1|CREB1|HSP90AA1|HSPA8|KIF4A|MED20|MYH10|MYH14|NRTN|PTK2|RAC1|RAF1|ROCK1|SPTAN \\
\hline $\begin{array}{l}\text { Metabolism of } \\
\text { proteins }\end{array}$ & 12 & CTSD|DDIT3|DNAJB9|EEF1A1|EEF1D|EEF1G|EIF5A|HSPD1|RPL10|RPL13A|RPL14|SHC1 \\
\hline \multicolumn{3}{|r|}{ Arsenic interacting common E2- and NRF1-target genes } \\
\hline KEGG Pathway & $\begin{array}{l}\text { Number of } \\
\text { Genes: }\end{array}$ & Annotated Genes \\
\hline Metabolism & 33 & $\begin{array}{c}\text { ADCY9|ALDOA|CYCS|ENO1|ERCC2|ETFB|ETFDH|FASN|GCLC|GSR|GSS|GSTM3|HSD17B4|HSP90AA1|INPP5A|I } \\
\text { P6K2|LDHA|MMADHC|NUBP1|P4HB|PAICS|PLCG1|PLD1|POM121|PPAT|PRKAR2A|SDHA|SGMS1|SLC44A2|STX } \\
\text { 1A|SUCLA2|TPMT|UBA52 }\end{array}$ \\
\hline Immune System & 30 & $\begin{array}{l}\text { ADCY9|ARPC2|CASP2|CDC42|CDK1|CREB1|CTSD|FZR1|HRAS|HSP90AA1|IP6K2|JAK2|KIF4A|MAPK7|PCBP2|PE } \\
\text { LI1|PLCG1|PLD1|POM121|PRKAR2A|PRKDC|PTK2|PVR|RAF1|SOS1|SRC|TUBB4B|UBA52|UBE2D4|XRCC5 }\end{array}$ \\
\hline Disease & 27 & $\begin{array}{l}\text { ADCY9|ALDOA|CDC42|CDK1|CDKN2B|CREB1|CTBP1|ENO1|ERCC2|FASN|HDAC4|HRAS|HSP90AA1|MMADHC } \\
\text { |NPM1|P4HB|PLCG1|POM121|PRKAR2A|RAF1|RPL36|SMARCA4|SOS1|SRC|STX1A|UBA52|XRCC5 }\end{array}$ \\
\hline $\begin{array}{l}\text { Signal } \\
\text { Transduction }\end{array}$ & 24 & $\begin{array}{r}\text { ADCY9|CASP2|CDC42|CDK1|CDKN2B|CREB1|CTBP1|E2F1|HDAC4|HRAS|HSP90AA1|JAK2|MAPK7|P4HB|PLCG } \\
\text { 1|PRKAR2A|PTCH1|PTK2|RAF1|ROCK1|SMARCA4|SOS1|SRC|UBA52 }\end{array}$ \\
\hline $\begin{array}{l}\text { Developmental } \\
\text { Biology }\end{array}$ & 22 & $\begin{array}{l}\text { ABLIM2|ARPC2|CDC42|CDK1|CREB1|HRAS|HSP90AA1|HSPA8|KIF4A|MED18|MYH10|MYH14|NRTN|PLCG1|PT } \\
\text { K2|RAF1|ROCK1|SCN3B|SIAH2|SOS1|SRC|TUBB4B }\end{array}$ \\
\hline $\begin{array}{l}\text { Metabolic } \\
\text { pathways }\end{array}$ & 20 & $\begin{array}{c}\text { ALDOA|COX6A2|DNMT1|ENO1|FASN|FUT8|GCLC|GSS|HSD17B4|INPP5A|LAP3|LDHA|PAICS|PLCG1|PLD1|PPA } \\
\text { T|SDHA|SGMS1|SUCLA2|SUFU }\end{array}$ \\
\hline Cell Cycle & 19 & $\begin{array}{c}\text { ATM|AURKA|CCNB1|CCNE1|CDK1|CDKN2B|CDKN2D|E2F1|FZR1|HSP90AA1|MAD2L1|NPM1|PCNA|POM121|S } \\
\text { ET|SPDL1|TUBB|TUBB4B|UBA52 }\end{array}$ \\
\hline $\begin{array}{l}\text { Gene } \\
\text { Expression }\end{array}$ & 19 & $\begin{array}{c}\text { CDKN2B|CNOT1|DNMT1|EEF1G|ERCC2|EZH2|HNRNPA1|HSPA8|LSM2|PCBP2|PTBP1|RNPS1|RPL36|RUNX2|SE } \\
\text { T|UBA52|UPF1|VARS2|ZNF610 }\end{array}$ \\
\hline $\begin{array}{l}\text { Pathways in } \\
\text { cancer }\end{array}$ & 17 & $\begin{array}{l}\text { CCNE1|CDC42|CDKN2B|CTBP1|CYCS|E2F1|HRAS|HSP90AA1|MSH2|PLCG1|PLD1|PTCH1|PTK2|RAF1|RASSF1|S } \\
\text { OS1|SUFU }\end{array}$ \\
\hline $\begin{array}{l}\text { Cellular } \\
\text { responses to } \\
\text { stress }\end{array}$ & 16 & 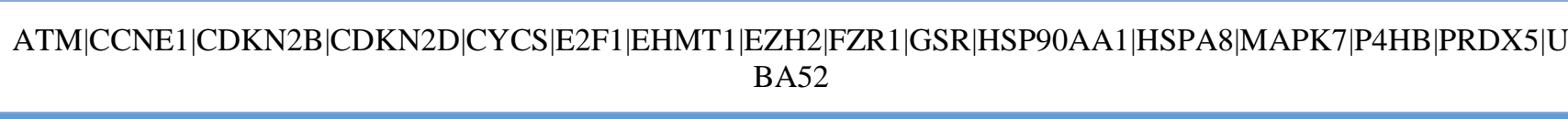 \\
\hline
\end{tabular}


Manganese interacting common E2- and NRF1-target genes

\begin{tabular}{|c|c|c|}
\hline KEGG Pathway & $\begin{array}{l}\text { Number of } \\
\text { Genes: }\end{array}$ & Annotated Genes \\
\hline $\begin{array}{l}\text { Cellular } \\
\text { responses to } \\
\text { stress }\end{array}$ & (2) & ATM|CYCS|GSR|HSPA8|P4HB|RELA \\
\hline $\begin{array}{c}\text { Developmental } \\
\text { Biology }\end{array}$ & 5 & CREB1|HSPA8|SCN3B|SPTAN1|SRC \\
\hline Tuberculosis & 4 & CREB 1|CYCS|RELA|SRC \\
\hline $\begin{array}{l}\text { p53 signaling } \\
\text { pathway }\end{array}$ & 3 & ATM|CYCS|PPM1D \\
\hline $\begin{array}{c}\text { Small cell lung } \\
\text { cancer }\end{array}$ & 3 & CKS2|CYCS|RELA \\
\hline Apoptosis & 3 & ATM|CYCS|RELA \\
\hline Cell cycle & 3 & ATM|BUB 1|MCM4 \\
\hline
\end{tabular}


Table 2: Interaction of Estrogenic Endocrine Disrupting Chemicals Modified Genes with

Estrogen Signaling and NRF1 network genes in the Individual Neurodegenerative Disease

Endocrine Disrupting Individual EDC responsive modified genes common to both NRF1 Chemical (EDC) and E2 target. * Indicates E2 responsive.

Alzheimer's Disease (AD)

\begin{tabular}{|c|c|}
\hline 17 beta-estradiol & 6 genes: APBB2 | DPYSL2 | EIF2S1|ENO1|MAPT|PAXIP1 \\
\hline Ethinyl Estradiol & 6 genes: $A P B B 2^{*}\left|E I F 2 S 1^{*}\right| E N O 1^{*}|I D E| M A P T^{*} \mid P A X I P 1^{*}$ \\
\hline Bisphenol A & $\begin{array}{c}8 \text { genes: } A P B B 2 *|D P Y S L 2 *| E I F 2 S 1^{*}\left|E N O 1^{*}\right| I D E\left|M A P T^{*}\right| \\
P A X I P 1 * \mid S L C 30 A 4\end{array}$ \\
\hline Dibutyl Phthalate & 4 genes: $D P Y S L 2 *|E I F 2 S 1 *| E N O 1 * \mid I D E$ \\
\hline Diethylhexyl Phthalate & 2 genes: $D P Y S L 2 * \mid M A P T^{*}$ \\
\hline Cadmium & 2 genes: $E N O 1^{*} \mid S L C 3 O A 4$ \\
\hline Arsenic & 2 genes: $E N O 1 * M A P T$ \\
\hline Manganese & 1 gene: $E N O 1^{*}$ \\
\hline \multicolumn{2}{|r|}{ Parkinson's Disease (PD) } \\
\hline 17 beta-estradiol & 8 genes: $H S P A 9|M A P T| R P L 14$ \\
\hline Ethinyl Estradiol & 3 genes: $H S P A 9^{*}\left|M A P T^{*}\right|$ PINK1 \\
\hline Bisphenol A & $\begin{array}{c}8 \text { genes: GAK | HSPA9*|MAPT*|PARK2 | PARK7| PINK1 } \mid \\
\text { RPL14*|VPS35 }\end{array}$ \\
\hline Dibutyl Phthalate & 4 genes: HSPA9*|PARK2 | PARK7|RPL14* \\
\hline Diethylhexyl Phthalate & 3 genes: HSPA9* $\left|M A P T^{*}\right| P A R K 2$ \\
\hline Cadmium & 3 genes: PARK2 | PINK1|RPL14* \\
\hline Arsenic & 4 genes: $G A K\left|H S P A 9^{*}\right| M A P T^{*} \mid P A R K 2$ \\
\hline Manganese & 2 genes: PARK2 | PARK7 \\
\hline
\end{tabular}




\begin{tabular}{|c|c|}
\hline \multicolumn{2}{|c|}{ Huntington's Disease (HD) } \\
\hline 17 beta-estradiol & 2 genes: $A I F M 1 \mid I P 6 K 2$ \\
\hline Ethinyl Estradiol & 1 gene: $A I F M 1 *$ \\
\hline Bisphenol A & 2 genes: $A I F M 1 * \mid I P 6 K 2 *$ \\
\hline Dibutyl Phthalate & 1 gene: $I P 6 K 2 *$ \\
\hline Cadmium & 1 gene: $A I F M 1 *$ \\
\hline Arsenic & 1 gene: $I P 6 K 2 *$ \\
\hline \multicolumn{2}{|c|}{ Amyotrophic Lateral Sclerosis (ALS) } \\
\hline 17 beta-estradiol & 1 gene: $G S R \mid C H M P 2 B$ \\
\hline Ethinyl Estradiol & 1 gene: $G S R^{*}$ \\
\hline Bisphenol A & 2 genes: $G S R^{*}\left|C H M P 2 B^{*}\right| U N C 13 A$ \\
\hline Dibutyl Phthalate & 1 gene: $G S R^{*}$ \\
\hline Diethylhexyl Phthalate & 1 gene: $G S R^{*}$ \\
\hline Cadmium & 1 gene: $G S R^{*}$ \\
\hline Arsenic & 1 gene: $G S R^{*}$ \\
\hline Manganese & 1 gene: $G S R$ \\
\hline \multicolumn{2}{|c|}{ Autism Spectrum Disorder (ASD) } \\
\hline 17 beta-estradiol & 3 genes: $C I R B P|P C D H 9| G T F 2 I$ \\
\hline Ethinyl Estradiol & 2 genes: $C I R B P * \mid G T F 2 I^{*}$ \\
\hline Bisphenol A & 3 genes: $C I R B P^{*}\left|G T F 2 I^{*}\right| P C D H 9^{*}$ \\
\hline Polychlorinated Biphenyls & 1 gene: $C I R B P^{*}$ \\
\hline
\end{tabular}




\begin{tabular}{|c|c|}
\hline Cadmium & 1 gene: $C I R B P^{*}$ \\
\hline Arsenic & 1 gene: $P C D H 9^{*}$ \\
\hline $\mathbf{1 7}$ beta-estradiol & Brain Neoplasms \\
\hline Ethinyl Estradiol & 3 genes: $P C N A|P T C H 1| R E L A$ \\
\hline Bisphenol A & 2 genes: $P C N A^{*} \mid P T C H 1^{*}$ \\
\hline Dibutyl Phthalate & 4 genes: $E M L 4\left|P C N A^{*}\right| P T C H 1^{*} \mid R E L A^{*}$ \\
\hline Polychlorinated Biphenyls & 2 genes: $P C N A^{*} \mid P T C H 1^{*}$ \\
\hline Cadmium & 1 gene: $P C N A$ \\
\hline Arsenic & 1 gene: $R E L A^{*}$ \\
\hline Manganese & 2 genes: $P C N A^{*} \mid P T C H 1^{*}$ \\
\hline & 1 gene: $R E L A^{*}$ \\
\hline & \\
\hline
\end{tabular}

References

1. Kiyama R, Wada-Kiyama Y. Estrogenic endocrine disruptors: Molecular mechanisms of action. Environ Int. 2015. doi:10.1016/j.envint.2015.05.012.

2. Diamanti-Kandarakis E, Bourguignon J-P, Giudice LC, et al. Endocrine-disrupting chemicals: an Endocrine Society scientific statement. Endocr Rev. 2009;30(4):293342. doi:10.1210/er.2009-0002.

3. Chen A, Dietrich KN, Huo X, Ho SM. Developmental neurotoxicants in e-waste: An emerging health concern. Environ Health Perspect. 2011. doi:10.1289/ehp.1002452. 
4. Bergman A, Heindel JJ, Jobling S, Kidd KA, Zoeller TR. State of the science of endocrine disrupting chemicals. World Heal Organ United Nations Environ Program. 2012:1-38. http://apps.who.int/iris/handle/10665/78102.

5. Weiss B. Endocrine disruptors as a threat to neurological function. J Neurol Sci. 2011;305(1-2):11-21. doi:10.1016/j.jns.2011.03.014.

6. Kajta M, Wojtowicz AK. Impact of endocrine-disrupting chemicals on neural development and the onset of neurological disorders. Pharmacol Reports. 2013;65(6):1632-1639. doi:10.1016/S1734-1140(13)71524-X.

7. León-Olea M, Martyniuk CJ, Orlando EF, et al. Current concepts in neuroendocrine disruption. Gen Comp Endocrinol. 2014;203:158-173. doi:10.1016/j.ygcen.2014.02.005.

8. Masuo Y, Ishido M. Neurotoxicity of endocrine disruptors: possible involvement in brain development and neurodegeneration. J Toxicol Environ Health B Crit Rev. 2011. doi:10.1080/10937404.2011.578557.

9. Gillies GE, McArthur S. Estrogen Actions in the Brain and the Basis for Differential Action in Men and Women: A Case for Sex-Specific Medicines. Pharmacol Rev. 2010;62(2):155-198. doi:10.1124/pr.109.002071.

10. Chakrabarti M, Haque A, Banik NL, Nagarkatti P, Nagarkatti M, Ray SK. Estrogen receptor agonists for attenuation of neuroinflammation and neurodegeneration. Brain Res Bull. 2014;109:22-31. doi:10.1016/j.brainresbull.2014.09.004.

11. Hristian C, Ruber JG, Alter W, et al. Production and actions of estrogens. $N$ Engl J Med. 2002;346(5). doi:10.1016/S0960-0760(01)00184-4.

12. Green PS, Simpkins JW. Neuroprotective effects of estrogens: Potential mechanisms of action. Int J Dev Neurosci. 2000;18(4-5):347-358. doi:10.1016/S0736-5748(00)00017-4.

13. Wise PM, Dubal DB, Wilson ME, Rau SW, Liu Y. Estrogens: trophic and protective factors in the adult brain. Front Neuroendocrinol. 2001;22(1):33-66. doi:10.1006/frne.2000.0207. 
14. Pozzi S, Benedusi V, Maggi A, Vegeto E. Estrogen action in neuroprotection and brain inflammation. Ann N Y Acad Sci. 2006;1089(2006):302-323. doi:10.1196/annals.1386.035.

15. Arevalo M-A, Azcoitia I, Garcia-Segura LM. The neuroprotective actions of oestradiol and oestrogen receptors. Neuroscience. 2015;16(1):17-29. doi:10.1038/nrn3856.

16. Leranth C, Petnehazy O, MacLusky NJ. Gonadal hormones affect spine synaptic density in the CA1 hippocampal subfield of male rats. J Neurosci. 2003;23(5):1588-1592.

17. MacLusky NJ, Hajszan T, Prange-Kiel J, Leranth C. Androgen modulation of hippocampal synaptic plasticity. Neuroscience. 2006;138(3):957-965. doi:10.1016/j.neuroscience.2005.12.054.

18. Parducz a., Hajszan T, MacLusky NJ, et al. Synaptic remodeling induced by gonadal hormones: Neuronal plasticity as a mediator of neuroendocrine and behavioral responses to steroids. Neuroscience. 2006;138(3):977-985. doi:10.1016/j.neuroscience.2005.07.008.

19. Ogiue-Ikeda M, Tanabe N, Mukai $\mathrm{H}$, et al. Rapid modulation of synaptic plasticity by estrogens as well as endocrine disrupters in hippocampal neurons. Brain Res Rev. 2008;57(2):363-375. doi:10.1016/j.brainresrev.2007.06.010.

20. Hojo Y, Murakami G, Mukai H, et al. Estrogen synthesis in the brain-Role in synaptic plasticity and memory. Mol Cell Endocrinol. 2008;290(1-2):31-43. doi:10.1016/j.mce.2008.04.017.

21. Simpkins JW, Yi KD, Yang S, Dykens J a. Mitochondrial mechanisms of estrogen neuroprotection. Biochim Biophys Acta. 2011;1800(10):1113-1120. doi:10.1016/j.bbagen.2009.11.013.Mitochondrial.

22. Arnold S, Beyer C. Neuroprotection by estrogen in the brain: The mitochondrial compartment as presumed therapeutic target. J Neurochem. 2009;110(1):1-11. doi:10.1111/j.1471-4159.2009.06133.x. 
23. Grimm A, Schmitt K, Lang UE, Mensah-Nyagan AG, Eckert A. Improvement of neuronal bioenergetics by neurosteroids: Implications for age-related neurodegenerative disorders. Biochim Biophys Acta - Mol Basis Dis. 2014;1842(12):2427-2438. doi:10.1016/j.bbadis.2014.09.013.

24. Barrett-Connor E, Goodman-Gruen D. Cognitive function and endogenous sex hormones in older women. J Am Geriatr Soc. 1999;47(11):1289-1293.

25. Low LF. Reproductive period and cognitive function in a representative sample of naturally postmenopausal women aged 60-64 years. Climacteric. 2005;8(4):380389. doi:10.1080/13697130500345240.

26. Heys M, Jiang C, Cheng KK, et al. Life long endogenous estrogen exposure and later adulthood cognitive function in a population of naturally postmenopausal women from Southern China: The Guangzhou Biobank Cohort Study. Psychoneuroendocrinology. 2011;36(6):864-873. doi:10.1016/j.psyneuen.2010.11.009.

27. Zimmerman ME, Lipton RB, Santoro N, et al. Endogenous estradiol is associated with verbal memory in nondemented older men. Brain Cogn. 2011;76(1):158-165. doi:10.1016/j.bandc.2011.01.011.

28. Cereda E, Barichella M, Cassani E, Caccialanza R, Pezzoli G. Reproductive factors and clinical features of Parkinson's disease. Parkinsonism Relat Disord. 2013;19(12):1094-1099. doi:10.1016/j.parkreldis.2013.07.020.

29. Manly JJ, Merchant CA, Jacobs DM, et al. Endogenous estrogen levels and Alzheimer's disease among postmenopausal women. Neurology. 2000;54(4):833837.

30. De Jong S, Huisman M, Sutedja N, et al. Endogenous female reproductive hormones and the risk of amyotrophic lateral sclerosis. J Neurol. 2013;260(2):507512. doi:10.1007/s00415-012-6665-5.

31. Geerlings MI, Ruitenberg A, Witteman JC, et al. Reproductive period and risk of dementia in postmenopausal women. JAMA. 2001;285(11):1475-1481. doi:10.1001/jama.285.11.1475. 
32. Schupf N, Winsten S, Patel B, et al. Bioavailable estradiol and age at onset of Alzheimer's disease in postmenopausal women with Down syndrome. Neurosci Lett. 2006;406(3):298-302. doi:10.1016/j.neulet.2006.07.062.

33. Fox M, Berzuini C, Knapp L a. Cumulative estrogen exposure, number of menstrual cycles, and Alzheimer's risk in a cohort of British women.

Psychoneuroendocrinology. 2013;38(12):2973-2982. doi:10.1016/j.psyneuen.2013.08.005.

34. Griksiene R, Ruksenas O. Effects of hormonal contraceptives on mental rotation and verbal fluency. Psychoneuroendocrinology. 2011;36(8):1239-1248. doi:10.1016/j.psyneuen.2011.03.001.

35. Beltz AM, Hampson E, Berenbaum SA. Oral Contraceptives and Cognition: A Role for Ethinyl Estradiol. Horm Behav. 2015. doi:10.1016/j.yhbeh.2015.06.012.

36. Egan KR, Gleason CE. Longer Duration of Hormonal Contraceptive Use Predicts Better Cognitive Outcomes Later in Life. J Women's Heal. 2012;21(12):12591266. doi:10.1089/jwh.2012.3522.

37. Warren AM, Gurvich C, Worsley R, Kulkarni J. A systematic review of the impact of oral contraceptives on cognition. Contraception. 2014;90(2):111-116. doi:10.1016/j.contraception.2014.03.015.

38. Maki PM, Henderson VW. Hormone therapy, dementia, and cognition: the Women's Health Initiative ten years on. Climacteric. 2012;15(3):256-262. doi:10.1016/j.biotechadv.2011.08.021.Secreted.

39. Xiao M, Cao GL, Marshall C, Hu G. Hypothesis: Multiple factors are associated with the lack of any beneficial effects of oestrogen-replacement therapy in the late postmenopausal stage. Clin Exp Pharmacol Physiol. 2010;37(8):873-876. doi:10.1111/j.1440-1681.2010.05400.x.

40. Shumaker S a, Legault C, Rapp SR, et al. Estrogen Plus Progestin and the Incidence of Dementia and Mild Cognitive Impairment in Postmenopausal Women. JAMA. 2003;289(20):2651-2662. 
41. Shumaker S a, Legault C, Kuller L, et al. Conjugated equine estrogens and incidence of probable dementia and mild cognitive impairment in postmenopausal women: Women's Health Initiative Memory Study. JAMA. 2004;291(24):29472958. doi:10.1097/01.ogx.0000140470.91959.32.

42. Resnick SM, Maki PM, Rapp SR, et al. Effects of combination estrogen plus progestin hormone treatment on cognition and affect. J Clin Endocrinol Metab. 2006;91(5):1802-1810. doi:10.1210/jc.2005-2097.

43. Resnick SM, Espeland M a., An Y, et al. Effects of conjugated equine estrogens on cognition and affect in postmenopausal women with prior hysterectomy. J Clin Endocrinol Metab. 2009;94(11):4152-4161. doi:10.1210/jc.2009-1340.

44. Almeida OP, Lautenschlager NT, Vasikaran S, Leedman P, Gelavis A, Flicker L. A 20-week randomized controlled trial of estradiol replacement therapy for women aged 70 years and older: Effect on mood, cognition and quality of life.

Neurobiology. 2006;27:141-149. doi:10.1016/j.neurobiolaging.2004.12.012.

45. Pefanco MA, Kenny AM, Kaplan RF, et al. The effect of 3-year treatment with $0.25 \mathrm{mg} /$ day of micronized 17??-estradiol on cognitive function in older postmenopausal women. J Am Geriatr Soc. 2007;55(3):426-431. doi:10.1111/j.1532-5415.2007.01085.x.

46. Viscoli CM, Brass LM, Kernan WN, Sarrel PM, Suissa S, Horwitz RI. Estrogen therapy and risk of cognitive decline: Results from the Women's Estrogen for Stroke Trial (WEST). Am J Obstet Gynecol. 2005;192(2):387-393. doi:10.1016/j.ajog.2004.08.017.

47. Yaffe K, Vittinghoff E, Ensrud KE, et al. Effects of ultra-low-dose transdermal estradiol on cognition and health-related quality of life. Arch Neurol. 2006;63(7):945-950. doi:10.1001/archneur.63.7.945.

48. Shao H, Breitner JCS, Whitmer R a., et al. Hormone therapy and Alzheimer disease dementia: New findings from the Cache County Study. Neurology. 2012;79(18):1846-1852. doi:10.1212/WNL.0b013e318271f823.

49. O’Brien J, Jackson JW, Grodstein F, Blacker D, Weuve J. Postmenopausal hormone therapy is not associated with risk of all-cause dementia and alzheimer's disease. Epidemiol Rev. 2014. doi:10.1093/epirev/mxt008. 
50. Lundin JI, Ton TG, LaCroix AZ, et al. Formulations of Hormone Therapy and Risk of Parkinson's Disease. Mov Disord. 2014;29(13):1631-. doi:10.1002/mds.26037.

51. ATSDR. Toxicological Profile for Phenol. Agency Toxic Subst Dis Regist. 2008:1269. doi:10.3109/15569529909037564.

52. Calafat AM, Ye X, Wong LY, Reidy JA, Needham LL. Exposure of the U.S. population to Bisphenol A and 4-tertiary-octylphenol: 2003-2004. Environ Health Perspect. 2008;116(1):39-44. doi:10.1289/ehp.10753.

53. Alonso-Magdalena P, Ropero AB, Soriano S, et al. Bisphenol-A acts as a potent estrogen via non-classical estrogen triggered pathways. Mol Cell Endocrinol. 2012;355(2):201-207. doi:10.1016/j.mce.2011.12.012.

54. Preciados M, Yoo C, Roy D. Estrogenic Endocrine Disrupting Chemicals Influencing NRF1 Regulated Gene Networks in the Development of Complex Human Brain Diseases. Int J Mol Sci. 2016;17(12):2086. doi:10.3390/ijms17122086.

55. Sun Y, Nakashima MN, Takahashi M, Kuroda N, Nakashima K. Determination of bisphenol A in rat brain by microdialysis and column switching high-performance liquid chromatography with fluorescence detection. Biomed Chromatogr. 2002;16(5):319-326. doi:10.1002/bmc.161.

56. Kim CS, Sapienza PP, Ross I a, Johnson W, Luu HMD, Hutter JC. Distribution of bisphenol A in the neuroendocrine organs of female rats. Toxicol Ind Health. 2004;20:41-50. doi:10.1191/0748233704th186oa.

57. Bowman RE, Luine V, Diaz Weinstein S, Khandaker H, DeWolf S, Frankfurt M. Bisphenol-A exposure during adolescence leads to enduring alterations in cognition and dendritic spine density in adult male and female rats. Horm Behav. 2015;69:89-97. doi:10.1016/j.yhbeh.2014.12.007.

58. Diaz Weinstein S, Villafane JJ, Juliano N, Bowman RE. Adolescent exposure to Bisphenol-A increases anxiety and sucrose preference but impairs spatial memory in rats independent of sex. Brain Res. 2013;1529:56-65. doi:10.1016/j.brainres.2013.07.018. 
59. Elsworth JD, Jentsch JD, VandeVoort CA, Roth RH, Eugene Redmond D, Leranth C. Prenatal exposure to bisphenol A impacts midbrain dopamine neurons and hippocampal spine synapses in non-human primates. Neurotoxicology. 2013;35(1):113-120. doi:10.1016/j.neuro.2013.01.001.

60. Fan Y, Ding S, Ye X, et al. Does preconception paternal exposure to a physiologically relevant level of bisphenol A alter spatial memory in an adult rat? Horm Behav. 2013;64(4):598-604. doi:10.1016/j.yhbeh.2013.08.014.

61. Inagaki T, Frankfurt M, Luine V. Estrogen-induced memory enhancements are blocked by acute bisphenol A in adult female rats: Role of dendritic spines. Endocrinology. 2012;153(7):3357-3367. doi:10.1210/en.2012-1121.

62. Jang YJ, Park HR, Kim TH, et al. High dose bisphenol A impairs hippocampal neurogenesis in female mice across generations. Toxicology. 2012;296(1-3):73-82. doi:10.1016/j.tox.2012.03.007.

63. Johnson SA, Javurek AB, Painter MS, et al. Effects of developmental exposure to bisphenol A on spatial navigational learning and memory in rats: A CLARITYBPA study. Horm Behav. 2015. doi:10.1016/j.yhbeh.2015.09.005.

64. Kumar D, Kumar Thakur M. Perinatal exposure to bisphenol-A impairs spatial memory through upregulation of neurexin 1 and neuroligin 3 expression in male mouse brain. PLoS One. 2014;9(10):1-9. doi:10.1371/journal.pone.0110482.

65. Matsuda S, Matsuzawa D, Ishii D, Tomizawa H, Sajiki J, Shimizu E. Perinatal exposure to bisphenol A enhances contextual fear memory and affects the serotoninergic system in juvenile female mice. Horm Behav. 2013;63(5):709-716. doi:10.1016/j.yhbeh.2013.03.016.

66. Wang C, Niu R, Zhu Y, et al. Changes in memory and synaptic plasticity induced in male rats after maternal exposure to bisphenol A. Toxicology. 2014;322:51-60. doi:10.1016/j.tox.2014.05.001.

67. Wang C, Li Z, Han H, et al. Impairment of object recognition memory by maternal bisphenol A exposure is associated with inhibition of Akt and ERK/CREB/BDNF pathway in the male offspring hippocampus. Toxicology. 2016;341-343:56-64. doi:10.1016/j.tox.2016.01.010. 
68. Xu X Bin, He Y, Song C, et al. Bisphenol a regulates the estrogen receptor alpha signaling in developing hippocampus of male rats through estrogen receptor. Hippocampus. 2014;24(12):1570-1580. doi:10.1002/hipo.22336.

69. Xu X, Liu X, Zhang Q, et al. Sex-specific effects of bisphenol-A on memory and synaptic structural modification in hippocampus of adult mice. Horm Behav. 2013;63(5):766-775. doi:10.1016/j.yhbeh.2013.03.004.

70. Zhang Q, Xu X, Li T, et al. Exposure to bisphenol-A affects fear memory and histone acetylation of the hippocampus in adult mice. Horm Behav. 2014;65(2):106-113. doi:10.1016/j.yhbeh.2013.12.004.

71. Eilam-Stock T, Serrano P, Frankfurt M, Luine V. Bisphenol-A impairs memory and reduces dendritic spine density in adult male rats. Behav Neurosci. 2012;126(1):175-185. doi:10.1037/a0025959.

72. Kim ME, Park HR, Gong EJ, Choi SY, Kim HS, Lee J. Exposure to bisphenol A appears to impair hippocampal neurogenesis and spatial learning and memory. Food Chem Toxicol. 2011;49(12):3383-3389. doi:10.1016/j.fct.2011.09.017.

73. Elsworth JD, Jentsch JD, Groman SM, Roth RH, Redmond ED, Leranth C. Low circulating levels of bisphenol-A induce cognitive deficits and loss of asymmetric spine synapses in dorsolateral prefrontal cortex and hippocampus of adult male monkeys. J Comp Neurol. 2015;523(8):1248-1257. doi:10.1002/cne.23735.

74. Neese SL, Bandara SB, Schantz SL. Working memory in bisphenol-A treated middle-aged ovariectomized rats. Neurotoxicol Teratol. 2013;35(1):46-53. doi:10.1016/j.ntt.2013.01.002.

75. Kuwahara R, Kawaguchi S, Kohara Y, Jojima T, Yamashita K. Bisphenol A does not affect memory performance in adult male rats. Cell Mol Neurobiol. 2014;34(3):333-342. doi:10.1007/s10571-013-0017-6.

76. Sadowski RN, Park P, Neese SL, Ferguson DC, Schantz SL, Juraska JM. Effects of perinatal bisphenol A exposure during early development on radial arm maze behavior in adult male and female rats. Neurotoxicol Teratol. 2014;42:17-24. doi:10.1016/j.ntt.2014.01.002. 
77. Ishido M, Masuo Y, Terasaki M, Morita M. Rat hyperactivity by bisphenol A, but not by its derivatives, 3-hydroxybisphenol A or bisphenol A 3,4-quinone. Toxicol Lett. 2011;206(3):300-305. doi:10.1016/j.toxlet.2011.08.011.

78. Viberg H, Fredriksson A, Buratovic S, Eriksson P. Dose-dependent behavioral disturbances after a single neonatal Bisphenol A dose. Toxicology. 2011;290(23):188-195. doi:10.1016/j.tox.2011.09.006.

79. Kimura E, Matsuyoshi C, Miyazaki W, et al. Prenatal exposure to bisphenol A impacts neuronal morphology in the hippocampal CA1 region in developing and aged mice. Arch Toxicol. 2015:691-700. doi:10.1007/s00204-015-1485-x.

80. Yin N, Yao X, Qin Z, Wang YL, Faiola F. Assessment of Bisphenol A (BPA) neurotoxicity in vitro with mouse embryonic stem cells. J Environ Sci (China). 2015;36:181-187. doi:10.1016/j.jes.2015.06.004.

81. Mersha MD, Patel BM, Patel D, Richardson BN, Dhillon HS. Effects of BPA and BPS exposure limited to early embryogenesis persist to impair non-associative learning in adults. Behav Brain Funct. 2015;11(1):27. doi:10.1186/s12993-0150071-y.

82. Kim K, Son TG, Park HR, et al. Potencies of bisphenol A on the neuronal differentiation and hippocampal neurogenesis. J Toxicol Environ Health A. 2009;72(21-22):1343-1351. doi:10.1080/15287390903212501.

83. U.S. Environmental Protection Agency. Bisphenol A; CASRN 80-05-7. 1988:1-7. http://cfpub.epa.gov/ncea/iris/iris_documents/documents/subst/0356_summary.pdf

84. Inagaki T, Frankfurt M, Luine V. Estrogen-induced memory enhancements are blocked by acute bisphenol A in adult female rats: Role of dendritic spines. Endocrinology. 2012;153(7):3357-3367. doi:10.1210/en.2012-1121.

85. Braun JM, Yolton K, Dietrich KN, et al. Prenatal bisphenol A exposure and early childhood behavior. Environ Health Perspect. 2009;117(12):1945-1952. doi:10.1289/ehp.0900979. 
86. Rebuli ME, Camacho L, Adonay ME, Reif DM, Aylor DL, Patisaul HB. Impact of low-dose oral exposure to bisphenol A (BPA) on Juvenile and adult rat exploratory and anxiety behavior: A CLARITY-BPA Consortium Study. Toxicol Sci. 2015;148(2):341-354. doi:10.1093/tox sci/kfv163.

87. Luo G, Wang S, Li Z, et al. Maternal bisphenol a diet induces anxiety-like behavior in female juvenile with neuroimmune activation. Toxicol Sci. 2014;140(2):364-373. doi:10.1093/toxsci/kfu085.

88. Carr R, Bertasi F, Betancourt A, et al. Effect of neonatal rat bisphenol a exposure on performance in the Morris water maze. J Toxicol Environ Health A. 2003;66(21):2077-2088. http://www.ncbi.nlm.nih.gov/pubmed/14555403. Accessed September 18, 2016.

89. Miyagawa K, Narita M, Narita M, Akama H, Suzuki T. Memory impairment associated with a dysfunction of the hippocampal cholinergic system induced by prenatal and neonatal exposures to bisphenol-A. Neurosci Lett. 2007;418(3):236241. doi:10.1016/j.neulet.2007.01.088.

90. Kawai K, Nozaki T, Nishikata H, Aou S, Takii M, Kubo C. Aggressive behavior and serum testosterone concentration during the maturation process of male mice: The effects of fetal exposure to bisphenol A. Environ Health Perspect. 2003;111(2):175-178. doi:10.1289/ehp.5440.

91. Nagao T, Saito Y, Usumi K, Kuwagata M, Imai K. Reproductive function in rats exposed neonatally to bisphenol A and estradiol benzoate. Reprod Toxicol. 1999;13(4):303-311. doi:10.1016/S0890-6238(99)00017-9.

92. Narita M, Miyagawa K, Mizuo K, Yoshida T, Suzuki T. Prenatal and neonatal exposure to low-dose of bisphenol-A enhance the morphine-induced hyperlocomotion and rewarding effect. Neurosci Lett. 2006;402(3):249-252. doi:10.1016/j.neulet.2006.04.014.

93. Narita M, Miyagawa K, Mizuo K, Yoshida T, Suzuki T. Changes in central dopaminergic systems and morphine reward by prenatal and neonatal exposure to bisphenol-A in mice: Evidence for the importance of exposure period. Addict Biol. 2007;12(2):167-172. doi:10.1111/j.1369-1600.2007.00048.x. 
94. Harley KG, Gunier RB, Kogut K, et al. Prenatal and early childhood bisphenol A concentrations and behavior in school-aged children. Environ Res. 2013;126:4350. doi:10.1016/j.envres.2013.06.004.

95. Kondolot M, Ozmert EN, Asc1 A, et al. Plasma phthalate and bisphenol a levels and oxidant-antioxidant status in autistic children. Environ Toxicol Pharmacol. 2016;43:149-158. doi:10.1016/j.etap.2016.03.006.

96. Arbuckle TE, Davis K, Boylan K, Fisher M, Fu J. Bisphenol A, phthalates and lead and learning and behavioral problems in Canadian children 6-11 years of age: CHMS 2007-2009. Neurotoxicology. 2016;54:89-98.

doi:10.1016/j.neuro.2016.03.014.

97. Yolton K, Xu Y, Strauss D, Altaye M, Calafat AM, Khoury J. Prenatal exposure to bisphenol A and phthalates and infant neurobehavior. Neurotoxicol Teratol. 2011;33(5):558-566. doi:10.1016/j.ntt.2011.08.003.

98. Roen EL, Wang Y, Calafat AM, et al. Bisphenol A exposure and behavioral problems among inner city children at 7-9 years of age. Environ Res. 2015;142:739-745. doi:10.1016/j.envres.2015.01.014.

99. Braun JM, Kalkbrenner AE, Calafat AM, et al. Impact of early-life bisphenol A exposure on behavior and executive function in children. Pediatrics. 2011;128(5):873-882. doi:10.1542/peds.2011-1335.

100. Miodovnik A, Engel SM, Zhu C, et al. Endocrine disruptors and childhood social impairment. Neurotoxicology. 2011;32(2):261-267.

doi:10.1016/j.neuro.2010.12.009.

101. Agency for Toxic Substances and Disease Registry (ATSDR). Toxicological Profile for Diethyl Phthalate. Agency Toxic Subst Dis Regist. 1995;(June):1-158.

102. Agency for Toxic Substances and Disease Registry (ATSDR). TOXICOLOGICAL PROFILE FOR DI-n-BUTYL PHTHALATE. Agency Toxic Subst Dis Regist. 2001;(September):225.

103. Agency for Toxic Substances and Disease Registry (ATSDR). Toxicological profile: di(2-ethylhexyl)phthalate (DEHP). Agency Toxic Subst Dis Regist. 2002;(September). http://www.atsdr.cdc.gov/toxprofiles/tp.asp?id=684\&tid=65. 
104. Heudorf U, Mersch-Sundermann V, Angerer J. Phthalates: Toxicology and exposure. Int J Hyg Environ Health. 2007;210(5):623-634.

doi:10.1016/j.ijheh.2007.07.011.

105. Miodovnik A, Edwards A, Bellinger DC, Hauser R. Developmental neurotoxicity of ortho-phthalate diesters: Review of human and experimental evidence. Neurotoxicology. 2014;41:112-122. doi:10.1016/j.neuro.2014.01.007.

106. Silva MJ, Barr DB, Reidy JA, et al. Urinary levels of seven phthalate metabolites in the U.S. population from the National Health and Nutrition Examination Survey (NHANES) 1999-2000. Environ Health Perspect. 2004;112(3):331-338. doi:10.1289/ehp.6723.

107. Blair RM, Fang H, Branham WS, et al. The estrogen receptor relative binding affinities of 188 natural and xenochemicals: structural diversity of ligands. Toxicol Sci. 2000;54(1):138-153. doi:10.1093/toxsci/54.1.138.

108. Jobling S, Reynolds T, White R, Parker MG, Sumpter JP. A variety of environmentally persistent chemicals, including some phthalate plasticizers, are weakly estrogenic. Environ Health Perspect. 1995;103(6):582-587. doi:10.1289/ehp.95103582.

109. Ma N, Liu S, Gao P, Cao P, Xu H. [Effect of diisobutyl phthalate on learning and memory behavior and apoptosis of hippocampus cells in mice]. Wei Sheng Yan Jiu. 2013;42(1):57-60. http://www.ncbi.nlm.nih.gov/pubmed/23596708. Accessed May 15, 2016.

110. Zeliger HI. Exposure to lipophilic chemicals as a cause of neurological impairments, neurodevelopmental disorders and neurodegenerative diseases. Interdiscip Toxicol. 2013;6(3):103-110. doi:10.2478/intox-2013-0018.

111. Lin H, Yuan K, Li L, et al. In utero exposure to diethylhexyl phthalate affects rat brain development: A behavioral and genomic approach. Int J Environ Res Public Health. 2015;12(11):13696-13710. doi:10.3390/ijerph121113696.

112. Betz AJ, Jayatilaka S, Joshi J, et al. Chronic exposure to benzyl butyl phthalate (BBP) alters social interaction and fear conditioning in male adult rats: Alterations in amygdalar MeCP2, ERK1/2 and ER?? Neuroendocrinol Lett. 2013;34(5):347358. 
113. Dai Y, Yang Y, Xu X, Hu Y. Effects of uterine and lactational exposure to di-(2ethylhexyl) phthalate on spatial memory and NMDA receptor of hippocampus in mice. Horm Behav. 2015;71:41-48. doi:10.1016/j.yhbeh.2015.03.008.

114. Li XJ, Jiang L, Chen L, Chen HS, Li X. Neurotoxicity of dibutyl phthalate in brain development following perinatal exposure: A study in rats. Environ Toxicol Pharmacol. 2013;36(2):392-402. doi:10.1016/j.etap.2013.05.001.

115. Li Y, Li T, Zhuang M, Wang K, Zhang J, Shi N. High-dose dibutyl phthalate improves performance of $\mathrm{F} 1$ generation male rats in spatial learning and increases hippocampal BDNF expression independent on p-CREB immunocontent. Environ Toxicol Pharmacol. 2010;29(1):32-38. doi:10.1016/j.etap.2009.09.003.

116. Li Y, Zhuang M, Li T, Shi N. Neurobehavioral toxicity study of dibutyl phthalate on rats following in utero and lactational exposure. J Appl Toxicol. 2009;29(7):603-611. doi:10.1002/jat.1447.

117. Holahan MR, Smith CA. Phthalates and neurotoxic effects on hippocampal network plasticity. Neurotoxicology. 2015;48:21-34.

doi:10.1016/j.neuro.2015.02.008.

118. Masuo Y, Morita M, Oka S, Ishido M. Motor hyperactivity caused by a deficit in dopaminergic neurons and the effects of endocrine disruptors: A study inspired by the physiological roles of PACAP in the brain. Regul Pept. 2004;123(1-3 SPEC. ISS.):225-234. doi:10.1016/j.regpep.2004.05.010.

119. Larsson M, Weiss B, Janson S, Sundell J, Bornehag CG. Associations between indoor environmental factors and parental-reported autistic spectrum disorders in children 6-8 years of age. Neurotoxicology. 2009;30(5):822-831. doi:10.1016/j.neuro.2009.01.011.

120. Sun W, Ban J-B, Zhang N, Zu Y-K, Sun W-X. Perinatal exposure to Di-(2ethylhexyl)-Phthalate leads to cognitive dysfunction and phospho-tau level increase in aged rats. Environ Toxicol. 2014;29(5):596-603. doi:10.1002/tox.21785.

121. Agency for Toxic Substances and Disease Registry (ATSDR). TOXICOLOGICAL PROFILE FOR POLYCHLORINATED BIPHENYLS ( PCBs ). Agency Toxic Subst Dis Regist. 2000;(November):1-948. 
122. Reilly MP, Weeks CD, Topper VY, Thompson LM, Crews D, Gore AC. The effects of prenatal PCBs on adult female paced mating reproductive behaviors in rats. Horm Behav. 2007;51(3):364-372. doi:10.1016/j.yhbeh.2006.12.004.

123. Lee DW, Notter SA, Thiruchelvam M, et al. Subchronic polychlorinated biphenyl (aroclor 1254) exposure produces oxidative damage and neuronal death of ventral midbrain dopaminergic systems. Toxicol Sci. 2012. doi:10.1093/toxsci/kfr313.

124. Zahara ARD, Michel NL, Flahr LM, Ejack LE, Morrissey C a. Latent cognitive effects from low-level polychlorinated biphenyl exposure in juvenile European starlings ( Sturnus vulgaris ). Environ Toxicol Chem. 2015;34(11):2513-252. doi:10.1002/etc.3084.

125. Elnar AA, Allouche A, Desor F, Yen FT, Soulimani R, Oster T. Lactational exposure of mice to low levels of non-dioxin-like polychlorinated biphenyls increases susceptibility to neuronal stress at a mature age. Neurotoxicology. 2015. doi:10.1016/j.neuro.2015.10.003.

126. Hilgier W, Łazarewicz JW, Strużynska L, Frontczak-Baniewicz M, Albrecht J. Repeated exposure of adult rats to Aroclor 1254 induces neuronal injury and impairs the neurochemical manifestations of the NMDA receptor-mediated intracellular signaling in the hippocampus. Neurotoxicology. 2012;33(1):16-22. doi:10.1016/j.neuro.2011.10.005.

127. Formisano L, Guida N, Laudati G, Mascolo L, Di Renzo G, Canzoniero LMT. MS-275 inhibits aroclor 1254-induced SH-SY5Y neuronal cell toxicity by preventing the formation of the HDAC3/REST complex on the synapsin-1 promoter. J Pharmacol Exp Ther. 2015;352(2):236-243. doi:10.1124/jpet.114.219345.

128. Bang Y, Lim J, Kim SS, et al. Aroclor1254 interferes with estrogen receptormediated neuroprotection against beta-amyloid toxicity in cholinergic SN56 cells. Neurochem Int. 2011;59(5):582-590. doi:10.1016/j.neuint.2011.04.006.

129. Orenstein STC, Thurston SW, Bellinger DC, et al. Prenatal organochlorine and methylmercury exposure and memory and learning in school-age children in communities near the new bedford harbor superfund site, Massachusetts. Environ Health Perspect. 2014;122(11):1253-1259. doi:10.1289/ehp.1307804. 
130. Boucher O, Muckle G, Jacobson JL, et al. Domain-specific effects of prenatal exposure to PCBs, mercury, and lead on infant cognition: Results from the environmental contaminants and child development study in nunavik. Environ Health Perspect. 2014;122(3):310-316. doi:10.1289/ehp.1206323.

131. Fitzgerald EF, Shrestha S, Gomez MI, et al. Polybrominated diphenyl ethers (PBDEs), polychlorinated biphenyls (PCBs) and neuropsychological status among older adults in New York. Neurotoxicology. 2012;33(1):8-15. doi:10.1016/j.neuro.2011.10.011.

132. Fitzgerald EF, Belanger EE, Gomez MI, et al. Polychlorinated biphenyl exposure and neuropsychological status among older residents of upper Hudson River communities. Environ Health Perspect. 2008;116(2):209-215. doi:10.1289/ehp.10432.

133. Schantz SL, Gasior DM, Polverejan E, et al. Impairments of memory and learning in older adults exposed to polychlorinated biphenyls via consumption of Great Lakes Fish. Environ Health Perspect. 2001;109(6):605-611. doi:10.1289/ehp.01109605.

134. Peper M, Klett M, Morgenstern R. Neuropsychological effects of chronic low-dose exposure to polychlorinated biphenyls (PCBs): a cross-sectional study. Environ Health. 2005;4:22. doi:10.1186/1476-069X-4-22.

135. Weisskopf M, Knekt P, O'Reilly E, et al. Polychlorinated biphenyls in prospectively collected serum and parkinson's disease risk. Mov Disord. 2012;27(13):1659-1665. doi:10.1016/j.surg.2006.10.010.Use.

136. Steenland K, Hein MJ, Cassinelli RT, et al. Polychlorinated biphenyls and neurodegenerative disease mortality in an occupational cohort. Epidemiology. 2006;17(1):8-13. doi:10.1097/01.ede.0000190707.51536.2b.

137. Hatcher-Martin JM, Gearing M, Steenland K, Levey AI, Miller GW, Pennell KD. Association between polychlorinated biphenyls and Parkinson's disease neuropathology. Neurotoxicology. 2012;33(5):1298-1304. doi:10.1016/j.neuro.2012.08.002. 
138. Agency for Toxic Substances and Disease Registry (ATSDR). Toxicological Profile for Cadmium. Agency Toxic Subst Dis Regist. 2012;(September). http://www.atsdr.cdc.gov/toxprofiles/tp5.pdf\%5Cnhttp://www.ncbi.nlm.nih.gov/bo oks/NBK158845/.

139. Choe SY, Kim SJ, Kim HG, et al. Evaluation of estrogenicity of major heavy metals. Sci Total Environ. 2003;312(1-3):15-21. doi:10.1016/S00489697(03)00190-6.

140. Wang S, Hu P, Wang H-L, et al. Effects of $\mathrm{Cd}(2+)$ on AMPA receptor-mediated synaptic transmission in rat hippocampal CA1 area. Toxicol Lett. 2008;176(3):215222. doi:10.1016/j.toxlet.2007.11.008.

141. Chen L, Liu L, Luo Y, Huang S. MAPK and mTOR pathways are involved in cadmium-induced neuronal apoptosis. J Neurochem. 2008;105(1):251-261. doi:10.1111/j.1471-4159.2007.05133.x.

142. Jiang JH, Ge G, Gao K, et al. Calcium Signaling Involvement in CadmiumInduced Astrocyte Cytotoxicity and Cell Death Through Activation of MAPK and PI3K/Akt Signaling Pathways. Neurochem Res. 2015;40(9):1929-1944. doi:10.1007/s11064-015-1686-y.

143. Chow ESH, Hui MNY, Lin CC, Cheng SH. Cadmium inhibits neurogenesis in zebrafish embryonic brain development. Aquat Toxicol. 2008;87(3):157-169. doi:10.1016/j.aquatox.2008.01.019.

144. Yuan $\mathrm{Y}$, Jiang $\mathrm{C}, \mathrm{Xu} \mathrm{H}$, et al. Cadmium-induced apoptosis in primary rat cerebral cortical neurons culture is mediated by a calcium signaling pathway. PLoS One. 2013;8(5):e64330. doi:10.1371/journal.pone.0064330.

145. Hossain S, Liu H-N, Nguyen M, Shore G, Almazan G. Cadmium exposure induces mitochondria-dependent apoptosis in oligodendrocytes. Neurotoxicology. 2009;30(4):544-554. doi:10.1016/j.neuro.2009.06.001.

146. Notarachille G, Arnesano F, Calò V, Meleleo D. Heavy metals toxicity: effect of cadmium ions on amyloid beta protein 1-42. Possible implications for Alzheimer's disease. Biometals. 2014;27(2):371-388. doi:10.1007/s10534-014-9719-6. 
147. Roos PM, Vesterberg O, Syversen T, Flaten TP, Nordberg M. Metal concentrations in cerebrospinal fluid and blood plasma from patients with amyotrophic lateral sclerosis. Biol Trace Elem Res. 2013. doi:10.1007/s12011012-9547-x.

148. Komatsu F, Kagawa Y, Kawabata T, et al. A high accumulation of hair minerals in Mongolian people: 2(nd) report; influence of manganese, iron, lead, cadmium and aluminum to oxidative stress, Parkinsonism and arthritis. Curr Aging Sci. 2011;4(1):42-56. http://www.ncbi.nlm.nih.gov/pubmed/21204778. Accessed September 19, 2016.

149. Jumpponen M, Rönkkömäki H, Pasanen P, Laitinen J. Occupational exposure to solid chemical agents in biomass-fired power plants and associated health effects. Chemosphere. 2014. doi:10.1016/j.chemosphere.2013.10.025.

150. Agency for Toxic Substances and Disease Registry (ATSDR). TOXICOLOGICAL PROFILE FOR ARSENIC. Health Effects. Agency Toxic Subst Dis Regist. 2007;(August).

151. Jing J, Zheng G, Liu M, et al. Changes in the synaptic structure of hippocampal neurons and impairment of spatial memory in a rat model caused by chronic arsenite exposure. Neurotoxicology. 2012;33(5):1230-1238. doi:10.1016/j.neuro.2012.07.003.

152. Luo J, Qiu Z, Zhang L, Shu W. Arsenite exposure altered the expression of NMDA receptor and postsynaptic signaling proteins in rat hippocampus. Toxicol Lett. 2012;211(1):39-44. doi:10.1016/j.toxlet.2012.02.021.

153. Luo J, Qiu Z, Shu W, Zhang Y, Zhang L, Chen J. Effects of arsenic exposure from drinking water on spatial memory, ultra-structures and NMDAR gene expression of hippocampus in rats. Toxicol Lett. 2009;184(2):121-125. doi:10.1016/j.toxlet.2008.10.029.

154. Qu L, Gao Y, Sun H, Wang H, Liu X, Sun D. Role of PTEN-Akt-CREB Signaling Pathway in Nervous System impairment of Rats with Chronic Arsenite Exposure. Biol Trace Elem Res. 2015;(23618504). doi:10.1007/s12011-015-0478-1. 
155. Shavali S, Sens DA. Synergistic Neurotoxic Effects of Arsenic and Dopamine in Human Dopaminergic Neuroblastoma SH-SY5Y Cells. Toxicol Sci. 2007;102(2):254-261. doi:10.1093/toxsci/kfm302.

156. Zarazúa S, Bürger S, Delgado JM, Jiménez-Capdeville ME, Schliebs R. Arsenic affects expression and processing of amyloid precursor protein (APP) in primary neuronal cells overexpressing the Swedish mutation of human APP. Int J Dev Neurosci. 2011;29(4):389-396. doi:10.1016/j.ijdevneu.2011.03.004.

157. O’Bryant SE, Edwards M, Menon C, Gong G, Barber R. Long-Term Low-Level Arsenic Exposure Is Associated with Poorer Neuropsychological Functioning: A Project FRONTIER Study. Int J Environ Res Public Health. 2011;8(12):861-874. doi:10.3390/ijerph8030861.

158. Edwards M, Hall J, Gong G, O’Bryant SE. Arsenic exposure, AS3MT polymorphism, and neuropsychological functioning among rural dwelling adults and elders: a cross-sectional study. Environ Health. 2014;13(1):15. doi:10.1186/1476-069X-13-15.

159. Park J-H, Lee D-W, Park KS, Joung H. Serum trace metal levels in Alzheimer's disease and normal control groups. Am J Alzheimers Dis Other Demen. 2014;29(1):76-83. doi:10.1177/1533317513506778.

160. Edwards M, Johnson L, Mauer C, Barber R, Hall J, O'Bryant S. Regional specific groundwater arsenic levels and neuropsychological functioning: a cross-sectional study. Int J Environ Health Res. 2014;24(6):546-557.

doi:10.1080/09603123.2014.883591.

161. Agency for Toxic Substances and Disease Registry (ATSDR). Toxicological Profile for Manganese. ATSDR - Toxicol Profiles. 2000;(September):504.

162. Hozumi I, Hasegawa T, Honda A, et al. Patterns of levels of biological metals in CSF differ among neurodegenerative diseases. J Neurol Sci. 2011;303(1-2):95-99. doi:10.1016/j.jns.2011.01.003.

163. Wasserman GA, Liu X, Parvez F, et al. Arsenic and manganese exposure and children's intellectual function. Neurotoxicology. 2011;32(4):450-457. doi:10.1016/j.neuro.2011.03.009. 
164. Kim Y, Bowler RM, Abdelouahab N, Harris M, Gocheva V, Roels HA. Motor function in adults of an Ohio community with environmental manganese exposure. Neurotoxicology. 2011;32(5):606-614. doi:10.1016/j.neuro.2011.07.011.

165. Carvalho CF, Menezes-Filho JA, Matos VP de, et al. Elevated airborne manganese and low executive function in school-aged children in Brazil. Neurotoxicology. 2014;45:301-308. doi:10.1016/j.neuro.2013.11.006.

166. Oulhote Y, Mergler D, Barbeau B, et al. Neurobehavioral function in school-age children exposed to manganese in drinking water. Environ Health Perspect. 2014;122(12):1343-1350. doi:10.1289/ehp.1307918.

167. Koc ER, Ilhan A, Ayturk Z, et al. A comparison of hair and serum trace elements in patients with Alzheimer disease and healthy participants. Turkish J Med Sci Turk J Med Sci, Turk J Med, Turk J Med Sci. 2015;45(5):1034-1039. doi:10.3906/sag-1407-67.

168. Miyake Y, Tanaka K, Fukushima W, et al. Dietary intake of metals and risk of Parkinson's disease: A case-control study in Japan. J Neurol Sci. 2011;306(12):98-102. doi:10.1016/j.jns.2011.03.035.

169. Kumudini N, Uma A, Prameela Devi Y, et al. Association of Parkinson's disease with altered serum levels of lead and transition metals among South Indian subjects. Indian J Biochem Biophys. 2014;51(2):121-126.

170. Garzillo EM, Lamberti M, Genovese G, et al. Blood lead, manganese, and aluminum levels in a regional Italian cohort of ALS patients: does aluminum have an influence? J Occup Environ Med. 2014;56(10):1062-1066. doi:10.1097/JOM.0000000000000266.

171. Kihira T, Sakurai I, Yoshida S, et al. Neutron \{Activation $\}$ Analysis $\}$ of $\{$ Scalp $\}$ \{Hair\} from \{ALS $\{$ Patients and \{Residents $\}$ in the $\{$ Kii $\}$ PPeninsula $\},\{$ Japan . Biol Trace Elem Res. 2015;164(1):36-42. doi:10.1007/s12011-014-0202-6.

172. Arain MS, Afridi HI, Kazi TG, et al. Correlation of aluminum and manganese concentration in scalp hair samples of patients having neurological disorders. Environ Monit Assess. 2015;187(2). doi:10.1007/s10661-014-4172-0. 
173. Bowler RM, Gocheva V, Harris M, et al. Prospective study on neurotoxic effects in manganese-exposed bridge construction welders. Neurotoxicology. 2011;32(5):596-605. doi:10.1016/j.neuro.2011.06.004.

174. Baker MG, Criswell SR, Racette BA, et al. Neurological outcomes associated with low-level manganese exposure in an inception cohort of asymptomatic welding trainees. Scand J Work Environ Heal. 2015;41(1):94-101. doi:10.5271/sjweh.3466.

175. Guilarte TR, McGlothan JL, Degaonkar M, et al. Evidence for cortical dysfunction and widespread manganese accumulation in the nonhuman primate brain following chronic manganese exposure: a 1H-MRS and MRI study. Toxicol Sci. 2006;94(2):351-358. doi:10.1093/toxsci/kfl106.

176. Guilarte TR, Chen M-K. Manganese inhibits NMDA receptor channel function: implications to psychiatric and cognitive effects. Neurotoxicology. 2007;28(6):1147-1152. doi:10.1016/j.neuro.2007.06.005.

177. Neal AP, Guilarte TR. Mechanisms of lead and manganese neurotoxicity. Toxicol Res (Camb). 2013;2(2):99-114. doi:10.1039/C2TX20064C.

178. Guilarte TR, Burton NC, McGlothan JL, et al. Impairment of nigrostriatal dopamine neurotransmission by manganese is mediated by pre-synaptic mechanism(s): implications to manganese-induced parkinsonism. J Neurochem. 2008;107(5):1236-1247. doi:10.1111/j.1471-4159.2008.05695.x.

179. Hojo Y, Hattori T-A, Enami T, et al. Adult male rat hippocampus synthesizes estradiol from pregnenolone by cytochromes P45017alpha and P450 aromatase localized in neurons. Proc Natl Acad Sci U S A. 2004;101(3):865-870. doi:10.1073/pnas.2630225100.

180. MacLusky NJ, Naftolin F, Goldman-Rakic PS. Estrogen formation and binding in the cerebral cortex of the developing rhesus monkey. Proc Natl Acad Sci. 1986;83(2):513-516. doi:10.1073/pnas.83.2.513.

181. Roy D, Palangat M, Chen CW, et al. Biochemical and molecular changes at the cellular level in response to exposure to environmental estrogen-like chemicals. $J$ Toxicol Environ Health. 1997;50(1):1-29. http://www.ncbi.nlm.nih.gov/pubmed/9015129. Accessed March 21, 2018. 
182. Ronchetti SA, Novack G V, Bianchi MS, Crocco MC, Duvilanski BH, Cabilla JP. In vivo xenoestrogenic actions of cadmium and arsenic in anterior pituitary and uterus. Reproduction. 2016;152(1):1-10. doi:10.1530/REP-16-0115.

183. Dearth RK, Hiney JK, Srivastava VK, Hamilton AM, Dees WL. Prepubertal exposure to elevated manganese results in estradiol regulated mammary gland ductal differentiation and hyperplasia in female rats. Exp Biol Med (Maywood). 2014;239(7):871-882. doi:10.1177/1535370214531865.

184. Wu Y, Li K, Zuo H, Yuan Y, Sun Y, Yang X. Primary neuronal-astrocytic coculture platform for neurotoxicity assessment of di-(2-ethylhexyl) phthalate. $J$ Environ Sci (China). 2014;26(5):1145-1153. doi:10.1016/S1001-0742(13)60504-5.

185. Bake S, Sohrabji F. 17B-Estradiol Differentially Regulates Blood-Brain Barrier Permeability in Young and Aging Female Rats. Endocrinology. 2004;145(12):5471-5475. doi:10.1210/en.2004-0984.

186. Van Der Meer TP, Artacho-Cordón F, Swaab DF, et al. Distribution of nonpersistent endocrine disruptors in two different regions of the human brain. Int $J$ Environ Res Public Health. 2017;14(9):1-11. doi:10.3390/ijerph14091059.

187. Lee S, Suk K, Kim IK, et al. Signaling pathways of bisphenol A-induced apoptosis in hippocampal neuronal cells: Role of calcium-induced reactive oxygen species, mitogen-activated protein kinases, and nuclear factor-??B. J Neurosci Res. 2008;86(13):2932-2942. doi:10.1002/jnr.21739.

188. Lee S, Kim YK, Shin TY, Kim SH. Neurotoxic effects of bisphenol AF on calcium-induced ROS and MAPKs. Neurotox Res. 2013;23(3):249-259. doi:10.1007/s12640-012-9353-4.

189. Scarpulla R. Transcriptional paradigms in mammalian mitochondrial biogenesis and function. Physiol Rev. 2008;88:611-638. doi:10.1152/physrev.00025.2007.

190. Satoh JI, Kawana N, Yamamoto Y. Pathway Analysis of ChIP-Seq-Based NRF1 Target Genes Suggests a Logical Hypothesis of their Involvement in the pathogenesis of Neurodegenerative Diseases. Gene Regul Syst Bio. 2013;2013(7):139-152. doi:10.4137/GRSB.S13204. 
191. Johri A, Beal F. Mitochondrial dysfunction in neurodegenerative diseases. $J$ Pharmacol Exp Ther. 2012;342(3):619-630.

192. Pennacchio LA, Loots GG, Nobrega MA, Ovcharenko I. Predicting tissue-specific enhancers in the human genome. Genome Res. 2007;17(2):201-211. doi:10.1101/gr.5972507.

193. Osellame LD, Blacker TS, Duchen MR. Cellular and molecular mechanisms of mitochondrial function. Best Pract Res Clin Endocrinol Metab. 2012;26(6):711723. doi:10.1016/j.beem.2012.05.003.

194. Corona JC, Duchen MR. Impaired mitochondrial homeostasis and neurodegeneration: towards new therapeutic targets? J Bioenerg Biomembr. 2014;47(1-2):89-99. doi:10.1007/s10863-014-9576-6.

195. Velarde MC. Mitochondrial and sex steroid hormone crosstalk during aging. Longev Heal. 2014;3(1):2. doi:10.1186/2046-2395-3-2.

196. Brannval K, Korhonen L, Lindholm D. Estrogen-Receptor-Dependent Regulation of Neural Stem Cell Proliferation and Differentiation. Mol Cell Neurosci. 2002;21(3):512-520. doi:10.1006/mcne.2002.1194.

197. Okada M, Murase K, Makino A, et al. Effects of estrogens on proliferation and differentiation of neural stem/progenitor cells. Biomed Res. 2008;29(3):163-170. doi:10.2220/biomedres.29.163.

198. Okada M, Makino A, Nakajima M, Okuyama S, Furukawa S, Furukawa Y. Estrogen stimulates proliferation and differentiation of neural stem/progenitor cells through different signal transduction pathways. Int J Mol Sci. 2010;11(10):41144123. doi:10.3390/ijms11104114.

199. Kinch CD, Ibhazehiebo K, Jeong J-H, Habibi HR, Kurrasch DM. Low-dose exposure to bisphenol $\mathrm{A}$ and replacement bisphenol $\mathrm{S}$ induces precocious hypothalamic neurogenesis in embryonic zebrafish. Proc Natl Acad Sci U S A. 2015;112(5):1475-1480. doi:10.1073/pnas.1417731112.

200. Ishido M, Suzuki J. Classification of phthalates based on an in vitro neurosphere assay using rat mesencephalic neural stem cells. J Toxicol Sci. 2014;39(1):25-32. doi:10.2131/jts.39.25. 
201. Tofighi R, Wan Ibrahim WN, Rebellato P, Andersson PL, Uhlén P, Ceccatelli S. Non-dioxin-like polychlorinated biphenyls interfere with neuronal differentiation of embryonic neural stem cells. Toxicol Sci. 2011;124(1):192-201. doi:10.1093/toxsci/kfr221.

202. Ivanov VN, Hei TK. Induction of apoptotic death and retardation of neuronal differentiation of human neural stem cells by sodium arsenite treatment. Exp Cell Res. 2013;319(6):875-887. doi:10.1016/j.yexcr.2012.11.019.

203. Tamm C, Sabri F, Ceccatelli S. Mitochondrial-mediated apoptosis in neural stem cells exposed to manganese. Toxicol Sci. 2008;101(2):310-320. doi:10.1093/toxsci/kfm267.

204. Wang J-L, Chang W-T, Tong C-W, Kohno K, Huang A-M. Human synapsin I mediates the function of nuclear respiratory factor 1 in neurite outgrowth in neuroblastoma IMR-32 cells. J Neurosci Res. 2009;87(10):2255-2263. doi:10.1002/jnr.22059.

205. Tong C-W, Wang J-L, Jiang M-S, Hsu C-H, Chang W-T, Huang A-M. Novel genes that mediate nuclear respiratory factor 1-regualted neurite outgrowth in neuroblastoma IMR-32 cells. Gene. 2013;515(1):62-70. doi:10.1016/j.gene.2012.11.026.

206. Wang JL, Tong CW, Chang WT, Huang AM. Novel genes FAM134C, C3orf10 and ENOX1 are regulated by NRF-1 and differentially regulate neurite outgrowth in neuroblastoma cells and hippocampal neurons. Gene. 2013;529(1):7-15. doi:10.1016/j.gene.2013.08.006.

207. Alzheimer's Association. 2017 Alzheimer's Disease Facts and Figures. Alzheimers Dement . 2017;13:325-373. doi:10.1016/j.jalz.2017.02.001.

208. Sladeczek J, Hartemink AJ, Robinson J. Banjo Users Guide. Gov Publ Rev. 2006;6(2). http://www.cs.duke.edu/ amink/software/banjo/documentation/banjo.user.pdf.

209. Hokama M, Oka S, Leon J, et al. Altered expression of diabetes-related genes in Alzheimer's disease brains: the Hisayama study. Cereb Cortex. 2014;24(9):24762488. doi:10.1093/cercor/bht101. 


\section{CHAPTER III}

\section{HYPOTHESIS AND SPECIFIC AIMS}

\section{Hypothesis}

Exposure to estrogenic endocrine disrupting chemicals (EEDCs) result in adverse brain health outcomes.

\section{Specific Aims}

Specific Aim 1: Assess exposure to phthalates and bisphenol-A (BPA) using urinary biomarkers from the CDC NHANES 2011-2014 datasets to find associations between phthalate and BPA bioburden and adverse brain health using surrogate indicators of brain health.

Specific Aim 2: Assess exposure to the metalloestrogens, cadmium (Cd), arsenic (As), and manganese (Mn) using urinary biomarkers from the CDC NHANES 2011-2014 datasets to find associations between phthalate and BPA bioburden and adverse brain health using surrogate indicators of brain health.

Specific Aim 3: Assess exposure to oral contraceptives (OC) and hormonal replacement therapy (HRT) using urinary biomarkers from the CDC NHANES 2011-2014 datasets to find associations between phthalate and BPA bioburden and adverse brain health using surrogate indicators of brain health.

Specific aim 4: Assess estrogen-responsive genes networks common to the EEDCs, phthalates, BPA, Cd, As, and Mn, NRF1, and neurodegenerative disease using bioinformatics methods. 


\section{CHAPTER IV}

\section{METHODS}

Study Design and Population: NHANES is a continuous cross-sectional data collection utilizing a complex multi-stage sampling design that creates a survey representative of the non-institutionalized population of the United States ${ }^{1,2}$. The survey has been conducted since 1999 and consists of an at-home questionnaires followed by a standardized physical examination and specimen collection conducted in mobile examination centers (MEC) ${ }^{1,2}$. Eligibility is determined using preset selection probabilities for the desired demographic subdomains ${ }^{2}$. A household screener is performed before to determine if any household members are eligible for the interview and examination ${ }^{2}$. The interview collects demographic, health, nutrition, and household information, while the physical examination includes physical measurements, dental examination, and the collection of blood and urine specimens for laboratory testing ${ }^{2}$. Prior to any to interviews and examinations, informed consent was obtained and all procedures were approved by the CDC Institutional Review Board ${ }^{3}$. In our study, we merged the NHANES 2011-2012 and 2013-2014 data cycles. All our analyses were limited to individuals 60 years of age and older who have recorded responses to cognitive test scores and/or memory and taste/smell questions and have EEDC urine measurements. 


\section{Inclusion/Exclusion Criteria}

\section{Inclusion criteria:}

1. Males and females, 60 years of age and older

2. Available EEDC urine measurements (Phthalates, BPA, Mn, As, Cd, OC use, HRT use)

3. Urine creatinine measurements $>30 \mathrm{mg} / \mathrm{dl}$ and $<300 \mathrm{mg} / \mathrm{dl}$.

4. Complete responses to identified outcome variables.

\section{Exclusion criteria:}

1. Males and females, 59 years of age and younger

2. Unavailable EEDC urine measurements (Phthalates, BPA, Mn, As, Cd, OC use, HRT use)

3. Urine creatinine measurements $<30 \mathrm{mg} / \mathrm{dl}$ and $>300 \mathrm{mg} / \mathrm{dl}$

4. Incomplete responses to identified outcome variables.

\section{Phthalate Exposure Assessment and Measurements}

Phthalates were measured from urine samples taken from a representative and random one-third subsample of individuals 6 years of age and older in the 2011-2012 and

2013-2014 survey cycles ${ }^{4,5}$. The laboratory utilized high performance liquid chromatography-electrospray ionization-tandem mass spectrometry to analyze urine phthalate levels and was consistently used in both survey cycles ${ }^{4,5}$. Phthalate levels were provided in $\mathrm{ng} / \mathrm{ml}$ and was used in our analyses. The following phthalates were used in our analyses: Mono (carboxynonyl) Phthalate (CNP); Mono (carboxyoctyl) Phthalate (COP); Mono-(2-ethyl-5-carboxypentyl) Phthalate (ECP); Mono-n-butyl Phthalate (MBP); Mono-(3-carboxypropyl) Phthalate (MC1); Mono-ethyl Phthalate (MEP); Mono 
(2-ethyl-5-hydroxy hexyl) Phthalate (MHH); Mono-(2-ethyl-5-oxohexyl) Phthalate

(MOH); Mono-benzyl Phthalate (MZP); Mono-isobutyl Phthalate (MIB).

The limit of detection variables indicates if subjects have urine phthalate levels above the limit of detection. A value of "0" indicates above the limit of detection and a value of "1" indicates below the limit of detection. Individuals with a below the limit of detection were given a dummy phthalate level of the LOD divided by the square root of two ${ }^{6}$. Some LODs differ between survey cycles and a conservative approach was used to account for differing LODs per survey cycle ${ }^{7}$. The specific phthalate metabolites were selected as $>60 \%$ of study subjects had urine phthalate levels above the LOD. A total of 5,175 subjects had available urinary phthalate measurements in the 2011-2012 and 20132014 datasets.

\section{BPA Exposure Assessment and Measurements}

BPA was measured from urine samples taken from a representative and random one-third subsample of individuals 6 years of age and older in the 2011-2012 and 20132014 survey cycles ${ }^{8,9}$. The laboratory on-line solid phase extraction coupled to high performance liquid chromatography and tandem mass spectrometry to analyze urine BPA levels and was consistently used in both survey cycles ${ }^{8,9}$. BPA levels were provided in $\mathrm{ng} / \mathrm{ml}$.

The limit of detection variables indicates if subjects have urine phthalate levels above the limit of detection. A value of " 0 " indicates above the limit of detection and a value of "1" indicates below the limit of detection. Individuals with a below the limit of

detection were given a dummy BPA level of the LOD divided by the square root of two ${ }^{6}$. Some LODs differ between survey cycles and a conservative approach was used to 
account for differing LODs per survey cycle ${ }^{7}$. A total of 5,175 subjects had available urinary BPA measurements in the 2011-2012 and 2013-2014 datasets.

\section{Cadmium Exposure Assessment and Measurements}

Cadmium were measured from urine samples taken from a representative and random one-third subsample of individuals 6 years of age and older in the 2011-2012 and 20132014 survey cycles ${ }^{10-12}$. The laboratory inductively coupled-plasma dynamic reaction cell-mass spectrometry to analyze urine Cadmium levels and was consistently used in both survey cycles ${ }^{10-12}$. Cadmium levels were provided in ug/L and was used in our analyses. Urinary cadmium was used in our analyses and coded as URXUCD, with a limit of detection variable coded as URDUCDLC. The limits of detection were 0.056 ug/L for 2011-2012 and 0.036 ug/L for 2013-2014.

The limit of detection variables indicates if subjects have urine Cadmium levels above the limit of detection. A value of "0" indicates above the limit of detection and a value of "1" indicates below the limit of detection. Individuals with a below the limit of detection were given a dummy Cadmium level of the LOD divided by the square root of two ${ }^{6}$. Some LODs differ between survey cycles and a conservative approach was used to account for differing LODs per survey cycle ${ }^{7}$.

\section{Manganese Exposure Assessment and Measurements}

Manganese were measured from urine samples taken from a representative and random one-third subsample of individuals 6 years of age and older in the 2011-2012 and 2013-2014 survey cycles ${ }^{10-12}$. The laboratory inductively coupled-plasma dynamic reaction cell-mass spectrometry to analyze urine Manganese levels and was consistently

used in both survey cycles ${ }^{10-12}$. Manganese levels were provided in $u g / L$ and was used in 
our analyses. The following Manganese metabolites were used in our analyses. Urinary manganese was used in our analyses and coded as URXUMN, with a limit of detection variable coded as URDUMNLC. The limits of detection were $0.08 \mathrm{ug} / \mathrm{L}$ for 2011-2012 and $0.013 \mathrm{ug} / \mathrm{L}$ for $2013-2014$.

The limit of detection variables indicates if subjects have urine Manganese levels above the limit of detection. A value of "0" indicates above the limit of detection and a value of "1" indicates below the limit of detection. Individuals with a below the limit of detection were given a dummy Manganese level of the LOD divided by the square root of two ${ }^{6}$. Some LODs differ between survey cycles and a conservative approach was used to account for differing LODs per survey cycle ${ }^{7}$.

\section{Arsenic Exposure Assessment and Measurements}

Arsenic were measured from urine samples taken from a representative and random onethird subsample of individuals 6 years of age and older in the 2011-2012 and 2013-2014 survey cycles ${ }^{10-12}$. The laboratory inductively coupled-plasma dynamic reaction cellmass spectrometry to analyze urine arsenic levels and was consistently used in both survey cycles ${ }^{10-12}$. Arsenic levels were provided in ug/L and was used in our analyses. Total urinary arsenic was used in our analyses and coded as URXUAS, with a limit of detection variable coded as URDUASLC. The limits of detection were $1.25 \mathrm{ug} / \mathrm{L}$ for 2011-2012 and 0.26 ug/L for 2013-2014.

Only total arsenic was analyzed in the study. The limit of detection variables indicates if subjects have urine arsenic levels above the limit of detection. A value of " 0 " indicates above the limit of detection and a value of "1" indicates below the limit of detection. Individuals with a below the limit of detection were given a dummy arsenic 
level of the LOD divided by the square root of two ${ }^{6}$. Some LODs differ between survey cycles and a conservative approach was used to account for differing LODs per survey cycle ${ }^{7}$. The specific arsenic metabolites were selected as $>60 \%$ of study subjects had urine arsenic levels above the LOD.

\section{Oral Contraceptive Use Assessment and Measurements}

Oral contraceptive use was recorded by the question yes/no question, "Have you ever taken birth control pills for any reason?". Female participants aged 12 years and older were eligible ${ }^{13,14}$. These questions were administered in the mobile examination center (MEC) by trainer interviewers ${ }^{13,14}$. A total of 1670 female subjects over the age of 60, provided a response, with 952 participants responding "yes" and 718 participants responding "no".

\section{Hormonal Replacement Therapy Use Assessment and Measurements}

Oral contraceptive use was recorded by the question yes/no question, "Have you ever used female hormones such as estrogen and progesterone?". Female participants aged 12 years and older were eligible ${ }^{13,14}$. These questions were administered in the mobile examination center (MEC) by trainer interviewers ${ }^{13,14}$. A total of 1662 female subjects over the age of 60 , provided a response, with 628 participants responding "yes" and "1034" participants responding "no".

\section{Assessment of Surrogate Brain Health Indicators - Cognitive Scores}

\section{CERAD Word Learning Subtest - Immediate Recall and Delayed Recall: The} Consortium to Establish a Registry for Alzheimer's Disease (CERAD) Word Learning Subtest assesses both immediate and delayed learning ${ }^{15,16}$. The delayed and immediate recall tests available in NHANES assess the ability to process new verbal information 
15,16. The tests are part of the neuropsychological assessment for the entire CERAD testing protocol, which was initially created to standardize Alzheimer's disease (AD) assessment and diagnosis ${ }^{17}$. The tests in the neuropsychological assessment itself were chosen because of their ability to assess cognitive functions inherent in $\mathrm{AD}^{17}$. The assessments have the ability to differentiate those of adequate cognitive status versus those who have mild cognitive impairment or dementia ${ }^{17-20}$. Although developed for use in the assessment of $\mathrm{AD}$, the CERAD assessments have shown utility in use for Parkinson's disease ${ }^{20}$ and frontotemporal lobar degeneration ${ }^{21}$.

Immediate Recall: For immediate recall, the subjects are asked to read aloud a sequence of 10 unrelated words as they are presented to them and immediately after, they are asked to recall as many words as possible ${ }^{15,16}$. This is done in three trials with the order of the words differing in each trial. ${ }^{15,16}$. Each trial has a maximum score of 10 , with a maximum overall score of $30^{15,16,18}$.

In our study, we included subjects 60 years of age and older who completed all immediate recall word list trials identified as: CFDCST1, CFDCST2, and CFDCST3 in the 2011-2012 and 2013-2014 NHANES data cycles. Those who did not have three trials completed were not included in the immediate recall analysis. We summed the total of the three trials and created a new variable with cut-off scores named

IMMEDIATERECALL. A cut-off score of $\leq 13$ and $\geq 14$ was used as it is the standard in other assessments ${ }^{18,22}$. A total of 3,149 subjects from the 2011-2012 and 2013-2014 responded with complete immediate recall trials.

We then accounted for subjects who had urinary phthalates and BPA samples taken and accounted for those with extreme creatinine scores ( $<30$ and $>300 \mathrm{mg} / \mathrm{dl}$ ), as 
these extreme values can affect analyses by being too dilute or concentrated ${ }^{23}$. After, our study population consisted of 940 subjects, 146 subjects with cut-off scores $\leq 13$ and 794 subjects with cut-off scores $\geq 14$. We then accounted for those who were measured for metalloestrogens and accounted for those with extreme creatinine scores $(<30$ and $>300$ $\mathrm{mg} / \mathrm{dl}$ ), as these extreme values can affect analyses by being too dilute or concentrated 23. After, our immediate recall study population consisted of 940 subjects, 146 subjects $\leq$ 13 and 794 subjects $\geq 14$. We then accounted for those who provided responses to OC and HRT use. After, our OC/immediate recall study population consisted of 1576 subjects, 193 subjects $\leq 13$ and 1383 subjects $\geq 14$. Our HRT/immediate recall study population consisted 1567 subjects, with 189 subjects $\leq 13$ and 1378 subjects $\geq 14$. Delayed Recall: For delayed recall, the subject is asked to repeat the sequence of 10 unrelated words after the other cognitive tests are completed, which is typical 8 to 10 minutes after the start of the word learning trials ${ }^{15,16}$. The maximum score is 10 for delayed recall ${ }^{15,16}$.

In our study, we included subjects 60 years of age and older who completed the delayed recall trial, identified as CFDCSR, for the 2011-2012 and 2013-2014 NHANES data cycles. After we created a new variable with the cut-off scores named DELAYEDRECALL. A cut-off score of $\leq 3$ and $\geq 4$ was used as it is the standard in other assessments ${ }^{18,22,24}$.

A total of 3,126 subjects from the 2011-2012 and 2013-2014 responded with a complete delayed recall trial. We then accounted for subjects who had urinary phthalates and BPA samples taken and accounted for those with extreme creatinine scores $(<30$ and $>300 \mathrm{mg} / \mathrm{dl}$ ), as these extreme values can affect analyses by being too dilute or 
concentrated $^{23}$. After, our study population consisted of 930 subjects, 157 subjects $\leq 3$ and 776 subjects $\geq 4$. We then accounted for those who were measured for metalloestrogens and accounted for those with extreme creatinine scores $(<30$ and $>300$ $\mathrm{mg} / \mathrm{dl}$ ), as these extreme values can affect analyses by being too dilute or concentrated 23. After, our delayed recall study population consisted of 930 subjects, 157 subjects $\leq 3$ and 776 subjects $\geq 4$. We then accounted for those who provided responses to OC and HRT use. After, our OC/delayed recall study population consisted of 1568 subjects, 218 subjects $\leq 3$ and 1350 subjects $\geq 4$. Our HRT/delayed recall study population consisted of 1560 subjects with 213 subjects $\leq 3$ and 1347 subjects $\geq 4$.

Animal Fluency: The animal fluency test is used to determine categorical verbal fluency, which is part of executive function and can differentiate between with normal cognition versus those with $\mathrm{MCI}$ and more severe cognitive impairment, such as $\mathrm{AD}{ }^{15,16}$. Since the test uses animal names, it does not require cultural consideration or formal education experience ${ }^{15,16}$. In the test, subjects are asked to name as many animals in a one minute span, with a maximum range of 40 words in the NHANES 2011-2014 data set. ${ }^{15,16}$. A sample test is given to each subject before the actual test ${ }^{15,16}$.

In our study, we included subjects 60 years of age and older who completed the animal fluency trial, identified as CFDAST, for the 2011-2012 and 2013-2014 NHANES data cycles. After we created a new variable with cut-off scores named VERBALFLUENCY. cut-off score of $\leq 11$ and $\geq 12$ was used as it is the standard in other assessments $18,22,24,25$.

A total of 3,110 subjects from the 2011-2012 and 2013-2014 responded with complete animal fluency scores. We then accounted for subjects who had urinary 
phthalates and BPA samples taken and accounted for those with extreme creatinine scores $(<30$ and $>300 \mathrm{mg} / \mathrm{dl})$, as these extreme values can affect analyses by being too dilute or concentrated ${ }^{23}$. After, our study population consisted of 931 subjects, 187 subjects $\leq 11$ and 744 subjects $\geq 12$. We then accounted for those who were measured for metalloestrogens and accounted for those with extreme creatinine scores $(<30$ and $>300$ $\mathrm{mg} / \mathrm{dl}$ ), as these extreme values can affect analyses by being too dilute or concentrated 23. After, our study population consisted of 931 subjects, 187 subjects $\leq 11$ and 744 subjects $\geq 12$. We then accounted for those who provided responses to OC and HRT use. After, our OC/Animal Fluency study population consisted of 1565 subjects, 321 subjects $\leq 321$ and 1244 subjects $\geq 12$. Our HRT/Animal Fluency study population consisted of 1557 subjects with 318 subjects $\leq 11$ and 1239 subjects $\geq 12$.

Digit Symbol Substitution Test: The Digit Symbol Substitution Test (DSST) is part of the Wechsler Adult Intelligence Scale (WAIS III) ${ }^{15,16,26}$. The test measures processing speed, sustained attention, and working memory ${ }^{15,16,26}$. The subtests have shown utility in the identification of dementia and other neurodegenerative disorders such as AD $^{27-29}$. The test is given in paper form, with a key that has 9 numbers paired to different symbols. The subject has 2 minutes to match each symbol to 133 boxes with a number associated to it, with the score as the total correct matches with a maximum score of 105 in the 2011-2014 NHANES dataset. ${ }^{15,16}$. A sample test is given to each subject before the actual test ${ }^{15,16}$.

In our study, we included subjects 60 years of age and older who completed the animal fluency trial, identified as CFDDS, for the 2011-2012 and 2013-2014 NHANES data cycles. After we created a new variable with cut-off scores named DSST, cut-off 
score of $\leq 27$ and $\geq 28$ was used as it is the standard in other assessments ${ }^{30-32}$. A total of 3,014 subjects from the 2011-2012 and 2013-2014 responded with complete DSST. We then accounted for subjects who had urinary phthalates and BPA samples taken and accounted for those with extreme creatinine scores ( $<30$ and $>300 \mathrm{mg} / \mathrm{dl})$, as these extreme values can affect analyses by being too dilute or concentrated ${ }^{23}$. After, our study population consisted of 891 subjects, 129 subjects $\leq 27$ and 762 subjects $\geq 28$. We then accounted for those who were measured for metalloestrogens and accounted for those with extreme creatinine scores ( $<30$ and $>300 \mathrm{mg} / \mathrm{dl}$ ), as these extreme values can affect analyses by being too dilute or concentrated ${ }^{23}$. After, our study population consisted of 891 subjects, 129 subjects $\leq 27$ and 744 subjects $\geq 28$. We then accounted for those who provided responses to OC and HRT use. After, our OC/DSST study population consisted of 1511 subjects, 227 subjects $\leq 27$ and 1284 subjects $\geq 28$. Our HRT/DSST study population consisted of 1505 subjects with 226 subjects $\leq 27$ and 1279 subjects $\geq$ 28. We then accounted for those who were measured for metalloestrogens and accounted for those with extreme creatinine scores $(<30$ and $>300 \mathrm{mg} / \mathrm{dl})$, as these extreme values can affect analyses by being too dilute or concentrated ${ }^{23}$. After, our study population consisted of 1,074 subjects, 188 subjects answered " 1 " and 783 subjects answered " 2 ". We then accounted for those who provided responses to OC and HRT use. After, our OC/MCQ084 study population consisted of 1669 subjects, 274 subjects responded "yes" and 1395 subjects responded "no". Our HRT/MCQ084 study population consisted of 1661 subjects with 272 subjects responded "yes" and 1389 subjects responded "no". 


\section{Assessment of Surrogate Brain Health Indicators - Memory Function}

\section{During the past 12 months, have you experienced confusion or memory loss that is}

happening more often or getting worse?: In our study we included subjects 60 years of age and older who had phthalate and BPA samples taken and responded to the yes/no question, "During the past 12 months, have you experienced confusion or memory loss that is happening more often or is getting worse?" 33 . As memory loss and confusion are early indicators of cognitive decline, dementia, and $\mathrm{AD}^{34} .3,628$ subjects responded to this question in the 2011-2012 and 2013-2014 survey cycle. We then accounted for subjects who had urinary phthalates and BPA samples taken and accounted for those with extreme creatinine scores $(<30$ and $>300 \mathrm{mg} / \mathrm{dl})$, as these extreme values can affect analyses by being too dilute or concentrated ${ }^{23}$. After, our study population consisted of 1,025 subjects, 181 subjects answered "yes" and 844 subjects answered "no". We then accounted for those who provided responses to OC and HRT use. After, our OC/MCQ084 study population consisted of 1669 subjects, 274 subjects responded "yes" and 1395 subjects responded "no". Our HRT/MCQ084 study population consisted of 1661 subjects with 272 subjects responded "yes" and 1389 subjects responded "no".

\section{During the past 7 days, how often have you had trouble remembering where you put}

things?: In our study we included subjects 60 years of age and older who had phthalate and BPA samples taken and responded to the question," During the past 7 days, how often have you had trouble remembering where you put things, like you keys or your wallet?" ${ }^{33} .3,448$ subjects responded to this question in the 2011-2012 and 2013-2014 survey cycle. 
The question is multi-leveled, where 3,448 subjects answered "Never" equaling the value " 0 ", 809 subjects answered "About once" equaling the value " 1 ", 544 subjects answered "Two or three times" equaling the value "2", 175 subjects answered "Nearly every day" equaling the value "3", and 102 subjects answered "Several times a day" equaling the value "4". We created a new variable named MCQ380_WK, which combines responses coded as "Never" equaling " 0 " and "About once" equaling " 1 " into a variable, "No" equaling "1", and "Two or Three Times" equaling “2”, "Nearly Every day" equaling "3", and "Several times a day" equaling "4", into a new variable, "Yes" equaling " 1 ".

We then accounted for subjects who had urinary phthalates and BPA samples taken and accounted for those with extreme creatinine scores ( $<30$ and $>300 \mathrm{mg} / \mathrm{dl}$ ), as these extreme values can affect analyses by being too dilute or concentrated ${ }^{23}$. After, our study population consisted of 971 subjects, 188 subjects answered " 1 " and 783 subjects answered " 2 ". We then accounted for those who were measured for metalloestrogens and accounted for those with extreme creatinine scores $(<30$ and $>300 \mathrm{mg} / \mathrm{dl})$, as these extreme values can affect analyses by being too dilute or concentrated ${ }^{23}$. After, our study population consisted of 1,074 subjects, 188 subjects answered " 1 " and 783 subjects answered " 2 ".

We then accounted for those who provided responses to OC and HRT use. After, our OC/MCQ380_WK study population consisted of 1576 subjects, 335 subjects responded "yes" and 1241 subjects responded "no". Our HRT/MCQ380_WK study population consisted of 1568 subjects with 332 subjects responded "yes" and 1236 subjects responded "no". 


\section{Are you limited in any way because of difficulty remembering or because you}

experience periods of confusion?: In our study we included subjects 60 years of age and older who had phthalate and BPA samples taken and responded to the question, "Are you limited in any way because of difficulty remembering or because you experience periods of confusion?" ${ }^{33}$. Limitations in physical movement due to difficulty remembering and confusion can indicate the development of cognition issues ${ }^{35} .11,323$ subjects responded to this question in the 2011-2012 and 2013-2014 survey cycle.

We then accounted for subjects who had urinary phthalates and BPA samples taken and accounted for those with extreme creatinine scores ( $<30$ and $>300 \mathrm{mg} / \mathrm{dl}$ ), as these extreme values can affect analyses by being too dilute or concentrated ${ }^{23}$. After, our study population consisted of 1,024 subjects, 165 subjects answered "yes" and 859 subjects answered "no". We then accounted for those who were measured for metalloestrogens and accounted for those with extreme creatinine scores $(<30$ and $>300$ $\mathrm{mg} / \mathrm{dl}$ ), as these extreme values can affect analyses by being too dilute or concentrated 23. After, our study population consisted of 1,133 subjects, 181 subjects answered yes and 952 subjects answered no. We then accounted for those who provided responses to OC and HRT use. After, our OC/PFQ057 study population consisted of 1670 subjects, 259 subjects responded "yes" and 1411 subjects responded "no". Our HRT/PFQ057 study population consisted of 1662 subjects with 254 subjects responded "yes" and 1408 subjects responded "no".

\section{Assessment of Surrogate Brain Health Indicators - Taste and Smell Function}

It has been observed that neurodegenerative disease has been shown to be preceded by smell and taste disorders ${ }^{36-39}$. The causes of these disorders have been 
linked to genetic alterations ${ }^{36}$, overexpression of key proteins ${ }^{37}$, and direct effect of some environmental chemicals on the olfactory mucosa ${ }^{40}$, which can have associations with exposure to EEDCs ${ }^{36,37,39,40}$. However, issues with olfaction can also be caused by upper respiratory tract infections, sino-nasal disease, head trauma, idiopathic causes, surgery of the nasal area, and congenital loss of smell ${ }^{41}$.

The two most common and prevalent neurodegenerative diseases, $\mathrm{AD}$ and $\mathrm{PD}$ have been shown to be preceded by smell disorders ${ }^{42-47}$. These disorders manifest themselves when evidence of pathological changes in the olfactory system are evident ${ }^{47}$. These are characterized by the build-up of pathological proteins, which cause the death of olfactory cells ${ }^{47}$. Several human epidemiological studies have also alluded to the utility of using sensory biomarkers as an early detection for neurodegenerative diseases ${ }^{48-51}$.

\section{Do you sometimes smell and unpleasant, bad, or burning odor when nothing is}

there?: In our study we included subjects 60 years of age and older who had phthalate and BPA samples taken and responded to the question, "Do you sometimes smell an unpleasant, bad or burning odor when nothing is there?" ${ }^{33} .7,399$ subjects responded to this question in the 2011-2012 and 2013-2014 survey cycle.

We then accounted for subjects who had urinary phthalates and BPA samples taken and accounted for those with extreme creatinine scores ( $<30$ and $>300 \mathrm{mg} / \mathrm{dl}$ ), as these extreme values can affect analyses by being too dilute or concentrated ${ }^{23}$. After, our study population consisted of 1,024 subjects, 74 subjects answered "yes" and "950" subjects answered no. We then accounted for those who were measured for metalloestrogens and accounted for those with extreme creatinine scores $(<30$ and $>300$ $\mathrm{mg} / \mathrm{dl}$ ), as these extreme values can affect analyses by being too dilute or concentrated 
23. After, our study population consisted of 1,050 subjects, 74 subjects answered yes and 950 subjects answered no. We then accounted for those who provided responses to OC and HRT use. After, our OC/CSQ040 study population consisted of 1664 subjects, 123 subjects responded "yes" and 1541 subjects responded "no". Our HRT/CSQ040 study population consisted of 1655 subjects with 123 subjects responded "yes" and 1532 subjects responded "no".

\section{During the past 12 months have you had a taste or other sensation in your mouth}

that does not go away?: In our study we included subjects 60 years of age and older who had phthalate and BPA samples taken and responded to the question," During the past 12 months have you had a taste or other sensation in your mouth that does not go away?"33. 7,407 subjects responded to this question in the 2011-2012 and 2013-2014 survey cycle.

We then accounted for subjects who had urinary phthalates and BPA samples taken and accounted for those with extreme creatinine scores $(<30$ and $>300 \mathrm{mg} / \mathrm{dl})$, as these extreme values can affect analyses by being too dilute or concentrated ${ }^{23}$. After, our study population consisted of 1,024 subjects, 66 subjects answered yes and 958 subjects answered no. We then accounted for those who were measured for metalloestrogens and accounted for those with extreme creatinine scores $(<30$ and $>300 \mathrm{mg} / \mathrm{dl})$, as these extreme values can affect analyses by being too dilute or concentrated ${ }^{23}$. After, our study population consisted of 1,024 subjects, 66 subjects answered yes and 958 subjects answered no. We then accounted for those who provided responses to OC and HRT use. After, our OC/CSQ110 study population consisted of 1667 subjects, 134 subjects responded "yes" and 1533 subjects responded "no". Our HRT/CSQ110 study population 
consisted of 1658 subjects with 134 subjects responded "yes" and 1524 subjects responded "no".

\section{Covariates and Confounding Variables}

In our study we included a number of covariates, based off a review of literature and well-known risk factors for neurodegenerative diseases, if they were available in the NHANES datasets. Confounding variables were controlled for in our logistic regression models.

The demographic variables are as follows: gender (male, female), age (60-69, 779, 80+), Race/Ethnicity (Hispanic, Non-Hispanic White, Non-Hispanic Black, NonHispanic Asian/Other), Family Income (Under 24k, 25k to 54,999k, 55k to 74,999k, over $75 \mathrm{k})$, Education $\left(<12^{\text {th }} \text { grade, completed high school, }>12^{\text {th }} \text { grade }\right)^{33}$.

Modifiable health variables and risk factors are as follows: ever smoked (yes, no), blood pressure (normal/high), diabetes (yes, no, borderline), coronary heart disease (yes, no), stroke (yes, no), heart attack (yes, no), head trauma (yes, no), alcohol use (yes, no), ever use birth control (yes, no), every use hormonal replacement therapy (yes, no) ${ }^{33}$.

\section{Statistical Analysis}

Statistical analysis was performed using SAS software ${ }^{52}$. The 2011-2012 and 2013-2014 survey cycles were merged and a four-year sampling weight was calculated to account for the complex sampling design in order to calculate correct statistical estimates and standard errors when calculating means, geometric means, and other statistics ${ }^{53}$.

For phthalate and BPA variables, a value of " 0 " indicates above the limit of detection and a value of " 1 " indicates below the limit of detection. Individuals with a 
below the limit of detection were given a dummy phthalate level of the LOD divided by the square root of two ${ }^{6}$. Some LODs differ between survey cycles and a conservative approach was used to account for differing LODs per survey cycle, where the LOD for that year was used to make a determination if the EEDC was above or below the LOD. ${ }^{7}$. We log-transformed and then adjusted for creatinine all phthalate and BPA variables ${ }^{54-56}$ since environmental chemical data is not normally distributed and urine dilution varies from person to person. We used the SAS Survey procedures to account for the complex sampling design of the NHANES data sets ${ }^{57}$.

We used PROC SURVEYFREQ was used to obtain descriptive statistics for the different populations we were examining in our study which accounts for the complex survey design of the NHANES data sets ${ }^{57}$. Descriptive statistics were organized based on the following categories per variable: gender (male, female), age (60-69, 7-79, 80+), Race/Ethnicity (Hispanic, Non-Hispanic White, Non-Hispanic Black, Non-Hispanic Asian/Other), Family Income (Under 24k, 25k to 54,999k, 55k to 74,999k, over 75k), Education $\left(<12^{\text {th }}\right.$ grade, completed high school, $>12^{\text {th }}$ grade), ever smoked (yes, no), blood pressure (normal/high), diabetes (yes, no, borderline), coronary heart disease (yes, no), stroke (yes, no), heart attack (yes, no), head trauma (yes, no), alcohol use (yes, no), ever use birth control (yes, no), every use hormonal replacement therapy (yes, no) ${ }^{33}$.

We used PROC SURVEYREG and guidance provided by the SAS institute to directly to determine the geometric mean of the EEDC to test if they were significant between the responses of our outcome variables ${ }^{57,58}$. The standard errors were calculated using the Taylor Series linearization method, which is the default method in the survey procedures to calculate standard error ${ }^{57}$. Geometric means (GM), geometric standard 
errors (GSE), and number of subjects were reported for the results of the outcome variables for all subjects that had EEDCs over the LOD. We looked at geometric means between the surrogate of brain health (yes vs. no, low test score vs. high test score), and also performed age-specific, gender-specific, and race/ethnicity-specific geometric means between the responses to the outcome variable. Due to the smaller range of ages in our dataset, 60 years and older, we calculated age-specific rates in lieu of age-standardized rates.

For OC and HRT use, which are both dichotomous variables, we performed a chi-square test of independence using PROC SURVEYREG with the CHISQ command to look for any relationships between OC and HRT use and our surrogate brain health indicators. We used PROC SURVEYLOGISTIC to find the unadjusted and adjusted odds ratios (ORs) and the $95 \%$ confidence intervals (CI) to examine the association between the surrogates of brain health and exposures to phthalates and BPA ${ }^{57}$. Analysis was done per EEDC per outcome variable. We presented three logistic regression models which were stratified by gender and examined phthalate and BPA exposures in the following groups, variable $<$ LOD to $50^{\text {th }}$ percentile (reference) and $\geq 50^{\text {th }}$ percentile. The three logistic regression models are as follows: unadjusted, adjusted for known risk factors, age, education, race/ethnicity, adjusted for known and suspected risk factors, age, education, race/ethnicity, smoking, blood pressure history, history of coronary heart disease, stroke, heart attack, diabetes status, head trauma, and alcohol use. We did not include income, OC and HRT use in our models as they significantly reduced the size of the population. 


\section{References}

1. Johnson CL, Paulose-Ram R, Ogden CL, et al. National health and nutrition examination survey: analytic guidelines, 1999-2010. Vital Health Stat 2. 2013;(161):1-24. http://www.ncbi.nlm.nih.gov/pubmed/25090154.

2. Johnson CL, Dohrmann SM, Burt VL, Mohadjer LK. National Health and Nutrition Examination Survey: Sample Design , 2011 - 2014. Vital Heal Stat. 2014;2(162):1-33. http://www.cdc.gov/nchs/data/series/sr_02/sr02_162.pdf.

3. Centers for Disease Control and Prevention (CDC). National Center for Health Statistics (NCHS). National Health and Nutrition Examination Survey NCHS Research Ethics Review Board (ERB) Approval. Hyattsville, MD: U.S. Department of Health and Human Services, Centers for Disease Control and Prevention. https://www.cdc.gov/nchs/nhanes/irba98.htm. Published 2016.

4. Centers for Disease Control and Prevention (CDC). National Center for Health Statistics (NCHS). National Health and Nutrition Examination Survey - 20112012 Data Documentation, Codebook, and Frequencies Phthalates \& Plasticizers Metabolites - Urine (PHTHTE_G). Hyattsville, MD: U.S. Department of Health and Human Services, Centers for Disease Control and Prevention. https://wwwn.cdc.gov/Nchs/Nhanes/2011-2012/PHTHTE_G.htm. Published 2016.

5. Centers for Disease Control and Prevention (CDC). National Center for Health Statistics (NCHS). National Health and Nutrition Examination Survey - National Health and Nutrition Examination Survey 2013-2014 Data Documentation, Codebook, and Frequencies Phthalates and Plasticizers Metabolites - Urine (PHTHTE_H). Hyattsville, MD: U.S. Department of Health and Human Services, Centers for Disease Control and Prevention. https://wwwn.cdc.gov/Nchs/Nhanes/2013-2014/PHTHTE_H.htm. Published 2016.

6. Centers for Disease Control and Prevention (CDC). National Center for Health Statistics (NCHS). National Health and Nutrition Examination Survey Data, Questionnaires, and Related Documentation and Information. National Health and Nutrition Examination Survey Data. Hyattsville, MD: U.S. Department of Health and Human Services, Centers for Disease Control and Prevention. https://www.cdc.gov/nchs/nhanes/index.htm. Published 2016. 
7. Centers for Disease Control and Prevention (CDC). National Center for Health Statistics (NCHS). National Health and Nutrition Examination Survey - Task 2: How to Address Different Fill Values and the Limit of Detection (LOD) in NHANES Environmental Chemical Data. Hyattsville, MD: U.S. Department of Health and Human Services, Centers for Disease Control and Prevention. https://www.cdc.gov/nchs/tutorials/environmental/critical_issues/limitations/Task 2.htm. Published 2016.

8. Centers for Disease Control and Prevention (CDC). National Center for Health Statistics (NCHS). National Health and Nutrition Examination Survey - 20132014 Data Documentation, Codebook, and Frequencies Personal Care and Consumer Product Chemicals and Metabolites (EPHPP_H). Hyattsville, MD: U.S. Department of Health and Human Services, Centers for Disease Control and Prevention. https://wwwn.cdc.gov/Nchs/Nhanes/2013-2014/EPHPP_H.htm. Published 2016.

9. Centers for Disease Control and Prevention (CDC). National Center for Health Statistics (NCHS). National Health and Nutrition Examination Survey 2011-2012 Data Documentation, Codebook, and Frequencies Environmental Phenols \& Parabens (EPH_G). Hyattsville, MD: U.S. Department of Health and Human Services, Centers for Disease Control and Prevention. https://wwwn.cdc.gov/Nchs/Nhanes/2011-2012/EPH_G.htm. Published 2016.

10. Centers for Disease Control and Prevention (CDC). National Center for Health Statistics (NCHS). National Health and Nutrition Examination Survey 2013-2014 Data Documentation, Codebook, and Frequencies Urinary Total Arsenic (UTAS_H). Hyattsville, MD: U.S. Department of Health and Human Services, Centers for Disease Control and Prevention. https://wwwn.cdc.gov/Nchs/Nhanes/2013-2014/UTAS_H.htm. Published 2016.

11. Centers for Disease Control and Prevention (CDC). National Center for Health Statistics (NCHS). National Health and Nutrition Examination Survey 2011-2012 Data Documentation, Codebook, and Frequencies Arsenics - Total \& Speciated Urine (UAS_G). Hyattsville, MD: U.S. Department of Health and Human Services, Centers for Disease Control and Prevention. https://wwwn.cdc.gov/Nchs/Nhanes/2011-2012/UAS_G.htm. Published 2016. 
12. Centers for Disease Control and Prevention (CDC). National Center for Health Statistics (NCHS). National Health and Nutrition Examination Survey 2013-2014 Data Documentation, Codebook, and Frequencies Urinary Speciated Arsenics (UAS_H). Hyattsville, MD: U.S. Department of Health and Human Services, Centers for Disease Control and Prevention. https://wwwn.cdc.gov/Nchs/Nhanes/2013-2014/UAS_H.htm. Published 2016.

13. Centers for Disease Control and Prevention (CDC). National Center for Health Statistics (NCHS). No TitleNational Health and Nutrition Examination Survey 2011-2012 Data Documentation, Codebook, and Frequencies Reproductive Health (RHQ_G). Hyattsville, MD: U.S. Department of Health and Human Services, Centers for Disease Control and Prevention. https://wwwn.cdc.gov/nchs/nhanes/2011-2012/RHQ_G.htm\#RHQ420. Published 2017.

14. Centers for Disease Control and Prevention (CDC). National Center for Health Statistics (NCHS). National Health and Nutrition Examination Survey 2013-2014 Data Documentation, Codebook, and Frequencies Reproductive Health (RHQ_H). https://wwwn.cdc.gov/Nchs/Nhanes/2013-2014/RHQ_H.htm. Published 2017.

15. Centers for Disease Control and Prevention (CDC). National Center for Health Statistics (NCHS). National Health and Nutrition Examination Survey 2013-2014 Data Documentation, Codebook, and Frequencies Cognitive Functioning (CFQ_H). Hyattsville, MD: U.S. Department of Health and Human Services, Centers for Disease Control and Prevention. https://wwwn.cdc.gov/Nchs/Nhanes/2013-2014/CFQ_H.htm. Published 2017.

16. Centers for Disease Control and Prevention (CDC). National Center for Health Statistics (NCHS). National Health and Nutrition Examination Survey 2011-2012 Data Documentation, Codebook, and Frequencies Cognitive Functioning (CFQ_G). Hyattsville, MD: U.S. Department of Health and Human Services, Centers for Disease Control and Prevention. https://wwwn.cdc.gov/Nchs/Nhanes/2011-2012/CFQ_G.htm\#CFDCST3. Published 2017.

17. Fillenbaum GG, van Belle G, Morris JC, et al. Consortium to Establish a Registry for Alzheimer's Disease (CERAD): The first twenty years. Alzheimer's Dement. 2008;4(2):96-109. doi:10.1016/j.jalz.2007.08.005. 
18. Bertolucci PHF, Okamoto IH, Brucki SMD, Siviero MO, Neto JT, Ramos LR. Applicability of the CERAD neuropsychological battery to Brazilian elderly. Arq Neuropsiquiatr. 2001;59(3 A):532-536. doi:10.1590/S0004282 X2001000400009.

19. Karrasch M, Sinervä E, Grönholm P, Rinne J, Laine M. CERAD test performances in amnestic mild cognitive impairment and Alzheimer's disease. Acta Neurol Scand. 2005;111(3):172-179. doi:10.1111/j.16000404.2005.00380.x.

20. Karrasch M, Laatu S, Martikainen K, Marttila R. CERAD test performance and cognitive impairment in Parkinson's disease. Acta Neurol Scand. 2013;128(6):409-413. doi:10.1111/ane.12138.

21. R.M. H, N.-M. S, P. H, et al. The CERAD neuropsychological battery in patients with frontotemporal lobar degeneration. Dement Geriatr Cogn Dis Extra. 2015;5(1):147-154. doi:10.1159/000380815.

22. Berres M, Monsch AU, Bernasconi F, Thalmann B, Stähelin HB. Normal ranges of neuropsychological tests for the diagnosis of Alzheimer's disease. Stud Health Technol Inform. 2000;77:195-199.

http://www.ncbi.nlm.nih.gov/pubmed/11187541. Accessed January 21, 2018.

23. Sata F, Araki S, Yokoyama K, Murata K. Adjustment of creatinine-adjusted values in urine to urinary flow rate: a study of eleven heavy metals and organic substances. Int Arch Occup Environ Health. 1995;68(1):64-68. http://www.ncbi.nlm.nih.gov/pubmed/8847115. Accessed November 15, 2017.

24. Alegret M, Espinosa A, Valero S, et al. Cut-off Scores of a Brief Neuropsychological Battery (NBACE) for Spanish Individual Adults Older than 44 Years Old. Brucki S, ed. PLoS One. 2013;8(10):e76436. doi:10.1371/journal.pone.0076436.

25. American Academy of Family Physicians. BB, Hartmann B, DeJoseph D. American Family Physician. Vol 84. American Academy of Family Physicians; 1970. https://www.aafp.org/afp/2011/1015/p895.html. Accessed January 21, 2018. 
26. Axelrod BN. Administration duration for the Wechsler Adult Intelligence ScaleIII and Wechsler Memory Scale-III. Arch Clin Neuropsychol. 2001;16(3):293301. doi:10.1016/S0887-6177(00)00048-2.

27. Matsuda O, Saito M, Kato M, Azami H, Shido E. Wechsler Adult Intelligence Scale-III profile in the early stages of Alzheimer's disease: Performance in subtests sensitive to and resistant to normal decline with ageing. Psychogeriatrics. 2015;15(1):1-6. doi:10.1111/psyg.12066.

28. Donnell AJ, Pliskin N, Holdnack J, Axelrod B, Randolph C. Rapidlyadministered short forms of the Wechsler Adult Intelligence Scale-3rd edition. Arch Clin Neuropsychol. 2007;22(8):917-924. doi:10.1016/j.acn.2007.06.007.

29. Nielsen NP, Ringström R, Wiig EH, Minthon L. Associations between AQT processing speed and neuropsychological tests in neuropsychiatric patients. Am J Alzheimers Dis Other Demen. 2011;22(3):202-210. doi:10.1177/1089253206300417.

30. Proust-Lima C, Amieva H, Dartigues JF, Jacqmin-Gadda H. Sensitivity of four psychometric tests to measure cognitive changes in brain aging-population-based studies. Am J Epidemiol. 2007;165(3):344-350. doi:10.1093/aje/kwk017.

31. Rosano C, Perera S, Inzitari M, Newman AB, Longstreth WT, Studenski S. Digit symbol substitution test and future clinical and subclinical disorders of cognition, mobility and mood in older adults. Age Ageing. 2016;45(5):687-694. doi:10.1093/ageing/afw116.

32. Saxton J, Lopez OL, Ratcliff G, et al. Preclinical Alzheimer disease: neuropsychological test performance 1.5 to 8 years prior to onset. Neurology. 2004;63(Mh 46643):2341-2347. doi:10.1212/01.WNL.0000147470.58328.50.

33. Centers for Disease Control and Prevention (CDC). National Center for Health Statistics (NCHS). National Health and Nutrition Examination Survey Questionnaires, Datasets, and Related Documentation. Hyattsville, MD: U.S. Department of Health and Human Services, Centers for Disease Control and Prevention. https://www.cdc.gov/nchs/nhanes/nhanes_questionnaires.htm. Published 2015. 
34. Alzheimer's Association. 2017 Alzheimer's Disease Facts and Figures. Alzheimers Dement . 2017;13:325-373. doi:10.1016/j.jalz.2017.02.001.

35. Tolea MI, Morris JC, Galvin JE. Longitudinal associations between physical and cognitive performance among community-dwelling older adults. PLoS One. 2015;10(4):1-12. doi:10.1371/journal.pone.0122878.

36. Ansoleaga B, Garcia-Esparcia P, Llorens F, Moreno J, Aso E, Ferrer I. Dysregulation of brain olfactory and taste receptors in AD, PSP and CJD, and AD-related model. Neuroscience. 2013;248:369-382. doi:10.1016/j.neuroscience.2013.06.034.

37. Macknin JB, Higuchi M, Lee VM-Y, Trojanowski JQ, Doty RL. Olfactory dysfunction occurs in transgenic mice overexpressing human $\tau$ protein. Brain Res. 2004;1000(1-2):174-178. doi:10.1016/j.brainres.2004.01.047.

38. Lee S, Manly J, Andrews $\mathrm{H}$, et al. Olfactory deficits predict cognitive decline and Alzheimer dementia in an urban community. 2014.

39. Hummel T, Landis B, Huttenbrink K. Smell and taste disorders. GMS Curr Tpoics Otorhinolaryngol Head Neck Surg. 2011;10:1-15. doi:10.3205/cto000077.

40. Doty RL. The olfactory vector hypothesis of neurodegenerative disease: Is it viable? Ann Neurol. 2008;63(1):7-15. doi:10.1002/ana.21327.

41. Liu J. Clinical features of olfactory disorders in patients seeking medical consultation. Med Sci Monit. 2013;19:444-450. doi:10.12659/MSM.883938.

42. Hüttenbrink K-B, Hummel T, Berg D, Gasser T, Hähner A. Olfactory dysfunction: common in later life and early warning of neurodegenerative disease. Dtsch Arztebl Int. 2013;110(1-2):1-7, e1. doi:10.3238/arztebl.2013.0001.

43. Barresi M, Ciurleo R, Giacoppo S, et al. Evaluation of olfactory dysfunction in neurodegenerative diseases. J Neurol Sci. 2012;323(1-2):16-24. doi:10.1016/j.jns.2012.08.028. 
44. Ruan Y, Zheng XY, Zhang HL, Zhu W, Zhu J. Olfactory dysfunctions in neurodegenerative disorders. J Neurosci Res. 2012;90(9):1693-1700.

doi:10.1002/jnr.23054.

45. Albers MW, Tabert MH, Devanand DP. Olfactory dysfunction as a predictor of neurodegenerative disease. Curr Neurol Neurosci Rep. 2006;6(5):379-386.

doi:10.1007/s11910-996-0018-7.

46. Winner B, Kohl Z, Gage FH. Neurodegenerative disease and adult neurogenesis. Eur J Neurosci. 2011;33(6):1139-1151. doi:10.1111/j.1460-9568.2011.07613.x.

47. Attems J, Walker L, Jellinger KA. Olfactory bulb involvement in neurodegenerative diseases. Acta Neuropathol. 2014;127(4):459-475. doi:10.1007/s00401-014-1261-7.

48. Makowska I, Kloszewska I, Grabowska A, Szatkowska I, Rymarczyk K. Olfactory deficits in normal aging and Alzheimer's disease in the polish elderly population. Arch Clin Neuropsychol. 2011;26(3):270-279. doi:10.1093/arclin/acr011.

49. Sohrabi HR, Bates K a, Weinborn MG, et al. Olfactory discrimination predicts cognitive decline among community-dwelling older adults. Transl Psychiatry. 2012;2(5):e118. doi:10.1038/tp.2012.43.

50. Driver-Dunckley E, Adler CH, Hentz JG, et al. Olfactory dysfunction in incidental Lewy body disease and Parkinson's disease. Parkinsonism Relat Disord. 2014;20(11):1260-1262. doi:10.1016/j.parkreldis.2014.08.006.

51. Ward AM, Calamia M, Thiemann E, Dunlap J, Tranel D. Association between olfaction and higher cortical functions in Alzheimer's disease, mild cognitive impairment, and healthy older adults. J Clin Exp Neuropsychol. November 2016:1-15. doi:10.1080/13803395.2016.1253667.

52. SAS 9.4. 2017. 
53. Centers for Disease Control and Prevention (CDC). National Center for Health Statistics (NCHS). National Health and Nutrition Examination Survey - Overview of NHANES Survey Design and Weights. Hyattsville, MD: U.S. Department of Health and Human Services, Centers for Disease Control and Prevention. https://www.cdc.gov/nchs/tutorials/environmental/orientation/sample_design/. Published 2013.

54. Centers for Disease Control and Prevention (CDC). National Center for Health Statistics (NCHS). National Health and Nutrition Examination Survey - Using Blood Lipid or Urine Creatinine Adjustments of Environmental Chemical Data. Hyattsville, MD: U.S. Department of Health and Human Services, Centers for Disease Control and Prevention.

https://www.cdc.gov/nchs/tutorials/environmental/critical_issues/adjustments/Info 1.htm. Published 2017.

55. Centers for Disease Control and Prevention (CDC). National Center for Health Statistics (NCHS). National Health and Nutrition Examination Survey - Using Blood Lipid or Urine Creatinine Adjustments in the Analysis of Environmental Chemical Data. Hyattsville, MD: U.S. Department of Health and Human Services, Centers for Disease Control and Prevention. https://www.cdc.gov/nchs/tutorials/environmental/critical_issues/adjustments/ind ex.htm. Published 2013.

56. Centers for Disease Control and Prevention (CDC). National Center for Health Statistics (NCHS). National Health and Nutrition Examination Survey - Data Normality and Transformations. Hyattsville, MD: U.S. Department of Health and Human Services, Centers for Disease Control and Prevention. https://www.cdc.gov/nchs/tutorials/environmental/critical_issues/normality/index. htm. Published 2010.

57. SAS Institute Inc. Sas/Stat ${ }^{\circledR}$ 9.3. 2011:User's Guide: The GLIMMIX Procedure (Chapter).

58. SAS Institute Inc. Estimating Geometric Means Using Data from a Complex Survey Sampling Design. http://citeseerx.ist.psu.edu/viewdoc/download?rep=rep1\&type=pdf\&doi=10.1.1.1 92.593. Published 2017. 


\title{
CHAPTER V
}

\section{MANUSCRIPT II}

\section{EXPOSURE TO PHTHALATES AND BISPHENOL-A AND ASSOCIATIONS}

\section{WITH BRAIN HEALTH: NHANES 2011-2014}

\begin{abstract}
BACKGROUND: The role of estrogenic endocrine disrupting chemicals (EEDCs) and their role in the development of neurodegenerative disease is of great public health concern, due to increasing exposures to these chemicals and increasingly aging population. Evidence suggests EEDCs exposure plays a role in the development of neurodegenerative disease, although epidemiological evidence is lacking in this area. Phthalates and Bisphenol-A are two of the most widespread EEDCs with demonstrated estrogenic activity, which affects brain health.

OBJECTIVE: The objective of this study is to investigate the relationship between surrogate brain health indicators and exposure to phthalates and BPA among the older individuals of the United States (US) population.
\end{abstract}

METHODS: In this study, we analyzed participants from the Center for Disease Control and Prevention (CDC) National Health and Nutrition Examination Survey (NHANES) in the survey cycles 2011-2012 and 2013-2014. The participants were 60 years of age and older who had phthalates and BPA urine samples taken during the examination portion of the survey. Other data pertaining to covariates and demographics were also obtained. In total, ten phthalate metabolites and Bisphenol-A were selected since $>60 \%$ of participants had levels over the established limit of detection (LOD). The ten analyzed EEDCs were the following: Mono (carboxynonyl) Phthalate (CNP); Mono (carboxyoctyl) Phthalate 
(COP); Mono-(2-ethyl-5-carboxypentyl) Phthalate (ECP); Mono-n-butyl Phthalate (MBP); Mono-(3-carboxypropyl) Phthalate (MC1); Mono-ethyl Phthalate (MEP); Mono (2-ethyl-5-hydroxy hexyl) Phthalate (MHH); Mono-(2-ethyl-5-oxohexyl) Phthalate (MOH); Mono-benzyl Phthalate (MZP); Mono-isobutyl Phthalate (MIB); Bisphenol-A (BPA). These EEDCs were analyzed versus surrogate brain health indicators available in the form of administered cognitive tests and self-reported questions, available in the NHANES datasets. The surrogate brain health indicators were as follows: immediate recall test; delayed recall test; animal fluency test; digit symbol substitution test. The surrogate brain health indicator self-reported questions were as follows: worsening memory over the past 12 months; trouble remembering over the past week; difficulty remembering or because you experience periods of confusion. The following smell and taste questions were also included as surrogate brain health indicators due to their potential as pre-clinical indicators of cognitive impairment: phantom odor (phantosmia) and problems with ability to taste sweet, sour, salty, or bitter foods over the past 12 months. Geometric means were calculated to compare the surrogate brain health indicators versus the EEDC concentrations. Logistic regression was then used to calculate odds ratios (ORs) and 95\% confidence intervals (CIs) to examine the associations between the surrogate brain health indicators and EEDC concentration. Three logistic regression models were presented in our study, stratified by gender: unadjusted; age, race, education; age, race, education, body mass index (BMI), smoking status, blood pressure, diabetes status, alcohol use, coronary heart disease status, heart attack status, stroke status, head injury status, and physical activity status. 
RESULTS: Increased levels of phthalate metabolites were observed in those who had lower cognitive test scores, reported having memory issues, and reported taste and smell deficits compared to those who did not have any observed brain health issues. Females have a greater bioburden of phthalates compared to males. In females, the phthalates ECP, MBP, MOH, MZP, and MIB were observed to have significantly higher bioburdens among two or more of the surrogate brain health indicators. In males, the phthalates ECP, $\mathrm{MHH}, \mathrm{MOH}$, and MIB were observed to have significantly higher bioburdens among two or more of the surrogate brain health indicators. BPA did not have any significant results in any of our tests. When controlling for known and suspected covariates of AD in males in our final logistic regression model, COP, ECP, MBP, MC1, MEP, MHH, MOH, and MIB were associated with one or more of the surrogate brain health indicators. When controlling for known and suspected covariates of $\mathrm{AD}$ in females in our final logistic regression model, ECP, MBP, MHH, MOH, MZP, and MIB were associated with one or more of the surrogate brain health indicators. BPA did not have any significant results in any of our tests.

CONCLUSION: Our study takes a novel approach to assessing cognitive dysfunction neurodegenerative disease and exposures to phthalate and BPA. It appears there is a link between exposure to phthalates and adverse brain health. Further research is needed with the use of clinical endpoints to further establish the relationship between neurodegenerative disease and phthalate/BPA exposure. 


\section{MANUSCRIPT II}

\section{EXPOSURE TO PHTHALATES AND BISPHENOL-A AND ASSOCIATIONS WITH BRAIN HEALTH: NHANES 2011-2014 INTRODUCTION}

The role of exposure to EEDCs and neurodegenerative disease development is of great public health concern as stated by the World Health Organization (WHO) ${ }^{1}$, due to an increasingly aging population ${ }^{2}$. Exposures to EEDCs have been linked to neurodegenerative diseases and other adverse brain health conditions, such as Alzheimer's disease (AD). In this study, we examine the associations of two of the mostly widely present phthalate and phenol compounds, phthalates and BPA, and their associations with brain health in an older US population.

Phthalates are class of estrogenic endocrine disrupting chemicals that have a role in the development of neurodegenerative disease. Phthalates, also known as plasticizers, are chemicals used in plastics to make them flexible and resilient ${ }^{3-5}$. Exposure to phthalates comes through ingestion, inhalation, and to a lesser extent, dermal contact with phthalate-containing products ${ }^{6}$. Phthalates are found in most consumer products. These include the following: wall coverings, tablecloths, floor tiles, furniture upholstery, shower curtains, garden hoses, baby products, toys, shoes, packing materials, medical devices, paints, glue, nail polish, hair spray, insect repellents, food packaging materials, cosmetics, insecticides, and drug products ${ }^{3-5}$. Phthalates have short biological half-lives and do not accumulate, with urine as the primary route of excretion ${ }^{7}$. Specific phthalates are also found in high levels among the US population. One study, found the body burden of the phthalates mono-ethyl phthalate (MEP), mono-n-butyl phthalate (MBP) and and 
mono-benzyl phthalate (MBzp) in 97\% of samples tested from the CDC NHANES 19992000 datasets $^{8}$. As such, most of the US population has some measurable levels of phthalates in their bodies ${ }^{7}$.

Phthalates have also been demonstrated to have estrogenic activity and affinity for estrogen receptors ${ }^{9,10}$ and have been shown to interact with estrogen-responsive genes implicated in various neurodegenerative disorders ${ }^{11}$.

There is limited information regarding human epidemiological studies with neurodegenerative disease and phthalate bioburden. Animal studies are numerous and have shown phthalates to adversely affect brain function. These adverse effects include negatively affecting learning ${ }^{12,13}$, negatively affecting memory ${ }^{13,14}$, interfering with locomotion ${ }^{14}$, negative affecting social behavior ${ }^{12}$, and producing cellular effects such as cell death, synaptic loss, and synaptic dysfunction ${ }^{15}$. Some animal studies have reported an improvement in memory ${ }^{16}$, and possible dose-dependent effects, where memory improves one dose, but degrades it in another dose ${ }^{17}$.

Human epidemiological studies have focused on pre-natal and early life exposure with regards to behavior, IQ, reproductive, and cognitive development with few focusing on older populations. Studies have indicated phthalate exposure to be associated with social deficits ${ }^{18}$, decreased visual recognition memory ${ }^{19}$ and decreased IQ ${ }^{20}$. However, there is evidence to suggest inconsistent cognitive and behavioral effects in children with regards to phthalate type and gender ${ }^{21,22}$. Only one study, using the NHANES 2011-2012 datasets found higher bioburdens of phthalates in individuals with memory issues ${ }^{23}$. BPA is a synthetic chemical that is widely used to make polycarbonate plastics and resins ${ }^{24}$. BPA is found in many consumer products and plastics. These products 
include the following: baby bottles, compact discs, impact-resistant safety equipment, medical devices, food cans and tops, water supply pipes, ATM receipts and dental sealants and composites ${ }^{24}$. Food containers made with BPA can cause BPA to leech into foods by the use of high heat ${ }^{24}$. It is of note that during the 2003-2004 NHANES data cycle, BPA was detected in $93 \%$ of urine samples collected from subjects 6 years of age and older ${ }^{25}$.

BPA has been shown to be weakly estrogenic and have a low affinity for binding to ER receptors, but is speculated to exert its effects through other non-classical pathways ${ }^{26}$. BPA has also been shown to interact with estrogen-responsive genes that are implicated in neurodegenerative disease pathways ${ }^{11}$.

Animal studies are numerous and have demonstrated BPA's negative effects on brain health and function. In animal studies BPA has been shown to negatively affect memory ${ }^{27-41}$, negatively affect neurogenesis ${ }^{33,42}$, negatively affect the structure of dendritic spines and synaptogenesis ${ }^{27,29,32,38,40,43}$, and negatively affect cellular processes, protein expression and expression $31,36,39,41$. There are also animal studies that shows BPA exerts no negative effects on spatial and working memory ${ }^{44-46}$.

The few human epidemiological studies have concentrated on pre-natal BPA exposure and exposure in children which have been associated with significant behavioral issues in children ${ }^{35,47-50}$. 


\section{OBJECTIVE}

There is limited information regarding exposures to phthalates and BPA the development of cognitive dysfunction and neurodegenerative disease in older populations. In this study we examine the relationship between 10 different phthalate metabolites and BPA with surrogate brain health indicators, from the CDC's NHANES 2011-2012 and 2013-2014 data cycles. The objectives of the study are as follows: 1) to assess the mean phthalate and BPA levels in older adults in the US, 60 years of age and above with the surrogates of brain health indicators in the US population, 2) assess the association between phthalate and BPA levels and surrogate brain health indicators in older adults in the US, to find the risk of poor cognitive function and development of mild cognitive impairment, dementia, and AD.

\section{METHODS}

Study Design and Population: NHANES is a continuous cross-sectional data collection utilizing a complex multi-stage sampling design that creates a survey representative of the non-institutionalized population of the United States ${ }^{51,52}$. The survey has been conducted since 1999 and consists of an at-home questionnaires followed by a standardized physical examination and specimen collection conducted in mobile examination centers (MEC) ${ }^{51,52}$. Eligibility is determined using preset selection probabilities for the desired demographic subdomains ${ }^{52}$. A household screener is performed before to determine if any household members are eligible for the interview and examination ${ }^{52}$. The interview collects demographic, health, nutrition, and household information, while the physical examination includes physical measurements, dental examination, and the collection of blood and urine specimens for laboratory testing 52 . 
Prior to any to interviews and examinations, informed consent was obtained and all procedures were approved by the CDC Institutional Review Board ${ }^{53}$.

In our study, we merged the NHANES 2011-2012 and 2013-2014 data cycles. All our analyses were limited to individuals 60 years of age and older who have recorded responses to cognitive test scores and/or memory and taste/smell questions and have EEDC urine measurements.

\section{Inclusion/Exclusion Criteria}

\section{Inclusion criteria:}

5. Males and females, 60 years of age and older

6. Available EEDC urine measurements (Phthalates, BPA)

7. Urine creatinine measurements $>30 \mathrm{mg} / \mathrm{dl}$ and $<300 \mathrm{mg} / \mathrm{dl}$.

8. Complete responses to identified outcome variables.

\section{Exclusion criteria:}

5. Males and females, 59 years of age and younger

6. Unavailable EEDC urine measurements (Phthalates, BPA)

7. Urine creatinine measurements $<30 \mathrm{mg} / \mathrm{dl}$ and $>300 \mathrm{mg} / \mathrm{dl}$

8. Incomplete responses to identified outcome variables.

\section{Phthalate Exposure Assessment and Measurements}

Phthalates were measured from urine samples taken from a representative and random one-third subsample of individuals 6 years of age and older in the 2011-2012 and 2013-2014 survey cycles ${ }^{54,55}$. The laboratory utilized high performance liquid chromatography-electrospray ionization-tandem mass spectrometry to analyze urine phthalate levels and was consistently used in both survey cycles ${ }^{54,55}$. Phthalate levels 
were provided in $\mathrm{ng} / \mathrm{ml}$ and was used in our analyses. The following phthalates were used in our analyses: Mono (carboxynonyl) Phthalate (CNP); Mono (carboxyoctyl) Phthalate (COP); Mono-(2-ethyl-5-carboxypentyl) Phthalate (ECP); Mono-n-butyl Phthalate (MBP); Mono-(3-carboxypropyl) Phthalate (MC1); Mono-ethyl Phthalate (MEP); Mono (2-ethyl-5-hydroxy hexyl) Phthalate (MHH); Mono-(2-ethyl-5-oxohexyl) Phthalate (MOH); Mono-benzyl Phthalate (MZP); Mono-isobutyl Phthalate (MIB).

The limit of detection variables indicates if subjects have urine phthalate levels above the limit of detection. A value of "0" indicates above the limit of detection and a value of "1" indicates below the limit of detection. Individuals with a below the limit of detection were given a dummy phthalate level of the LOD divided by the square root of two ${ }^{56}$. Some LODs differ between survey cycles and a conservative approach was used to account for differing LODs per survey cycle ${ }^{57}$. The specific phthalate metabolites were selected as $>60 \%$ of study subjects had urine phthalate levels above the LOD. A total of 5,175 subjects had available urinary phthalate measurements in the 2011-2012 and 2013-2014 datasets.

\section{BPA Exposure Assessment and Measurements}

BPA was measured from urine samples taken from a representative and random one-third subsample of individuals 6 years of age and older in the 2011-2012 and 20132014 survey cycles ${ }^{58,59}$. The laboratory on-line solid phase extraction coupled to high performance liquid chromatography and tandem mass spectrometry to analyze urine BPA

levels and was consistently used in both survey cycles ${ }^{58,59}$. BPA levels were provided in $\mathrm{ng} / \mathrm{ml}$. 
The limit of detection variables indicates if subjects have urine phthalate levels above the limit of detection. A value of "0" indicates above the limit of detection and a value of "1" indicates below the limit of detection. Individuals with a below the limit of detection were given a dummy BPA level of the LOD divided by the square root of two ${ }^{56}$. Some LODs differ between survey cycles and a conservative approach was used to account for differing LODs per survey cycle ${ }^{57}$. A total of 5,175 subjects had available urinary BPA measurements in the 2011-2012 and 2013-2014 datasets.

\section{Assessment of Surrogate Brain Health Indicators - Cognitive Scores}

\section{CERAD Word Learning Subtest - Immediate Recall and Delayed Recall: The} Consortium to Establish a Registry for Alzheimer's Disease (CERAD) Word Learning Subtest assesses both immediate and delayed learning ${ }^{60,61}$. The delayed and immediate recall tests available in NHANES assess the ability to process new verbal information ${ }^{60,61}$. The tests are part of the neuropsychological assessment for the entire CERAD testing protocol, which was initially created to standardize Alzheimer's disease (AD) assessment and diagnosis ${ }^{62}$. The tests in the neuropsychological assessment itself were chosen because of their ability to assess cognitive functions inherent in $\mathrm{AD}^{62}$. The assessments have the ability to differentiate those of adequate cognitive status versus those who have mild cognitive impairment or dementia ${ }^{62-65}$. Although developed for use in the assessment of $\mathrm{AD}$, the CERAD assessments have shown utility in use for Parkinson's disease ${ }^{65}$ and frontotemporal lobar degeneration ${ }^{66}$.

Immediate Recall: For immediate recall, the subjects are asked to read aloud a sequence of 10 unrelated words as they are presented to them and immediately after, they are asked to recall as many words as possible ${ }^{60,61}$. This is done in three trials with the order of the 
words differing in each trial. ${ }^{60,61}$. Each trial has a maximum score of 10 , with a maximum overall score of $30^{60,61,63}$.

In our study, we included individuals 60 years of age and older who completed all immediate recall word list trials identified as: CFDCST1, CFDCST2, and CFDCST3 in the 2011-2012 and 2013-2014 NHANES data cycles. Those who did not have three trials completed were not included in the immediate recall analysis. We summed the total of the three trials and created a new variable with cut-off scores named IMMEDIATERECALL. A cut-off score of $\leq 13$ and $\geq 14$ was used as it is the standard in other assessments ${ }^{63,67}$. A total of 3,149 subjects from the 2011-2012 and 2013-2014 responded with complete immediate recall trials. We then accounted for subjects who had urinary phthalates and BPA samples taken and accounted for those with extreme creatinine scores $(<30$ and $>300 \mathrm{mg} / \mathrm{dl})$, as these extreme values can affect analyses by being too dilute or concentrated ${ }^{68}$. After, our study population consisted of 940 subjects, 146 subjects with cut-off scores $\leq 13$ and 794 subjects with cut-off scores $\geq 14$.

Delayed Recall: For delayed recall, the subject is asked to repeat the sequence of 10 unrelated words after the other cognitive tests are completed, which is typical 8 to 10 minutes after the start of the word learning trials ${ }^{60,61}$. The maximum score is 10 for delayed recall ${ }^{60,61}$.

In our study, we included subjects 60 years of age and older who completed the delayed recall trial, identified as CFDCSR, for the 2011-2012 and 2013-2014 NHANES data cycles. After we created a new variable with the cut-off scores named DELAYEDRECALL. A cut-off score of $\leq 3$ and $\geq 4$ was used as it is the standard in other assessments ${ }^{63,67,69}$. A total of 3,126 subjects from the 2011-2012 and 2013-2014 
responded with a complete delayed recall trial. We then accounted for subjects who had urinary phthalates and BPA samples taken and accounted for those with extreme creatinine scores $(<30$ and $>300 \mathrm{mg} / \mathrm{dl})$, as these extreme values can affect analyses by being too dilute or concentrated ${ }^{68}$. After, our study population consisted of 930 subjects, 157 subjects $\leq 3$ and 776 subjects $\geq 4$.

Animal Fluency: The animal fluency test is used to determine categorical verbal fluency, which is part of executive function and can differentiate between with normal cognition versus those with $\mathrm{MCI}$ and more severe cognitive impairment, such as $\mathrm{AD}{ }^{60,61}$. Since the test uses animal names, it does not require cultural consideration or formal education experience ${ }^{60,61}$. In the test, subjects are asked to name as many animals in a one minute span, with a maximum range of 40 words in the NHANES 2011-2014 data set. ${ }^{60,61}$. A sample test is given to each subject before the actual test ${ }^{60,61}$.

In our study, we included subjects 60 years of age and older who completed the animal fluency trial, identified as CFDAST, for the 2011-2012 and 2013-2014 NHANES data cycles. After we created a new variable with cut-off scores named VERBALFLUENCY. cut-off score of $\leq 11$ and $\geq 12$ was used as it is the standard in other assessments ${ }^{63,67,69,70}$. A total of 3,110 subjects from the 2011-2012 and 2013-2014 responded with complete animal fluency scores. We then accounted for subjects who had urinary phthalates and BPA samples taken and accounted for those with extreme creatinine scores $(<30$ and $>300 \mathrm{mg} / \mathrm{dl})$, as these extreme values can affect analyses by being too dilute or concentrated ${ }^{68}$. After, our study population consisted of 931 subjects, 187 subjects $\leq 11$ and 744 subjects $\geq 12$. 
Digit Symbol Substitution Test: The Digit Symbol Substitution Test (DSST) is part of the Wechsler Adult Intelligence Scale (WAIS III) ${ }^{60,61,71}$. The test measures processing speed, sustained attention, and working memory ${ }^{60,61,71}$. The subtests have shown utility in the identification of dementia and other neurodegenerative disorders such as $\mathrm{AD}^{72-74}$. The test is given in paper form, with a key that has 9 numbers paired to different symbols. The subject has 2 minutes to match each symbol to 133 boxes with a number associated to it, with the score as the total correct matches with a maximum score of 105 in the 2011-2014 NHANES dataset. ${ }^{60,61}$. A sample test is given to each subject before the actual test ${ }^{60,61}$.

In our study, we included subjects 60 years of age and older who completed the animal fluency trial, identified as CFDDS, for the 2011-2012 and 2013-2014 NHANES data cycles. After we created a new variable with cut-off scores named DSST, cut-off score of $\leq 27$ and $\geq 28$ was used as it is the standard in other assessments ${ }^{75-77}$. A total of 3,014 subjects from the 2011-2012 and 2013-2014 responded with complete DSST. We then accounted for subjects who had urinary phthalates and BPA samples taken and accounted for those with extreme creatinine scores $(<30$ and $>300 \mathrm{mg} / \mathrm{dl})$, as these extreme values can affect analyses by being too dilute or concentrated ${ }^{68}$. After, our study population consisted of 891 subjects, 129 subjects $\leq 27$ and 762 subjects $\geq 28$.

\section{Assessment of Surrogate Brain Health Indicators - Memory Function}

\section{During the past 12 months, have you experienced confusion or memory loss that is}

happening more often or getting worse?: In our study we included subjects 60 years of age and older who had phthalate and BPA samples taken and responded to the yes/no question, "During the past 12 months, have you experienced confusion or memory loss 
that is happening more often or is getting worse?" 78 . As memory loss and confusion are early indicators of cognitive decline, dementia, and $\mathrm{AD}^{2} .3,628$ subjects responded to this question in the 2011-2012 and 2013-2014 survey cycle. We then accounted for subjects who had urinary phthalates and BPA samples taken and accounted for those with extreme creatinine scores ( $<30$ and $>300 \mathrm{mg} / \mathrm{dl}$ ), as these extreme values can affect analyses by being too dilute or concentrated ${ }^{68}$. After, our study population consisted of 1,025 subjects, 181 subjects answered "yes" and 844 subjects answered "no".

\section{During the past 7 days, how often have you had trouble remembering where you put}

things?: In our study we included subjects 60 years of age and older who had phthalate and BPA samples taken and responded to the question," During the past 7 days, how often have you had trouble remembering where you put things, like you keys or your wallet?" $78.3,448$ subjects responded to this question in the 2011-2012 and 2013-2014 survey cycle.

The question is multi-leveled, where 3,448 subjects answered "Never" equaling the value " 0 ", 809 subjects answered "About once" equaling the value " 1 ", 544 subjects answered "Two or three times" equaling the value "2", 175 subjects answered "Nearly every day" equaling the value "3", and 102 subjects answered "Several times a day" equaling the value "4". We created a new variable named MCQ380_WK, which combines responses coded as "Never" equaling " 0 " and "About once" equaling " 1 " into a variable, "No" equaling “1", and "Two or Three Times" equaling “2", "Nearly Every day" equaling "3", and "Several times a day" equaling "4", into a new variable, "Yes" equaling " 1 ". 
We then accounted for subjects who had urinary phthalates and BPA samples taken and accounted for those with extreme creatinine scores ( $<30$ and $>300 \mathrm{mg} / \mathrm{dl})$, as these extreme values can affect analyses by being too dilute or concentrated ${ }^{68}$. After, our study population consisted of 971 subjects, 188 subjects answered " 1 " and 783 subjects answered " 2 ".

\section{$\underline{\text { Are you limited in any way because of difficulty remembering or because you }}$}

experience periods of confusion?: In our study we included subjects 60 years of age and older who had phthalate and BPA samples taken and responded to the question, "Are you limited in any way because of difficulty remembering or because you experience periods of confusion?" 78 . Limitations in physical movement due to difficulty remembering and confusion can indicate the development of cognition issues ${ }^{79} \cdot 11,323$ subjects responded to this question in the 2011-2012 and 2013-2014 survey cycle.

We then accounted for subjects who had urinary phthalates and BPA samples taken and accounted for those with extreme creatinine scores ( $<30$ and $>300 \mathrm{mg} / \mathrm{dl}$ ), as these extreme values can affect analyses by being too dilute or concentrated ${ }^{68}$. After, our study population consisted of 1,024 subjects, 165 subjects answered "yes" and 859 subjects answered "no".

\section{Assessment of Surrogate Brain Health Indicators - Taste and Smell Function}

It has been observed that neurodegenerative disease has been shown to be preceded by smell and taste disorders ${ }^{80-83}$. The causes of these disorders have been linked to genetic alterations ${ }^{80}$, overexpression of key proteins ${ }^{81}$, and direct effect of some environmental chemicals on the olfactory mucosa ${ }^{84}$, which can have associations with exposure to EEDCs ${ }^{80,81,83,84}$. However, issues with olfaction can also be caused by upper 
respiratory tract infections, sino-nasal disease, head trauma, idiopathic causes, surgery of the nasal area, and congenital loss of smell ${ }^{85}$.

The two most common and prevalent neurodegenerative diseases, AD and PD have been shown to be preceded by smell disorders ${ }^{86-91}$. These disorders manifest themselves when evidence of pathological changes in the olfactory system are evident ${ }^{91}$. These are characterized by the build-up of pathological proteins, which cause the death of olfactory cells ${ }^{91}$. Several human epidemiological studies have also alluded to the utility of using sensory biomarkers as an early detection for neurodegenerative diseases ${ }^{92-95}$.

\section{Do you sometimes smell and unpleasant, bad, or burning odor when nothing is}

there?: In our study we included subjects 60 years of age and older who had phthalate and BPA samples taken and responded to the question, "Do you sometimes smell an unpleasant, bad or burning odor when nothing is there?" ${ }^{78} .7,399$ subjects responded to this question in the 2011-2012 and 2013-2014 survey cycle.

We then accounted for subjects who had urinary phthalates and BPA samples taken and accounted for those with extreme creatinine scores $(<30$ and $>300 \mathrm{mg} / \mathrm{dl})$, as these extreme values can affect analyses by being too dilute or concentrated ${ }^{68}$. After, our delayed recall study population consisted of 1,024 subjects, 74 subjects answered "yes" and "950" subjects answered no.

\section{During the past 12 months have you had a taste or other sensation in your mouth}

that does not go away?: In our study we included subjects 60 years of age and older who had phthalate and BPA samples taken and responded to the question," During the past 12

months have you had a taste or other sensation in your mouth that does not go away?"78. 7,407 subjects responded to this question in the 2011-2012 and 2013-2014 survey cycle. 
We then accounted for subjects who had urinary phthalates and BPA samples taken and accounted for those with extreme creatinine scores ( $<30$ and $>300 \mathrm{mg} / \mathrm{dl}$ ), as these extreme values can affect analyses by being too dilute or concentrated ${ }^{68}$. After, our delayed recall study population consisted of 1,024 subjects, 66 subjects answered yes and 958 subjects answered no.

\section{Covariates}

In our study we included a number of covariates, based off a review of literature and well-known risk factors for neurodegenerative diseases, if they were available in the NHANES datasets.

The demographic variables are as follows: gender (male, female), age (60-69, 779, 80+), Race/Ethnicity (Hispanic, Non-Hispanic White, Non-Hispanic Black, NonHispanic Asian/Other), Family Income (Under 24k, 25k to 54,999k, 55k to 74,999k, over $75 \mathrm{k})$, Education $\left(<12^{\text {th }} \text { grade, completed high school, }>12^{\text {th }} \text { grade }\right)^{78}$.

Modifiable health variables and risk factors are as follows: ever smoked (yes, no), blood pressure (normal/high), diabetes (yes, no, borderline), coronary heart disease (yes, no), stroke (yes, no), heart attack (yes, no), head trauma (yes, no), alcohol use (yes, no), ever use birth control (yes, no), every use hormonal replacement therapy (yes, no) ${ }^{78}$.

\section{Statistical Analysis}

Statistical analysis was performed using SAS software ${ }^{96}$. The 2011-2012 and 2013-2014 survey cycles were merged and a four-year sampling weight was calculated to account for the complex sampling design in order to calculate correct statistical estimates and standard errors when calculating means, geometric means, and other statistics ${ }^{97}$. 
For phthalate and BPA variables, a value of " 0 " indicates above the limit of detection and a value of " 1 " indicates below the limit of detection. Individuals with a below the limit of detection were given a dummy phthalate level of the LOD divided by the square root of two ${ }^{56}$. Some LODs differ between survey cycles and a conservative approach was used to account for differing LODs per survey cycle, where the LOD for that year was used to make a determination if the EEDC was above or below the LOD. ${ }^{57}$. We log-transformed and then adjusted for creatinine all phthalate and BPA variables ${ }^{98-}$ ${ }^{100}$ since environmental chemical data is not normally distributed and urine dilution varies from person to person.

We used the SAS Survey procedures to account for the complex sampling design of the NHANES data sets ${ }^{101}$.

We used PROC SURVEYFREQ was used to obtain descriptive statistics for the different populations we were examining in our study which accounts for the complex survey design of the NHANES data sets ${ }^{101}$. Descriptive statistics were organized based on the following categories per variable: gender (male, female), age (60-69, 7-79, 80+), Race/Ethnicity (Hispanic, Non-Hispanic White, Non-Hispanic Black, Non-Hispanic Asian/Other), Family Income (Under 24k, 25k to 54,999k, 55k to 74,999k, over 75k), Education $\left(<12^{\text {th }}\right.$ grade, completed high school, $>12^{\text {th }}$ grade), ever smoked (yes, no), blood pressure (normal/high), diabetes (yes, no, borderline), coronary heart disease (yes, no), stroke (yes, no), heart attack (yes, no), head trauma (yes, no), alcohol use (yes, no), ever use birth control (yes, no), every use hormonal replacement therapy (yes, no) ${ }^{78}$.

We used PROC SURVEYREG and guidance provided by the SAS institute to directly to determine the geometric mean of the EEDC to test if they were significant 
between the responses of our outcome variables ${ }^{101,102}$. The standard errors were calculated using the Taylor Series linearization method, which is the default method in the survey procedures to calculate standard error ${ }^{101}$. Geometric means (GM), geometric standard errors (GSE), and number of subjects were reported for the results of the outcome variables for all subjects that had EEDCs over the LOD. We looked at geometric means between the surrogate of brain health (yes vs. no, low test score vs. high test score), and also performed age-specific, gender-specific, and race/ethnicity-specific geometric means between the responses to the outcome variable. Due to the smaller range of ages in our dataset, 60 years and older, we calculated age-specific rates in lieu of agestandardized rates.

We used PROC SURVEYLOGISTIC to find the unadjusted and adjusted odds ratios (ORs) and the $95 \%$ confidence intervals $(\mathrm{CI})$ to examine the association between the surrogates of brain health and exposures to phthalates and BPA ${ }^{101}$. Analysis was done per EEDC per outcome variable. We presented three logistic regression models which were stratified by gender and examined phthalate and BPA exposures in the following groups, variable $<$ LOD to $50^{\text {th }}$ percentile (reference) and $\geq 50^{\text {th }}$ percentile. The three logistic regression models are as follows: unadjusted, adjusted for known risk factors, age, education, race/ethnicity, adjusted for known and suspected risk factors, age, education, race/ethnicity, smoking, blood pressure history, history of coronary heart disease, stroke, heart attack, diabetes status, head trauma, and alcohol use. We did not include income, OC and HRT use in our models as they significantly reduced the size of the population. 


\section{RESULTS}

\section{Descriptive statistics of Surrogates of Brain Health Indicators and Covariates:}

Descriptive statistics of the study populations are described in table 1.1 for each of our 9 outcomes with their respective covariates. Urinary phthalate and BPA levels were available for 5,175 subjects in the 2011-2012 and 2013-2014 NHANES data cycles. Immediate Recall test scores were available for 940 subjects with $146(12.43 \%)$ having lower immediate recall test scores. Delayed recall scores were available for 930 subjects with 157 (13.14\%) having lower delayed recall scores. Animal Fluency scores were available for 931 subjects with 187 (12.85\%) having lower animal fluency scores. DSST scores were available for 891 subjects with 129 (7.10\%) having lower DSST scores. 1,025 subjects had yes/no responses to "Past 12 months, memory getting worse" with 181 (15.50\%) responding "yes" to having worsening memory over the past 12 months. 971 subjects had yes/no responses to "Past 7 days, trouble remembering?" with 188 (18.16\%) responding "yes" to having memory issues in the past 7 days. 1,024 subjects had yes/no responses to, "Limited due to difficulty remembering or confusion", with 165 (3.14\%) responding "yes". 1,024 subjects had yes/no responses to experiencing phantom odor, with $74(2.42 \%)$ responding "yes". 1,024 subjects had yes/no responses to experiencing sensation in their mouths that does not go away, with 66 (2.11\%) answering "yes".

Among all the outcome variables and covariates, gender was fairly distributed between males and females. (Table 1.1). A majority of subjects fell with the 60-69-year age group (>50\%), were predominantly non-Hispanic White (>70\%), have completed $>12^{\text {th }}$ grade education (>55\%), fall mostly in the overweight or obese range of BMI (> 70\%), have reported using alcohol (>70\%), are fairly distributed by smoking status, 
and are more physically inactive (>55\%) (Table 1.1). Most subjects reported not having diabetes $(>70 \%)$, having normal blood pressure $(>65 \%)$, have not had a stroke $(>90 \%)$, have not been diagnosed with coronary heart disease $(>80 \%)$, and have not experienced significant head trauma (>85\%). Among females, a majority reported using female hormones (>65\%) and were fairly distributed in regards to birth control use. 


\begin{tabular}{|c|c|c|c|c|c|c|c|c|c|c|c|c|c|c|c|c|c|c|c|c|c|c|c|c|c|c|c|c|c|c|c|c|c|c|c|c|}
\hline \multirow{2}{*}{$\begin{array}{c}\text { Table } 1.1 \text { - Descriptive } \\
\text { Statistics -- Surrogate brain } \\
\text { health indicators and } \\
\text { covariates for Pthalates and } \\
\text { BPA }\end{array}$} & \multicolumn{4}{|c|}{$\begin{array}{c}\text { Immediate Recall Score } \\
(\mathrm{n}=940)\end{array}$} & & $\begin{array}{r}\text { yed } \mathrm{Re} \\
(\mathrm{n}=9\end{array}$ & (ecall S & Score & & $\begin{array}{r}\text { mal Flue } \\
(\mathrm{n}=9\end{array}$ & ency & & Substi & $\begin{array}{c}\text { Digit } \mathrm{S} \\
\text { titution } \\
\text { (n=8 }\end{array}$ & 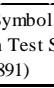 & & $\begin{array}{r}\begin{array}{r}\mathrm{Pa} \\
\text { memor }\end{array} \\
\end{array}$ & $\begin{array}{l}\text { ast } 12 \\
\text { ory get } \\
\quad(1,0 \\
\end{array}$ & $\begin{array}{l}\text { month } \\
\text { ting w } \\
\text { 225) }\end{array}$ & $\begin{array}{l}\text { hs, } \\
\text { worse? }\end{array}$ & & $\begin{array}{l}\text { th } 7 \text { day } \\
\text { emberin }\end{array}$ & & & & $\begin{array}{l}\text { dd due t } \\
\text { emembe } \\
\text { fusion? }\end{array}$ & $\begin{array}{l}\text { to difficitis } \\
\text { ering } \\
? \text { ? (n=1,0 }\end{array}$ & $\begin{array}{l}\text { ficulty } \\
\text { or } \\
.0244\end{array}$ & & & & & $\begin{array}{r}\text { Sensati } \\
\text { does }\end{array}$ & $\begin{array}{l}\text { tion in } \\
\text { s not } \\
(\mathrm{n}=1\end{array}$ & $\begin{array}{l}1 \text { mouth } \\
\text { go awa } \\
\text { 1024) }\end{array}$ & $\begin{array}{l}\text { th that } \\
\text { vay? }\end{array}$ \\
\hline & $\mathrm{n}$ & $\%$ & $\mathrm{n}$ & $\%$ & $\mathrm{n}$ & $\%$ & $\mathrm{n}$ & $\%$ & $\mathrm{n}$ & $\%$ & $\mathrm{n}$ & $\%$ & $\mathrm{n}$ & $\%$ & $\mathrm{n}$ & $\%$ & $\mathrm{n}$ & $\%$ & $\mathrm{n}$ & $\%$ & $\mathrm{n}$ & $\%$ & $\mathrm{n}$ & & $\mathrm{n}$ & $\%$ & $\mathrm{n}$ & & $\mathrm{n}$ & $\%$ & $\mathrm{n}$ & $\%$ & & $\%$ & $\mathrm{n}$ & $\%$ \\
\hline Total Population & $\begin{array}{r}\leq \\
146\end{array}$ & $\begin{array}{c}13 \\
12.43\end{array}$ & $\begin{aligned} 21 \\
\end{aligned}$ & $\begin{array}{l}14 \\
87.57\end{array}$ & $\begin{array}{r}\leq 3 \\
157\end{array}$ & $\begin{array}{l}3 \\
13.14\end{array}$ & $\begin{array}{r}\geq \\
776\end{array}$ & 24 & $\begin{array}{r}\leq 1 \\
187\end{array}$ & \begin{tabular}{|c|}
11 \\
1288
\end{tabular} & & & $\begin{array}{c}\leq 2 \\
129\end{array}$ & \begin{tabular}{|c|}
27 \\
7.10 \\
\end{tabular} & & \begin{tabular}{|l|}
28 \\
92.90
\end{tabular} & $\begin{aligned} \mathrm{Ye} \\
181\end{aligned}$ & $\begin{array}{l}\text { Yes } \\
15.50\end{array}$ & & $\begin{array}{l}\text { No } \\
\end{array}$ & $\begin{array}{l}\mathrm{Ye} \\
188\end{array}$ & $\begin{array}{l}\text { les } \\
18.16\end{array}$ & & $\begin{array}{l}\text { No } \\
81.84\end{array}$ & & & $\begin{array}{r}\text { No } \\
859\end{array}$ & 23.09 & & & $\begin{aligned} \text { No } \\
95\end{aligned}$ & 39.04 & $\begin{array}{r}\text { Yes } \\
66\end{array}$ & $\begin{array}{l}\mathrm{es} \\
2.11\end{array}$ & $\begin{array}{c}\mathrm{No} \\
958\end{array}$ & $\begin{array}{l}\text { No } \\
39.35\end{array}$ \\
\hline Gender (n, \%) & & & & & & & & & & & & & & & & & & & & & & & & & & & & & & & & & & & & \\
\hline & & 7.01 & 44 & 41.97 & 89 & 7.60 & 81 & 41.48 & & 5.49 & & 43.23 & & 3.11 & 887 & 46.16 & 80 & 5.68 & 438 & 43.08 & & 5.81 & \begin{tabular}{|l|l|}
426 \\
\end{tabular} & 42.96 & & 4.85 & 4454 & 43.87 & & 2.61 & 3646 & 46.1 & 25 & 1.91 & 493 & 46.87 \\
\hline Female & 66 & 5.42 & 4 & 45.61 & 68 & 5.54 & 5 & 45.38 & 2 & 7.35 & 62 & 43.92 & 60 & 3.99 & 375 & 46.75 & 101 & 9.82 & 406 & 41.42 & 117 & 12.35 & 357 & 38.88 & 93 & 7.12 & 4144 & 44.16 & 43 & 3.23 & $464 \quad 48$ & 48.03 & 41 & & 465 & 48.05 \\
\hline Age (years) (n, & & & & & & & & & & & & & & & & & & & & & & & & & & & & & & & & & & & & \\
\hline & 56 & 3.46 & & & 61 & 4.10 & & 48.18 & 84 & 4.06 & & 48.38 & 53 & 2.16 & 29 & & 69 & 5.11 & 464 & & \begin{tabular}{|l}
89 \\
89
\end{tabular} & & 419 & & 75 & & 4584 & & 50 & & 48247 & & & & 492 & 48.73 \\
\hline & 37 & 4.39 & & & 43 & 4.57 & 235 & 27.45 & 56 & 5.04 & 218 & 26.56 & 40 & 2.08 & 30 & & 52 & 5.37 & 250 & 26.15 & 59 & 6.26 & 229 & 24.85 & 36 & 2.98 & 2662 & 28.57 & 17 & 1.25 & 28530 & & & & 2893 & 30.52 \\
\hline 80 & 53 & 4.58 & 3 & 11.33 & 53 & 4.47 & 1 & 11.23 & \begin{tabular}{|l|l|}
47 \\
\end{tabular} & 3.75 & 118 & 12.22 & 36 & 2.85 & 103 & 11.69 & 60 & 5.02 & 130 & 11.66 & 40 & 4.06 & 135 & 12.33 & 54 & 4.30 & $135 \quad 1$ & 12.31 & 7 & 0.53 & 183 if & & & & 177 & 15.67 \\
\hline Race/Ethni & & & & & & & & & & & & & & & & & & & & & & & & & & & & & & & & & & & & \\
\hline & & & & 62 & & & & 6.04 & & & & & & & & 5.23 & & 1.51 & & 6.79 & & & & & & & & & & & & & & & & 1.38 \\
\hline Non-H & & 8.45 & 6 & & 53 & 8.86 & & 69.32 & & 6.79 & & 71.25 & 23 & 49 & & 76.45 & 86 & 11.58 & 56 & 65.36 & 89 & 13. & & 63.64 & 58 & & 336 & & 25 & & 177 & & & & & 73. \\
\hline & & 1.31 & & & 48 & & 195 & 7.49 & & 2.96 & 169 & 6.41 & 48 & 1.90 & 182 & 7.04 & 31 & 1.12 & 231 & 8.25 & 39 & 1.52 & 216 & 8.11 & 35 & 1.39 & 227 & 7.99 & 21 & 0.75 & & 63 & & 0.26 & & 9.11 \\
\hline Non-Hisp & 18 & 0.98 & 67 & 3.69 & 14 & 0.63 & \begin{tabular}{|l|} 
\\
\end{tabular} & 4.65 & 22 & 1.27 & 60 & 3.35 & & & 74 & 4.18 & 26 & & 80 & 4.11 & 18 & & 84 & 4.13 & 22 & 1.14 & 84 & 4.25 & 7 & 0.33 & 98 & & & & 98 & 5.04 \\
\hline & & & & & & & & & & & & & & & & & & & & & & & & & & & & & & & & & & & & \\
\hline & & & & & 79 & & & 16.90 & & 6.08 & & & & & & & & & & & & & & & & & & & & & & & & & 3092 & \\
\hline Complet & 29 & 3.28 & 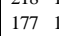 & 18.10 & 31 & 3.29 & & 18.11 & & $\begin{array}{l}3.00 \\
3.32\end{array}$ & & 18.14 & 15 & & & 20.27 & 38 & & & 18.05 & 45 & & & $\begin{array}{l}16.20 \\
16.20\end{array}$ & & & & 17.90 & & & $\begin{array}{ll}0 & 1 \\
01 & 1\end{array}$ & & & & 209 & \\
\hline & 37 & & 75 & 52.48 & & 5.14 & & 51.84 & & 3.4 & & 53.59 & & & & 56.49 & & 7.02 & & 48.51 & & & 365 & & & & & & 20 & & & & & & & \\
\hline BMI & & & & & & & & & & & & & & & & & & & & & & & & & & & & & & & & & & & & \\
\hline & & & & & 8 & & & & & & & & & & & & & & & & & & & & & & & & & & & & & & 38 & 3.88 \\
\hline Normal W & 45 & 3.73 & 2 & 20.90 & 41 & & 19 & 20.95 & 5 & 4.12 & 18 & 20.53 & 31 & 94 & 192 & 22.73 & 53 & 4.95 & 210 & 19.96 & 45 & .07 & 204 & & 47 & 4.13 & 152 & 20 & 17 & 82 & $245 \quad 2$ & & 18 & 20 & 2442 & 23. \\
\hline & 56 & 4.45 & 28 & 32.16 & 62 & 5.09 & 274 & 31.61 & 62 & 3.87 & 72 & 32.54 & 43 & 2.06 & 281 & 34.56 & 57 & 4.46 & 308 & 31.91 & 66 & 6.26 & 281 & 30.56 & 49 & 3.12 & 3163 & 33.28 & 21 & 2.38 & $\begin{array}{lll}344 & 3\end{array}$ & 34.01 & & 1.04 & $346 ?$ & 35.34 \\
\hline & 36 & 3.00 & 53 & 31.64 & 46 & 3.68 & & 31.08 & & 3.75 & & 31.03 & 46 & 2.36 & 270 & 32.55 & 63 & 5.53 & 293 & 29.05 & 70 & & 270 & 28.95 & 59 & 4.01 & 2973 & & 34 & & 3223 & & & & $330 ?$ & \\
\hline & & & & & & & & & & & & & & & & & & & & & & & & & & & & & & & & & & & & \\
\hline & & & & & & & & 64.93 & & & & 64.8 & & & & 69. & & 9.9 & & 61. & & & & & & & & & & & & & & & & 67 \\
\hline & & 5.13 & 2 & 22.27 & 62 & & & 22.05 & & & & 22.55 & & & & 23.29 & & 4.5 & & 23.80 & & & & 22.27 & & & & & 2 & & 62 & & & & 08 & \\
\hline Ever & & & & & & & & & & & & & & & & & & & & & & & & & & & & & & & & & & & & \\
\hline & & & & 46.55 & 78 & & & 45.45 & & & & 45.72 & & & & 49.45 & & & & 44.02 & & & & 43.76 & & & & & & & & & & & & 49.04 \\
\hline & & 6.38 & 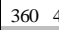 & 41.00 & 78 & 5.83 & & 41.43 & \begin{tabular}{|l|}
96 \\
\end{tabular} & & & 41.42 & & & & & & & & & 105 & & 359 & & 75 & & & & 29 & & $\begin{array}{llll}458 \\
4\end{array}$ & & 32 & & 454 & 45. \\
\hline Physically A & & & & & & & & & & & & & & & & & & & & & & & & & & & & & & & & & & & & \\
\hline & & & & & & & & 36.50 & 55 & 3.50 & & 38.03 & & & & & & & & 36 & & & & 33.66 & 44 & & & & & & & & & & & 39 \\
\hline & & 8.2 & 5 & 50.35 & & 8.04 & & 50.36 & & 9. & & 49.12 & & & & 52.61 & & 11 & & 48.37 & 129 & & & 48.17 & 121 & 8.97 & & & 49 & 67 & $95 \quad 5$ & & & & 596 : & \\
\hline Diabet & & & & & & & & & & & & & & & & & & & & & & & & & & & & & & & & & & & & \\
\hline & & & & & & & & & & & & & & & & & & & & & & & & & & & & & & & & & & & & 19 \\
\hline & & & $\epsilon$ & 68.73 & 10 & & & 64.77 & & & & 66.07 & & & & 70.90 & & & & 63.96 & 31 & & & 61.08 & 105 & & & & 43 & & & & & & 70 & \\
\hline & & 0.72 & & & 10 & & & 3.63 & & & & 3.92 & & & 39 & 4.01 & & & 3 & 3.85 & & & \begin{tabular}{|l|l}
40 \\
\end{tabular} & 3.81 & 9 & & 41 & & 3 & & $47 \quad 4$ & & 3 & & 47 & 4.18 \\
\hline od P & & & & & & & & & & & & & & & & & & & & & & & & & & & & & & & & & & & & \\
\hline & & & & 62.58 & 92 & & & 62.54 & & & & 62.22 & & & & & & & & 59.23 & & & & & & & & & & & & & & & & \\
\hline & & 4.96 & 2 & 25.00 & 65 & 5.54 & & 24.32 & 80 & 5.02 & & 24.94 & 64 & 3. & 8 & 26.08 & 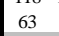 & 5.06 & 70 & 25.28 & 3 & 5.60 & & 24.72 & 68 & 4.8 & 2642 & 25.4 & 25 & 1. & $07 \quad 2$ & 28.89 & 1 & 1.52 & 304 & \\
\hline Myoca & & & & & & & & & & & & & & & & & & & & & & & & & & & & & & & & & & & & \\
\hline & & & & & & & & & & & & & & & & & & & & & & & & & & & & & & & & & & & & \\
\hline & & 10.2 & 257 & 79.15 & 142 & 11.0 & 05 & 78.40 & 166 & 10.8 & 680 & 78.58 & 5 & 6.08 & 696 & 83.4 & 158 & & 77 & 76.2 & 173 & 16.5 & 714 & 74.1 & 143 & & 7877 & 79.6 & 62 & & & & & & 8698 & 85. \\
\hline & & & & & & & & & & & & & & & & & & & & & & & & & & & & & & & & & & & & \\
\hline & & & & & & & & & & & & & & & & & & & & & & & & & & & & & & & & & & & & \\
\hline & & & 73 & 82.77 & 141 & & & 82 & & 11.08 & 76 & 83.32 & 3 & 6.2 & 18 & 88.77 & & 14.06 & 77 & 79.59 & 8 & 16.49 & 720 & 77.17 & & 10 & 7968 & & 6 & & 708 & 88.31 & & 4.95 & & \\
\hline Coronary Hear & & & & & & & & & & & & & & & & & & & & & & & & & & & & & & & & & & & & \\
\hline & & & & & & & & & & & & & & & & & & & & & & & & & & & & & & & & & & & & \\
\hline & & & & & 141 & & & 78.11 & & & & & & & & & & & & & & & & & & & & & & & & & & & & \\
\hline & & & & & & & & & & & & & & & & & & & & & & & & & & & & & & & & & & & & \\
\hline & & & & & & & & & & & & & & & & & & & & & & & & & & & & & & & & & & & & \\
\hline & & & 69 & 75. & 1421 & 11 & & 74. & & & 64 & 74.76 & & 6. & & & & & 757 & 73. & 156 & & 704 & 71. & 133 & & 7707 & & 61 & & & & & 4.18 & & \\
\hline Used Fer & & & & & & & & & & & & & & & & & & & & & & & & & & & & & & & & & & & & \\
\hline & & & & & 24 & & & & & & & & & & & & & & & & & & & 51 & & & & & & & & & & & & \\
\hline & & & & & 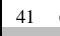 & & & & & & & & & & & & & & & & & & & & & & & & & & & & & & & \\
\hline $\mathrm{U}$ & & & & & & & & & & & & & & & & & & & & & & & & & & & & & & & & & & & & \\
\hline & & & & & 8 & & & & & & & & & & & & & & & & & & & & & & & & & & & & 1 & 7 & & \\
\hline & & & & 4. & 54 & 9. & & & & & & & & & & & & & & & & & & & & & & & & & & 4 & 24 & 3.96 & 9 & \\
\hline
\end{tabular}




\section{Associations Between Exposures to Phthalate and BPA and Cognitive Test Scores}

Exposures to the 12 phthalate metabolites and BPA and four cognitive scores (immediate and delayed recall, animal fluency, and DSST score) are summarized in tables 1.2 to 1.21. The cognitive test scores have been used as a surrogate indicator of brain health to assess cognitive decline and the possible development of mild cognitive impairment, dementia, and/or AD elderly patients as part of neuropsychological testing. Immediate Recall Scores and Exposure to BPA and Phthalates: Tables 1.2 to 1.5 present the GMs and GSEs of urinary phthalates and BPA levels among subjects with immediate recall scores. Table 1.2 presents GMs and GSEs of subjects who have measurable phthalate and BPA levels over the LOD by immediate recall cut-off scores. The phthalate metabolite, ECP, was significantly higher in subjects with immediate recall scores $\leq 13$ compared to subjects who scored $\geq 14(\mathrm{p}<0.05)$. Table 1.3 presents the age-specific GMs and GSEs for subjects who have measurable phthalate and BPA levels over the LOD by immediate recall cut-off score. In the 60-69 age group, the phthalate metabolite MEP was significantly higher in subjects who scored $\geq 14$ than subjects who scored $\leq 13$ $(\mathrm{p}<0.05)$. In the 70-79 age group, the GM mean of the phthalate metabolite MBP to be higher in subjects who scored $\leq 13$ than in subjects who scored $\geq 14$. $(\mathrm{p}<0.05)$. Table 1.4 presents the gender-specific GMs and GSEs for subjects who have measurable phthalate and BPA levels over the LOD by immediate recall cut-off score. Several phthalates were significantly higher in females with immediate recall scores $\leq 13$ than in females who scored $\geq 14$. ECP and $\mathrm{MOH}$ were found to be significantly higher in females with immediate recall scores $\leq 13$ than in the $\geq 14$ group $(\mathrm{p}<0.05)$, while MBP was found to be very significant in females who scored $\leq 13$ than those who scored $\geq 14$ group 
( $\mathrm{p}<0.001)$. Table 1.5 presents race-specific geometric GMs and GSEs for subjects who have measurable phthalate and BPA levels over the LOD by immediate recall cut-off score. Only one significant result was found with phthalate metabolite MIB being significantly higher in subjects who scored $\geq 14$ score compared to subjects who scored $\leq$ 13 ( $\mathrm{p}<0.05)$, among Asian/Others racial group. Table 1.6 presents the estimated ORs of phthalate and BPA levels by immediate recall scores. For ECP in the $\geq 50$ th percentile among females compared to the reference group, ECP was significantly associated with lower immediate recall scores in the unadjusted model $(\mathrm{OR}=2.591,95 \% \mathrm{CI}: 1.240$ 5.412), in adjusted model \#1 (OR=2.361, 95\% $\mathrm{CI}=1.0998-5.078)$, and adjusted model \#2 $(\mathrm{OR}=2.402,95 \% \mathrm{CI}: 1.036-5.566)$. In the $\geq$ 50th percentile among females compared to the reference group, MBP was found to be significantly associated with lower immediate test scores in the unadjusted $(\mathrm{OR}=2.669,95 \% \mathrm{CI}: 1.394-5.111)$ and adjusted model \#1 $(\mathrm{OR}=2.157,95 \%$ CI: 1.086-4.283). 
Table 1.2 Geometric Mean Urinary Phthalate and BPA Levels by Immediate Recall Cut-Off Score for Subjects >= 60 years of age, NHANES 2011-2014, over the LOD

\begin{tabular}{|c|ccc|ccc|}
\hline \multirow{2}{*}{ EEDC } & \multicolumn{5}{|c|}{ Geometric Mean (ng/ml) (GSE, N) } \\
\cline { 2 - 7 } & \multicolumn{2}{|c|}{ Immediate Recall Score $\leq \mathbf{1 3}$} & Immediate Recall Score $\geq \mathbf{1 4}$ \\
\cline { 2 - 7 } & $\mathbf{G M}$ & $\mathbf{G S E}$ & $\mathbf{N}$ & $\mathbf{G M}$ & $\mathbf{G S E}$ & $\mathbf{N}$ \\
\hline Mono (carboxynonyl) Phthalate - CNP & 2.57 & 0.2472, & 141 & 2.65 & 0.25 & 792 \\
Mono (carboxyoctyl) Phthalate - COP & 17.25 & 2.14 & 143 & 19.03 & 1.58 & 793 \\
Mono-(2-ethyl-5-carboxypentyl) Phthalate -ECP & $\mathbf{1 4 . 6 5}$ & $\mathbf{1 . 2 1}$ & $\mathbf{1 4 2} *$ & 11.72 & 0.61 & 793 \\
Mono-n-butyl Phthalate - MBP & 11.27 & 1.10 & 140 & 9.71 & 0.53 & 783 \\
Mono-(3-carboxypropyl) Phthalate - MC1 & 2.91 & 0.37 & 138 & 2.68 & 0.18 & 755 \\
Mono-ethyl Phthalate -MEP & 45.78 & 7.12 & 143 & 57.46 & 5.22 & 794 \\
Mono (2-ethyl-5-hydroxy hexyl) Phthalate - MHH & 8.44 & 0.77 & 143 & 7.12 & 0.35 & 793 \\
Mono-(2-ethyl-5-oxohexyl) Phthalate -MOH & 5.75 & 0.53 & 142 & 4.80 & 0.25 & 793 \\
Mono-benzyl Phthalate - MZP & 4.80 & 0.54 & 140 & 4.10 & 0.21 & 784 \\
Mono-isobutyl Phthalate - MIB & 5.84 & 0.67 & 142 & 6.28 & 0.28 & 785 \\
\hline Bisphenol A - BPA & 1.41 & 0.15 & 130 & 1.55 & 0.08 & 755 \\
\hline
\end{tabular}

NHANES sampling weight applied before calculating the geometric mean.

The EEDCs were creatinine-adjusted then log transformed prior to analyses. Extreme creatinine values $(<30 \mathrm{mg} / \mathrm{dL}$ and $>300 \mathrm{mg} / \mathrm{dL}$ ) and EEDCs levels below the LOD were taken into account using the appropriate domain statement in SAS.

$* \mathrm{p}<0.05, * * \mathrm{p}<0.01 * * * \mathrm{p}<0.001$

\begin{tabular}{|c|c|c|c|c|c|c|c|c|c|c|c|c|c|c|c|c|c|c|}
\hline \multicolumn{19}{|c|}{ Table 1.3 Age-Specific Geometric Mean Urinary Phthalate and BPA Levels by Immediate Recall Cut-0ff Scores for Subjects >= 60 years of age, NHANES 2011-2014, over the LOD } \\
\hline \multirow{3}{*}{ EEDC } & \multicolumn{6}{|c|}{ Geometric Mean (ng/ml) (GSE,N), Age 60-69 } & \multicolumn{6}{|c|}{ Geometric Mean (ng/ml) (GSE, N), Age 70-79 } & \multicolumn{6}{|c|}{ Geometric Mean (ng/ml) (GSE, N), Age 80 + } \\
\hline & \multicolumn{3}{|c|}{ Immediate Recall Score $\leq 13$} & \multicolumn{3}{|c|}{ Immediate Recall Score $\geq 14$} & \multicolumn{3}{|c|}{ Immediate Recall Score $\leq 13$} & \multicolumn{3}{|c|}{ Immediate Recall Score $\geq 14$} & \multicolumn{3}{|c|}{ Immediate Recall Score $\leq 13$} & \multicolumn{3}{|c|}{ Immediate Recall Score $\geq 14$} \\
\hline & GM & GSE & $\mathrm{N}$ & GM & GSE & $\mathrm{N}$ & GM & GSE & $\mathrm{N}$ & GM & GSE & $\mathrm{N}$ & GM & GSE & $\mathrm{N}$ & GM & GSE & $\mathrm{N}$ \\
\hline Mono (carboxynonyl) Phthalate - CNP & 2.38 & 0.27 & 53 & 2.54 & 0.18 & 439 & 3.34 & 0.74 & 36 & 2.85 & 0.25 & 240 & 2.12 & 0.21 & 52 & 2.66 & 0.24 & 113 \\
\hline Mono (carboxyoctyl) Phthalate - COP & 17.15 & 3.06 & 55 & 18.12 & 2.07 & 438 & 20.93 & 5.40 & 36 & 21.85 & 2.02 & 242 & 14.33 & 2.12 & 52 & 16.81 & 2.04 & 113 \\
\hline Mono-(2-ethyl-5-carboxypentyl) Phthalate -ECP & 12.99 & 1.92 & 54 & 10.70 & 0.79 & 438 & 15.40 & 2.41 & 36 & 13.30 & 0.76 & 242 & 15.31 & 1.99 & 52 & 12.68 & 0.76 & 113 \\
\hline Mono-n-butyl Phthalate - MBP & 8.23 & 1.49 & 54 & 9.31 & 0.65 & 432 & 12.66 & 1.25 & $36 *$ & 9.81 & 0.83 & 239 & 12.93 & 1.92 & 50 & 11.30 & 1.27 & 112 \\
\hline Mono-(3-carboxypropyl) Phthalate - MCl & 2.55 & 0.25 & 50 & 2.50 & 0.22 & 418 & 4.04 & 1.30 & 36 & 2.91 & 0.29 & 229 & 2.32 & 0.26 & 52 & 2.93 & 0.31 & 108 \\
\hline Mono-ethyl Phthalate -MEP & 41.12 & 6.06 & 55 & 60.05 & 7.05 & $439 *$ & 64.48 & 14.11 & 36 & 58.66 & 7.45 & 242 & 35.60 & 8.22 & 52 & 45.23 & 5.30 & 113 \\
\hline Mono (2-ethyl-5-hydroxy hexyl) Phthalate - МHH & 8.03 & 1.08 & 55 & 6.59 & 0.48 & 438 & 9.51 & 1.81 & 36 & 8.04 & 0.44 & 242 & 7.81 & 0.96 & 52 & 7.35 & 0.61 & 113 \\
\hline Mono-(2-ethyl-5-0xohexyl) Phthalate -MOH & 4.80 & 0.81 & 54 & 4.33 & 0.31 & 439 & 6.53 & 1.08 & 36 & 5.48 & 0.28 & 242 & 5.84 & 0.72 & 52 & 5.43 & 0.44 & 112 \\
\hline Mono-benzyl Phthalate - MZP & 3.84 & 1.06 & 54 & 4.05 & 0.32 & 434 & 4.12 & 0.83 & 36 & 3.82 & 0.30 & 239 & 5.02 & 0.89 & 50 & 5.13 & 0.54 & 111 \\
\hline Mono-isobutyl Phthalate - MIB & 5.31 & 1.10 & 54 & 6.53 & 0.37 & 436 & 7.37 & 0.93 & 36 & 6.05 & 0.36 & 238 & 5.01 & 0.61 & 52 & 5.83 & 0.54 & 111 \\
\hline Bisphenol A-BPA & 1.52 & 0.20 & 50 & 1.50 & 0.10 & 421 & 1.68 & 0.38 & 31 & 1.66 & 0.11 & 227 & 1.14 & 0.18 & 49 & 1.51 & 0.15 & 107 \\
\hline The EEDCs were creatinine-adjusted then log tra & 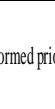 & & 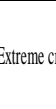 & $\mathrm{NHA}$ & ES samp & g weight ap & plied befo & ecalculat & the geomet & ric mean. & & 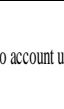 & & & & & & \\
\hline
\end{tabular}




\begin{tabular}{|c|c|c|c|c|}
\hline \multirow{2}{*}{ EEDC } & \multicolumn{2}{|c|}{ Geometric Mean (ng/ml) (GSE, N), Hispanic } & \multicolumn{2}{|c|}{ Geometric Mean (ng/ml) (GSE, N), Non-Hispanic White } \\
\hline & Immediate Recall Score $\leq 13$ & Immediate Recall Score $\geq 14$ & Immediate Recall Score $\leq 13$ & Immediate Recall Score $\geq 14$ \\
\hline \multirow{10}{*}{$\begin{array}{c}\text { Mono (carboxynonyl) Phthalate - CNP } \\
\text { Mono (carboxyoctyl) Phthalate - COP } \\
\text { Mono-(2-ethyl-5-carboxypentyl) Phthalate -ECP } \\
\text { Mono-n-butyl Phthalate - MBP } \\
\text { Mono-(3-carboxypropyl) Phthalate - MC1 } \\
\text { Mono-ethyl Phthalate -MEP } \\
\text { Mono (2-ethyl-5-hydroxy hexyl) Phthalate - MHH } \\
\text { Mono-(2-ethyl-5-oxohexyl) Phthalate -MOH } \\
\text { Mono-benzyl Phthalate - MZP } \\
\text { Mono-isobutyl Phthalate - MIB } \\
\end{array}$} & $2.2965(0.1286,37)$ & $2.2965(0.1286,158)$ & $2.4676(0.2737,54)$ & $2.8334(0.1894,360)$ \\
\hline & $23.8754(3.4422,37)$ & $19.3870(1.7161,158)$ & $17.3626(3.1119,54)$ & $19.9950(2.0021,360)$ \\
\hline & $21.0143(2.3875,37)$ & $17.0325(1.2343,158)$ & $13.9929(1.6572,54)$ & $11.4191(0.6811,359)$ \\
\hline & $15.0088(1.3451,37)$ & $13.4964(1.0022,156)$ & $10.2638(1.2350,53)$ & $9.1497(0.6380,354)$ \\
\hline & $2.9123(0.5823,36)$ & $2.7870(0.2652,149)$ & $2.8808(0.5064,53)$ & $2.7439(0.2397,344)$ \\
\hline & $96.4011(37.1953,37)$ & $103.54(10.1955,158)$ & $37.1518(7.5889,54)$ & $52.6929(5.5850,360)$ \\
\hline & $12.1871(1.5294,37)$ & $9.8256(0.8115,158)$ & $7.9610(1.0074,54)$ & $6.9275(0.3958,359)$ \\
\hline & $7.8263(0.8892,37)$ & $6.3021(0.5447,158)$ & $5.6054(0.7171,53)$ & $4.6890(0.2825,359)$ \\
\hline & $4.5260(1.0839,37)$ & $3.7164(0.2857,158)$ & $4.4628(0.7253,53)$ & $4.0416(0.2188,354)$ \\
\hline & $8.9347(0.6799,37)$ & $9.2662(0.5774,158)$ & $5.0993(0.7162,54)$ & $5.7985(0.2729,356)$ \\
\hline Bisphenol A - BPA & $1.7034(0.3919,32)$ & $1.5965(0.1383,153)$ & $1.2953(0.1527,52)$ & $1.5743(0.1014,343)$ \\
\hline \multirow{2}{*}{ EEDC } & \multicolumn{2}{|c|}{ Geometric Mean (ng/ml) (GSE, N), Non-Hispanic Black } & \multicolumn{2}{|c|}{ Geometric Mean (ng/ml) (GSE, N), Asian/Other } \\
\hline & Immediate Recall Score $\leq 13$ & Immediate Recall Score $\geq 14$ & Immediate Recall Score $\leq 13$ & Immediate Recall Score $\geq 14$ \\
\hline \multirow{10}{*}{$\begin{array}{c}\text { Mono (carboxynonyl) Phthalate - CNP } \\
\text { Mono (carboxyoctyl) Phthalate - COP } \\
\text { Mono-(2-ethyl-5-carboxypentyl) Phthalate -ECP } \\
\text { Mono-n-butyl Phthalate - MBP } \\
\text { Mono-(3-carboxypropyl) Phthalate - MC2 } \\
\text { Mono-ethyl Phthalate -MEP } \\
\text { Mono (2-ethyl-5-hydroxy hexyl) Phthalate - MHH } \\
\text { Mono-(2-ethyl-5-oxohexyl) Phthalate -MOH } \\
\text { Mono-benzyl Phthalate - MZP } \\
\text { Mono-isobutyl Phthalate - MIB } \\
\end{array}$} & $2.2298(0.1864,35)$ & $1.9424(0.1190,209)$ & $4.9033(2.8533,15)$ & $1.8193(0.1971,65)$ \\
\hline & $16.7325(2.3525,35)$ & $14.3818(1.2288,208)$ & $9.5483(2.7300,17)$ & $13.2066(2.7433,67)$ \\
\hline & $12.4860(2.8316,34)$ & $10.7172(0.7589,209)$ & $14.0561(1.7512,17)$ & $12.1370(0.8803,67)$ \\
\hline & $10.3880(1.6886,35)$ & $10.7279(0.5481,206)$ & $17.1321(1.9694,15)$ & $13.5408(2.8390,67)$ \\
\hline & $2.7616(0.3375,32)$ & $2.1801(0.1632,204)$ & $3.3319(0.7287,17)$ & $2.4152(0.4367,58)$ \\
\hline & $59.8544(12.6913,35)$ & $83.9700(8.4832,209)$ & $49.8820(13.4898,17)$ & $48.2367(15.6196,67)$ \\
\hline & $8.9757(1.6401,35)$ & $7.1347(0.4916,209)$ & $6.6561(0.8808,17)$ & $6.8298(0.8575,67)$ \\
\hline & $5.4584(1.1728,35)$ & $4.8532(0.3093,209)$ & $4.4377(0.4585,17)$ & $4.5599(0.5473,67)$ \\
\hline & $4.2698(1.3594,34)$ & $4.6965(0.3901,207)$ & $3.1107(0.9491,16)$ & $4.7131(1.8994,65)$ \\
\hline & $7.6339(0.6272,34)$ & $7.5526(0.4351,205)$ & $6.1213(1.2606,17)$ & $9.8494(1.8704,66) *$ \\
\hline Bisphenol A - BPA & $1.4074(0.2446,35)$ & $1.4106(0.06072,201)$ & $2.3009(0.8958,11)$ & $1.2806(0.1923,58)$ \\
\hline \multicolumn{5}{|c|}{ NHANES sampling weight applied before calculating the geon } \\
\hline
\end{tabular}

\begin{tabular}{|c|c|c|c|c|c|c|c|c|c|c|c|c|}
\hline \multirow{4}{*}{ EEDC } & \multicolumn{6}{|c|}{ Geometric Mean (ng/ml) (GSE, N) } & \multicolumn{6}{|c|}{ Geometric Mean (ng/ml) (GSE, N) } \\
\hline & \multicolumn{6}{|c|}{ Male } & \multicolumn{6}{|c|}{ Female } \\
\hline & \multicolumn{3}{|c|}{ Immediate Recall Score $\leq 13$} & \multicolumn{3}{|c|}{ Immediate Recall Score $\geq 14$} & \multicolumn{3}{|c|}{ Immediate Recall Score $\leq 13$} & \multicolumn{3}{|c|}{ Immediate Recall Score $\geq 14$} \\
\hline & GM & GSE & $\mathbf{N}$ & GM & GSE & $\mathbf{N}$ & GM & GSE & $\mathbf{N}$ & GM & GSE & $\mathbf{N}$ \\
\hline Mono (carboxynonyl) Phthalate - CNP & 2.50 & 0.35 & 78 & 2.34 & 0.15 & 392 & 2.69 & 0.37 & 63 & 2.97 & 0.25 & 400 \\
\hline Mono (carboxyoctyl) Phthalate - COP & 14.97 & 1.82 & 79 & 16.83 & 1.27 & 393 & 20.97 & 4.18 & 64 & 21.32 & 2.72 & 400 \\
\hline Mono-(2-ethyl-5-carboxypentyl) Phthalate -ECP & 12.68 & 1.27 & 79 & 10.56 & 0.60 & 394 & 17.87 & 2.05 & $63 *$ & 12.90 & 0.84 & 399 \\
\hline Mono-n-butyl Phthalate - MBP & 9.11 & 1.20 & 77 & 9.33 & 0.74 & 387 & 15.01 & 1.25 & $63 * * *$ & 10.07 & 0.54 & 396 \\
\hline Mono-(3-carboxypropyl) Phthalate - MC1 & 2.72 & 0.47 & 77 & 2.49 & 0.20 & 376 & 3.19 & 0.53 & 61 & 2.87 & 0.26 & 379 \\
\hline Mono-ethyl Phthalate -MEP & 41.95 & 8.92 & 79 & 54.22 & 6.17 & 394 & 51.64 & 9.54 & 64 & 60.62 & 6.80 & 400 \\
\hline Mono (2-ethyl-5-hydroxy hexyl) Phthalate - MHH & 7.64 & 0.84 & 79 & 6.69 & 0.41 & 394 & 9.69 & 1.20 & 64 & 7.53 & 0.43 & 399 \\
\hline Mono-(2-ethyl-5-oxohexyl) Phthalate -MOH & 5.12 & 0.60 & 78 & 4.52 & 0.29 & 393 & 6.73 & 0.82 & $64 *$ & 5.07 & 0.30 & 400 \\
\hline Mono-benzyl Phthalate - MZP & 3.46 & 0.43 & 77 & 3.63 & 0.28 & 389 & 5.83 & 1.11 & 63 & 4.58 & 0.25 & 395 \\
\hline Mono-isobutyl Phthalate - MIB & 4.99 & 0.64 & 79 & 5.87 & 0.32 & 390 & 7.27 & 0.87 & 63 & 6.69 & 0.34 & 395 \\
\hline Bisphenol A-BPA & 1.34 & 0.20 & 69 & 1.39 & 0.08 & 379 & 1.50 & 0.21 & 61 & 1.71 & 0.11 & 376 \\
\hline The EEDCs were creatinine-adjusted then log tra & $\begin{array}{l}\text { NHA } \\
\text { med prior } \\
\text { using the }\end{array}$ & $\begin{array}{l}\text { S samp } \\
\text { analyse } \\
\text { propria }\end{array}$ & $\begin{array}{l}\text { weight ap } \\
\text { treme cr } \\
\text { main sta }\end{array}$ & $\begin{array}{l}\text { lied befo } \\
\text { eatinine va } \\
\text { ement in } \mathrm{S}\end{array}$ & $\begin{array}{l}\text { alculat } \\
\mathrm{s}(<30 \\
{ }^{*} \mathrm{p}<0 \\
\end{array}$ & he geomet & ic mean. & & & & & ount \\
\hline
\end{tabular}




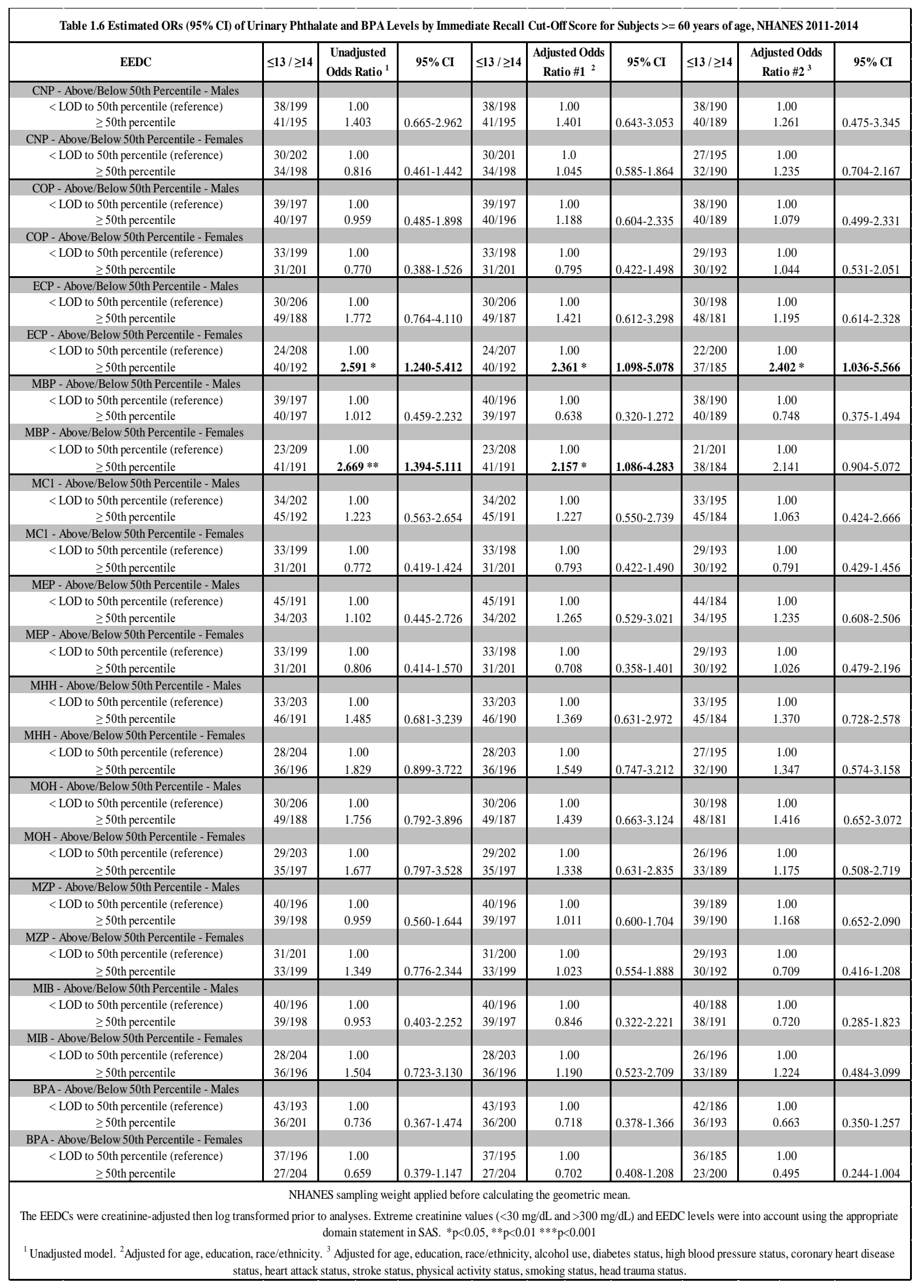


Delayed Recall Scores and Exposure to BPA and Phthalates: Tables 1.7 to 1.11 present the GMs and GSEs of urinary phthalates and BPA levels among subjects with delayed recall scores. Table 1.7 presents GMs and GSEs of subjects who have measurable phthalate and BPA levels over the LOD by delayed recall cut-off scores. The crude GM levels of 6 of the 10 phthalate metabolites, ECP, MBP, MEP, MHH, MOH, and MZP, are higher in subjects that scored $\leq 3$. No significant differences were found. Table 1.8 presents the age-specific GMs and GSEs for subjects who have measurable phthalate and BPA levels over the LOD by delayed recall cut-off score. No significant differences were found in any of the age groups. Table 1.9 presents the gender-specific GMs and GSEs for subjects who have measurable phthalate and BPA levels over the LOD by delayed recall cut-off score. There were several significant findings amongst females, with GM mean levels of ECP ( $\mathrm{p}<0.05), \mathrm{MBP}(\mathrm{p}<0.001), \mathrm{MOH}(\mathrm{p}<0.05)$, and MZP $(\mathrm{p}<0.05)$ being higher in the subjects with a delayed recall score $\leq 3$ compared to subjects with a delayed recall score $\geq 4$. Table 1.10 presents race-specific geometric GMs and GSEs for subjects who have measurable phthalate and BPA levels over the LOD by delayed recall cut-off score. Significant findings were found, with the GM for MIB among Asian/other being significantly higher in subjects who scored $\geq 14$ compared to those who scored $\leq 13$ $(\mathrm{p}<0.05)$. The GMs for $\mathrm{MHH}, \mathrm{MOH}$, and MZP among Hispanics were significantly higher $(\mathrm{p}<0.05)$ in those who scored $\leq 3$ compared to those who scored $\geq 4$.

Table 1.11 presents estimated ORs of phthalate and BPA levels by delayed recall cut-off scores. For the phthalate ECP in the $\geq 50$ th percentile among females compared to the reference group, ECP was significantly associated with lower delayed recall scores in the unadjusted model $(\mathrm{OR}=2.218,95 \% \mathrm{CI}: 1.084-4.538)$, but not in the adjusted 
models. For the phthalate MBP in the $\geq 50$ th percentile among females compared to the reference group, MBP was significantly associated with lower delayed recall scores $(\mathrm{OR}=2.174,95 \%$ CI: 1.026-4.6605) in the unadjusted model. MBP was not found to be significantly associated with lower test scores in the adjusted models. For the phthalate MEP among females in the $\geq$ 50th percentile compared to the reference group, MEP concentrations were found to be significantly associated with lower delayed recall scores in the unadjusted model (OR=2.019, 95\% CI: 1.027-3.969), adjusted model \#1 $(\mathrm{OR}=2.409,95 \%$ CI: $1.214-4.778)$, and adjusted model \#2 (OR=2.443, 95\% CI: 1.358 4.393). For the phthalate $\mathrm{MOH}$ among females in the $\geq 5$ th percentile compared to the reference group, $\mathrm{MOH}$ concentrations were found to be significantly associated with lower delayed recall scores in the unadjusted model ( $\mathrm{OR}=2.386,95 \%$ CI: 1.134-5.021). No significant associations were found in the adjusted models. For the phthalate MZP in females in the $\geq 50$ th percentile compared to the reference group, MZP concentrations were found to be significantly associated with lower delayed recall scores in the unadjusted model (OR=1.798, 95\% CI: 1.068-3.026). No significant associations were found in the adjusted models. For the phthalate MIB among females in the $\geq 50$ th percentile, MIB concentrations were found to be significantly associated with lower delayed recall scores $(\mathrm{OR}=1.844,95 \%$ CI: 1.045-3.252) when compared to the reference group in the unadjusted model. No significant associations were found in the adjusted models. 
Table 1.7 Geometric Mean Urinary Phthalate and BPA Levels by Delayed Recall Cut-Off Score for Subjects > 60 years of age, NHANES 2011-2014, over the LOD

\begin{tabular}{|c|c|c|}
\hline \multirow{2}{*}{ EEDC } & \multicolumn{2}{|c|}{ Geometric Mean (ng/ml) (GSE, N) } \\
\hline & Delayed Recall Score $\leq 3$ & Delayed Recall Score $\geq 4$ \\
\hline Mono (carboxynonyl) Phthalate - CNP & $2.3874(0.2028,156)$ & $2.6792(0.1433,773)$ \\
\hline Mono (carboxyoctyl) Phthalate - COP & $16.3583(2.4210,157)$ & $19.1739(1.5721,775)$ \\
\hline Mono-(2-ethyl-5-carboxypentyl) Phthalate -ECP & $13.9383(1.1101,156)$ & $11.7602(0.5800,775)$ \\
\hline Mono-n-butyl Phthalate - MBP & $11.7959(1.1573,154)$ & $9.6276(0.5050,765)$ \\
\hline Mono-(3-carboxypropyl) Phthalate - MC1 & $2.6840(0.2796,152)$ & $2.7018(0.1851,737)$ \\
\hline Mono-ethyl Phthalate -MEP & $61.6129(8.4627,157)$ & $55.1030(4.6833,776)$ \\
\hline Mono (2-ethyl-5-hydroxy hexyl) Phthalate - MHH & $8.2016(0.7581,157)$ & $7.1290(0.3341,775)$ \\
\hline Mono-(2-ethyl-5-oxohexyl) Phthalate -MOH & $5.7034(0.4356,157)$ & $4.7910(0.2419,774)$ \\
\hline Mono-benzyl Phthalate - MZP & $4.7526(0.6688,153)$ & $4.0388(0.1976,767)$ \\
\hline Mono-isobutyl Phthalate - MIB & $5.8055(0.6299,156)$ & $6.2925(0.2863,767)$ \\
\hline Bisphenol A - BPH & $1.4799(0.1033,150)$ & $1.5383(0.07611,732)$ \\
\hline \multicolumn{3}{|c|}{ NHANES sampling weight applied before calculating the geometric mean. } \\
\hline \multicolumn{3}{|c|}{$\begin{array}{l}\text { The EEDCs were creatinine-adjusted then log transformed prior to analyses. Extreme creatinine values }(<30 \\
\mathrm{mg} / \mathrm{dL} \text { and }>300 \mathrm{mg} / \mathrm{dL}) \text { and EEDC levels below the LOD were taken into account using the appropriate } \\
\text { domain statement in SAS. } * \mathrm{p}<0.05, * * \mathrm{p}<0.01 * * * \mathrm{p}<0.001\end{array}$} \\
\hline
\end{tabular}

\begin{tabular}{|c|c|c|c|c|c|c|c|c|c|c|c|c|c|c|c|c|c|c|}
\hline \multirow{3}{*}{ EEDC } & \multicolumn{6}{|c|}{ Geometric Mean (ng/ml) (GSE, N), Age 60-69 } & \multicolumn{6}{|c|}{ Geometric Mean (ng/ml) (GSE, N), Age 70-79 } & \multicolumn{6}{|c|}{ Geometric Mean (ng/ml) (GSE, N), Age $80+$} \\
\hline & \multicolumn{3}{|c|}{ Delayed Recall Score $\leq 3$} & \multicolumn{3}{|c|}{ Delayed Recall Score $\geq 4$} & \multicolumn{3}{|c|}{ Delayed Recall Score $\leq 3$} & \multicolumn{3}{|c|}{ Delayed Recall Score $\geq 4$} & \multicolumn{3}{|c|}{ Delayed Recall Score $\leq 3$} & \multicolumn{3}{|c|}{ Delayed Recall Score $\geq 4$} \\
\hline & GM & GSE & $\mathrm{N}$ & GM & GSE & $\mathrm{N}$ & GM & GSE & $\mathrm{N}$ & GM & GSE & $\mathrm{N}$ & GM & GSE & $\mathrm{N}$ & GM & GSE & $\mathrm{N}$ \\
\hline Mono (carboxynonyl) Phthalate - CNP & 2.24 & 0.39 & 60 & 2.55 & 0.16 & 429 & 2.40 & 0.45 & 43 & 3.01 & 0.28 & 233 & 2.52 & 0.29 & 53 & 2.50 & 0.22 & 111 \\
\hline Mono (carboxyoctyl) Phthalate - COP & 16.69 & 4.73 & 61 & 18.12 & 2.04 & 429 & 15.60 & 4.41 & 43 & 22.95 & 2.25 & 235 & 16.86 & 2.28 & 53 & 15.73 & 2.12 & 111 \\
\hline Mono-(2-ethyl-5-carboxypentyl) Phthalate -ECP & 12.81 & 1.83 & 60 & 10.68 & 0.75 & 429 & 13.88 & 2.04 & 43 & 13.51 & 0.73 & 235 & 15.12 & 1.66 & 53 & 12.65 & 0.72 & 111 \\
\hline Mono-n-butyl Phthalate - MBP & 12.42 & 3.42 & 60 & 9.01 & 0.59 & 423 & 11.18 & 1.00 & 43 & 9.99 & 0.87 & 232 & 11.90 & 1.47 & 51 & 11.66 & 1.34 & 110 \\
\hline Mono-(3-carboxypropyl) Phthalate - $\mathrm{MCl}$ & 2.53 & 0.21 & 58 & 2.50 & 0.21 & 407 & 2.85 & 0.81 & 43 & 3.09 & 0.30 & 222 & 2.66 & 0.36 & 51 & 2.75 & 0.28 & 108 \\
\hline Mono-ethyl Phthalate -MEP & 100.17 & 31.88 & 61 & 56.05 & 5.83 & 430 & 64.46 & 11.90 & 43 & 58.60 & 8.01 & 235 & 37.64 & 9.16 & 53 & 44.05 & 5.32 & 111 \\
\hline Mono (2-ethyl-5-hydroxy hexyl) Phthalate - MHH & 8.09 & 1.02 & 61 & 6.57 & 0.46 & 429 & 8.69 & 1.60 & 43 & 8.15 & 0.42 & 235 & 7.83 & 0.97 & 53 & 7.30 & 0.54 & 111 \\
\hline Mono-(2-ethyl-5-oxohexyl) Phthalate -MOH & 5.12 & 0.63 & 61 & 4.30 & 0.30 & 429 & 6.03 & 0.91 & 43 & 5.54 & 0.26 & 235 & 5.95 & 0.64 & 53 & 5.36 & 0.44 & 110 \\
\hline Mono-benzyl Phthalate - MZP & 4.83 & 1.49 & 60 & 3.98 & 0.31 & 425 & 4.09 & 0.90 & 42 & 3.83 & 0.31 & 233 & 5.50 & 0.88 & 51 & 4.93 & 0.53 & 109 \\
\hline Mono-isobutyl Phthalate - MIB & 5.14 & 1.30 & 60 & 6.56 & 0.35 & 427 & 7.17 & 0.80 & 43 & 6.06 & 0.37 & 231 & 5.22 & 0.73 & 53 & 5.73 & 0.65 & 109 \\
\hline Bisphenol A-BPA & 1.57 & 0.22 & 61 & 1.49 & 0.09 & 408 & 1.54 & 0.23 & 39 & 1.68 & 0.12 & 219 & 1.35 & 0.18 & 50 & 1.41 & 0.18 & 105 \\
\hline \multicolumn{19}{|c|}{$\begin{array}{l}\text { NHANES sampling weight applied before calculating the geometric mean. } \\
\text { The EEDCs were creatinine-adjusted then log transformed prior to analyses. Extreme creatinine values }(<30 \mathrm{mg} / \mathrm{dL} \text { and }>300 \mathrm{mg} / \mathrm{dL}) \text { and EEDC levels below the LOD were taken into account using the appropriate domain statement } \\
\text { SAS. }{ }^{*}<0.05,{ }^{* *} \times<0.01{ }^{* * *} \mathrm{p}<0.001\end{array}$} \\
\hline
\end{tabular}




\begin{tabular}{|c|c|c|c|c|c|c|c|c|c|c|c|c|}
\hline \multicolumn{13}{|c|}{$\begin{array}{l}\text { Table 1.9 Gender-specific Geometric Mean Urinary Phthalate and BPA Levels by Delayed Recall Cut-Off Score for Subjects }>=60 \text { years of age, NHANES 2011- } \\
\qquad 2014 \text {, over the LOD }\end{array}$} \\
\hline \multirow{4}{*}{ EEDC } & \multicolumn{6}{|c|}{ Geometric Mean (ng/ml) (GSE, N) } & \multicolumn{6}{|c|}{ Geometric Mean (ng/ml) (GSE, N) } \\
\hline & \multicolumn{6}{|c|}{ Male } & \multicolumn{6}{|c|}{ Female } \\
\hline & \multicolumn{3}{|c|}{ Delayed Recall Score $\leq \mathbf{3}$} & \multicolumn{3}{|c|}{ Delayed Recall Score $\geq 4$} & \multicolumn{3}{|c|}{ Delayed Recall Score $\leq 3$} & \multicolumn{3}{|c|}{ Delayed Recall Score $\geq 4$} \\
\hline & GM & GSE & $\mathbf{N}$ & GM & GSE & $\mathbf{N}$ & GM & GSE & $\mathbf{N}$ & GM & GSE & $\mathbf{N}$ \\
\hline Mono (carboxynonyl) Phthalate - CNP & 2.06 & 0.17 & 89 & 2.43 & 0.16 & 378 & 2.94 & 0.40 & 67 & 2.93 & 0.27 & 395 \\
\hline Mono (carboxyoctyl) Phthalate - COP & 13.30 & 2.07 & 89 & 17.19 & 1.37 & 380 & 21.74 & 4.04 & 68 & 21.18 & 2.71 & 395 \\
\hline Mono-(2-ethyl-5-carboxypentyl) Phthalate -ECP & 11.68 & 1.18 & 89 & 10.67 & 0.61 & 381 & 17.79 & 2.05 & $67 *$ & 12.86 & 0.83 & 394 \\
\hline Mono-n-butyl Phthalate - MBP & 10.33 & 1.54 & 87 & 9.12 & 0.69 & 374 & 14.09 & 1.51 & $67 * * *$ & 10.11 & 0.53 & 391 \\
\hline Mono-(3-carboxypropyl) Phthalate - MC1 & 2.49 & 0.25 & 88 & 2.52 & 0.22 & 362 & 2.99 & 0.57 & 64 & 2.88 & 0.25 & 375 \\
\hline Mono-ethyl Phthalate -MEP & 64.55 & 13.13 & 89 & 50.28 & 4.94 & 381 & 57.80 & 11.19 & 68 & 59.91 & 6.77 & 395 \\
\hline Mono (2-ethyl-5-hydroxy hexyl) Phthalate - MHH & 7.22 & 0.81 & 88 & 6.74 & 0.40 & 381 & 9.77 & 1.28 & 68 & 7.51 & 0.42 & 394 \\
\hline Mono-(2-ethyl-5-oxohexyl) Phthalate -MOH & 4.87 & 0.49 & 89 & 4.55 & 0.29 & 379 & 7.08 & 0.79 & $68 *$ & 5.02 & 0.30 & 395 \\
\hline Mono-benzyl Phthalate - MZP & 3.72 & 0.54 & 86 & 3.58 & 0.28 & 377 & 6.59 & 1.23 & $67 *$ & 4.51 & 0.23 & 390 \\
\hline Mono-isobutyl Phthalate - MIB & 4.88 & 0.64 & 89 & 5.91 & 0.34 & 377 & 7.40 & 0.75 & 67 & 6.67 & 0.32 & 390 \\
\hline Bisphenol A - BPA & 1.36 & 0.12 & 85 & 1.39 & 0.08 & 361 & 1.65 & 0.21 & 65 & 1.69 & 0.11 & 371 \\
\hline Isted then log tran & ANES & pling v & $\begin{array}{l}\text { ht appl } \\
\text { s. Extr } \\
\text { te dom }\end{array}$ & befor & $\begin{array}{l}\text { Iculatir } \\
\text { ne valu }\end{array}$ & $\begin{array}{l}\text { e geor } \\
<30 \mathrm{~m} \\
\mathrm{n}<0 \mathrm{n}\end{array}$ & ric mes & $\mathrm{P}$ & $\begin{array}{l}\text { and } \mathrm{EE} \\
01\end{array}$ & 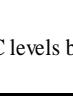 & & \\
\hline
\end{tabular}

\begin{tabular}{|c|c|c|c|c|c|c|c|c|c|c|c|c|}
\hline \multicolumn{13}{|c|}{$\begin{array}{l}\text { Table 1.10 Race-specific Geometric Mean Urinary Phthalate and BPA Levels by Delayed Recall Cut-Off Scores for Subjects >=60 years of age, NHANES 2011- } \\
\qquad 2014 \text {, over the LOD }\end{array}$} \\
\hline \multirow{3}{*}{ EEDC } & \multicolumn{6}{|c|}{ Geometric Mean (ng/ml) (GSE, N), Hispanic } & \multicolumn{6}{|c|}{ Geometric Mean (ng/ml) (GSE, N), Non-Hispanic White } \\
\hline & \multicolumn{3}{|c|}{ Delayed Recall Score $\leq \mathbf{3}$} & \multicolumn{3}{|c|}{ Delayed Recall Score $\geq 4$} & \multicolumn{3}{|c|}{ Delayed Recall Score $\leq 3$} & \multicolumn{3}{|c|}{ Delayed Recall Score $\geq 4$} \\
\hline & GM & GSE & $\mathbf{N}$ & GM & GSE & $\mathbf{N}$ & GM & GSE & $\mathbf{N}$ & GM & GSE & $\mathbf{N}$ \\
\hline Mono (carboxynonyl) Phthalate - CNP & 2.49 & 0.31 & 42 & 2.29 & 0.11 & 152 & 2.47 & 0.28 & 53 & 2.84 & 0.18 & 360 \\
\hline Mono (carboxyoctyl) Phthalate - COP & 20.00 & 2.90 & 42 & 20.20 & 1.66 & 152 & 16.36 & 3.32 & 53 & 20.17 & 2.02 & 360 \\
\hline Mono-(2-ethyl-5-carboxypentyl) Phthalate -ECP & 21.78 & 2.08 & 42 & 16.78 & 1.22 & 152 & 12.95 & 1.29 & 53 & 11.50 & 0.65 & 359 \\
\hline Mono-n-butyl Phthalate - MBP & 14.98 & 1.23 & 42 & 13.51 & 1.07 & 150 & 11.53 & 1.60 & 52 & 9.00 & 0.60 & 354 \\
\hline Mono-(3-carboxypropyl) Phthalate - MC1 & 2.69 & 0.52 & 40 & 2.85 & 0.24 & 144 & 2.73 & 0.36 & 52 & 2.76 & 0.24 & 344 \\
\hline Mono-ethyl Phthalate -MEP & 132.82 & 49.20 & 42 & 94.33 & 10.34 & 152 & 54.81 & 10.04 & 53 & 50.27 & 4.92 & 360 \\
\hline Mono (2-ethyl-5-hydroxy hexyl) Phthalate - MHH & 13.09 & 1.18 & $42 *$ & 9.55 & 0.76 & 152 & 7.53 & 0.90 & 53 & 6.96 & 0.37 & 359 \\
\hline Mono-(2-ethyl-5-oxohexyl) Phthalate -MOH & 8.30 & 0.76 & $42 *$ & 6.15 & 0.53 & 152 & 5.41 & 0.53 & 53 & 4.70 & 0.27 & 358 \\
\hline Mono-benzyl Phthalate - MZP & 5.38 & 1.13 & $42 *$ & 3.51 & 0.30 & 152 & 5.10 & 0.97 & 52 & 3.97 & 0.20 & 354 \\
\hline Mono-isobutyl Phthalate - MIB & 8.95 & 0.72 & 42 & 9.32 & 0.56 & 152 & 5.16 & 0.76 & 53 & 5.80 & 0.27 & 356 \\
\hline Bisphenol A - BPA & 1.75 & 0.27 & 39 & 1.58 & 0.15 & 145 & 1.44 & 0.14 & 52 & 1.56 & 0.10 & 342 \\
\hline \multirow{3}{*}{ EEDC } & \multicolumn{6}{|c|}{ Geometric Mean (ng/ml) (GSE, N), Non-Hispanic Black } & \multicolumn{6}{|c|}{ Geometric Mean (ng/ml) (GSE, N), Asian/Other } \\
\hline & \multicolumn{3}{|c|}{ Delayed Recall Score $\leq 3$} & \multicolumn{3}{|c|}{ Delayed Recall Score $\geq 4$} & \multicolumn{3}{|c|}{ Delayed Recall Score $\leq 3$} & \multicolumn{3}{|c|}{ Delayed Recall Score $\geq 4$} \\
\hline & GM & GSE & $\mathbf{N}$ & GM & GSE & $\mathbf{N}$ & GM & GSE & $\mathbf{N}$ & GM & GSE & $\mathbf{N}$ \\
\hline Mono (carboxynonyl) Phthalate - CNP & 1.87 & 0.18 & 48 & 1.98 & 0.11 & 195 & 2.50 & 0.89 & 13 & 2.19 & 0.39 & 66 \\
\hline Mono (carboxyoctyl) Phthalate - COP & 15.71 & 2.54 & 48 & 14.25 & 1.10 & 194 & 9.92 & 3.30 & 14 & 12.83 & 2.65 & 69 \\
\hline Mono-(2-ethyl-5-carboxypentyl) Phthalate -ECP & 12.48 & 2.26 & 47 & 10.58 & 0.78 & 195 & 13.48 & 1.61 & 14 & 12.35 & 0.77 & 69 \\
\hline Mono-n-butyl Phthalate - MBP & 9.15 & 1.61 & 48 & 11.08 & 0.64 & 192 & 16.09 & 3.54 & 12 & 13.99 & 2.70 & 69 \\
\hline Mono-(3-carboxypropyl) Phthalate - MC2 & 2.37 & 0.28 & 46 & 2.20 & 0.15 & 189 & 2.90 & 0.97 & 14 & 2.56 & 0.43 & 60 \\
\hline Mono-ethyl Phthalate -MEP & 59.65 & 10.61 & 48 & 85.98 & 8.94 & 195 & 33.44 & 11.36 & 14 & 52.11 & 15.46 & 69 \\
\hline Mono (2-ethyl-5-hydroxy hexyl) Phthalate - MHH & 8.05 & 1.40 & 48 & 7.23 & 0.55 & 195 & 6.90 & 1.09 & 14 & 6.75 & 0.76 & 69 \\
\hline Mono-(2-ethyl-5-oxohexyl) Phthalate -MOH & 5.21 & 0.95 & 48 & 4.88 & 0.33 & 195 & 4.82 & 0.78 & 14 & 4.48 & 0.50 & 69 \\
\hline Mono-benzyl Phthalate - MZP & 3.48 & 0.89 & 46 & 4.94 & 0.44 & 194 & 2.66 & 0.41 & 13 & 4.74 & 1.73 & 67 \\
\hline Mono-isobutyl Phthalate - MIB & 7.03 & 0.88 & 47 & 7.70 & 0.51 & 191 & 4.94 & 1.67 & 14 & 9.85 & 1.69 & $68 *$ \\
\hline Bisphenol A - BPA & 1.36 & 0.14 & 47 & 1.42 & 0.07 & 188 & 1.69 & 0.53 & 12 & 1.40 & 0.23 & 57 \\
\hline taken into acc & ANES & pling u & ht appl & before & Iculati & e geon & tric me & $k * *$ & & & & \\
\hline
\end{tabular}




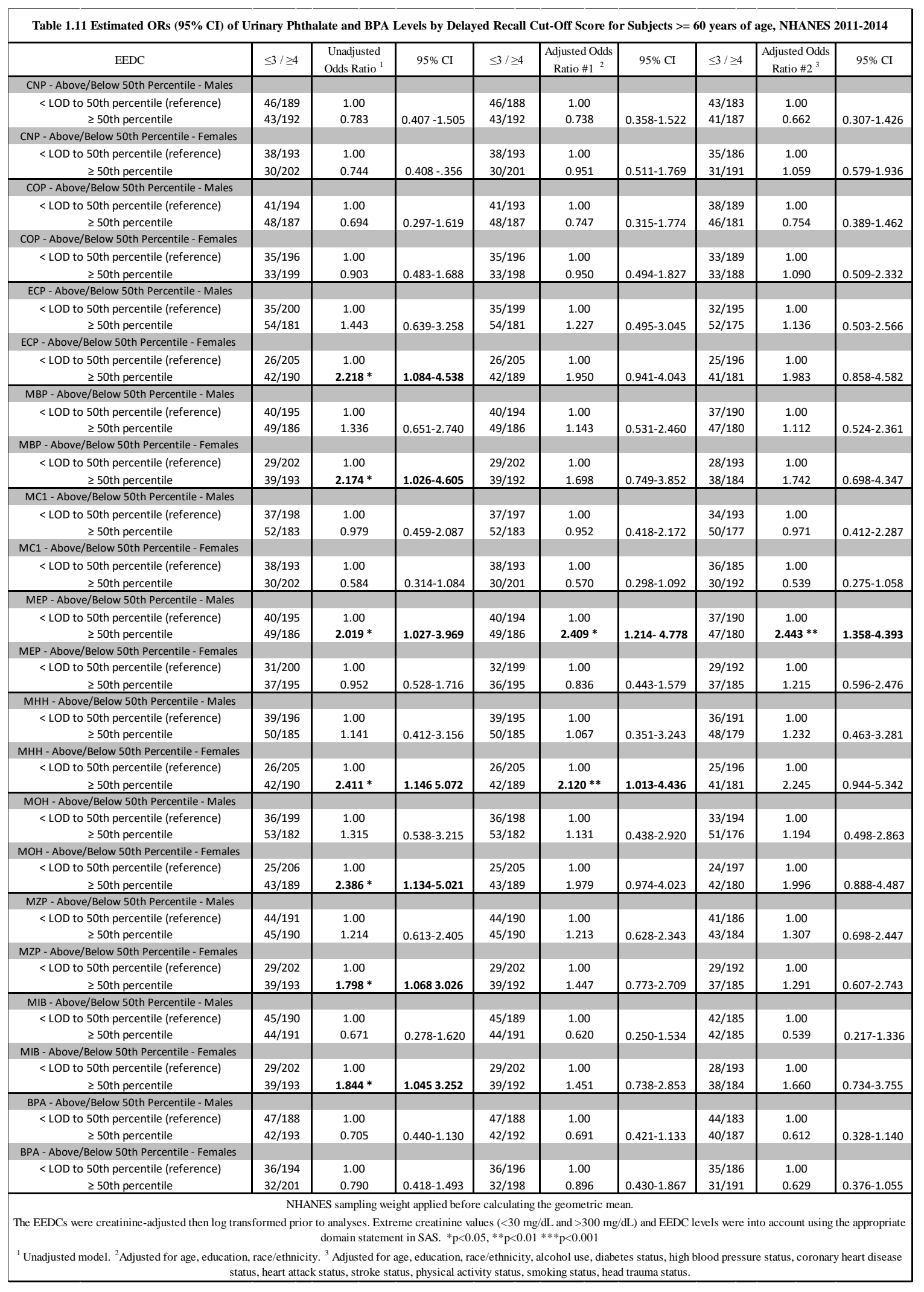


Animal Fluency Scores and Exposure to BPA and Phthalates: Tables 1.12 to 1.16 present the GMs and GSEs of urinary phthalates and BPA levels among subjects with animal fluency scores. Table 1.12 presents GMs and GSEs of subjects who have measurable phthalate and BPA levels over the LOD by animal fluency cut-off scores. The crude GM mean of 7 of the 10 phthalates (ECP, MBP, MC1, MHH, MOH, MZP, MIB) and BPA are higher in subjects with a score of $\leq 11$ compared to those with a score $\geq 12$. 4 of the 10 phthalates GM means were found to be significant higher in subjects with an animal fluency score $\leq 11$ : ECP $(p<0.05), \operatorname{MBP}(p<0.05), \operatorname{MZP}(p<0.05)$, and MIB $(\mathrm{p}<0.05)$. Table 1.13 presents the age-specific GMs and GSEs for subjects who have measurable phthalate and BPA levels over the LOD by animal fluency cut-off score. In the 60-69-year age group, 3 phthalates had significantly higher GMs in subjects with a animal fluency score $\leq 11$ compared to subjects with a score of $\geq 12$ : ECP $(p<0.05)$, MBP $(\mathrm{p}<0.01)$, and MIB $(\mathrm{p}<0.05)$. The phthalate COP has a significantly higher GM in the in subjects with an animal fluency score $\geq 12(\mathrm{p}<0.05)$. In the $70-79$-year age group, MIB had a significantly higher GM in subjects with a score of $\leq 11(\mathrm{p}<0.05)$. In the $80+$ years age group, 5 phthalates were found to have significantly higher GM mean levels in the Animal fluency Score $\leq 11$ group: COP $(p<0.05)$, ECP $(p<0.01)$, MBP $(p<0.05)$, MHH $(\mathrm{p}<0.01), \mathrm{MOH}(\mathrm{p}<0.05)$. Table 1.14 presents gender-specific geometric GMs and GSEs for subjects who have measurable phthalate and BPA levels over the LOD by animal fluency cut-off score. In females, 5 of the phthalates had significantly higher GM means in subjects with an animal fluency score $\leq 11$ : $\mathrm{ECP}(\mathrm{p}<0.01), \mathrm{MBP}(\mathrm{p}<0.001), \mathrm{MOH}$ $(\mathrm{p}<0.05)$, MZP $(\mathrm{p}<0.01)$, and MIB $(\mathrm{p}<0.05)$. Table 1.15 presents race-specific geometric GMs and GSEs for subjects who have measurable phthalate and BPA levels over the 
LOD by animal fluency cut-off score. In Hispanics, the GM level of MZP was significantly higher in the Animal fluency Score $\leq 11$ group $(\mathrm{p}<0.001)$.

Table 1.16 presents the estimated ORs and 95\% CIs of phthalate and BPA levels by animal fluency scores. For the phthalate MBP, with females in the $\geq 50$ th percentile group compared to the reference group, MBP concentration is associated with lower animal fluency scores in the unadjusted model (OR=2.369, 95\% CI: 1.392-4.031), adjusted model \#1 (OR=2.080, 95\% CI: 1.151-3.757), and adjusted model \#2 (OR=2.437, 95\% CI: 1.190-4.989). For the phthalate MHH with females in the $\geq 50$ th percentile group, the MHH concentration is associated with lower animal fluency scores compared to the reference group in the unadjusted model (1.861, 95\% CI: 1.078-3.212). The adjusted models were not significant (Table 1.16). For the phthalate MZP, with females in the $\geq 50$ th percentile, MZP concentration is significantly associated with lower animal fluency scores in the unadjusted model (OR=2.610, 95\% CI: 1.573-4.332) and adjusted model \#1 (OR=2.175, 95\% CI: 1.281-3.692) compared to the reference group (Table 1.16). For the phthalate MIB, for females in the $\geq 50$ th percentile, MIB concentration is associated with lower animal fluency scores in the adjusted model $(\mathrm{OR}=2.205,95 \% \mathrm{CI}$ : 1.323-3.675) and adjusted model \#1 (2.051, 95\% CI: 1.111-3.786), compared to the reference group (Table 1.16). 


\begin{tabular}{|c|c|c|c|c|c|c|}
\hline \multirow{3}{*}{ EEDC } & \multicolumn{6}{|c|}{ Geometric Mean (ng/ml) (GSE, N) } \\
\hline & \multicolumn{3}{|c|}{ Animal Fluency Score $\leq 11$} & \multicolumn{3}{|c|}{ Animal Fluency Score $\geq 12$} \\
\hline & GM & GSE & $\mathbf{N}$ & GM & GSE & $\mathbf{N}$ \\
\hline Mono (carboxynonyl) Phthalate - CNP & 2.51 & 0.18 & 185 & 2.68 & 0.16 & 742 \\
\hline Mono (carboxyoctyl) Phthalate - COP & 17.14 & 2.05 & 186 & 19.24 & 1.71 & 744 \\
\hline Mono-(2-ethyl-5-carboxypentyl) Phthalate -ECP & 14.26 & 0.97 & $187 *$ & 11.73 & 0.61 & 742 \\
\hline Mono-n-butyl Phthalate - MBP & 11.58 & 0.80 & $186 *$ & 9.67 & 0.54 & 730 \\
\hline Mono-(3-carboxypropyl) Phthalate - MC1 & 2.75 & 0.43 & 177 & 2.71 & 0.21 & 711 \\
\hline Mono-ethyl Phthalate -MEP & 54.00 & 6.58 & 187 & 56.91 & 28.20 & 744 \\
\hline Mono (2-ethyl-5-hydroxy hexyl) Phthalate - MHH & 8.15 & 0.50 & 187 & 7.14 & 0.35 & 743 \\
\hline Mono-(2-ethyl-5-oxohexyl) Phthalate -MOH & 5.67 & 0.36 & 187 & 4.79 & 0.25 & 742 \\
\hline Mono-benzyl Phthalate - MZP & 5.51 & 0.66 & $185 *$ & 3.94 & 0.21 & 733 \\
\hline Mono-isobutyl Phthalate - MIB & 7.45 & 0.37 & $184 *$ & 6.08 & 0.30 & 737 \\
\hline Bisphenol A - BPA & 1.55 & 0.12 & 174 & 1.53 & 0.08 & 705 \\
\hline
\end{tabular}

NHANES sampling weight applied before calculating the geometric mean.

The EEDCs were creatinine-adjusted then log transformed prior to analyses. Extreme creatinine values $(<30$ $\mathrm{mg} / \mathrm{dL}$ and $>300 \mathrm{mg} / \mathrm{dL}$ ) and EEDC levels below the LOD were taken into account using the appropriate domain statement in SAS. *p $<0.05, * * \mathrm{p}<0.01 * * * \mathrm{p}<0.001$

\begin{tabular}{|c|c|c|c|c|c|c|c|c|c|c|c|c|c|c|c|c|c|c|}
\hline \multirow{3}{*}{ EEDC } & \multicolumn{6}{|c|}{ Geometric Mean (ng/ml) (GSE, N), Age 60-69 } & \multicolumn{6}{|c|}{ Geometric Mean (ng/ml) (GSE, N), Age 70-79 } & \multicolumn{6}{|c|}{ Geometric Mean (ng/ml) (GSE, N), Age $80+$} \\
\hline & \multicolumn{3}{|c|}{ Animal Fluency Score $\leq 11$} & \multicolumn{3}{|c|}{ Animal Fluency Score $\geq 12$} & \multicolumn{3}{|c|}{ Animal Fluency Score $\leq 11$} & \multicolumn{3}{|c|}{ Animal Fluency Score $\geq 12$} & \multicolumn{3}{|c|}{ Animal Fluency Score $\leq 11$} & \multicolumn{3}{|c|}{ Animal Fluency Score $\geq 12$} \\
\hline & GM & GSE & $\mathrm{N}$ & GM & GSE & $\mathrm{N}$ & GM & GSE & $\mathrm{N}$ & GM & GSE & $\mathrm{N}$ & GM & GSE & $\mathrm{N}$ & GM & GSE & $\mathrm{N}$ \\
\hline Mono (carboxynonyl) Phthalate - CNP & 2.26 & 0.20 & 82 & 2.55 & 0.18 & 408 & 2.39 & 0.53 & 56 & 3.07 & 0.31 & 216 & 2.99 & 0.58 & 47 & 2.42 & 0.17 & 118 \\
\hline Mono (carboxyoctyl) Phthalate - COP & 12.59 & 1.73 & 83 & 18.60 & 2.20 & $408 *$ & 17.55 & 4.16 & 56 & 22.95 & 2.61 & 218 & 23.14 & 3.57 & $47 *$ & 15.01 & 1.87 & 118 \\
\hline Mono-(2-ethyl-5-carboxypentyl) Phthalate -ECP & 14.39 & 1.57 & $84 *$ & 10.57 & 0.78 & 406 & 12.55 & 1.51 & 56 & 13.70 & 0.81 & 218 & 16.75 & 1.35 & $47^{* * *}$ & 12.59 & 0.85 & 118 \\
\hline Mono-n-butyl Phthalate - MBP & 12.31 & 1.02 & $83^{* *}$ & 9.01 & 0.61 & 400 & 8.88 & 0.96 & 56 & 10.40 & 0.95 & 215 & 15.52 & 2.33 & $47 *$ & 10.87 & 1.11 & 115 \\
\hline Mono-(3-carboxypropyl) Phthalate - MCl & 2.24 & 0.34 & 79 & 2.52 & 0.23 & 387 & 2.70 & 0.88 & 52 & 3.13 & 0.36 & 210 & 3.54 & 0.49 & 46 & 2.62 & 0.29 & 114 \\
\hline Mono-ethyl Phthalate -MEP & 46.72 & 9.39 & 84 & 59.47 & 6.99 & 408 & 54.94 & 9.76 & 56 & 61.26 & 8.77 & 218 & 61.72 & 13.91 & 47 & 37.56 & 4.22 & 118 \\
\hline Mono (2-ethyl-5-hydroxy hexyl) Phthalate - MHH & 8.11 & 0.89 & 84 & 6.56 & 0.48 & 407 & 7.42 & 0.79 & 56 & 8.36 & 0.54 & 218 & 9.31 & 0.75 & $47 * *$ & 7.08 & 0.52 & 118 \\
\hline Mono-(2-ethyl-5-0xohexyl) Phthalate -MOH & 5.55 & 0.64 & 84 & 4.26 & 0.30 & 407 & 5.04 & 0.59 & 56 & 5.69 & 0.35 & 218 & 6.81 & 0.37 & $47 *$ & 5.25 & 0.43 & 117 \\
\hline Mono-benzyl Phthalate - MZP & 5.56 & 1.09 & 83 & 3.93 & 0.31 & 403 & 5.06 & 0.80 & 55 & 3.65 & 0.32 & 216 & 6.11 & 0.97 & 47 & 4.77 & 0.44 & 114 \\
\hline Mono-isobutyl Phthalate - MIB & 8.11 & 0.78 & $83 *$ & 6.30 & 0.37 & 405 & 7.38 & 0.55 & $54 *$ & 6.03 & 0.37 & 216 & 6.87 & 0.83 & 47 & 5.34 & 0.54 & 116 \\
\hline Bisphenol A-BPA & 1.69 & 0.14 & 79 & 1.48 & 0.10 & 390 & 1.55 & 0.26 & 49 & 1.69 & 0.12 & 205 & 1.40 & 0.19 & 46 & 1.41 & 0.16 & 110 \\
\hline \multicolumn{19}{|c|}{$\begin{array}{l}\text { NHANES sampling weight applied before calculating the geometric mean. } \\
\text { The EEDCs were creatinine-adjusted then log transformed prior to analyses. Extreme creatinine values ( }<30 \mathrm{mg} / \mathrm{dL} \text { and }>300 \mathrm{mg} / \mathrm{dL}) \text { and EEDC levels below the LOD were taken into account using the appropriate domain statement in } \\
\qquad \text { SAS. }{ }^{*} \mathrm{p}<0.05,{ }^{*} \mathrm{p}<0.01{ }^{* * *} \mathrm{p}<0.001\end{array}$} \\
\hline
\end{tabular}




\begin{tabular}{|c|c|c|c|c|c|c|c|c|c|c|c|c|}
\hline \multirow{4}{*}{ EEDC } & \multicolumn{6}{|c|}{ Geometric Mean (ng/ml) (GSE, N) } & \multicolumn{6}{|c|}{ Geometric Mean (ng/ml) (GSE, N) } \\
\hline & \multicolumn{6}{|c|}{ Male } & \multicolumn{6}{|c|}{ Female } \\
\hline & \multicolumn{3}{|c|}{ Animal Fluency Score $\leq 11$} & \multicolumn{3}{|c|}{ Animal Fluency Score $\geq 12$} & \multicolumn{3}{|c|}{ Animal Fluency Score $\leq 11$} & \multicolumn{3}{|c|}{ Animal Fluency Score $\geq 12$} \\
\hline & GM & GSE & $\mathrm{N}$ & GM & GSE & $\mathrm{N}$ & GM & GSE & $\mathrm{N}$ & GM & GSE & $\mathrm{N}$ \\
\hline Mono (carboxynonyl) Phthalate - CNP & 2.69 & 0.35 & 84 & 2.34 & 0.15 & 380 & 2.38 & 0.31 & 101 & 3.06 & 0.28 & 362 \\
\hline Mono (carboxyoctyl) Phthalate - COP & 15.03 & 2.28 & 84 & 16.85 & 1.34 & 382 & 18.90 & 3.20 & 102 & 21.93 & 2.87 & 362 \\
\hline Mono-(2-ethyl-5-carboxypentyl) Phthalate -ECP & 11.92 & 1.26 & 85 & 10.66 & 0.56 & 382 & 16.30 & 1.10 & $102 * *$ & 12.90 & 0.87 & 360 \\
\hline Mono-n-butyl Phthalate - MBP & 8.73 & 0.86 & 85 & 9.35 & 0.73 & 372 & 14.33 & 1.08 & $101 * * *$ & 9.98 & 0.56 & 358 \\
\hline Mono-(3-carboxypropyl) Phthalate - $\mathrm{MCl}$ & 2.68 & 0.75 & 83 & 2.51 & 0.21 & 364 & 2.82 & 0.35 & 94 & 2.92 & 0.28 & 347 \\
\hline Mono-ethyl Phthalate -MEP & 44.21 & 6.89 & 85 & 53.83 & 5.62 & 382 & 62.70 & 9.49 & 102 & 58.77 & 6.80 & 362 \\
\hline Mono (2-ethyl-5-hydroxy hexyl) Phthalate - MHH & 7.24 & 0.67 & 85 & 6.74 & 0.38 & 382 & 8.92 & 0.79 & 102 & 7.56 & 0.43 & 361 \\
\hline Mono-(2-ethyl-5-oxohexyl) Phthalate -MOH & 4.89 & 0.49 & 85 & 4.54 & 0.26 & 380 & 6.34 & 0.49 & $102 *$ & 5.06 & 0.30 & 362 \\
\hline Mono-benzyl Phthalate - MZP & 4.02 & 0.62 & 84 & 3.54 & 0.29 & 376 & 6.97 & 0.86 & $101 * *$ & 4.38 & 0.25 & 357 \\
\hline Mono-isobutyl Phthalate - MIB & 6.75 & 0.57 & 84 & 5.60 & 0.32 & 379 & 7.99 & 0.58 & $100 *$ & 6.59 & 0.35 & 358 \\
\hline Bisphenol A-BPA & 1.54 & 0.23 & 76 & 1.37 & 0.07 & 366 & 1.55 & 0.10 & 98 & 1.71 & 0.11 & 339 \\
\hline \multicolumn{13}{|c|}{$\begin{array}{l}\text { NHANES sampling weight applied before calculating the geometric mean. } \\
\text { The EEDCs were creatinine-adjusted then log transformed prior to analyses. Extreme creatinine values }(<30 \mathrm{mg} / \mathrm{dL} \text { and }>300 \mathrm{mg} / \mathrm{dL} \text { ) and EEDC levels below the LOD were } \\
\text { taken into account using the appropriate domain statement in SAS. }{ }^{*} \mathrm{p}<0.05,{ }^{*} \mathrm{p}<0.01{ }^{*} * \mathrm{p}_{\mathrm{p}}<0.001\end{array}$} \\
\hline
\end{tabular}

\begin{tabular}{|c|c|c|c|c|c|c|c|c|c|c|c|c|}
\hline \multirow{3}{*}{ EEDC } & \multicolumn{6}{|c|}{ Geometric Mean (ng/ml) (GSE, N), Hispanic } & \multicolumn{6}{|c|}{ Geometric Mean (ng/ml) (GSE, N), Non-Hispanic White } \\
\hline & \multicolumn{3}{|c|}{ Animal Fluency Score $\leq 11$} & \multicolumn{3}{|c|}{ Animal Fluency Score $\geq 12$} & \multicolumn{3}{|c|}{ Animal Fluency Score $\leq 11$} & \multicolumn{3}{|c|}{ Animal Fluency Score $\geq 12$} \\
\hline & GM & GSE & $\mathbf{N}$ & GM & GSE & $\mathbf{N}$ & GM & GSE & $\mathbf{N}$ & GM & GSE & $\mathbf{N}$ \\
\hline Mono (carboxynonyl) Phthalate - CNP & 2.42 & 0.31 & 43 & 2.31 & 0.17 & 151 & 2.72 & 0.36 & 47 & 2.83 & 0.20 & 364 \\
\hline Mono (carboxyoctyl) Phthalate - COP & 25.51 & 5.19 & 43 & 19.01 & 1.45 & 151 & 18.33 & 3.09 & 47 & 20.09 & 2.12 & 364 \\
\hline Mono-(2-ethyl-5-carboxypentyl) Phthalate -ECP & 20.88 & 2.23 & 43 & 16.97 & 1.03 & 151 & 13.97 & 1.62 & 47 & 11.47 & 0.66 & 363 \\
\hline Mono-n-butyl Phthalate - MBP & 15.33 & 2.05 & 43 & 13.36 & 0.89 & 149 & 10.65 & 1.19 & 47 & 9.17 & 0.64 & 357 \\
\hline Mono-(3-carboxypropyl) Phthalate - MC1 & 3.21 & 0.61 & 41 & 2.72 & 0.28 & 143 & 2.91 & 0.82 & 45 & 2.77 & 0.26 & 350 \\
\hline Mono-ethyl Phthalate -MEP & 102.55 & 38.82 & 43 & 101.55 & 13.06 & 151 & 42.85 & 7.74 & 47 & 51.94 & 5.22 & 364 \\
\hline Mono (2-ethyl-5-hydroxy hexyl) Phthalate - MHH & 10.63 & 1.56 & 43 & 10.14 & 0.67 & 151 & 8.17 & 0.86 & 47 & 6.93 & 0.37 & 363 \\
\hline Mono-(2-ethyl-5-oxohexyl) Phthalate -MOH & 7.07 & 0.91 & 43 & 6.45 & 0.45 & 151 & 5.69 & 0.64 & 47 & 4.69 & 0.27 & 362 \\
\hline Mono-benzyl Phthalate - MZP & 6.02 & 0.94 & $43 * * *$ & 3.41 & 0.29 & 151 & 6.25 & 1.32 & 47 & 3.92 & 0.23 & 357 \\
\hline Mono-isobutyl Phthalate - MIB & 10.39 & 1.04 & 43 & 8.85 & 0.46 & 151 & 6.83 & 0.69 & 46 & 5.64 & 0.28 & 361 \\
\hline Bisphenol A-BPA & 1.60 & 0.22 & 40 & 1.62 & 0.17 & 144 & 1.59 & 0.20 & 43 & 1.54 & 0.10 & 349 \\
\hline \multirow{3}{*}{ EEDC } & \multicolumn{6}{|c|}{ Geometric Mean (ng/ml) (GSE, N), Non-Hispanic Black } & \multicolumn{6}{|c|}{ Geometric Mean (ng/ml) (GSE, N), Asian/Other } \\
\hline & \multicolumn{3}{|c|}{ Animal Fluency Score $\leq 11$} & \multicolumn{3}{|c|}{ Animal Fluency Score $\geq 12$} & \multicolumn{3}{|c|}{ Animal Fluency Score $\leq 11$} & \multicolumn{3}{|c|}{ Animal Fluency Score $\geq 12$} \\
\hline & GM & GSE & $\mathbf{N}$ & GM & GSE & $\mathbf{N}$ & GM & GSE & $\mathbf{N}$ & GM & GSE & $\mathbf{N}$ \\
\hline Mono (carboxynonyl) Phthalate - CNP & 1.79 & 0.15 & 75 & 2.03 & 0.13 & 169 & 3.85 & 1.80 & 20 & 1.86 & 0.20 & 58 \\
\hline Mono (carboxyoctyl) Phthalate - COP & 14.28 & 1.90 & 74 & 14.47 & 1.38 & 169 & 10.28 & 3.12 & 22 & 13.62 & 2.80 & 60 \\
\hline Mono-(2-ethyl-5-carboxypentyl) Phthalate -ECP & 12.65 & 1.20 & 75 & 10.12 & 0.84 & 168 & 12.10 & 1.26 & 22 & 12.80 & 0.90 & 60 \\
\hline Mono-n-butyl Phthalate - MBP & 10.99 & 0.92 & 75 & 10.43 & 0.67 & 165 & 13.81 & 2.37 & 21 & 14.13 & 3.07 & 59 \\
\hline Mono-(3-carboxypropyl) Phthalate - MC2 & 2.16 & 0.26 & 73 & 2.24 & 0.19 & 163 & 2.96 & 0.71 & 18 & 2.52 & 0.46 & 55 \\
\hline Mono-ethyl Phthalate -MEP & 67.52 & 10.35 & 75 & 81.93 & 9.22 & 169 & 43.72 & 11.08 & 22 & 50.84 & 15.95 & 60 \\
\hline Mono (2-ethyl-5-hydroxy hexyl) Phthalate - MHH & 8.08 & 0.75 & 75 & 7.02 & 0.64 & 169 & 5.62 & 0.83 & 22 & 7.39 & 0.84 & 60 \\
\hline Mono-(2-ethyl-5-oxohexyl) Phthalate -MOH & 5.67 & 0.52 & 75 & 4.59 & 0.37 & 169 & 4.06 & 0.55 & 22 & 4.79 & 0.59 & 60 \\
\hline Mono-benzyl Phthalate - MZP & 5.29 & 0.95 & 73 & 4.30 & 0.37 & 168 & 2.71 & 0.45 & 22 & 5.03 & 2.21 & 57 \\
\hline Mono-isobutyl Phthalate - MIB & 7.64 & 0.70 & 74 & 7.46 & 0.61 & 165 & 6.74 & 1.27 & 21 & 9.90 & 2.14 & 60 \\
\hline Bisphenol A - BPA & 1.37 & 0.11 & 73 & 1.42 & 0.06 & 163 & 1.70 & 0.66 & 18 & 1.37 & 0.23 & 49 \\
\hline then log tran & $\begin{array}{l}\text { HANES s } \\
\text { formed p }\end{array}$ & npling $\mathrm{v}$ & ight applic & ed before & alculatin & he geom & etric mea & 1 & $\square$ & lev & & were \\
\hline
\end{tabular}




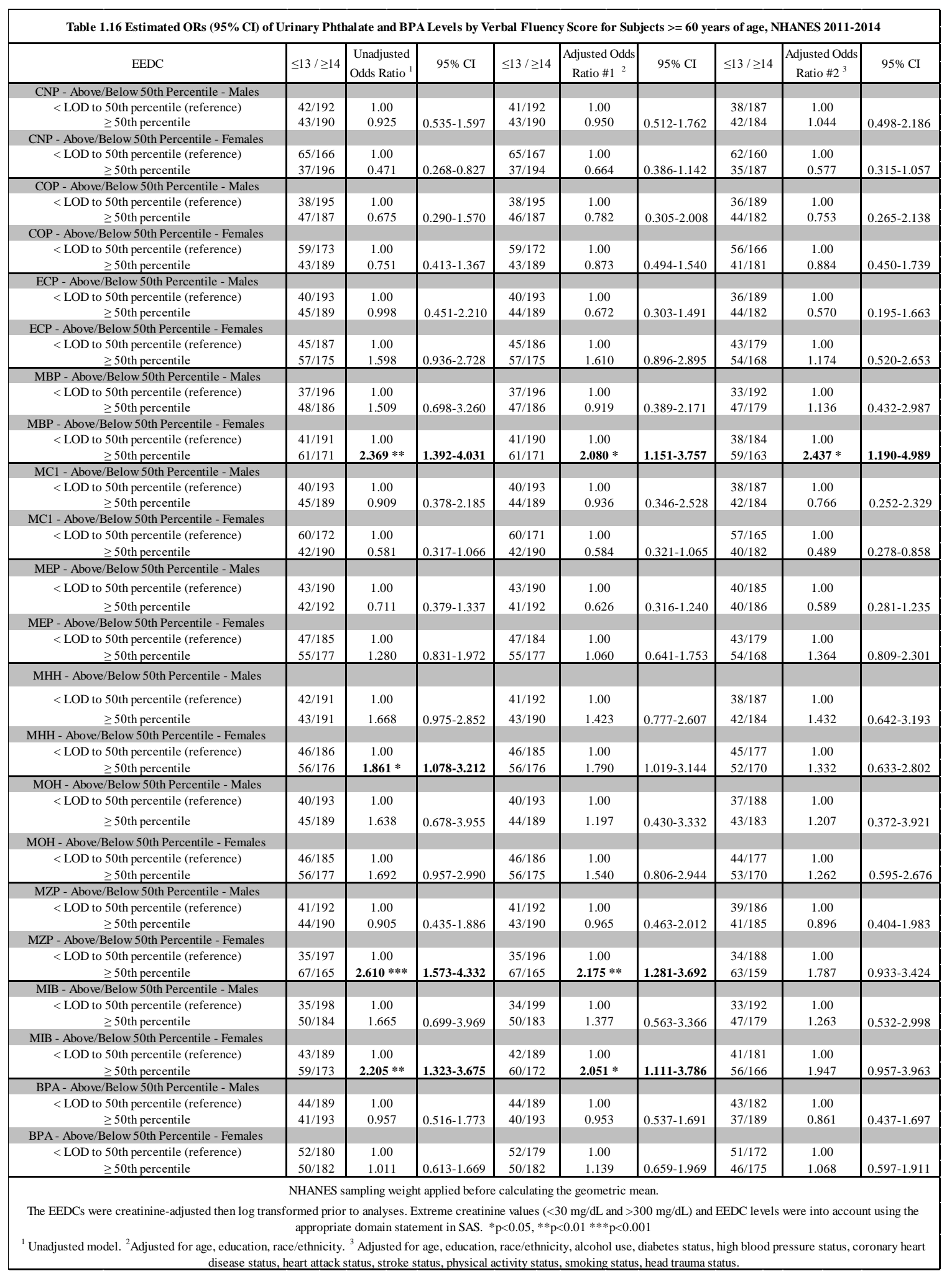




\section{Digit Symbol Substitution Test (DSST) Scores and Exposure to BPA and Phthalates:}

Tables 1.17 to 1.21 present the GMs and GSEs of urinary phthalates and BPA levels among subjects with DSST scores. Table 1.17 presents GMs and GSEs for subjects who have measurable phthalate and BPA levels over the LOD, by DSST cut-off scores. Of the ten phthalates, seven of the phthalates have GMs that were higher in subjects with a DSST score $\leq 27$ than with a DSST score $\geq 28$. These phthalates are ECP, MBP, MEP, MHH, MOH, MZP, and MIB. Furthermore, GMs were significant for ECP $(\mathrm{p}<0.01)$, MBP ( $\mathrm{p}<0.05), \mathrm{MHH}(\mathrm{p}<0.05)$, and MOH $(\mathrm{p}<0.05)$, in subjects with a DSST score $\leq 27$ compared to those with a DSST score $\geq 28$. Table 1.18 gives age-specific GMs and GSEs for phthalate and BPA levels by DSST cut-off scores. In the 60-69-year group, the GM for phthalate MBP is significantly higher in subjects with scores $\leq 27$ compared to subjects who scored $\geq 28$ group $(\mathrm{p}<0.05)$. For the phthalates CNP and COP, they are significantly higher in subjects with scores $\geq 28$ compared to those with scores $\leq 27$ $(\mathrm{p}<0.001$ and $\mathrm{p}<0.01)$. No significant findings were found in the $70-79$ age group. In the $80+$ age group, the concentrations for the phthalate MEP were significantly higher subjects with scores $\leq 27$ compared to those with scores $\geq 28(p<0.05)$ (Table 1.18). Table 1.19 gives gender-specific GM and GSE for phthalate and BPA concentrations by DSST cut-off score. In males, ECP (p<0.01), MHH ( $<<0.05)$, and $\mathrm{MOH}(\mathrm{p}<0.05)$ concentrations are significantly higher in the DSST Score $\leq 27$ group compared to the DSST Score $\geq 28$ group. In females, MBP $(\mathrm{p}<0.05)$ concentrations were significantly higher in the DSST Score $\leq 27$ group compared to the DSST Score $\geq 28$ group. Table 1.20 presents race-specific GMs and GSEs for phthalates and BPA concentrations by DSST cut-off scores. In Hispanics, MEP is significantly higher $(\mathrm{p}<0.05)$ in subjects with 
a score of $\leq 27$ compared to those with a score of $\geq 28$. CNP and COP are significantly higher in subjects with a score of $\geq 28$ compared to those with a score of $\leq 27$ (CNP, $\mathrm{p}<0.05 ; \mathrm{COP}, \mathrm{p}<0.05)$. In Non-Hispanic Whites, ECP, MOH, and MZP are significantly higher in subjects with a score of $\leq 27$ compared to those with a score of $\geq 28$ (ECP, $\mathrm{p}<0.05 ; \mathrm{MOH}, \mathrm{p}<0.05 ; \mathrm{MZP}, \mathrm{p}<0.05)$. In the Asian/others, MEP and MZP are significantly higher in subjects with a score of $\geq 28$ compared to those with a score of $\leq$ 27 (MEP, $\mathrm{p}<0.01$; MZP, $\mathrm{p}<0.05$ ). Table 1.21 presents estimated ORs and 95\% CI of phthalate and BPA concentrations by DSST cut-off scores. For the phthalate ECP, males in the $\geq 50$ th percentile compared to the reference group, ECP concentration was significantly associated with lower DSST scores adjusted model \#2 (OR=2.302, 95\% CI: 1.031-5.141). For MBP, females in the $\geq 50$ th percentile compared to the reference group were found to have MBP levels significantly associated with lower DSST scores $(\mathrm{OR}=2.650,95 \% \mathrm{CI}: 1.342-5.236)$. MBP in females was not significant in the adjusted models. For $\mathrm{MOH}$, males in the $\geq 50$ th percentile compared to the reference group were found to have $\mathrm{MOH}$ concentrations significantly associated with lower DSST scores in adjusted model \#2 (OR=2.132, 95\% CI: 1.019-4.462). For MZP, females in the $\geq 50$ th percentile compared to the reference group were found to have MZP concentrations that were associated with lower DSST scores in the unadjusted model (OR=1.763, 95\% CI: 1.003-3.099). Subsequent adjusted models were not significant. 


\begin{tabular}{|c|c|c|c|c|c|c|}
\hline \multicolumn{7}{|c|}{$\begin{array}{l}\text { Table 1.17 Geometric Mean Urinary Phthalate and BPA Levels by Digit Symbol Substitution Test (DSST) } \\
\text { Cut-Off Scores for Subjects >= } 60 \text { years of age, NHANES 2011-2014, over the LOD }\end{array}$} \\
\hline \multirow{3}{*}{ EEDC } & \multicolumn{6}{|c|}{ Geometric Mean (ng/ml) (GSE, N) } \\
\hline & \multicolumn{3}{|c|}{ DSST Score $\leq 27$} & \multicolumn{3}{|c|}{ DSST Score $\geq 28$} \\
\hline & GM & GSE & $\mathbf{N}$ & GM & GSE & $\mathbf{N}$ \\
\hline Mono (carboxynonyl) Phthalate - CNP & 2.14 & 0.22 & 128 & 2.67 & 0.14 & 759 \\
\hline Mono (carboxyoctyl) Phthalate - COP & 15.48 & 1.67 & 129 & 19.00 & 1.58 & 761 \\
\hline Mono-(2-ethyl-5-carboxypentyl) Phthalate -ECP & 15.86 & 1.65 & $129 * *$ & 11.59 & 0.56 & 760 \\
\hline Mono-n-butyl Phthalate - MBP & 12.55 & 1.09 & $127 *$ & 9.63 & 0.54 & 749 \\
\hline Mono-(3-carboxypropyl) Phthalate - MC1 & 2.69 & 0.36 & 120 & 2.70 & 0.19 & 727 \\
\hline Mono-ethyl Phthalate -MEP & 71.91 & 13.31 & 129 & 53.51 & 4.57 & 762 \\
\hline Mono (2-ethyl-5-hydroxy hexyl) Phthalate - MHH & 9.11 & 0.91 & $129 *$ & 7.05 & 0.32 & 761 \\
\hline Mono-(2-ethyl-5-oxohexyl) Phthalate -MOH & 6.31 & 0.39 & $129 *$ & 4.74 & 0.22 & 760 \\
\hline Mono-benzyl Phthalate - MZP & 4.74 & 0.60 & 129 & 4.03 & 0.21 & 750 \\
\hline Mono-isobutyl Phthalate - MIB & 6.89 & 0.61 & 127 & 6.14 & 0.27 & 755 \\
\hline Bisphenol A - BPA & 1.62 & 0.17 & 121 & 1.52 & 0.08 & 720 \\
\hline \multicolumn{7}{|c|}{$\begin{array}{l}\text { NHANES sampling weight applied before calculating the geometric mean. } \\
\text { The EEDCs were creatinine-adjusted then log transformed prior to analyses. Extreme creatinine values }(<30 \mathrm{mg} / \mathrm{dL} \\
\text { and }>300 \mathrm{mg} / \mathrm{dL}) \text { and EEDC levels below the LOD were taken into account using the appropriate domain statement in } \\
\text { SAS. } * \mathrm{p}<0.05, * * \mathrm{p}<0.01 * * \mathrm{p}<0.001\end{array}$} \\
\hline
\end{tabular}

\begin{tabular}{|c|c|c|c|c|c|c|c|c|c|c|c|c|c|c|c|c|c|c|}
\hline \multicolumn{19}{|c|}{ Table 1.18 Age-Specific Geometric Mean Urinary Phthalate and BPALevels by Digit Symbol Substitution (DSST) Cut-Off Scores for Subjects >= 60 years of age, NHANES 2011-2014, over the LOD } \\
\hline \multirow{3}{*}{ EEDC } & \multicolumn{6}{|c|}{ Geometric Mean (ng/ml) (GSE, N), Age 60-69 } & \multicolumn{6}{|c|}{ Geometric Mean (ng/ml) (GSE, N), Age 70-79 } & \multicolumn{6}{|c|}{ Geometric Mean (ng/ml) (GSE, N), Age $80+$} \\
\hline & \multicolumn{3}{|c|}{ DSSTSCore $\leq 27$} & \multicolumn{3}{|c|}{ DSST Score $\geq 28$} & \multicolumn{3}{|c|}{ DSST Score $\leq 27$} & \multicolumn{3}{|c|}{ DSST Score $\geq 28$} & \multicolumn{3}{|c|}{ DSSTScore $\leq 27$} & \multicolumn{3}{|c|}{ DSST Score $\geq 28$} \\
\hline & GM & GSE & $\mathrm{N}$ & GM & GSE & $\mathrm{N}$ & GM & GSE & $\mathrm{N}$ & GM & GSE & $\mathrm{N}$ & GM & GSE & $\mathrm{N}$ & GM & GSE & $\mathrm{N}$ \\
\hline Mono (carboxynonyl) Phthalate - CNP & 1.62 & 0.13 & 52 & 2.56 & 0.18 & $428^{4 \text { 䋨 }}$ & 2.41 & 0.31 & 40 & 2.95 & 0.28 & 228 & 2.42 & 0.44 & 36 & 2.45 & 0.19 & 103 \\
\hline Mono (carboxyoctyl) Phthalate - COP & 11.80 & 1.66 & 53 & 18.27 & 2.07 & 428 蜼 & 20.21 & 3.56 & 40 & 22.08 & 2.27 & 230 & 15.66 & 2.59 & 36 & 15.32 & 1.79 & 103 \\
\hline Mono-(2-ethyl-5-carboxypenty)) Phthalate - ECP & 14.90 & 2.45 & 53 & 10.59 & 0.75 & 427 & 16.35 & 3.00 & 40 & 13.35 & 0.72 & 230 & 16.26 & 2.37 & 36 & 11.90 & 0.71 & 103 \\
\hline Mono-n-butyl Phthalate - MBP & 12.83 & 1.58 & $51^{*}$ & 9.10 & 0.61 & 422 & 11.94 & 2.02 & 40 & 10.02 & 0.85 & 227 & 12.81 & 1.97 & 36 & 11.22 & 1.32 & 100 \\
\hline Mono-(3-carboxypropyl) Phthalate - $\mathrm{MCl}$ & 2.06 & 0.36 & 47 & 2.50 & 0.21 & 409 & 3.12 & 0.91 & 37 & 3.09 & 0.32 & 220 & 2.89 & 0.38 & 36 & 2.68 & 0.33 & 98 \\
\hline Mono-ethyl Phthalate -MEP & 91.95 & 19.23 & 53 & 58.27 & 6.55 & 429 & 77.66 & 24.26 & 40 & 56.85 & 6.79 & 230 & 56.43 & 12.84 & $36 *$ & 31.55 & 2.83 & 103 \\
\hline Mono (2-ethyl-5-hydroxy hexyl) Phthalate - МHH & 8.77 & 1.44 & 53 & 6.56 & 0.46 & 428 & 10.20 & 2.39 & 40 & 8.11 & 0.45 & 230 & 8.63 & 0.89 & 36 & 6.69 & 0.53 & 103 \\
\hline Mono-(2-ethyl-5-oxohexyl) Phthalate -MOH & 5.65 & 0.97 & 53 & 4.27 & 0.29 & 428 & 6.99 & 1.57 & 40 & 5.53 & 0.27 & 230 & 6.36 & 0.64 & 36 & 5.04 & 0.42 & 102 \\
\hline Mono-benzyl Phthalate - MZP & 5.18 & 1.37 & 53 & 3.98 & 0.31 & 424 & 4.78 & 0.91 & 40 & 3.80 & 0.29 & 227 & 4.42 & 0.75 & 36 & 4.97 & 0.49 & 99 \\
\hline Mono-isobutyl Phthalate - MIB & 8.21 & 1.07 & 52 & 6.37 & 0.37 & 427 & 7.42 & 0.96 & 40 & 6.14 & 0.36 & 226 & 5.70 & 0.91 & 35 & 5.25 & 0.49 & 102 \\
\hline Bisphenol A-BPA & 1.71 & 0.21 & 51 & 1.48 & 0.10 & 408 & 1.71 & 0.36 & 36 & 1.66 & 0.11 & 215 & 1.50 & 0.29 & 34 & 1.39 & 0.16 & 97 \\
\hline EEDCs were creatinine-adjusted then log transfo & 1 & & $\mathrm{NHA}$ & NES sam & ling weig & $\begin{array}{l}\text { Iht applied be } \\
0 \mathrm{mg} / \mathrm{dL} \text { and } \\
0.05, *{ }^{*} \mathrm{p}<0\end{array}$ & efore cal & ulating the & cometr & cmean. & & & & & & & & \\
\hline
\end{tabular}




\begin{tabular}{|c|c|c|c|c|c|c|c|c|c|c|c|c|}
\hline \multicolumn{13}{|c|}{$\begin{array}{l}\text { Table 1.19 Geometric Mean Urinary Phthalate and BPA Levels by Digit Symbol Substitution (DSST) Cut-Off Scores for Subjects >=60 years of age, NHANES 2011- } \\
2014 \text {, over the LOD }\end{array}$} \\
\hline \multirow{4}{*}{ EEDC } & \multicolumn{6}{|c|}{ Geometric Mean $(\mathrm{ng} / \mathrm{ml})(\mathrm{GSE}, \mathrm{N})$} & \multicolumn{6}{|c|}{ Geometric Mean (ng/ml) (GSE, N) } \\
\hline & \multicolumn{6}{|c|}{ Male } & \multicolumn{6}{|c|}{ Female } \\
\hline & \multicolumn{3}{|c|}{ DSST Score $\leq 27$} & \multicolumn{3}{|c|}{ DSST Score $\geq 28$} & \multicolumn{3}{|c|}{ DSST Score $\leq 27$} & \multicolumn{3}{|c|}{ DSST Score $\geq 28$} \\
\hline & GM & GSE & $\mathbf{N}$ & GM & GSE & $\mathbf{N}$ & GM & GSE & $\mathbf{N}$ & GM & GSE & $\mathbf{N}$ \\
\hline Mono (carboxynonyl) Phthalate - CNP & 1.95 & 0.23 & 69 & 2.38 & 0.13 & 384 & 2.31 & 0.30 & 59 & 2.98 & 0.27 & 375 \\
\hline Mono (carboxyoctyl) Phthalate - COP & 13.79 & 2.22 & 69 & 16.71 & 1.233 & 86 & 16.94 & 2.38 & 60 & 21.56 & 2.83 & 375 \\
\hline Mono-(2-ethyl-5-carboxypentyl) Phthalate -ECP & 15.28 & 1.95 & $69 * *$ & 10.50 & 0.50 & 387 & 16.32 & 2.40 & 60 & 12.78 & 0.83 & 373 \\
\hline Mono-n-butyl Phthalate - MBP & 10.29 & 1.41 & 68 & 9.23 & 0.71 & 378 & 14.67 & 1.57 & $59 *$ & 10.05 & 0.59 & 371 \\
\hline Mono-(3-carboxypropyl) Phthalate - MC1 & 2.67 & 0.52 & 64 & 2.50 & 0.20 & 372 & 2.70 & 0.35 & 56 & 2.92 & 0.29 & 355 \\
\hline Mono-ethyl Phthalate -MEP & 63.90 & 14.22 & 69 & 51.25 & 5.44 & 387 & 78.84 & 19.27 & 60 & 55.84 & 6.21 & 375 \\
\hline Mono (2-ethyl-5-hydroxy hexyl) Phthalate - MHH & 8.69 & 1.10 & $69 *$ & 6.66 & 0.36 & 387 & 9.45 & 1.24 & 60 & 7.44 & 0.42 & 374 \\
\hline Mono-(2-ethyl-5-oxohexyl) Phthalate -MOH & 6.01 & 0.75 & $69 *$ & 4.48 & 0.23 & 385 & 6.54 & 0.89 & 60 & 5.02 & 0.30 & 375 \\
\hline Mono-benzyl Phthalate - MZP & 3.88 & 0.51 & 69 & 3.58 & 0.29 & 380 & 5.55 & 1.01 & 60 & 4.53 & 0.25 & 370 \\
\hline Mono-isobutyl Phthalate - MIB & 6.01 & 0.75 & 68 & 4.48 & 0.23 & 384 & 6.54 & 0.89 & 59 & 5.02 & 0.30 & 371 \\
\hline Bisphenol A-BPA & 1.66 & 0.17 & 65 & 1.36 & 0.07 & 366 & 1.59 & 0.23 & 56 & 1.71 & 0.11 & 354 \\
\hline $\begin{array}{r}\text { The EEDCs were creatinine-adjusted then log transfor } \\
\text { into accoun }\end{array}$ & ES sam & ng weig & applied & fore ca & ulating th & reome & mean. & $<0.001$ & $\mathrm{DC}$ & dent & 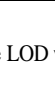 & \\
\hline
\end{tabular}

\begin{tabular}{|c|c|c|c|c|c|c|c|c|c|c|c|c|}
\hline \multirow{3}{*}{ EEDC } & \multicolumn{6}{|c|}{ Geometric Mean (ng/ml) (GSE, N), Hispanic } & \multicolumn{6}{|c|}{ Geometric Mean (ng/ml) (GSE, N), Non-Hispanic White } \\
\hline & \multicolumn{3}{|c|}{ Animal Fluency Score $\leq 11$} & \multicolumn{3}{|c|}{ Animal Fluency Score $\geq 12$} & \multicolumn{3}{|c|}{ Animal Fluency Score $\leq 11$} & \multicolumn{3}{|c|}{ Animal Fluency Score $\geq 12$} \\
\hline & GM & GSE & $\mathbf{N}$ & GM & GSE & $\mathbf{N}$ & GM & GSE & $\mathbf{N}$ & GM & GSE & $\mathbf{N}$ \\
\hline Mono (carboxynonyl) Phthalate - CNP & 1.89 & 0.16 & 49 & 2.49 & 0.18 & $129 *$ & 2.77 & 0.48 & 23 & 2.77 & 0.17 & 377 \\
\hline Mono (carboxyoctyl) Phthalate - COP & 17.64 & 2.56 & 49 & 20.22 & 2.08 & $129 *$ & 17.77 & 3.28 & 23 & 19.67 & 1.91 & 377 \\
\hline Mono-(2-ethyl-5-carboxypentyl) Phthalate -ECP & 20.06 & 2.68 & 49 & 16.34 & 1.01 & 129 & 19.10 & 3.50 & $23 *$ & 11.33 & 0.61 & 376 \\
\hline Mono-n-butyl Phthalate - MBP & 13.68 & 1.29 & 49 & 13.80 & 1.18 & 127 & 12.98 & 2.48 & 23 & 9.10 & 0.60 & 370 \\
\hline Mono-(3-carboxypropyl) Phthalate - MC1 & 2.53 & 0.38 & 44 & 2.85 & 0.33 & 124 & 3.82 & 0.78 & 23 & 2.72 & 0.23 & 360 \\
\hline Mono-ethyl Phthalate -MEP & 169.71 & 38.53 & $49 * *$ & 83.07 & 9.44 & 129 & 48.78 & 11.50 & 23 & 49.43 & 4.83 & 377 \\
\hline Mono (2-ethyl-5-hydroxy hexyl) Phthalate - MHH & 11.70 & 2.01 & 49 & 9.66 & 0.69 & 129 & 10.02 & 1.80 & 23 & 6.86 & 0.35 & 376 \\
\hline Mono-(2-ethyl-5-oxohexyl) Phthalate -MOH & 7.22 & 1.20 & 49 & 6.18 & 0.44 & 129 & 7.65 & 1.33 & $23 *$ & 4.65 & 0.24 & 375 \\
\hline Mono-benzyl Phthalate - MZP & 3.94 & 0.68 & 49 & 3.69 & 0.26 & 129 & 6.71 & 1.71 & $23 *$ & 3.97 & 0.22 & 270 \\
\hline Mono-isobutyl Phthalate - MIB & 10.00 & 0.80 & 49 & 8.84 & 0.57 & 129 & 4.58 & 0.80 & 23 & 5.73 & 0.25 & 373 \\
\hline Bisphenol A - BPA & 1.75 & 0.18 & 43 & 1.53 & 0.15 & 127 & 1.66 & 0.42 & 22 & 1.54 & 0.09 & 359 \\
\hline \multirow{3}{*}{ EEDC } & \multicolumn{6}{|c|}{ Geometric Mean (ng/ml) (GSE, N), Non-Hispanic Black } & \multicolumn{6}{|c|}{ Geometric Mean (ng/ml) (GSE, N), Asian/Other } \\
\hline & \multicolumn{3}{|c|}{ Animal Fluency Score $\leq 11$} & \multicolumn{3}{|c|}{ Animal Fluency Score $\geq 12$} & \multicolumn{3}{|c|}{ Animal Fluency Score $\leq 11$} & \multicolumn{3}{|c|}{ Animal Fluency Score $\geq 12$} \\
\hline & GM & GSE & $\mathbf{N}$ & GM & GSE & $\mathbf{N}$ & GM & GSE & $\mathbf{N}$ & GM & GSE & $\mathbf{N}$ \\
\hline Mono (carboxynonyl) Phthalate - CNP & 1.81 & 0.14 & 48 & 1.96 & 0.12 & 182 & 1.92 & 0.59 & 8 & 2.35 & 0.38 & 71 \\
\hline Mono (carboxyoctyl) Phthalate - COP & 12.98 & 2.20 & 48 & 14.70 & 1.16 & 181 & 8.60 & 3.00 & 9 & 14.35 & 2.98 & 74 \\
\hline Mono-(2-ethyl-5-carboxypentyl) Phthalate -ECP & 9.96 & 1.09 & 48 & 10.62 & 0.81 & 181 & 13.12 & 4.13 & 9 & 13.14 & 0.76 & 74 \\
\hline Mono-n-butyl Phthalate - MBP & 9.91 & 1.34 & 47 & 11.12 & 0.69 & 179 & 18.37 & 4.63 & 8 & 13.69 & 2.69 & 73 \\
\hline Mono-(3-carboxypropyl) Phthalate - MC2 & 1.94 & 0.21 & 45 & 2.25 & 0.18 & 177 & 1.80 & 0.58 & 8 & 2.92 & 0.43 & 66 \\
\hline Mono-ethyl Phthalate -MEP & 64.41 & 17.59 & 48 & 84.59 & 8.39 & 182 & 17.26 & 6.05 & 9 & 60.95 & 16.85 & $74 * *$ \\
\hline Mono (2-ethyl-5-hydroxy hexyl) Phthalate - MHH & 6.32 & 0.82 & 48 & 7.30 & 0.58 & 182 & 7.54 & 2.88 & 9 & 7.19 & 0.74 & 74 \\
\hline Mono-(2-ethyl-5-oxohexyl) Phthalate -MOH & 4.45 & 0.61 & 48 & 4.82 & 0.33 & 182 & 5.00 & 1.81 & 9 & 4.73 & 0.47 & 74 \\
\hline Mono-benzyl Phthalate - MZP & 4.78 & 0.96 & 48 & 4.57 & 0.42 & 180 & 1.89 & 0.49 & 9 & 4.92 & 1.81 & $71 *$ \\
\hline Mono-isobutyl Phthalate - MIB & 7.93 & 0.84 & 46 & 7.49 & 0.47 & 180 & 6.09 & 1.61 & 9 & 9.86 & 1.79 & 73 \\
\hline Bisphenol A - BPA & 1.53 & 0.17 & 47 & 1.39 & 0.06 & 175 & 1.38 & 0.46 & 9 & 1.43 & 0.23 & 59 \\
\hline int & INES sam & ing weig & applied b & efore cal & lating th & geometri & ic mean. & $<0.001$ & & & & \\
\hline
\end{tabular}




\begin{tabular}{|c|c|c|c|c|c|c|c|c|c|}
\hline EEDC & $\leq \mathbf{2 7} / \geq \mathbf{2 8}$ & $\begin{array}{c}\text { Unadjusted } \\
\text { Odds Ratio }^{1}\end{array}$ & $95 \% \mathrm{CI}$ & $\leq \mathbf{2 7} / \geq \mathbf{2 8}$ & $\begin{array}{c}\text { Adjusted Odds } \\
\text { Ratio \#1 }{ }^{2}\end{array}$ & $95 \%$ CI & $\leq 27 / \geq 28$ & $\begin{array}{c}\text { Adjusted Odds } \\
{\text { Ratio } \# 2^{3}}^{3}\end{array}$ & $95 \% \mathrm{CI}$ \\
\hline \multicolumn{10}{|l|}{ CNP - Above/Below 50th Percentile - Males } \\
\hline$<$ LOD to 50th percentile (reference) & $38 / 190$ & 1.00 & \multirow{3}{*}{$0.436-1.337$} & $37 / 190$ & 1.00 & \multirow{3}{*}{$0.487-1.767$} & $36 / 186$ & 1.00 & \multirow[b]{2}{*}{$0.639-2.750$} \\
\hline$\geq 50$ th percentile & $31 / 197$ & 0.764 & & $31 / 197$ & 0.928 & & $30 / 191$ & 1.326 & \\
\hline \multicolumn{8}{|l|}{ CNP - Above/Below 50th Percentile - Females } & & \\
\hline$<$ LOD to 50th percentile (reference) & $37 / 180$ & 1.00 & \multirow[b]{2}{*}{$0.209-0.789$} & $38 / 179$ & 1.00 & \multirow[b]{2}{*}{$0.214-1.061$} & $33 / 177$ & 1.00 & \multirow[b]{2}{*}{$0.248-1.300$} \\
\hline$\geq 50$ th percentile & $23 / 195$ & 0.406 & & $22 / 195$ & 0.477 & & $22 / 187$ & 0.568 & \\
\hline \multicolumn{10}{|l|}{ COP - Above/Below 50th Percentile - Males } \\
\hline$<$ LOD to 50th percentile (reference) & $39 / 189$ & 1.00 & & $39 / 188$ & 1.00 & & $37 / 184$ & 1.00 & \\
\hline$\geq 50$ th percentile & $30 / 198$ & 0.704 & $0.342-1.452$ & 29/199 & 0.687 & $0.318-1.484$ & $29 / 193$ & 0.889 & $0.386-2.046$ \\
\hline COP - Above/Below 50th Percentile - Femal & & & & & & & & & \\
\hline$<$ LOD to 50th percentile (reference) & $29 / 188$ & 1.00 & & $29 / 188$ & 1.00 & & $25 / 184$ & 1.00 & \\
\hline$\geq 50$ th percentile & $31 / 187$ & 0.827 & $0.440-1.555$ & $31 / 186$ & 1.029 & $0.543-1.953$ & $30 / 180$ & 1.087 & $0.495-2.388$ \\
\hline ECP - Above/Below 50th Percentile - Male & & & & & & & & & \\
\hline$<$ LOD to 50th percentile (reference) & $26 / 202$ & 1.00 & & $26 / 201$ & 1.00 & & $24 / 197$ & 1.00 & \\
\hline$\geq 50$ th percentile & $43 / 185$ & 2.095 & $0.965-4.547$ & $42 / 186$ & 1.593 & $0.621-4.088$ & $42 / 180$ & $2.302 *$ & $1.031-5.141$ \\
\hline ECP - Above/Below 50th Percentile - Femal & & & & & & & & & \\
\hline$<$ LOD to 50th percentile (reference) & $27 / 190$ & 1.00 & & $27 / 190$ & 1.00 & & $24 / 185$ & 1.00 & \\
\hline$\geq 50$ th percentile & $33 / 185$ & 1.539 & $0.662-3.579$ & $33 / 184$ & 1.295 & $0.469-3.578$ & $31 / 179$ & 1.118 & $0.359-3.485$ \\
\hline MBP - Above/Below 50th Percentile - Male & & & & & & & & & \\
\hline$<$ LOD to 50th percentile (reference) & $31 / 197$ & 1.00 & & $30 / 197$ & 1.00 & & $29 / 192$ & 1.00 & \\
\hline$\geq 50$ th percentile & $38 / 190$ & 1.608 & $0.732-3.532$ & $38 / 190$ & 0.902 & $0.388-2.098$ & $37 / 185$ & 0.839 & $0.366-1.924$ \\
\hline MBP - Above/Below 50th Percentile - Femal & & & & & & & & & \\
\hline$<$ LOD to 50th percentile (reference) & $25 / 192$ & 1.00 & & $25 / 192$ & 1.00 & & $23 / 186$ & 1.00 & \\
\hline$\geq 50$ th percentile & $35 / 183$ & $2.650 * *$ & $1.342-5.236$ & $35 / 182$ & 2.106 & $0.973-4.555$ & $32 / 178$ & 2.366 & $0.824-6.790$ \\
\hline MC1 - Above/Below 50th Percentile - Male & & & & & & & & & \\
\hline$<$ LOD to 50th percentile (reference) & $37 / 191$ & 1.00 & & $37 / 190$ & 1.00 & & $36 / 185$ & 1.00 & \\
\hline$\geq 50$ th percentile & $32 / 196$ & 0.949 & $0.382-2.359$ & $31 / 197$ & 1.252 & $0.571-2.744$ & $30 / 192$ & 1.197 & $0.518-2.763$ \\
\hline MC1 - Above/Below 50th Percentile - Femal & & & & & & & & & \\
\hline$<$ LOD to 50th percentile (reference) & $33 / 184$ & 1.00 & & $33 / 184$ & 1.00 & & $29 / 180$ & 1.00 & \\
\hline$\geq 50$ th percentile & $27 / 191$ & 0.706 & $0.327-1.523$ & $27 / 190$ & 0.723 & $0.269-1.941$ & $26 / 184$ & 0.608 & $0.270-1.367$ \\
\hline MEP - Above/Below 50th Percentile - Male & & & & & & & & & \\
\hline$<$ LOD to 50th percentile (reference) & $27 / 201$ & 1.00 & & $27 / 200$ & 1.00 & & $26 / 195$ & 1.00 & \\
\hline$\geq 50$ th percentile & $42 / 186$ & 1.329 & $0.622-2.842$ & $41 / 187$ & 1.071 & $0.458-2.508$ & $40 / 182$ & 0.956 & $0.416-2.193$ \\
\hline MEP - Above/Below 50th Percentile - Femal & & & & & & & & & \\
\hline$<$ LOD to 50th percentile (reference) & $27 / 190$ & 1.00 & & $27 / 190$ & 1.00 & & $24 / 185$ & 1.00 & \\
\hline$\geq 50$ th percentile & $33 / 185$ & 1.246 & $0.599-2.591$ & $33 / 184$ & 1.121 & $0.466-2.698$ & $31 / 179$ & 2.504 & $0.725-8.643$ \\
\hline MHH - Above/Below 50th Percentile - Mal & & & & & & & & & \\
\hline$<$ LOD to 50th percentile (reference) & $30 / 198$ & 1.00 & & $29 / 198$ & 1.00 & & $27 / 194$ & 1.00 & \\
\hline$\geq 50$ th percentile & $39 / 189$ & 1.353 & $0.727-2.517$ & $39 / 189$ & 1.012 & $0.500-2.048$ & $39 / 183$ & 1.538 & $0.658-3.596$ \\
\hline MHH - Above/Below 50th Percentile - Fema & & & & & & & & & \\
\hline$<$ LOD to 50th percentile (reference) & $30 / 187$ & 1.00 & & $30 / 187$ & 1.00 & & $27 / 182$ & 1.00 & \\
\hline$\geq 50$ th percentile & $30 / 188$ & 1.559 & $0.778-3.125$ & $30 / 187$ & 1.266 & $0.646-2.481$ & $28 / 182$ & 1.150 & $0.479-2.760$ \\
\hline MOH - Above/Below 50th Percentile - Mal & & & & & & & & & \\
\hline$<$ LOD to 50th percentile (reference) & $28 / 200$ & 1.00 & & $27 / 200$ & 1.00 & & $26 / 195$ & 1.00 & \\
\hline$\geq 50$ th percentile & $41 / 187$ & 1.840 & $0.932-3.635$ & $41 / 187$ & 1.444 & $0.730-2.856$ & $40 / 182$ & $2.132 *$ & $1.019-4.462$ \\
\hline MOH - Above/Below 50th Percentile - Fema & & & & & & & & & \\
\hline$<$ LOD to 50th percentile (reference) & $27 / 190$ & 1.00 & & $27 / 190$ & 1.00 & & $24 / 185$ & 1.00 & \\
\hline$\geq 50$ th percentile & $33 / 185$ & 1.880 & $0.901-3.923$ & $33 / 184$ & 1.533 & $0.739-3.182$ & $31 / 179$ & 1.359 & $0.526-3.509$ \\
\hline MZP - Above/Below 50th Percentile - Male & & & & & & & & & \\
\hline$<$ LOD to 50th percentile (reference) & $30 / 198$ & 1.00 & & $30 / 197$ & 1.00 & & $29 / 192$ & 1.00 & \\
\hline$\geq 50$ th percentile & $39 / 189$ & 1.230 & $0.628-2.409$ & $38 / 190$ & 0.955 & $0.471-1.934$ & $37 / 185$ & 0.960 & $0.519-1.775$ \\
\hline MZP - Above/Below 50th Percentile - Femal & & & & & & & & & \\
\hline$<$ LOD to 50th percentile (reference) & $25 / 192$ & 1.00 & & $25 / 192$ & 1.00 & & $23 / 186$ & 1.00 & \\
\hline$\geq 50$ th percentile & $35 / 183$ & $1.763 *$ & $1.003-3.099$ & $35 / 182$ & 1.356 & $0.722-2.544$ & $32 / 178$ & 1.148 & $0.441-2.985$ \\
\hline MIB - Above/Below 50th Percentile - Male & & & & & & & & & \\
\hline$<$ LOD to 50th percentile (reference) & $29 / 199$ & 1.00 & & 28/199 & 1.00 & & $26 / 195$ & 1.00 & \\
\hline$\geq 50$ th percentile & $40 / 188$ & 1.281 & $0.733-2.239$ & $40 / 188$ & 0.789 & $0.474-1.315$ & $40 / 182$ & 1.073 & $0.554-2.079$ \\
\hline MIB - Above/Below 50th Percentile - Femal & & & & & & & & & \\
\hline$<$ LOD to 50th percentile (reference) & $25 / 192$ & 1.00 & & $25 / 192$ & 1.00 & & $23 / 186$ & 1.00 & \\
\hline$\geq 50$ th percentile & $35 / 183$ & 1.421 & $0.825-2.446$ & $35 / 182$ & 0.882 & $0.469-1.658$ & $32 / 178$ & 1.234 & $0.501-3.037$ \\
\hline BPA - Above/Below 50th Percentile - Male & & & & & & & & & \\
\hline$<$ LOD to 50th percentile (reference) & $30 / 198$ & 1.00 & & $30 / 198$ & 1.00 & & $30 / 191$ & 1.00 & $0.515-1.885$ \\
\hline$\geq 50$ th percentile & $39 / 189$ & 1.069 & $0.573-1.996$ & $38 / 189$ & 1.111 & $0.523-2.362$ & $36 / 186$ & 0.885 & $0.444-1.763$ \\
\hline BPA- Above/Below 50th Percentile - Femal & & & & & & & & & \\
\hline$<$ LOD to 50th percentile (reference) & $30 / 187$ & 1.00 & & $30 / 187$ & 1.00 & & $28 / 181$ & 1.00 & \\
\hline$\geq 50$ th percentile & $30 / 188$ & 0.820 & $0.397-1.692$ & $30 / 187$ & 0.810 & $0.333-1.966$ & $27 / 183$ & 0.446 & $0.193-1.027$ \\
\hline & NHANE & sampling weigl & ht applied befor & calculating & the geometric & & & & \\
\hline The EEDCs were creatinine-adjusted then log tr & & $\begin{array}{l}\text { nalyses. Extren } \\
\text { main statement }\end{array}$ & $\begin{array}{l}\text { ne creatinine va } \\
\text { tin SAS. }{ }^{*} p<0\end{array}$ & $\begin{array}{l}\text { lues }(<30 \mathrm{~ms} \\
05, * * \mathrm{p}<0.0\end{array}$ & $1 * * * p<0.001$ & $/ \mathrm{dL})$ and $\mathrm{EED}$ & vels $\mathrm{w}$ & Hertite & he appropriate \\
\hline${ }^{1}$ Unadjusted model. ${ }^{2}$ Adjusted for age, educati & t attack & Adjusted ror a & $\begin{array}{l}\text { e, education, ra } \\
\text { us, physical acti }\end{array}$ & $\begin{array}{l}\text { ce/ethnicity, } \\
\text { vity status, si }\end{array}$ & moking status, hea & $\begin{array}{l}\text { etes status, hi } \\
\text { ad trauma stat }\end{array}$ & $\operatorname{lood} \mathrm{p}$ & tatus, coror & heart disease \\
\hline
\end{tabular}




\section{Associations between Exposures to Phthalate and BPA and Memory Function}

Exposures to the 12 phthalate metabolites and BPA and three memory function indicators: 1) During the past 12 months, have you experienced confusion or memory loss that is happening more often or getting worse?; 2) During the past 7 days, how often have you had trouble remembering where you put things?; 3) Are you limited in any way because of difficulty remembering or because you experience periods of confusion?) summarized in tables 1.22 to 1.36 . Memory function have been included as a surrogate indicator of brain health as declining memory function can indicate the development of mild cognitive impairment, dementia, $\mathrm{AD}$, or other memory-related neurodegenerative disease.

\section{Worsening memory past 12 months and Exposure to BPA and Phthalates: Table}

1.22 presents crude GMs and GSEs for subjects with phthalates and BPA concentrations over the LOD, by responses to the question, "During the past 12 months, have you experience confusion or memory loss that is happening more often or getting worse?". The GMs of three of the phthalate metabolites, MBP, MZP, and MIB, were found to be significantly higher in those who responded "yes" to experiencing confusion or memory loss in the past year than those who responded "no" (MBP, MZP, MIB, p<0.05).

Table 1.23 presents age-specific GMs and GSEs for subjects with phthalates and BPA concentrations over the LOD who responded to having memory loss or confusion over the past year. In the 60-69-year age group, three phthalate metabolites, $\mathrm{MHH}, \mathrm{MOH}$, MIB, were found to have GMs significantly higher $(\mathrm{p}<0.05)$ in subjects who answered "yes" versus those who answered "no". In the 70-79-year age group, the phthalate metabolite, COP, was found to have a significantly higher GM in the "no" group 
compared to the "yes" group $(\mathrm{p}<0.05)$. In the $80+$ age group, the phthalate metabolite, MIB, was found to have a significantly higher GM in the "yes" group compared to the “no" group $(\mathrm{p}<0.05)$.

Table 1.24 presents gender-specific GMs and GSEs for subjects with phthalate and BPA concentrations over the LOD who responded having memory loss or confusion over the past year. Among females, the phthalate metabolites MZP and MIB had significantly higher GMs in those who experienced memory issues in the past year, than those who did not $(\mathrm{p}<0.05)$.

Table 1.25 presents race-specific GMS and GSEs for subjects with phthalates and BPA concentrations over the LOD who to having memory loss or confusion over the past year. In the Non-Hispanic White group, the phthalate metabolite MIB was found to have a higher GM among the "yes" group compared to the "no" group $(\mathrm{p}<0.05)$. No other significant observations were observed.

Table 1.26 presents estimated ORs and 95\% CIs for phthalate metabolites and BPA concentrations by responses to experiencing memory and confusion problems the last 12 months. For the phthalate metabolite MBP, concentrations among males in the $\geq$ 50th percentile compared to the reference group, were found to be significantly associated with experiencing memory loss or confusion over the past year in the unadjusted model (OR=2.844, 95\% CI: $1.170-6.912)$ and the $1^{\text {st }}$ adjusted model $(\mathrm{OR}=3.184,95 \%$ CI: 1.081-9.381). For the phthalate metabolite MZP, among females in the $\geq$ 50th percentile group compared to the reference group, MZP concentrations were found to be significantly associated with experiencing memory loss or confusion over the past year in the unadjusted model $(\mathrm{OR}=2.277,95 \% \mathrm{CI} 1.183-4.383)$ and the $1^{\text {st }}$ adjusted 
model (OR=2.214, 95\% CI: 1.055-4.647). For the phthalate metabolite MIB among females in the $\geq 50$ th percentile group compared to the reference group, concentrations were found to be significantly associated with experiencing memory loss or confusion over the past year in the $2^{\text {nd }}$ adjusted model (OR=2.180, 95\% CI: $\left.1.137-1.332\right)$. 


\begin{tabular}{|c|c|c|c|c|c|c|}
\hline \multicolumn{7}{|c|}{$\begin{array}{l}\text { Table 1.22 Geometric Mean Urinary Phthalate and BPA Levels by responses, "I } \\
\text { experienced confusion or memory loss that is happening more often or is gettin } \\
60 \text { years of age, NHANES 2011-2014, over the LOD }\end{array}$} \\
\hline \multirow{3}{*}{ EEDC } & \multicolumn{6}{|c|}{ Geometric Mean (ng/ml) (GSE, N) } \\
\hline & \multicolumn{3}{|c|}{ Yes } & \multicolumn{3}{|c|}{ No } \\
\hline & GM & GSE & $\mathbf{N}$ & GM & GSE & $\mathbf{N}$ \\
\hline Mono (carboxynonyl) Phthalate - CNP & 2.82 & 0.43 & 178 & 2.63 & 0.13 & 843 \\
\hline Mono (carboxyoctyl) Phthalate - COP & 18.63 & 2.34 & 181 & 19.14 & 1.55 & 843 \\
\hline Mono-(2-ethyl-5-carboxypentyl) Phthalate -ECP & 13.51 & 1.12 & 180 & 12.02 & 0.63 & 843 \\
\hline Mono-n-butyl Phthalate - MBP & 11.80 & 0.81 & $179 *$ & 9.80 & 0.55 & 831 \\
\hline Mono-(3-carboxypropyl) Phthalate - MC1 & 2.79 & 0.28 & 173 & 2.74 & 0.18 & 805 \\
\hline Mono-ethyl Phthalate -MEP & 54.91 & 6.40 & 181 & 56.30 & 4.95 & 844 \\
\hline Mono (2-ethyl-5-hydroxy hexyl) Phthalate - MHH & 8.27 & 0.64 & 181 & 7.25 & 0.35 & 843 \\
\hline Mono-(2-ethyl-5-oxohexyl) Phthalate -MOH & 5.63 & 0.39 & 181 & 4.88 & 0.24 & 842 \\
\hline Mono-benzyl Phthalate - MZP & 5.24 & 0.65 & $179 *$ & 4.06 & 0.22 & 831 \\
\hline Mono-isobutyl Phthalate - MIB & 7.29 & 0.38 & $180 *$ & 6.19 & 0.30 & 835 \\
\hline Bisphenol A - BPA & 1.46 & 0.11 & 166 & 1.54 & 0.08 & 800 \\
\hline
\end{tabular}

NHANES sampling weight applied before calculating the geometric mean.

The EEDCs were creatinine-adjusted then log transformed prior to analyses. Extreme creatinine values $(<30$ $\mathrm{mg} / \mathrm{dL}$ and $>300 \mathrm{mg} / \mathrm{dL}$ ) and EEDC levels below the LOD were taken into account using the appropriate domain statement in SAS. $* \mathrm{p}<0.05, * * \mathrm{p}<0.01 * * * \mathrm{p}<0.001$

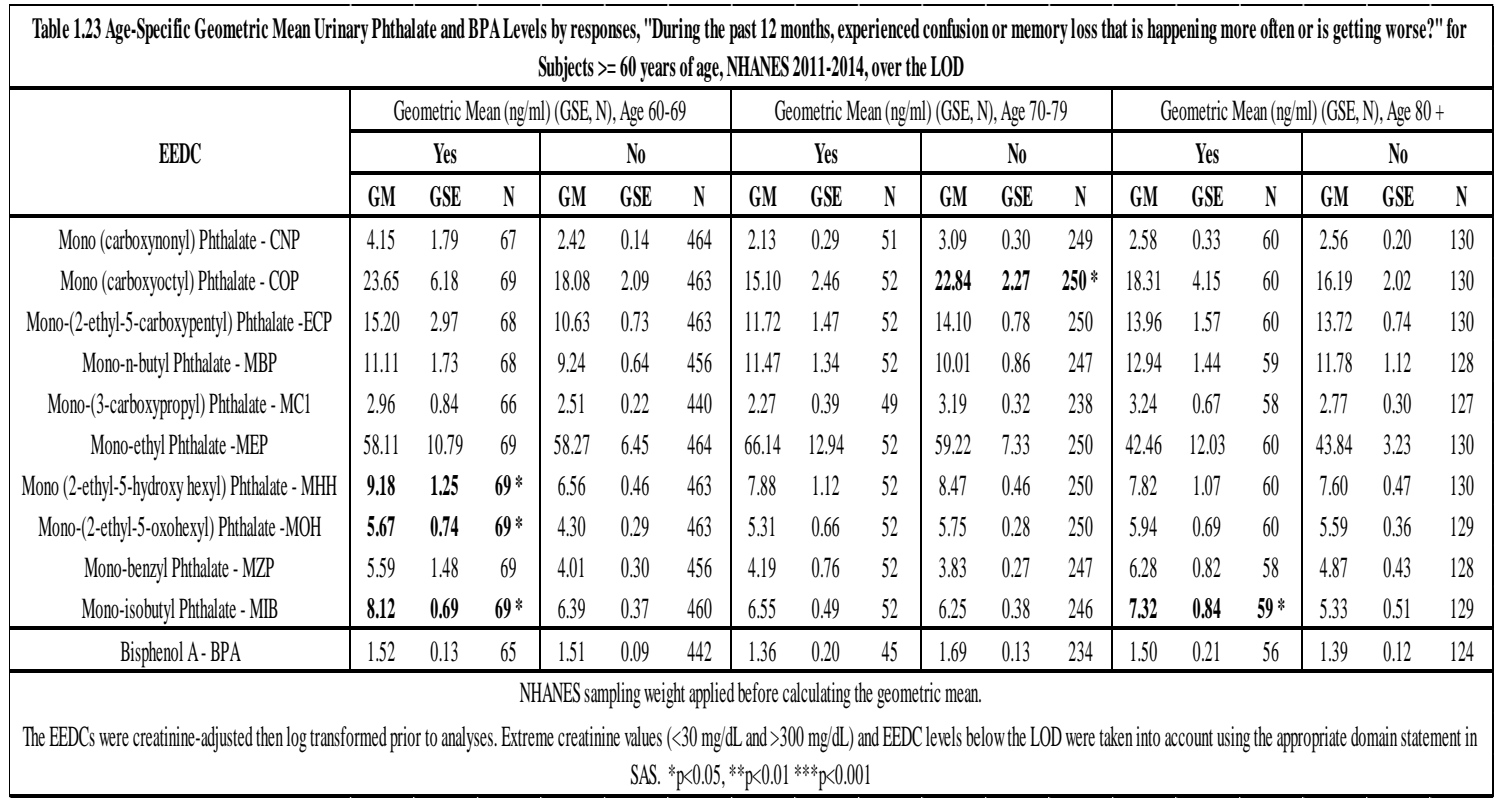




\begin{tabular}{|c|c|c|c|c|c|c|c|c|c|c|c|c|}
\hline \multirow{4}{*}{ EEDC } & \multicolumn{6}{|c|}{ Geometric Mean (ng/ml) (GSE, N) } & \multicolumn{6}{|c|}{ Geometric Mean (ng/ml) (GSE, N) } \\
\hline & \multicolumn{6}{|c|}{ Male } & \multicolumn{6}{|c|}{ Female } \\
\hline & \multicolumn{3}{|c|}{ Yes } & \multicolumn{3}{|c|}{ No } & \multicolumn{3}{|c|}{ Yes } & \multicolumn{3}{|c|}{ No } \\
\hline & GM & GSE & $\mathrm{N}$ & GM & GSE & $\mathrm{N}$ & GM & GSE & $\mathrm{N}$ & GM & GSE & $\mathrm{N}$ \\
\hline Mono (carboxynonyl) Phthalate - CNP & 2.05 & 0.29 & 78 & 2.40 & 0.14 & 437 & 3.40 & 0.85 & 100 & 2.89 & 0.21 & 406 \\
\hline Mono (carboxyoctyl) Phthalate - COP & 11.94 & 2.06 & 80 & 17.41 & 1.31 & 437 & 24.11 & 4.08 & 101 & 21.13 & 2.65 & 406 \\
\hline Mono-(2-ethyl-5-carboxypentyl) Phthalate -ECP & 11.80 & 1.46 & 80 & 10.86 & 0.57 & 438 & 14.62 & 1.94 & 100 & 13.38 & 0.91 & 405 \\
\hline Mono-n-butyl Phthalate - MBP & 10.25 & 1.03 & 79 & 9.25 & 0.67 & 429 & 12.81 & 1.22 & 100 & 10.38 & 0.60 & 402 \\
\hline Mono-(3-carboxypropyl) Phthalate - MC1 & 2.32 & 0.42 & 77 & 2.56 & 0.19 & 420 & 3.12 & 0.51 & 96 & 2.96 & 0.26 & 385 \\
\hline Mono-ethyl Phthalate -MEP & 49.63 & 8.64 & 80 & 53.52 & 5.07 & 438 & 58.21 & 8.77 & 101 & 59.36 & 7.04 & 406 \\
\hline Mono (2-ethyl-5-hydroxy hexyl) Phthalate - MHH & 7.24 & 0.86 & 80 & 6.80 & 0.38 & 438 & 8.93 & 0.86 & 101 & 7.74 & 0.46 & 405 \\
\hline Mono-(2-ethyl-5-oxohexyl) Phthalate -MOH & 4.92 & 0.58 & 80 & 4.59 & 0.25 & 436 & 6.08 & 0.53 & 101 & 5.19 & 0.33 & 406 \\
\hline Mono-benzyl Phthalate - MZP & 3.40 & 0.67 & 78 & 3.74 & 0.31 & 432 & 6.70 & 1.13 & $101 *$ & 4.42 & 0.24 & 399 \\
\hline Mono-isobutyl Phthalate - MIB & 6.18 & 0.58 & 79 & 5.80 & 0.32 & 435 & 8.00 & 0.60 & $101 *$ & 6.62 & 0.37 & 400 \\
\hline Bisphenol A- BPA & 1.33 & 0.19 & 70 & 1.40 & 0.08 & 419 & 1.53 & 0.13 & 96 & 1.72 & 0.11 & 381 \\
\hline $\begin{array}{l}\text { The EEDCs were creatinine-adjusted then log trans } \\
\text { taken into acce }\end{array}$ & ANES sa & ling we & t appl & before $\mathrm{c}$ & culating & e geom & ric mean. & & 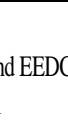 & 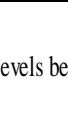 & & \\
\hline
\end{tabular}

\begin{tabular}{|c|c|c|c|c|c|c|c|c|c|c|c|c|}
\hline \multirow[t]{2}{*}{ EEDC } & \multicolumn{3}{|c|}{ Yes } & \multicolumn{3}{|c|}{ No } & \multicolumn{3}{|c|}{ Yes } & \multicolumn{3}{|c|}{ No } \\
\hline & GM & GSE & $\mathrm{N}$ & GM & GSE & $\mathrm{N}$ & GM & GSE & $\mathbf{N}$ & GM & GSE & $\mathbf{N}$ \\
\hline Mono (carboxynonyl) Phthalate - CNP & 2.62 & 0.34 & 38 & 2.31 & 0.15 & 177 & 2.98 & 0.57 & 86 & 2.79 & 0.18 & 356 \\
\hline Mono (carboxyoctyl) Phthalate - COP & 19.08 & 3.69 & 38 & 19.89 & 2.27 & 177 & 19.05 & 2.72 & 86 & 20.33 & 2.06 & 356 \\
\hline Mono-(2-ethyl-5-carboxypentyl) Phthalate -ECP & 19.61 & 2.27 & 38 & 17.72 & 1.01 & 177 & 12.69 & 1.29 & 86 & 11.66 & 0.70 & 355 \\
\hline Mono-n-butyl Phthalate - MBP & 13.60 & 1.89 & 38 & 13.73 & 0.89 & 175 & 11.03 & 0.92 & 86 & 9.11 & 0.65 & 349 \\
\hline Mono-(3-carboxypropyl) Phthalate - MC1 & 2.69 & 0.34 & 36 & 2.85 & 0.30 & 167 & 2.81 & 0.35 & 82 & 2.82 & 0.24 & 342 \\
\hline Mono-ethyl Phthalate -MEP & 80.38 & 11.12 & 38 & 99.65 & 14.07 & 17 & 52.33 & 7.80 & 86 & 50.66 & 5.16 & 356 \\
\hline Mono (2-ethyl-5-hydroxy hexyl) Phthalate - MHH & 11.31 & 1.56 & 38 & 10.22 & 0.69 & 177 & 7.88 & 0.70 & 86 & 6.99 & 0.39 & 355 \\
\hline Mono-(2-ethyl-5-oxohexyl) Phthalate -MOH & 7.66 & 0.90 & 38 & 6.53 & 0.45 & 177 & 5.37 & 0.43 & 86 & 4.74 & 0.28 & 354 \\
\hline Mono-benzyl Phthalate - MZP & 4.42 & 0.98 & 38 & 3.90 & 0.30 & 177 & 5.41 & 0.85 & 85 & 4.00 & 0.23 & 350 \\
\hline Mono-isobutyl Phthalate - MIB & 8.66 & 0.92 & 38 & 9.31 & 0.58 & 177 & 6.78 & 0.40 & $85 *$ & 5.65 & 0.28 & 353 \\
\hline Bisphenol A-BPA & 1.80 & 0.35 & 35 & 1.57 & 0.14 & 170 & 1.44 & 0.13 & 79 & 1.57 & 0.10 & 340 \\
\hline \multirow{3}{*}{ EEDC } & \multicolumn{6}{|c|}{ Geometric Mean (ng/ml) (GSE, N), Non-Hispanic Black } & \multicolumn{6}{|c|}{ Geometric Mean (ng/ml) (GSE, N), Asian/Other } \\
\hline & \multicolumn{3}{|c|}{ Yes } & \multicolumn{3}{|c|}{ No } & \multicolumn{3}{|c|}{ Yes } & \multicolumn{3}{|c|}{ No } \\
\hline & GM & GSE & $\mathrm{N}$ & GM & GSE & $\mathbf{N}$ & GM & GSE & $\mathbf{N}$ & GM & GSE & $\mathbf{N}$ \\
\hline Mono (carboxynonyl) Phthalate - CNP & 1.88 & 0.21 & 31 & 1.98 & 0.10 & 231 & 2.65 & 0.73 & 23 & 2.25 & 0.36 & 79 \\
\hline Mono (carboxyoctyl) Phthalate - COP & 15.13 & 2.09 & 31 & 15.13 & 2.09 & 230 & 17.86 & 7.65 & 26 & 12.78 & 1.76 & 80 \\
\hline Mono-(2-ethyl-5-carboxypentyl) Phthalate -ECP & 12.22 & 2.32 & 30 & 10.85 & 0.63 & 231 & 16.71 & 2.96 & 26 & 12.62 & 0.92 & 80 \\
\hline Mono-n-butyl Phthalate - MBP & 12.34 & 1.85 & 31 & 10.84 & 0.57 & 227 & 18.31 & 4.03 & 24 & 14.37 & 2.68 & 80 \\
\hline Mono-(3-carboxypropyl) Phthalate - MC2 & 2.28 & 0.31 & 29 & 2.19 & 0.15 & 225 & 3.19 & 0.90 & 26 & 2.65 & 0.41 & 71 \\
\hline Mono-ethyl Phthalate -MEP & 59.29 & 14.35 & 31 & 83.59 & 7.95 & 231 & 50.46 & 17.02 & 26 & 53.22 & 14.57 & 80 \\
\hline Mono (2-ethyl-5-hydroxy hexyl) Phthalate - MHH & 8.87 & 1.61 & 31 & 7.28 & 0.45 & 231 & 8.28 & 1.87 & 26 & 7.15 & 0.78 & 80 \\
\hline Mono-(2-ethyl-5-oxohexyl) Phthalate -MOH & 6.13 & 1.27 & 31 & 4.87 & 0.26 & 231 & 5.50 & 1.06 & 26 & 4.74 & 0.56 & 80 \\
\hline Mono-benzyl Phthalate - MZP & 5.56 & 1.29 & 31 & 4.60 & 0.44 & 228 & 4.59 & 1.11 & 25 & 4.31 & 1.49 & 76 \\
\hline Mono-isobutyl Phthalate - MIB & 8.99 & 1.74 & 31 & 7.46 & 0.38 & 226 & 9.51 & 2.05 & 26 & 9.12 & 1.70 & 79 \\
\hline Bisphenol A- BPA & 1.34 & 0.13 & 30 & 1.42 & 0.06 & 224 & 1.40 & 0.26 & 22 & 1.31 & 0.23 & 66 \\
\hline $\mathrm{NH}$ & ANES sa & pling we & appli & before $\mathrm{c}$ & culating & geom & ric mean & 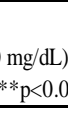 & & & & \\
\hline
\end{tabular}




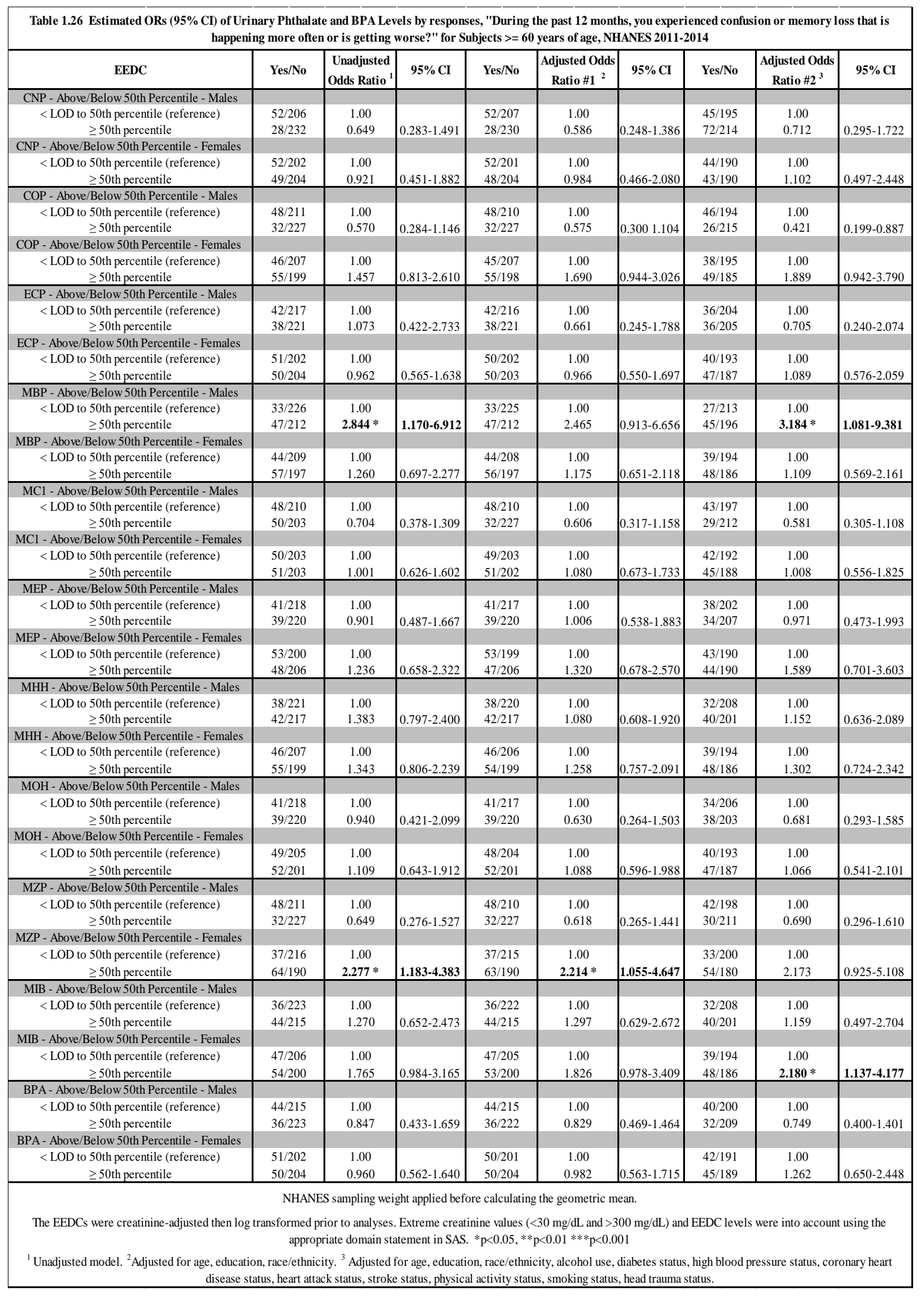




\section{Trouble remembering past 7 days and Exposure to BPA and Phthalates:}

Table 1.27 presents crude GMs and GSEs for phthalate and BPA concentrations over the LOD for subjects who provided a response to the question "During the past 7 days, how often have you had trouble remembering where you put things?" The phthalate metabolite, MIB, was found to be significantly higher in those who experienced memory issues in the past week $(\mathrm{p}<0.05)$. Table 1.28 presents age-specific GMs and GSEs. No significant findings were observed. Table 1.29 presents gender-specific GMs and GSEs. Among males, GMs the for-phthalate metabolites ECP, $\mathrm{MHH}$, and $\mathrm{MOH}$ were found to be significantly higher in those that experienced memory issues in the past week (ECP, $\mathrm{p}<0.05 ; \mathrm{MHH}, \mathrm{p}<0.05 ; \mathrm{MOH}, \mathrm{p}<0.05)$. Table 1.30 presents race-specific GMs and GSEs. Among Hispanics, the phthalate metabolite MBP was found to be significantly higher $(\mathrm{p}<0.05)$ in subjects that experienced memory issues in the past week.

Table 1.31 presents the estimated ORs and 95\% CIs. For the phthalate metabolite ECP, among females in the $\geq 50$ th percentile group compared to the reference group, ECP concentrations were found to be significantly associated with the having trouble remembering in the past week in adjusted model \#1 (OR=1.673, 95\% CI: 1.007-2.780) and adjusted model \#2 (OR=1.927, 95\% CI: 1.131-3.283). For the phthalate metabolite MBP, among females in the $\geq 50$ th percentile group compared to the reference group, MBP concentrations were found to be significantly associated with having trouble remembering in the past week $(\mathrm{OR}=1.955,95 \% \mathrm{CI}: 1.030-3.711)$ in the $2^{\text {nd }}$ adjusted model. 
For the phthalate metabolite $\mathrm{MHH}$, among females in the $\geq$ 50th percentile group, $\mathrm{MHH}$ concentrations were found to be significantly associated with having trouble remembering in the past week (OR=1.821, 95\% CI: 1.104-3.005) in the $2^{\text {nd }}$ adjusted model. 


\begin{tabular}{|c|c|c|c|c|c|c|}
\hline $\begin{array}{r}\text { Table 1.27 Geometric Mean Urinary Phthalate } \\
\text { how often have you had trouble remembering w } \\
\text { Subjects }>=60 \text { years of age, }\end{array}$ & $\begin{array}{l}\text { and BP } \\
\text { lere you } \\
\text { NHANE }\end{array}$ & $\begin{array}{l}\text { evels } \\
\text { ut thin } \\
\text { 2011-2 }\end{array}$ & $\begin{array}{l}\text { Respon } \\
\text { like yo } \\
\text { 4, over }\end{array}$ & $\begin{array}{l}\text { "Duri } \\
\text { keys o } \\
\text { LOD }\end{array}$ & the $n$ & $\begin{array}{l}\text { days, } \\
\text { ?" for }\end{array}$ \\
\hline & & Geon & ic Mea & $\mathrm{ng} / \mathrm{ml})($ & $\mathrm{E}, \mathrm{N})$ & \\
\hline EEDC & & Yes & & & No & \\
\hline & GM & GSE & $\mathbf{N}$ & GM & GSE & $\mathbf{N}$ \\
\hline Mono (carboxynonyl) Phthalate - CNP & 2.64 & 0.18 & 187 & 2.67 & 0.15 & 780 \\
\hline Mono (carboxyoctyl) Phthalate - COP & 17.79 & 1.83 & 188 & 19.45 & 1.66 & 782 \\
\hline Mono-(2-ethyl-5-carboxypentyl) Phthalate -ECP & 12.55 & 0.58 & 188 & 11.94 & 0.64 & 781 \\
\hline Mono-n-butyl Phthalate - MBP & 10.41 & 0.78 & 186 & 9.95 & 0.57 & 770 \\
\hline Mono-(3-carboxypropyl) Phthalate - MC1 & 2.36 & 0.24 & 178 & 2.84 & 0.19 & 748 \\
\hline Mono-ethyl Phthalate -MEP & 63.99 & 8.74 & 188 & 53.71 & 4.47 & 783 \\
\hline Mono (2-ethyl-5-hydroxy hexyl) Phthalate - MHH & 7.60 & 0.41 & 188 & 7.19 & 0.38 & 782 \\
\hline Mono-(2-ethyl-5-oxohexyl) Phthalate -MOH & 5.07 & 0.30 & 187 & 4.86 & 0.26 & 782 \\
\hline Mono-benzyl Phthalate - MZP & 4.38 & 0.38 & 186 & 4.12 & 0.24 & 771 \\
\hline Mono-isobutyl Phthalate - MIB & 7.04 & 0.47 & $187 *$ & 6.15 & 0.30 & 775 \\
\hline Bisphenol A - BPA & 1.42 & 0.12 & 180 & 1.53 & 0.07 & 736 \\
\hline $\begin{array}{r}\text { NHANES sampling weight appli } \\
\text { The EEDCs were creatinine-adjusted then log tran } \\
\mathrm{mg} / \mathrm{dL} \text { and }>300 \mathrm{mg} / \mathrm{dL} \text { ) and EEDCs levels below th } \\
\text { statement in SAS. *p }\end{array}$ & $\begin{array}{l}\text { before } \\
\text { formed } \\
\text { LOD w } \\
0.05, *\end{array}$ & $\begin{array}{l}\text { lculatil } \\
\text { or to a } \\
\text { taken } \\
0.01 *\end{array}$ & $\begin{array}{l}\text { he geon } \\
\text { yses. Ex } \\
\text { accou } \\
p<0.001\end{array}$ & $\begin{array}{l}\text { ric mea } \\
\text { me cre } \\
\text { ising th }\end{array}$ & $\begin{array}{l}\text { ne va } \\
\text { propr }\end{array}$ & $\begin{array}{l}<30 \\
\text { lomain }\end{array}$ \\
\hline
\end{tabular}

\begin{tabular}{|c|c|c|c|c|c|c|c|c|c|c|c|c|}
\hline \multirow{3}{*}{ EEDC } & \multicolumn{6}{|c|}{ Geometric Mean (ng/ml) (GSE, N), Hispanic } & \multicolumn{6}{|c|}{ Geometric Mean (ng/ml) (GSE, N), Non-Hispanic White } \\
\hline & \multicolumn{3}{|c|}{ Yes } & \multicolumn{3}{|c|}{ No } & \multicolumn{3}{|c|}{ Yes } & \multicolumn{3}{|c|}{ No } \\
\hline & GM & GSE & $\mathbf{N}$ & GM & GSE & $\mathbf{N}$ & GM & GSE & $\mathbf{N}$ & GM & GSE & $\mathbf{N}$ \\
\hline Mono (carboxynonyl) Phthalate - CNP & 2.56 & 0.30 & 42 & 2.37 & 0.18 & 150 & 2.70 & 0.20 & 89 & 2.85 & 0.20 & 333 \\
\hline Mono (carboxyoctyl) Phthalate - COP & 21.13 & 2.39 & 42 & 20.10 & 2.41 & 150 & 18.83 & 2.65 & 89 & 20.58 & 2.15 & 333 \\
\hline Mono-(2-ethyl-5-carboxypentyl) Phthalate -ECP & 20.53 & 1.85 & 42 & 17.62 & 1.11 & 150 & 11.71 & 0.66 & 89 & 11.60 & 0.71 & 332 \\
\hline Mono-n-butyl Phthalate - MBP & 17.40 & 1.96 & $41 *$ & 13.72 & 0.90 & 149 & 9.22 & 0.76 & 89 & 9.32 & 0.70 & 326 \\
\hline Mono-(3-carboxypropyl) Phthalate - MC1 & 3.06 & 0.48 & 39 & 2.96 & 0.35 & 143 & 2.24 & 0.29 & 85 & 2.94 & 0.26 & 319 \\
\hline Mono-ethyl Phthalate -MEP & 104.73 & 24.56 & 42 & 93.49 & 13.03 & 150 & 53.54 & 6.98 & 89 & 49.61 & 4.96 & 333 \\
\hline Mono (2-ethyl-5-hydroxy hexyl) Phthalate - MHH & 12.24 & 1.33 & 42 & 10.12 & 0.78 & 150 & 7.11 & 0.47 & 89 & 6.95 & 0.43 & 332 \\
\hline Mono-(2-ethyl-5-oxohexyl) Phthalate -MOH & 7.80 & 0.81 & 42 & 6.52 & 0.48 & 150 & 4.82 & 0.36 & 88 & 4.72 & 0.30 & 332 \\
\hline Mono-benzyl Phthalate - MZP & 4.31 & 0.68 & 42 & 3.87 & 0.35 & 150 & 4.30 & 0.47 & 88 & 4.09 & 0.26 & 327 \\
\hline Mono-isobutyl Phthalate - MIB & 10.41 & 0.87 & 42 & 9.20 & 0.67 & 150 & 6.22 & 0.44 & 89 & 5.67 & 0.29 & 329 \\
\hline Bisphenol A - BPA & 1.69 & 0.33 & 39 & 1.56 & 0.12 & 144 & 1.36 & 0.14 & 85 & 1.57 & 0.10 & 316 \\
\hline \multirow{3}{*}{ EEDC } & \multicolumn{6}{|c|}{ Geometric Mean (ng/ml) (GSE, N), Non-Hispanic Black } & \multicolumn{6}{|c|}{ Geometric Mean (ng/ml) (GSE, N), Asian/Other } \\
\hline & \multicolumn{3}{|c|}{ Yes } & \multicolumn{3}{|c|}{ No } & \multicolumn{3}{|c|}{ Yes } & \multicolumn{3}{|c|}{ No } \\
\hline & GM & GSE & $\mathbf{N}$ & GM & GSE & $\mathbf{N}$ & GM & GSE & $\mathbf{N}$ & GM & GSE & $\mathbf{N}$ \\
\hline Mono (carboxynonyl) Phthalate - CNP & 2.02 & 0.23 & 39 & 1.93 & 0.09 & 216 & 3.09 & 1.76 & 17 & 2.15 & 0.24 & 81 \\
\hline Mono (carboxyoctyl) Phthalate - COP & 14.77 & 1.89 & 39 & 13.82 & 1.05 & 215 & 9.67 & 2.84 & 18 & 15.14 & 2.25 & 84 \\
\hline Mono-(2-ethyl-5-carboxypentyl) Phthalate -ECP & 12.30 & 1.61 & 39 & 10.58 & 0.72 & 215 & 13.88 & 2.39 & 18 & 13.39 & 1.06 & 84 \\
\hline Mono-n-butyl Phthalate - MBP & 12.07 & 1.58 & 39 & 10.72 & 0.52 & 212 & 16.55 & 2.98 & 17 & 14.82 & 3.01 & 83 \\
\hline Mono-(3-carboxypropyl) Phthalate - MC2 & 2.11 & 0.24 & 38 & 2.18 & 0.15 & 209 & 3.29 & 1.04 & 16 & 2.63 & 0.37 & 77 \\
\hline Mono-ethyl Phthalate -MEP & 106.02 & 19.93 & 39 & 77.01 & 8.27 & 216 & 121.49 & 80.49 & 18 & 40.44 & 8.13 & 84 \\
\hline Mono (2-ethyl-5-hydroxy hexyl) Phthalate - MHH & 8.59 & 1.14 & 39 & 7.16 & 0.50 & 216 & 7.11 & 1.44 & 18 & 7.42 & 0.83 & 84 \\
\hline Mono-(2-ethyl-5-oxohexyl) Phthalate -MOH & 5.43 & 0.81 & 39 & 4.87 & 0.31 & 216 & 4.47 & 0.94 & 18 & 4.99 & 0.56 & 84 \\
\hline Mono-benzyl Phthalate - MZP & 5.77 & 0.96 & 39 & 4.48 & 0.44 & 214 & 3.81 & 0.91 & 17 & 4.29 & 1.55 & 80 \\
\hline Mono-isobutyl Phthalate - MIB & 8.32 & 0.91 & 39 & 7.38 & 0.44 & 212 & 12.99 & 4.20 & 17 & 8.55 & 1.56 & 84 \\
\hline Bisphenol A - BPA & 1.39 & 0.16 & 39 & 1.43 & 0.06 & 209 & 1.97 & 0.46 & 17 & 1.14 & 0.17 & 67 \\
\hline $\begin{array}{l}\text { EEDCs were creatinıne-adjusted then log tra } \\
\text { taken into a }\end{array}$ & orme & he app & ies. E & $\mathrm{d}$ before & nt in $S$ & e geo & tric me & $* * * \mathrm{p}$ & 1 & Ieve & & Der \\
\hline
\end{tabular}




\begin{tabular}{|c|c|c|c|c|c|c|c|c|c|c|c|c|c|c|c|c|c|c|}
\hline \multirow{3}{*}{ EEDC } & \multicolumn{6}{|c|}{ Geometric Mean (ng/ml) (GSE, N), Age 60-69 } & \multicolumn{6}{|c|}{ Geometric Mean (ng/ml) (GSE, N), Age 70-79 } & \multicolumn{6}{|c|}{ Geometric Mean (ng/ml) (GSE, N), Age $80+$} \\
\hline & \multicolumn{3}{|c|}{ Yes } & \multicolumn{3}{|c|}{ No } & \multicolumn{3}{|c|}{ Yes } & \multicolumn{3}{|c|}{\begin{tabular}{|c|} 
No \\
\end{tabular}} & \multicolumn{3}{|c|}{ Yes } & \multicolumn{3}{|c|}{ No } \\
\hline & GM & GSE & $\mathbf{N}$ & GM & GSE & $\mathbf{N}$ & GM & GSE & $\mathbf{N}$ & GM & GSE & $\mathbf{N}$ & GM & GSE & $\mathbf{N}$ & GM & GSE & $\mathbf{N}$ \\
\hline Mono (carboxynonyl) Phthalate - CNP & 2.08 & 0.22 & 88 & 2.66 & 0.20 & 418 & 3.29 & 0.41 & 59 & 2.81 & 0.26 & 227 & 2.99 & 0.37 & 40 & 2.42 & 0.23 & 135 \\
\hline Mono (carboxyoctyl) Phthalate - COP & 13.62 & 3.26 & 89 & 19.52 & 2.29 & 418 & 22.11 & 4.08 & 59 & 21.49 & 2.29 & 229 & 21.35 & 5.33 & 40 & 15.67 & 2.08 & 135 \\
\hline Mono-(2-ethyl-5-carboxypentyl) Phthalate -ECP & 11.59 & 1.25 & 89 & 10.81 & 0.84 & 417 & 13.68 & 1.09 & 59 & 13.36 & 0.88 & 229 & 12.81 & 1.55 & 40 & 13.58 & 0.79 & 135 \\
\hline Mono-n-butyl Phthalate - MBP & 9.91 & 1.07 & 87 & 9.33 & 0.67 & 412 & 9.92 & 1.07 & 59 & 10.20 & 0.94 & 226 & 12.33 & 1.92 & 40 & 11.98 & 1.13 & 132 \\
\hline Mono-(3-carboxypropyl) Phthalate - MC1 & 2.02 & 0.38 & 83 & 2.66 & 0.24 & 400 & 2.54 & 0.41 & 56 & 3.14 & 0.36 & 217 & 2.80 & 0.67 & 39 & 2.94 & 0.39 & 131 \\
\hline Mono-ethyl Phthalate -MEP & 67.84 & 17.75 & 89 & 55.88 & 5.88 & 419 & 70.60 & 17.55 & 59 & 58.83 & 8.50 & 229 & 49.12 & 11.23 & 40 & 38.75 & 2.85 & 135 \\
\hline Mono (2-ethyl-5-hydroxy hexyl) Phthalate - $\mathrm{MHH}$ & 6.95 & 0.77 & 89 & 6.68 & 0.51 & 418 & 8.88 & 0.67 & 59 & 8.01 & 0.55 & 229 & 7.13 & 0.94 & 40 & 7.55 & 0.56 & 135 \\
\hline Mono-(2-ethyl-5-oxohexyl) Phthalate -MOH & 4.33 & 0.47 & 89 & 4.39 & 0.32 & 418 & 5.90 & 0.48 & 59 & 5.47 & 0.34 & 229 & 5.46 & 0.76 & 39 & 5.53 & 0.36 & 135 \\
\hline Mono-benzyl Phthalate - MZP & 4.87 & 0.83 & 88 & 3.99 & 0.32 & 413 & 3.51 & 0.42 & 59 & 3.91 & 0.35 & 226 & 5.03 & 0.92 & 39 & 5.17 & 0.47 & 132 \\
\hline Mono-isobutyl Phthalate - MIB & 7.70 & 0.82 & 89 & 6.29 & 0.36 & 416 & 6.98 & 0.59 & 58 & 6.09 & 0.39 & 226 & 5.98 & 0.95 & 40 & 5.81 & 0.47 & 133 \\
\hline Bisphenol A-BPA & 1.44 & 0.16 & 87 & 1.50 & 0.10 & 396 & 1.55 & 0.17 & 54 & 1.63 & 0.12 & 212 & 1.21 & 0.27 & 39 & 1.48 & 0.11 & 128 \\
\hline
\end{tabular}

\begin{tabular}{|c|c|c|c|c|c|c|c|c|c|c|c|c|}
\hline \multirow{4}{*}{ EEDC } & \multicolumn{6}{|c|}{ Geometric Mean (ng/ml) (GSE, N) } & \multicolumn{6}{|c|}{ Geometric Mean (ng/ml) (GSE, N) } \\
\hline & \multicolumn{6}{|c|}{ Male } & \multicolumn{6}{|c|}{$\begin{array}{l}\text { Female } \\
\end{array}$} \\
\hline & \multicolumn{3}{|c|}{ Yes } & \multicolumn{3}{|c|}{ No } & \multicolumn{3}{|c|}{ Yes } & \multicolumn{3}{|c|}{ No } \\
\hline & GM & GSE & $\mathbf{N}$ & GM & GSE & $\mathbf{N}$ & GM & GSE & $\mathbf{N}$ & GM & GSE & $\mathbf{N}$ \\
\hline Mono (carboxynonyl) Phthalate - CNP & 2.65 & 0.40 & 71 & 2.35 & 0.14 & 423 & 2.64 & 0.26 & 116 & 3.06 & 0.30 & 357 \\
\hline Mono (carboxyoctyl) Phthalate - COP & 15.02 & 2.58 & 71 & 16.96 & 1.25 & 425 & 19.27 & 3.21 & 117 & 22.61 & 3.13 & 357 \\
\hline Mono-(2-ethyl-5-carboxypentyl) Phthalate -ECP & 13.27 & 1.21 & $71 *$ & 10.47 & 0.55 & 426 & 12.22 & 0.86 & 117 & 13.82 & 1.06 & 355 \\
\hline Mono-n-butyl Phthalate - MBP & 9.71 & 1.28 & 71 & 9.36 & 0.69 & 416 & 10.76 & 0.88 & 115 & 10.64 & 0.72 & 354 \\
\hline Mono-(3-carboxypropyl) Phthalate - MC1 & 2.28 & 0.42 & 68 & 2.57 & 0.22 & 408 & 2.39 & 0.37 & 110 & 3.18 & 0.30 & 340 \\
\hline Mono-ethyl Phthalate -MEP & 56.35 & 11.19 & 71 & 52.19 & 4.99 & 426 & 67.94 & 11.77 & 117 & 55.44 & 5.65 & 357 \\
\hline Mono (2-ethyl-5-hydroxy hexyl) Phthalate - $\mathrm{MHH}$ & 8.06 & 0.75 & $71 *$ & 6.53 & 0.37 & 426 & 7.40 & 0.53 & 117 & 7.99 & 0.52 & 356 \\
\hline Mono-(2-ethyl-5-oxohexyl) Phthalate -MOH & 5.66 & 0.51 & $70 *$ & 4.40 & 0.25 & 425 & 4.82 & 0.39 & 117 & 5.42 & 0.36 & 357 \\
\hline Mono-benzyl Phthalate - MZP & 3.41 & 0.43 & 70 & 3.74 & 0.33 & 419 & 4.91 & 0.58 & 116 & 4.58 & 0.31 & 352 \\
\hline Mono-isobutyl Phthalate-MIB & 6.31 & 0.82 & 71 & 5.73 & 0.31 & 422 & 7.41 & 0.59 & 116 & 6.66 & 0.38 & 353 \\
\hline Bisphenol A-BPA & 1.38 & 0.24 & 65 & 1.37 & 0.08 & 404 & 1.44 & 0.12 & 115 & 1.75 & 0.12 & 332 \\
\hline
\end{tabular}




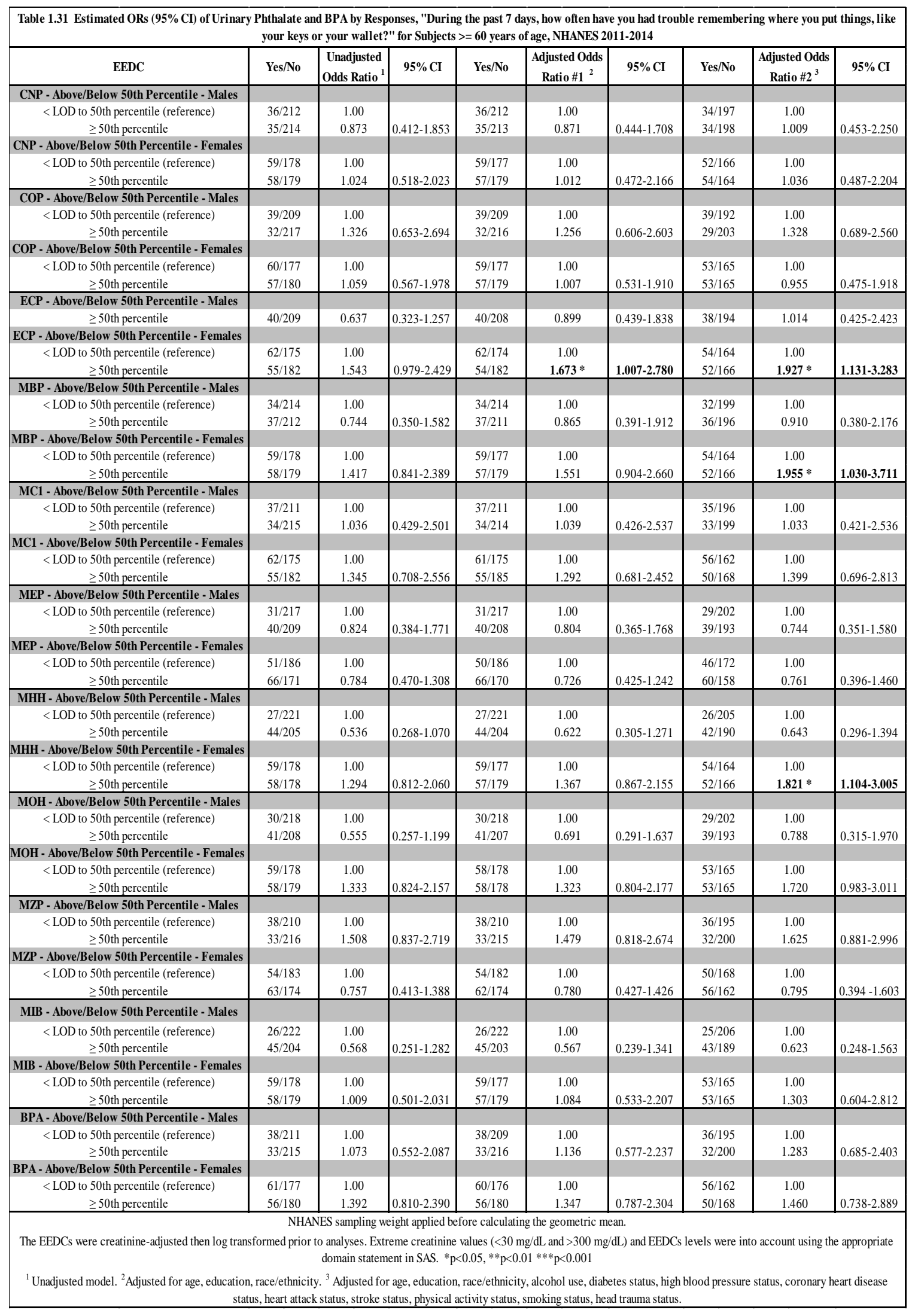




\section{Limitations Due to Difficulty Remembering or Confusion and Exposure to BPA and}

Phthalates: Table 1.32 presents crude GMs and GSEs for subjects with phthalate and BPA levels above the LOD who responded to the question, "Are you limited in any way because of difficulty remembering or because you experience periods of confusion?". The phthalate metabolites ECP, MBP, MHH, MOH, MZP, and MIB were found to be significantly higher among individuals who are limited because of difficulty remembering or confusion $(\mathrm{ECP}, \mathrm{p}<0.05 ; \mathrm{MBP}, \mathrm{p}<0.01 ; \mathrm{MHH}, \mathrm{p}<0.01 ; \mathrm{MOH}, \mathrm{p}<0.05$; $\mathrm{MZP}, \mathrm{p}<0.01 ; \mathrm{MIB}, \mathrm{p}<0.001)($

Table 1.33 presents age-specific GMs and GSEs. For the age group 60-69, the phthalate metabolites ECP, MBP, MHH, MOH, MZP, and MIB were found to be significantly higher in those who are limited due to difficulty remembering or confusion (ECP, $\mathrm{p}<0.01 ; \mathrm{MBP}, \mathrm{p}<0.05 ; \mathrm{MHH}, \mathrm{p}<0.01 ; \mathrm{MOH}, \mathrm{p}<0.05 ; \mathrm{MZP}, \mathrm{p}<0.05 ; \mathrm{MIB}, \mathrm{p}<0.01)$. In the age group 70-79, the phthalate metabolite MIB was found to be significantly higher in those who are limited due to difficulty remembering or confusion $(\mathrm{p}<0.05)$. In the age group $80+$, the phthalate metabolite MIB was found to be significantly higher in those who experienced memory issues or confusion $(\mathrm{p}<0.05)$. Table 1.34 presents genderspecific GMs and GSEs. For males, the phthalate metabolites ECP, MHH, MOH, and MIB were found to be significantly higher in those who are limited due to memory issues or confusion (ECP, p<0.05; MHH, p<0.05; MOH, p<0.05; MIB, $\mathrm{p}<0.001$ ). For females, the phthalate metabolites MBP, MZP, and MIB were found to be significantly higher in those in those who are limited due to memory issues or confusion (MBP, $\mathrm{p}<0.05$; MZP, $\mathrm{p}<0.001 ; \mathrm{MIB}, \mathrm{p}<0.05)$. In females, BPA was found to be significantly higher in those that significantly higher in those in those who are not limited due to memory issues or 
confusion $(\mathrm{p}<0.05)$. Table 1.35 presents race-specific GMs and GSEs. Among nonHispanic whites, the phthalate metabolites MZP and MIB were found to be significantly higher among those that are limited due to memory issues or confusion (MZP, $\mathrm{p}<0.01$; MIB, $\mathrm{p}<0.05)$. Table 1.36 presents estimated ORs and $95 \% \mathrm{CI}$ for phthalate metabolites and BPA among subjects who responded to "Are you limited in any way because of difficulty remembering or because you experience periods of confusion?". For the phthalate metabolite MBP among females in the $\geq 50$ th percentile group compared to the reference group, MBP concentrations were found to be significantly associated with having limitations due to memory issues or confusion $(\mathrm{OR}=1.721,95 \% \mathrm{CI}: 1.083-2.735)$ in the unadjusted model. The adjusted models were not significant. For the phthalate metabolite $\mathrm{MHH}$, among males in the $\geq 50$ th percentile group compared to the reference group, $\mathrm{MHH}$ concentrations were found to be significantly associate with limitations due to memory issues or confusion in the unadjusted model, adjusted model \#1, and adjusted model \#2 (OR=2.460, 95\% CI: 1.392-4.350, OR=2.444, 95\% CI: 1.278-4.675, $\mathrm{OR}=3.029,95 \% \mathrm{CI}: 1.441=6.366)$. For the phthalate metabolite $\mathrm{MOH}$, among males in the $\geq 50$ th percentile group compared to the reference group, $\mathrm{MOH}$ concentrations were significantly associated with limitations due to memory issues or confusion the unadjusted model $(\mathrm{OR}=2.050,95 \% \mathrm{CI}: 1.016-4.136)$.

For the phthalate metabolite MZP, among females in the $\geq 50$ th percentile group compared to the reference group, MZP concentrations were significantly associated with limitations due to memory issues or confusion in the unadjusted and both adjusted models $(\mathrm{OR}=3.135,95 \%$ CI: 1.965-5.001, OR=2.863, 95\% CI: $1.719-4.768, \mathrm{OR}=2.124$, 95\% CI: 1.151-3.922). For the phthalate metabolite MIB, among males in the $\geq 50$ th 
percentile group compared to the reference group, MIB concentrations were significantly associated with limitations due to memory issues or confusion in the unadjusted and both adjusted models (OR=3.779, 95\% CI: 1.756-8.131; OR=3.818, 95\% CI: $1.742-8.369$; $\mathrm{OR}=3.953,95 \%$ CI: $1.691-9.242$ ). Among females in the $\geq$ 50th percentile compared to the reference group, MIB concentrations were significantly associated with limitations due to memory issues or confusion in only the unadjusted model $(\mathrm{OR}=1.786,95 \% \mathrm{CI}$ : 1.046-3.049). 


\begin{tabular}{|c|c|c|c|c|c|c|}
\hline \multirow{3}{*}{ EEDC } & \multicolumn{6}{|c|}{ Geometric Mean (ng/ml) (GSE, N) } \\
\hline & \multicolumn{3}{|c|}{ Yes } & \multicolumn{3}{|c|}{ No } \\
\hline & GM & GSE & $\mathbf{N}$ & GM & GSE & $\mathbf{N}$ \\
\hline Mono (carboxynonyl) Phthalate - CNP & 2.44 & 0.20 & 164 & 269 & 0.15 & 856 \\
\hline Mono (carboxyoctyl) Phthalate - COP & 17.74 & 2.76 & 165 & 19.29 & 1.56 & 858 \\
\hline Mono-(2-ethyl-5-carboxypentyl) Phthalate -ECP & 14.51 & 0.94 & $164 *$ & 11.98 & 0.59 & 858 \\
\hline Mono-n-butyl Phthalate - MBP & 12.25 & 0.81 & $164 * *$ & 9.83 & 0.51 & 845 \\
\hline Mono-(3-carboxypropyl) Phthalate-MC1 & 2.63 & 0.37 & 160 & 2.77 & 0.19 & 818 \\
\hline Mono-ethyl Phthalate-MEP & 55.64 & 7.67 & 165 & 56.20 & 4.65 & 859 \\
\hline Mono (2-ethyl-5-hydroxy hexyl) Phthalate - MHH & 8.62 & 0.53 & $165 * *$ & 7.26 & 0.33 & 858 \\
\hline Mono-(2-ethyl-5-oxohexyl) Phthalate -MOH & 5.86 & 0.36 & $165 *$ & 4.89 & 0.23 & 857 \\
\hline Mono-benzylPhthalate - MZP & 5.73 & 0.59 & $165 *$ & 4.05 & 0.21 & 845 \\
\hline Mono-isobutyl Phthalate - MIB & 8.42 & 0.65 & $165 * * *$ & 6.11 & 0.27 & 850 \\
\hline Bisphenol A - BPA & 1.42 & 0.13 & 154 & 1.55 & 0.07 & 811 \\
\hline
\end{tabular}

\begin{tabular}{|c|c|c|c|c|c|c|c|c|c|c|c|c|}
\hline \multirow[b]{2}{*}{ EEDC } & \multicolumn{6}{|c|}{ Geometric Mean (ng/ml) (GSE, N), Hispanic } & \multicolumn{6}{|c|}{ Geometric Mean $(\mathrm{ng} / \mathrm{ml})(\mathrm{GSE}, \mathrm{N})$, Non-Hispanic White } \\
\hline & \multicolumn{3}{|c|}{ Yes } & \multicolumn{3}{|c|}{ No } & \multicolumn{3}{|c|}{ Yes } & \multicolumn{3}{|c|}{ No } \\
\hline Mono (carboxynonyl) Phthalate - CNP & 2.54 & 0.22 & 50 & 2.32 & 0.14 & 165 & 2.62 & 0.26 & 58 & 2.84 & 0.20 & 383 \\
\hline Mono (carboxyoctyl) Phthalate - COP & 20.87 & 3.32 & 50 & 1939 & 2.13 & 165 & 18.46 & 3.70 & 58 & 20.37 & 2.02 & 383 \\
\hline Mono-(2-ethyl-5-carboxypentyl) Phthalate -ECP & 19.36 & 1.52 & 50 & 17.66 & 1.10 & 165 & 13.47 & 1.32 & 58 & 11.66 & 0.66 & 382 \\
\hline Mono-(3-carboxypropyl) Phthalate -MC1 & 2.72 & 0.42 & 46 & 2.85 & 0.32 & 157 & 2.61 & 0.52 & 57 & 2.84 & 0.24 & 367 \\
\hline Mono-ethyl Phthalate -MEP & 97.76 & 15.35 & 50 & 9522 & 14.73 & 165 & 44.74 & 7.32 & 58 & 51.67 & 5.12 & 383 \\
\hline Mono (2-ethyl-5-hydroxy hexyl) Phthalate - MHH & 11.61 & 1.46 & 50 & 10.06 & 0.75 & 165 & 7.74 & 0.66 & 58 & 7.08 & 0.37 & 382 \\
\hline Mono-(2-ethyl-5-oxohexyl) Phthalate -MOH & 7.58 & 0.80 & 50 & 6.47 & 0.48 & 165 & 5.41 & 0.42 & 58 & 4.79 & 0.26 & 381 \\
\hline Mono-benzyl Phthalate - MZP & 4.50 & 0.92 & 50 & 3.84 & 0.30 & 165 & 6.12 & 0.97 & $58 * *$ & 4.01 & 0.22 & 377 \\
\hline Mono-isobutyl Phthalate - MIB & 8.86 & 0.64 & 50 & 9.30 & 0.63 & 165 & 7.71 & 0.84 & 58 * & 5.64 & 0.26 & 380 \\
\hline EEDC & GM & GSE & $\mathbf{N}$ & GM & GSE & $\mathbf{N}$ & GM & GSE & $\mathbf{N}$ & GM & GSE & $\mathbf{N}$ \\
\hline Mono (carboxynonyl) Phthalate - CNP & 1.77 & 0.26 & 35 & 2.01 & 0.10 & 227 & 2.08 & 0.58 & 21 & 2.40 & 0.38 & 81 \\
\hline Mono (carboxyoctyl) Phthalate - COP & 16.00 & 3.51 & 35 & 13.90 & 0.89 & 226 & 11.67 & 3.88 & 22 & 14.48 & 2.23 & 84 \\
\hline Mono-(2-ethyl-5-carboxypentyl) Phthalate -ECP & 13.47 & 2.27 & 34 & 10.63 & 0.58 & 227 & 15.54 & 2.61 & 22 & 12.98 & 0.96 & 84 \\
\hline Mono-n-butyl Phthalate - MBP & 11.89 & 1.51 & 35 & 10.86 & 0.65 & 223 & 22.74 & 4.89 & 21 & 13.66 & 2.47 & 83 \\
\hline Mono-(3-carboxypropyl) Phthalate -MC2 & 2.58 & 0.43 & 35 & 2.14 & 0.14 & 219 & 2.64 & 0.52 & 22 & 2.82 & 0.45 & 75 \\
\hline Mono-ethyl Phthalate-MEP & 49.05 & 13.22 & 35 & 8739 & 7.79 & 227 & 100.51 & 62.86 & 22 & 44.15 & 8.71 & 84 \\
\hline Mono (2-ethyl-5-hydroxy hexyl) Phthalate - MHH & 9.08 & 1.69 & 35 & 7.20 & 0.42 & 227 & 9.70 & 2.15 & 22 & 6.88 & 0.72 & 84 \\
\hline Mono-(2-ethyl-5-oxohexyl) Phthalate -MOH & 6.42 & 0.14 & 35 & 4.79 & 0.25 & 227 & 5.59 & 1.42 & 22 & 4.74 & 0.50 & 84 \\
\hline Mono-benzyl Phthalate - MZP & 6.17 & 0.98 & 35 & 4.49 & 0.44 & 224 & 521 & 1.31 & 22 & 4.16 & 1.41 & 79 \\
\hline Mono-isobutyl Phthalate-MIB & 8.88 & 1.06 & 35 & 7.43 & 0.42 & 222 & 12.81 & 4.31 & 22 & 8.42 & 1.41 & 83 \\
\hline
\end{tabular}




\begin{tabular}{|c|c|c|c|c|c|c|c|c|c|c|c|c|c|c|c|c|c|c|}
\hline \multicolumn{19}{|c|}{$\begin{array}{l}\text { Table 1.33 Age-Specific Geometric Mean Urinary Phthalate and BPA Levels by Responses, "Are you limited in any way because of difficulty remembering or because you experience periods of confusion?" for Subjects >= 60 year } \\
\text { of age, NHANES 2011-2014, over the LOD }\end{array}$} \\
\hline \multirow{3}{*}{ EFDC } & \multicolumn{6}{|c|}{ Geometric Mean (ng/ml) (GSE, N), Age 60-69 } & \multicolumn{6}{|c|}{ Geometric Mean (ng/ml) (GSE, N), Age 70-79 } & \multicolumn{6}{|c|}{ Geometric Mean (ng/ml) (GSE, N), Age $80+$} \\
\hline & \multicolumn{3}{|c|}{ Yes } & \multicolumn{3}{|c|}{ No } & \multicolumn{3}{|c|}{ Yes } & \multicolumn{3}{|c|}{ No } & \multicolumn{3}{|c|}{ Yes } & \multicolumn{3}{|c|}{ No } \\
\hline & GM & GSE & $\mathbf{N}$ & GM & GSE & $\mathbf{N}$ & GM & GSE & $\mathbf{N}$ & GM & GSE & $\mathbf{N}$ & GM & GSE & $\mathbf{N}$ & GM & GSE & $\mathbf{N}$ \\
\hline Mono (carboxynonyl) Phthalate - CNP & 2.26 & 0.19 & 74 & 2.58 & 0.19 & 457 & 2.34 & 0.31 & 36 & 2.97 & 0.27 & 264 & 2.72 & 0.41 & 54 & 2.54 & 0.17 & 135 \\
\hline Mono (carboxyoctyl) Phthalate - COP & 17.00 & 2.86 & 75 & 18.73 & 2.12 & 457 & 15.80 & 3.19 & 36 & 21.95 & 2.09 & 266 & 20.14 & 5.55 & 54 & 15.98 & 1.80 & 135 \\
\hline Mono-(2-ethyl-5-carboxypentyl) Phthalate -ECP & 15.04 & 1.28 & $74 * *$ & 10.67 & 0.78 & 457 & 13.81 & 1.71 & 36 & 13.65 & 0.73 & 266 & 14.43 & 1.51 & 54 & 13.72 & 0.7918 & 135 \\
\hline Mono-n-butyl Phthalate - MBP & 12.01 & 1.24 & $74 *$ & 9.19 & 0.62 & 450 & 10.96 & 1.18 & 36 & 10.17 & 0.81 & 263 & 13.50 & 1.62 & 54 & 11.75 & 1.09 & 132 \\
\hline Mono-(3-carboxypropyl) Phthalate - $\mathrm{MC1}$ & 2.39 & 0.32 & 73 & 2.57 & 0.24 & 433 & 2.49 & 0.73 & 34 & 3.08 & 0.30 & 253 & 3.03 & 0.74 & 53 & 2.86 & 0.32 & 132 \\
\hline Mono-ethyl Phthalate -MEP & 60.87 & 18.15 & 75 & 58.00 & 6.47 & 458 & 78.19 & 16.52 & 36 & 58.73 & 6.76 & 266 & 39.85 & 9.80 & 54 & 44.95 & 3.76 & 135 \\
\hline Mono (2-ethyl-5-hydroxy hexyl) Phthalate - MHH & 9.27 & 0.93 & $75 * *$ & 6.57 & 0.46 & 457 & 9.27 & 1.26 & 36 & 8.28 & 0.45 & 266 & 7.57 & 0.89 & 54 & 7.84 & 0.48 & 135 \\
\hline Mono-(2-ethyl-5-oxohexyl) Phthalate -MOH & 5.81 & 0.61 & $75 *$ & 4.30 & 0.30 & 457 & 6.08 & 0.76 & 36 & 5.63 & 0.27 & 266 & 5.76 & 0.55 & 54 & 5.76 & 0.38 & 134 \\
\hline Mono-benzyl Phthalate - MZP & 6.16 & 1.00 & $75 *$ & 3.98 & 0.30 & 450 & 5.01 & 0.78 & 36 & 3.79 & 0.30 & 263 & 5.81 & 0.77 & 54 & 5.07 & 0.45 & 132 \\
\hline Mono-isobutyl Phthalate - MIB & 8.96 & 1.09 & $75 * *$ & 6.34 & 0.34 & 454 & 8.73 & 1.26 & $36 *$ & 6.09 & 0.34 & 262 & 7.66 & 0.83 & $54 * *$ & 5.34 & 0.46 & 134 \\
\hline Bisphenol A - BPA & 1.62 & 0.20 & 73 & 1.50 & 0.10 & 434 & 1.28 & 0.28 & 30 & 1.66 & 0.10 & 249 & 1.30 & 0.19 & 51 & 1.48 & 0.14 & 128 \\
\hline
\end{tabular}

\begin{tabular}{|c|c|c|c|c|c|c|c|c|c|c|c|c|}
\hline \multirow{4}{*}{ EEDC } & \multicolumn{6}{|c|}{ Geometric Mean (ng/ml) (GSE, N) } & \multicolumn{6}{|c|}{ Geometric Mean (ng/ml) (GSE, N) } \\
\hline & \multicolumn{6}{|c|}{ Male } & \multicolumn{6}{|c|}{ Female } \\
\hline & \multicolumn{3}{|c|}{ Yes } & \multicolumn{3}{|c|}{ No } & \multicolumn{3}{|c|}{ Yes } & \multicolumn{3}{|c|}{ No } \\
\hline & GM & GSE & $\mathrm{N}$ & GM & GSE & $\mathrm{N}$ & GM & GSE & $\mathrm{N}$ & GM & GSE & $\mathrm{N}$ \\
\hline Mono (carboxynonyl) Phthalate - CNP & 2.22 & 0.25 & 72 & 2.38 & 0.14 & 442 & 2.60 & 0.34 & 92 & 3.05 & 0.27 & 414 \\
\hline Mono (carboxyoctyl) Phthalate - COP & 15.30 & 3.29 & 72 & 16.88 & 1.30 & 444 & 19.62 & 3.96 & 93 & 22.02 & 2.68 & 414 \\
\hline Mono-(2-ethyl-5-carboxypentyl) Phthalate-ECP & 14.22 & 1.45 & $72 *$ & 10.68 & 0.55 & 445 & 14.70 & 1.19 & 92 & 13.44 & 0.90 & 413 \\
\hline Mono-n-butyl Phthalate - MBP & 9.91 & 0.84 & 72 & 9.32 & 0.68 & 435 & 14.16 & 1.28 & $92 *$ & 10.35 & 0.55 & 410 \\
\hline Mono-(3-carboxypropyl) Phthalate - MC1 & 2.49 & 0.69 & 71 & 2.53 & 0.19 & 426 & 2.73 & 0.37 & 89 & 3.03 & 0.29 & 392 \\
\hline Mono-ethyl Phthalate -MEP & 61.56 & 7.92 & 72 & 52.27 & 5.08 & 445 & 51.93 & 10.58 & 93 & 60.39 & 6.37 & 414 \\
\hline Mono (2-ethyl-5-hydroxy hexyl) Phthalate - MHH & 8.70 & 0.77 & $72 *$ & 6.70 & 0.36 & 445 & 8.56 & 0.84 & 93 & 7.86 & 0.45 & 413 \\
\hline Mono-(2-ethyl-5-oxohexyl) Phthalate -MOH & 5.87 & 0.53 & $72 *$ & 4.53 & 0.24 & 443 & 5.85 & 0.53 & 93 & 5.28 & 0.33 & 414 \\
\hline Mono-benzyl Phthalate - MZP & 4.31 & 0.87 & 72 & 3.64 & 0.30 & 438 & 6.96 & 0.73 & $93 * * *$ & 4.51 & 0.25 & 407 \\
\hline Mono-isobutyl Phthalate - MIB & 8.47 & 0.82 & $72 * * *$ & 5.61 & 0.29 & 442 & 8.38 & 0.88 & $93 *$ & 6.65 & 0.33 & 408 \\
\hline Bisphenol A - BPA & 1.61 & 0.28 & 64 & 1.38 & 0.07 & 424 & 1.32 & 0.12 & 90 & 1.75 & 0.11 & $387 *$ \\
\hline
\end{tabular}




\begin{tabular}{|c|c|c|c|c|c|c|c|c|c|}
\hline EEDC & Yes/No & $\begin{array}{l}\text { Unadjusted } \\
\text { Odds Ratio }^{1}\end{array}$ & $95 \% \mathrm{CI}$ & Yes/No & $\begin{array}{c}\text { Adjusted Odds } \\
\text { Ratio \#1 }\end{array}$ & $95 \% \mathrm{CI}$ & Yes $/$ No & \begin{tabular}{|c|} 
Adjusted Odds \\
Ratio $\# 2^{3}$
\end{tabular} & $95 \% \mathrm{CI}$ \\
\hline \multicolumn{10}{|l|}{ CNP - Above / Below 50 th Percentile - Males } \\
\hline <LOD to 50th percentile (reference) & $42 / 217$ & 1.00 & & $42 / 216$ & 1.00 & & $37 / 202$ & 1.00 & \\
\hline$\geq 50$ th percentile & 30228 & 0.645 & $0.348-1.194$ & 30228 & 0.614 & $0.352-1.069$ & $27 / 214$ & 0.642 & $0.337-1.220$ \\
\hline \multicolumn{10}{|l|}{ CNP - Above Below 50th Percentile - Females } \\
\hline$<\mathrm{LOD}$ to 50 th percentile (reference) & $48 / 206$ & 1.00 & & $48 / 205$ & 1.00 & & 42192 & 1.00 & \\
\hline$\geq 50$ th percentile & $45 / 208$ & 1.018 & $0.555-1.870$ & $44 / 208$ & 1.389 & $0.747-2.584$ & 42191 & 1.649 & $0.833-3.264$ \\
\hline \multicolumn{10}{|l|}{ COP - Above / Below 50th Percentile - Males } \\
\hline <LOD to 50th percentile (reference) & $41 / 217$ & 1.00 & & $41 / 217$ & 1.00 & & $38 / 202$ & 1.00 & \\
\hline$\geq 50$ th percentile & $31 / 228$ & 1.031 & $0.445-2.393$ & $31 / 227$ & 1.129 & $0.525-2.431$ & $26 / 214$ & 1.074 & $0.504-2.291$ \\
\hline \multicolumn{10}{|l|}{ COP - Above Below 50 th Percentile - Females } \\
\hline$<$ LOD to 50 th percentile (reference) & $46 / 207$ & 1.00 & & $45 / 207$ & 1.00 & & 40193 & 1.00 & \\
\hline$\geq 50$ th percentile & $47 / 207$ & 0.923 & $0.467-1.823$ & $47 / 206$ & 1.177 & $0.568-2.437$ & 44190 & 1.288 & $0.553-3.002$ \\
\hline \multicolumn{10}{|l|}{ ECP - Above Below 50 th Percentile - Males } \\
\hline <LOD to 50th percentile (reference) & $35 / 223$ & 1.00 & & $35 / 223$ & 1.00 & & 30210 & 1.00 & \\
\hline$\geq 50$ th percentile & $37 / 222$ & 1.537 & $0.770-3.068$ & $37 / 221$ & 1.275 & $0.564-2.887$ & 34206 & 1.405 & $0.504-3.917$ \\
\hline \multicolumn{10}{|l|}{ ECP - Above/Below 50th Percentile - Females } \\
\hline$<$ LOD to 50th percentile (reference) & $43 / 210$ & 1.00 & & $42 / 210$ & 1.00 & & $35 / 198$ & 1.00 & \\
\hline$\geq 50$ th percentile & 50204 & 1.128 & $0.666-1.909$ & 50203 & 1.027 & $0.554-1.903$ & $49 / 185$ & 1.097 & $0.599-2.008$ \\
\hline \multicolumn{10}{|l|}{ MBP - Above / Below 50th Percentile - Males } \\
\hline <LOD to 50th percentile (reference) & $31 / 227$ & 1.00 & & $31 / 227$ & 1.00 & & $25 / 215$ & 1.00 & \\
\hline$\geq 50$ th percentile & $41 / 218$ & 1.800 & $0.803-4.033$ & $41 / 217$ & 1.562 & $0.679-3.591$ & $39 / 201$ & 2.272 & $0.853-6.049$ \\
\hline \multicolumn{10}{|l|}{ MBP - Above Below 50th Percentile - Females } \\
\hline$<$ LOD to 50th percentile (reference) & $36 / 217$ & 1.00 & & $36 / 216$ & 1.00 & & $33 / 200$ & 1.00 & \\
\hline$\geq 50$ th percentile & $57 / 197$ & $1.721 *$ & $1,083-2.735$ & 56197 & 1.350 & $0.820-2.221$ & $51 / 183$ & 1.183 & $0.728-1.923$ \\
\hline \multicolumn{10}{|l|}{$\mathrm{MCl}$ - Above Below 50th Percentile - Males } \\
\hline$<$ LOD to 50th percentile (reference) & $43 / 216$ & 1.00 & & $43 / 214$ & 1.00 & & $39 / 201$ & 1.00 & \\
\hline$\geq 50$ th percentile & $29 / 229$ & 0.943 & $0.323-2.750$ & $29 / 230$ & 0.907 & $0.331-2.487$ & $25 / 215$ & 0.917 & $0.290-2.901$ \\
\hline $\mathrm{MCl}$ - Above Below 50th Percentile - Femal & & & & & & & & & \\
\hline$<$ LOD to 50 th percentile (reference) & $46 / 207$ & 1.00 & & $45 / 207$ & 1.00 & & $41 / 193$ & 1.00 & \\
\hline$\geq 50$ th percentile & $47 / 207$ & 0.877 & $0.466-1.650$ & $47 / 206$ & 0.994 & $0.516-1.915$ & $43 / 190$ & 0.808 & $0.408-1.600$ \\
\hline MEP - Above / Below 50th Percentile - Male & & & & & & & & & \\
\hline$<$ LOD to 50th percentile (reference) & $28 / 230$ & 1.00 & & $28 / 230$ & 1.00 & & $27 / 213$ & 1.00 & \\
\hline$\geq 50$ th percentile & $44 / 215$ & 1.605 & $0.955-2.698$ & $44 / 214$ & 1.765 & $0.948-3.287$ & $37 / 203$ & 1.866 & $0.927-3.757$ \\
\hline MEP - Above Below 50th Percentile - Femal & & & & & & & & & \\
\hline$<$ LOD to 50th percentile (reference) & $48 / 205$ & 1.00 & & $47 / 205$ & 1.00 & & $41 / 192$ & 1.00 & \\
\hline$\geq 50$ th percentile & $45 / 209$ & 0.915 & $0.511-1.640$ & $45 / 208$ & 0.900 & $0.467-1.733$ & $43 / 191$ & 1.215 & $0.557-2.652$ \\
\hline MHH-Above/Below 50th Percentile - Male & & & & & & & & & \\
\hline$<$ LOD to 50 th percentile (reference) & $27 / 231$ & 1.00 & & $27 / 231$ & 1.00 & & $23 / 217$ & 1.00 & \\
\hline$\geq 50$ th percentile & $45 / 214$ & $2.460 *$ & $1.392-4.350$ & $45 / 213$ & $2.444 * *$ & $1278-4.675$ & $41 / 199$ & $3.029 \div *$ & $1.441-6.366$ \\
\hline MHH - A bove Below 50 th Percentile - Femal & & & & & & & & & \\
\hline$<$ LOD to 50 th percentile (reference) & $38 / 215$ & 1.00 & & $38 / 214$ & 1.00 & & 34199 & 1.00 & \\
\hline$\geq 50$ th percentile & $55 / 199$ & 1.560 & $0.845-2.879$ & $54 / 199$ & 1.297 & $0.667-2.523$ & 50184 & 1.173 & $0.593-2.321$ \\
\hline $\mathrm{MOH}-\mathrm{Above} /$ Below 50 th Percentile - Male & & & & & & & & & \\
\hline$<$ LOD to 50 th percentile (reference) & $28 / 230$ & 1.00 & & $28 / 230$ & 1.00 & & $25 / 215$ & 1.00 & \\
\hline$\geq 50$ th percentile & $44 / 215$ & $2.050 *$ & $1.016-4.136$ & $44 / 214$ & 1.744 & $0.868-3.504$ & $39 / 201$ & 1.767 & 0.7564 .128 \\
\hline MOH - A bove Below 50th Percentile - Femal & & & & & & & & & \\
\hline$<$ LOD to 50 th percentile (reference) & $42 / 212$ & 1.00 & & $41 / 211$ & 1.00 & & $36 / 197$ & 1.00 & \\
\hline$\geq 50$ th percentile & $51 / 202$ & 1.172 & $0.677-2.029$ & $51 / 202$ & 1.008 & $0.557-1.827$ & $48 / 186$ & 0.922 & $0.511-1.663$ \\
\hline MZP - Above Below 50th Percentile - Male & & & & & & & & & \\
\hline <LOD to 50th percentile (reference) & $38 / 220$ & 1.00 & & $38 / 220$ & 1.00 & & 34206 & 1.00 & \\
\hline$\geq 50$ th percentile & 34225 & 1.179 & $0.489-2.844$ & 34224 & 1.084 & $0.491-2.395$ & 30210 & 1.117 & $0.465-2.683$ \\
\hline MZP - Above Below 50th Percentile - Femal & & & & & & & & & \\
\hline$<$ LOD to 50 th percentile (reference) & $29 / 224$ & 1.00 & & $29 / 223$ & 1.00 & & $27 / 206$ & 1.00 & \\
\hline$\geq 50$ th percentile & 64190 & $3.135 * \pi \hbar$ & $1965-5.001$ & $63 / 190$ & $2.863 * \pi *$ & $1.719-4.768$ & $57 / 177$ & $2.124 * \pi$ & 1.151 .3922 \\
\hline MIB - Above / Below 50th Percentile - Male & & & & & & & & & \\
\hline <LOD to 50 th percentile (reference) & $24 / 234$ & 1.00 & & $24 / 234$ & 1.00 & & $21 / 219$ & 1.00 & \\
\hline$\geq 50$ th percentile & $48 / 211$ & $3.779 \div \div$ & $1.756-8.131$ & $48 / 210$ & $3.818 * *$ & $1.742-8.369$ & $43 / 197$ & $3.953 \div$ & $1.691-9.242$ \\
\hline MIB - Above/ Below 50th Percentile - Female & & & & & & & & & \\
\hline$<$ LOD to 50 th percentile (reference) & $39 / 214$ & 1.00 & & $39 / 213$ & 1.00 & & $35 / 198$ & 1.00 & \\
\hline$\geq 50$ th percentile & 54200 & $1.786 *$ & $1.046-3.049$ & $53 / 200$ & 1.487 & $0.759-2.913$ & $49 / 185$ & 1.541 & $0.768-3.090$ \\
\hline BPA - Above Below 50th Percentile - Male & & & & & & & & & \\
\hline <LOD to 50th percentile (reference) & $37 / 221$ & 1.00 & & $37 / 221$ & 1.00 & & 32208 & 1.00 & \\
\hline$\geq 50$ th percentile & $35 / 224$ & 1.044 & $0.496-2.200$ & $35 / 223$ & 0.959 & $0.512-1.797$ & 32208 & 0.945 & $0.443-2.014$ \\
\hline BPA - Above Below 50th Percentile - Female & & & & & & & & & \\
\hline$<$ LOD to 50 th percentile (reference) & $53 / 200$ & 1.00 & & 52199 & 1.00 & & $46 / 187$ & 1.00 & \\
\hline$\geq 50$ th percentile & $40: 214$ & 0.513 & $0.249-1.058$ & 40214 & 0.544 & $0249-1.187$ & $38 / 196$ & 0.567 & $0.254-1.270$ \\
\hline & NHANE & ampling wei & it applied bef & cakulatir & the geometric & & & & \\
\hline The EEDCs were creatinine-adjusted then & $\begin{array}{r}\text { formed } \\
\text { app }\end{array}$ & to analyses. & $\begin{array}{l}\text { Extreme creati } \\
\text { atement in SAS }\end{array}$ & values: & $\begin{array}{l}0 \mathrm{mg} / \mathrm{dL} \text { and }>30 \\
<0.01 * * * \mathrm{p}<0.00\end{array}$ & $00 \mathrm{mg} / \mathrm{dL}$ ) an & DCs leve & e into accou & tusing the \\
\hline Unadjusted model ${ }^{2}$ Adjusted for age, educa & ato $a$ & ${ }^{3}$ Adjusted & rage, educe & celet & ; alcoholuse & diabetes $\mathrm{s}$ & gh bloc & ssure status & conary hear \\
\hline
\end{tabular}




\section{Associations Between Exposures to Phthalate and BPA and Taste/Smell Function}

Exposures to the 12 phthalate metabolites and BPA and two indicators of taste/smell function: 1) Do you sometimes smell an unpleasant, bad, or burning odor when nothing is there (phantosmia), and 2) During the past 12 months have you had a taste or other sensation in your mouth that does not go away? Taste and smell indicators have been included as a surrogate indicator of brain health as taste and smell dysfunction is a possible pre-clinical indicator in the development of $\mathrm{AD}$ and other memory-related neurodegenerative diseases.

Smell Dysfunction and Exposure to BPA and Phthalates: Table 1.37 presents crude GMs and GSEs for subjects with phthalate metabolite and BPA measurements over the LOD who provided a response to the question, "Do you sometimes smell an unpleasant, bad, or burning odor when nothing is there?". The GM for the phthalate metabolite MIB was found to be significantly higher in those that experienced phantosmia $(\mathrm{p}<0.05)$. Table 1.38 presents age-specific GMs and GSEs. In the 60-69 age group, the GM for the phthalate MIB was found to be significantly higher in those that experienced phantom odors $(\mathrm{p}<0.05)$. In the $70-79$ age group, the GM for the phthalate MEP was found to be significantly higher in those that experienced phantom odors $(\mathrm{p}<0.01)$. Table 1.39 presents gender-specific GMs and GSEs. In females, the GMs for the phthalate metabolites MBP and MOH were significantly higher in those that experienced phantom odors (MBP, $\mathrm{p}<0.01 ; \mathrm{MOH}, \mathrm{p}<0.05)$. Table 1.40 presents race-specific GMs and GSEs. Among non-Hispanic blacks, the GM for phthalate metabolite MC1 was significantly higher in those that experienced phantom odors $(\mathrm{p}<0.01)$. The GM for BPA among Asian/other was significantly higher in those that experienced phantom odors $(\mathrm{p}<0.05)$. 
Table 1.41 presents estimated ORs and 95\% CI for subjects with measurable phthalate metabolite and BPA levels who responded to the question "Do you sometimes smell and unpleasant, bad, or burning odor when nothing is there?". For the phthalate metabolite MEP, among females in the $\geq 50$ th percentile compared to the reference group, MEP concentrations are significantly associated with phantom odor $(\mathrm{OR}=2.036$, 95\% CI: 1.047-3.958) in the unadjusted model (Table 1.41). 


\begin{tabular}{|c|c|c|c|c|c|c|}
\hline \multirow{3}{*}{ EEDC } & \multicolumn{6}{|c|}{ Geometric Mean (ng/ml) (GSE, N) } \\
\hline & \multicolumn{3}{|c|}{ Yes } & \multicolumn{3}{|c|}{ No } \\
\hline & GM & GSE & $\mathbf{N}$ & GM & GSE & $\mathbf{N}$ \\
\hline Mono (carboxynonyl) Phthalate - CNP & 2.51 & 0.27 & 73 & 2.67 & 0.14 & 947 \\
\hline Mono (carboxyoctyl) Phthalate - COP & 21.90 & 3.90 & 74 & 18.91 & 1.48 & 949 \\
\hline Mono-(2-ethyl-5-carboxypentyl) Phthalate -ECP & 13.63 & 1.69 & 74 & 12.16 & 0.56 & 948 \\
\hline Mono-n-butyl Phthalate - MBP & 11.55 & 1.32 & 74 & 10.00 & 0.52 & 935 \\
\hline Mono-(3-carboxypropyl) Phthalate - MC1 & 2.50 & 0.37 & 70 & 2.77 & 0.18 & 907 \\
\hline Mono-ethyl Phthalate -MEP & 75.21 & 16.90 & 74 & 55.11 & 4.33 & 950 \\
\hline Mono (2-ethyl-5-hydroxy hexyl) Phthalate - MHH & 8.36 & 1.16 & 73 & 7.34 & 0.31 & 950 \\
\hline Mono-(2-ethyl-5-oxohexyl) Phthalate -MOH & 5.51 & 0.74 & 74 & 4.96 & 0.23 & 948 \\
\hline Mono-benzyl Phthalate - MZP & 4.84 & 0.73 & 73 & 4.19 & 0.23 & 936 \\
\hline Mono-isobutyl Phthalate - MIB & 7.57 & 0.66 & $74 *$ & 6.28 & 0.28 & 940 \\
\hline Bisphenol A - BPA & 1.51 & 0.18 & 70 & 1.53 & 0.07 & 895 \\
\hline
\end{tabular}

NHANES sampling weight applied before calculating the geometric mean.

The EEDCs were creatinine-adjusted then log transformed prior to analyses. Extreme creatinine values $(<30$ $\mathrm{mg} / \mathrm{dL}$ and $>300 \mathrm{mg} / \mathrm{dL}$ ) and EEDCs levels below the LOD were taken into account using the appropriate domain statement in SAS. $* \mathrm{p}<0.05, * * \mathrm{p}<0.01 * * * \mathrm{p}<0.001$

\begin{tabular}{|c|c|c|c|c|c|c|c|c|c|c|c|c|}
\hline \multirow{3}{*}{ EEDC } & \multicolumn{6}{|c|}{ Geometric Mean (ng/ml) (GSE, N), Hispanic } & \multicolumn{6}{|c|}{ Geometric Mean (ng/ml) (GSE, N), Non-Hispanic White } \\
\hline & \multicolumn{3}{|c|}{ Yes } & \multicolumn{3}{|c|}{ No } & \multicolumn{3}{|c|}{ Yes } & \multicolumn{3}{|c|}{ No } \\
\hline & GM & GSE & $\mathbf{N}$ & GM & GSE & $\mathbf{N}$ & GM & GSE & $\mathbf{N}$ & GM & GSE & $\mathbf{N}$ \\
\hline Mono (carboxynonyl) Phthalate - CNP & 1.93 & 0.30 & 21 & 2.43 & 0.13 & 194 & 2.68 & 0.34 & 25 & 2.82 & 0.18 & 417 \\
\hline Mono (carboxyoctyl) Phthalate - COP & 15.73 & 3.97 & 21 & 20.28 & 1.65 & 194 & 24.01 & 5.79 & 25 & 19.94 & 1.90 & 417 \\
\hline Mono-(2-ethyl-5-carboxypentyl) Phthalate -ECP & 16.79 & 1.91 & 21 & 18.21 & 1.05 & 194 & 13.28 & 2.47 & 25 & 11.74 & 0.60 & 416 \\
\hline Mono-n-butyl Phthalate - MBP & 12.02 & 2.26 & 21 & 13.93 & 0.99 & 192 & 11.13 & 1.85 & 25 & 9.29 & 0.60 & 410 \\
\hline Mono-(3-carboxypropyl) Phthalate - MC1 & 2.49 & 0.37 & 18 & 2.86 & 0.27 & 185 & 2.45 & 0.52 & 24 & 2.84 & 0.23 & 400 \\
\hline Mono-ethyl Phthalate -MEP & 141.12 & 46.34 & 21 & 91.49 & 11.53 & 194 & 68.51 & 22.09 & 25 & 50.11 & 4.51 & 417 \\
\hline Mono (2-ethyl-5-hydroxy hexyl) Phthalate - MHH & 9.96 & 1.25 & 21 & 10.47 & 0.73 & 194 & 8.19 & 1.63 & 24 & 7.07 & 0.34 & 417 \\
\hline Mono-(2-ethyl-5-oxohexyl) Phthalate -MOH & 6.42 & 0.82 & 21 & 6.76 & 0.48 & 194 & 5.38 & 1.04 & 25 & 4.81 & 0.25 & 415 \\
\hline Mono-benzyl Phthalate - MZP & 4.09 & 0.87 & 21 & 3.98 & 0.36 & 194 & 5.24 & 1.18 & 24 & 4.13 & 0.26 & 411 \\
\hline Mono-isobutyl Phthalate - MIB & 9.59 & 1.13 & 21 & 9.14 & 0.48 & 194 & 7.31 & 0.92 & 25 & 5.74 & 0.27 & 413 \\
\hline Bisphenol A - BPA & 1.57 & 0.33 & 21 & 1.62 & 0.17 & 184 & 1.61 & 0.26 & 23 & 1.55 & 0.09 & 396 \\
\hline \multirow{3}{*}{ EEDC } & \multicolumn{6}{|c|}{ Geometric Mean (ng/ml) (GSE, N), Non-Hispanic Black } & \multicolumn{6}{|c|}{ Geometric Mean (ng/ml) (GSE, N), Asian/Other } \\
\hline & \multicolumn{3}{|c|}{ Yes } & \multicolumn{3}{|c|}{ No } & \multicolumn{3}{|c|}{ Yes } & \multicolumn{3}{|c|}{ No } \\
\hline & GM & GSE & $\mathrm{N}$ & GM & GSE & $\mathrm{N}$ & GM & GSE & $\mathrm{N}$ & GM & GSE & $\mathrm{N}$ \\
\hline Mono (carboxynonyl) Phthalate - CNP & 2.06 & 0.46 & 21 & 1.96 & 0.10 & 241 & 3.91 & 1.70 & $\overline{6}$ & 2.23 & 0.34 & 95 \\
\hline Mono (carboxyoctyl) Phthalate - COP & 22.09 & 6.62 & 21 & 13.66 & 0.79 & 240 & 17.73 & 5.57 & 7 & 13.74 & 2.11 & 98 \\
\hline Mono-(2-ethyl-5-carboxypentyl) Phthalate -ECP & 12.44 & 2.33 & 21 & 10.89 & 0.63 & 240 & 12.93 & 2.83 & 7 & 13.53 & 0.96 & 98 \\
\hline Mono-n-butyl Phthalate - MBP & 10.82 & 1.83 & 21 & 11.03 & 0.58 & 237 & 18.60 & 5.19 & 7 & 15.02 & 2.59 & 96 \\
\hline Mono-(3-carboxypropyl) Phthalate - MC1 & 3.30 & 0.56 & $21 * *$ & 2.12 & 0.12 & 233 & 1.70 & 0.62 & 7 & 2.90 & 0.40 & 89 \\
\hline Mono-ethyl Phthalate -MEP & 64.66 & 17.91 & 21 & 81.72 & 8.27 & 241 & 58.36 & 35.50 & 7 & 52.76 & 11.50 & 98 \\
\hline Mono (2-ethyl-5-hydroxy hexyl) Phthalate - MHH & 8.05 & 1.81 & 21 & 7.40 & 0.46 & 241 & 7.27 & 2.00 & 7 & 7.41 & 0.77 & 98 \\
\hline Mono-(2-ethyl-5-oxohexyl) Phthalate -MOH & 5.44 & 1.08 & 21 & 4.97 & 0.27 & 241 & 5.00 & 1.26 & 7 & 4.90 & 0.54 & 98 \\
\hline Mono-benzyl Phthalate - MZP & 4.93 & 0.77 & 21 & 4.69 & 0.44 & 238 & 2.94 & 0.89 & 7 & 4.55 & 1.36 & 93 \\
\hline Mono-isobutyl Phthalate - MIB & 6.76 & 0.82 & 21 & 7.72 & 0.46 & 236 & 7.87 & 0.78 & 7 & 9.46 & 1.56 & 97 \\
\hline Bisphenol A - BPA & 1.30 & 0.29 & 21 & 1.42 & 0.06 & 233 & 0.78 & 0.20 & 5 & 1.37 & 0.16 & $82 *$ \\
\hline
\end{tabular}




\begin{tabular}{|c|ccc|cc|c|ccc|cc|c|c|c|}
\hline \multicolumn{10}{|c|}{ Table 1.38 Age-specific Geometric Mean Urinary Phthalate and BPA Levels by Responses, "Do you sometimes smell an unpleasant, bad or burning odor when nothing is there?" for Subjects >= 60 years of age, NHANES 2011- } \\
\hline
\end{tabular}

Table 1.39 Gender-specific Geometric Mean Urinary Phthalate and BPA Levels by Responses, "Do you sometimes smell an unpleas ant, bad or burning odor when nothing is there?" for Subjects >= 60 years of age, NHANES 2011-2014, over the LOD

\begin{tabular}{|c|c|c|c|c|c|c|c|c|c|c|c|c|}
\hline \multirow{4}{*}{ EEDC } & \multicolumn{6}{|c|}{ Geometric Mean (ng/ml) (GSE, N) } & \multicolumn{6}{|c|}{ Geometric Mean (ng/ml) (GSE, N) } \\
\hline & \multicolumn{6}{|c|}{$\begin{array}{l}\text { Male } \\
\end{array}$} & \multicolumn{6}{|c|}{$\begin{array}{c}\text { Female } \\
\end{array}$} \\
\hline & \multicolumn{3}{|c|}{ Yes } & \multicolumn{3}{|c|}{ No } & \multicolumn{3}{|c|}{ Yes } & \multicolumn{3}{|c|}{ No } \\
\hline & GM & GSE & $\mathrm{N}$ & GM & GSE & $\mathrm{N}$ & GM & GSE & $\mathrm{N}$ & GM & GSE & $\mathrm{N}$ \\
\hline Mono (carboxynonyl) Phthalate - CNP & 2.62 & 0.36 & 30 & 2.34 & 0.12 & 484 & 2.42 & 0.35 & 43 & 3.02 & 0.25 & 463 \\
\hline Mono (carboxyoctyl) Phthalate - COP & 21.12 & 7.05 & 31 & 16.45 & 1.15 & 485 & 22.56 & 4.12 & 43 & 21.61 & 2.53 & 464 \\
\hline Mono-(2-ethyl-5-carboxypentyl) Phthalate -ECP & 11.11 & 2.70 & 31 & 10.95 & 0.50 & 486 & 16.07 & 2.01 & 43 & 13.45 & 0.86 & 462 \\
\hline Mono-n-butyl Phthalate - MBP & 8.03 & 1.66 & 31 & 9.45 & 0.66 & 476 & 15.49 & 1.62 & $43 * *$ & 10.55 & 0.57 & 459 \\
\hline Mono-(3-carboxypropyl) Phthalate - MC1 & 2.48 & 0.68 & 30 & 2.53 & 0.18 & 466 & 2.52 & 0.35 & 40 & 3.02 & 0.26 & 441 \\
\hline Mono-ethyl Phthalate -MEP & 84.50 & 39.48 & 31 & 51.73 & 4.49 & 486 & 68.45 & 9.74 & 43 & 58.56 & 5.94 & 464 \\
\hline Mono (2-ethyl-5-hydroxy hexyl) Phthalate - MHH & 7.08 & 1.88 & 31 & 6.84 & 0.33 & 486 & 9.62 & 1.34 & 42 & 7.86 & 0.43 & 464 \\
\hline Mono-(2-ethyl-5-oxohexyl) Phthalate -MOH & 4.57 & 1.27 & 31 & 4.63 & 0.23 & 484 & 6.42 & 0.64 & $43 *$ & 5.29 & 0.31 & 464 \\
\hline Mono-benzyl Phthalate - MZP & 4.22 & 1.24 & 31 & 3.68 & 0.31 & 478 & 5.43 & 0.53 & 42 & 4.75 & 0.27 & 458 \\
\hline Mono-isobutyl Phthalate - MIB & 7.26 & 1.13 & 31 & 5.78 & 0.31 & 482 & 7.83 & 0.64 & 43 & 6.81 & 0.35 & 458 \\
\hline Bisphenol A - BPA & 1.33 & 0.26 & 29 & 1.40 & 0.07 & 459 & 1.68 & 0.22 & 41 & 1.68 & 0.10 & 436 \\
\hline
\end{tabular}

The EEDCs were creatinine-adjusted then log transformed prior to analyses. Extreme creatinine values ( $<30 \mathrm{mg} / \mathrm{dL}$ and $>300 \mathrm{mg} / \mathrm{dL})$ and EEDCs levels below the LOD were taken into account using the appropriate domain statement in SAS. *p $<0.05, * * \mathrm{p}<0.01 * * * \mathrm{p}<0.001$ 


\begin{tabular}{|c|c|c|c|c|c|c|c|c|c|}
\hline EHDC & Yes/No & $\begin{array}{c}\text { Unadjusted } \\
\text { Odds Ratio }^{1}\end{array}$ & $95 \% \mathrm{CI}$ & Yes/No & $\begin{array}{l}\text { Adjusted Odds } \\
\text { Ratio \#1 }{ }^{2}\end{array}$ & $95 \% \mathrm{CI}$ & Yes/No & 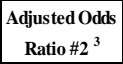 & $95 \% \mathrm{CI}$ \\
\hline \multicolumn{10}{|l|}{ CNP - Above/Below 50th Percentile - Males } \\
\hline $\begin{array}{l}<\text { LOD to } 50 \text { th percentile (reference) } \\
\quad \geq 50 \text { th percentile }\end{array}$ & $\begin{array}{l}15 / 243 \\
16 / 243\end{array}$ & $\begin{array}{c}1.00 \\
1.254\end{array}$ & $0.483-3.256$ & $\begin{array}{l}15 / 242 \\
16 / 243\end{array}$ & $\begin{array}{c}1.00 \\
1.532\end{array}$ & $0.510-4.600$ & $\begin{array}{l}13 / 227 \\
13 / 227\end{array}$ & $\begin{array}{c}1.00 \\
1.601\end{array}$ & $0.659-3.890$ \\
\hline \multicolumn{10}{|l|}{ CNP - Above/Below 50th Percentile - Females } \\
\hline $\begin{array}{c}<\text { LOD to 50th percentile (reference) } \\
\geq 50 \text { th percentile }\end{array}$ & $\begin{array}{l}26 / 228 \\
17 / 236\end{array}$ & $\begin{array}{c}1.00 \\
0.649\end{array}$ & $0.274-1.537$ & $\begin{array}{l}26 / 227 \\
17 / 235\end{array}$ & $\begin{array}{c}1.00 \\
0.694\end{array}$ & $0.312-1.540$ & $\begin{array}{l}23 / 211 \\
17 / 216\end{array}$ & $\begin{array}{c}1.00 \\
0.906\end{array}$ & $0.396-2.075$ \\
\hline \multicolumn{10}{|l|}{ COP - Above/Below 50th Percentile - Males } \\
\hline $\begin{array}{c}<\text { LOD to 50th percentile (reference) } \\
\quad \geq 50 \text { th percentile }\end{array}$ & $\begin{array}{l}12 / 246 \\
19 / 240\end{array}$ & $\begin{array}{l}1.00 \\
1.403\end{array}$ & $0.292-6.732$ & $\begin{array}{l}12 / 246 \\
19 / 239\end{array}$ & $\begin{array}{c}1.00 \\
1.258\end{array}$ & $0.253-6.258$ & $\begin{array}{l}10 / 230 \\
16 / 224\end{array}$ & $\begin{array}{c}1.00 \\
0.839\end{array}$ & $0.129-5.478$ \\
\hline \multicolumn{10}{|l|}{ COP - Above/Below 50th Percentile - Females } \\
\hline $\begin{array}{l}<\text { LOD to 50th percentile (reference) } \\
\geq 50 \text { th percentile }\end{array}$ & $\begin{array}{l}17 / 236 \\
26 / 228\end{array}$ & $\begin{array}{l}1.00 \\
1.880\end{array}$ & $0.814-4.339$ & $\begin{array}{l}17 / 235 \\
26 / 227\end{array}$ & $\begin{array}{c}1.00 \\
1.981\end{array}$ & $0.892-4.401$ & $\begin{array}{l}16 / 217 \\
24 / 210\end{array}$ & $\begin{array}{c}1.00 \\
2.317\end{array}$ & $0.891-6.022$ \\
\hline \multicolumn{10}{|l|}{ ECP - Above/Below 50th Percentile - Males } \\
\hline $\begin{array}{l}<\text { LOD to 50th percentile (reference) } \\
\quad \geq 50 \text { th percentile }\end{array}$ & $\begin{array}{l}13 / 245 \\
18 / 241\end{array}$ & $\begin{array}{c}1.00 \\
1.741\end{array}$ & $0.449-6.744$ & $\begin{array}{l}13 / 245 \\
18 / 240\end{array}$ & $\begin{array}{c}1.00 \\
2.210\end{array}$ & $0.582-8.389$ & $\begin{array}{l}12 / 228 \\
14 / 226\end{array}$ & $\begin{array}{l}1.00 \\
1.264\end{array}$ & $0.305-5.247$ \\
\hline \multicolumn{10}{|l|}{ ECP - Above/Below 50th Percentile - Females } \\
\hline$<$ LOD to 50th percentile (reference) & $18 / 235$ & 1.00 & & $18 / 234$ & 1.00 & & $15 / 218$ & 1.00 & \\
\hline$\geq 50$ th percentile & $25 / 229$ & 1.724 & $0.701-4.240$ & $25 / 228$ & 1.708 & $0.672-4.341$ & $25 / 209$ & 1.755 & $0.702-4.388$ \\
\hline \multicolumn{10}{|l|}{ MBP - Above/Below 50th Percentile - Males } \\
\hline $\begin{array}{l}<\text { LOD to 50th percentile (reference) } \\
\geq 50 \text { th percentile }\end{array}$ & $\begin{array}{l}17 / 241 \\
14 / 245\end{array}$ & $\begin{array}{c}1.00 \\
0.980\end{array}$ & $0.252-3.817$ & $\begin{array}{l}17 / 241 \\
14 / 244\end{array}$ & $\begin{array}{c}1.00 \\
1.056\end{array}$ & $0.222-5.026$ & $\begin{array}{l}14 / 226 \\
12 / 228\end{array}$ & $\begin{array}{c}1.00 \\
1.577\end{array}$ & $0.315-7.881$ \\
\hline \multicolumn{10}{|l|}{ MBP - Above/Below 50th Percentile - Females } \\
\hline$<$ LOD to 50th percentile (reference) & $18 / 235$ & 1.00 & & $18 / 234$ & 1.00 & & $18 / 215$ & 1.00 & \\
\hline$\geq 50$ th percentile & $25 / 229$ & 1.945 & $0.907-4.168$ & $25 / 228$ & 1.843 & $0.912-3.724$ & $22 / 215$ & 1.539 & $0.719-3.294$ \\
\hline \multicolumn{10}{|l|}{ MC1 - Above/Below 50th Percentile - Males } \\
\hline $\begin{array}{c}<\text { LOD to 50th percentile (reference) } \\
\geq 50 \text { th percentile }\end{array}$ & $\begin{array}{l}17 / 242 \\
14 / 244\end{array}$ & $\begin{array}{l}1.00 \\
1.016\end{array}$ & $0.265-3.899$ & $\begin{array}{l}17 / 240 \\
14 / 245\end{array}$ & $\begin{array}{c}1.00 \\
1.095\end{array}$ & $0.251-4.771$ & $\begin{array}{l}15 / 225 \\
11 / 229\end{array}$ & $\begin{array}{c}1.00 \\
0.887\end{array}$ & $0.167-4.702$ \\
\hline \multicolumn{10}{|l|}{ MC1 - Above/Below 50th Percentile - Females } \\
\hline$<$ LOD to 50th percentile (reference) & $21 / 232$ & 1.00 & & $21 / 231$ & 1.00 & & $18 / 216$ & 1.00 & \\
\hline$\geq 50$ th percentile & $23 / 232$ & 0.806 & $0.436-1.492$ & $22 / 231$ & 0.807 & $0.424-1.537$ & $22 / 211$ & 0.939 & $0.471-1.871$ \\
\hline \multicolumn{10}{|l|}{ MEP - Above/Below 50th Percentile - Males } \\
\hline $\begin{array}{l}<\text { LOD to 50th percentile (reference) } \\
\geq 50 \text { th percentile }\end{array}$ & $\begin{array}{l}10 / 248 \\
21 / 238\end{array}$ & $\begin{array}{l}1.00 \\
1.749\end{array}$ & $0.431-7.096$ & $\begin{array}{l}10 / 248 \\
21 / 237\end{array}$ & $\begin{array}{l}1.00 \\
1.457\end{array}$ & $0.387-5.484$ & $\begin{array}{c}8 / 232 \\
18 / 222\end{array}$ & $\begin{array}{c}1.00 \\
1.318\end{array}$ & $0.220-7.899$ \\
\hline \multicolumn{10}{|l|}{ MEP - Above/Below 50th Percentile - Females } \\
\hline$<$ LOD to 50th percentile (reference) & $16 / 237$ & 1.00 & & $17 / 235$ & 1.00 & & $16 / 217$ & 1.00 & \\
\hline$\geq 50$ th percentile & $27 / 227$ & $2.036 *$ & $1.047-3.958$ & $26 / 227$ & 1.693 & $0.811-3.535$ & $24 / 210$ & 1.819 & $0.706-4.685$ \\
\hline \multicolumn{10}{|l|}{ MHH - Above/Below 50th Percentile - Males } \\
\hline$<$ LOD to 50th percentile (reference) & $14 / 244$ & 1.00 & & $14 / 244$ & 1.00 & & $12 / 228$ & 1.00 & \\
\hline$\geq 50$ th percentile & 17/242 & 1.390 & $0.392-4.936$ & $17 / 241$ & 1.596 & $0.416-6.118$ & $14 / 226$ & 1.420 & $0.348-5.800$ \\
\hline MHH - Above/Below 50th Percentile - Fema & & & & & & & & & \\
\hline$<$ LOD to 50th percentile (reference) & 19/234 & 1.00 & & $19 / 233$ & 1.00 & & $17 / 216$ & 1.00 & \\
\hline$\geq 50$ th percentile & $24 / 230$ & 1.143 & $0.449-2.905$ & $24 / 229$ & 1.080 & $0.422-2.764$ & $23 / 211$ & 1.060 & $0.380-2.957$ \\
\hline MOH - Above/Below 50th Percentile - Mal & & & & & & & & & \\
\hline < LOD to 50th percentile (reference) & $15 / 243$ & 1.00 & & $15 / 243$ & 1.00 & & $13 / 227$ & 1.00 & \\
\hline$\geq 50$ th percentile & $16 / 243$ & 1.014 & $0.270-3.813$ & $16 / 242$ & 1.155 & $0.287-4.645$ & $13 / 227$ & 0.962 & $0.172-5.377$ \\
\hline MOH - Above/Below 50th Percentile - Fema & & & & & & & & & \\
\hline < LOD to 50th percentile (reference) & $18 / 236$ & 1.00 & & $18 / 234$ & 1.00 & & $15 / 218$ & 1.00 & \\
\hline$\geq 50$ th percentile & $25 / 228$ & 1.715 & $0.782-3.761$ & $25 / 228$ & 1.630 & $0.690-3.855$ & $25 / 209$ & 1.871 & $0.823-4.253$ \\
\hline MZP - Above/Below 50th Percentile - Mal & & & & & & & & & \\
\hline < LOD to 50th percentile (reference) & $16 / 242$ & 1.00 & & $16 / 242$ & 1.00 & & $15 / 225$ & 1.00 & \\
\hline$\geq 50$ th percentile & $15 / 244$ & 0.633 & $0.159-2.519$ & $15 / 243$ & 0.504 & $0.144-1.757$ & $11 / 229$ & 0.303 & $0.064-1.442$ \\
\hline MZP - Above/Below 50th Percentile - Fema & & & & & & & & & \\
\hline$<$ LOD to 50th percentile (reference) & $18 / 235$ & 1.00 & & $18 / 234$ & 1.00 & & $16 / 217$ & 1.00 & \\
\hline$\geq 50$ th percentile & $25 / 229$ & 1.539 & $0.652-3.636$ & $25 / 228$ & 1.474 & $0.577-3.766$ & $24 / 210$ & 1.493 & $0.444-5.021$ \\
\hline MIB - Above/Below 50th Percentile - Male & & & & & & & & & \\
\hline$<$ LOD to 50th percentile (reference) & $11 / 247$ & 1.00 & & $11 / 247$ & 1.00 & & $11 / 229$ & 1.00 & \\
\hline$\geq 50$ th percentile & $20 / 239$ & 1.664 & $0.402-6.880$ & $20 / 238$ & 1.330 & $0.363-4.877$ & $15 / 225$ & 1.032 & $0.263-4.048$ \\
\hline MIB - Above/Below 50th Percentile - Femal & & & & & & & & & \\
\hline$<$ LOD to 50th percentile (reference) & $21 / 232$ & 1.00 & & 20/232 & 1.00 & & $18 / 215$ & 1.00 & \\
\hline$\geq 50$ th percentile & $22 / 232$ & 1.452 & $0.710-2.969$ & $23 / 230$ & 1.331 & $0.620-2.859$ & $22 / 212$ & 1.449 & $0.709-2.960$ \\
\hline BPA - Above/Below 50th Percentile - Male & & & & & & & & & \\
\hline$<$ LOD to 50th percentile (reference) & $11 / 248$ & 1.00 & & $11 / 246$ & 1.00 & & 9/231 & 1.00 & \\
\hline$\geq 50$ th percentile & $20 / 238$ & 2.216 & $0.511-9.613$ & $20 / 239$ & 2.079 & $0.426-10.141$ & $17 / 223$ & 1.352 & $0.257-7.104$ \\
\hline BPA - Above/Below 50th Percentile - Femal & & & & & & & & & \\
\hline < LOD to 50th percentile (reference) & $24 / 229$ & 1.00 & & $24 / 227$ & 1.00 & & $22 / 211$ & 1.00 & \\
\hline$\geq 50$ th percentile & $19 / 235$ & 0.756 & $0.327-1.750$ & $19 / 235$ & 0.783 & $0.321-1.910$ & $18 / 216$ & 0.879 & $0.337-2.295$ \\
\hline & INES san & g weight app & lied before ca & ating the & metric mean. & & & & \\
\hline The EEDCs were creatinine-adjusted then log $t$ & $\begin{aligned} \text { to anal } \\
\text { dom }\end{aligned}$ & tatement in S & $\begin{array}{l}\text { tinine values } \\
\text { AS. }{ }^{*} p<0.05 \text {, }\end{array}$ & $0<0.01 *$ & $\begin{array}{l}-300 \mathrm{mg} / \mathrm{dL}) \\
0.001\end{array}$ & EEDCs le & re in & using tl & propriate \\
\hline${ }^{1}$ Unadjusted model. ${ }^{2}$ Adjusted for age, educati & $\begin{array}{l}\text { ty. }{ }^{3} \text { Adj } \\
\text {, stroke }\end{array}$ & $\mathrm{d}$ for age, $\mathrm{e}$ & ucation, race/e & icity, a & use, , high & d pressure & & 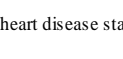 & us, heart \\
\hline
\end{tabular}


Taste Dysfunction and Exposure to BPA and Phthalates: Table 1.42 presents crude GMs and GSEs for subjects who have phthalate metabolite and BPA levels over the LOD who responded to the question, "During the past 12 months, you had a problem with your ability to taste sweet, sour, salty or bitter foods and drinks?'. The GMs phthalate metabolites COP, ECP, MBP, MHH, MOH, and MIB were found to be significantly higher in those that had persistent sensation in their mouth the past 12 months compared to those that did not have it (COP, MIB, $\mathrm{p}<0.05$; MBP, MHH, MOH, $\mathrm{p}<0.01$; ECP, $\mathrm{p}<0.001$ ) (Table 1.42). Table 1.43 presents age-specific GMs and GSEs. Among the 60-69 age group, GMs for the phthalate metabolites ECP, MBP, MHH, MOH, and MIB were significantly higher in those with persistent sensation in their mouths compared to those who did not have persistent sensation (ECP, MHH, MOH, $\mathrm{p}<0.01 ; \mathrm{MBP}, \mathrm{MIB}, \mathrm{p}<0.05)$. Among the $80+$ age group, GMs for the phthalate metabolites $\mathrm{CNP}, \mathrm{ECP}, \mathrm{MHH}$, and $\mathrm{MOH}$ were significantly higher in those with persistent sensation in their mouths compared to those who did not have persistent sensation (CNP, ECP, MHH, p<0.05; MOH, p<0.01) (Table 1.43). Table 1.44 presents gender-specific GMs and GSEs. Among males, the phthalate metabolites MBP and MIB were significantly higher in those that experienced persistent sensations in their mouths compared to those who did not have those sensations (MBP, MIB, p<0.05). In females, ECP, MBP, MHH, and $\mathrm{MOH}$ were observed to be significantly higher in those that experience persistent sensation in their mouths (ECP, MBP, MHH, MOH, p<0.01) (Table 1.44). Table 1.45 presents race-specific GMs and GSEs. Among non-Hispanic Whites, the phthalate metabolites COP, ECP, MBP, MHH, MOH, and MIB were found to be significantly higher in 
those with persistent sensation in their mouths (ECP, MBP, $\mathrm{p}<0.001 ; \mathrm{MHH}, \mathrm{p}<0.01$; $\mathrm{COP}, \mathrm{MOH}, \mathrm{MIB}, \mathrm{p}<0.05)$. Among Asians/Others, the phthalate metabolite $\mathrm{MHH}$ was found to be significantly higher in those with persistent taste or sensation in their mouths $(\mathrm{MHH}, \mathrm{p}<0.05)$.

Table 1.46 presents ORs and 95\% CI for those with Yes/No responses to the question "During the past 12 months have you had a taste or other sensation in your mouth that does not go away?" and phthalates and BPA stratified by gender with two groups: $<$ LOD to 50 th percentile (reference) and $\geq 50$ th percentile. For the phthalate COP, males in the $\geq 50$ th percentile compared to the reference group were found to have COP concentrations significantly associated gustatory sensations that would not go away in the unadjusted model and both adjusted models $(\mathrm{OR}=3.649,95 \% \mathrm{CI}$ :

1.054-12.639; OR=4.1.280, 95\% CI: 1.386-12.603; OR=4.608, 95\% CI: $1.130-$ 18.787). For the phthalate ECP, males in the $\geq 50$ th percentile compared to the reference group were found to have ECP concentrations significantly associated with gustatory sensations that would not go away in the unadjusted model and both adjusted models $(\mathrm{OR}=8.673,95 \%$ CI: 2.471-30.448; OR=4.180, 95\% CI: $1.386-$ 12.603; OR=4.608, 95\% CI: $1.130-18.787$ ) and in females ECP concentrations were significant in the second adjusted model (OR=3.411; 95\% CI: 1.013-11.483). For the phthalate MBP, males in the $\geq 50$ th percentile compared to the reference group were found to have MBP concentrations significantly associated with gustatory sensations that would not go away in the unadjusted model and second adjusted model $(\mathrm{OR}=4.548,95 \%$ CI: 1.339-15.445; OR=5.547, 95\% CI: 1.137-27.073), and in females MBP concentrations were significant in the unadjusted and first adjusted 
model $(\mathrm{OR}=2.153,95 \%$ CI: $1.063-4.358 ; \mathrm{OR}=2.229,95 \% \mathrm{CI}: 1.055-4.706)$. For the phthalate $\mathrm{MC1}$, males in the $\geq 50$ th percentile compared to the reference group were found to have MC1 concentrations significantly associated with gustatory sensations that would not go away in the second adjusted model (OR=4.545, 95\% CI: 1.488 13.877). For the phthalate $\mathrm{MHH}$, males in the $\geq 50$ th percentile compared to the reference group were found to have $\mathrm{MHH}$ concentrations significantly associated with gustatory sensations that would not go away in the unadjusted model, first adjusted model, and second adjusted model (OR=4.611, 95\% CI: 1.360-15.633; $\mathrm{OR}=4.628,95 \%$ CI: $1.068-20.065 ; \mathrm{OR}=5.525,95 \% \mathrm{CI}: 1.487-20.519)$ with the same observation in females $(\mathrm{OR}=3.255,95 \% \mathrm{CI}: 1.261-8.401$; OR=3.386, 95\% CI: 1.313 8.734; $\mathrm{OR}=3.025,95 \% \mathrm{CI}: 1.223-7.480)$. For the phthalate $\mathrm{MOH}$, females in the $\geq$ 50th percentile compared to the reference group were found to have $\mathrm{MOH}$ concentrations significantly associated with gustatory sensations that would not go away in the unadjusted model, first adjusted model, and second adjusted model $(\mathrm{OR}=3.576,95 \%$ CI: 1.345-9.506; OR=3.671, 95\% CI: $1.341-10.047 ; \mathrm{OR}=3.571$, 95\% CI: $1.343-9.494)$. For the phthalate $\mathrm{MOH}$, females in the $\geq 50$ th percentile compared to the reference group were found to have MZP concentrations significantly associated with gustatory sensations that would not go away in the unadjusted model, first adjusted model, and second adjusted model (OR=2.743, 95\% CI: 1.003-7.498; OR=3.055, 95\% CI: 1.088-8.581; OR=4.161, 95\% CI: 1.299 13.324). 


\begin{tabular}{|c|c|c|c|c|c|c|}
\hline $\begin{array}{r}\text { Table } 1.42 \text { Geometric Mean Urinary Phthalate a } \\
\text { have you had a taste or other sensation in your mo } \\
\text { NHANES } 20\end{array}$ & $\begin{array}{l}\text { A BPA } \\
\text { h that o } \\
-2014 \text {, }\end{array}$ & $\begin{array}{l}\text { els by } 1 \\
\text { not gc } \\
\text { r the I }\end{array}$ & $\begin{array}{l}\text { ponses } \\
\text { way?" } 1 \\
\text { D }\end{array}$ & Ibje & 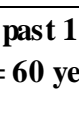 & $\begin{array}{l}\text { nths } \\
\text { f age, }\end{array}$ \\
\hline & & Geor & ric Mea & $\mathrm{gg} / \mathrm{ml})$ & $\mathrm{N})$ & \\
\hline EEDC & & Yes & & & No & \\
\hline & GM & GSE & $\mathbf{N}$ & GM & GSE & $\mathbf{N}$ \\
\hline Mono (carboxynonyl) Phthalate - CNP & 3.30 & 0.45 & 66 & 2.63 & 0.13 & 954 \\
\hline Mono (carboxyoctyl) Phthalate - COP & 25.12 & 3.25 & $66 *$ & 18.77 & 1.46 & 957 \\
\hline Mono-(2-ethyl-5-carboxypentyl) Phthalate -ECP & $\mathbf{1 7 . 8 3}$ & 1.63 & $66 * * *$ & 11.99 & 0.56 & 956 \\
\hline Mono-n-butyl Phthalate - MBP & 14.00 & 1.19 & $65 * *$ & 9.91 & 0.51 & 944 \\
\hline Mono-(3-carboxypropyl) Phthalate - MC1 & 3.22 & 0.59 & 63 & 2.72 & 0.17 & 914 \\
\hline Mono-ethyl Phthalate -MEP & 68.42 & 18.48 & 66 & 55.46 & 4.35 & 958 \\
\hline Mono (2-ethyl-5-hydroxy hexyl) Phthalate - MHH & 10.19 & 1.04 & $66 * *$ & 7.27 & 0.31 & 957 \\
\hline Mono-(2-ethyl-5-oxohexyl) Phthalate -MOH & 7.01 & 0.72 & $66 * *$ & 4.89 & 0.22 & 956 \\
\hline Mono-benzyl Phthalate - MZP & 5.05 & 0.68 & 66 & 4.18 & 0.22 & 943 \\
\hline Mono-isobutyl Phthalate - MIB & 8.30 & 0.89 & $66 *$ & 6.25 & 0.28 & 948 \\
\hline Bisphenol A - BPA & 1.72 & 0.19 & 64 & 1.52 & 0.07 & 901 \\
\hline
\end{tabular}

NHANES sampling weight applied before calculating the geometric mean.

The EEDCs were creatinine-adjusted then log transformed prior to analyses. Extreme creatinine values $(<30$ $\mathrm{mg} / \mathrm{dL}$ and $>300 \mathrm{mg} / \mathrm{dL}$ ) and EEDCs levels below the LOD were taken into account using the appropriate domain statement in SAS. $* \mathrm{p}<0.05, * * \mathrm{p}<0.01 * * * \mathrm{p}<0.001$

\begin{tabular}{|c|c|c|c|c|c|c|c|c|c|c|c|c|}
\hline \multirow[b]{2}{*}{ EEDC } & \multicolumn{6}{|c|}{ Geometric Mean (ng/ml) (GSE, N), Hispanic } & \multicolumn{6}{|c|}{ Geometric Mean (ng/ml) (GSE, N), Non-Hispanic White } \\
\hline & \multicolumn{3}{|c|}{ Yes } & \multicolumn{3}{|c|}{ No } & \multicolumn{3}{|c|}{ Yes } & \multicolumn{3}{|c|}{ No } \\
\hline Mono (carboxynonyl) Phthalate - CNP & 2.2342 & 0.2408 & 26 & 2.3859 & 0.1460 & 189 & 3.9106 & 0.6435 & 25 & 2.7724 & 0.1817 & 417 \\
\hline Mono (carboxyoctyl) Phthalate - COP & 17.9850 & 2.9303 & 26 & 19.9700 & 1.6055 & 189 & 30.6082 & 4.9858 & $25 *$ & 19.7210 & 1.9011 & 417 \\
\hline Mono-(3-carboxypropyl) Phthalate - MC1 & 2.2833 & 0.4231 & 23 & 2.8941 & 0.2699 & 180 & 3.7968 & 0.8898 & 25 & 2.7757 & 0.2247 & 399 \\
\hline Mono-ethyl Phthalate -MEP & 109.36 & 29.9294 & 26 & 94.2364 & 12.4476 & 189 & 63.4905 & 23.6845 & 25 & 50.3623 & 4.6046 & 417 \\
\hline Mono (2-ethyl-5-hydroxy hexyl) Phthalate - MHH & 8.8961 & 1.3089 & 26 & 10.6238 & 0.7541 & 189 & 10.6166 & 1.362 & $25 * *$ & 6.9825 & 0.3365 & 416 \\
\hline Mono-(2-ethyl-5-oxohexyl) Phthalate -MOH & 6.184 & 0.8758 & 26 & 6.791 & 0.4969 & 189 & 7.3103 & 0.9166 & $25 *$ & 4.7364 & 0.2406 & 415 \\
\hline Mono-benzyl Phthalate - MZP & 3.2295 & 0.4627 & 26 & 4.0989 & 0.4304 & 189 & 5.6186 & 0.9783 & 25 & 4.1235 & 0.2376 & 410 \\
\hline EEDC & GM & GSE & $\mathbf{N}$ & GM & GSE & $\mathbf{N}$ & GM & GSE & $\mathbf{N}$ & GM & GSE & $\mathbf{N}$ \\
\hline Mono (carboxynonyl) Phthalate - CNP & 2.4360 & 0.8530 & 8 & 1.9588 & 0.0971 & 254 & 1.9807 & 0.7439 & 7 & 2.3450 & 0.3556 & 94 \\
\hline Mono (carboxyoctyl) Phthalate - COP & 14.5821 & 4.3951 & 8 & 14.1855 & 0.9590 & 253 & 11.3284 & 5.0000 & 7 & 13.8170 & 2.1139 & 98 \\
\hline Mono-(2-ethyl-5-carboxypentyl) Phthalate -ECP & 11.9787 & 2.2455 & 8 & 10.9773 & 0.697 & 253 & 15.592 & 2.713 & 7 & 13.245 & 0.9117 & 98 \\
\hline Mono-n-butyl Phthalate - MBP & 10.838 & 1.5849 & 8 & 11.0157 & 0.5595 & 250 & 14.1343 & 4.2605 & 7 & 15.1841 & 2.4725 & 96 \\
\hline Mono-(3-carboxypropyl) Phthalate - MC1 & 1.9788 & 0.4841 & 8 & 2.2092 & 0.1326 & 246 & 1.8634 & 0.7431 & 7 & 2.7594 & 0.369 & 89 \\
\hline Mono-ethyl Phthalate -MEP & 74.1442 & 33.8916 & 8 & 80.3933 & 7.68 & 254 & 37.2185 & 10.0307 & 7 & 53.0442 & 11.4011 & 98 \\
\hline Mono (2-ethyl-5-hydroxy hexyl) Phthalate - MHH & 7.2754 & 2.0263 & 8 & 7.458 & 0.4859 & 254 & 12.7003 & 3.0819 & $8 *$ & 7.0862 & 0.7082 & 98 \\
\hline Mono-(2-ethyl-5-oxohexyl) Phthalate -MOH & 5.2183 & 1.4067 & 8 & 4.9967 & 0.2934 & 254 & 8.0265 & 2.3301 & 8 & 4.7063 & 0.5009 & 96 \\
\hline Mono-benzyl Phthalate - MZP & 6.338 & 0.8961 & 8 & 4.669 & 0.4342 & 251 & 4.6039 & 1.1814 & 7 & 4.3551 & 1.2852 & 93 \\
\hline Mono-isobutyl Phthalate - MIB & 7.0111 & 1.7604 & 8 & 7.6527 & 0.4326 & 249 & 12.6603 & 4.9063 & 7 & 8.975 & 1.4887 & 97 \\
\hline Bisphenol A - BPA & 1.1639 & 0.2892 & 8 & 1.4181 & 0.05666 & 246 & 1.024 & 0.2724 & 6 & 1.3599 & 0.1736 & 81 \\
\hline
\end{tabular}


Table 1.43 Age-Specific Geometric Urinary Mean Phthalate and BPA Levels by Responses, "During the past 12 months have you had a taste or other sensation in your mouth that does not go away?" for Subjects >= 60 years of age,

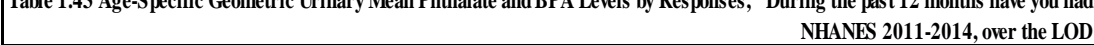

\begin{tabular}{|c|c|c|c|c|c|c|c|c|c|c|c|c|c|c|c|c|c|c|}
\hline \multirow[b]{2}{*}{ EWD Metabolite } & \multicolumn{6}{|c|}{ Geometric Mean (ng/ml) (GSE, N), Age 60-69 } & \multicolumn{6}{|c|}{ Geometric Mean (ng/ml) (GSE, N), Age 70-79 } & \multicolumn{6}{|c|}{ Geometric Mean (ng/ml) (GSE, N), Age $80+$} \\
\hline & \multicolumn{3}{|c|}{ Yes } & \multicolumn{3}{|c|}{ No } & \multicolumn{3}{|c|}{ Yes } & \multicolumn{3}{|c|}{ No } & \multicolumn{3}{|c|}{ Yes } & \multicolumn{3}{|c|}{$\mathrm{N}_{0}$} \\
\hline Mono (carboxynonyl) Phthalate - CNP & 3.35 & 0.68 & 40 & 2.51 & 0.18 & 490 & 2.26 & 0.48 & 13 & 2.92 & 0.23 & 287 & 4.62 & 1.26 & $13 *$ & 2.47 & 0.14 & 177 \\
\hline Mono-(2-ethyl-5-carboxypentyl) Phthalate -ECP & 17.18 & 2.06 & 40 ** & 10.69 & 0.77 & 490 & 15.91 & 1.72 & 13 & 13.59 & 0.70 & 289 & 22.35 & 4.65 & $13 *$ & 13.37 & 0.65 & 177 \\
\hline Mono-n-butyl Phthalate - MBP & 12.49 & 1.18 & 39 * & 9.24 & 0.61 & 484 & 16.00 & 3.58 & 13 & 10.10 & 0.77 & 286 & 17.20 & 4.02 & 13 & 11.85 & 1.08 & 174 \\
\hline Mono-(3-carboxypropyl) Phthalate - $\mathrm{MCl}$ & 3.14 & 0.82 & 38 & 2.51 & 0.23 & 467 & 3.50 & 1.83 & 12 & 3.00 & 0.27 & 275 & 3.20 & 0.67 & 13 & 2.88 & 0.29 & 172 \\
\hline Mono-ethyl Phthalate -MEP & 46.34 & 12.77 & 40 & 59.02 & 6.59 & 492 & 115.71 & 48.63 & 13 & 59.06 & 7.01 & 289 & 131.42 & 86.95 & 13 & 40.42 & 3.06 & 177 \\
\hline Mono-(2-ethyl-5-oxohexyl) Phthalate -MOH & 6.75 & 1.01 & 40 ** & 4.30 & 0.29 & 491 & 6.29 & 0.81 & 13 & 5.65 & 0.27 & 289 & 8.72 & 1.36 & $13 * *$ & 5.54 & 0.31 & 176 \\
\hline Mono-benzyl Phthalate - MZP & 4.79 & 0.92 & 40 & 4.10 & 0.30 & 484 & 4.70 & 1.12 & 13 & 3.87 & 0.30 & 286 & 3.69 & 0.49 & 13 & 4.28 & 0.15 & 173 \\
\hline Mono-isobutyl Phthalate - MIB & 8.83 & 1.23 & 40 * & 6.41 & 0.36 & 488 & 8.33 & 1.45 & 13 & 6.24 & 0.35 & 285 & 6.84 & 2.00 & 13 & 5.80 & 0.45 & 175 \\
\hline Bisphenol A - BPA & 1.78 & 0.29 & 39 & 1.49 & 0.09 & 467 & 1.44 & 0.33 & 13 & 1.64 & 0.10 & 266 & 1.85 & 0.54 & 12 & 1.40 & 0.12 & 168 \\
\hline
\end{tabular}

NHANES sampling weight applied before calculating the geometric mean.

The EEDCs were creatinine-adjusted then log transformed prior to analyses. Extreme creatinine values $(<30 \mathrm{mg} / \mathrm{dL}$ and $>300 \mathrm{mg} / \mathrm{dL})$ and EEDCs levels below the LOD were taken into account using the appropriate domain statement in SAS. ${ }^{*} \mathrm{p}<0.05,{ }^{* *} \mathrm{p}<0.01 * * * \mathrm{p}<0.001$

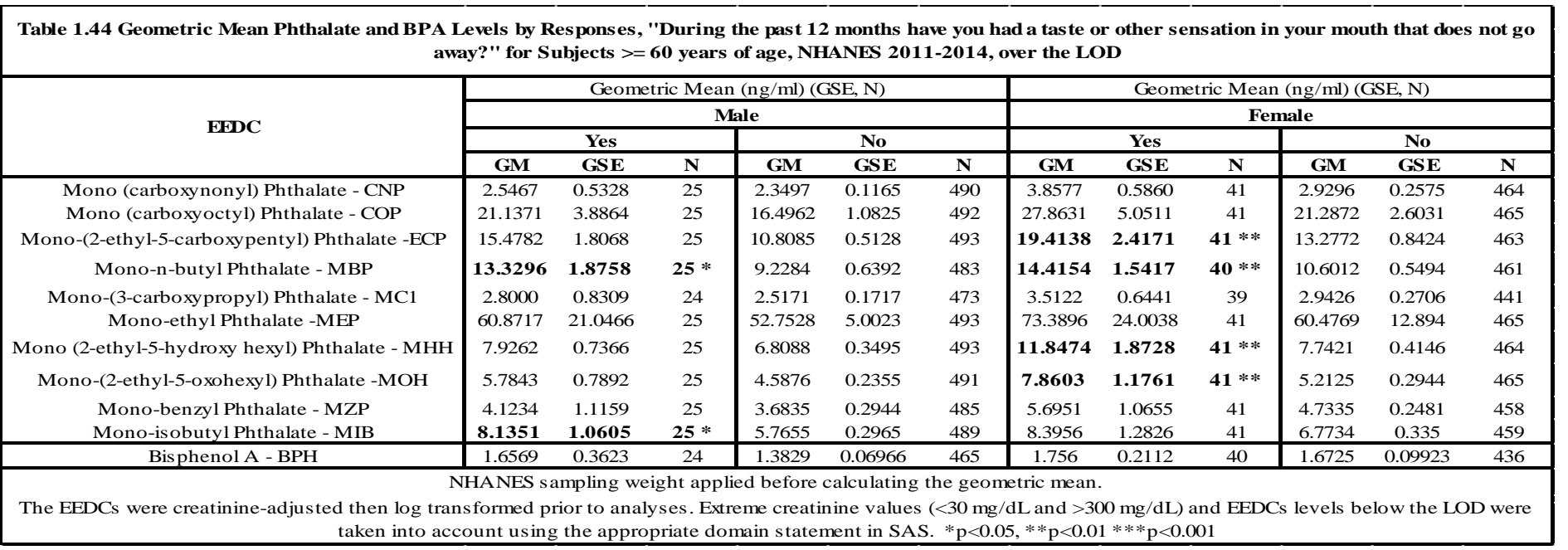




\begin{tabular}{|c|c|c|c|c|c|c|c|c|c|}
\hline EEDC & Yes / No & $\begin{array}{l}\text { Unadjusted } \\
\text { Odds Ratio }\end{array}$ & $95 \% \mathrm{CI}$ & Yes / No & $\begin{array}{c}\text { Adjusted } \\
\text { Odds Ratio }\end{array}$ & $95 \% \mathrm{CI}$ & Yes / No & $\begin{array}{c}\text { Adjusted } \\
\text { Odds Ratio }\end{array}$ & $95 \% \mathrm{CI}$ \\
\hline \multicolumn{10}{|l|}{ CNP - Above/Below 50th Percentile - Males } \\
\hline $\begin{array}{l}<\text { LOD to 50th percentile (reference) } \\
\quad \geq 50 \text { th percentile }\end{array}$ & $\begin{array}{l}15 / 243 \\
10 / 250\end{array}$ & $\begin{array}{c}1.00 \\
0.679\end{array}$ & $0.185-2.493$ & $\begin{array}{l}15 / 244 \\
10 / 248\end{array}$ & $\begin{array}{c}1.00 \\
0.751\end{array}$ & $0.211-2.678$ & $\begin{array}{l}11 / 162 \\
4 / 169\end{array}$ & $\begin{array}{c}1.00 \\
0.511\end{array}$ & $0.101-2.581$ \\
\hline \multicolumn{10}{|l|}{ CNP - Above/Below 50th Percentile - Females } \\
\hline $\begin{array}{l}<\text { LOD to 50th percentile (reference) } \\
\geq 50 \text { th percentile }\end{array}$ & $\begin{array}{l}19 / 233 \\
22 / 232 \\
\end{array}$ & $\begin{array}{c}1.00 \\
1.703 \\
\end{array}$ & $0.699-4.147$ & $\begin{array}{l}19 / 232 \\
22 / 231 \\
\end{array}$ & $\begin{array}{l}1.00 \\
1.685\end{array}$ & $0.719-3.950$ & $\begin{array}{l}13 / 165 \\
13 / 165 \\
\end{array}$ & $\begin{array}{c}1.00 \\
2.005 \\
\end{array}$ & $0.757-5.306$ \\
\hline \multicolumn{10}{|l|}{ COP - Above/Below 50th Percentile - Males } \\
\hline $\begin{array}{l}<\text { LOD to 50th percentile (reference) } \\
\quad \geq 50 \text { th percentile }\end{array}$ & $\begin{array}{l}11 / 248 \\
14 / 245\end{array}$ & $\begin{array}{c}1.00 \\
\mathbf{3 . 6 4 9} *\end{array}$ & $1.054-12.639$ & $\begin{array}{l}11 / 247 \\
14 / 245\end{array}$ & $\begin{array}{c}1.00 \\
4.180 *\end{array}$ & $1.386-12.603$ & $\begin{array}{l}8 / 165 \\
7 / 166\end{array}$ & $\begin{array}{c}1.00 \\
4.608 *\end{array}$ & $1.130-18.787$ \\
\hline \multicolumn{10}{|l|}{ COP - Above/Below 50th Percentile - Females } \\
\hline $\begin{array}{l}<\text { LOD to 50th percentile (reference) } \\
\geq 50 \text { th percentile }\end{array}$ & $\begin{array}{l}19 / 234 \\
22 / 231 \\
\end{array}$ & $\begin{array}{l}1.00 \\
1.696 \\
\end{array}$ & $0.732-3.928$ & $\begin{array}{l}19 / 233 \\
22 / 230 \\
\end{array}$ & $\begin{array}{l}1.00 \\
1.620\end{array}$ & $0.735-3.571$ & $\begin{array}{l}10 / 168 \\
16 / 162\end{array}$ & $\begin{array}{c}1.00 \\
2.850 \\
\end{array}$ & $0.937-8.670$ \\
\hline \multicolumn{10}{|l|}{ ECP - Above/Below 50th Percentile - Males } \\
\hline $\begin{array}{l}<\text { LOD to 50th percentile (reference) } \\
\quad \geq 50 \text { th percentile }\end{array}$ & $\begin{array}{c}7 / 252 \\
18 / 241\end{array}$ & $\begin{array}{c}1.00 \\
8.673 * *\end{array}$ & 2.471-30.448 & $\begin{array}{c}7 / 251 \\
18 / 241\end{array}$ & $\begin{array}{c}1.00 \\
9.359\end{array}$ & $1.753-49.967$ & $\begin{array}{c}5 / 168 \\
10 / 163\end{array}$ & $\begin{array}{c}1.00 \\
\mathbf{1 3 . 3 1 1} * *\end{array}$ & $2.090-84.762$ \\
\hline \multicolumn{10}{|l|}{ ECP - Above/Below 50th Percentile - Females } \\
\hline $\begin{array}{l}<\text { LOD to 50th percentile (reference) } \\
\geq 50 \text { th percentile }\end{array}$ & $\begin{array}{l}15 / 238 \\
26 / 227 \\
\end{array}$ & $\begin{array}{r}1.00 \\
2.195\end{array}$ & $0.927-5.199$ & $\begin{array}{l}15 / 237 \\
26 / 226 \\
\end{array}$ & $\begin{array}{c}1.00 \\
2.128\end{array}$ & $0.831-5.448$ & $\begin{array}{c}6 / 172 \\
20 / 158 \\
\end{array}$ & $\begin{array}{c}1.00 \\
\mathbf{3 . 4 1 1} * \\
\end{array}$ & $1.013-11.483$ \\
\hline \multicolumn{10}{|l|}{ MBP - Above/Below 50th Percentile - Males } \\
\hline $\begin{array}{l}<\text { LOD to 50th percentile (reference) } \\
\quad \geq 50 \text { th percentile }\end{array}$ & $\begin{array}{l}12 / 247 \\
13 / 246\end{array}$ & $\begin{array}{c}1.00 \\
4.548 *\end{array}$ & $1.339-15.445$ & $\begin{array}{l}12 / 246 \\
13 / 246\end{array}$ & $\begin{array}{c}1.00 \\
4.517\end{array}$ & $0.970-21.040$ & $\begin{array}{l}10 / 230 \\
12 / 229\end{array}$ & $\begin{array}{c}1.00 \\
\mathbf{5 . 5 4 7}\end{array}$ & $1.137-27.073$ \\
\hline \multicolumn{10}{|l|}{ MBP - Above/Below 50th Percentile - Females } \\
\hline $\begin{array}{l}<\text { LOD to 50th percentile (reference) } \\
\quad \geq 50 \text { th percentile }\end{array}$ & $\begin{array}{l}16 / 237 \\
25 / 228 \\
\end{array}$ & $\begin{array}{r}1.00 \\
2.153\end{array}$ & $1.063-4.358$ & $\begin{array}{l}16 / 236 \\
25 / 227 \\
\end{array}$ & $\begin{array}{c}1.00 \\
\mathbf{2 . 2 2 9} * \\
\end{array}$ & $1.055-4.706$ & $\begin{array}{l}15 / 228 \\
24 / 209 \\
\end{array}$ & $\begin{array}{c}1.00 \\
2.257 \\
\end{array}$ & $0.919-5.541$ \\
\hline \multicolumn{10}{|l|}{$\mathrm{MCl}$ - Above/Below 50th Percentile - Males } \\
\hline $\begin{array}{l}<\text { LOD to 50th percentile (reference) } \\
\quad \geq 50 \text { th percentile }\end{array}$ & $\begin{array}{l}11 / 247 \\
14 / 246\end{array}$ & $\begin{array}{c}1.00 \\
2.848\end{array}$ & $0.941-8.623$ & $\begin{array}{l}11 / 247 \\
14 / 245\end{array}$ & $\begin{array}{c}1.00 \\
3.084\end{array}$ & $0.987-9.635$ & $\begin{array}{l}9 / 231 \\
13 / 228\end{array}$ & $\begin{array}{c}1.00 \\
4.545 * *\end{array}$ & $1.488-13.877$ \\
\hline \multicolumn{10}{|l|}{ MCl - Above/Below 50th Percentile - Females } \\
\hline $\begin{array}{c}<\text { LOD to 50th percentile (reference) } \\
\geq 50 \text { th percentile }\end{array}$ & $\begin{array}{l}21 / 232 \\
20 / 233 \\
\end{array}$ & $\begin{array}{l}1.00 \\
1.554\end{array}$ & $0.660-3.657$ & $\begin{array}{l}21 / 231 \\
20 / 232 \\
\end{array}$ & $\begin{array}{c}1.00 \\
1.513\end{array}$ & $0.641-3.570$ & $\begin{array}{l}20 / 212 \\
19 / 215\end{array}$ & $\begin{array}{l}1.00 \\
1.602\end{array}$ & $0.652-3.938$ \\
\hline \multicolumn{10}{|l|}{ MEP - Above/Below 50th Percentile - Males } \\
\hline $\begin{array}{l}<\text { LOD to 50th percentile (reference) } \\
\quad \geq 50 \text { th percentile }\end{array}$ & $\begin{array}{l}11 / 248 \\
14 / 245\end{array}$ & $\begin{array}{c}1.00 \\
1.915\end{array}$ & $0.474-7.743$ & $\begin{array}{l}11 / 247 \\
14 / 245\end{array}$ & $\begin{array}{c}1.00 \\
1.831\end{array}$ & $0.403-8.330$ & $\begin{array}{l}9 / 231 \\
13 / 228\end{array}$ & $\begin{array}{c}1.00 \\
1.738\end{array}$ & $0.396-7.627$ \\
\hline \multicolumn{10}{|l|}{ MEP - Above/Below 50th Percentile - Females } \\
\hline $\begin{array}{l}<\text { LOD to 50th percentile (reference) } \\
\geq 50 \text { th percentile }\end{array}$ & $\begin{array}{l}19 / 234 \\
22 / 231\end{array}$ & $\begin{array}{l}1.00 \\
1.517\end{array}$ & $0.633-3.638$ & $\begin{array}{l}19 / 233 \\
22 / 230 \\
\end{array}$ & $\begin{array}{c}1.00 \\
1.317\end{array}$ & $0.492-3.526$ & $\begin{array}{l}18 / 215 \\
21 / 212 \\
\end{array}$ & $\begin{array}{c}1.00 \\
1.599\end{array}$ & $0.519-4.924$ \\
\hline \multicolumn{10}{|l|}{ MHH - Above/Below 50th Percentile - Males } \\
\hline $\begin{array}{l}<\text { LOD to 50th percentile (reference) } \\
\quad \geq 50 \text { th percentile }\end{array}$ & $\begin{array}{l}10 / 249 \\
15 / 244\end{array}$ & $\begin{array}{c}1.00 \\
4.611 *\end{array}$ & $1.360-15.633$ & $\begin{array}{l}10 / 248 \\
15 / 244\end{array}$ & $\begin{array}{c}1.00 \\
4.628 *\end{array}$ & $1.068-20.065$ & $\begin{array}{c}8 / 232 \\
14 / 227\end{array}$ & $\begin{array}{c}1.00 \\
\mathbf{5 . 5 2 5} *\end{array}$ & $1.487-20.519$ \\
\hline \multicolumn{10}{|l|}{ MHH - Above/Below 50th Percentile - Females } \\
\hline $\begin{array}{l}<\text { LOD to 50th percentile (reference) } \\
\geq 50 \text { th percentile }\end{array}$ & $\begin{array}{l}13 / 240 \\
28 / 225\end{array}$ & $\begin{array}{c}1.00 \\
3.255 *\end{array}$ & $1.261-8.401$ & $\begin{array}{l}13 / 239 \\
28 / 224\end{array}$ & $\begin{array}{c}1.00 \\
\mathbf{3 . 3 8 6} *\end{array}$ & $1.313-8.734$ & $\begin{array}{l}13 / 220 \\
26 / 207\end{array}$ & $\begin{array}{c}1.00 \\
\mathbf{3 . 0 2 5} *\end{array}$ & $1.223-7.480$ \\
\hline \multicolumn{10}{|l|}{ MOH - Above/Below 50th Percentile - Males } \\
\hline $\begin{array}{l}<\text { LOD to 50th percentile (reference) } \\
\quad \geq 50 \text { th percentile }\end{array}$ & $\begin{array}{l}10 / 249 \\
15 / 244\end{array}$ & $\begin{array}{c}1.00 \\
2.375\end{array}$ & $0.571-9.871$ & $\begin{array}{l}10 / 248 \\
15 / 244\end{array}$ & $\begin{array}{c}1.00 \\
2.193\end{array}$ & $0.490-9.814$ & $\begin{array}{c}8 / 232 \\
14 / 227\end{array}$ & $\begin{array}{c}1.00 \\
2.159\end{array}$ & $0.533-8.743$ \\
\hline \multicolumn{10}{|l|}{ MOH - Above/Below 50th Percentile - Females } \\
\hline $\begin{array}{l}<\text { LOD to 50th percentile (reference) } \\
\geq 50 \text { th percentile }\end{array}$ & $\begin{array}{l}12 / 240 \\
29 / 225 \\
\end{array}$ & $\begin{array}{r}1.00 \\
\mathbf{3 . 5 7 6} * \\
\end{array}$ & $1.345-9.506$ & $\begin{array}{l}12 / 240 \\
29 / 223 \\
\end{array}$ & $\begin{array}{r}1.00 \\
\mathbf{3 . 6 7 1} * \\
\end{array}$ & $1.341-10.047$ & $\begin{array}{l}12 / 221 \\
27 / 206 \\
\end{array}$ & $\begin{array}{r}1.00 \\
3.571 * \\
\end{array}$ & $1.343-9.494$ \\
\hline \multicolumn{10}{|l|}{ MZP - Above/Below 50th Percentile - Males } \\
\hline $\begin{array}{l}<\text { LOD to 50th percentile (reference) } \\
\quad \geq 50 \text { th percentile }\end{array}$ & $\begin{array}{l}14 / 245 \\
11 / 248\end{array}$ & $\begin{array}{l}1.00 \\
2.145\end{array}$ & $0.746-6.168$ & $\begin{array}{l}14 / 244 \\
11 / 248\end{array}$ & $\begin{array}{c}1.00 \\
1.990\end{array}$ & $0.747-5.303$ & $\begin{array}{l}12 / 228 \\
10 / 231\end{array}$ & $\begin{array}{c}1.00 \\
2.089\end{array}$ & $0.777-5.619$ \\
\hline \multicolumn{10}{|l|}{ MZP - Above/Below 50th Percentile - Females } \\
\hline $\begin{array}{l}<\text { LOD to 50th percentile (reference) } \\
\geq 50 \text { th percentile }\end{array}$ & $\begin{array}{l}15 / 238 \\
26 / 227 \\
\end{array}$ & $\begin{array}{c}1.00 \\
\mathbf{2 . 7 4 3} *\end{array}$ & $1.003-7.498$ & $\begin{array}{l}15 / 237 \\
26 / 226 \\
\end{array}$ & $\begin{array}{c}1.00 \\
\mathbf{3 . 0 5 5} * \\
\end{array}$ & $1.088-8.581$ & $\begin{array}{l}13 / 220 \\
26 / 207 \\
\end{array}$ & $\begin{array}{r}1.00 \\
\mathbf{4 . 1 6 1} * \\
\end{array}$ & $1.299-13.324$ \\
\hline \multicolumn{10}{|l|}{ MIB - Above/Below 50th Percentile - Males } \\
\hline $\begin{array}{l}<\text { LOD to } 50 \text { th percentile (reference) } \\
\quad \geq 50 \text { th percentile }\end{array}$ & $\begin{array}{l}12 / 247 \\
13 / 246\end{array}$ & $\begin{array}{c}1.00 \\
2.789\end{array}$ & $0.923-8.426$ & $\begin{array}{l}12 / 246 \\
13 / 246\end{array}$ & $\begin{array}{c}1.00 \\
2.165\end{array}$ & $0.816-5.742$ & $\begin{array}{l}10 / 230 \\
12 / 229\end{array}$ & $\begin{array}{l}1.00 \\
2.442\end{array}$ & $0.787-7.580$ \\
\hline \multicolumn{10}{|l|}{ MIB - Above/Below 50th Percentile - Females } \\
\hline $\begin{array}{l}<\text { LOD to 50th percentile (reference) } \\
\geq 50 \text { th percentile }\end{array}$ & $\begin{array}{l}14 / 239 \\
27 / 226 \\
\end{array}$ & $\begin{array}{c}1.00 \\
2.048\end{array}$ & $0.880-4.766$ & $\begin{array}{l}14 / 238 \\
27 / 225 \\
\end{array}$ & $\begin{array}{c}1.00 \\
2.049\end{array}$ & $0.768-5.466$ & $\begin{array}{l}13 / 220 \\
26 / 207 \\
\end{array}$ & $\begin{array}{c}1.00 \\
1.995\end{array}$ & $0.656-6.071$ \\
\hline \multicolumn{10}{|l|}{ BPA - Above/Below 50th Percentile - Males } \\
\hline $\begin{array}{l}<\text { LOD to 50th percentile (reference) } \\
\quad \geq 50 \text { th percentile }\end{array}$ & $\begin{array}{l}12 / 247 \\
13 / 246\end{array}$ & $\begin{array}{l}1.00 \\
1.232\end{array}$ & $0.317-4.792$ & $\begin{array}{l}12 / 247 \\
13 / 245\end{array}$ & $\begin{array}{l}1.00 \\
1.172\end{array}$ & $0.290-4.740$ & $\begin{array}{l}10 / 230 \\
12 / 229\end{array}$ & $\begin{array}{c}1.00 \\
1.199\end{array}$ & $0.233-6.165$ \\
\hline \multicolumn{10}{|l|}{ BPA - Above/Below 50th Percentile - Females } \\
\hline $\begin{array}{l}<\text { LOD to 50th percentile (reference) } \\
\quad \geq 50 \text { th percentile }\end{array}$ & $\begin{array}{l}18 / 234 \\
23 / 231\end{array}$ & $\begin{array}{c}1.00 \\
1.619\end{array}$ & $0.729-3.595$ & $\begin{array}{l}18 / 235 \\
23 / 228\end{array}$ & $\begin{array}{l}1.00 \\
1.661\end{array}$ & $0.718-3.843$ & $\begin{array}{l}16 / 217 \\
23 / 210\end{array}$ & $\begin{array}{c}1.00 \\
2.362\end{array}$ & $0.918-5.484$ \\
\hline
\end{tabular}

NHANES sampling weight applied before calculating the geometric mean.

The EEDs were creatinine-adjusted then log transformed prior to analyses. Extreme creatinine values ( $<30 \mathrm{mg} / \mathrm{dL}$ and $>300 \mathrm{mg} / \mathrm{dL}$ and metalloestrogen levels were into account using the appropriate domain statement in SAS. ${ }^{*} \mathrm{p}<0.05,{ }^{* *} \mathrm{p}<0.01 * * * \mathrm{p}<0.001$

${ }^{1}$ Unadjusted model. ${ }^{2}$ Adjusted for age, education, race/ethnicity. ${ }^{3}$ Adjusted for age, education, race/ethnicity, alcohol use, high blood pressure status, coronary heart disease status, heart attack status, stroke status, physical activity status, smoking status, head trauma status. 


\section{DISCUSSION}

This study takes a novel approach in the assessment of exposure to phthalates and BPA in the development of cognitive dysfunction and neurodegenerative disease using surrogates of brain health. The study examined an older geriatric-aged population of US adults 60 years of age and older. We first assessed the bioburden of urinary phthalates and BPA by calculating and comparing the GMs of each EEDCs versus each surrogate of brain health, looking at age, gender, and race and subsequently used ORs and 95\% CI to determine the risk of developing cognitive dysfunction.

Major Findings: Overall, we observed a higher bioburden of phthalates in the GMs of subjects who scores lower on the four cognitive tests, had memory function deficits from our three memory questions, and had taste/smell deficits on our two taste and smell questions (Tables 1.2, 1.7, 1.12, 1.17, 1.22, 1.27, 1.32, 1.37, and 1.47). In our analyses of GMs by age, gender, and race, we accounted for subjects that have extreme creatinine measurements and examined subjects who had phthalate and BPA levels over the LOD. A higher bioburden of phthalates was observed according to gender (Tables 1.3, 1.9, 1.14, 1.19, 1.24, 1.29, 1.34, 1.39, 1.33, 1.48 and 1.49). This trend was more prominent in females (Table 1.48 and 1.49). We did not observe any meaningful trends when examining GMs by age, as the age grouping was skewed towards the 60-69-year-old age group, with the number of subjects decreasing in the 70-79-year-old and 80+ year old age group. We also did not observe any meaningful observations in our analyses of race, where large majority of participants were in the Non-Hispanic White age group. BPA was not observed to be significantly higher in 
those who experienced adverse brain health versus those who did not in any of analyses.

\begin{tabular}{|c|c|c|c|c|c|c|c|c|c|c|c|c|c|c|c|c|c|c|}
\hline \multirow[t]{2}{*}{$\begin{array}{l}\text { Table } 1.47 \text { - Summary Table of EEDCs overall } \\
\text { signficance of GMs by Surrogate of Brain Health } \\
\text { Indicators }\left(* \mathrm{p}<0.05,{ }^{*} \mathrm{p}<0.01,{ }^{*} * * \mathrm{p}<0.001\right)\end{array}$} & \multicolumn{2}{|c|}{$\begin{array}{c}\text { Immediate Recall } \\
\text { Score }(\mathrm{n}=940)\end{array}$} & \multicolumn{2}{|c|}{$\begin{array}{l}\text { Delayed Recall } \\
\text { Score }(\mathrm{n}=930)\end{array}$} & \multicolumn{2}{|c|}{$\begin{array}{l}\text { Animal Fluency } \\
\text { Score }(\mathrm{n}=931)\end{array}$} & \multicolumn{2}{|c|}{$\begin{array}{l}\text { Digit Symbol } \\
\text { Substitution Test } \\
\text { Score (n=891) }\end{array}$} & \multicolumn{2}{|c|}{$\begin{array}{l}\text { Past } 12 \text { months, } \\
\text { memory getting } \\
\text { worse? }(\mathrm{n}=1,025)\end{array}$} & \multicolumn{2}{|c|}{$\begin{array}{l}\text { Past } 7 \text { days, } \\
\quad \text { trouble } \\
\text { remembering? } \\
\quad(\mathrm{n}=971)\end{array}$} & \multicolumn{2}{|c|}{$\begin{array}{l}\text { Limited due to } \\
\text { difficulty } \\
\text { remembering or } \\
\text { confusion? } \\
(\mathrm{n}=1,024)\end{array}$} & \multicolumn{2}{|c|}{$\begin{array}{c}\text { Phantom Odor? } \\
(\mathrm{n}=1,024)\end{array}$} & \multicolumn{2}{|c|}{$\begin{array}{c}\text { Sensation in } \\
\text { mouth that does } \\
\text { not go away? } \\
(\mathrm{n}=1024)\end{array}$} \\
\hline & $\leq 13$ & $\geq 14$ & $\leq 3$ & $>4$ & $\leq 11$ & $\geq 12$ & $\leq 27$ & $\geq 28$ & Yes & No & Yes & No & Yes & No & Yes & № & Yes & No \\
\hline \multirow{2}{*}{$\begin{array}{l}\text { Mono (carboxynonyl) Phthalate - CNP } \\
\text { Mono (carboxyoctyl) Phthalate - COP }\end{array}$} & & & & & & & & & & & & & & & & & & \\
\hline & & & & & & & & & & & & & & & & & * & \\
\hline $\begin{array}{l}\text { Mono-(2-ethyl-5-carboxypentyl) Phthalate -ECP } \\
\text { Mono-n-butyl Phthalate - MBP } \\
\text { Mono-(3-carboxypropyl) Phthalate - MCl } \\
\text { Mono-ethyl Phthalate -MEP }\end{array}$ & * & & & & * & & *** & & & & & & * & & & & $* * *$ & \\
\hline \multirow{4}{*}{$\begin{array}{c}\text { Mono (2-ethyl-5-hydroxy hexyl) Phthalate - MHH } \\
\text { Mono-(2-ethyl-5-oxohexyl) Phthalate -MOH } \\
\text { Mono-benzyl Phthalate - MZP } \\
\text { Mono-isobutyl Phthalate - MIB }\end{array}$} & & & & & & & * & & & & & & $* *$ & & & & $* *$ & \\
\hline & & & & & & & * & & & & & & * & & & & & \\
\hline & & & & & $*$ & & & & * & & & & *** & & & & $*$ & \\
\hline & & & & & * & & & & * & & $*$ & & $* * *$ & & * & & * & \\
\hline Bisphenol A - BPA & & & & & & & & & & & & & & & & & & \\
\hline
\end{tabular}

\begin{tabular}{|c|c|c|c|c|c|c|c|c|c|c|c|c|c|c|c|c|c|c|}
\hline $\begin{array}{l}\text { Table } 1.48 \text { - Summary Table of EEDCs overall } \\
\text { signficance of GMs by Surrogate of Brain Health } \\
\text { Indicators in males (* } \mathrm{p}<0.05, * * \mathrm{p}<0.01, * * \mathrm{p}<0.001)\end{array}$ & \multicolumn{2}{|c|}{$\begin{array}{l}\text { Immediate Recall } \\
\text { Score }(\mathrm{n}=940)\end{array}$} & \multicolumn{2}{|c|}{$\begin{array}{l}\text { Delayed Recall } \\
\text { Score }(\mathrm{n}=930)\end{array}$} & \multicolumn{2}{|c|}{$\begin{array}{l}\text { Animal Fluency } \\
\text { Score (n=931) }\end{array}$} & \multicolumn{2}{|c|}{$\begin{array}{c}\text { Digit Symbol } \\
\text { Substitution Test } \\
\text { Score }(\mathrm{n}=891)\end{array}$} & \multicolumn{2}{|c|}{$\begin{array}{l}\text { Past } 12 \text { months, } \\
\text { memory getting } \\
\text { worse? ( } \mathrm{n}=1,025)\end{array}$} & \multicolumn{2}{|c|}{$\begin{array}{l}\text { Past } 7 \text { days, } \\
\text { trouble } \\
\text { remembering? } \\
\quad(\mathrm{n}=971)\end{array}$} & \multicolumn{2}{|c|}{$\begin{array}{l}\text { Limited due to } \\
\text { difficulty } \\
\text { remembering or } \\
\text { confusion? } \\
(\mathrm{n}=1,024)\end{array}$} & \multicolumn{2}{|c|}{$\begin{array}{c}\text { Phantom Odor? } \\
\quad(\mathrm{n}=1,024)\end{array}$} & \multicolumn{2}{|c|}{$\begin{array}{l}\text { Sensation in } \\
\text { mouth that does } \\
\text { not go away? } \\
\quad(\mathrm{n}=1024)\end{array}$} \\
\hline Mono (carboxynonyl) Phthalate - CNP & & & & & & & & & & & & & & & & & & \\
\hline Mono (carboxyoctyl) Phthalate - COP & & & & & & & & & & & & & & & & & & \\
\hline Mono-(2-ethyl-5-carboxypentyl) Phthalate-ECP & & & & & & & ** & & & & * & & * & & & & & \\
\hline Mono-n-butyl Phthalate - MBP & & & & & & & & & & & & & & & & & * & \\
\hline Mono (2-ethyl-5-hydroxy hexyl) Phthalate - MHH & & & & & & & * & & & & * & & * & & & & & \\
\hline Mono-(2-ethyl-5-oxohexyl) Phthalate -MOH & & & & & & & * & & & & ${ }^{*}$ & & * & & & & & \\
\hline Mono-benzyl Phthalate - MZP & & & & & & & & & & & & & & & & & & \\
\hline Mono-isobutyl Phthalate - MIB & & & & & & & & & & & & & **** & & & & * & \\
\hline Bisphenol A-BPA & & & & & & & & & & & & & & & & & & \\
\hline
\end{tabular}

\begin{tabular}{|c|c|c|c|c|c|c|c|c|c|c|c|c|c|c|c|c|c|c|}
\hline \multirow[t]{2}{*}{$\begin{array}{l}\text { Table } 1.49 \text { - Summary Table of EEDCs overall } \\
\text { signficance of GMs by Surrogate of Brain Health } \\
\text { Indicators in females }(* \mathrm{*}<0.05 \text {, ** } \mathrm{p}<0.01 \text {, *** } \\
\mathrm{p}<0.001)\end{array}$} & \multicolumn{2}{|c|}{$\begin{array}{c}\text { Immediate Recall } \\
\text { Score (n=940) }\end{array}$} & \multicolumn{2}{|c|}{$\begin{array}{c}\text { Delayed Recall } \\
\text { Score }(n=930)\end{array}$} & \multicolumn{2}{|c|}{$\begin{array}{c}\text { Animal Fluency } \\
\text { Score }(\mathrm{n}=931)\end{array}$} & \multicolumn{2}{|c|}{$\begin{array}{c}\text { Digit Symbol } \\
\text { Substitution Test } \\
\text { Score (n=891) }\end{array}$} & \multicolumn{2}{|c|}{$\begin{array}{c}\text { Past } 12 \text { months, } \\
\text { memory getting } \\
\text { worse? (n=1,025) }\end{array}$} & \multicolumn{2}{|c|}{$\begin{array}{l}\text { Past } 7 \text { days, } \\
\quad \text { trouble } \\
\text { remembering? } \\
\quad(\mathrm{n}=971)\end{array}$} & \multicolumn{2}{|c|}{$\begin{array}{l}\text { Limited due to } \\
\text { difficulty } \\
\text { remembering or } \\
\text { confusion? } \\
(\mathrm{n}=1,024)\end{array}$} & \multicolumn{2}{|c|}{$\begin{array}{l}\text { Phantom Odor? } \\
\quad(\mathrm{n}=1,024)\end{array}$} & \multicolumn{2}{|c|}{$\begin{array}{l}\text { Sensation in } \\
\text { mouth that does } \\
\text { not go away? } \\
(\mathrm{n}=1024)\end{array}$} \\
\hline & $\leq 13$ & $\geq 14$ & $\leq 3$ & $\geq 4$ & $\leq 11$ & $\geq 12$ & $\leq 27$ & $\geq 28$ & Yes & No & Yes & No & Yes & No & Yes & No & Yes & No \\
\hline \multirow{2}{*}{\multicolumn{19}{|c|}{$\begin{array}{l}\text { Mono (carboxynonyl) Phthalate - CNP } \\
\text { Mono (carboxyoctyl) Phthalate - COP }\end{array}$}} \\
\hline & & & & & & & & & & & & & & & & & & \\
\hline \multirow{2}{*}{$\begin{array}{l}\text { Mono-(2-ethyl-5-carboxypentyl) Phthalate -ECP } \\
\text { Mono-n-butyl Phthalate - MBP } \\
\text { Mono-(3-carboxypropyl) Phthalate - MCl } \\
\text { Mono-ethyl Phthalate -MEP }\end{array}$} & * & & * & & $* *$ & & & & & & & & & & & & *** $^{* *}$ & \\
\hline & & & & & & & & & & & & & & & & & & \\
\hline \multirow{3}{*}{$\begin{array}{l}\text { Mono (2-ethyl-5-hydroxy hexyl) Phthalate - MHH } \\
\text { Mono-(2-ethyl-5-oxohexyl) Phthalate -MOH } \\
\text { Mono-benzyl Phthalate - MZP } \\
\text { Mono-isobutyl Phthalate - MIB }\end{array}$} & * & & * & & $*$ & & & & & & & & & & * & & *** & \\
\hline & & & * & & *** & & & & * & & & & ${ }^{* * * *}$ & & & & & \\
\hline & & & & & $*$ & & & & $*$ & & & & $*$ & & & & & \\
\hline Bisphenol A - BPA & & & & & & & & & & & & & & & & & & \\
\hline
\end{tabular}

The analysis of odds of having a cognitive dysfunction among our nine surrogates of brain health varied by the surrogate and type of model. Although a number of the phthalates examined were significant in our unadjusted models and first adjusted model (controlling for age, education, and gender) a number of them were still significant after adjusting for all the known and suspected risk factors of cognitive dysfunction and AD. The final adjusted model accounts for the following 
covariates: age, education, race/ethnicity, alcohol use, diabetes status, high blood pressure status, coronary heart disease status, heart attack status, stroke status, physical activity status, smoking status, and head trauma status.

For immediate recall, ECP in females was the only phthalate metabolite to be found significantly associated with lower immediate recall scores after controlling for the known and suspected risk factors. For delayed recall, MEP in males was the only phthalate to be found significantly associated with lower delayed recall scores after controlling for the known and suspected risk factors. For animal fluency, the phthalate MBP in females was the only phthalate to be found significantly associated with lower animal fluency scores. For DSST, the phthalate metabolites ECP and $\mathrm{MOH}$ in males were found to be significantly associated with lower DSST scores after controlling for the known and suspected risk factors.

Among those who had reported issues with memory in the past year, the phthalate metabolites MBP in males and MIB in females were found to be significantly associated with having memory issues in the past year after controlling for the known and suspected risk factors. Those who had reported memory issues in the past week, the phthalate metabolites ECP, MBP, and MHH in females were found to be significantly associated with having memory issues in the past year after controlling for the known and suspected risk factors.

Among those who had reported experiencing phantom odors, no phthalate or BPA was found to be significant in the second adjusted models. Those who had reported experiencing gustatory sensations that do not go away, the phthalate metabolites $\mathrm{COP}, \mathrm{ECP}, \mathrm{MBP}, \mathrm{MC} 1$, and $\mathrm{MHH}$ in males were significantly associated 
with experiencing gustatory sensations that did not go away. In females, this was observed with the phthalate metabolites ECP, MHH, MOH, and MZP. It should be noted that due to a smaller number of cases with taste and smell variables, diabetes status was left out of the final model since it contributed to a quasi-separation of data, making the model validity questionable, due to having zero cases among those who had diabetes. The large standard errors among the taste and smell variables indicate that the results should be interpreted with caution.

The higher bioburden of phthalates among females are in line with an earlier study that examined the urinary levels of phthalate metabolites in the US population using the NHANES 1999-2000 data sets ${ }^{8}$, which found females to have a higher bioburden of phthalates overall. Another study using the NHANES 2003-2004 data sets found BPA to be higher in females than in males ${ }^{25}$. It is speculated that this increase is due to the use of phthalate containing products marketed to a female population in addition to everyday exposures to phthalates ${ }^{8}$. A recent study of NHANES 2009 to 2012 cycle years found gender-specific increases in product use and phthalate bioburden in males and females ${ }^{103}$.

There are few epidemiological studies that specifically look at an older population with respect to phthalate bioburden and brain health. Shiue (2015) found a higher bioburden of phthalates, but not BPA in those having difficulties thinking or remembering and those with memory issues in the past week ${ }^{23}$ from the 2011-2012 NHANES data sets. These findings are consistent with our study. Human epidemiological studies regarding phthalates and BPA have focused on the pre-natal, post-natal, and childhood exposures. These studies have found phthalate exposure to 
be associated with social deficits ${ }^{18}$, decreased visual recognition memory ${ }^{19}$ and decreased IQ ${ }^{20}$. However there is evidence to suggests inconsistent cognitive and behavioral effects in children with regards to phthalate type and gender ${ }^{21,22}$. The few human epidemiological studies have concentrated on pre-natal BPA exposure and exposure in children which have been associated with significant behavioral issues in children ${ }^{35,47-50}$. These studies support the hypothesis of phthalates and BPA having an effect on the brain.

We also observed an increase in EEDC bioburden in females vs. males. Among females, geometric means of the EEDCs were generally higher in females than males, both significant and insignificant. Gender plays large role in the development of neurodegenerative diseases ${ }^{104}$ and estrogen has been suspected to play a large role in the development of neurodegenerative disease ${ }^{105}$, possibly due to its many neuroprotective effects it has on the brain ${ }^{106}$. The increase in bioburden is females versus males was observed in all surrogates of brain health that were examined. Studies have greater bioburden of phthalates in the mother can is associated with developmental dysfunction in children ${ }^{11}$, which give credence to a higher bioburden of EEDCs in women. An increase in the bioburden of EEDCs in females, can affect estrogen balance in the female body, which can contribute to a higher incidence of neurodegenerative disease in females. Geometric means of EEDCs were also observed to generally increase as age increases.

Our study also observed specific phthalates reoccurring with significant associations in our surrogates of brain health. The phthalates ECP (Mono-(2-ethyl-5carboxypentyl) Phthalate), MOH (Mono-(2-ethyl-5-oxohexyl) Phthalate), MBP 
(Mono-n-butyl Phthalate), MHH, and MIB were found to be have more significant results among our surrogates of brain health compared to the other phthalates and BPA. Literature is limited regarding the specific phthalates and their effects on gender and neurodegenerative disease.

Biological Mechanisms and Brain Health: With respect to biological mechanisms, phthalate and BPA's effects have focused on mechanisms affecting the pre, peri, and postnatal stages of development and much of what is known in older human populations is based off of animal and neuronal cell line studies ${ }^{11,107}$. Both phthalates and BPA have been demonstrated to have affinity for estrogen receptors ${ }^{9,10,26}$. BPA, although having a low affinity for ER receptor binding, is also thought to exert effects through other non-classical pathways ${ }^{26}$, which allows for phthalates and BPA to mimic and interfere with estrogen. Estrogen itself has been demonstrated to have roles in neuroprotection as well as exert anti-inflammatory effects on the brain ${ }^{106,108-}$ 112. Animal studies have also demonstrated estrogens significant role in the modulation and promotion of neuroplasticity and synaptogenesis ${ }^{113-117}$. Estrogen has also been demonstrated to protect and regulate mitochondrial function in the brain, where mitochondrial dysfunction is implicated as one of the causes of neurodegenerative disease ${ }^{118-120}$. This leads to the possibility of phthalates and BPA interfering with the function of estrogen and its protective and restorative effects on the brain.

Animal studies are numerous and have shown phthalates to adversely affect brain function and gives an idea of the possible mechanisms involved. These adverse effects include negatively affecting learning ${ }^{12,13}$, negatively affecting 
memory ${ }^{13,14}$, interfering with locomotion ${ }^{14}$, negative affecting social behavior ${ }^{12}$, and producing cellular effects such as cell death, synaptic loss, and synaptic dysfunction ${ }^{15}$. Some animal studies have reported an improvement in memory ${ }^{16}$, and possible dose-dependent effects, where memory improves one dose, but degrades it in another dose ${ }^{17}$. A recent studies, examining phthalates effects on neuronal cells found an increase in ROS concentrations after exposure ${ }^{121}$, increase neuronal cell death ${ }^{122}$, and disturbances to dendritic outgrowth.

Animal studies have shown BPA to also adversely brain function. Animal studies are numerous and have demonstrated BPA's negative effects on brain health and function. In animal studies BPA has been shown to negatively affect memory ${ }^{27-}$ ${ }^{41}$, negatively affect neurogenesis ${ }^{33,42}$, negatively affect the structure of dendritic spines and synaptogenesis ${ }^{27,29,32,38,40,43}$, and negatively affect cellular processes, protein expression and expression $31,36,39,41$. There are also animal studies that shows BPA exerts no negative effects on spatial and working memory ${ }^{44-46}$.

BPA has been found is post-mortem brain tissue ${ }^{123}$ and phthalates have shown to adversely affect brain neuroplasticity and affect neurodevelopment ${ }^{107}$. Although the mechanisms are not completely understood, both EEDCs appear to have the ability to cross the blood brain barrier.

Furthermore, genes that have been implicated in the development of neurodegenerative disease such as $\mathrm{AD}$ which are also estrogen-responsive are also pathway for BPA and phthalates to exert their effects on the aging brain. Genes that are estrogen-responsive, BPA-responsive, interfere with mitochondrial energetics, and implicated in AD include APBB2, DPYSL2, EIF2S1, ENO1, MAPT, and 
PAXIP1 ${ }^{11}$. Genes that are estrogen-responsive, phthalate-responsive, interfere with mitochondrial energetics, and implicated in AD include DPYSL2, EIFS1, ENO1, and MAPT ${ }^{11}$. Additionally, a recent search of the Comparative Toxicogenomic Database (CTD) reveals that from 88 genes implicated in $\mathrm{AD}, 61$ of those genes are estrogen and BPA responsive ${ }^{124}$. A search of the phthalates most associated with adverse brain health in men and women from our analyses, ECP, MBP, MHH, MOH, and $\mathrm{MIB}$, for any estrogen-responsive $\mathrm{AD}$ genes found none for $\mathrm{ECP}, \mathrm{MOH}, \mathrm{MHH}$, and MIB and two for MBP (EPHA1 and PPARG), which may indicate previously unknown gene/chemical interactions that affect brain health ${ }^{124}$. The results from our study can result from any of the possible biological mechanisms observations that have been referenced.

Strengths and Limitations: Our study has several limitations. The cross-sectional design of our study with self-reported data lends itself to misclassification bias. We cannot account for day to day variability with those who responded to our different brain health indicators. Neurodegeneration and neurodegenerative disease have a number of other risk factors. Genetic susceptibility as well as other direct measures of risk factors such as blood lipid profiles, were either not included, lacked the sufficient number of subjects, or did not use the appropriate sampling weights. We could also not account for estrogen levels as there were not measurements and we lacked the subjects required for oral contraceptive and hormone replacement therapy in our analyses. We used surrogates of brain health to link neurodegenerative disease development to EEDC exposure. The surrogates, as valid as they can be, are not clinical endpoints which cannot be used solely to diagnose neurodegenerative 
conditions. Taste and smell variables have a large number of confounders associated with them and including all of them in the regression model testing would have affected the overall validity of the model.

Our study had a number of strengths. The ability to combine data cycles greatly increased our sample size and the availability of EEDC measurements allowed us a way to compare large groups together. The availability of surrogate brain health indicators made it possible to assess bioburdens and odds of adverse brain health and generalize it to the US population. The study is novel in approach and is one of the first studies to examine the role of EEDCs in an older US population.

\section{CONCLUSION}

Based on our findings for our study, increased levels of specific phthalates play a role in the development of neurodegenerative disease. Surprisingly, BPA did not appear to be significant in any of our brain health surrogates. The examination of this older population can give us a sense of a possible lifetime of exposure and how it can lead to the pre-conditions of Alzheimer's disease, Parkinson's disease, and others. Further research is needed with biologically plausible clinical endpoints and robust epidemiological studies in order to fully assess the effects of phthalate exposure on brain health and the development of neurodegenerative disease.

\section{REFERENCES}

1. Bergman A, Heindel JJ, Jobling S, Kidd KA, Zoeller TR. State of the science of endocrine disrupting chemicals. World Heal Organ United Nations Environ Program. 2012:1-38. http://apps.who.int/iris/handle/10665/78102. 
2. Alzheimer's Association. 2017 Alzheimer's Disease Facts and Figures. Alzheimers Dement . 2017;13:325-373. doi:10.1016/j.jalz.2017.02.001.

3. Agency for Toxic Substances and Disease Registry (ATSDR). Toxicological Profile for Diethyl Phthalate. Agency Toxic Subst Dis Regist. 1995;(June):1158.

4. Agency for Toxic Substances and Disease Registry (ATSDR). TOXICOLOGICAL PROFILE FOR DI-n-BUTYL PHTHALATE. Agency Toxic Subst Dis Regist. 2001;(September):225.

5. Agency for Toxic Substances and Disease Registry (ATSDR). Toxicological profile: di(2-ethylhexyl)phthalate (DEHP). Agency Toxic Subst Dis Regist. 2002; (September). http://www.atsdr.cdc.gov/toxprofiles/tp.asp?id=684\&tid=65.

6. Heudorf U, Mersch-Sundermann V, Angerer J. Phthalates: Toxicology and exposure. Int J Hyg Environ Health. 2007;210(5):623-634.

doi:10.1016/j.ijheh.2007.07.011.

7. Miodovnik A, Edwards A, Bellinger DC, Hauser R. Developmental neurotoxicity of ortho-phthalate diesters: Review of human and experimental evidence. Neurotoxicology. 2014;41:112-122. doi:10.1016/j.neuro.2014.01.007.

8. Silva MJ, Barr DB, Reidy JA, et al. Urinary levels of seven phthalate metabolites in the U.S. population from the National Health and Nutrition Examination Survey (NHANES) 1999-2000. Environ Health Perspect. 2004;112(3):331-338. doi:10.1289/ehp.6723.

9. Blair RM, Fang H, Branham WS, et al. The estrogen receptor relative binding affinities of 188 natural and xenochemicals: structural diversity of ligands. Toxicol Sci. 2000;54(1):138-153. doi:10.1093/toxsci/54.1.138.

10. Jobling S, Reynolds T, White R, Parker MG, Sumpter JP. A variety of environmentally persistent chemicals, including some phthalate plasticizers, are weakly estrogenic. Environ Health Perspect. 1995;103(6):582-587. doi:10.1289/ehp.95103582. 
11. Preciados M, Yoo C, Roy D. Estrogenic Endocrine Disrupting Chemicals Influencing NRF1 Regulated Gene Networks in the Development of Complex Human Brain Diseases. Int J Mol Sci. 2016;17(12):2086. doi:10.3390/ijms 17122086.

12. Betz AJ, Jayatilaka S, Joshi J, et al. Chronic exposure to benzyl butyl phthalate (BBP) alters social interaction and fear conditioning in male adult rats: Alterations in amygdalar MeCP2, ERK1/2 and ER?? Neuroendocrinol Lett. 2013;34(5):347-358.

13. Lin H, Yuan K, Li L, et al. In utero exposure to diethylhexyl phthalate affects rat brain development: A behavioral and genomic approach. Int J Environ Res Public Health. 2015;12(11):13696-13710. doi:10.3390/ijerph121113696.

14. Dai Y, Yang Y, Xu X, Hu Y. Effects of uterine and lactational exposure to di(2-ethylhexyl) phthalate on spatial memory and NMDA receptor of hippocampus in mice. Horm Behav. 2015;71:41-48. doi:10.1016/j.yhbeh.2015.03.008.

15. Li XJ, Jiang L, Chen L, Chen HS, Li X. Neurotoxicity of dibutyl phthalate in brain development following perinatal exposure: A study in rats. Environ Toxicol Pharmacol. 2013;36(2):392-402. doi:10.1016/j.etap.2013.05.001.

16. Li Y, Li T, Zhuang M, Wang K, Zhang J, Shi N. High-dose dibutyl phthalate improves performance of $\mathrm{F} 1$ generation male rats in spatial learning and increases hippocampal BDNF expression independent on $\mathrm{p}-\mathrm{CREB}$ immunocontent. Environ Toxicol Pharmacol. 2010;29(1):32-38. doi:10.1016/j.etap.2009.09.003.

17. Li Y, Zhuang M, Li T, Shi N. Neurobehavioral toxicity study of dibutyl phthalate on rats following in utero and lactational exposure. J Appl Toxicol. 2009;29(7):603-611. doi:10.1002/jat.1447.

18. Miodovnik A, Engel SM, Zhu C, et al. Endocrine disruptors and childhood social impairment. Neurotoxicology. 2011;32(2):261-267. doi:10.1016/j.neuro.2010.12.009. 
19. Ipapo KN, Factor-Litvak P, Whyatt RM, et al. Maternal prenatal urinary phthalate metabolite concentrations and visual recognition memory among infants at 27 weeks. Environ Res. 2017;155(September 2016):7-14. doi:10.1016/j.envres.2017.01.019.

20. Factor-Litvak P, Insel B, Calafat AM, et al. Persistent associations between maternal prenatal exposure to phthalates on child IQ at age 7 years. PLoS One. 2014;9(12):1-15. doi:10.1371/journal.pone.0114003.

21. Yolton K, Xu Y, Strauss D, Altaye M, Calafat AM, Khoury J. Prenatal exposure to bisphenol A and phthalates and infant neurobehavior. Neurotoxicol Teratol. 2011;33(5):558-566. doi:10.1016/j.ntt.2011.08.003.

22. Doherty BT, Engel SM, Buckley JP, Silva MJ, Calafat AM, Wolff MS. Prenatal phthalate biomarker concentrations and performance on the Bayley Scales of Infant Development-II in a population of young urban children. Environ Res. 2017;152(September 2016):51-58.

doi:10.1016/j.envres.2016.09.021.

23. Shiue I. Arsenic, heavy metals, phthalates, pesticides, hydrocarbons and polyfluorinated compounds but not parabens or phenols are associated with adult remembering condition: US NHANES, 2011-2012. Environ Sci Pollut Res Int. 2015;22(8):6381-6386. doi:10.1007/s11356-015-4261-9.

24. ATSDR. Toxicological Profile for Phenol. Agency Toxic Subst Dis Regist. 2008:1-269. doi:10.3109/15569529909037564.

25. Calafat AM, Ye X, Wong LY, Reidy JA, Needham LL. Exposure of the U.S. population to Bisphenol A and 4-tertiary-octylphenol: 2003-2004. Environ Health Perspect. 2008;116(1):39-44. doi:10.1289/ehp.10753.

26. Alonso-Magdalena P, Ropero AB, Soriano S, et al. Bisphenol-A acts as a potent estrogen via non-classical estrogen triggered pathways. Mol Cell Endocrinol. 2012;355(2):201-207. doi:10.1016/j.mce.2011.12.012.

27. Bowman RE, Luine V, Diaz Weinstein S, Khandaker H, DeWolf S, Frankfurt $\mathrm{M}$. Bisphenol-A exposure during adolescence leads to enduring alterations in cognition and dendritic spine density in adult male and female rats. Horm Behav. 2015;69:89-97. doi:10.1016/j.yhbeh.2014.12.007. 
28. Diaz Weinstein S, Villafane JJ, Juliano N, Bowman RE. Adolescent exposure to Bisphenol-A increases anxiety and sucrose preference but impairs spatial memory in rats independent of sex. Brain Res. 2013;1529:56-65. doi:10.1016/j.brainres.2013.07.018.

29. Eilam-Stock T, Serrano P, Frankfurt M, Luine V. Bisphenol-A impairs memory and reduces dendritic spine density in adult male rats. Behav Neurosci. 2012;126(1):175-185. doi:10.1037/a0025959.

30. Elsworth JD, Jentsch JD, VandeVoort CA, Roth RH, Eugene Redmond D, Leranth C. Prenatal exposure to bisphenol A impacts midbrain dopamine neurons and hippocampal spine synapses in non-human primates. Neurotoxicology. 2013;35(1):113-120. doi:10.1016/j.neuro.2013.01.001.

31. Fan Y, Ding S, Ye X, et al. Does preconception paternal exposure to a physiologically relevant level of bisphenol A alter spatial memory in an adult rat? Horm Behav. 2013;64(4):598-604. doi:10.1016/j.yhbeh.2013.08.014.

32. Inagaki T, Frankfurt M, Luine V. Estrogen-induced memory enhancements are blocked by acute bisphenol $\mathrm{A}$ in adult female rats: Role of dendritic spines. Endocrinology. 2012;153(7):3357-3367. doi:10.1210/en.2012-1121.

33. Jang YJ, Park HR, Kim TH, et al. High dose bisphenol A impairs hippocampal neurogenesis in female mice across generations. Toxicology. 2012;296(13):73-82. doi:10.1016/j.tox.2012.03.007.

34. Johnson SA, Javurek AB, Painter MS, et al. Effects of developmental exposure to bisphenol A on spatial navigational learning and memory in rats: A CLARITY-BPA study. Horm Behav. 2015. doi:10.1016/j.yhbeh.2015.09.005.

35. Braun JM, Kalkbrenner AE, Calafat AM, et al. Impact of early-life bisphenol A exposure on behavior and executive function in children. Pediatrics. 2011;128(5):873-882. doi:10.1542/peds.2011-1335.

36. Kumar D, Kumar Thakur M. Perinatal exposure to bisphenol-A impairs spatial memory through upregulation of neurexin1 and neuroligin3 expression in male mouse brain. PLoS One. 2014;9(10):1-9. doi:10.1371/journal.pone.0110482. 
37. Matsuda S, Matsuzawa D, Ishii D, Tomizawa H, Sajiki J, Shimizu E. Perinatal exposure to bisphenol A enhances contextual fear memory and affects the serotoninergic system in juvenile female mice. Horm Behav. 2013;63(5):709716. doi:10.1016/j.yhbeh.2013.03.016.

38. Wang C, Niu R, Zhu Y, et al. Changes in memory and synaptic plasticity induced in male rats after maternal exposure to bisphenol A. Toxicology. 2014;322:51-60. doi:10.1016/j.tox.2014.05.001.

39. $\mathrm{Xu} \mathrm{X} \mathrm{Bin,} \mathrm{He} \mathrm{Y,} \mathrm{Song} \mathrm{C,} \mathrm{et} \mathrm{al.} \mathrm{Bisphenol} \mathrm{a} \mathrm{regulates} \mathrm{the} \mathrm{estrogen} \mathrm{receptor}$ alpha signaling in developing hippocampus of male rats through estrogen receptor. Hippocampus. 2014;24(12):1570-1580. doi:10.1002/hipo.22336.

40. Xu X, Liu X, Zhang Q, et al. Sex-specific effects of bisphenol-A on memory and synaptic structural modification in hippocampus of adult mice. Horm Behav. 2013;63(5):766-775. doi:10.1016/j.yhbeh.2013.03.004.

41. Zhang Q, Xu X, Li T, et al. Exposure to bisphenol-A affects fear memory and histone acetylation of the hippocampus in adult mice. Horm Behav. 2014;65(2):106-113. doi:10.1016/j.yhbeh.2013.12.004.

42. Kim ME, Park HR, Gong EJ, Choi SY, Kim HS, Lee J. Exposure to bisphenol A appears to impair hippocampal neurogenesis and spatial learning and memory. Food Chem Toxicol. 2011;49(12):3383-3389. doi:10.1016/j.fct.2011.09.017.

43. Elsworth JD, Jentsch JD, Groman SM, Roth RH, Redmond ED, Leranth C. Low circulating levels of bisphenol-A induce cognitive deficits and loss of asymmetric spine synapses in dorsolateral prefrontal cortex and hippocampus of adult male monkeys. J Comp Neurol. 2015;523(8):1248-1257. doi:10.1002/cne.23735.

44. Kuwahara R, Kawaguchi S, Kohara Y, Jojima T, Yamashita K. Bisphenol A does not affect memory performance in adult male rats. Cell Mol Neurobiol. 2014;34(3):333-342. doi:10.1007/s10571-013-0017-6.

45. Neese SL, Bandara SB, Schantz SL. Working memory in bisphenol-A treated middle-aged ovariectomized rats. Neurotoxicol Teratol. 2013;35(1):46-53. doi:10.1016/j.ntt.2013.01.002. 
46. Sadowski RN, Park P, Neese SL, Ferguson DC, Schantz SL, Juraska JM. Effects of perinatal bisphenol A exposure during early development on radial arm maze behavior in adult male and female rats. Neurotoxicol Teratol. 2014;42:17-24. doi:10.1016/j.ntt.2014.01.002.

47. Roen EL, Wang Y, Calafat AM, et al. Bisphenol A exposure and behavioral problems among inner city children at 7-9 years of age. Environ Res. 2015;142:739-745. doi:10.1016/j.envres.2015.01.014.

48. Harley KG, Gunier RB, Kogut K, et al. Prenatal and early childhood bisphenol A concentrations and behavior in school-aged children. Environ Res. 2013;126:43-50. doi:10.1016/j.envres.2013.06.004.

49. Braun JM, Yolton K, Dietrich KN, et al. Prenatal bisphenol A exposure and early childhood behavior. Environ Health Perspect. 2009;117(12):1945-1952. doi:10.1289/ehp.0900979.

50. Braun JM, Muckle G, Arbuckle T, et al. Associations of prenatal urinary bisphenol a concentrations with child behaviors and cognitive abilities. Environ Health Perspect. 2017;125(6):1-9. doi:10.1289/EHP984.

51. Johnson CL, Paulose-Ram R, Ogden CL, et al. National health and nutrition examination survey: analytic guidelines, 1999-2010. Vital Health Stat 2. 2013;(161):1-24. http://www.ncbi.nlm.nih.gov/pubmed/25090154.

52. Johnson CL, Dohrmann SM, Burt VL, Mohadjer LK. National Health and Nutrition Examination Survey: Sample Design , 2011 - 2014. Vital Heal Stat. 2014;2(162):1-33. http://www.cdc.gov/nchs/data/series/sr_02/sr02_162.pdf.

53. Centers for Disease Control and Prevention (CDC). National Center for Health Statistics (NCHS). National Health and Nutrition Examination Survey NCHS Research Ethics Review Board (ERB) Approval. Hyattsville, MD: U.S. Department of Health and Human Services, Centers for Disease Control and Prevention. https://www.cdc.gov/nchs/nhanes/irba98.htm. Published 2016. 
54. Centers for Disease Control and Prevention (CDC). National Center for Health Statistics (NCHS). National Health and Nutrition Examination Survey - 20112012 Data Documentation, Codebook, and Frequencies Phthalates \& Plasticizers Metabolites - Urine (PHTHTE_G). Hyattsville, MD: U.S. Department of Health and Human Services, Centers for Disease Control and Prevention. https://wwwn.cdc.gov/Nchs/Nhanes/2011-2012/PHTHTE_G.htm. Published 2016.

55. Centers for Disease Control and Prevention (CDC). National Center for Health Statistics (NCHS). National Health and Nutrition Examination Survey National Health and Nutrition Examination Survey 2013-2014 Data Documentation, Codebook, and Frequencies Phthalates and Plasticizers Metabolites - Urine (PHTHTE_H). Hyattsville, MD: U.S. Department of Health and Human Services, Centers for Disease Control and Prevention. https://wwwn.cdc.gov/Nchs/Nhanes/2013-2014/PHTHTE_H.htm. Published 2016.

56. Centers for Disease Control and Prevention (CDC). National Center for Health Statistics (NCHS). National Health and Nutrition Examination Survey Data, Questionnaires, and Related Documentation and Information. National Health and Nutrition Examination Survey Data. Hyattsville, MD: U.S. Department of Health and Human Services, Centers for Disease Control and Prevention. https://www.cdc.gov/nchs/nhanes/index.htm. Published 2016.

57. Centers for Disease Control and Prevention (CDC). National Center for Health Statistics (NCHS). National Health and Nutrition Examination Survey - Task 2: How to Address Different Fill Values and the Limit of Detection (LOD) in NHANES Environmental Chemical Data. Hyattsville, MD: U.S. Department of Health and Human Services, Centers for Disease Control and Prevention. https://www.cdc.gov/nchs/tutorials/environmental/critical_issues/limitations/T ask2.htm. Published 2016.

58. Centers for Disease Control and Prevention (CDC). National Center for Health Statistics (NCHS). National Health and Nutrition Examination Survey - 20132014 Data Documentation, Codebook, and Frequencies Personal Care and Consumer Product Chemicals and Metabolites (EPHPP_H). Hyattsville, MD: U.S. Department of Health and Human Services, Centers for Disease Control and Prevention. https://wwwn.cdc.gov/Nchs/Nhanes/20132014/EPHPP_H.htm. Published 2016. 
59. Centers for Disease Control and Prevention (CDC). National Center for Health Statistics (NCHS). National Health and Nutrition Examination Survey 20112012 Data Documentation, Codebook, and Frequencies Environmental Phenols \& Parabens (EPH_G). Hyattsville, MD: U.S. Department of Health and Human Services, Centers for Disease Control and Prevention. https://wwwn.cdc.gov/Nchs/Nhanes/2011-2012/EPH_G.htm. Published 2016.

60. Centers for Disease Control and Prevention (CDC). National Center for Health Statistics (NCHS). National Health and Nutrition Examination Survey 20132014 Data Documentation, Codebook, and Frequencies Cognitive Functioning (CFQ_H). Hyattsville, MD: U.S. Department of Health and Human Services, Centers for Disease Control and Prevention.

https://wwwn.cdc.gov/Nchs/Nhanes/2013-2014/CFQ_H.htm. Published 2017.

61. Centers for Disease Control and Prevention (CDC). National Center for Health Statistics (NCHS). National Health and Nutrition Examination Survey 20112012 Data Documentation, Codebook, and Frequencies Cognitive Functioning (CFQ_G). Hyattsville, MD: U.S. Department of Health and Human Services, Centers for Disease Control and Prevention.

https://wwwn.cdc.gov/Nchs/Nhanes/2011-2012/CFQ_G.htm\#CFDCST3.

Published 2017.

62. Fillenbaum GG, van Belle G, Morris JC, et al. Consortium to Establish a Registry for Alzheimer's Disease (CERAD): The first twenty years. Alzheimer's Dement. 2008;4(2):96-109. doi:10.1016/j.jalz.2007.08.005.

63. Bertolucci PHF, Okamoto IH, Brucki SMD, Siviero MO, Neto JT, Ramos LR. Applicability of the CERAD neuropsychological battery to Brazilian elderly. Arq Neuropsiquiatr. 2001;59(3 A):532-536. doi:10.1590/S0004282X2001000400009.

64. Karrasch M, Sinervä E, Grönholm P, Rinne J, Laine M. CERAD test performances in amnestic mild cognitive impairment and Alzheimer's disease. Acta Neurol Scand. 2005;111(3):172-179. doi:10.1111/j.16000404.2005.00380.x.

65. Karrasch M, Laatu S, Martikainen K, Marttila R. CERAD test performance and cognitive impairment in Parkinson's disease. Acta Neurol Scand. 2013;128(6):409-413. doi:10.1111/ane.12138. 
66. R.M. H, N.-M. S, P. H, et al. The CERAD neuropsychological battery in patients with frontotemporal lobar degeneration. Dement Geriatr Cogn Dis Extra. 2015;5(1):147-154. doi:10.1159/000380815.

67. Berres M, Monsch AU, Bernasconi F, Thalmann B, Stähelin HB. Normal ranges of neuropsychological tests for the diagnosis of Alzheimer's disease. Stud Health Technol Inform. 2000;77:195-199. http://www.ncbi.nlm.nih.gov/pubmed/11187541. Accessed January 21, 2018.

68. Sata F, Araki S, Yokoyama K, Murata K. Adjustment of creatinine-adjusted values in urine to urinary flow rate: a study of eleven heavy metals and organic substances. Int Arch Occup Environ Health. 1995;68(1):64-68. http://www.ncbi.nlm.nih.gov/pubmed/8847115. Accessed November 15, 2017.

69. Alegret M, Espinosa A, Valero S, et al. Cut-off Scores of a Brief Neuropsychological Battery (NBACE) for Spanish Individual Adults Older than 44 Years Old. Brucki S, ed. PLoS One. 2013;8(10):e76436. doi:10.1371/journal.pone.0076436.

70. American Academy of Family Physicians. BB, Hartmann B, DeJoseph D. American Family Physician. Vol 84. American Academy of Family Physicians; 1970. https://www.aafp.org/afp/2011/1015/p895.html. Accessed January 21, 2018.

71. Axelrod BN. Administration duration for the Wechsler Adult Intelligence Scale-III and Wechsler Memory Scale-III. Arch Clin Neuropsychol. 2001;16(3):293-301. doi:10.1016/S0887-6177(00)00048-2.

72. Matsuda O, Saito M, Kato M, Azami H, Shido E. Wechsler Adult Intelligence Scale-III profile in the early stages of Alzheimer's disease: Performance in subtests sensitive to and resistant to normal decline with ageing. Psychogeriatrics. 2015;15(1):1-6. doi:10.1111/psyg. 12066.

73. Donnell AJ, Pliskin N, Holdnack J, Axelrod B, Randolph C. Rapidlyadministered short forms of the Wechsler Adult Intelligence Scale-3rd edition. Arch Clin Neuropsychol. 2007;22(8):917-924. doi:10.1016/j.acn.2007.06.007. 
74. Nielsen NP, Ringström R, Wiig EH, Minthon L. Associations between AQT processing speed and neuropsychological tests in neuropsychiatric patients. Am J Alzheimers Dis Other Demen. 2011;22(3):202-210. doi:10.1177/1089253206300417.

75. Proust-Lima C, Amieva H, Dartigues JF, Jacqmin-Gadda H. Sensitivity of four psychometric tests to measure cognitive changes in brain aging-populationbased studies. Am J Epidemiol. 2007;165(3):344-350. doi:10.1093/aje/kwk017.

76. Rosano C, Perera S, Inzitari M, Newman AB, Longstreth WT, Studenski S. Digit symbol substitution test and future clinical and subclinical disorders of cognition, mobility and mood in older adults. Age Ageing. 2016;45(5):687-694. doi:10.1093/ageing/afw116.

77. Saxton J, Lopez OL, Ratcliff G, et al. Preclinical Alzheimer disease: neuropsychological test performance 1.5 to 8 years prior to onset. Neurology. 2004;63(Mh 46643):2341-2347. doi:10.1212/01.WNL.0000147470.58328.50.

78. Centers for Disease Control and Prevention (CDC). National Center for Health Statistics (NCHS). National Health and Nutrition Examination Survey Questionnaires, Datasets, and Related Documentation. Hyattsville, MD: U.S. Department of Health and Human Services, Centers for Disease Control and Prevention. https://www.cdc.gov/nchs/nhanes/nhanes_questionnaires.htm. Published 2015.

79. Tolea MI, Morris JC, Galvin JE. Longitudinal associations between physical and cognitive performance among community-dwelling older adults. PLoS One. 2015;10(4):1-12. doi:10.1371/journal.pone.0122878.

80. Ansoleaga B, Garcia-Esparcia P, Llorens F, Moreno J, Aso E, Ferrer I. Dysregulation of brain olfactory and taste receptors in AD, PSP and CJD, and AD-related model. Neuroscience. 2013;248:369-382. doi:10.1016/j.neuroscience.2013.06.034.

81. Macknin JB, Higuchi M, Lee VM-Y, Trojanowski JQ, Doty RL. Olfactory dysfunction occurs in transgenic mice overexpressing human $\tau$ protein. Brain Res. 2004;1000(1-2):174-178. doi:10.1016/j.brainres.2004.01.047. 
82. Lee S, Manly J, Andrews H, et al. Olfactory deficits predict cognitive decline and Alzheimer dementia in an urban community. 2014.

83. Hummel T, Landis B, Huttenbrink K. Smell and taste disorders. GMS Curr Tpoics Otorhinolaryngol Head Neck Surg. 2011;10:1-15. doi:10.3205/cto000077.

84. Doty RL. The olfactory vector hypothesis of neurodegenerative disease: Is it viable? Ann Neurol. 2008;63(1):7-15. doi:10.1002/ana.21327.

85. Liu J. Clinical features of olfactory disorders in patients seeking medical consultation. Med Sci Monit. 2013;19:444-450. doi:10.12659/MSM.883938.

86. Hüttenbrink K-B, Hummel T, Berg D, Gasser T, Hähner A. Olfactory dysfunction: common in later life and early warning of neurodegenerative disease. Dtsch Arztebl Int. 2013;110(1-2):1-7, e1. doi:10.3238/arztebl.2013.0001.

87. Barresi M, Ciurleo R, Giacoppo S, et al. Evaluation of olfactory dysfunction in neurodegenerative diseases. J Neurol Sci. 2012;323(1-2):16-24. doi:10.1016/j.jns.2012.08.028.

88. Ruan Y, Zheng XY, Zhang HL, Zhu W, Zhu J. Olfactory dysfunctions in neurodegenerative disorders. J Neurosci Res. 2012;90(9):1693-1700. doi:10.1002/jnr.23054.

89. Albers MW, Tabert MH, Devanand DP. Olfactory dysfunction as a predictor of neurodegenerative disease. Curr Neurol Neurosci Rep. 2006;6(5):379-386. doi:10.1007/s11910-996-0018-7.

90. Winner B, Kohl Z, Gage FH. Neurodegenerative disease and adult neurogenesis. Eur J Neurosci. 2011;33(6):1139-1151. doi:10.1111/j.14609568.2011.07613.x.

91. Attems J, Walker L, Jellinger KA. Olfactory bulb involvement in neurodegenerative diseases. Acta Neuropathol. 2014;127(4):459-475. doi:10.1007/s00401-014-1261-7. 
92. Makowska I, Kloszewska I, Grabowska A, Szatkowska I, Rymarczyk K. Olfactory deficits in normal aging and Alzheimer's disease in the polish elderly population. Arch Clin Neuropsychol. 2011;26(3):270-279. doi:10.1093/arclin/acr011.

93. Sohrabi HR, Bates K a, Weinborn MG, et al. Olfactory discrimination predicts cognitive decline among community-dwelling older adults. Transl Psychiatry. 2012;2(5):e118. doi:10.1038/tp.2012.43.

94. Driver-Dunckley E, Adler CH, Hentz JG, et al. Olfactory dysfunction in incidental Lewy body disease and Parkinson's disease. Parkinsonism Relat Disord. 2014;20(11):1260-1262. doi:10.1016/j.parkreldis.2014.08.006.

95. Ward AM, Calamia M, Thiemann E, Dunlap J, Tranel D. Association between olfaction and higher cortical functions in Alzheimer's disease, mild cognitive impairment, and healthy older adults. J Clin Exp Neuropsychol. November 2016:1-15. doi:10.1080/13803395.2016.1253667.

96. SAS 9.4. 2017.

97. Centers for Disease Control and Prevention (CDC). National Center for Health Statistics (NCHS). National Health and Nutrition Examination Survey Overview of NHANES Survey Design and Weights. Hyattsville, MD: U.S. Department of Health and Human Services, Centers for Disease Control and Prevention. https://www.cdc.gov/nchs/tutorials/environmental/orientation/sample_design/. Published 2013.

98. Centers for Disease Control and Prevention (CDC). National Center for Health Statistics (NCHS). National Health and Nutrition Examination Survey - Using Blood Lipid or Urine Creatinine Adjustments of Environmental Chemical Data. Hyattsville, MD: U.S. Department of Health and Human Services, Centers for Disease Control and Prevention. https://www.cdc.gov/nchs/tutorials/environmental/critical_issues/adjustments/I nfo1.htm. Published 2017. 
99. Centers for Disease Control and Prevention (CDC). National Center for Health Statistics (NCHS). National Health and Nutrition Examination Survey - Using Blood Lipid or Urine Creatinine Adjustments in the Analysis of Environmental Chemical Data. Hyattsville, MD: U.S. Department of Health and Human Services, Centers for Disease Control and Prevention. https://www.cdc.gov/nchs/tutorials/environmental/critical_issues/adjustments/i ndex.htm. Published 2013.

100. Centers for Disease Control and Prevention (CDC). National Center for Health Statistics (NCHS). National Health and Nutrition Examination Survey - Data Normality and Transformations. Hyattsville, MD: U.S. Department of Health and Human Services, Centers for Disease Control and Prevention. https://www.cdc.gov/nchs/tutorials/environmental/critical_issues/normality/ind ex.htm. Published 2010.

101. SAS Institute Inc. Sas/Stat ${ }^{\circledR}$ 9.3. 2011:User's Guide: The GLIMMIX Procedure (Chapter).

102. SAS Institute Inc. Estimating Geometric Means Using Data from a Complex Survey Sampling Design. http://citeseerx.ist.psu.edu/viewdoc/download?rep=rep1 \&type=pdf\&doi=10.1. 1.192.593. Published 2017.

103. Ferguson KK, Colacino JA, Lewis RC, John D, Arbor A. and use of mouthwash and sunscreen Study population. J Expo Sci Environ Epidemiol. 2017;27(3):326-332. doi:10.1038/jes.2016.27.Personal.

104. Zagni E, Simoni L, Colombo D. Sex and Gender Differences in Central Nervous System-Related Disorders. Neurosci J. 2016;2016:1-13. doi:10.1155/2016/2827090.

105. Czlonkowska A, Ciesielska A, Gromadzka G. Gender Differences in Neurological Disease. 2006;29(2):243-256.

106. Arevalo M-A, Azcoitia I, Garcia-Segura LM. The neuroprotective actions of oestradiol and oestrogen receptors. Neuroscience. 2015;16(1):17-29. doi:10.1038/nrn3856. 
107. Holahan MR, Smith CA. Phthalates and neurotoxic effects on hippocampal network plasticity. Neurotoxicology. 2015;48:21-34.

doi:10.1016/j.neuro.2015.02.008.

108. Chakrabarti M, Haque A, Banik NL, Nagarkatti P, Nagarkatti M, Ray SK. Estrogen receptor agonists for attenuation of neuroinflammation and neurodegeneration. Brain Res Bull. 2014;109:22-31. doi:10.1016/j.brainresbull.2014.09.004.

109. Green PS, Simpkins JW. Neuroprotective effects of estrogens: Potential mechanisms of action. Int J Dev Neurosci. 2000;18(4-5):347-358. doi:10.1016/S0736-5748(00)00017-4.

110. Hristian $\mathrm{C}$, Ruber JG, Alter W, et al. Production and actions of estrogens. $N$ Engl J Med. 2002;346(5). doi:10.1016/S0960-0760(01)00184-4.

111. Pozzi S, Benedusi V, Maggi A, Vegeto E. Estrogen action in neuroprotection and brain inflammation. Ann N Y Acad Sci. 2006;1089(2006):302-323. doi:10.1196/annals.1386.035.

112. Wise PM, Dubal DB, Wilson ME, Rau SW, Liu Y. Estrogens: trophic and protective factors in the adult brain. Front Neuroendocrinol. 2001;22(1):33-66. doi:10.1006/frne.2000.0207.

113. Hojo Y, Murakami G, Mukai H, et al. Estrogen synthesis in the brain-Role in synaptic plasticity and memory. Mol Cell Endocrinol. 2008;290(1-2):31-43. doi:10.1016/j.mce.2008.04.017.

114. Leranth C, Szigeti-Buck K, MacLusky NJ, Hajszan T. Bisphenol A prevents the synaptogenic response to testosterone in the brain of adult male rats. Endocrinology. 2008;149(3):988-994. doi:10.1210/en.2007-1053.

115. Parducz a., Hajszan T, MacLusky NJ, et al. Synaptic remodeling induced by gonadal hormones: Neuronal plasticity as a mediator of neuroendocrine and behavioral responses to steroids. Neuroscience. 2006;138(3):977-985. doi:10.1016/j.neuroscience.2005.07.008. 
116. Leranth C, Petnehazy O, MacLusky NJ. Gonadal hormones affect spine synaptic density in the CA1 hippocampal subfield of male rats. J Neurosci. 2003;23(5):1588-1592.

117. Ogiue-Ikeda M, Tanabe N, Mukai H, et al. Rapid modulation of synaptic plasticity by estrogens as well as endocrine disrupters in hippocampal neurons. Brain Res Rev. 2008;57(2):363-375. doi:10.1016/j.brainresrev.2007.06.010.

118. Arnold S, Beyer C. Neuroprotection by estrogen in the brain: The mitochondrial compartment as presumed therapeutic target. J Neurochem. 2009;110(1):1-11. doi:10.1111/j.1471-4159.2009.06133.x.

119. Grimm A, Schmitt K, Lang UE, Mensah-Nyagan AG, Eckert A. Improvement of neuronal bioenergetics by neurosteroids: Implications for age-related neurodegenerative disorders. Biochim Biophys Acta - Mol Basis Dis. 2014;1842(12):2427-2438. doi:10.1016/j.bbadis.2014.09.013.

120. Simpkins JW, Yi KD, Yang S, Dykens J a. Mitochondrial mechanisms of estrogen neuroprotection. Biochim Biophys Acta. 2011;1800(10):1113-1120. doi:10.1016/j.bbagen.2009.11.013.Mitochondrial.

121. Wu Y, Li K, Zuo H, Yuan Y, Sun Y, Yang X. Primary neuronal-astrocytic coculture platform for neurotoxicity assessment of di-(2-ethylhexyl) phthalate. $J$ Environ Sci (China). 2014;26(5):1145-1153. doi:10.1016/S10010742(13)60504-5.

122. Guida N, Laudati G, Galgani M, et al. Histone deacetylase 4 promotes ubiquitin-dependent proteasomal degradation of $\mathrm{Sp} 3$ in SH-SY5Y cells treated with di(2-ethylhexyl)phthalate (DEHP), determining neuronal death. Toxicol Appl Pharmacol. 2014;280(1):190-198. doi:10.1016/j.taap.2014.07.014.

123. Van Der Meer TP, Artacho-Cordón F, Swaab DF, et al. Distribution of nonpersistent endocrine disruptors in two different regions of the human brain. Int J Environ Res Public Health. 2017;14(9):1-11. doi:10.3390/ijerph14091059.

124. Davis AP, Grondin CJ, Lennon-Hopkins K, et al. The Comparative Toxicogenomics Database's 10th year anniversary: Update 2015. Nucleic Acids Res. 2015;43(D1):D914-D920. doi:10.1093/nar/gku935. 


\title{
CHAPTER VI
}

\section{MANUSCRIPT III}

\section{EXPOSURE TO METALLOESTROGENS AND ASSOCIATIONS WITH}

\section{BRAIN HEALTH: NHANES 2011-2014}

\author{
ABSTRACT \\ BACKGROUND: The role of estrogenic endocrine disrupting chemicals (EEDCs) \\ and their role in the development of neurodegenerative disease is of great public \\ health concern, due to increasing exposures to these chemicals and increasingly aging \\ population. Evidence suggests EEDCs exposure plays a role in the development of \\ neurodegenerative disease. Metalloestrogens are known estrogenic endocrine \\ disrupting chemicals (EEDCs) that are also heavy metals, and have been shown to \\ exert estrogenic activity, which affects estrogen and its protective effects on brain \\ health.
}

OBJECTIVE: The objective of this study is to investigate the relationship between surrogate brain health indicators and exposure to metalloestrogens among the older individuals of the United States (US) population.

METHODS: In this study, we analyzed participants from the Center for Disease Control and Prevention (CDC) National Health and Nutrition Examination Survey (NHANES) in the survey cycles 2011-2012 and 2013-2014. The participants were 60 years of age and older who had urine samples taken during the examination. Other data pertaining to covariates and demographics were also obtained. In total, three metallogestrogens were selected. The three analyzed metalloestrogens were the 
following: cadmium $(\mathrm{Cd})$, manganese $(\mathrm{Mn})$, and arsenic (As). These EEDCs were analyzed versus various brain health indicators available in the form of test scores and questionnaires, available in the NHANES datasets. The brain health indicators test scores were the following: immediate recall test scores; delayed recall test scores; animal fluency test scores; digit symbol substitution test scores. The brain health indicator questions were as follows: worsening memory over the past 12 months; trouble remembering over the past week; difficulty remembering or because you experience periods of confusion. The following smell and taste questions were also included as brain health indicators due to their potential as pre-clinical indicators of cognitive impairment: phantom odor (phantosmia) and persistent taste in mouth over the past 12 months. Geometric means were calculated to compare yes/no or low score/high score dichotomous responses to the brain health indicators versus the EEDC concentrations. Logistic regression was then used to calculate odds ratios (ORs) and 95\% confidence intervals (CIs). Three logistic regression models were presented in our study with all of them stratified by gender: unadjusted; age, race, education; age, race, education, body mass index (BMI), smoking status, blood pressure, diabetes status, alcohol use, coronary heart disease status, heart attack status, stroke status, head injury status, and physical activity status.

RESULTS: $\mathrm{Cd}$ and Mn bioburdens were found to be more evident in individuals who have lower cognitive scores and/or have memory cognition issues. In our logistic regression models, $\mathrm{Cd}$ stood out as the metalloestrogen that plays a larger role in the development of neurodegenerative disease and adverse brain health in an older population. 
CONCLUSION: $\mathrm{Cd}$ and to a lesser extent, $\mathrm{Mn}$, may play a role in adverse brain health and the development of some neurodegenerative condition. Further research is necessary due to the cross-sectional nature of our study.

\section{Exposure to Metalloestrogens and Associations with Brain Health: NHANES 2011-2014 INTRODUCTION}

The role of exposure to EEDCs and neurodegenerative disease development is of great public health concern as stated by the World Health Organization (WHO) ${ }^{1}$, due to cognitive health and neurodegenerative disease emerging as great public health concerns due to an increasingly aging population ${ }^{2}$. Exposures to EEDCs have been linked to neurodegenerative diseases and other adverse brain health conditions, such as Alzheimer's disease (AD). In this study, we examine the associations of three of the mostly widely present Metalloestrogens, $\mathrm{Cd}, \mathrm{Mn}$, and As, and their associations with brain health in an older US population.

The role of exposure to EEDCs, brain health, and neurodegenerative disease development is of great public health concern as stated by the World Health Organization (WHO) since exposure to endocrine disrupting chemicals can lead to the development of various human disease, which include neurodegenerative diseases 1.

Cadmium is a naturally occurring metal in the earth's crust and a natural constituent of ocean water ${ }^{3}$. Populations are exposed through food, cigarettes, smoke, drinking water, and air ${ }^{3}$. Cadmium is introduced into the food chain through soil and food 
contact surfaces, and through foods such as leafy vegetables, grains, legumes, and organ meats ${ }^{3}$. Occupation exposures are highest in occupations involving cadmiumcontaining products, and occupations involved in alloy, battery, plastics, and coloring production ${ }^{3}$. Inhalation and oral routes of exposure are predominant, followed by dermal exposure ${ }^{3}$. Current evidence supports the toxicity of cadmium and its effects on the developing organism, reproductive toxicity, hepatic effects, hematological effects, and immunological effects ${ }^{3}$.

Studies on human populations have also shown cadmium's adverse effects on adult brain function. A cross-sectional study using NHANES III data from 1988 to 1994 compared neurocognitive test scores and urinary cadmium concentrations found individuals with no smoking history, or known occupational cadmium exposure were found to have lower attention/perception scores with increasing urinary cadmium levels ${ }^{4}$. In a cross-sectional study of rural elderly Chinese persons, it was found that increasing serum cadmium and copper levels was significant associated with lower composite cognitive scores ${ }^{5}$ Another cross-sectional study examined the cerebrospinal fluid of ALS patients found elevated levels of various metals, including cadmium, which her higher than the measured blood levels and indicative of bioaccumulation ${ }^{6}$. A case-control study examining heavy metal levels in a group of Mongolian people found elevated cadmium, as well as other heavy metals in those with Parkinson-like symptoms in hair samples taken ${ }^{7}$.

Arsenic is an element in the environment, found in the earth's crust, and is considered a metalloid ${ }^{8}$. Populations are usually exposed through the air, drinking water, and food, with food being the main source of arsenic in a population, with some areas 
having naturally high levels of arsenic ${ }^{8}$. Occupational exposure occurs through individuals working in metal smelting, wood treatment, and those in working in the production and application of pesticides and herbicides ${ }^{8}$. Arsenic is also used in the animal and poultry feed as an antimicrobial additive ${ }^{8}$. The major routes of exposure are inhalation and oral, with dermal exposure being considered a minor route ${ }^{8}$. Arsenic has been associated with various health conditions, which include respiratory disorders, cardiovascular outcomes, diabetes, ocular effects, immune response disturbances, impaired neurological function, developmental effects, and cancers ${ }^{8}$ Genetic polymorphisms are suspected of contributing to the sensitivity towards $\operatorname{arsenic}^{8}$. In cross-sectional study consisting of 133 men and 201 women from the Project FRONTIER, a rural healthcare study, long-term low level exposure to arsenic from groundwater was found to be associated with poorer scores in language, visuospatial skills, and executive functioning, global cognition, processing speed, and immediate memory ${ }^{9}$. In another cross-sectional study from Project Frontier, consisting of 526 subjects genotyped according to the AS3MT gene, exposure to higher low level arsenic in groundwater reduced cognitive functioning, but the results differed with amongst the different SNPs ${ }^{10}$. In a cross-sectional study measuring heavy metal serum levels in 89 Ad patients and 188 cognitively normal controls, there was no difference in serum arsenic levels between the AD group and controls ${ }^{11}$. Another cross-sectional study conducted by Edwards et al. ${ }^{12}$ with a cohort consisting of 733 AD patients, 127 individuals with mild CI, and 530 individuals of normal cognition, 
found that exposure to low level arsenic exposure from groundwater was found to be associated with poorer neuropsychological performance.

Manganese is a metal that is an essential nutrient required as a cofactor for various enzymes and is found naturally in grains and fruit ${ }^{13}$. Manganese is found in various industrial processes and products and exposure can occur through inhalation, oral, dermal, and occupational routes ${ }^{13}$. Manganese has the potential to accumulate in lower level organisms in the food chain and has been linked to various health issues including inflammation, impaired lung function, and adverse neurological effects ${ }^{13}$.

Recent epidemiological studies have assessed the effects of manganese on brain health. A cross-sectional study by Hozumi et al. ${ }^{14}$, analyzed the cerebrospinal fluid of various neurodegenerative disease patients and found a higher level of manganese among PD patients. In another cross-sectional study assessing children's intellectual functioning and arsenic and manganese exposure, blood manganese levels were negatively associated with full scale IQ test scores, working memory, and perceptual memory ${ }^{15}$. A cross-sectional study by Kim et al. ${ }^{16}$ examining low-level manganese exposure in adults of a Ohio community found subtle subclinical effects in UPDRS and postural sway test for PD. A cross-sectional study of school-aged children in Brazil found inverse scores on executive function and attention tests with manganese levels ${ }^{17}$. A study amongst school-children in Canada found low-level manganese exposure in drinking water was associated with poorer neurobehavioral functions ${ }^{18}$. Koc et al. ${ }^{19}$ found higher levels of metal, including manganese, in hair samples of $\mathrm{AD}$ patients compared to controls. 
A case-control study Miyake et al. ${ }^{20}$, assessing dietary intake of heavy metals amongst PD patients found no association with manganese intake. A casecontrol study by Roos et al. ${ }^{6}$ found elevated manganese levels in cerebrospinal fluid of ALS patients. A study by Kumudini et al. ${ }^{21}$ found not correlation between manganese blood levels in PD patients compared to controls. A case control study by Garzillo et al. ${ }^{22}$ found no association between manganese levels in ALS patients vs

controls. A study by Kihira et al. ${ }^{23}$ found elevated manganese levels in ALS patients vs controls from hair samples. Another study by Arain et al. ${ }^{24}$ found higher levels of manganese and aluminum in hair samples of patients suffering from neurodegenerative disease.

A small cohort study that followed 26 welders exposed to manganese found after a 3.5-year follow-up found worsened olfactory, extrapyramidal, and mood disturbances ${ }^{25}$. A cohort study following asymptomatic welder trainees with no previous manganese exposure found low-level exposure to cause sub-clinical brain changes in subjects before any measurable learning deficits may occur ${ }^{26}$.

Cadmium, arsenic, and manganese have been found to all have estrogenic activity and affinity for estrogen receptors ${ }^{27}$ and have been found to interact with estrogen-responsive genes implicated in various neurodegenerative disorders 28 Heavy metals have also been demonstrated to affect and cross the blood brain barrier $29-31$.

\section{OBJECTIVE}

There is limited information regarding exposures to metalloestrogens the development of cognitive dysfunction and neurodegenerative disease in older 
populations. In this study we examine the relationship between three metalloestrogens, $\mathrm{Cd}, \mathrm{Mn}$, and As, with surrogate brain health indicators, from the CDC's NHANES 2011-2012 and 2013-2014 data cycles. The objectives of the study are as follows: 1) to assess the mean $\mathrm{Cd}, \mathrm{Mn}$, and As levels in older adults in the US, 60 years of age and above with the surrogate brain health indicators in the US population, 2) assess the association between $\mathrm{Cd}, \mathrm{Mn}$, and As levels and surrogate brain health indicators in older adults in the US, to find the risk of poor cognitive function and possible development of mild cognitive impairment, dementia, and AD.

\section{METHODS}

Study Design and Population: NHANES is a continuous cross-sectional data collection utilizing a complex multi-stage sampling design that creates a survey representative of the non-institutionalized population of the United States ${ }^{32,33}$. The survey has been conducted since 1999 and consists of an at-home questionnaires followed by a standardized physical examination and specimen collection conducted in mobile examination centers (MEC) ${ }^{32,33}$. Eligibility is determined using preset selection probabilities for the desired demographic subdomains ${ }^{33}$. A household screener is performed before to determine if any household members are eligible for the interview and examination ${ }^{33}$. The interview collects demographic, health, nutrition, and household information, while the physical examination includes physical measurements, dental examination, and the collection of blood and urine specimens for laboratory testing ${ }^{33}$. Prior to any to interviews and examinations, informed consent was obtained and all procedures were approved by the CDC Institutional Review Board ${ }^{34}$ 
In our study, we merged the NHANES 2011-2012 and 2013-2014 data cycles. All our analyses were limited to individuals 60 years of age and older who have recorded responses to cognitive test scores and/or memory and taste/smell questions and have select metalloestrogen urine measurements.

\section{Inclusion/Exclusion Criteria}

\section{Inclusion criteria:}

9. Males and females, 60 years of age and older

10. Available metalloestrogen urine measurements (Cd, Mn, As)

11. Urine creatinine measurements $>30 \mathrm{mg} / \mathrm{dl}$ and $>300 \mathrm{mg} / \mathrm{dl}$.

12. Complete responses to identified outcome variables.

\section{Exclusion criteria:}

9. Males and females, 59 years of age and younger

10. Unavailable metalloestrogen urine measurements (Cd, Mn, As)

11. Urine creatinine measurements $<30 \mathrm{mg} / \mathrm{dl}$ and $>300 \mathrm{mg} / \mathrm{dl}$

12. Complete responses to identified outcome variables.

\section{Cadmium Exposure Assessment Measurements}

Cadmium were measured from urine samples taken from a representative and random one-third subsample of individuals 6 years of age and older in the 2011-2012 and 2013-2014 survey cycles ${ }^{35-37}$. The laboratory inductively coupled-plasma dynamic reaction cell-mass spectrometry to analyze urine Cadmium levels and was consistently used in both survey cycles ${ }^{35-37}$. Cadmium levels were provided in ug/L and was used in our analyses. Urinary cadmium was used in our analyses and coded 
as URXUCD, with a limit of detection variable coded as URDUCDLC. The limits of detection were 0.056 ug/L for 2011-2012 and 0.036 ug/L for 2013-2014.

The limit of detection variables indicates if subjects have urine Cadmium levels above the limit of detection. A value of " 0 " indicates above the limit of detection and a value of " 1 " indicates below the limit of detection. Individuals with a below the limit of detection were given a dummy Cadmium level of the LOD divided by the square root of two ${ }^{38}$. Some LODs differ between survey cycles and a conservative approach was used to account for differing LODs per survey cycle ${ }^{39}$.

\section{Manganese Exposure Assessment and Measurements}

Manganese were measured from urine samples taken from a representative and random one-third subsample of individuals 6 years of age and older in the 2011-2012 and 2013-2014 survey cycles ${ }^{35-37}$. The laboratory inductively coupled-plasma dynamic reaction cell-mass spectrometry to analyze urine Manganese levels and was

consistently used in both survey cycles ${ }^{35-37}$. Manganese levels were provided in ug/L and was used in our analyses. The following Manganese metabolites were used in our analyses. Urinary manganese was used in our analyses and coded as URXUMN, with a limit of detection variable coded as URDUMNLC. The limits of detection were $0.08 \mathrm{ug} / \mathrm{L}$ for 2011-2012 and 0.013 ug/L for 2013-2014.

The limit of detection variables indicates if subjects have urine Manganese levels above the limit of detection. A value of " 0 " indicates above the limit of detection and a value of "1" indicates below the limit of detection. Individuals with a below the limit of detection were given a dummy Manganese level of the LOD divided by the 
square root of two ${ }^{38}$. Some LODs differ between survey cycles and a conservative approach was used to account for differing LODs per survey cycle ${ }^{39}$.

\section{Arsenic Exposure Assessment and Measurements}

Arsenic were measured from urine samples taken from a representative and random one-third subsample of individuals 6 years of age and older in the 2011-2012 and 2013-2014 survey cycles ${ }^{35-37}$. The laboratory inductively coupled-plasma dynamic reaction cell-mass spectrometry to analyze urine arsenic levels and was consistently used in both survey cycles ${ }^{35-37}$. Arsenic levels were provided in ug/L and was used in our analyses. Total urinary arsenic was used in our analyses and coded as URXUAS, with a limit of detection variable coded as URDUASLC. The limits of detection were $1.25 \mathrm{ug} / \mathrm{L}$ for $2011-2012$ and $0.26 \mathrm{ug} / \mathrm{L}$ for 2013-2014.

Only total arsenic was analyzed in the study. The limit of detection variables indicates if subjects have urine arsenic levels above the limit of detection. A value of " 0 " indicates above the limit of detection and a value of "1" indicates below the limit of detection. Individuals with a below the limit of detection were given a dummy arsenic level of the LOD divided by the square root of two ${ }^{38}$. Some LODs differ between survey cycles and a conservative approach was used to account for differing LODs per survey cycle ${ }^{39}$. The specific arsenic metabolites were selected as $>60 \%$ of study subjects had urine arsenic levels above the LOD.

\section{Assessment of Surrogate Brain Health Indicators - Cognitive Scores}

\section{CERAD Word Learning Subtest - Immediate Recall and Delayed Recall:}

The Consortium to Establish a Registry for Alzheimer's Disease (CERAD)

Word assesses both immediate and delayed learning ${ }^{40,41}$. The delayed and immediate 
recall tests available in NHANES assess the ability to process new verbal information 40,41. The tests are part of the neuropsychological assessment for the entire CERAD testing protocol, which was initially created to standardize AD assessment and diagnosis ${ }^{42}$. The tests in the neuropsychological assessment itself were chose because of their ability to assess cognitive functions inherent in $\mathrm{AD}^{42}$. The assessments have the ability to differentiate those of adequate cognitive status versus those who have mild cognitive impairment or dementia ${ }^{42-45}$. Although developed for use in the assessment of $\mathrm{AD}$, the CERAD assessments have shown utility in use for Parkinson's disease $^{45}$ and frontotemporal lobar degeneration ${ }^{46}$.

Immediate Recall: For immediate recall, , the subjects are asked to read aloud a sequence of 10 unrelated words as they are presented to them and immediately after, they are asked to recall as many words as possible ${ }^{40,41}$. This is done in three trials with the order of the words differing in each trial. ${ }^{40,41}$. Each trial has a maximum score of 10 , with a maximum overall score of $30^{40,41,43}$.

In our study, we included individuals 60 years of age and older who completed all immediate recall word list trials identified as: CFDCST1, CFDCST2, and CFDCST3 in the 2011-2012 and 2013-2014 NHANES data cycles. Those who did not have three trials completed were not included in the immediate recall analysis. We summed the total of the three trials and created a new variable with cut-off scores named IMMEDIATERECALL. A cut-off score of $\leq 13$ and $\geq 14$ was used as it is the standard in other assessments ${ }^{43,47}$. A total of 3,149 subjects from the 2011-2012 and 2013-2014 responded with complete immediate recall trials. We then accounted for those who were measured for metalloestrogens and accounted for those with extreme 
creatinine scores $(<30$ and $>300 \mathrm{mg} / \mathrm{dl})$, as these extreme values can affect analyses by being too dilute or concentrated ${ }^{48}$. After, our immediate recall study population consisted of 940 subjects, 146 subjects $\leq 13$ and 794 subjects $\geq 14$.

Delayed Recall: For delayed recall, the subject is asked to repeat the sequence of 10 unrelated words after the other cognitive tests are completed, which is typical 8 to 10 minutes after the start of the word learning trials ${ }^{40,41}$. The maximum score is 10 for delayed recall ${ }^{40,41}$.

In our study, we included subjects 60 years of age and older who completed the delayed recall trial, identified as CFDCSR, for the 2011-2012 and 2013-2014 NHANES data cycles. After we created a new variable with the cut-off scores named DELAYEDRECALL. A cut-off score of $\leq 13$ and $\geq 14$ was used as it is the standard in other assessments ${ }^{43,47,49}$. A total of 3,126 subjects from the 2011-2012 and 20132014 responded with a complete delayed recall trial. We then accounted for those who were measured for metalloestrogens and accounted for those with extreme creatinine scores $(<30$ and $>300 \mathrm{mg} / \mathrm{dl})$, as these extreme values can affect analyses by being too dilute or concentrated ${ }^{48}$. After, our study population consisted of 930 subjects, 157 subjects $\leq 3$ and 776 subjects $\geq 4$.

Animal Fluency: The animal fluency test is used to determine categorical verbal fluency, which is part of executive function and can differentiate between with normal cognition versus those with MCI and more severe cognitive impairment ${ }^{40,41}$. Since the test uses animal names, it does not require cultural consideration or formal education experience ${ }^{40,41}$. In the test, subjects are asked to name as many animals in a 
one minute span, with a maximum range of 40 words in the NHANES 2011-2014 data set. ${ }^{40,41}$. A sample test is given to each subject before the actual test ${ }^{40,41}$. In our study, we included subjects 60 years of age and older who completed the animal fluency trial, identified as CFDAST, for the 2011-2012 and 2013-2014 NHANES data cycles. After we created a new variable with cut-off scores named VERBALFLUENCY. cut-off score of $\leq 11$ and $\geq 12$ was used as it is the standard in other assessments ${ }^{43,47,49,50}$. A total of 3,110 subjects from the 2011-2012 and 20132014 responded with a complete delayed recall trial. We then accounted for those who were measured for metalloestrogens and accounted for those with extreme creatinine scores $(<30$ and $>300 \mathrm{mg} / \mathrm{dl})$, as these extreme values can affect analyses by being too dilute or concentrated ${ }^{48}$. After, our study population consisted of 931 subjects, 187 subjects $\leq 11$ and 744 subjects $\geq 12$.

Digit Symbol Substitution Test: The Digit Symbol Substitution Test (DSST) is part of the Wechsler Adult Intelligence Scale (WAIS III) ${ }^{40,41,51}$. The test measures processing speed, sustained attention, and working memory ${ }^{40,41,51}$. The subtests have shown utility in the identification of dementia and other neurodegenerative disorders ${ }^{52-54}$. The test is given in paper form, with a key that has 9 numbers paired to different symbols. The subject has 2 minutes to match each symbol to 133 boxes with a number associated to it, with the score as the total correct matches with a maximum score of 105 in the 2011-2014 NHANES dataset. ${ }^{40,41}$. A sample test is given to each subject before the actual test ${ }^{40,41}$.

In our study, we included subjects 60 years of age and older who completed the animal fluency trial, identified as CFDDS, for the 2011-2012 and 2013-2014 
NHANES data cycles. After we created a new variable with cut-off scores named DSST. cut-off score of $\leq 27$ and $\geq 28$ was used as it is the standard in other assessments ${ }^{55-57}$. A total of 3,104 subjects from the 2011-2012 and 2013-2014 responded with a complete delayed recall trial. We then accounted for those who were measured for metalloestrogens and accounted for those with extreme creatinine scores ( $<30$ and $>300 \mathrm{mg} / \mathrm{dl}$ ), as these extreme values can affect analyses by being too dilute or concentrated ${ }^{48}$. After, our study population consisted of 891 subjects, 129 subjects $\leq 27$ and 744 subjects $\geq 28$.

\section{Assessment of Surrogate Brain Health Indicators - Memory Function}

\section{During the past 12 months, have you experienced confusion or memory loss that}

is happening more often or getting worse?: In our study we included subjects 60 years of age and older who had metalloestrogens samples taken and responded to the yes $=0 /$ no $=1$ question," During the past 12 months, you experienced confusion or memory loss that is happening more often or is getting worse?" ${ }^{58}$. Memory issues, such as confusion and memory loss often precede the development of dementia and neurodegeneration ${ }^{2,59}$, which makes this question a potential surrogate for the development of memory issues. 3,628 subjects responded to this question in the 20112012 and 2013-2014 survey cycle. We then accounted for those who were measured for metalloestrogens and accounted for those with extreme creatinine scores $(<30$ and $>300 \mathrm{mg} / \mathrm{dl}$ ), as these extreme values can affect analyses by being too dilute or

concentrated $^{48}$. After, our delayed recall study population consisted of 1,025 subjects, 181 subjects answered "yes" and 844 subjects answered "no". 


\section{During the past 7 days, how often have you had trouble remembering where you}

put things?: In our study we included subjects 60 years of age and older who had metalloestrogens samples taken and responded to the question," During the past 7 days, how often $\{$ have you/has SP $\}$ had trouble remembering where $\{$ you/he/she $\}$ put things, like $\left\{\right.$ your/his/her\} keys or $\{$ your/his/her $\}$ wallet?" ${ }^{58}$. Memory issues, such as confusion and memory loss often precede the development of dementia and neurodegeneration ${ }^{2,59}$, which makes this question a potential surrogate for the development of memory issues. 3,448 subjects responded to this question in the 20112012 and 2013-2014 survey cycle.

The question is multi-leveled, where 1,993 subjects answered "Never" $=0,809$ subjects answered "About once" $=1,544$ subjects answered "Two or three times" $=2$, 175 subjects answered "Nearly every day" $=3$, and 102 subjects answered "Several times a day" $=4$. We created a new variable named MCQ380_WK, which combines responses coded as $0 / \mathrm{Never}$ and $1 /$ About once into a variable $=2$ and $2 /$ Two or three times, 3/Nearly every day and 4/Several times a day into a variable $=1$.

We then accounted for those who were measured for metalloestrogens and accounted for those with extreme creatinine scores $(<30$ and $>300 \mathrm{mg} / \mathrm{dl})$, as these extreme values can affect analyses by being too dilute or concentrated ${ }^{48}$. After, our study population consisted of 1,074 subjects, 188 subjects answered " 1 " and 783 subjects answered "2".

$\underline{\text { Are you limited in any way because of difficulty remembering or because you }}$ experience periods of confusion?: In our study we included subjects 60 years of age and older who had metalloestrogens samples taken and responded to the question," 
\{Are you/Is SP $\}$ limited in any way because of difficulty remembering or because $\{\mathrm{you} / \mathrm{s} / \mathrm{he}\}$ experience $\{\mathrm{s}\}$ periods of confusion?" ${ }^{58}$. Limitations in physical movement due to difficulty remembering and confusion can indicate the development of cognition issues ${ }^{60} .3,629$ subjects responded to this question in the 2011-2012 and 2013-2014 survey cycle.

We then accounted for those who were measured for metalloestrogens and accounted for those with extreme creatinine scores ( $<30$ and $>300 \mathrm{mg} / \mathrm{dl}$ ), as these extreme values can affect analyses by being too dilute or concentrated ${ }^{48}$. After, our study population consisted of 1,133 subjects, 181 subjects answered yes and 952 subjects answered no.

\section{Assessment of Surrogate Brain Health Indicators - Taste and Smell Function}

It has been observed that neurodegenerative disease has been shown to be preceded by smell and taste disorders ${ }^{61-64}$. The causes of these disorders have been linked to genetic alterations ${ }^{61}$, overexpression of key proteins ${ }^{62}$, and direct effect of some environmental chemicals on the olfactory mucosa ${ }^{65}$, which can have associations with exposure to EEDCs ${ }^{61,62,64,65}$. However, issues with olfaction can also be caused by upper respiratory tract infections, sino-nasal disease, head trauma, idiopathic causes, surgery of the nasal area, and congenital loss of smell ${ }^{66}$.

The two most common and prevalent neurodegenerative diseases, $\mathrm{AD}$ and $\mathrm{PD}$ have been shown to be preceded by smell disorders ${ }^{67-72}$. These disorders manifest themselves when evidence of pathological changes in the olfactory system are evident

${ }^{72}$. These are characterized by the build-up of pathological proteins, which cause the death of olfactory cells ${ }^{72}$. Several human epidemiological studies have also alluded 
to the utility of using sensory biomarkers as an early detection for neurodegenerative diseases ${ }^{73-76}$

\section{Do you sometimes smell and unpleasant, bad, or burning odor when nothing is}

there?: In our study we included subjects 60 years of age and older who had metalloestrogens samples taken and responded to the question," $\{$ Do you/Does SP $\}$

sometimes smell an unpleasant, bad or burning odor when nothing is there?" $58.3,617$ subjects responded to this question in the 2011-2012 and 2013-2014 survey cycle. We then accounted for those who were measured for metalloestrogens and accounted for those with extreme creatinine scores $(<30$ and $>300 \mathrm{mg} / \mathrm{dl})$, as these extreme values can affect analyses by being too dilute or concentrated ${ }^{48}$. After, our study population consisted of 1,050 subjects, 74 subjects answered yes and 950 subjects answered no.

\section{During the past 12 months have you had a taste or other sensation in your} mouth that does not go away?: In our study we included subjects 60 years of age and older who had metalloestrogens samples taken and responded to the question," During the past 12 months $\{$ have you/has SP had a taste or other sensation in \{your/his/her\} mouth that does not go away?"58. 3,623 subjects responded to this question in the 2011-2012 and 2013-2014 survey cycle.

We then accounted for those who were measured for metalloestrogens and accounted for those with extreme creatinine scores $(<30$ and $>300 \mathrm{mg} / \mathrm{dl})$, as these extreme values can affect analyses by being too dilute or concentrated ${ }^{48}$. After, our study population consisted of 1,024 subjects, 66 subjects answered yes and 958 subjects answered no. 


\section{Covariates}

In our study we included a number of covariates, based off a review of literature and well-known risk factors for neurodegenerative diseases, if they were available in the NHANES datasets.

The demographic variables are as follows: gender (male, female), age (60-69, 7-79, 80+), Race/Ethnicity (Hispanic, Non-Hispanic White, Non-Hispanic Black, NonHispanic Asian/Other), Family Income (Under 24k, 25k to 54,999k, 55k to 74,999k, over $75 \mathrm{k})$, Education $\left(<12^{\text {th }} \text { grade, completed high school, }>12^{\text {th }} \text { grade }\right)^{58}$. Modifiable health variables and risk factors are as follows: ever smoked (yes, no), blood pressure (normal/high), diabetes (yes, no, borderline), coronary heart disease (yes, no), stroke (yes, no), heart attack (yes, no), head trauma (yes, no), alcohol use (yes, no), ever use birth control (yes, no), every use hormonal replacement therapy (yes, no $)^{58}$.

\section{Statistical Analysis}

Statistical analysis was performed using SAS software ${ }^{77}$. The 20112012 and 2013-2014 survey cycles were merged and a four-year sampling weight was calculated to account for the complex sampling design in order to calculate correct statistical estimates and standard errors when calculating means, geometric means, and other statistics ${ }^{78}$.

For metalloestrogens variables, a value of " 0 " indicates above the limit of detection and a value of " 1 " indicates below the limit of detection. Individuals with a below the limit of detection were given a dummy phthalate level of the LOD divided by the

square root of two ${ }^{38}$. Some LODs differ between survey cycles and a conservative 
approach was used to account for differing LODs per survey cycle, where the LOD for that year was used to make a determination if the EEDC was above or below the LOD. ${ }^{39}$. We log-transformed and then adjusted for creatinine all metalloestrogens variables ${ }^{79-81}$ since environmental chemical data is not normally distributed and urine dilution varies from person to person.

We used the SAS Survey procedures to account for the complex sampling design of the NHANES data sets ${ }^{82}$.

We used PROC SURVEYFREQ was used to obtain descriptive statistics for the different populations we were examining in our study which accounts for the complex survey design of the NHANES data sets ${ }^{82}$. Descriptive statistics were organized based on the following categories per variable: gender (male, female), age (60-69, 7 79, 80+), Race/Ethnicity (Hispanic, Non-Hispanic White, Non-Hispanic Black, NonHispanic Asian/Other), Family Income (Under 24k, 25k to 54,999k, 55k to 74,999k, over $75 \mathrm{k})$, Education ( $<12^{\text {th }}$ grade, completed high school, $>12^{\text {th }}$ grade), ever smoked (yes, no), blood pressure (normal/high), diabetes (yes, no, borderline), coronary heart disease (yes, no), stroke (yes, no), heart attack (yes, no), head trauma (yes, no), alcohol use (yes, no), ever use birth control (yes, no), every use hormonal replacement therapy (yes, no) ${ }^{58}$.

We used PROC SURVEYREG and guidance provided by the SAS institute to directly to determine the geometric mean of the EEDC to test if they were significant between the responses of our outcome variables ${ }^{82,83}$. The standard errors were calculated using the Taylor Series linearization method, which is the default method in the survey procedures to calculate standard error ${ }^{82}$. Geometric means (GM), 
geometric standard errors (GSE), and number of subjects were reported for the results of the outcome variables for all subjects that had EEDCs over the LOD. We looked at geometric means between the outcome variables (yes vs. no, low test score vs. high test score), and also performed age-specific, gender-specific, and race/ethnicityspecific geometric means between the responses to the outcome variable. Due to the smaller range of ages in our dataset, 60 years and older, we calculated age-specific rates in lieu of age-standardized rates.

We used PROC SURVEYLOGISTIC to find the unadjusted and adjusted odds ratios (ORs) and the 95\% confidence intervals (CI) to examine the association between our outcome variables and exposures to metalloestrogens ${ }^{82}$. Analysis was done per EEDC per outcome variable. We presented three logistic regression models which were stratified by gender and presented the EEDC as a continuous variable as well as a ranked variable $<\mathrm{LOD}$ to $50^{\text {th }}$ percentile (reference) and $\geq 50^{\text {th }}$ percentile: unadjusted, adjusted for known risk factors, age, education, race/ethnicity, adjusted for known and suspected risk factors, age, education, race/ethnicity, smoking, blood pressure history, history of coronary heart disease, stroke, heart attack, diabetes status, head trauma, and alcohol use. We did not include income, OC and HRT use as variables as they significantly reduced the size of the population.

\section{RESULTS}

\section{Descriptive statistics for the Surrogates of Brain Health Indicators and}

Covariates: Descriptive statistics of the study populations are described in table 1.1 and 1.1a for each of our 9 outcomes with their respective covariates. 


\begin{tabular}{|c|c|c|c|c|c|c|c|c|c|c|c|c|c|c|c|c|c|c|c|c|c|c|c|c|c|c|c|c|c|c|c|c|c|c|c|c|}
\hline \multirow{2}{*}{$\begin{array}{c}\text { Table } 1.1 \text { - Descriptive } \\
\text { Statistics }- \text { Surrogate brain } \\
\text { health indicators and covariates } \\
\text { for } \mathrm{Cd} \text { and } \mathrm{Mn}\end{array}$} & \multicolumn{4}{|c|}{$\begin{array}{c}\text { Immediate Recall Score } \\
(\mathrm{n}=1378)\end{array}$} & & $\begin{array}{l}\text { tayed } \mathrm{B} \\
(\mathrm{n}=\mathrm{s}\end{array}$ & Recall 19 & IScore & & imal Flv & $\begin{array}{l}\text { vency Score } \\
=931)\end{array}$ & $\begin{array}{r}\text { D } \\
\text { Substit } \\
\end{array}$ & $\begin{array}{c}\begin{array}{c}\text { Digits } \\
\text { itution } \\
(\mathrm{n}-\mathrm{s}\end{array} \\
\end{array}$ & $\begin{array}{l}\text { Symbc } \\
\text { n Test } \\
\text { 891) }\end{array}$ & sol & & $\begin{array}{l}512 \mathrm{mo} \\
\mathrm{etting} \text { wo }\end{array}$ & $\begin{array}{l}\text { onths, n } \\
\text { orsse?'(1) }\end{array}$ & $\begin{array}{l}\text { menory } \\
(1,025)\end{array}$ & & $\begin{array}{l}\text { Past } 7 \mathrm{day} \\
\text { ememberin }\end{array}$ & ays, trou & $\begin{array}{l}\text { ouble } \\
=971)\end{array}$ & $\begin{array}{c}\text { Limite } \\
r \\
\text { conf }\end{array}$ & $\begin{array}{l}\text { tied due } \\
\text { rememb } \\
\text { nfussion? }\end{array}$ & $\begin{array}{l}\text { to doff } \\
\text { bering } \\
n(n=1.1\end{array}$ & $\begin{array}{l}\text { Sifficulty } \\
\text { gor } \\
1,024)\end{array}$ & & $\begin{array}{l}\text { Phantom } \\
\text { ( } \mathrm{n}=1,\end{array}$ & $\begin{array}{l}0 \text { Odor } \\
1,024)\end{array}$ & & & $\begin{array}{c}\text { ensation } \\
\text { does no } \\
(n-m\end{array}$ & $\begin{array}{l}n=\operatorname{lin} \\
\text { not } g 0 \\
n=102\end{array}$ & $\begin{array}{l}\text { south } \\
\text { purat } \\
\text { 24) }\end{array}$ & \\
\hline & $n$ & & $\mathrm{n}$ & $\%$ & $n$ & $\%$ & $n$ & $\%$ & $n$ & $\%$ & $\mathrm{n} \%$ & $n$ & $\%$ & $\mathrm{n}$ & & $\mathrm{n}$ & $\%$ & $n$ & $\%$ & a & $\%$ & $n$ & & $n$ & $\%$ & $n$ & $\%$ & $n$ & $\%$ & $n$ & $\%$ & & $\%$ & $n$ & & $\%$ \\
\hline Total Population & $\begin{array}{c}\leq 13 \\
17389\end{array}$ & & 212 & 9107 & & & & $\begin{array}{l}24 \\
48586\end{array}$ & $282^{3}$ & & \begin{tabular}{|c|}
212 \\
1078 \\
\end{tabular} & $\begin{array}{r}\leq 27 \\
2319\end{array}$ & 9.75 & & $\begin{array}{l}288 \\
39025\end{array}$ & & & 97 & $\begin{array}{l}N_{0} \\
83.61\end{array}$ & & \begin{tabular}{l|l|} 
Yes \\
8 \\
15.44
\end{tabular} & & $\begin{array}{l}\text { No } \\
8436\end{array}$ & & $\begin{array}{l}\text { Yes } \\
11.81\end{array}$ & & $\begin{array}{l}\text { No } \\
988.19\end{array}$ & & $\begin{array}{l}\text { Yes } \\
4.19\end{array}$ & & $\begin{array}{l}N_{0} \\
59581\end{array}$ & & $\begin{array}{l}\text { Yes } \\
{ }_{3} \quad 509\end{array}$ & & $\begin{array}{l}\mathrm{N}_{0} \\
494\end{array}$ & 8491 \\
\hline Gender (n, \%) & & & & & & & & & & & & & & & & & & & & & & & & & & & & & & & & & & & & \\
\hline & 1085.3 & & 3924 & & & & & 41.18 & 139 & 6.90 & $\left|\begin{array}{ll}355 & 42.55\end{array}\right|$ & 1314 & & 550 & 44.46 & 6) 135 & 8.98 & 616 & 40.07 & & $\begin{array}{ll}11 & 6.93\end{array}$ & 610 & 4237 & & 3.26 & 664 & 4 43.97 & 30 & 1.80 & & 47.45 & & 112.20 & & & 47,04 \\
\hline Femile & $\begin{array}{ll}65 & 3.6\end{array}$ & & 6134 & 47.30 & & 6.10 & 1 & 44.68 & 143 & 7.62 & {$\left[\begin{array}{ll}523 & 42.93\end{array}\right]$} & 1004 & 4.84 & 545 & 45.78 & 124 & 7,41 & 591 & 43.54 & & 278.51 & 542 & 42.00 & 130 & 6.55 & 585 & 34.22 & 40 & 2.39 & & 48.36 & & $2 \quad 2.89$ & 8966 & $\begin{array}{ll}62 & 47\end{array}$ & 47.87 \\
\hline Age (yea & & & & & & & & & & & & & & & & & & & & & & & & & & & & & & & & & & & & \\
\hline & $\begin{array}{ll}69 & 2.4\end{array}$ & & 6635 & 51.06 & & 4.89 & & 48.69 & 109 & 437 & 349.00 & 993 & 3.35 & 619 & 51.04 & a & 1. 6.47 & 661 & 46.48 & 115 & $15 \quad 7.20$ & 617 & 46.01 & 106 & 4.70 & 668 & 8.48 .41 & 54 & 3.25 & & 49.81 & & ? 3.13 & 13 & 1649 & 49.97 \\
\hline & $\begin{array}{ll}52 & 3.3\end{array}$ & & 3822 & & & 4.58 & & 27.85 & 100 & 5.69 & & 803 & 3.43 & & 28.87 & & 5.08 & 389 & 27.19 & 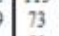 & $\begin{array}{lll}3 & 4.85\end{array}$ & 367 & 27.44 & 52 & 3.10 & 407 & 729.06 & 12 & 0.73 & 447 & & & $\begin{array}{ll}4 & 1.37\end{array}$ & & & 30.80 \\
\hline $80+$ & $52 \quad 3.1$ & & $160 \mathrm{i}$ & 11.00 & 75 & 4.67 & 135 & 9.32 & 73 & 4.45 & {$\left[\begin{array}{ll}23 \\
138 & 9.79\end{array}\right]$} & 522 & 297 & 143 & 10.3 & 78 & 4.84 & 157 & 9.94 & 30 & $\begin{array}{ll}0 & 3.39\end{array}$ & 168 & 11.11 & 61 & 4.01 & 174 & 410,72 & 4 & 0.21 & 231 & 1453 & & $2 \quad 0.60$ & 5022 & 2314 & 14.13 \\
\hline RaceEthricity (n, \%) & & & & & & & & & & & & & & & & & & & & & & & & & & & & & & & & & & & & \\
\hline Hisp: & & & 2278 & & & & & 5.80 & 58 & 1.69 & & 842 & 2.49 & 163 & 4.81 & 58 & 1.72 & 228 & 6.28 & & $\begin{array}{ll}0 & 1.47\end{array}$ & 207 & 6.09 & & & 227 & $7 \quad 6.37$ & & 0.42 & 272 & 7.57 & & 20.90 & $\begin{array}{l}90 \\
95\end{array}$ & 547. & \\
\hline Non-Hin & 6.1 & & 5847 & 73.00 & & & 01 & 5.80 & 103 & 9.42 & $\begin{array}{l}3 \\
3\end{array} 69.94$ & 634 & $\begin{array}{l}2.47 \\
4.88\end{array}$ & $\begin{array}{l}1039 \\
579\end{array}$ & & 131 & & 46 & & 112 & $12 \quad 11.66$ & 595 & & 94 & & 585 & $5 \quad 69.74$ & $\begin{array}{l}14 \\
25\end{array}$ & $\begin{array}{l}0.42 \\
293\end{array}$ & 654 & 75.26 & 32 & $\begin{array}{l}0.90 \\
3.37\end{array}$ & & 74 & \\
\hline Non-H & $\begin{array}{ll}7 & 0 \\
37 & 0.8\end{array}$ & & 287 & 8.06 & 59 & 1.49 & 548 & 68.97 & 91 & 2.65 & {$\left[\begin{array}{ll}224 & 6.08\end{array}\right]$} & 691 & $\begin{array}{l}7.86 \\
1.92\end{array}$ & 235 & 6.72 & 32 & 0.81 & 310 & 8.23 & ${ }_{52}^{112}$ & $\begin{array}{ll}2 & 1.55\end{array}$ & 279 & 7.60 & 32 & 0.83 & 310 & $\begin{array}{l}0 \\
0.18\end{array}$ & 26 & 0.68 & 314 & 8.28 & & 100.61 & 61 & 22 & 8.41 \\
\hline Non-Hispanic & $\begin{array}{ll}27 & 0.6\end{array}$ & & 107 & & 19 & 0.49 & 62 & 7.43 & 30 & 0.76 & $\mid \begin{array}{ll}2 & 3.40 \\
101 & 3.40\end{array}$ & 150 & 0.47 & 118 & 3.75 & 38 & 1.18 & $l_{123}^{10}$ & 3.73 & 24 & $\begin{array}{ll}4 & 0.75\end{array}$ & 131 & 4.20 & 34 & 1.00 & $\begin{array}{l}3127 \\
127\end{array}$ & $\begin{array}{l}7.90 \\
7 \\
7\end{array}$ & $\begin{array}{c}20 \\
5\end{array}$ & 0.18 & $\mid \begin{array}{l}159 \\
155\end{array}$ & 4.70 & & & $\left.22\right|_{15} ^{3}$ & & $\begin{array}{l}0.71 \\
4.66\end{array}$ \\
\hline & & & & & & & & & & & & & & & & & & & & & & & & & & & & & & & & & & & & \\
\hline & & & 2951 & 14.30 & & 3.64 & & 13.88 & 124 & 4.82 & $\begin{array}{ll}52 & 12.58\end{array}$ & 1354 & 435 & 216 & & & 3.91 & 349 & 15.55 & & $8 \quad 2.56$ & & & & 3.85 & & 915.54 & 23 & 0.80 & 15 & & & $4 \quad 1.35$ & $35 \mid 40$ & 0518 & \\
\hline Complet & 2.3 & & $278 \mathrm{i}$ & 18.69 & 58 & 4.37 & 50 & 16.57 & 90 & 5.03 & 7.55 & 532 & 259 & 247 & 18.13 & 36 & 3.39 & 275 & 17.59 & 73 & 4.17 & 243 & 16.69 & 64 & 3.47 & 267 & $7 \quad 17.44$ & 20 & 0.96 & 311 & 19.98 & 26 & 1.67 & & 19 & 19.25 \\
\hline & $\begin{array}{ll}46 & 3.2\end{array}$ & & 6305 & 58.12 & 80 & 6.10 & 594 & 55.44 & 65 & 4.62 & {$\left[\begin{array}{ll}1 & 17.36 \\
6008 & 57.36\end{array}\right]$} & 422 & 2.80 & 631 & 59.78 & 110 & 9.06 & 581 & 50.49 & 97 & $\begin{array}{ll}7.73 \\
7\end{array} 8.73$ & 362 & 51.23 & 62 & 4.46 & 631 & $\begin{array}{l}155.23 \\
153\end{array}$ & 2 & 2.44 & 665 & 57.29 & & 32.08 & 0866 & 6057 & 57.63 \\
\hline BMIO & & & 2000 & & 800 & 0.10 & 197 & 25,4 & 102 & +.02 & & & & 031 & & 100 & 9.00 & j81 & 200.4 & 91 & 78.12 & T202. & 12.20 & 02 & 4.40 & 031 & & & $2 .+4$ & & | & & & & & 31.09 \\
\hline $\mathrm{U}$ & & & $36: 2$ & & & 0.88 & 27 & 224 & 13 & 0.71 & $\begin{array}{ll}29 & 2.43\end{array}$ & 100 & 0.69 & 28 & 239 & 16 & 0.93 & 35 & & 9 & 0.47 & 33 & 231 & 20 & & 31 & 219 & 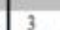 & 0.17 & 48 & & & 6030 & 30 & 2 & \\
\hline & & & 2892 & & & & & & 69 & & 79 & $32 \mathrm{1}$ & & 279 & & 73 & & 02 & & 55 & & & & & & & & & & & & & & & & \\
\hline erwer & $\begin{array}{lll}68 & 3.1\end{array}$ & & 4193 & 35.19 & 104 & 6.11 & 80 & 32.31 & 102 & 4.76 & $\begin{array}{ll}81 & 33.81\end{array}$ & 843 & $\begin{array}{l}3.49 \\
3.49\end{array}$ & 387 & 34.73 & 82 & 5.42 & 426 & 32.15 & 83 & $\begin{array}{ll}3 & 5.78\end{array}$ & 403 & 32.44 & 60 & 2.81 & 448 & 834.63 & 18 & 0.98 & 490 & 36.49 & & 31.80 & $80-47$ & 75 & 35.65 \\
\hline Obes & $47 \quad 2.4$ & & 4613 & 32.83 & & 4.06 & 33 & 31.16 & 98 & 5.28 & $98 \quad 29.45$ & 853 & 3.66 & 401 & 31.62 & 88 & 5.38 & 444 & 29.61 & 91 & 3.61 & 415 & 29.32 & 70 & 4.01 & 464 & 431.19 & 40 & 2.76 & 492 & 32.41 & 37 & 72.29 & $29 \sqrt{49}$ & $97 \quad 32$ & \\
\hline Alcohol & & & & & & & & & & & & & & & & & & & & & & & & & & & & & & & & & & & & \\
\hline & 935.0 & & $816 \quad 6$ & 67.98 & 143 & 9.13 & & 64.03 & 148 & 798 & $\begin{array}{ll}56 & 65.61\end{array}$ & 1425 & 5.73 & 753 & 67.96 & 157 & 71133 & 766 & 60.51 & 1. & $16 \quad 11.08$ & 729 & 60.60 & 105 & 6.26 & & 065.70 & 50 & 3.27 & & & & $8 \quad 3.58$ & & & \\
\hline & $\begin{array}{ll}66 & 3.4\end{array}$ & & 3662 & 23.47 & 89 & 4.78 & 38 & 22.06 & 119 & 6.16 & $\left.\begin{array}{ll}02 & 20.25\end{array}\right]$ & 823 & 3.88 & 321 & 22.4 & 83 & 4.70 & 384 & 23.4 & 73 & $\begin{array}{lll}3 & 3.93\end{array}$ & 369 & 24.39 & 90 & 4.91 & & $7 \quad 23.13$ & 16 & 0.91 & 449 & & & $10 \quad 1.49$ & 4943 & & 26.56 \\
\hline Ever Smok & & & & & & & & & 年 & & & & & & & & & {$[504$} & & & & & & & & & & & & & & & & & & \\
\hline & & & 6184 & 48.44 & & 691 & & 46.11 & 140 & 6.74 & $\left|\begin{array}{ll}558 & 46.39\end{array}\right|$ & 1295 & & 549 & 47.23 & & 69.39 & 601 & & 122 & 12. 791 & 588 & 44.73 & 106 & & 643 & & 38 & & 710 & & & 3.32 & & & \\
\hline $\mathrm{N}$ & $85 \quad 4.5$ & & 5874 & $\begin{array}{l}70.63 \\
42.63\end{array}$ & & 7.23 & 34 & 39.75 & 142 & 7.78 & $\left|\begin{array}{ll}520 & 39.10\end{array}\right|$ & 1024 & 431 & 546 & 43.02 & 113 & 7.00 & 606 & 40.36 & 5116 & {$\left[\begin{array}{ll}6 & 753\end{array}\right.$} & 564 & 39.83 & & 5.68 & 606 & 641.53 & 32 & 1.48 & 685 & & & $\begin{array}{ll}2 & 1.77\end{array}$ & & & \\
\hline Physically A & & & & & & & & & & & & & & & & & & & & & & & & & & & & & & & & & & & & \\
\hline & 26 & & 4503 & 37.21 & & 4.51 & & 35.39 & 64 & 3.10 & 3 3. 37.05 & 522 & 2.18 & 433 & & & 4.88 & 437 & & & $7 \quad 4.49$ & & & & 3.29 & & & 20 & 1.35 & & & & $4 \quad 1.04$ & & & \\
\hline $\mathrm{N}$ & $\begin{array}{ll}123 & 6.3 \\
12\end{array}$ & & $755 \quad 5$ & 53.86 & & 9.63 & 997 & 50.47 & 218 & 11.42 & $\begin{array}{lll}45 & 48.44\end{array}$ & 1797 & 7.57 & 662 & 52.43 & 179 & 911. & 770 & 49.53 & 171 & $\begin{array}{ll}1 & 10.95\end{array}$ & 728 & 30.23 & 154 & 8.52 & 797 & 752.66 & 50 & 2.84 & 898 & 58,30 & 69 & 69.05 & 058 & & 57. \\
\hline Diabete & & & & & & & & & & & & & & & & & & & & & & & & & & & & & & & & & & & & \\
\hline & & & 2721 & & & & & & 90 & & & & & & & & & 291 & & & & & & & & & & & & & & & & & & \\
\hline & 6.3 & & 8706 & 69 & & & & 65.75 & 173 & & $\begin{array}{l}0 \\
0\end{array} 66.45$. & $\begin{array}{lll}89 & 3 \\
135 & 6\end{array}$ & & $\frac{2}{8}$ & & & & ${ }_{55}^{91}$ & & 173 & $\begin{array}{ll}4 & 2.7 .29 \\
3 & 12.29\end{array}$ & 15 & & & & & & 39 & & & & & & & & \\
\hline & $\begin{array}{ll}3 & 0.3\end{array}$ & & 63 & 5.03 & & 0.89 & & 4.50 & 19 & 0.88 & $50 \quad 4.42$ & $\begin{array}{ll}7 & 0 \\
7 & 0\end{array}$ & 0.23 & 60 & 5.05 & & 0.72 & & & 1 & $\begin{array}{ll}1 & 0.41\end{array}$ & & & & 7) & & & & 0] & & 4.70 & & $\begin{array}{ll}7 & 0.56\end{array}$ & & & \\
\hline Blood $P_{r}$ & & & & & & & & & & & & & & & & & & & & & & & & & & & & & & & & & & & & \\
\hline & & & 176 & 67. & & 9.36 & & & & & & & & & & & & 4822 & & & 811.46 & & & & & & & 54 & & & & & $6 \quad 3.49$ & & & \\
\hline & 3.2 & & 3882 & 23.99 & 89 & 4.78 & & 22.40 & 103 & 5.41 & $136 \quad 22.02$ & 994 & 4.08 & 324 & 22.99 & 88 & 4.95 & 385 & & 7 & 3.98 & 377 & & 6 & 3.62 & 413 & 323.96 & 16 & & 458 & 26.76 & 37 & 71.60 & $6 0 \longdiv { 4 3 }$ & & 25.9 \\
\hline Myocardial ith & & & & & & & & & & & & & & & & & & & & & & & & & & & & & & & & & & & & \\
\hline $\mathrm{Y}$ & $26 \quad 1.6$ & & 897 & 795 & & & & 6.74 & 28 & & $\begin{array}{ll}84 & 7.75\end{array}$ & 261 & & & & & & 77 & & & $\begin{array}{ll}0 & 205\end{array}$ & & & & & & & & & & & & & & & \\
\hline & 1477.3 & & 1168 & 83.12 & & 11.27 & 1043 & 79.12 & 254 & 12.64 & $994 \quad 77.73$ & 2058 & 8.33 & 1014 & 482.20 & 215 & 513 & 1130 & 76.84 & 208 & $8 \begin{array}{ll}8 & 13.39\end{array}$ & 1074 & 77.92 & 90 & 9.40 & & & & & & & & & & & \\
\hline & & & & & & & & & & & & & & & & & & & & & & & & & & & & & & & & & & & & \\
\hline & & & 725 & 5.2 & & 1.92 & & 3.87 & & 1.82 & & & 16 & & & & 1.88 & & & & $2 \quad 204$ & & & & & & & & & & & & & & & \\
\hline & 1568.4 & & 1308 & 85.81 & & 1224 & 1061 & 181.97 & 245 & 12.53 & 22681.73 & 2028 & 8.60 & 1044 & 485.94 & 230 & 14 & 1126 & 678 & 2 & $5 \quad 13.43$ & 1073 & 80 & & & & & 58 & 3 & & & & & & & \\
\hline vonary Hear & & & & & & & & & & & & & & & & & & & & & & & & & & & & & & & & & & & & \\
\hline & $\begin{array}{|ll|}27 & 1.8 \\
\end{array}$ & & 93 & 7.06 & & & & 6.05 & 31 & 1.85 & 7.12 & 251 & & & & & & 88 & & 25 & $\begin{array}{ll}5 & 1.54\end{array}$ & & & & & & & & & & & & 00.45 & & & \\
\hline & 1427.1 & & 1058 & 84.04 & & 11.28 & 1039 & 99.82 & 247 & 12.67 & $\mid \begin{array}{ll}982 & 78.37\end{array}$ & 2038 & 823 & & 283.03 & & & (ii. & 77.41 & 209 & $\begin{array}{ll}9 & 13.58\end{array}$ & 1048 & & & 10 & & 3780 & 1258 & 3.87 & & & & $3 \quad 4.67$ & & & \\
\hline Head Tra & & & & & & & & & & & & & & & & & & & & & & & & & & & & & & & & & & & & \\
\hline & 2 & & 1531 & 15 & & 2.7 & & & 22 & 1. & & 191 & 1.22 & & & & & & & & 93.60 & & & & & & & & & & & & & & & \\
\hline & 1477 & & ; & 75.48 & & & & & 258 & 83 & & & 8.53 & & & & & & 71 & 18 & 11.85 & & & & 8.44 & & & & & & & & 3.8 & & & \\
\hline$U_{s}$ & & & & & & & & & & & & & & & & & & & & & & & & & & & & & & & & & & & & \\
\hline & & & & & & & & & & & & & & & & & & & & & & & & & & & & & & & & & & & & \\
\hline & $43 \quad 4.6$ & & 2203 & 30.63 & 59 & 6.67 & & 28. & 70 & 8.57 & $\begin{array}{ll}187 & 26.90\end{array}$ & 626 & 6.28 & & & 64 & 6.87 & & & 46 & $\begin{array}{ll}6 & 5.39\end{array}$ & 207 & & & 7.49 & & & & 46 & & & & $3 \quad 1.00$ & & & \\
\hline ale Hor & & & & & & & & & & & & & & & & & & & & & & & & & & & & & & & & & & & & \\
\hline & & & 2575 & & & & 2 & & 35 & 4.16 & & & & & & 36 & 5.02 & 235 & & 35 & & & & 37 & 4.12 & & & & 2.38 & & & 19 & & & & \\
\hline & 46 & & 330 & 41.99 & 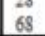 & 8.32 & 30 & 39.06 & 102 & 11.13 & $\left|\begin{array}{ll}251 \\
267 & 36.34\end{array}\right|$ & 707 & 7.3 & 2 & 38.83 & 75 & 8.53 & 320 & 39.75 & 79 & $\begin{array}{lll}9 & 9.83 \\
\end{array}$ & 289 & 3809 & 78 & 7.87 & 317 & & 259 & 49.41 & 368 & 45.82 & 30 & $\begin{array}{ll}30 & 3,32 \\
0 & 3.32\end{array}$ & 3236 & & \\
\hline
\end{tabular}




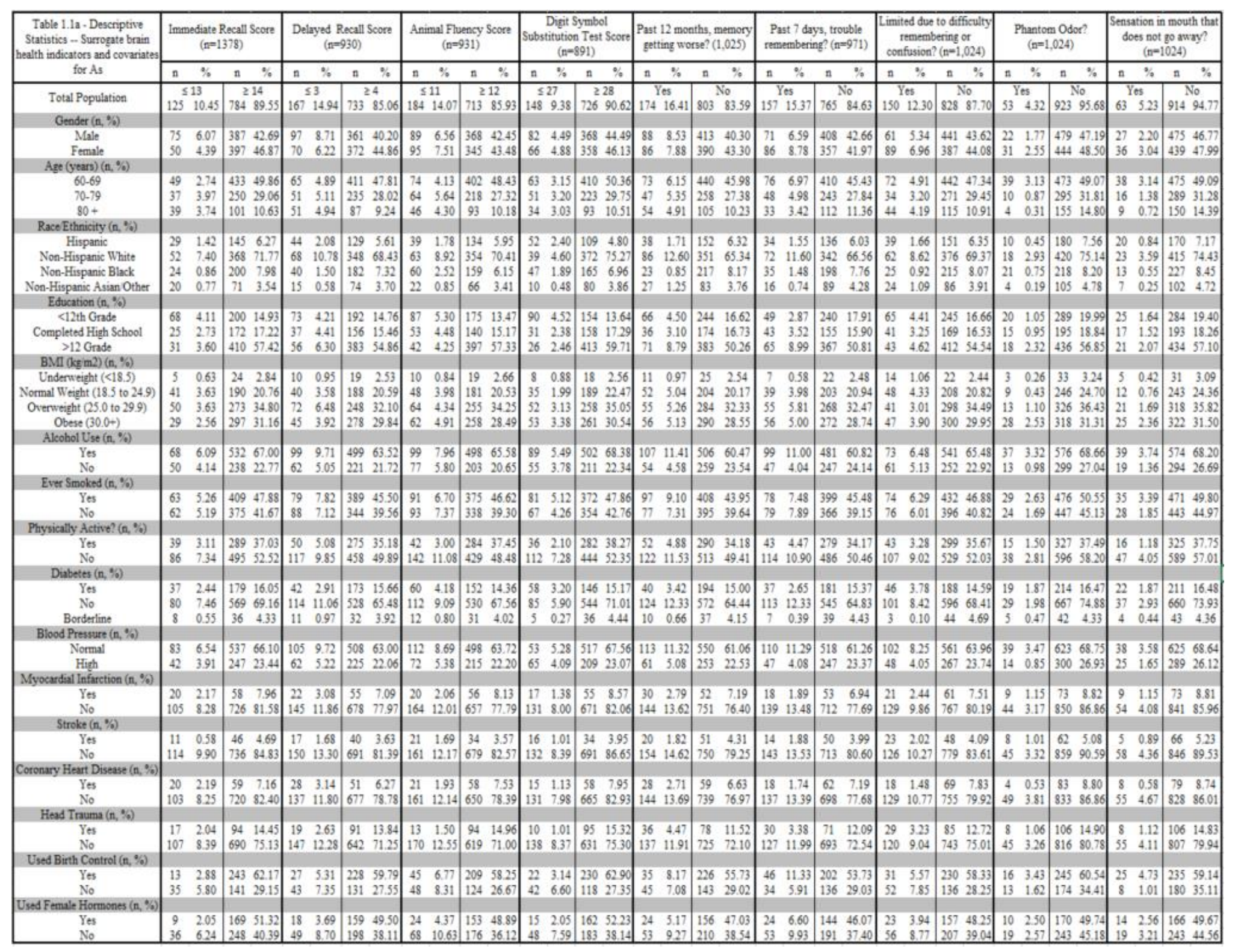




\section{Associations Between Exposures to Metalloestrogens and Cognitive Test Scores}

Exposures to the $\mathrm{Cd}, \mathrm{Mn}, \mathrm{As}$, and four cognitive scores (immediate and delayed recall, animal fluency, and DSST score) are summarized in tables 1.2 to 1.21. The cognitive test scores have been used as a surrogate indicator of brain health to assess cognitive decline and the possible development of mild cognitive impairment, dementia, and/or AD elderly patients as part of neuropsychological testing.

Immediate Recall Scores and Exposure to Metalloestrogens: Tables 1.2 to 1.5 present the GMs and GSEs of urinary Cd, Mn, and As levels among subjects with immediate recall scores. Table 1.2 presents GMs and GSEs of subjects who have measurable metalloestrogens levels over the LOD by immediate recall score. Cd was significantly higher in subjects with immediate recall scores $\leq 13$ compared to subjects who scored $\geq 14(\mathrm{p}<0.05)$. Table 1.3 presents age-specific GMs levels by age groups 60-69, 70-79, and 80+. No significant results were observed. Table 1.4 presents gender-specific GM levels. In females, the As GM levels were significantly higher in the $\leq 13$ group compared to the $\geq 14$. Table 1.5 presents race-specific GM levels among Hispanics, Non-Hispanic Whites, Non-Hispanic Blacks, and Asian/Other. Among all racial groups, no significant results were found.

Table 1.6 presents estimated ORs and 95\% CI for immediate recall scores and metalloestrogens stratified by gender with two groups: < LOD to 50th percentile (reference) and $\geq 50$ th percentile. In the unadjusted model, significant associations between lower immediate recall scores and $\mathrm{Cd}$ were observed in both males [OR=2.146, 95\% CI: 1.109-4.153] and females [OR=2.142, 95\% CI: 1.098-4.180] and between lower immediate recall scores and As in females only [OR $=2.507,95 \%$ 
CI: 1.170-5.372]. In model adjusted for age, education, and race/ethnicity, significant association between lower immediate recall scores and Cd were observed in females [OR=2.033, 95\% CI: 1.002-4.126] and between lower immediate recall scores and As in females [OR=2.569, 95\% CI: $1.103-5.987]$. No significant associations were observed in our second adjusted model.

\begin{tabular}{l} 
Table 1.2 Geometric Mean Urinary Metalloestrogen Levels by Immediate Recall Cut-off \\
Score for Subjects $>$ = 60 years of age, NHANES 2011-2014, over the LOD \\
\hline \multirow{3}{*}{ Metalloestrogen } \\
\cline { 2 - 7 }
\end{tabular}

\begin{tabular}{|c|c|c|c|c|c|c|}
\hline \multicolumn{7}{|c|}{$\begin{array}{l}\text { Table 1.3 Age-Specific Geometric Mean Urinary Metalloestrogen Levels by Immediate } \\
\text { Recall Cut-off Score for Subjects }>=60 \text { years of age, NHANES 2011-2014, over the LOD }\end{array}$} \\
\hline \multirow{3}{*}{ Metalloestrogen } & \multicolumn{6}{|c|}{ Geometric Mean (ug/ml) (GSE, N), Age 60-69 } \\
\hline & \multicolumn{3}{|c|}{ Immediate Recall Score $\leq 13$} & \multicolumn{3}{|c|}{ Immediate Recall Score $\geq 14$} \\
\hline & GM & GSE & $\mathrm{N}$ & GM & GSE & $\mathrm{N}$ \\
\hline Cadmium - Cd & 0.3572 & 0.04305 & 68 & 0.3106 & 0.01884 & 653 \\
\hline Manganese - Mn & 0.2146 & 0.08907 & 29 & 0.1941 & 0.01622 & 278 \\
\hline Arsenic - As & 8.5617 & 1.6511 & 49 & 8.759 & 0.692 & 429 \\
\hline \multirow{3}{*}{ Metalloestrogen } & \multicolumn{6}{|c|}{ Geometric Mean (ug/ml) (GSE, N), Age 70-79 } \\
\hline & \multicolumn{3}{|c|}{ Immediate Recall Score $\leq 13$} & \multicolumn{3}{|c|}{ Immediate Recall Score $\geq 14$} \\
\hline & GM & GSE & $\mathrm{N}$ & GM & GSE & $\mathrm{N}$ \\
\hline Cadmium - Cd & 0.366 & 0.04247 & 50 & 0.3462 & 0.02387 & 378 \\
\hline Manganese - Mn & 0.2187 & 0.04978 & 22 & 0.2009 & 0.02169 & 172 \\
\hline Arsenic - As & 8.0382 & 1.8811 & 37 & 10.7656 & 1.4646 & 249 \\
\hline \multirow{3}{*}{ Metalloestrogen } & \multicolumn{6}{|c|}{ Geometric Mean $(\mathrm{ug} / \mathrm{ml})(\mathrm{GSE}, \mathrm{N})$, Age $80+$} \\
\hline & \multicolumn{3}{|c|}{ Immediate Recall Score $\leq 13$} & \multicolumn{3}{|c|}{ Immediate Recall Score $\geq 14$} \\
\hline & GM & GSE & $\mathrm{N}$ & GM & GSE & $\mathrm{N}$ \\
\hline Cadmium - Cd & 0.4259 & 0.04196 & 51 & 0.381 & 0.03484 & 158 \\
\hline Manganese - Mn & 0.2101 & 0.01604 & 31 & 0.2028 & 0.02544 & 74 \\
\hline Arsenic - As & 12.5417 & 2.4947 & 38 & 11.5491 & 1.6923 & 100 \\
\hline \multicolumn{7}{|c|}{ NHANES sampling weight applied before calculating the geometric mean. } \\
\hline \multicolumn{7}{|c|}{$\begin{array}{l}\text { The EEDs were creatinine-adjusted then log transformed prior to analyses. Extreme creatinine } \\
\text { values }(<30 \mathrm{mg} / \mathrm{dL} \text { and }>300 \mathrm{mg} / \mathrm{dL}) \text { and metalloestrogen levels below the LOD were taken into } \\
\qquad \text { account using the appropriate domain statement in SAS. } \\
\qquad * \mathrm{p}<0.05,{ }^{* *} \mathrm{p}<0.01{ }^{* * *} \mathrm{p}<0.001\end{array}$} \\
\hline
\end{tabular}


Table 1.4 Gender-Specific Geometric Mean Urinary Metalloestrogen Levels by Immediate Recall Cut-off Score for Subjects $>=60$ years of age, NHANES 2011-2014, over the LOD

\begin{tabular}{|c|c|c|c|c|c|c|c|c|c|c|c|c|}
\hline \multirow{4}{*}{ Metalloestrogen } & \multicolumn{6}{|c|}{ Geometric Mean (ug ml) (GSE, N) } & \multicolumn{6}{|c|}{ Geometric Mean (ug/ml) (GSE, N) } \\
\hline & \multicolumn{6}{|c|}{ Males } & \multicolumn{6}{|c|}{ Females } \\
\hline & \multicolumn{3}{|c|}{ Immediate Recall Score $\leq 13$} & \multicolumn{3}{|c|}{ Immediate Recall Score $\geq 14$} & \multicolumn{3}{|c|}{ Immediate Recall Score $\leq 13$} & \multicolumn{3}{|c|}{ Immediate Recall Score $\geq 14$} \\
\hline & $\mathrm{GM}$ & GSE & $\mathrm{N}$ & $\mathrm{GM}$ & GSE & $\mathrm{N}$ & GM & GSE & $\mathrm{N}$ & $\mathrm{GM}$ & GSE & $\mathrm{N}$ \\
\hline Cadmium - $\mathrm{Cd}$ & 0.3182 & 0.03194 & 105 & 0.2669 & 0.0124 & 584 & 0.5057 & 0.05948 & 64 & 0.4008 & 0.01818 & 605 \\
\hline Manganese - Mn & 0.1727 & 0.0171 & 42 & 0.1409 & 0.01338 & 226 & 0.2651 & 0.05621 & 40 & 0.2547 & 0.0221 & 298 \\
\hline Arsenic - As & 6.9297 & 1.3437 & 75 & 9.2689 & 0.735 & 382 & 15.0262 * & 2.7989 & 49 & 10.0662 & 1.0643 & 396 \\
\hline
\end{tabular}

NHANES sampling weight applied before calculating the geometric mean.

The EEDs were creatinine-adjusted then log transformed prior to analyses. Extreme creatinine values ( $<30 \mathrm{mg} / \mathrm{dL}$ and $>300 \mathrm{mg} / \mathrm{dL}$ ) and metalloestrogen levels below the LOD were taken into account using the appropriate domain statement in SAS. ${ }^{*} \mathrm{p}<0.05, * *_{p}<0.01 * * *_{p}<0.001$

\begin{tabular}{|c|c|c|c|c|c|c|c|c|c|c|c|c|}
\hline \multirow{2}{*}{ Metalloestrogen } & \multicolumn{6}{|c|}{ Geometric Mean $(\mathrm{ng} / \mathrm{ml})(\mathrm{GSE}, \mathrm{N})$, Hispanic } & \multicolumn{6}{|c|}{ Geometric Mean (ng/ml) (GSE, N), Non-Hispanic White } \\
\hline & $\mathrm{GM}$ & GSE & $\mathrm{N}$ & GM & GSE & $\mathrm{N}$ & $\mathrm{GM}$ & GSE & $\mathrm{N}$ & GM & GSE & $\mathrm{N}$ \\
\hline Cadmium - Cd & 0.4278 & 0.04806 & 37 & 0.333 & 0.02753 & 223 & 0.3524 & 0.02767 & 69 & 0.3208 & 0.01402 & 575 \\
\hline Manganese - Mn & 0.1568 & 0.01751 & 17 & 0.1646 & 0.01525 & 105 & 0.2086 & 0.02603 & 31 & 0.2064 & 0.01373 & 250 \\
\hline \multirow[t]{2}{*}{ Metalloestrogen } & \multicolumn{3}{|c|}{ Immediate Recall Score $\leq 13$} & \multicolumn{3}{|c|}{ Immediate Recall Score $\geq 14$} & \multicolumn{3}{|c|}{ Immediate Recall Score $\leq 13$} & \multicolumn{3}{|c|}{ Immediate Recall Score $\geq 14$} \\
\hline & GM & GSE & $\mathrm{N}$ & $\mathrm{GM}$ & GSE & $\mathrm{N}$ & $\mathrm{GM}$ & GSE & $\mathrm{N}$ & GM & GSE & $\mathrm{N}$ \\
\hline Cadmium - Cd & 0.3789 & 0.04938 & 37 & 0.3359 & 0.0185 & 285 & 0.6829 & 0.111 & 26 & 0.5541 & 0.05086 & 105 \\
\hline Manganese - Mn & 0.3352 & 0.2217 & 18 & 0.1504 & 0.008246 & 117 & 0.2296 & 0.03706 & 16 & 0.1925 & 0.02882 & 52 \\
\hline Arsenic - As & 9.2215 & 1.8467 & 23 & 8.0595 & 0.754 & 199 & 33.7027 & 4.3825 & 20 & 29.7997 & 4.8305 & 71 \\
\hline
\end{tabular}

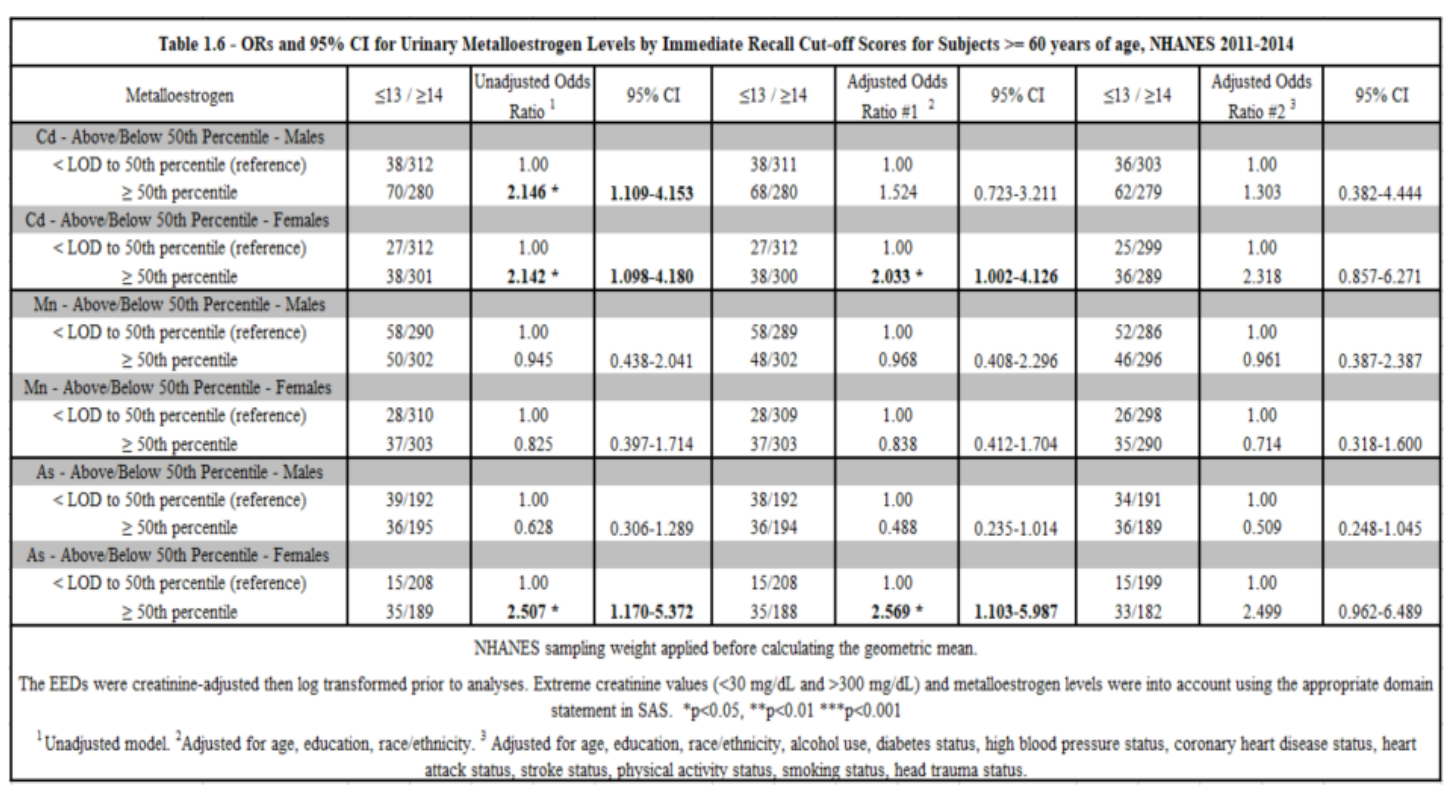




\section{Delayed Recall Scores and Exposure to Metalloestrogens: Tables 1.7 to 1.11}

present the GMs and GSEs of urinary metalloestrogen levels among subjects with delayed recall scores. Table 1.7 presents crude GM levels of metalloestrogens for of subjects 60 years of age and older who have delayed recall cognitive test scores and urinary metalloestrogen levels above the LOD. No significant differences were observed. Table 1.8 presents age-specific GMs among the age groups 60-69, 70-79, and $80+$. In the 70-79-year age group, the GM level for As was found to be significantly higher in the delayed recall score $\leq 4$ compared to the delayed recall $\geq 3$ group $(\mathrm{p}<0.05)$. Table 1.9 presents gender-specific GMs among males and females. In males, GM levels of $\mathrm{Cd}$ were found to be significantly higher in the delayed recall score $\leq 3$ compared to the delayed recall $\geq 4$ group $(\mathrm{p}<0.05)$.

Table 1.10 presents race-specific GMs. Among Hispanics, GM levels for As were found to be higher in the delayed recall score group $\leq 3$ compared to the delayed recall score $\geq 4$ group $(\mathrm{p}<0.05)$. The GM level for Mn was found to be significantly higher in the delayed recall score $\geq 4$ group compared to the delayed recall score group $\leq 3$ $(\mathrm{p}<0.05)$.

Table 1.11 presents estimated ORs and 95\% CI for delayed recall scores and metalloestrogens stratified by gender with two groups: < LOD to 50th percentile (reference) and $\geq 50$ th percentile. In the unadjusted model, among males, a significant association between Cd GM levels and lower delayed recall test scores was found [OR=2.588, 95\% CI: $1.343-4.9890]$. 
In the adjusted model \#1, among males, a significant association was found between

Cd GM levels and lower delayed recall test scores [OR=2.070, 95\% CI: 1.055-4060].

No significant associations were found in our final adjusted models.

Table 1.7 Geometric Mean Urinary Metalloestrogen Levels by Delayed Recall Cut-off Score for Subjects >= 60 year's of age, NHANES 2011-2014, over the LOD

\begin{tabular}{|c|ccc|ccc|}
\hline \multirow{2}{*}{ Metalloestrogen } & \multicolumn{5}{|c|}{ Geometric Mean (ug/ml) (GSE, N) } \\
\cline { 2 - 7 } & \multicolumn{2}{|c|}{ Delayed Recall Score $\leq 3$} & \multicolumn{3}{|c|}{ Delayed Recall Score $\geq 4$} \\
\cline { 2 - 7 } & GM & GSE & N & GM & GSE & N \\
\hline Cadmium & 0.366 & 0.02894 & 241 & 0.3282 & 0.01265 & 1106 \\
Manganese & 0.1772 & 0.01855 & 117 & 0.2015 & 0.01037 & 483 \\
Arsenic & 9.5258 & 1.5281 & 166 & 9.6271 & 0.7876 & 727 \\
\hline
\end{tabular}

NHANES sampling weight applied before calculating the geometric mean.

The EEDs were creatinine-adjusted then log transformed prior to analy ses. Extreme creatinine values $(<30$ $\mathrm{mg} / \mathrm{dL}$ and $>300 \mathrm{mg} / \mathrm{dL}$ and phthalate levels below the LOD were taken into account using the appropriate domain statement in SAS.

$* \mathrm{p}<0.05, * * \mathrm{p}<0.01 * * * \mathrm{p}<0.001$ 
Table 1.8 Age-Specific Geometric Mean Urinary Metalloestrogen Levels by Delayed Recall Cut-off Score for Subjects $>=60$ years of age, NHANES 2011-2014, over the LOD

\begin{tabular}{|c|c|c|c|c|c|c|}
\hline \multirow{3}{*}{ Metalloestrogen } & \multicolumn{6}{|c|}{ Geometric Mean (ug/ml) (GSE, N), Age 60-69 } \\
\hline & \multicolumn{3}{|c|}{ Delayed Recall Score $\leq 3$} & \multicolumn{3}{|c|}{ Delayed Recall Score $\geq 4$} \\
\hline & GM & GSE & $\mathrm{N}$ & GM & GSE & $\mathrm{N}$ \\
\hline Cadmium & 0.3006 & 0.04553 & 94 & 0.3133 & 0.01918 & 619 \\
\hline Manganese & 0.207 & 0.03832 & 49 & 0.1937 & 0.01678 & 255 \\
\hline Arsenic & 10.1671 & 2.7511 & 65 & 8.5695 & 0.6482 & 407 \\
\hline \multirow{3}{*}{ Metalloestrogen } & \multicolumn{6}{|c|}{ Geometric Mean (ug/ml) (GSE, N), Age 70-79 } \\
\hline & \multicolumn{3}{|c|}{ Delayed Recall Score $\leq 3$} & \multicolumn{3}{|c|}{ Delayed Recall Score $\geq 4$} \\
\hline & GM & GSE & $\mathrm{N}$ & GM & GSE & $\mathrm{N}$ \\
\hline Cadmium & 0.3588 & 0.0309 & 73 & 0.3459 & 0.02489 & 354 \\
\hline Manganese & 0.1585 & 0.03226 & 30 & 0.2083 & 0.02398 & 163 \\
\hline Arsenic & 6.352 & 1.386 & 51 & $11.37 *$ & 1.684 & 234 \\
\hline \multirow{3}{*}{ Metalloestrogen } & \multicolumn{6}{|c|}{ Geometric Mean (ug/ml) (GSE, N), Age $80+$} \\
\hline & \multicolumn{3}{|c|}{ Delayed Recall Score $\leq 3$} & \multicolumn{3}{|c|}{ Delayed Recall Score $\geq 4$} \\
\hline & GM & GSE & $\mathrm{N}$ & GM & GSE & $\mathrm{N}$ \\
\hline Cadmium & 0.4586 & 0.06833 & 74 & 0.3567 & 0.03288 & 133 \\
\hline Manganese & 0.1711 & 0.02048 & 38 & 0.2206 & 0.02557 & 65 \\
\hline Arsenic & 13.627 & 2.5889 & 50 & 10.5588 & 1.4187 & 86 \\
\hline \multicolumn{7}{|c|}{ NHANES sampling weight applied before calculating the geometric mean. } \\
\hline \multicolumn{7}{|c|}{$\begin{array}{l}\text { The EEDs were creatinine-adjusted then log transformed prior to analyses. Extreme creatinine value } \\
(<30 \mathrm{mg} / \mathrm{dL} \text { and }>300 \mathrm{mg} / \mathrm{dL} \text { and phthalate levels below the LOD were taken into account using the } \\
\text { appropriate domain statement in SAS. }\end{array}$} \\
\hline \multicolumn{7}{|c|}{$* \mathrm{p}<0.05, * * \mathrm{p}<0.01 * * * \mathrm{p}<0.001$} \\
\hline
\end{tabular}

Table 1.9 Gender-Specific Geometric Mean Urinary Metalloes trogen Levels by Delayed Recall Cut-off Score for Subjects $>=60$ years of age, NHANES 2011-2014, over the LOD

\begin{tabular}{|c|c|c|c|c|c|c|}
\hline \multirow{4}{*}{ Metalloestrogen } & \multicolumn{6}{|c|}{ Geometric Mean (ug/ml) (GSE, N) } \\
\hline & \multicolumn{6}{|c|}{ Males } \\
\hline & \multicolumn{3}{|c|}{ Delayed Recall Score $\leq 3$} & \multicolumn{3}{|c|}{ Delayed Recall Score $\geq 4$} \\
\hline & GM & GSE & $\mathrm{N}$ & GM & GSE & $\mathrm{N}$ \\
\hline Cadmium & $\mathbf{0 . 3 1 3 1} *$ & 0.02213 & 142 & 0.2638 & 0.01183 & 543 \\
\hline Manganese & 0.1415 & 0.01572 & 67 & 0.1446 & 0.01439 & 198 \\
\hline Arsenic & 7.3954 & 1.3287 & 97 & 9.2942 & 0.7997 & 356 \\
\hline \multirow{4}{*}{ Metalloestrogen } & \multicolumn{6}{|c|}{ Geometric Mean (ug/ml) (GSE, N) } \\
\hline & \multicolumn{6}{|c|}{ Females } \\
\hline & \multicolumn{3}{|c|}{ Delayed Recall Score $\leq 3$} & \multicolumn{3}{|c|}{ Delayed Recall Score $\geq 4$} \\
\hline & GM & GSE & $\mathrm{N}$ & GM & GSE & $\mathrm{N}$ \\
\hline Cadmium & 0.4519 & 0.07882 & 99 & 0.4007 & 0.01993 & 563 \\
\hline Manganese & 0.2372 & 0.03562 & 50 & 0.2551 & 0.02281 & 285 \\
\hline Arsenic & 13.6099 & 2.7464 & 69 & 9.9297 & 1.0512 & 371 \\
\hline
\end{tabular}

NHANES s ampling weight applied before calculating the geometric mean.

The EEDs were creatinine-adjusted then log transformed prior to analyses. Extreme creatinine values $(<30 \mathrm{mg} / \mathrm{dL}$ and $>300 \mathrm{mg} / \mathrm{dL}$ and phthalate levels below the LOD were taken into account using the appropriate domain statement in SAS.

$* \mathrm{p}<0.05, * * \mathrm{p}<0.01 * * * \mathrm{p}<0.001$ 


\begin{tabular}{|c|c|c|c|c|c|c|c|c|c|c|c|c|}
\hline \multicolumn{13}{|c|}{$\begin{array}{c}\text { Table 1.10 Race-specific Geometric Mean Urinary Metalloestrogen Levels by Delayed Recall Cut-off Score for Subjects >= } 60 \text { years of age, NHANES 2011-201 } \\
\text { over the LOD }\end{array}$} \\
\hline \multirow{3}{*}{ Metalloestrogens } & \multicolumn{6}{|c|}{ Geometric Mean (ng/ml) (GSE, N), Hispanic } & \multicolumn{6}{|c|}{ Geometric Mean (ng/ml) (GSE, N), Non-Hispanic White } \\
\hline & \multicolumn{3}{|c|}{ Delayed Recall Score $\leq 3$} & \multicolumn{3}{|c|}{ Delayed Recall Score $\geq 4$} & \multicolumn{3}{|c|}{ Delayed Recall Score $\leq 3$} & \multicolumn{3}{|c|}{ Delayed Recall Score $\geq 4$} \\
\hline & GM & GSE & $\mathrm{N}$ & GM & GSE & $\mathrm{N}$ & GM & GSE & $\mathrm{N}$ & GM & GSE & $\mathrm{N}$ \\
\hline Cadmium & 0.3603 & 0.04223 & 63 & 0.3403 & 0.02554 & 196 & 0.3658 & 0.04311 & 101 & 0.3161 & 0.01417 & 538 \\
\hline Manganese & 0.1297 & 0.01224 & 36 & $0.1807 *$ & 0.01909 & 85 & 0.1725 & 0.02077 & 46 & 0.2103 & 0.01312 & 232 \\
\hline Arsenic & $10.8211 *$ & 1.6028 & 44 & 8.7522 & 0.9843 & 129 & 9.2727 & 2.0764 & 68 & 9.2182 & 0.9256 & 343 \\
\hline \multirow{3}{*}{ Metalloestrogens } & \multicolumn{6}{|c|}{ Geometric Mean (ng/ml) (GSE, N), Non-Hispanic Black } & \multicolumn{6}{|c|}{ Geometric Mean (ng/ml) (GSE, N), Asian/Other } \\
\hline & \multicolumn{3}{|c|}{ Delayed Recall Score $\leq 3$} & \multicolumn{3}{|c|}{ Delayed Recall Score $\geq 4$} & \multicolumn{3}{|c|}{ Delayed Recall Score $\leq 3$} & \multicolumn{3}{|c|}{ Delayed Recall Score $\geq 4$} \\
\hline & GM & GSE & $\mathrm{N}$ & $\mathrm{GM}$ & GSE & $\mathrm{N}$ & GM & GSE & $\mathrm{N}$ & GM & GSE & $\mathrm{N}$ \\
\hline Cadmium & 0.3223 & 0.03307 & 59 & 0.3439 & 0.01894 & 261 & 0.5919 & 0.1442 & 18 & 0.5685 & 0.05876 & 111 \\
\hline Manganese & 0.3631 & 0.2185 & 22 & 0.144 & 0.009342 & 112 & 0.2046 & 0.03657 & 13 & 0.2 & 0.02938 & 54 \\
\hline Arsenic & 6.5296 & 0.8656 & 39 & 8.5349 & 0.8946 & 181 & 25.5786 & 4.3194 & 15 & 31.1416 & 4.9994 & 74 \\
\hline \multicolumn{13}{|c|}{$\begin{array}{l}\text { NHANES sampling weight applied before calculating the geometric mean. } \\
\text { The EEDs were creatinine-adjusted then log transformed prior to analyses. Extreme creatinine values }(<30 \mathrm{mg} / \mathrm{dL} \text { and }>300 \mathrm{mg} / \mathrm{dL} \text { and phthalate levels below the LOD } \\
\text { were taken into account using the appropriate domain statement in SAS. } \\
\qquad{ }^{*}<<0.05, * * p<0.01 * * * p<0.001\end{array}$} \\
\hline
\end{tabular}

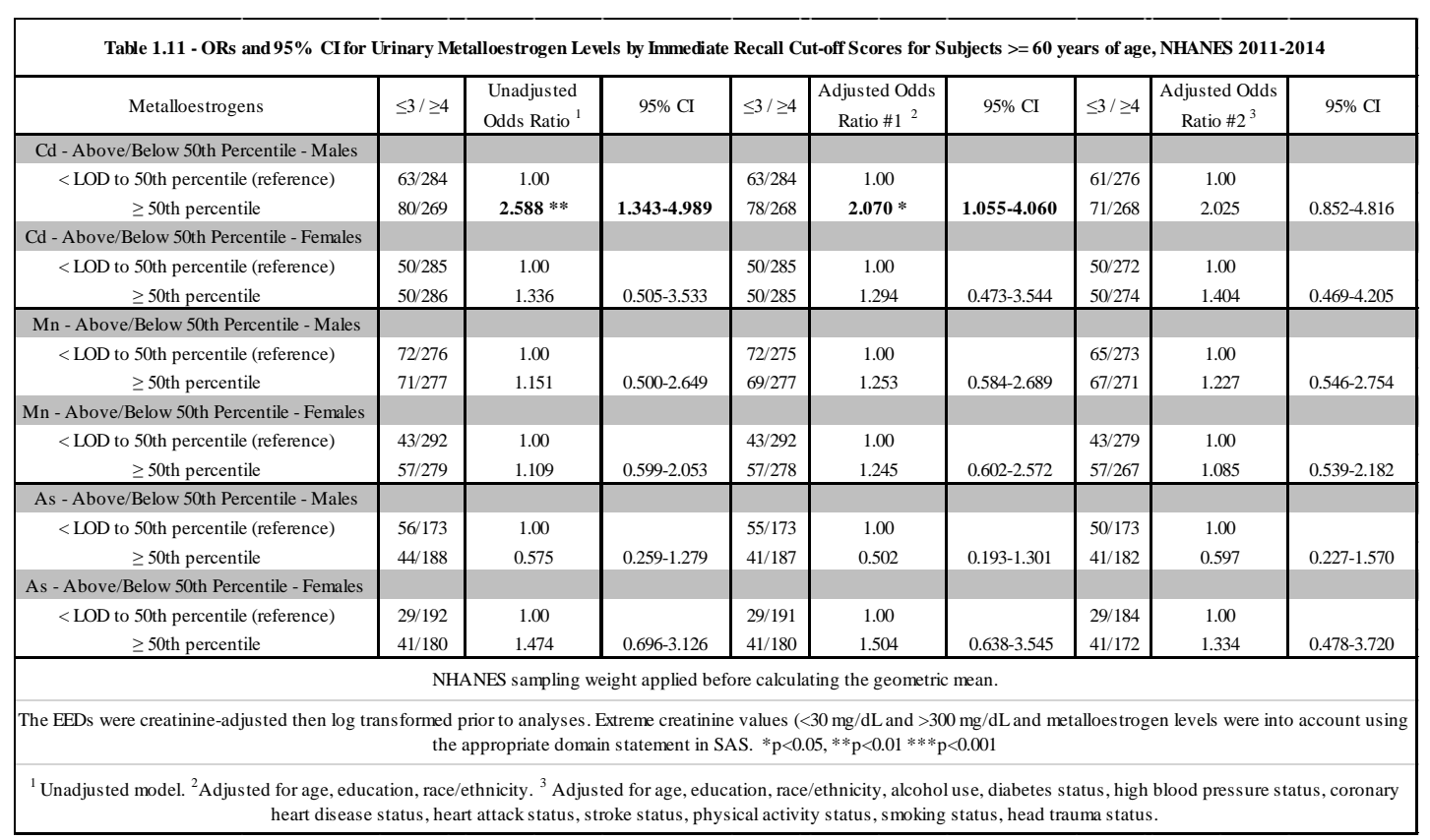

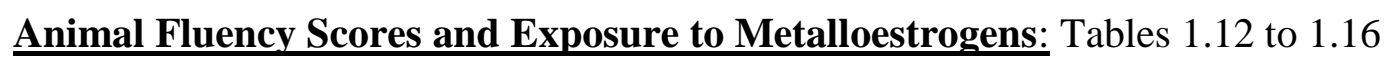

present the GMs and GSEs of urinary metalloestrogen levels among subjects with

animal fluency scores. Table 1.12 presents crude GM levels of metalloestrogens for

of subjects 60 years of age and older who have animal fluency cognitive test scores

and urinary metalloestrogen levels above the LOD. No significant differences were 
observed. Table 1.13 presents age-specific GMs for the ag groups 60-69, 70-79, and 80+. No significant results were observed. Table 1.14 presents gender-specific GMs. No significant results were observed. Table 1.15 presents race-specific GMs across the following groups: Hispanic, Non-Hispanic White, Non-Hispanic Black, and Asian/Other. For all racial groups, no significant results were observed.

Table 1.16 presents estimated ORs and 95\% CI for animal fluency scores and metalloestrogens stratified by gender with two groups: < LOD to 50th percentile (reference) and $\geq 50$ th percentile. In the unadjusted model, among males, a significant association between Cd GM levels and lower delayed recall test scores was found [OR=1.971, 95\% CI: 1.063-3.654].

Table 1.12 Geometric Mean Urinary Metalloestrogen Levels by Animal Fluency Cut-off Score for Subjects > 60 years of age, NHANES 2011-2014, over the LOD

\begin{tabular}{|c|ccc|ccc|}
\hline \multirow{2}{*}{ Metalloestrogen } & \multicolumn{5}{|c|}{ Geometric Mean (ug/ml) (GSE, N) } \\
\cline { 2 - 8 } & \multicolumn{2}{|c|}{ Animal Fluency Score $\leq 11$} & \multicolumn{3}{|c|}{ Animal Fluency Score $\geq 12$} \\
\cline { 2 - 8 } & GM & GSE & N & GM & GSE & N \\
\hline Cadmium & 0.3741 & 0.02301 & 280 & 0.3289 & 0.01218 & 1060 \\
Manganese & 0.2207 & 0.02332 & 119 & 0.1954 & 0.01067 & 479 \\
Arsenic & 9.1819 & 1.0455 & 183 & 9.8017 & 0.8204 & 707 \\
\hline
\end{tabular}

NHANES sampling weight applied before calculating the geometric mean.

The EEDs were creatinine-adjusted then log transformed prior to analyses. Extreme creatinine values $(<30 \mathrm{mg} / \mathrm{dL}$ and $>300 \mathrm{mg} / \mathrm{dL}$ and phthalate levels below the LOD were taken into account using the appropriate domain statement in SAS.

$* \mathrm{p}<0.05, * * \mathrm{p}<0.01 * * * \mathrm{p}<0.001$ 


\begin{tabular}{|c|c|c|c|c|c|c|c|c|c|c|c|c|}
\hline \multirow{3}{*}{ Metalloestrogen } & \multicolumn{6}{|c|}{ Geometric Mean (ng/ml) (GSE, N), Hispanic } & \multicolumn{6}{|c|}{ Geometric Mean (ng/ml) (GSE, N), Non-Hispanic White } \\
\hline & \multicolumn{3}{|c|}{ Animal Fluency Score $\leq 11$} & \multicolumn{3}{|c|}{ Animal Fluency Score $\geq 12$} & \multicolumn{3}{|c|}{ Animal Fluency Score $\leq 11$} & \multicolumn{3}{|c|}{ Animal Fluency Score $\geq 12$} \\
\hline & GM & GSE & $\mathrm{N}$ & GM & GSE & $\mathrm{N}$ & GM & GSE & $\mathrm{N}$ & GM & GSE & $\mathrm{N}$ \\
\hline Cadmium & 0.4236 & 0.05284 & 58 & 0.328 & 0.02682 & 200 & 0.3546 & 0.02884 & 102 & 0.32 & 0.01472 & 538 \\
\hline Manganese & 0.1734 & 0.02582 & 29 & 0.1602 & 0.01311 & 93 & 0.2278 & 0.02688 & 35 & 0.2028 & 0.01359 & 242 \\
\hline Arsenic & 8.8717 & 1.6535 & 39 & 9.3797 & 1.0026 & 134 & 8.4117 & 1.5886 & 62 & 9.4515 & 0.958 & 350 \\
\hline \multirow{3}{*}{ Metalloestrogen } & \multicolumn{6}{|c|}{ Geometric Mean (ng/ml) (GSE, N), Non-Hispanic Black } & \multicolumn{6}{|c|}{ Geometric Mean (ng/ml) (GSE, N), Asian/Other } \\
\hline & \multicolumn{3}{|c|}{ Animal Fluency Score $\leq 11$} & \multicolumn{3}{|c|}{ Animal Fluency Score $\geq 12$} & \multicolumn{3}{|c|}{ Animal Fluency Score $\leq 11$} & \multicolumn{3}{|c|}{ Animal Fluency Score $\geq 12$} \\
\hline & GM & GSE & $\mathrm{N}$ & GM & GSE & $\mathrm{N}$ & GM & GSE & $\mathrm{N}$ & GM & GSE & $\mathrm{N}$ \\
\hline Cadmium & 0.3712 & 0.04361 & 91 & 0.3315 & 0.02451 & 223 & 0.5692 & 0.07856 & 29 & 0.5697 & 0.04965 & 99 \\
\hline Manganese & 0.246 & 0.08075 & 37 & 0.1515 & 0.0135 & 97 & 0.2212 & 0.0408 & 18 & 0.1941 & 0.02797 & 47 \\
\hline Arsenic & 8.5702 & 1.2119 & 60 & 8.1649 & 0.825 & 157 & 30.0025 & 3.6506 & 22 & 30.7608 & 5.4181 & 66 \\
\hline \multicolumn{13}{|c|}{$\begin{array}{l}\text { NHANES sampling weight applied before calculating the geometric mean. } \\
\text { The EEDs were creatinine-adjusted then log transformed prior to analyses. Extreme creatinine values }(<30 \mathrm{mg} / \mathrm{dL} \text { and }>300 \mathrm{mg} / \mathrm{dL} \text { and phthalate levels below the LOD wer } \\
\text { taken into account using the appropriate domain statement in SAS. } \\
\qquad * \mathrm{p}<0.05, * * \mathrm{p}<0.01 * * * \mathrm{p}<0.001\end{array}$} \\
\hline
\end{tabular}

\begin{tabular}{|c|c|c|c|c|c|c|c|c|c|}
\hline Metalloestrogen & $\leq 11 / \geq 12$ & $\begin{array}{l}\text { Unadjusted } \\
\text { Odds Ratio }^{1}\end{array}$ & $95 \% \mathrm{CI}$ & $\leq 11 / \geq 12$ & \begin{tabular}{|c} 
Adjusted Odds \\
Ratio $\# 1^{2}$ \\
\end{tabular} & $95 \% \mathrm{CI}$ & $\leq 11 / \geq 12$ & \begin{tabular}{|c|} 
Adjusted Odds \\
Ratio $\# 2^{3}$ \\
\end{tabular} & $95 \% \mathrm{CI}$ \\
\hline \multicolumn{10}{|l|}{ Cd - Above/Below 50th Percentile - Males } \\
\hline $\begin{array}{c}<\text { LOD to 50th percentile (reference) } \\
\quad \geq 50 \text { th percentile }\end{array}$ & $\begin{array}{l}50 / 297 \\
89 / 258\end{array}$ & $\begin{array}{c}1.00 \\
\mathbf{1 . 9 7 1} *\end{array}$ & $1.063-3.654$ & $\begin{array}{l}50 / 295 \\
86 / 260\end{array}$ & $\begin{array}{c}1.00 \\
1.354\end{array}$ & $0.713-2.570$ & $\begin{array}{l}46 / 291 \\
81 / 256\end{array}$ & $\begin{array}{l}1.00 \\
1.505\end{array}$ & $0.653-3.467$ \\
\hline \multicolumn{10}{|l|}{$\mathrm{Cd}$ - Above/Below 50th Percentile - Females } \\
\hline $\begin{array}{c}<\text { LOD to 50th percentile (reference) } \\
\geq 50 \text { th percentile }\end{array}$ & $\begin{array}{l}56 / 277 \\
87 / 246 \\
\end{array}$ & $\begin{array}{l}1.00 \\
1.416\end{array}$ & $0.745-2.692$ & $\begin{array}{l}56 / 277 \\
87 / 245 \\
\end{array}$ & $\begin{array}{l}1.00 \\
1.353 \\
\end{array}$ & $0.640-2.860$ & $\begin{array}{l}56 / 264 \\
80 / 239 \\
\end{array}$ & $\begin{array}{l}1.00 \\
1.552\end{array}$ & $0.801-3.007$ \\
\hline \multicolumn{10}{|l|}{ Mn - Above/Below 50th Percentile - Males } \\
\hline $\begin{array}{l}<\text { LOD to 50th percentile (reference) } \\
\quad \geq 50 \text { th percentile }\end{array}$ & $\begin{array}{l}68 / 276 \\
71 / 279\end{array}$ & $\begin{array}{c}1.00 \\
1.144\end{array}$ & $0.635-2.061$ & $\begin{array}{l}67 / 276 \\
69 / 279\end{array}$ & $\begin{array}{l}1.00 \\
1.305\end{array}$ & $0.676-2.519$ & $\begin{array}{l}62 / 272 \\
65 / 275\end{array}$ & $\begin{array}{l}1.00 \\
1.155\end{array}$ & $0.473-2.822$ \\
\hline \multicolumn{10}{|l|}{ Mn - Above/Below 50th Percentile - Females } \\
\hline $\begin{array}{c}<\text { LOD to 50th percentile (reference) } \\
\geq 50 \text { th percentile }\end{array}$ & $\begin{array}{l}65 / 267 \\
78 / 256 \\
\end{array}$ & $\begin{array}{c}1.00 \\
1.046 \\
\end{array}$ & $0.599-1.826$ & $\begin{array}{l}65 / 266 \\
78 / 256 \\
\end{array}$ & $\begin{array}{r}1.00 \\
1.382 \\
\end{array}$ & $0.797-2.395$ & $\begin{array}{l}65 / 255 \\
71 / 248 \\
\end{array}$ & $\begin{array}{l}1.00 \\
1.173 \\
\end{array}$ & $0.600-2.291$ \\
\hline \multicolumn{10}{|l|}{ As - Above/Below 50th Percentile - Males } \\
\hline $\begin{array}{l}<\text { LOD to 50th percentile (reference) } \\
\quad \geq 50 \text { th percentile }\end{array}$ & $\begin{array}{l}49 / 179 \\
40 / 189\end{array}$ & $\begin{array}{c}1.00 \\
0.622\end{array}$ & $0.357-1.084$ & $\begin{array}{l}48 / 179 \\
39 / 189\end{array}$ & $\begin{array}{l}1.00 \\
0.483\end{array}$ & $0.255-0.915$ & $\begin{array}{l}44 / 178 \\
38 / 185\end{array}$ & $\begin{array}{c}1.00 \\
0.473\end{array}$ & $0.222-1.009$ \\
\hline \multicolumn{10}{|l|}{ As - Above/Below 50th Percentile - Females } \\
\hline $\begin{array}{c}<\text { LOD to 50th percentile (reference) } \\
\geq 50 \text { th percentile }\end{array}$ & $\begin{array}{l}42 / 178 \\
53 / 167 \\
\end{array}$ & $\begin{array}{c}1.00 \\
1.142 \\
\end{array}$ & $0.673-1.938$ & $\begin{array}{l}42 / 177 \\
53 / 167 \\
\end{array}$ & $\begin{array}{c}1.00 \\
1.149 \\
\end{array}$ & $0.659-2.002$ & $\begin{array}{l}40 / 171 \\
51 / 161 \\
\end{array}$ & $\begin{array}{l}1.00 \\
1.064 \\
\end{array}$ & $0.559-2.026$ \\
\hline The EEDs were creatinine-adjusted then lo & & $\begin{array}{r}\text { yses. Extreme } \\
\text { state }\end{array}$ & $\begin{array}{l}\text { tinine values } \\
\text { tin SAS. *p. }\end{array}$ & $\mathrm{ng} / \mathrm{dL}$ and & $\begin{array}{l}0 \mathrm{mg} / \mathrm{dL} \text { and } \mathrm{M} \\
0<0.001\end{array}$ & oestrogen & ere into & Int using the a & priate domain \\
\hline
\end{tabular}

\section{Digit Symbol Substitution Test (DSST) Scores and Exposure to Metalloestrogens:}

Tables 1.17 to 1.21 present the GMs and GSEs of urinary metalloestrogen levels among subjects with DSST scores. Table 1.17 presents crude GMs levels of metalloestrogens for subjects 60 years of age and older who have digit symbol substitution scores and urinary metalloestrogen levels above the LOD. The crude GM mean levels for Cd was found to be higher in those with a DSST score $\leq 27$ compared to those with a DSST score $\geq 28(\mathrm{p}<0.05)$. Table 1.18 presents age-specific GMs by the age groups $60-69$, 70-79, and 80+. For Mn in the 80+ year age group, the GM was significantly higher in 
those with a DSST score $\geq 28$ compared to those with a DSST score $\leq 27(\mathrm{p}<0.05)$.

Table 1.19 presents gender-specific GMs by male and female. No significant results were found. Table 1.20 presents race-specific GMs. No results among race-specific GMs were found to be significant.

Table 1.21 presents estimated ORs and 95\% CI for DSST scores and metalloestrogens stratified by gender with two groups: < LOD to 50th percentile (reference) and $\geq 50$ th percentile. In the unadjusted model, among males, a significant association between Cd GM levels and lower DSST test scores was found [OR=2.409, 95\% CI: 1.389-4.180].

Table 1.17 Geometric Mean Urinary Metalloestrogen Levels by Digit Symbol Subs titution (DSST) Cut-off Scores for Subjects $>=60$ years of age, NHANES 2011-2014, over the LOD

\begin{tabular}{|c|ccc|ccc|}
\hline \multirow{2}{*}{ Metalloestrogen } & \multicolumn{5}{|c|}{ Geometric Mean (ug/ml) (GSE, N) } \\
\cline { 2 - 7 } & \multicolumn{3}{|c|}{ DSST Score $\leq 27$} & \multicolumn{3}{c|}{ DSST Score $\geq 28$} \\
\cline { 2 - 7 } & GM & GSE & N & GM & GSE & $\mathrm{N}$ \\
\hline Cadmium & $\mathbf{0 . 3 9 9 9} *$ & $\mathbf{0 . 0 3 5 3 8}$ & $\mathbf{2 2 8}$ & 0.3266 & 0.01216 & 1078 \\
Manganese & 0.2586 & 0.09081 & 96 & 0.1905 & 0.009645 & 487 \\
Arsenic & 9.2296 & 1.0124 & 146 & 9.7471 & 0.8301 & 721 \\
\hline \multicolumn{3}{|c|}{ NHANES sampling weight applied before calculating the geometric mean. } \\
The EEDs were creatinine-adjusted then log transformed prior to analyses. Extreme creatinine \\
values (<30 mg/dL and $>300$ mg/dL and phthalate levels below the LOD were taken into account \\
using the appropriate domain statement in SAS. \\
$* \mathrm{p}<0.05, * * \mathrm{p}<0.01 * * * \mathrm{p}<0.001$ \\
\hline
\end{tabular}


Table 1.20 Race-specific Geometric Mean Urinary Metalloestrogen Levels by Digit Symbol Substitution (DSST) Cut-off Scores for Subjects >= 60 years of age, NHANES 2011-2014, over the LOD

\begin{tabular}{|c|c|c|c|c|c|c|c|c|c|c|c|c|}
\hline \multirow{3}{*}{ Metalloestrogen } & \multicolumn{6}{|c|}{ Geometric Mean (ng/ml) (GSE, N), Hispanic } & \multicolumn{6}{|c|}{ Geometric Mean (ng/ml) (GSE, N), Non-Hispanic White } \\
\hline & \multicolumn{3}{|c|}{ DSST Score $\leq 27$} & \multicolumn{3}{|c|}{ DSST Score $\geq 28$} & \multicolumn{3}{|c|}{ DSST Score $\leq 27$} & \multicolumn{3}{|c|}{ DSST Score $\geq 28$} \\
\hline & $\mathrm{GM}$ & GSE & $\mathrm{N}$ & GM & GSE & $\mathrm{N}$ & GM & GSE & $\mathrm{N}$ & GM & GSE & $\mathrm{N}$ \\
\hline Cadmium & 0.3817 & 0.05502 & 82 & 0.3345 & 0.02398 & 160 & 0.4167 & 0.07094 & 63 & 0.3166 & 0.01412 & 568 \\
\hline Manganese & 0.1468 & 0.02376 & 35 & 0.1653 & 0.0167 & 76 & 0.4278 & 0.2363 & 26 & 0.1954 & 0.01166 & 251 \\
\hline Arsenic & 9.5891 & 1.479 & 52 & 8.9243 & 1.1056 & 109 & 9.0462 & 1.8241 & 39 & 9.3658 & 0.9609 & 367 \\
\hline \multirow{3}{*}{ Metalloestrogen } & \multicolumn{6}{|c|}{ Geometric Mean (ng/ml) (GSE, N), Non-Hispanic Black } & \multicolumn{6}{|c|}{ Geometric Mean (ng/ml) (GSE, N), Asian/Other } \\
\hline & \multicolumn{3}{|c|}{ DSST Score $\leq 27$} & \multicolumn{3}{|c|}{ DSST Score $\geq 28$} & \multicolumn{3}{|c|}{ DSST Score $\leq 27$} & \multicolumn{3}{|c|}{ DSST Score $\geq 28$} \\
\hline & GM & GSE & $\mathrm{N}$ & GM & GSE & $\mathrm{N}$ & GM & GSE & $\mathrm{N}$ & GM & GSE & $\mathrm{N}$ \\
\hline Cadmium & 0.3551 & 0.03703 & 69 & 0.3328 & 0.01853 & 234 & 0.5458 & 0.1045 & 14 & 0.5651 & 0.04797 & 116 \\
\hline Manganese & 0.1323 & 0.01385 & 30 & 0.1517 & 0.0102 & 99 & 0.1467 & 0.02046 & 5 & 0.199 & 0.02579 & 61 \\
\hline Arsenic & 6.9908 & 1.0097 & 45 & 8.4954 & 0.8396 & 165 & 26.4609 & 6.4777 & 10 & 29.7625 & 4.3838 & 80 \\
\hline
\end{tabular}

NHANES sampling weight applied before calculating the geometric mean.

The EEDs were creatinine-adjusted then log transformed prior to analyses. Extreme creatinine values ( $<30 \mathrm{mg} / \mathrm{dL}$ and $>300 \mathrm{mg} / \mathrm{dL}$ and phthalate levels below the LOD were taken into account using the appropriate domain statement in SAS.

$* p<0.05, * * p<0.01 * * * p<0.001$

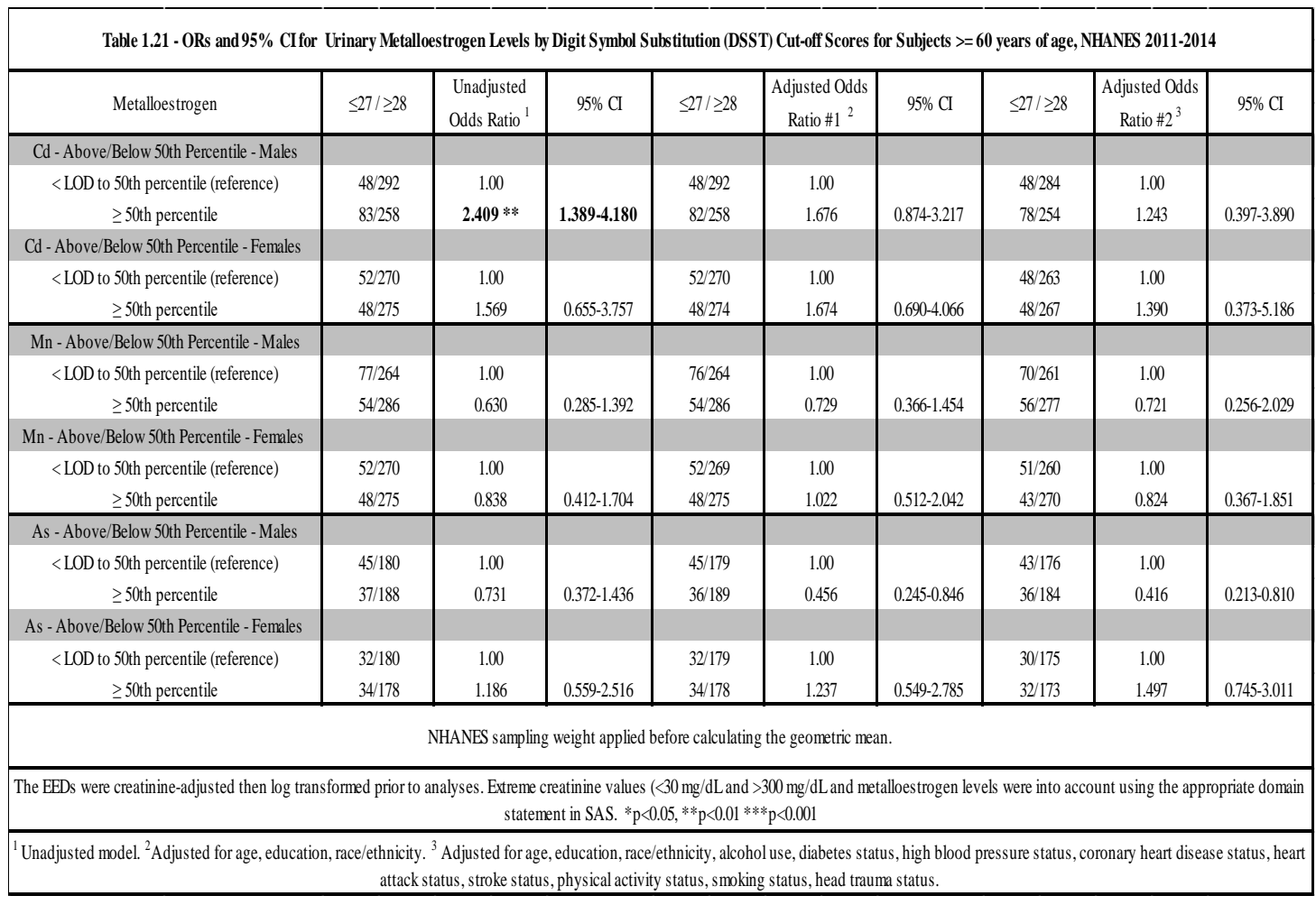




\section{Associations Between Exposures to Metalloestrogens and Memory Function}

Exposures to the metalloestrogens $\mathrm{Cd}, \mathrm{Mn}$, and As and three memory function indicators: 1) During the past 12 months, have you experienced confusion or memory loss that is happening more often or getting worse? 2) During the past 7 days, how often have you had trouble remembering where you put things? 3) Are you limited in any way because of difficulty remembering or because you experience periods of confusion?) are summarized in tables 1.22 to 1.36 . Memory function have been included as a surrogate indicator of brain health as declining memory function can indicate the development of mild cognitive impairment, dementia, AD, or other memory-related neurodegenerative disease.

\section{Worsening memory past 12 months and Exposure to Metalloestrogens: Table}

1.22 presents the crude GMs for subjects over the age of 60 years of age who responded $\mathrm{Y} / \mathrm{N}$ to the question "During the past 12 months, you experienced confusion or memory loss that is happening more often or is getting worse?" and have urinary metalloestrogen levels above the LOD. No significant results were found. Table 1.23 presents age-specific GM levels for the age groups 60-69, 70-79, and 80+. In the 60-69-year age group, Cd GM levels were higher in those who answered "yes" versus those who answered "no". Among the 70 to 79 -year age group, As GM mean levels were found to be significantly different in those that answered "no" compared to those that answered "yes" ( $<<0.05)$. Table 1.24 shows gender-specific GM among males and females. For males, As GM levels are significantly higher among those who answered "no" versus those who answered "no" ( $<<0.05)$. Table 1.25 shows. 
race-specific GM among Hispanics, Non-Hispanic White, Non-Hispanic Black, and Asian/Other. No significant findings were observed.

Table 1.26 presents estimated ORs and 95\% CI for Yes/No responses to the question "During the past 12 months, you experienced confusion or memory loss that is happening more often or is getting worse?" and metalloestrogens stratified by gender with two groups: $<$ LOD to 50th percentile (reference) and $\geq 50$ th percentile. Significant associations were found for $\mathrm{Cd}$ among women in the $\geq 50$ th percentile versus $<$ LOD to 50th percentile (reference), where those who had experience confusion or memory loss over the past 12 months was associated with Cadmium levels in the unadjusted model [OR=2.463, 95\% CI: 1.572-3.8610, model adjusted for age, education, and race/ethnicity [OR=2.517, 95\% CI: 1.483-4.272] and the model adjusted for age, education, race/ethnicity, alcohol use, diabetes status, high blood pressure status, coronary heart disease status, heart attack status, stroke status, physical activity status, smoking status, head trauma status [OR=2.175, 95\% CI: $1.207-3.921]$. 
Table 1.22 Geometric Mean Urinary Metalloestrogen Levels by $\mathrm{Y} / \mathrm{N}$ "During the past 12 months, you experienced confusion or memory loss that is happening more often or is getting worse?" for Subjects >= 60 years of age, NHANES 2011-2014, over the LOD

\begin{tabular}{|c|ccc|ccc|}
\hline \multirow{2}{*}{ Metalloestrogen } & \multicolumn{6}{|c|}{ Geometric Mean (ug/ml) (GSE, N) } \\
\cline { 2 - 8 } & \multicolumn{3}{|c|}{ Yes } & \multicolumn{4}{c|}{ No } \\
\cline { 2 - 8 } & GM & GSE & N & GM & GSE & N \\
\hline Cadmium & 0.3916 & 0.0325 & 257 & 0.3307 & 0.01173 & 1187 \\
Manganese & 0.1758 & 0.01473 & 124 & 0.2037 & 0.01154 & 530 \\
Arsenic & 8.2517 & 1.0081 & 171 & 9.9903 & 0.7947 & 799 \\
\hline
\end{tabular}

NHANES sampling weight applied before calculating the geometric mean.

The EEDs were creatinine-adjusted then log transformed prior to analyses. Extreme creatinine values $(<30 \mathrm{mg} / \mathrm{dL}$ and $>300 \mathrm{mg} / \mathrm{dL}$ and phthalate levels below the LOD were taken into account using the appropriate domain statement in SAS.

$* \mathrm{p}<0.05, * * \mathrm{p}<0.01 * * * \mathrm{p}<0.001$

Table 1.23 Age-Specific Geometric Mean Urinary Metalloestrogen Levels by Y/N " During the past 12 months, you experienced confusion or memory loss that is happening more often or is getting worse?" for Subjects >= 60 years of age, NHANES 2011-2014, over the LOD

\begin{tabular}{|c|c|c|c|c|c|c|}
\hline \multirow{3}{*}{ Metalloestrogen } & \multicolumn{6}{|c|}{ Geometric Mean (ug/ml) (GSE, N), Age 60-69 } \\
\hline & \multicolumn{3}{|c|}{ Yes } & \multicolumn{3}{|c|}{ No } \\
\hline & GM & GSE & $\mathrm{N}$ & GM & GSE & $\mathrm{N}$ \\
\hline Cadmium & 0.4161 & 0.05847 & 111 & 0.3083 & 0.01982 & 650 \\
\hline Manganese & 0.1851 & 0.03617 & 55 & 0.1958 & 0.01916 & 270 \\
\hline Arsenic & 7.2239 & 1.0657 & 72 & 9.0248 & 0.6698 & 437 \\
\hline \multirow{3}{*}{ Metalloestrogen } & \multicolumn{6}{|c|}{ Geometric Mean (ug/ml) (GSE, N), Age 70-79 } \\
\hline & \multicolumn{3}{|c|}{ Yes } & \multicolumn{3}{|c|}{ No } \\
\hline & GM & GSE & $\mathrm{N}$ & GM & GSE & $\mathrm{N}$ \\
\hline Cadmium & 0.3417 & 0.03614 & 69 & 0.3546 & 0.02321 & 382 \\
\hline Manganese & 0.1516 & 0.0245 & 29 & 0.212 & 0.02643 & 176 \\
\hline Arsenic & 6.5234 & 0.871 & 46 & $11.3961 *$ & 1.573 & 258 \\
\hline \multirow{3}{*}{ Metalloestrogen } & \multicolumn{6}{|c|}{ Geometric Mean (ug/ml) (GSE, N), Age $80+$} \\
\hline & \multicolumn{3}{|c|}{ Yes } & \multicolumn{3}{|c|}{ No } \\
\hline & GM & GSE & $\mathrm{N}$ & GM & GSE & $\mathrm{N}$ \\
\hline Cadmium & 0.4168 & 0.04176 & 77 & 0.3767 & 0.02928 & 155 \\
\hline Manganese & 0.1897 & 0.03105 & 40 & 0.2147 & 0.02026 & 84 \\
\hline Arsenic & 12.5252 & 2.5776 & 53 & 11.0389 & 1.4565 & 104 \\
\hline \multicolumn{7}{|c|}{ NHANES sampling weight applied before calculating the geometric mean. } \\
\hline \multicolumn{7}{|c|}{$\begin{array}{c}\text { The EEDs were creatinine-adjusted then log transformed prior to analys es. Extreme creatinine } \\
\text { values }(<30 \mathrm{mg} / \mathrm{dL} \text { and }>300 \mathrm{mg} / \mathrm{dL} \text { and phthalate levels below the LOD were taken into account } \\
\text { using the appropriate domain statement in SAS. }\end{array}$} \\
\hline \multicolumn{7}{|c|}{$* \mathrm{p}<0.05, * * \mathrm{p}<0.01 * * * \mathrm{p}<0.001$} \\
\hline
\end{tabular}




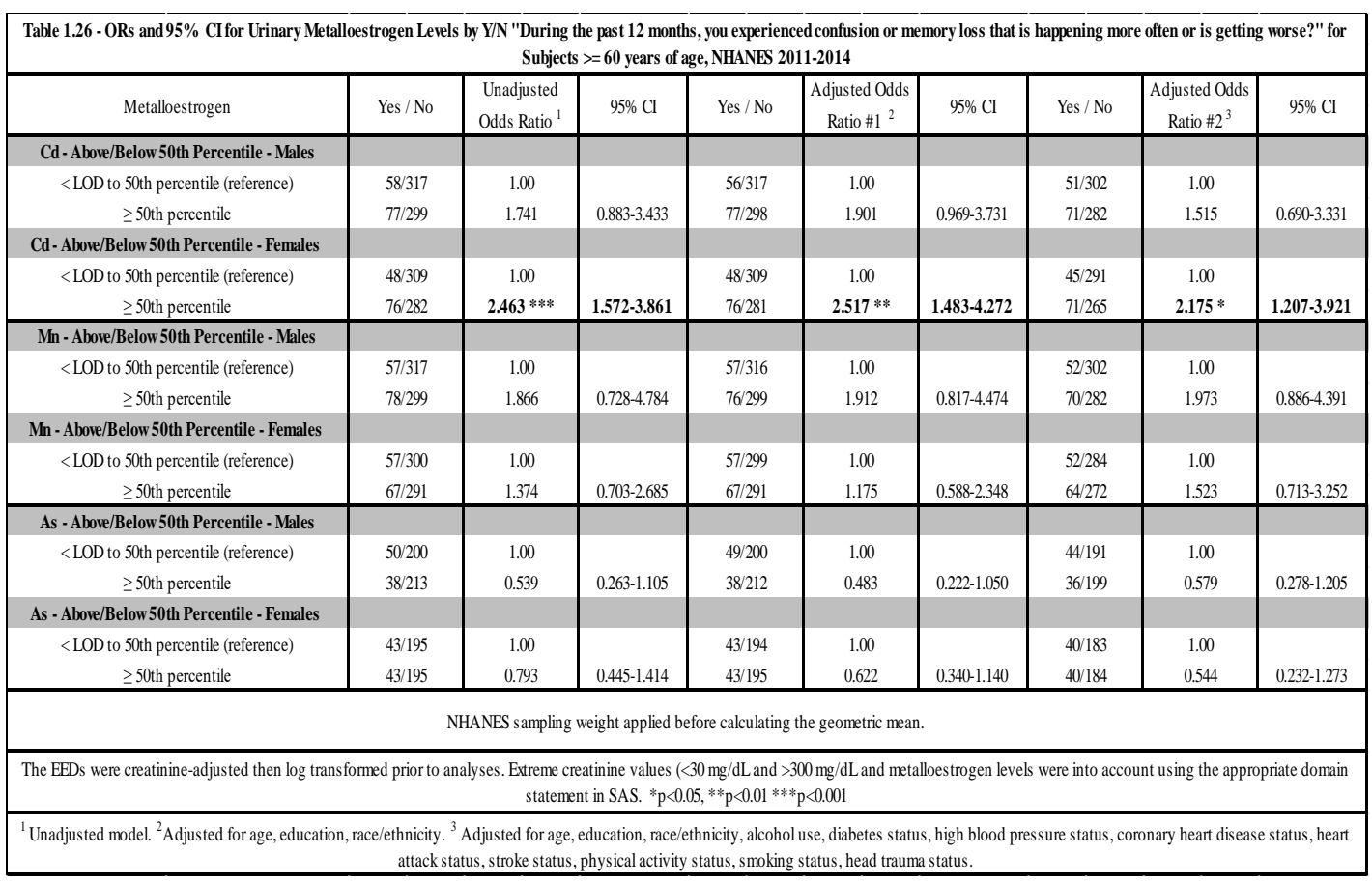

Trouble remembering past 7 days and Exposure to Metalloestrogens: Table 1.27 presents crude GM levels of metalloestrogens for subjects 60 years of age and older who have responded to the yes/no question, "During the past 7 days, how often have you had trouble remembering where you put things, like your keys or your wallet?" and have urinary metalloestrogen levels above the LOD. No significant findings were observed. Table 1.28 presents age-specific GMs by the age groups 60-69, 70-79, and $80+$ years of age. No significant findings were observed. Table 1.29 presents genderspecific GM levels. In males and females, no significant findings were observed.

Table 1.30 presents race-specific geometric means. Cd GM levels were higher in those that answered "yes" versus "no" among Hispanics, Non-Hispanic Whites, and Asian/Other. Cd and Mn GM levels were significantly higher in those that answered "no" among Non-Hispanic Blacks ( $<<0.05)$. 
Table 1.31 presents ORs and 95\% CI for those with Yes/No responses to the question "During the past 7 days, how often have you had trouble remembering where you put things, like your keys or your wallet?" and metalloestrogens stratified by gender with two groups: $<$ LOD to 50 th percentile (reference) and $\geq 50$ th percentile. No significant associations were found with our analysis of ORs.

Table 1.27 Geometric Mean Urinary Metalloestrogen Levels Y/N "During the past 7 days, how often have you had trouble remembering where you put things, like your keys or your wallet?" for Subjects >= 60 years of age, NHANES 2011-2014, over the LOD

\begin{tabular}{|c|ccc|ccc|}
\hline \multirow{2}{*}{ Metalloes trogen } & \multicolumn{5}{|c|}{ Geometric Mean (ug/ml) (GSE, N) } \\
\cline { 2 - 7 } & \multicolumn{3}{|c|}{ Yes } & \multicolumn{4}{c|}{ No } \\
\cline { 2 - 7 } & GM & GSE & N & GM & GSE & N \\
\hline Cadmium & 0.3568 & 0.03751 & 236 & 0.3387 & 0.01003 & 1132 \\
Manganese & 0.2371 & 0.04713 & 112 & 0.1958 & 0.01149 & 496 \\
Arsenic & 8.8395 & 1.0114 & 156 & 9.6986 & 0.7345 & 759 \\
\hline
\end{tabular}

NHANES sampling weight applied before calculating the geometric mean.

The EEDs were creatinine-adjusted then log transformed prior to analyses. Extreme creatinine values $(<30 \mathrm{mg} / \mathrm{dL}$ and $>300 \mathrm{mg} / \mathrm{dL}$ and phthalate levels below the LOD were taken into account using the appropriate domain statement in SAS.

$$
* \mathrm{p}<0.05, * * \mathrm{p}<0.01 * * * \mathrm{p}<0.001
$$




\begin{tabular}{|c|c|c|c|c|c|c|c|c|c|c|c|c|}
\hline \multirow{3}{*}{ Metalloestrogen } & \multicolumn{6}{|c|}{ Geometric Mean (ng/ml) (GSE, N), Hispanic } & \multicolumn{6}{|c|}{ Geometric Mean (ng/ml) (GSE, N), Non-Hispanic White } \\
\hline & \multicolumn{3}{|c|}{ Yes } & \multicolumn{3}{|c|}{ No } & \multicolumn{3}{|c|}{ Yes } & \multicolumn{3}{|c|}{ No } \\
\hline & $\mathrm{GM}$ & GSE & $\mathrm{N}$ & $\mathrm{GM}$ & GSE & $\mathrm{N}$ & GM & GSE & $\mathrm{N}$ & $\mathrm{GM}$ & GSE & $\mathrm{N}$ \\
\hline Cadmium & 0.4055 & 0.03021 & 48 & 0.3357 & 0.02963 & 204 & 0.3443 & 0.04775 & 112 & 0.3266 & 0.01149 & 524 \\
\hline Manganese & 0.1395 & 0.02234 & 26 & 0.1647 & 0.01718 & 93 & 0.2728 & 0.0661 & 53 & 0.2006 & 0.01459 & 220 \\
\hline Arsenic & 10.184 & 2.3493 & 34 & 8.9909 & 0.933 & 136 & 8.1171 & 1.1886 & 71 & 9.2188 & 0.8607 & 338 \\
\hline \multirow{3}{*}{ Metalloestrogen } & \multicolumn{6}{|c|}{ Geometric Mean (ng/ml) (GSE, N), Non-Hispanic Black } & \multicolumn{6}{|c|}{ Geometric Mean (ng/ml) (GSE, N), Asian/Other } \\
\hline & \multicolumn{3}{|c|}{ Yes } & \multicolumn{3}{|c|}{ No } & \multicolumn{3}{|c|}{ Yes } & \multicolumn{3}{|c|}{ No } \\
\hline & GM & GSE & $\mathrm{N}$ & GM & GSE & $\mathrm{N}$ & GM & GSE & $\mathrm{N}$ & GM & GSE & $\mathrm{N}$ \\
\hline Cadmium & 0.2827 & 0.02211 & 52 & $0.3517 *$ & 0.0197 & 278 & 0.7948 & 0.1396 & 24 & 0.5701 & 0.05043 & \\
\hline Manganese & 0.1073 & 0.02575 & 16 & $0.1822 *$ & 0.0211 & 122 & 0.2053 & 0.02623 & 17 & 0.1981 & 0.02566 & 61 \\
\hline Arsenic & 7.3151 & 1.4456 & 35 & 8.2592 & 0.7266 & 196 & 35.9647 & 7.3555 & 16 & 31.3738 & 4.2059 & 89 \\
\hline \multicolumn{13}{|c|}{$\begin{array}{l}\text { The EEDs were creatinine-adjusted then log transformed prior to analyses. Extreme creatinine values }(<30 \mathrm{mg} / \mathrm{dL} \text { and }>300 \mathrm{mg} / \mathrm{dL} \text { and phthalate levels below the } \\
\qquad \text { LOD were taken into account using the appropriate domain statement in SAS. } \\
\qquad{ }^{*}<0.05,{ }^{*} \mathrm{p}<0.01 *^{* *} \mathrm{p}<0.001\end{array}$} \\
\hline
\end{tabular}

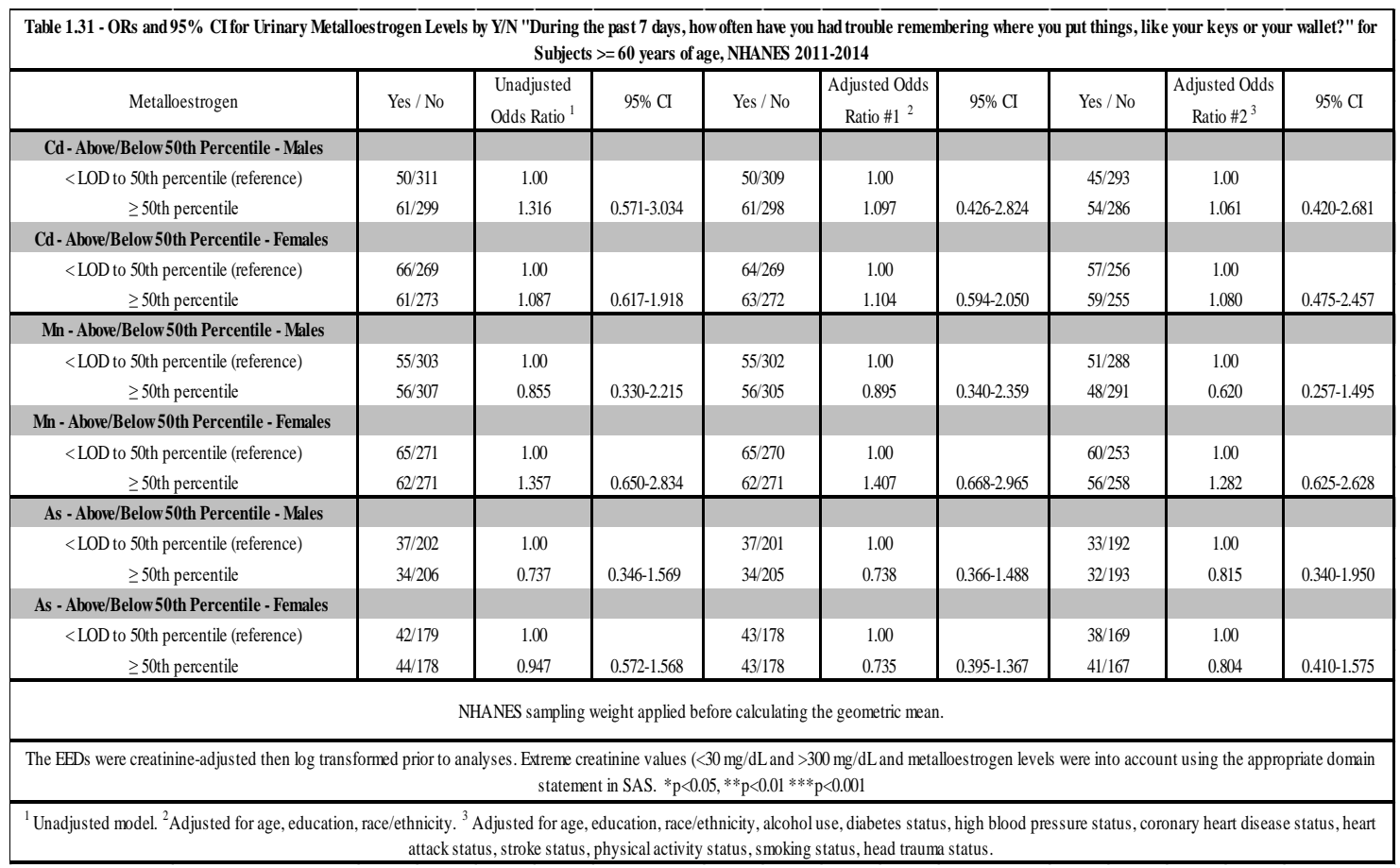

\section{Limitations Due to Difficulty Remembering or Confusion and Exposure to}

Metalloestrogens: Table 1.32 presents crude GM means for subjects who responded

to the yes/no question "Are you limited in any way because of difficulty remembering

or because you experience periods of confusion?" and who have measurable 
metalloestrogen urinary levels above the LOD. In the analysis, no significant associations were found. Table 1.33 presents age-specific geometric means divided amongst three age groups. Among all age groups, no significant associations were found. Table 1.34 presents gender-specific GM levels. Amongst males, $\mathrm{Cd}$ and $\mathrm{Mn}$ GM levels were higher amongst those who answered "yes" compared to those who answered "no". There were no significant findings in our analysis of gender-specific GM levels. Table 1.35 presents race-specific GMs by racial group. Cd GM levels were higher in those who answered "yes" compared to those who answered "no" amongst all racial groups. There were no significant findings among racial groups. Table 1.36 presents ORs and $95 \% \mathrm{CI}$ for those with Yes/No responses to the question "Are you limited in any way because of difficulty remembering or because you experience periods of confusion?" and metalloestrogens stratified by gender with two groups: $<$ LOD to 50th percentile (reference) and $\geq 50$ th percentile. In the unadjusted model, a significant association was found answering "yes" to being limited because difficulty remembering or because of periods of confusion and $\mathrm{Cd}$, among females in the $\geq 50$ th percentile group [OR=1.952, 95\% CI: 1.126-3.384]. The adjusted models were found not to be significant. Among males in the $\geq 50$ th percentile and $\mathrm{Cd}$ a significant association was observed $[\mathrm{OR}=2.075,95 \% \mathrm{CI}=1.029-4.185]$ in the first adjusted model controlling for age, education, and race/ethnicity. The second adjusted model was found to be not significant. 
Table 1.32 Geometric Mean Urinary Metalloestrogen Levels by Y/N "Are you limited in any way because of difficulty remembering or because you experience periods of confusion?" for Subjects $>=60$ years of age, NHANES $2011-2014$, over the LOD

\begin{tabular}{|c|ccc|ccc|}
\hline \multirow{2}{*}{ Metalloestrogen } & \multicolumn{4}{|c|}{ Geometric Mean (ug/ml) (GSE, N) } \\
\cline { 2 - 7 } & \multicolumn{3}{c|}{ Yes } & \multicolumn{3}{c|}{ No } \\
\cline { 2 - 7 } & GM & GSE & N & GM & GSE & N \\
\hline Cadmium & 0.3993 & 0.03364 & 217 & 0.3325 & 0.01176 & 1229 \\
Manganese & 0.2234 & 0.0431 & 106 & 0.195 & 0.007832 & 548 \\
Arsenic & 9.9504 & 1.3274 & 148 & 9.6526 & 0.7632 & 823 \\
\hline
\end{tabular}

NHANES s ampling weight applied before calculating the geometric mean.

The EEDs were creatinine-adjusted then log transformed prior to analys es. Extreme creatinine values $(<30 \mathrm{mg} / \mathrm{dL}$ and $>300 \mathrm{mg} / \mathrm{dL}$ and phthalate levels below the LOD were taken into account us ing the appropriate domain statement in SAS.

$$
* \mathrm{p}<0.05, * * \mathrm{p}<0.01 * * * \mathrm{p}<0.001
$$

\begin{tabular}{|c|c|c|c|c|c|c|}
\hline \multicolumn{7}{|c|}{$\begin{array}{l}\text { Table } 1.33 \text { Age-Specific Geometric Mean Urinary Metalloestrogen Levels by Y/N "Are you } \\
\text { limited in any way because of difficulty remembering or because you experience periods of } \\
\text { confusion?" for Subjects }>=60 \text { years of age, NHANES } 2011-2014 \text {, over the LOD }\end{array}$} \\
\hline \multirow{3}{*}{ Metalloestrogen } & \multicolumn{6}{|c|}{ Geometric Mean (ug/ml) (GSE, N), Age 60-69 } \\
\hline & \multicolumn{3}{|c|}{ Yes } & \multicolumn{3}{|c|}{ No } \\
\hline & GM & GSE & $\mathrm{N}$ & GM & GSE & $\mathrm{N}$ \\
\hline Cadmium & 0.3775 & 0.06417 & 106 & 0.3145 & 0.01879 & 657 \\
\hline Manganese & 0.3214 & 0.136 & 47 & 0.1844 & 0.01341 & 278 \\
\hline Arsenic & 9.3726 & 1.7523 & 71 & 8.7476 & 0.6515 & 439 \\
\hline \multirow{3}{*}{ Metalloestrogen } & \multicolumn{6}{|c|}{ Geometric Mean (ug/ml) (GSE, N), Age 70-79 } \\
\hline & \multicolumn{3}{|c|}{ Yes } & \multicolumn{3}{|c|}{ No } \\
\hline & GM & GSE & $\mathrm{N}$ & GM & GSE & $\mathrm{N}$ \\
\hline Cadmium & 0.3622 & 0.04967 & 50 & 0.3515 & 0.02173 & 401 \\
\hline Manganese & 0.1676 & 0.04507 & 21 & 0.2047 & 0.02122 & 184 \\
\hline Arsenic & 8.7666 & 1.9198 & 34 & 10.6219 & 1.4041 & 270 \\
\hline \multirow{3}{*}{ Metalloestrogen } & \multicolumn{6}{|c|}{ Geometric Mean (ug/ml) (GSE, N), Age 80 + } \\
\hline & \multicolumn{3}{|c|}{ Yes } & \multicolumn{3}{|c|}{ No } \\
\hline & GM & GSE & $\mathrm{N}$ & GM & GSE & $\mathrm{N}$ \\
\hline Cadmium & 0.4589 & 0.06375 & 61 & 0.366 & 0.03203 & 171 \\
\hline Manganese & 0.1891 & 0.0325 & 38 & 0.2145 & 0.01997 & 86 \\
\hline Arsenic & 11.7613 & 2.6919 & 43 & 11.4034 & 1.4413 & 114 \\
\hline \multicolumn{7}{|c|}{$\begin{array}{l}\text { NHANES s ampling weight applied before calculating the geometric mean. } \\
\text { The EEDs were creatinine-adjusted then log trans formed prior to analys es. Extreme creatinine } \\
\text { values }(<30 \mathrm{mg} / \mathrm{dL} \text { and }>300 \mathrm{mg} / \mathrm{dL} \text { and phthalate levels below the LOD were taken into account } \\
\text { using the appropriate domain statement in SAS. } \\
* \mathrm{p}<0.05, * * \mathrm{p}<0.01 * * * \mathrm{p}<0.001\end{array}$} \\
\hline
\end{tabular}

\begin{tabular}{|c|c|c|c|c|c|c|c|c|c|c|c|c|}
\hline \multirow{4}{*}{ Metalloestrogen } & \multicolumn{6}{|c|}{ Geometric Mean (ug/ml) (GSE, N) } & \multicolumn{6}{|c|}{ Geometric Mean (ug/ml) (GSE, N) } \\
\hline & \multicolumn{6}{|c|}{ Males } & \multicolumn{6}{|c|}{ Females } \\
\hline & \multicolumn{3}{|c|}{ Yes } & \multicolumn{3}{|c|}{ No } & \multicolumn{3}{|c|}{ Yes } & \multicolumn{3}{|c|}{ No } \\
\hline & GM & GSE & $\mathrm{N}$ & GM & GSE & $\mathrm{N}$ & GM & GSE & $\mathrm{N}$ & GM & GSE & $\mathrm{N}$ \\
\hline Cadmium & 0.3227 & 0.04037 & 89 & 0.2732 & 0.01181 & 651 & 0.4748 & 0.05024 & 128 & 0.4036 & 0.01925 & 528 \\
\hline Manganese & 0.1886 & 0.07547 & 38 & 0.139 & 0.009702 & 251 & 0.2502 & 0.03475 & 68 & 0.2531 & 0.02169 & 297 \\
\hline Arsenic & 8.9348 & 1.3318 & 60 & 9.0086 & 0.7082 & 437 & 10.7949 & 1.7682 & 88 & 10.3254 & 1.0542 & 386 \\
\hline The EEDs were creatinir & isted the & $\begin{array}{l}\text { NHANE } \\
\log \text { transfo } \\
\text { LOD were }\end{array}$ & $\begin{array}{l}\text { ampli } \\
\text { ed pri } \\
\text { en in }\end{array}$ & $\begin{array}{l}\text { reight ap } \\
\text { analyse } \\
\text { count us } \\
* \mathrm{p}<0.05\end{array}$ & $\begin{array}{l}\text { Extreme cre } \\
\mathrm{g} \text { the appr } \\
*_{\mathrm{p}}<0.01 * *\end{array}$ & $\begin{array}{l}\text { ulating } \\
\text { aine va } \\
\text { late do } \\
<0.001\end{array}$ & $\begin{array}{l}\text { geometr } \\
\text { s }(<30 \mathrm{mg}) \\
\text { n stateme }\end{array}$ & $\begin{array}{l}\text { mean. } \\
\text { L and >30 } \\
\text { in SAS. }\end{array}$ & $\mathrm{ng} / \mathrm{dl}$ & nd phthale & levels be & w the \\
\hline
\end{tabular}




\begin{tabular}{|c|c|c|c|c|c|c|c|c|c|c|c|c|}
\hline \multicolumn{13}{|c|}{$\begin{array}{l}\text { Table 1.35 Race-specific Geometric Mean Urinary Metalloestrogen Levels by Y/N "Are you limited in any way because of difficulty remembering or because } \\
\text { you experience periods of confusion?" for Subjects >=60 years of age, NHANES 2011-2014, over the LOD }\end{array}$} \\
\hline \multirow{3}{*}{ Metalloestrogen } & \multicolumn{6}{|c|}{ Geometric Mean (ng/ml) (GSE, N), Hispanic } & \multicolumn{6}{|c|}{ Geometric Mean (ng/ml) (GSE, N), Non-Hispanic White } \\
\hline & \multicolumn{3}{|c|}{ Yes } & \multicolumn{3}{|c|}{ No } & \multicolumn{3}{|c|}{ Yes } & \multicolumn{3}{|c|}{ No } \\
\hline & $\mathrm{GM}$ & GSE & $\mathrm{N}$ & GM & GSE & $\mathrm{N}$ & GM & GSE & $\mathrm{N}$ & GM & GSE & $\mathrm{N}$ \\
\hline Cadmium & 0.3839 & 0.04416 & 59 & 0.3466 & 0.02651 & 222 & 0.3775 & 0.04405 & 92 & 0.321 & 0.01409 & 576 \\
\hline Manganese & 0.1447 & 0.01979 & 30 & 0.1612 & 0.01221 & 104 & 0.2364 & 0.05618 & 46 & 0.2035 & 0.01078 & 247 \\
\hline Arsenic & 9.4822 & 1.1434 & 39 & 9.1964 & 1.1371 & 151 & 8.6561 & 1.5424 & 61 & 9.312 & 0.9191 & 372 \\
\hline \multirow{3}{*}{ Metalloestrogen } & \multicolumn{6}{|c|}{ Geometric Mean (ng/ml) (GSE, N), Non-Hispanic Black } & \multicolumn{6}{|c|}{ Geometric Mean (ng/ml) (GSE, N), Asian/Other } \\
\hline & \multicolumn{3}{|c|}{ Yes } & \multicolumn{3}{|c|}{ No } & \multicolumn{3}{|c|}{ Yes } & \multicolumn{3}{|c|}{ No } \\
\hline & $\mathrm{GM}$ & GSE & $\mathrm{N}$ & GM & GSE & $\mathrm{N}$ & GM & GSE & $\mathrm{N}$ & GM & GSE & $\mathrm{N}$ \\
\hline Cadmium & 0.3675 & 0.05186 & 32 & 0.3381 & 0.01684 & 309 & 0.726 & 0.1112 & 34 & 0.5625 & 0.04587 & 122 \\
\hline Manganese & 0.4235 & 0.3938 & 13 & 0.1523 & 0.007981 & 131 & 0.1873 & 0.02783 & 17 & 0.2014 & 0.02415 & 66 \\
\hline Arsenic & 12.4755 & 3.8178 & 24 & 7.7405 & 0.5951 & 214 & 26.4076 & 8.6919 & 24 & 30.8778 & 3.8751 & 86 \\
\hline \multicolumn{13}{|c|}{ NHANES sampling weight applied before calculating the geometric mean. } \\
\hline \multicolumn{13}{|c|}{$\begin{array}{l}\text { The EEDs were creatinine-adjusted then log transformed prior to analyses. Extreme creatinine values }(<30 \mathrm{mg} / \mathrm{dL} \text { and }>300 \mathrm{mg} / \mathrm{dL} \text { and phthalate levels below the } \\
\text { LOD were taken into account using the appropriate domain statement in SAS. } \\
\qquad * \mathrm{p}<0.05 *^{*} \mathrm{p}<0.01 * * * \mathrm{p}<0.001\end{array}$} \\
\hline
\end{tabular}

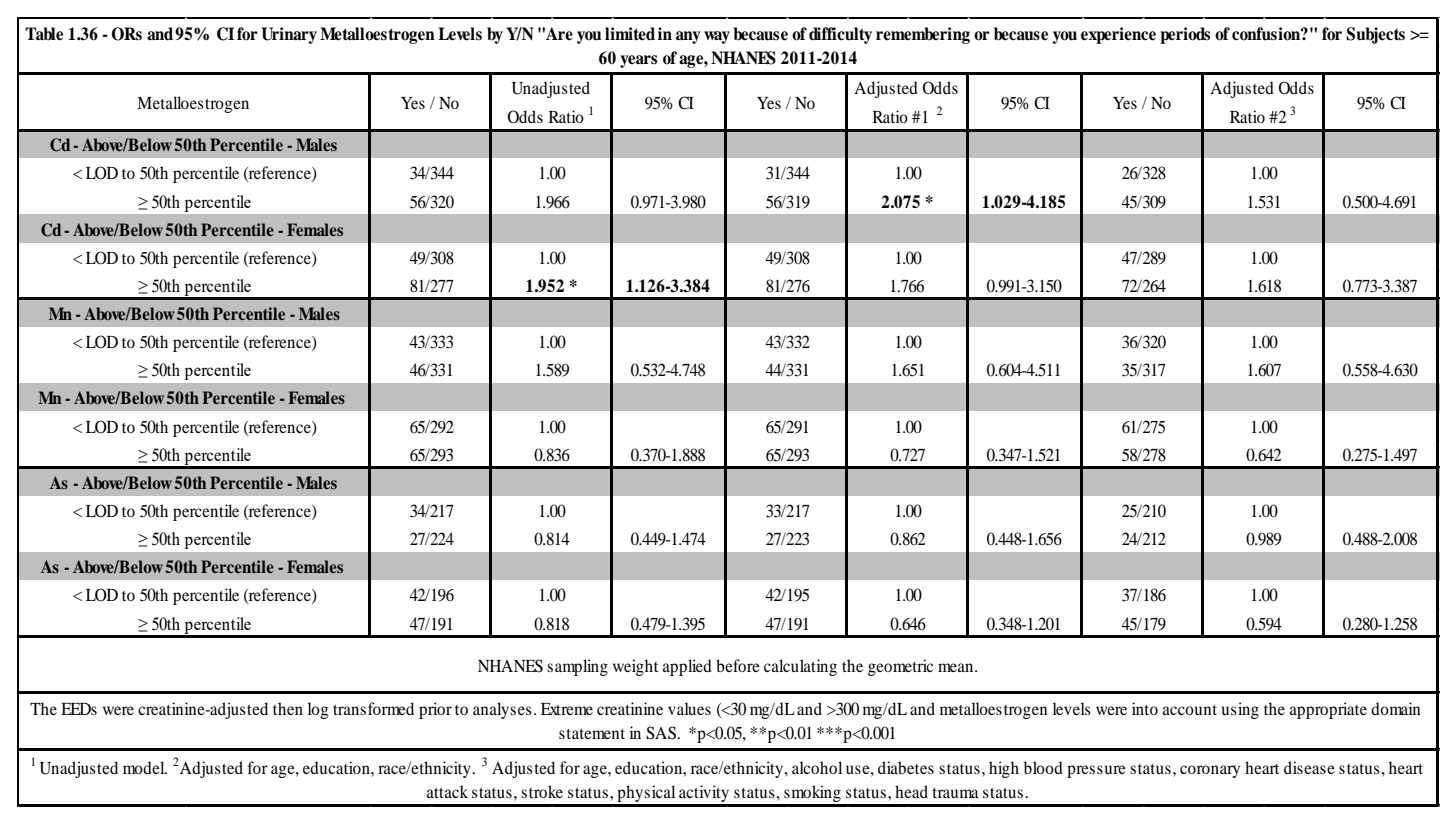

\section{Associations Between Exposures to Metalloestrogens and Taste/Smell Function}

Exposures to the metalloestrogens $\mathrm{Cd}, \mathrm{Mn}$, and As, and two indicators of taste/smell function: 1) Do you sometimes smell an unpleasant, bad, or burning odor when nothing is there (phantosmia), and 2) During the past 12 months have you had a taste or other sensation in your mouth that does not go away? Taste and smell indicators have been included as a surrogate indicator of brain health as taste and 
smell dysfunction is a possible pre-clinical indicator in the development of AD and other memory-related neurodegenerative diseases.

Smell Dysfunction and Exposure to Metalloestrogens: Table 1.37 presents crude GM means for subjects who responded to the yes/no "Do you sometimes smell an unpleasant, bad or burning odor when nothing is there?" and who have measurable metalloestrogen urinary levels above the LOD. Crude As GM levels were found to be significantly higher in those who responded "no", compared to those who responded “yes" ( $p<0.05)$. Table 1.38 presents age-specific GMs by the age groups 60-69, 7079, and 80+. Cd GM levels were significantly higher among those who responded "yes" compared to those who responded "no" in significantly higher in the $80+$ age group ( $\mathrm{p}<0.01)$. Table 1.39 presents gender-specific GMs. Among males, Mn GM levels were significantly higher among those who responded "yes" compared to those who responded "no" $(\mathrm{p}<0.01)$. Among females, Mn and As GM levels, were significantly higher for those who responded "no" compared to those who responded "yes" (Mn, p<0.05; As, $\mathrm{p}<0.01)$.

Table 1.40 presents race-specific GMs. Among Non-Hispanic Whites, As GM levels were found to be significantly higher in those that responded "no" compared to those who responded "yes" $(\mathrm{p}<0.01)$. Among Asian/Others, the Cd GM level was significantly higher among those who responded "yes" compared to those who responded "no" $(\mathrm{p}<0.001)$. The As GM level was found to be higher among those who responded "no" compared to those who responded "yes" $(p<0.05)$.

Table 1.41 presents ORs and 95\% CI for those with Yes/No responses to the question "Do you sometimes smell an unpleasant, bad or burning odor when nothing is there?" 
and metalloestrogens stratified by gender with two groups: < LOD to 50th percentile (reference) and $\geq 50$ th percentile. In the second adjusted model for males in the $\geq$ 50th percentile group, $\mathrm{Cd}$ and experiencing a phantom odor were found to be significantly associated when controlling for age, education, race/ethnicity, alcohol use, high blood pressure status, coronary heart disease status, heart attack status, stroke status, physical activity status, smoking status, head trauma status $[\mathrm{OR}=10.770,95 \% \mathrm{CI}: 2.826-41.047)$. We removed diabetes status from the final model since it contributed to a quasi-separation of data, making the model validity questionable.

\begin{tabular}{l} 
Table 1.37 Geometric Mean Urinary Metalloestrogen Levels by Y/N "Do you sometimes smell \\
an unpleasant, bad or burning odor when nothing is there?" for Subjects >= 60 years of age, \\
NHANES 2011-2014, over the LOD \\
\hline \multirow{3}{*}{ Metalloestrogen } \\
\cline { 2 - 8 }
\end{tabular}

NHANES sampling weight applied before calculating the geometric mean.

The EEDs were creatinine-adjusted then log transformed prior to analyses. Extreme creatinine values $(<30 \mathrm{mg} / \mathrm{dL}$ and $>300 \mathrm{mg} / \mathrm{dL})$ and metalloestrogen levels below the LOD were taken into account using the appropriate domain statement in SAS.

$* \mathrm{p}<0.05, * * \mathrm{p}<0.01 * * * \mathrm{p}<0.001$ 


\begin{tabular}{|c|c|c|c|c|c|c|}
\hline \multicolumn{7}{|c|}{$\begin{array}{l}\text { Table 1.38 Age-Specific Geometric Mean Urinary Metalloestrogen Levels by Y/N "Do you } \\
\text { sometimes smell an unpleasant, bad or burning odor when nothing is there?" for Subjects >= } 60 \\
\text { years of age, NHANES 2011-2014, over the LOD }\end{array}$} \\
\hline \multirow{3}{*}{ Metalloestrogen } & \multicolumn{6}{|c|}{ Geometric Mean (ug/ml) (GSE, N), Age 60-69 } \\
\hline & \multicolumn{3}{|c|}{ Yes } & \multicolumn{3}{|c|}{ No } \\
\hline & GM & GSE & $\mathrm{N}$ & GM & GSE & $\mathrm{N}$ \\
\hline Cadmium & 0.3729 & 0.0549 & 52 & 0.3169 & 0.01929 & 708 \\
\hline Manganese & 0.1891 & 0.02418 & 30 & 0.1915 & 0.01659 & 292 \\
\hline Arsenic & 6.2777 & 1.2596 & 39 & 8.9926 & 0.6798 & 469 \\
\hline \multirow{3}{*}{ Metalloestrogen } & \multicolumn{6}{|c|}{ Geometric Mean (ug/ml) (GSE, N), Age 70-79 } \\
\hline & \multicolumn{3}{|c|}{ Yes } & \multicolumn{3}{|c|}{ No } \\
\hline & GM & GSE & $\mathrm{N}$ & GM & GSE & $\mathrm{N}$ \\
\hline Cadmium & 0.2864 & 0.09536 & 11 & 0.3542 & 0.02177 & 440 \\
\hline Manganese & 0.1775 & 0.03617 & 6 & 0.2018 & 0.02004 & 199 \\
\hline Arsenic & 8.0468 & 1.9187 & 10 & 10.4974 & 1.2967 & 294 \\
\hline \multirow{3}{*}{ Metalloestrogen } & \multicolumn{6}{|c|}{ Geometric Mean (ug/ml) (GSE, N), Age $80+$} \\
\hline & \multicolumn{3}{|c|}{ Yes } & \multicolumn{3}{|c|}{ No } \\
\hline & GM & GSE & $\mathrm{N}$ & GM & GSE & $\mathrm{N}$ \\
\hline Cadmium & $0.4082 * *$ & 0.06266 & 4 & 0.3892 & 0.02754 & 228 \\
\hline Manganese & 0.2049 & 0.008173 & 2 & 0.2061 & 0.0157 & 122 \\
\hline Arsenic & 6.9858 & 2.1654 & 4 & 11.6256 & 1.4704 & 153 \\
\hline \multicolumn{7}{|c|}{ NHANES sampling weight applied before calculating the geometric mean. } \\
\hline \multicolumn{7}{|c|}{$\begin{array}{l}\text { The EEDs were creatinine-adjusted then log transformed prior to analyses. Extreme creatinine } \\
\text { values }(<30 \mathrm{mg} / \mathrm{dL} \text { and }>300 \mathrm{mg} / \mathrm{dL}) \text { and metalloestrogen levels below the LOD were taken into } \\
\qquad \begin{array}{l}\text { account using the appropriate domain statement in SAS. } \\
\qquad * \mathrm{p}<0.05, * * \mathrm{p}<0.01 * * * \mathrm{p}<0.001\end{array}\end{array}$} \\
\hline
\end{tabular}

Table 1.39 Gender-Specific Geometric Mean Urinary Metalloestrogen Levels by Y/N "Do you sometimes smell an unpleasant, bad or burning odor when nothing is there?" for Subjects >= 60 years of age, NHANES 2011-2014, over the LOD

\begin{tabular}{|c|c|c|c|c|c|c|c|c|c|c|c|c|}
\hline \multirow{4}{*}{ Metalloestrogen } & \multicolumn{6}{|c|}{ Geometric Mean (ug/ml) (GSE, N) } & \multicolumn{6}{|c|}{ Geometric Mean (ug/ml) (GSE, N) } \\
\hline & \multicolumn{6}{|c|}{ Males } & \multicolumn{6}{|c|}{ Females } \\
\hline & \multicolumn{3}{|c|}{ Yes } & \multicolumn{3}{|c|}{ No } & \multicolumn{3}{|c|}{ Yes } & \multicolumn{3}{|c|}{$\mathrm{N}_{0}$} \\
\hline & GM & GSE & $\mathrm{N}$ & GM & GSE & $\mathrm{N}$ & $\mathrm{GM}$ & GSE & $\mathrm{N}$ & $\mathrm{GM}$ & GSE & $\mathrm{N}$ \\
\hline Cadmium & 0.2224 & 0.02978 & 27 & 0.2776 & 0.01239 & 712 & 0.4132 & 0.08311 & 40 & 0.4129 & 0.01711 & 664 \\
\hline Manganese & $0.2258 * *$ & 0.02295 & 8 & 0.1425 & 0.01176 & 280 & 0.1794 & 0.02282 & 30 & $0.2559 *$ & 0.01982 & 333 \\
\hline Arsenic & 6.1009 & 1.5527 & 22 & 9.1335 & 0.6863 & 474 & 7.0641 & 1.0455 & 31 & $10.5977 * *$ & 1.0416 & 442 \\
\hline \multicolumn{13}{|c|}{ NHANES sampling weight applied before calculating the geometric mean. } \\
\hline \multicolumn{13}{|c|}{$\begin{array}{l}\text { The EEDs were creatinine-adjusted then log transformed prior to analyses. Extreme creatinine values }(<30 \mathrm{mg} / \mathrm{dL} \text { and }>300 \mathrm{mg} / \mathrm{dL}) \text { and metalloestrogen levels below } \\
\qquad \text { the LOD were taken into account using the appropriate domain statement in } S A S \text {. }\end{array}$} \\
\hline
\end{tabular}


Taste Dysfunction and Exposure to Metalloestrogens: Table 1.42 presents crude GM means for subjects who responded to the yes/no question "During the past 12 months have you had a taste or other sensation in your mouth that does not go away?" and who have measurable metalloestrogen urinary levels above the LOD. No significant increases were observed.

Table 1.43 presents age-specific GMs for the age groups 60-69, 70-79, and 80+. In the 79-79-year age group, the GM for As are higher in among subjects who answered "no" compared to those that answered "yes" $(\mathrm{p}<0.05)$. Table 1.44 presents genderspecific GMs. Among males, the GM for Cd was significantly higher among those that answered "yes" compared to those that answered "no" ( $p<0.01)$. Among females, the GM for Mn were found to be significantly higher among those who answered "no" compared to those that answered "yes" $(\mathrm{p}<0.01)$. Table 1.45 presents racespecific GMs. No significant results were observed among racial groups.

Table 1.46 presents ORs and 95\% CI for those with Yes/No responses to the question "During the past 12 months have you had a taste or other sensation in your mouth that does not go away?" and metalloestrogens stratified by gender with two groups: $<$ LOD to 50 th percentile (reference) and $\geq 50$ th percentile. For males in the $\geq$ 50th percentile group, $\mathrm{Cd}$ was found to be significantly associated with taste issues in the first adjusted model controlling for age, education, race/ethnicity [OR=4.444, 95\% CI: 1.564-12.630], and the second adjusted model controlling for age, education, race/ethnicity, alcohol use, high blood pressure status, coronary heart disease status, heart attack status, stroke status, physical activity status, smoking status, head trauma status $[\mathrm{OR}=3.751,95 \% \mathrm{CI}: 1.103-12.763]$. We removed diabetes status from the final 
model since it contributed to a quasi-separation of data, making the model validity questionable.

Table 1.42 Geometric Mean Urinary Metalloestrogen Levels by $\mathrm{Y} / \mathrm{N}$ "During the past 12 months have you had a taste or other sensation in your mouth that does not go away?" for Subjects $>=60$ years of age, NHANES 2011-2014, over the LOD

\begin{tabular}{|c|ccc|ccc|}
\hline \multirow{2}{*}{ Metalloestrogen } & \multicolumn{5}{|c|}{ Geometric Mean (ug/ml) (GSE, N) } \\
\cline { 2 - 7 } & \multicolumn{4}{|c|}{ Yes } & \multicolumn{4}{c|}{ No } \\
\cline { 2 - 7 } & GM & GSE & N & GM & GSE & N \\
\hline Cadmium & 0.4332 & 0.0528 & 91 & 0.3352 & 0.01164 & 1354 \\
Manganese & 0.1722 & 0.01624 & 45 & 0.2002 & 0.009317 & 608 \\
Arsenic & 7.6795 & 1.2809 & 62 & 9.8023 & 0.7702 & 908 \\
\hline NHANES sampling weight applied before calculating the geometric mean. \\
The EEDs were creatinine-adjusted then log transformed prior to analyses. Extreme creatinine \\
values (<30 mg/dL and $>300 \mathrm{mg} / \mathrm{dL}$ ) and metalloestrogen levels below the LOD were taken into \\
account using the appropriate domain statement in SAS. \\
$* \mathrm{p}<0.05, * * \mathrm{p}<0.01 * * * \mathrm{p}<0.001$ \\
\hline
\end{tabular}




\begin{tabular}{|c|c|c|c|c|c|c|}
\hline & \multicolumn{6}{|c|}{ Geometric Mean (ug/ml) (GSE, N), Age 60-69 } \\
\hline \multirow[t]{2}{*}{ Metalloestrogen } & \multicolumn{3}{|c|}{ Yes } & \multicolumn{3}{|c|}{ No } \\
\hline & GM & GSE & $\mathrm{N}$ & GM & GSE & $\mathrm{N}$ \\
\hline Cadmium & 0.3994 & 0.05737 & 55 & 0.315 & 0.01875 & 707 \\
\hline Manganese & 0.16 & 0.01746 & 32 & 0.1977 & 0.01679 & 292 \\
\hline Arsenic & 7.6542 & 1.8706 & 38 & 8.8675 & 0.6761 & 471 \\
\hline \multirow{3}{*}{ Metalloestrogen } & \multicolumn{6}{|c|}{ Geometric Mean (ug/ml) (GSE, N), Age 70-79 } \\
\hline & \multicolumn{3}{|c|}{ Yes } & \multicolumn{3}{|c|}{ No } \\
\hline & GM & GSE & $\mathrm{N}$ & GM & GSE & $\mathrm{N}$ \\
\hline Cadmium & 0.5432 & 0.1454 & 24 & 0.3457 & 0.02076 & 427 \\
\hline Manganese & 0.1788 & 0.04444 & 6 & 0.2019 & 0.02024 & 199 \\
\hline Arsenic & 7.0141 & 0.7715 & 15 & $10.588 *$ & 1.3343 & 289 \\
\hline \multirow{3}{*}{ Metalloestrogen } & \multicolumn{6}{|c|}{ Geometric Mean (ug/ml) (GSE, N), Age $80+$} \\
\hline & \multicolumn{3}{|c|}{ Yes } & \multicolumn{3}{|c|}{ No } \\
\hline & GM & GSE & $\mathrm{N}$ & GM & GSE & $\mathrm{N}$ \\
\hline Cadmium & 0.3911 & 0.08895 & 12 & 0.3894 & 0.02822 & 220 \\
\hline Manganese & 0.2505 & 0.06654 & 7 & 0.2044 & 0.015 & 117 \\
\hline Arsenic & 9.117 & 3.3521 & 9 & 11.637 & 1.4675 & 148 \\
\hline
\end{tabular}

NHANES sampling weight applied before calculating the geometric mean.

The EEDs were creatinine-adjusted then log transformed prior to analyses. Extreme creatinine values $(<30 \mathrm{mg} / \mathrm{dL}$ and $>300 \mathrm{mg} / \mathrm{dL})$ and metalloestrogen levels below the LOD were taken into account using the appropriate domain statement in SAS.

$* \mathrm{p}<0.05, * * \mathrm{p}<0.01 * * * \mathrm{p}<0.001$

\begin{tabular}{|c|c|c|c|c|c|c|c|c|c|c|c|c|}
\hline \multirow{4}{*}{ Metalloestrogen } & \multicolumn{6}{|c|}{ Geometric Mean (ug/ml) (GSE, N) } & \multicolumn{6}{|c|}{ Geometric Mean (ug/ml) (GSE, N) } \\
\hline & \multicolumn{6}{|c|}{ Males } & \multicolumn{6}{|c|}{ Females } \\
\hline & \multicolumn{3}{|c|}{ Yes } & \multicolumn{3}{|c|}{ No } & \multicolumn{3}{|c|}{ Yes } & \multicolumn{3}{|c|}{ No } \\
\hline & GM & GSE & $\mathrm{N}$ & GM & GSE & $\mathrm{N}$ & GM & GSE & $\mathrm{N}$ & $\mathrm{GM}$ & GSE & $\mathrm{N}$ \\
\hline Cadmium & $0.4285 * *$ & 0.07048 & 39 & 0.2727 & 0.0117 & 701 & 0.4367 & 0.07316 & 52 & 0.4103 & 0.01848 & 653 \\
\hline Manganese & 0.1915 & 0.06236 & 10 & 0.1436 & 0.01172 & 279 & 0.1699 & 0.01784 & 35 & $0.2627 * *$ & 0.0199 & 329 \\
\hline Arsenic & 8.2886 & 2.3636 & 26 & 9.0337 & 0.6802 & 471 & 7.2922 & 1.3284 & 36 & 10.6049 & 1.05 & 437 \\
\hline The EEDs were creati & $\begin{array}{r}\text { adjusted the } \\
\text { belo }\end{array}$ & $\begin{array}{l}\text { NHANE } \\
\text { an log trans } \\
\text { w the LOD }\end{array}$ & sampl & $\begin{array}{l}\text { weight ap } \\
\text { to analys } \\
\text { into acco } \\
{ }^{*} \mathrm{p}<0.05\end{array}$ & $\begin{array}{l}\text { lied before } \\
\text { s. Extreme } \\
\text { t using the } \\
* x<0.01 *\end{array}$ & $\begin{array}{l}\text { lculatir } \\
\text { atinine } \\
\text { ppropr } \\
p<0.00\end{array}$ & $\begin{array}{l}\text { le geomet } \\
\text { ues ( }<301 \\
\text { domain } \mathrm{s}\end{array}$ & $\begin{array}{l}\text { mean. } \\
\text { ement in and }>\end{array}$ & & ) and metall & estrogen 1 & \\
\hline
\end{tabular}




\begin{tabular}{|c|c|c|c|c|c|c|c|c|c|c|c|c|}
\hline \multirow{3}{*}{ Metalloestrogen } & \multicolumn{6}{|c|}{ Geometric Mean ( $\mathrm{ng} / \mathrm{ml})(\mathrm{GSE}, \mathrm{N})$, Hispanic } & \multicolumn{6}{|c|}{ Geometric Mean (ng/ml) (GSE, N), Non-Hispanic White } \\
\hline & \multicolumn{3}{|c|}{ Yes } & \multicolumn{3}{|c|}{ No } & \multicolumn{3}{|c|}{ Yes } & \multicolumn{3}{|c|}{ No } \\
\hline & GM & GSE & $\mathrm{N}$ & GM & GSE & $\mathrm{N}$ & GM & GSE & $\mathrm{N}$ & GM & GSE & $\mathrm{N}$ \\
\hline Cadmium & 0.4094 & 0.08975 & 30 & 0.348 & 0.02671 & 251 & 0.4425 & 0.07468 & 32 & 0.3221 & 0.01426 & 636 \\
\hline Manganese & 0.1252 & 0.01799 & 19 & 0.1625 & 0.0133 & 115 & 0.2037 & 0.02269 & 17 & 0.2073 & 0.01209 & 276 \\
\hline Arsenic & 10.1299 & 2.0136 & 20 & 9.1571 & 1.1248 & 170 & 6.4749 & 1.3872 & 22 & 9.3922 & 0.9167 & 411 \\
\hline \multirow{3}{*}{ Metalloestrogen } & \multicolumn{6}{|c|}{ Geometric Mean (ng/ml) (GSE, N), Non-Hispanic Black } & \multicolumn{6}{|c|}{ Geometric Mean (ng/ml) (GSE, N), Asian/Other } \\
\hline & \multicolumn{3}{|c|}{ Yes } & \multicolumn{3}{|c|}{ No } & \multicolumn{3}{|c|}{ Yes } & \multicolumn{3}{|c|}{ No } \\
\hline & $\mathrm{GM}$ & GSE & $\mathrm{N}$ & GM & GSE & $\mathrm{N}$ & GM & GSE & $\mathrm{N}$ & $\mathrm{GM}$ & GSE & $\mathrm{N}$ \\
\hline Cadmium & 0.345 & 0.08018 & 20 & 0.3404 & 0.01799 & 321 & 0.7206 & 0.1684 & 9 & 0.5826 & 0.04734 & 146 \\
\hline Manganese & 0.07289 & 0.01199 & 4 & 0.1739 & 0.0203 & 140 & 0.1949 & 0.02197 & 5 & 0.2009 & 0.02247 & 77 \\
\hline Arsenic & 7.2645 & 2.7402 & 13 & 8.1729 & 0.7111 & 225 & 35.2148 & 16.9838 & 7 & 29.2516 & 4.2144 & 102 \\
\hline \multicolumn{13}{|c|}{$\begin{array}{l}\text { The EEDs were creatinine-adjusted then log transformed prior to analyses. Extreme creatinine values ( }<30 \mathrm{mg} / \mathrm{dL} \text { and }>300 \mathrm{mg} / \mathrm{dL}) \text { and metalloestrogen levels } \\
\qquad \text { below the LOD were taken into account using the appropriate domain statement in SAS. }\end{array}$} \\
\hline \multicolumn{13}{|c|}{${ }^{*} \mathrm{p}<0.05,{ }^{* *} \mathrm{p}<0.01 * * * \mathrm{p}<0.001$} \\
\hline
\end{tabular}

\begin{tabular}{|c|c|c|c|c|c|c|c|c|c|}
\hline Metalloestrogen & Yes / No & \begin{tabular}{|c|}
$\begin{array}{c}\text { Unadjusted Odds } \\
\text { Ratio }^{1}\end{array}$ \\
\end{tabular} & $95 \% \mathrm{CI}$ & Yes / No & $\begin{array}{c}\text { Adjusted Odds } \\
\text { Ratio } \# 1^{2}\end{array}$ & $95 \% \mathrm{CI}$ & Yes / No & $\begin{array}{l}\text { Adjusted Odds } \\
\text { Ratio }=2^{3}\end{array}$ & $95 \% \mathrm{CI}$ \\
\hline \multicolumn{10}{|l|}{ Cd-Above/Below 50th Percentile - Males } \\
\hline$<$ LOD to 50th percentile (reference) & $15 / 362$ & 1.00 & & $13 / 362$ & 1.00 & & $11 / 343$ & 1.00 & \\
\hline$\geq 50$ th percentile & $26 / 350$ & 1.871 & $0.547-6.404$ & $28 / 347$ & $4.444^{* *}$ & $1.564-12.630$ & $27 / 327$ & $3.751^{*}$ & $1.103-12.763$ \\
\hline \multicolumn{10}{|l|}{ Cd-Above/Below 50th Percentile - Females } \\
\hline$<$ LOD to 50 th percentile (reference) & $23 / 334$ & 1.00 & & $23 / 333$ & 1.00 & & $22 / 314$ & 1.00 & \\
\hline$\geq 50$ th percentile & $29 / 328$ & 1.214 & $0.483-3.051$ & $29 / 328$ & 1.257 & $0.521-3.033$ & $28 / 307$ & 1.786 & $0.679-4.696$ \\
\hline \multicolumn{10}{|l|}{ Mn - Above/Below 50th Percentile - Males } \\
\hline$<$ LOD to S0th percentile (reference) & $19 / 357$ & 1.00 & & 19/356 & 1.00 & & $19 / 337$ & 1.00 & \\
\hline$\geq 50$ th percentile & $22 / 355$ & 2.458 & $0.691-8.735$ & $22 / 353$ & 2.478 & $0.800-7.675$ & 19/333 & 1.532 & $0.511-4.587$ \\
\hline \multicolumn{10}{|l|}{ Mn - Above/Below 50th Percentile - Females } \\
\hline$<$ LOD to 50 th percentile (reference) & $32 / 324$ & 1.00 & & $32 / 323$ & 1.00 & & $30 / 305$ & 1.00 & \\
\hline$\geq 50$ th percentile & $20 / 338$ & 0.657 & $0.244-1.767$ & $20 / 338$ & 0.670 & $0.230-1.954$ & $20 / 316$ & 0.855 & $0.356-2.056$ \\
\hline \multicolumn{10}{|l|}{ As - Above/Below 50th Percentile - Males } \\
\hline < LOD to 50th percentile (reference) & $15 / 236$ & 1.00 & & $15 / 235$ & 1.00 & & $15 / 220$ & 1.00 & \\
\hline$\geq$ S0th percentile & 12239 & 0.832 & $0.245-2.828$ & $12 / 238$ & 0.877 & $0.236-3.257$ & $9 / 227$ & 0.790 & $0.194-3.223$ \\
\hline \multicolumn{10}{|l|}{ As - Above/Below 50th Percentile - Females } \\
\hline$<$ LOD to 50th percentile (reference) & $24 / 213$ & 1.00 & & $24 / 213$ & 1.00 & & $24 / 199$ & 1.00 & \\
\hline$\geq 50$ th percentile & 12226 & 0.396 & $0.137-1.145$ & $12 / 225$ & 0.410 & $0.141-1.195$ & $10 / 213$ & 0.428 & $0.099-1.851$ \\
\hline \multicolumn{10}{|c|}{ 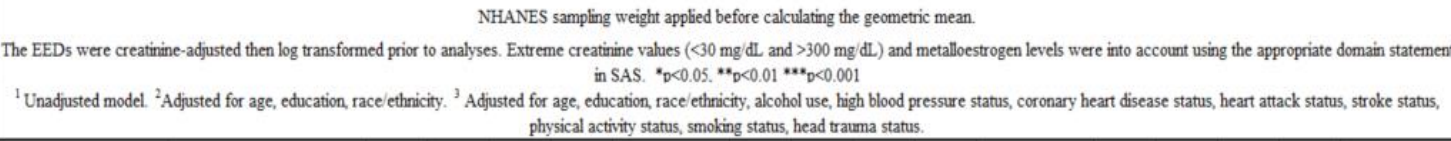 } \\
\hline
\end{tabular}

\section{DISCUSSION}

This study takes a novel approach in the assessment of exposure to metalloestrogens in the development of cognitive dysfunction and possible neurodegenerative disease using surrogate indicators of brain health. The study examined an older geriatric-aged population of US adults 60 years of age and older. We first assessed the bioburden of the urinary metalloestrogens, Cd, Mn, and As, by 
calculating and comparing the GMs of each EEDCs versus each surrogate indicator of brain health, looking at age, gender, and race and subsequently used ORs and 95\% CI to determine the risk of developing cognitive dysfunction.

Major Findings: Overall, we observed a higher bioburden of Cd in the GMs of subjects who scored lower on the four cognitive tests, who answered "yes" to having memory function issues on the four memory function surrogates and had taste/smell deficits on our taste/smell function surrogates (Tables 1.2, 1.7, 1.12, 1.17, 1.22, 1.27, $1.32,1.37$, and 1.47). In our analyses of GMs by age, gender, and race, we accounted for subjects that have extreme creatinine measurements and examined subjects who had Cd, Mn, and As levels over the LOD. A higher bioburden of Cd was observed according to gender (Tables 1.3, 1.9, 1.14, 1.19, 1.24, 1.29, 1.34, 1.39, 1.33, 1.48 and 1.49). This trend was higher in females, but the significant differences were observed in males (Tables 1.48 and 1.49). No meaningful trends were observed by age and race, although it was observed metalloestrogen bioburden increased with age. A higher bioburden of Mn was found in those who scored lower on cognitive tests, while bioburden trends for As were inconclusive (Tables, 1.2, 1.7, 1.12, and 1.17). In our logistic regression models, when controlling for all, known and suspected covariates of $\mathrm{AD}, \mathrm{Cd}$ levels in females were found to be significantly associated with worsening memory over the past 12 months (Table 1.26). Cd levels in females were also associated with adverse taste and smell functioning when controlling for all known and suspected covariates of AD (Table 1.41 and 1.46). Caution must be taken in interpreting the results of taste and smell dysfunction 
because of the large confidence interval and smaller number of causes with the covariates of the model.

The findings with cadmium and adverse brain function are consistent with other studies. A recent meta-analysis found higher Cd bioburden in AD patients ${ }^{84}$. Another study using NHANES survey data from 1988-1994 and 1999-2006, linked AD mortality with elevated $\mathrm{Cd}$ levels ${ }^{85}$. Higher circulating $\mathrm{Cd}$ levels were also associated with reduce attention and perception scores in a cross-sectional study of US adults ${ }^{86}$ and in a cohort of rural elderly Chinese person, increased serum cadmium was associated with lower composite cognitive scores ${ }^{5}$. Mn, although not significant, was still found in higher levels among those who experience memory issues and/or scored lower on cognitive exams. Since metalloestrogens can affect sensitive populations differently, can bioaccumulate, and may exhibit non-monotonic dose responses ${ }^{87}$, this may provide some basis for Mn to have higher bioburdens in subjects with memory issues.

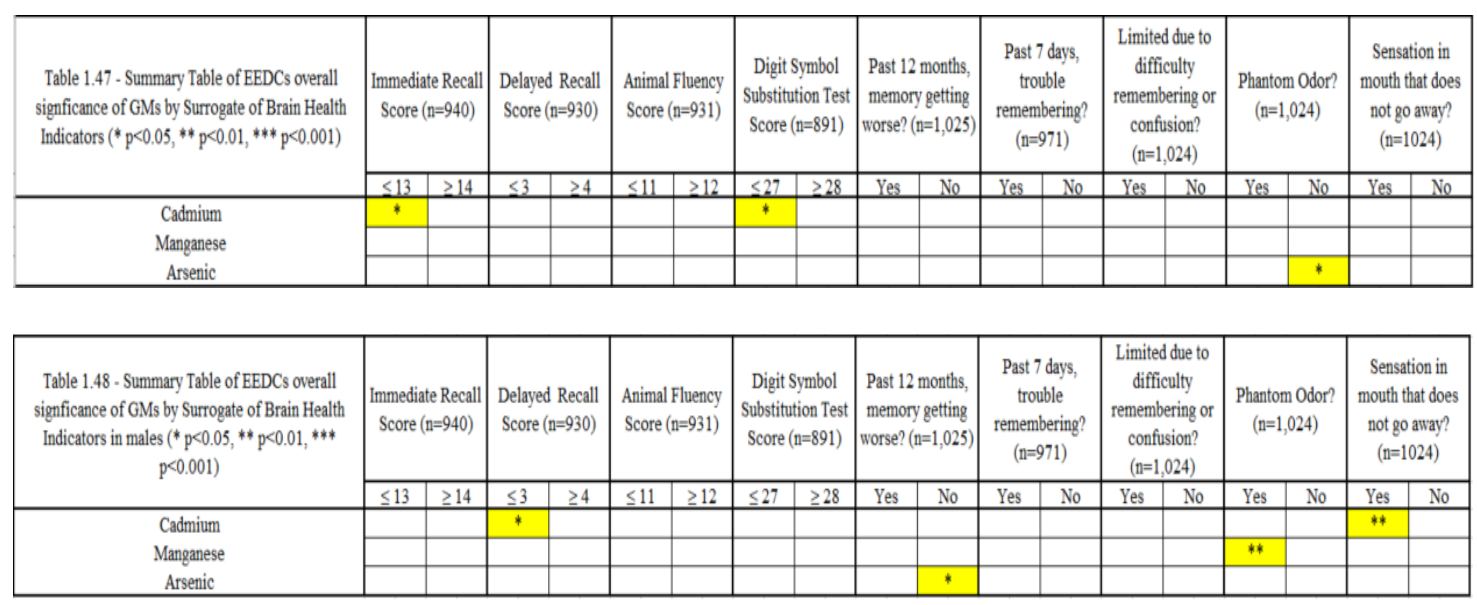




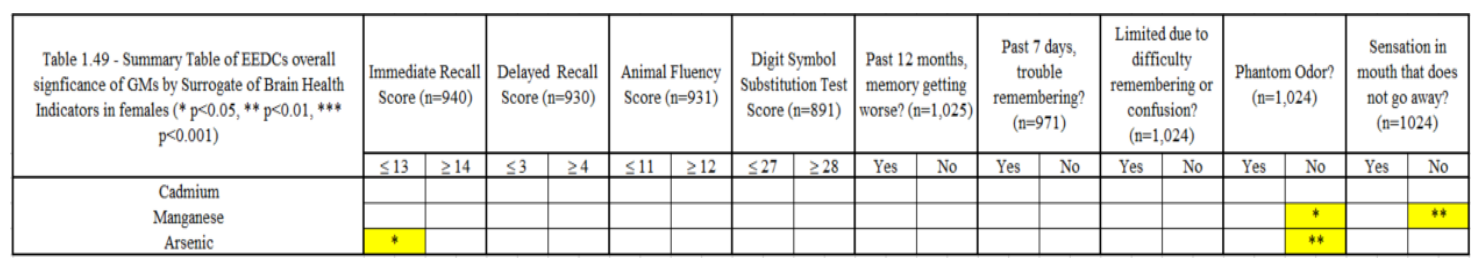

Biological Mechanisms and Brain Health: Our results show the effects of $\mathrm{Cd}$ exposure and its role in cognitive dysfunction. Numerous biological effects and mechanisms can result in cognitive dysfunction. Cadmium, arsenic, and manganese have been found to all have estrogenic activity and affinity for estrogen receptors ${ }^{27}$ and have been found to interact with estrogen-responsive genes implicated in various neurodegenerative disorders ${ }^{28}$ Heavy metals have also been demonstrated to affect and cross the blood brain barrier ${ }^{29-31}$.

Cell models have shown cadmium to have adverse effects on brain health. In a one study hippocampal CA1 neurons, Cd was demonstrated to affect synaptic transmission and short-term neural plasticity ${ }^{88}$. Another studied that used PC12 and SH-SY5Y neuronal cells, cadmium induced cell death and apoptosis ${ }^{89,90}$.

Animal models have shown cadmium to affect brain health. In a zebrafish model, cadmium inhibited neurogenesis in embryonic development ${ }^{91}$. Cadmium has been shown to induce apoptosis rat cerebellum cortical neurons by disrupting calcium homeostasis ${ }^{92}$ and also has been shown to induce apoptosis in vitro in through damage of mitochondria in rat oligodendrocytes ${ }^{93}$. Cadmium has also been shown to interact with beta amyloid peptides which is implicated in the development of $\mathrm{AD}{ }^{94}$.

Animal studies in rats have shown arsenic exposure to affect synaptic plasticity, by affecting the expression of NDMA receptors ${ }^{95,96}$ and downregulating the PTEN-AktCreb signaling pathway and damaging cerebral neurons ${ }^{97}$. In one cell study, arsenic 
was found to cause enhanced oxidative stress and cell death in cultured neuronal cells, when administered with dopamine ${ }^{98}$. In a study using a cholingeric neuronal cell line overexpressing amyloid precursor protein (APP) and exposing it to sodium arsenite and its' metabolite, dimethylarsenic acid, it was found to affect cleavage of APP and increase its' production ${ }^{99}$.

Evidence also suggests manganese having a role in neurotoxicity. Chronic manganese exposure has been shown to promote the build-up of the metal in the basal ganglia, white matter, and cortical structures of the brain ${ }^{100}$. Animal studies have shown manganese has also been shown to cause an inhibitory effect on NMDA receptors 101. Manganese appears to interfere with dopaminergic synaptic transmission, by possibly impairing presynaptic dopamine release. A study using a monkey model showed manganese exposure caused neurotoxicity by inhibiting dopamine neurotransmission ${ }^{102}$.

Furthermore, genes that have been implicated in the development of neurodegenerative disease such as $\mathrm{AD}$ which are also estrogen-responsive are also pathway for $\mathrm{Cd}, \mathrm{Mn}$, and As to exert their effects on the aging brain. Genes that are estrogen-responsive, metalloestrogen-responsive, and interfere with mitochondrial energetics, and implicated in AD include ENO1 ${ }^{28}$. Additionally, a recent search of the Comparative Toxicogenomic Database (CTD) reveals that from 88 genes implicated in AD, 39 of those genes are estrogen and Cd-responsive, 30 are estrogen and As-responsive, and 23 are estrogen and Mn-responsive ${ }^{103}$. These indicate other targets where metalloestrogens can exert their effects in the $\mathrm{AD}$ and neurodegenerative disease 
pathways. The results from our study can originate from any of the biological mechanisms that have been referenced.

Strengths and Limitations: There are strengths and limitations to our study. The largest limitations are the cross-sectional design of our study. The data is selfreported which makes inference difficult and prone to misclassification bias. Our outcome variables are surrogates rather than actual clinical endpoints, which limits our ability to say that our outcome will lead to neurodegenerative disease. The cut-off scores of our cognitive exams were based on previous studies, however, performance on those exams are heavily dependent on education, so there is a possibility that poor performance might be a function of lack of education. For our taste/smell indicators, the senses heavily affected by numerous confounders, some of which were not feasible in study due to making the sample smaller, or the confounder not being available. Strengths of our study include the novelty of such an analysis to be conducted on an older population. We had relatively large sample sizes available for our analyzes. The generalizability of our study to the US population and the large amount of chemical measurements available gives the study the ability to examine different aspects of chemical exposure.

\section{CONCLUSION}

Based on our findings, $\mathrm{Cd}$, and possibly Mn play a role in the development of neurodegenerative conditions in an older population. With an ever-increasing aging population, the study provides some insight in how metalloestrogen exposure can affect this sensitive population. 
Further research is need, particularly human epidemiological studies examining older populations, to shed more light into the development of neurodegenerative disease and exposures to metalloestrogens.

\section{References}

1. Bergman A, Heindel JJ, Jobling S, Kidd KA, Zoeller TR. State of the science of endocrine disrupting chemicals. World Heal Organ United Nations Environ Program. 2012:1-38. http://apps.who.int/iris/handle/10665/78102.

2. Alzheimer's Association. 2017 Alzheimer's Disease Facts and Figures. Alzheimers Dement . 2017;13:325-373. doi:10.1016/j.jalz.2017.02.001.

3. Agency for Toxic Substances and Disease Registry (ATSDR). Toxicological Profile for Cadmium. Agency Toxic Subst Dis Regist. 2012;(September). http://www.atsdr.cdc.gov/toxprofiles/tp5.pdf\%5Cnhttp://www.ncbi.nlm.nih.go v/books/NBK158845/.

4. Ciesielski T, Bellinger DC, Schwartz J, Hauser R, Wright RO. Associations between cadmium exposure and neurocognitive test scores in a cross-sectional study of US adults. Environ Health. 2013;12(1):13. doi:10.1186/1476-069X12-13.

5. Gao S, Jin Y, Unverzagt FW, et al. Trace element levels and cognitive function in rural elderly Chinese. Journals Gerontol Ser A-Biological Sci Med Sci. 2008;63(6):635-641.

http://shibboleth.ovid.com/secure/?T=JS\&CSC=Y\&NEWS=N\&PAGE=fullte xt\&D=med5\&AN=18559640\%5Cnhttp://sfx.kcl.ac.uk/kings?sid=OVID:medli ne\&id=pmid: \&id=doi:\&genre=article \&atitle=Trace+element+levels + and $+\operatorname{cog}$ nitive+function+in+rural+elderly+Chinese.\&title=Journals+o.

6. Roos PM, Vesterberg O, Syversen T, Flaten TP, Nordberg M. Metal concentrations in cerebrospinal fluid and blood plasma from patients with amyotrophic lateral sclerosis. Biol Trace Elem Res. 2013. doi:10.1007/s12011-012-9547-x. 
7. Komatsu F, Kagawa Y, Kawabata T, et al. A high accumulation of hair minerals in Mongolian people: 2(nd) report; influence of manganese, iron, lead, cadmium and aluminum to oxidative stress, Parkinsonism and arthritis. Curr Aging Sci. 2011;4(1):42-56. http://www.ncbi.nlm.nih.gov/pubmed/21204778. Accessed September 19, 2016.

8. Agency for Toxic Substances and Disease Registry (ATSDR). TOXICOLOGICAL PROFILE FOR ARSENIC. Health Effects. Agency Toxic Subst Dis Regist. 2007;(August).

9. O'Bryant SE, Edwards M, Menon C, Gong G, Barber R. Long-Term LowLevel Arsenic Exposure Is Associated with Poorer Neuropsychological Functioning: A Project FRONTIER Study. Int J Environ Res Public Health. 2011;8(12):861-874. doi:10.3390/ijerph8030861.

10. Edwards M, Hall J, Gong G, O’Bryant SE. Arsenic exposure, AS3MT polymorphism, and neuropsychological functioning among rural dwelling adults and elders: a cross-sectional study. Environ Health. 2014;13(1):15. doi:10.1186/1476-069X-13-15.

11. Park J-H, Lee D-W, Park KS, Joung H. Serum trace metal levels in Alzheimer's disease and normal control groups. Am J Alzheimers Dis Other Demen. 2014;29(1):76-83. doi:10.1177/1533317513506778.

12. Edwards M, Johnson L, Mauer C, Barber R, Hall J, O’Bryant S. Regional specific groundwater arsenic levels and neuropsychological functioning: a cross-sectional study. Int J Environ Health Res. 2014;24(6):546-557. doi:10.1080/09603123.2014.883591.

13. Agency for Toxic Substances and Disease Registry (ATSDR). Toxicological Profile for Manganese. ATSDR - Toxicol Profiles. 2000;(September):504.

14. Hozumi I, Hasegawa T, Honda A, et al. Patterns of levels of biological metals in CSF differ among neurodegenerative diseases. J Neurol Sci. 2011;303(12):95-99. doi:10.1016/j.jns.2011.01.003. 
15. Wasserman GA, Liu X, Parvez F, et al. Arsenic and manganese exposure and children's intellectual function. Neurotoxicology. 2011;32(4):450-457. doi:10.1016/j.neuro.2011.03.009.

16. Kim Y, Bowler RM, Abdelouahab N, Harris M, Gocheva V, Roels HA. Motor function in adults of an Ohio community with environmental manganese exposure. Neurotoxicology. 2011;32(5):606-614. doi:10.1016/j.neuro.2011.07.011.

17. Carvalho CF, Menezes-Filho JA, Matos VP de, et al. Elevated airborne manganese and low executive function in school-aged children in Brazil. Neurotoxicology. 2014;45:301-308. doi:10.1016/j.neuro.2013.11.006.

18. Oulhote Y, Mergler D, Barbeau B, et al. Neurobehavioral function in schoolage children exposed to manganese in drinking water. Environ Health Perspect. 2014;122(12):1343-1350. doi:10.1289/ehp.1307918.

19. Koc ER, Ilhan A, Ayturk Z, et al. A comparison of hair and serum trace elements in patients with Alzheimer disease and healthy participants. Turkish J Med Sci Turk J Med Sci, Turk J Med, Turk J Med Sci. 2015;45(5):10341039. doi:10.3906/sag-1407-67.

20. Miyake Y, Tanaka K, Fukushima W, et al. Dietary intake of metals and risk of Parkinson's disease: A case-control study in Japan. J Neurol Sci. 2011;306(12):98-102. doi:10.1016/j.jns.2011.03.035.

21. Kumudini N, Uma A, Prameela Devi Y, et al. Association of Parkinson's disease with altered serum levels of lead and transition metals among South Indian subjects. Indian J Biochem Biophys. 2014;51(2):121-126.

22. Garzillo EM, Lamberti M, Genovese G, et al. Blood lead, manganese, and aluminum levels in a regional Italian cohort of ALS patients: does aluminum have an influence? J Occup Environ Med. 2014;56(10):1062-1066. doi:10.1097/JOM.0000000000000266.

23. Kihira T, Sakurai I, Yoshida S, et al. Neutron \{Activation $\{$ Analysis $\}$ of \{Scalp $\{$ Hair $\}$ from $\{$ ALS $\}$ \{Patients $\}$ and $\{$ Residents $\}$ in the $\{$ Kii $\}$ \{Peninsula\}, \{Japan\}. Biol Trace Elem Res. 2015;164(1):36-42. doi:10.1007/s12011-014-0202-6. 
24. Arain MS, Afridi HI, Kazi TG, et al. Correlation of aluminum and manganese concentration in scalp hair samples of patients having neurological disorders. Environ Monit Assess. 2015;187(2). doi:10.1007/s10661-014-4172-0.

25. Bowler RM, Gocheva V, Harris M, et al. Prospective study on neurotoxic effects in manganese-exposed bridge construction welders. Neurotoxicology. 2011;32(5):596-605. doi:10.1016/j.neuro.2011.06.004.

26. Baker MG, Criswell SR, Racette BA, et al. Neurological outcomes associated with low-level manganese exposure in an inception cohort of asymptomatic welding trainees. Scand J Work Environ Heal. 2015;41(1):94-101. doi:10.5271/sjweh.3466.

27. Choe SY, Kim SJ, Kim HG, et al. Evaluation of estrogenicity of major heavy metals. Sci Total Environ. 2003;312(1-3):15-21. doi:10.1016/S00489697(03)00190-6.

28. Preciados M, Yoo C, Roy D. Estrogenic Endocrine Disrupting Chemicals Influencing NRF1 Regulated Gene Networks in the Development of Complex Human Brain Diseases. Int J Mol Sci. 2016;17(12):2086. doi:10.3390/ijms 17122086.

29. Kim JH, Byun HM, Chung EC, Chung HY, Bae ON. Loss of integrity: Impairment of the blood-brain barrier in heavy metal-associated ischemic stroke. Toxicol Res. 2013;29(3):157-164. doi:10.5487/TR.2013.29.3.157.

30. Wang B, Du Y. Cadmium and its neurotoxic effects. Oxid Med Cell Longev. 2013;2013. doi:10.1155/2013/898034.

31. Zheng W, Aschner M, Ghersi-Egea J-F. Brain barrier systems: a new frontier in metal neurotoxicological research. Toxicol Appl Pharmacol. 2003;192(1):111. doi:10.1016/S0041-008X(03)00251-5.

32. Johnson CL, Paulose-Ram R, Ogden CL, et al. National health and nutrition examination survey: analytic guidelines, 1999-2010. Vital Health Stat 2. 2013;(161):1-24. http://www.ncbi.nlm.nih.gov/pubmed/25090154. 
33. Johnson CL, Dohrmann SM, Burt VL, Mohadjer LK. National Health and Nutrition Examination Survey: Sample Design , 2011 - 2014. Vital Heal Stat. 2014;2(162):1-33. http://www.cdc.gov/nchs/data/series/sr_02/sr02_162.pdf.

34. Centers for Disease Control and Prevention (CDC). National Center for Health Statistics (NCHS). National Health and Nutrition Examination Survey NCHS Research Ethics Review Board (ERB) Approval. Hyattsville, MD: U.S. Department of Health and Human Services, Centers for Disease Control and Prevention. https://www.cdc.gov/nchs/nhanes/irba98.htm. Published 2016.

35. Centers for Disease Control and Prevention (CDC). National Center for Health Statistics (NCHS). National Health and Nutrition Examination Survey 2013-2014 Data Documentation, Codebook, and Frequencies Urinary Total Arsenic (UTAS_H). Hyattsville, MD: U.S. Department of Health and Human Services, Centers for Disease Control and Prevention. https://wwwn.cdc.gov/Nchs/Nhanes/2013-2014/UTAS_H.htm. Published 2016.

36. Centers for Disease Control and Prevention (CDC). National Center for Health Statistics (NCHS). National Health and Nutrition Examination Survey 2011-2012 Data Documentation, Codebook, and Frequencies Arsenics - Total \& Speciated - Urine (UAS_G). Hyattsville, MD: U.S. Department of Health and Human Services, Centers for Disease Control and Prevention. https://wwwn.cdc.gov/Nchs/Nhanes/2011-2012/UAS_G.htm. Published 2016.

37. Centers for Disease Control and Prevention (CDC). National Center for Health Statistics (NCHS). National Health and Nutrition Examination Survey 2013-2014 Data Documentation, Codebook, and Frequencies Urinary Speciated Arsenics (UAS_H). Hyattsville, MD: U.S. Department of Health and Human Services, Centers for Disease Control and Prevention. https://wwwn.cdc.gov/Nchs/Nhanes/2013-2014/UAS_H.htm. Published 2016.

38. Centers for Disease Control and Prevention (CDC). National Center for Health Statistics (NCHS). National Health and Nutrition Examination Survey Data, Questionnaires, and Related Documentation and Information. National Health and Nutrition Examination Survey Data. Hyattsville, MD: U.S. Department of Health and Human Services, Centers for Disease Control and Prevention. https://www.cdc.gov/nchs/nhanes/index.htm. Published 2016. 
39. Centers for Disease Control and Prevention (CDC). National Center for Health Statistics (NCHS). National Health and Nutrition Examination Survey - Task 2: How to Address Different Fill Values and the Limit of Detection (LOD) in NHANES Environmental Chemical Data. Hyattsville, MD: U.S. Department of Health and Human Services, Centers for Disease Control and Prevention.

https://www.cdc.gov/nchs/tutorials/environmental/critical_issues/limitations/T ask2.htm. Published 2016.

40. Centers for Disease Control and Prevention (CDC). National Center for Health Statistics (NCHS). National Health and Nutrition Examination Survey 2013-2014 Data Documentation, Codebook, and Frequencies Cognitive Functioning (CFQ_H). Hyattsville, MD: U.S. Department of Health and Human Services, Centers for Disease Control and Prevention. https://wwwn.cdc.gov/Nchs/Nhanes/2013-2014/CFQ_H.htm. Published 2017.

41. Centers for Disease Control and Prevention (CDC). National Center for Health Statistics (NCHS). National Health and Nutrition Examination Survey 2011-2012 Data Documentation, Codebook, and Frequencies Cognitive Functioning (CFQ_G). Hyattsville, MD: U.S. Department of Health and Human Services, Centers for Disease Control and Prevention. https://wwwn.cdc.gov/Nchs/Nhanes/2011-2012/CFQ_G.htm\#CFDCST3. Published 2017.

42. Fillenbaum GG, van Belle G, Morris JC, et al. Consortium to Establish a Registry for Alzheimer's Disease (CERAD): The first twenty years. Alzheimer's Dement. 2008;4(2):96-109. doi:10.1016/j.jalz.2007.08.005.

43. Bertolucci PHF, Okamoto IH, Brucki SMD, Siviero MO, Neto JT, Ramos LR. Applicability of the CERAD neuropsychological battery to Brazilian elderly. Arq Neuropsiquiatr. 2001;59(3 A):532-536. doi:10.1590/S0004282X2001000400009.

44. Karrasch M, Sinervä E, Grönholm P, Rinne J, Laine M. CERAD test performances in amnestic mild cognitive impairment and Alzheimer's disease. Acta Neurol Scand. 2005;111(3):172-179. doi:10.1111/j.16000404.2005.00380.x.

45. Karrasch M, Laatu S, Martikainen K, Marttila R. CERAD test performance and cognitive impairment in Parkinson's disease. Acta Neurol Scand. 2013;128(6):409-413. doi:10.1111/ane.12138. 
46. R.M. H, N.-M. S, P. H, et al. The CERAD neuropsychological battery in patients with frontotemporal lobar degeneration. Dement Geriatr Cogn Dis Extra. 2015;5(1):147-154. doi:10.1159/000380815.

47. Berres M, Monsch AU, Bernasconi F, Thalmann B, Stähelin HB. Normal ranges of neuropsychological tests for the diagnosis of Alzheimer's disease. Stud Health Technol Inform. 2000;77:195-199. http://www.ncbi.nlm.nih.gov/pubmed/11187541. Accessed January 21, 2018.

48. Sata F, Araki S, Yokoyama K, Murata K. Adjustment of creatinine-adjusted values in urine to urinary flow rate: a study of eleven heavy metals and organic substances. Int Arch Occup Environ Health. 1995;68(1):64-68. http://www.ncbi.nlm.nih.gov/pubmed/8847115. Accessed November 15, 2017.

49. Alegret M, Espinosa A, Valero S, et al. Cut-off Scores of a Brief Neuropsychological Battery (NBACE) for Spanish Individual Adults Older than 44 Years Old. Brucki S, ed. PLoS One. 2013;8(10):e76436. doi:10.1371/journal.pone.0076436.

50. American Academy of Family Physicians. BB, Hartmann B, DeJoseph D. American Family Physician. Vol 84. American Academy of Family Physicians; 1970. https://www.aafp.org/afp/2011/1015/p895.html. Accessed January 21, 2018.

51. Axelrod BN. Administration duration for the Wechsler Adult Intelligence Scale-III and Wechsler Memory Scale-III. Arch Clin Neuropsychol. 2001;16(3):293-301. doi:10.1016/S0887-6177(00)00048-2.

52. Matsuda O, Saito M, Kato M, Azami H, Shido E. Wechsler Adult Intelligence Scale-III profile in the early stages of Alzheimer's disease: Performance in subtests sensitive to and resistant to normal decline with ageing. Psychogeriatrics. 2015;15(1):1-6. doi:10.1111/psyg.12066.

53. Donnell AJ, Pliskin N, Holdnack J, Axelrod B, Randolph C. Rapidlyadministered short forms of the Wechsler Adult Intelligence Scale-3rd edition. Arch Clin Neuropsychol. 2007;22(8):917-924. doi:10.1016/j.acn.2007.06.007. 
54. Nielsen NP, Ringström R, Wiig EH, Minthon L. Associations between AQT processing speed and neuropsychological tests in neuropsychiatric patients. Am J Alzheimers Dis Other Demen. 2011;22(3):202-210. doi: $10.1177 / 1089253206300417$.

55. Proust-Lima C, Amieva H, Dartigues JF, Jacqmin-Gadda H. Sensitivity of four psychometric tests to measure cognitive changes in brain agingpopulation-based studies. Am J Epidemiol. 2007;165(3):344-350. doi:10.1093/aje/kwk017.

56. Rosano C, Perera S, Inzitari M, Newman AB, Longstreth WT, Studenski S. Digit symbol substitution test and future clinical and subclinical disorders of cognition, mobility and mood in older adults. Age Ageing. 2016;45(5):687694. doi:10.1093/ageing/afw116.

57. Saxton J, Lopez OL, Ratcliff G, et al. Preclinical Alzheimer disease: neuropsychological test performance 1.5 to 8 years prior to onset. Neurology. 2004;63(Mh 46643):2341-2347. doi:10.1212/01.WNL.0000147470.58328.50.

58. Centers for Disease Control and Prevention (CDC). National Center for Health Statistics (NCHS). National Health and Nutrition Examination Survey - Questionnaires, Datasets, and Related Documentation. Hyattsville, MD: U.S. Department of Health and Human Services, Centers for Disease Control and Prevention. https://www.cdc.gov/nchs/nhanes/nhanes_questionnaires.htm. Published 2015.

59. Brooks LG, Loewenstein DA. Assessing the progression of mild cognitive impairment to Alzheimer's disease: current trends and future directions. Alzheimers Res Ther. 2010;2(5):28. doi:10.1186/alzrt52.

60. Tolea MI, Morris JC, Galvin JE. Longitudinal associations between physical and cognitive performance among community-dwelling older adults. PLoS One. 2015;10(4):1-12. doi:10.1371/journal.pone.0122878.

61. Ansoleaga B, Garcia-Esparcia P, Llorens F, Moreno J, Aso E, Ferrer I. Dysregulation of brain olfactory and taste receptors in AD, PSP and CJD, and AD-related model. Neuroscience. 2013;248:369-382. doi:10.1016/j.neuroscience.2013.06.034. 
62. Macknin JB, Higuchi M, Lee VM-Y, Trojanowski JQ, Doty RL. Olfactory dysfunction occurs in transgenic mice overexpressing human $\tau$ protein. Brain Res. 2004;1000(1-2):174-178. doi:10.1016/j.brainres.2004.01.047.

63. Lee S, Manly J, Andrews H, et al. Olfactory deficits predict cognitive decline and Alzheimer dementia in an urban community. 2014.

64. Hummel T, Landis B, Huttenbrink K. Smell and taste disorders. GMS Curr Tpoics Otorhinolaryngol Head Neck Surg. 2011;10:1-15. doi:10.3205/cto000077.

65. Doty RL. The olfactory vector hypothesis of neurodegenerative disease: Is it viable? Ann Neurol. 2008;63(1):7-15. doi:10.1002/ana.21327.

66. Liu J. Clinical features of olfactory disorders in patients seeking medical consultation. Med Sci Monit. 2013;19:444-450. doi:10.12659/MSM.883938.

67. Hüttenbrink K-B, Hummel T, Berg D, Gasser T, Hähner A. Olfactory dysfunction: common in later life and early warning of neurodegenerative disease. Dtsch Arztebl Int. 2013;110(1-2):1-7, e1. doi:10.3238/arztebl.2013.0001.

68. Barresi M, Ciurleo R, Giacoppo S, et al. Evaluation of olfactory dysfunction in neurodegenerative diseases. J Neurol Sci. 2012;323(1-2):16-24. doi:10.1016/j.jns.2012.08.028.

69. Ruan Y, Zheng XY, Zhang HL, Zhu W, Zhu J. Olfactory dysfunctions in neurodegenerative disorders. J Neurosci Res. 2012;90(9):1693-1700. doi:10.1002/jnr.23054.

70. Albers MW, Tabert MH, Devanand DP. Olfactory dysfunction as a predictor of neurodegenerative disease. Curr Neurol Neurosci Rep. 2006;6(5):379-386. doi:10.1007/s11910-996-0018-7.

71. Winner B, Kohl Z, Gage FH. Neurodegenerative disease and adult neurogenesis. Eur J Neurosci. 2011;33(6):1139-1151. doi:10.1111/j.14609568.2011.07613.x. 
72. Attems J, Walker L, Jellinger KA. Olfactory bulb involvement in neurodegenerative diseases. Acta Neuropathol. 2014;127(4):459-475. doi:10.1007/s00401-014-1261-7.

73. Makowska I, Kloszewska I, Grabowska A, Szatkowska I, Rymarczyk K. Olfactory deficits in normal aging and Alzheimer's disease in the polish elderly population. Arch Clin Neuropsychol. 2011;26(3):270-279. doi:10.1093/arclin/acr011.

74. Sohrabi HR, Bates K a, Weinborn MG, et al. Olfactory discrimination predicts cognitive decline among community-dwelling older adults. Transl Psychiatry. 2012;2(5):e118. doi:10.1038/tp.2012.43.

75. Driver-Dunckley E, Adler CH, Hentz JG, et al. Olfactory dysfunction in incidental Lewy body disease and Parkinson's disease. Parkinsonism Relat Disord. 2014;20(11):1260-1262. doi:10.1016/j.parkreldis.2014.08.006.

76. Ward AM, Calamia M, Thiemann E, Dunlap J, Tranel D. Association between olfaction and higher cortical functions in Alzheimer's disease, mild cognitive impairment, and healthy older adults. J Clin Exp Neuropsychol. November 2016:1-15. doi:10.1080/13803395.2016.1253667.

77. SAS 9.4. 2017.

78. Centers for Disease Control and Prevention (CDC). National Center for Health Statistics (NCHS). National Health and Nutrition Examination Survey - Overview of NHANES Survey Design and Weights. Hyattsville, MD: U.S. Department of Health and Human Services, Centers for Disease Control and Prevention.

https://www.cdc.gov/nchs/tutorials/environmental/orientation/sample_design/. Published 2013.

79. Centers for Disease Control and Prevention (CDC). National Center for Health Statistics (NCHS). National Health and Nutrition Examination Survey - Using Blood Lipid or Urine Creatinine Adjustments of Environmental Chemical Data. Hyattsville, MD: U.S. Department of Health and Human Services, Centers for Disease Control and Prevention. https://www.cdc.gov/nchs/tutorials/environmental/critical_issues/adjustments/ Info1.htm. Published 2017. 
80. Centers for Disease Control and Prevention (CDC). National Center for Health Statistics (NCHS). National Health and Nutrition Examination Survey - Using Blood Lipid or Urine Creatinine Adjustments in the Analysis of Environmental Chemical Data. Hyattsville, MD: U.S. Department of Health and Human Services, Centers for Disease Control and Prevention. https://www.cdc.gov/nchs/tutorials/environmental/critical_issues/adjustments/ index.htm. Published 2013.

81. Centers for Disease Control and Prevention (CDC). National Center for Health Statistics (NCHS). National Health and Nutrition Examination Survey - Data Normality and Transformations. Hyattsville, MD: U.S. Department of Health and Human Services, Centers for Disease Control and Prevention. https://www.cdc.gov/nchs/tutorials/environmental/critical_issues/normality/in dex.htm. Published 2010.

82. SAS Institute Inc. Sas/Stat ${ }^{\circledR}$ 9.3. 2011:User's Guide: The GLIMMIX Procedure (Chapter).

83. SAS Institute Inc. Estimating Geometric Means Using Data from a Complex Survey Sampling Design.

http://citeseerx.ist.psu.edu/viewdoc/download?rep=rep1\&type=pdf\&doi=10.1. 1.192.593. Published 2017.

84. Xu L, Zhang W, Liu X, Zhang C, Wang P, Zhao X. Circulatory Levels of Toxic Metals (Aluminum, Cadmium, Mercury, Lead) in Patients with Alzheimer's Disease: A Quantitative Meta-Analysis and Systematic Review. J Alzheimer's Dis. 2018;62(1):361-372. doi:10.3233/JAD-170811.

85. Peng Q, Bakulski KM, Nan B, Park SK. Cadmium and Alzheimer's disease mortality in U.S. adults: Updated evidence with a urinary biomarker and extended follow-up time. Environ Res. 2017;157(May):44-51. doi:10.1016/j.envres.2017.05.011.

86. Ciesielski T, Bellinger DC, Schwartz J, Hauser R, Wright RO. Associations between cadmium exposure and neurocognitive test scores in a cross-sectional study of US adults. Environ Heal A Glob Access Sci Source. 2013;12(1):1. doi:10.1186/1476-069X-12-13. 
87. Bergman Å, Heindel J, Jobling S, Kidd K, Zoeller RT. State of the Science of Endocrine Disrupting Chemicals, 2012. Vol 211.; 2012. doi:10.1016/j.toxlet.2012.03.020.

88. Wang S, Hu P, Wang H-L, et al. Effects of $\mathrm{Cd}(2+)$ on AMPA receptormediated synaptic transmission in rat hippocampal CA1 area. Toxicol Lett. 2008;176(3):215-222. doi:10.1016/j.toxlet.2007.11.008.

89. Chen L, Liu L, Luo Y, Huang S. MAPK and mTOR pathways are involved in cadmium-induced neuronal apoptosis. J Neurochem. 2008;105(1):251-261. doi:10.1111/j.1471-4159.2007.05133.x.

90. Jiang JH, Ge G, Gao K, et al. Calcium Signaling Involvement in CadmiumInduced Astrocyte Cytotoxicity and Cell Death Through Activation of MAPK and PI3K/Akt Signaling Pathways. Neurochem Res. 2015;40(9):1929-1944. doi:10.1007/s11064-015-1686-y.

91. Chow ESH, Hui MNY, Lin CC, Cheng SH. Cadmium inhibits neurogenesis in zebrafish embryonic brain development. Aquat Toxicol. 2008;87(3):157-169. doi:10.1016/j.aquatox.2008.01.019.

92. Yuan $\mathrm{Y}$, Jiang $\mathrm{C}, \mathrm{Xu} \mathrm{H}$, et al. Cadmium-induced apoptosis in primary rat cerebral cortical neurons culture is mediated by a calcium signaling pathway. PLoS One. 2013;8(5):e64330. doi:10.1371/journal.pone.0064330.

93. Hossain S, Liu H-N, Nguyen M, Shore G, Almazan G. Cadmium exposure induces mitochondria-dependent apoptosis in oligodendrocytes. Neurotoxicology. 2009;30(4):544-554. doi:10.1016/j.neuro.2009.06.001.

94. Notarachille G, Arnesano F, Calò V, Meleleo D. Heavy metals toxicity: effect of cadmium ions on amyloid beta protein 1-42. Possible implications for Alzheimer's disease. Biometals. 2014;27(2):371-388. doi:10.1007/s10534014-9719-6.

95. Luo J, Qiu Z, Zhang L, Shu W. Arsenite exposure altered the expression of NMDA receptor and postsynaptic signaling proteins in rat hippocampus. Toxicol Lett. 2012;211(1):39-44. doi:10.1016/j.toxlet.2012.02.021. 
96. Luo J, Qiu Z, Shu W, Zhang Y, Zhang L, Chen J. Effects of arsenic exposure from drinking water on spatial memory, ultra-structures and NMDAR gene expression of hippocampus in rats. Toxicol Lett. 2009;184(2):121-125. doi:10.1016/j.toxlet.2008.10.029.

97. Qu L, Gao Y, Sun H, Wang H, Liu X, Sun D. Role of PTEN-Akt-CREB Signaling Pathway in Nervous System impairment of Rats with Chronic Arsenite Exposure. Biol Trace Elem Res. 2015;(23618504). doi:10.1007/s12011-015-0478-1.

98. Shavali S, Sens DA. Synergistic Neurotoxic Effects of Arsenic and Dopamine in Human Dopaminergic Neuroblastoma SH-SY5Y Cells. Toxicol Sci. 2007;102(2):254-261. doi:10.1093/toxsci/kfm302.

99. Zarazúa S, Bürger S, Delgado JM, Jiménez-Capdeville ME, Schliebs R. Arsenic affects expression and processing of amyloid precursor protein (APP) in primary neuronal cells overexpressing the Swedish mutation of human APP. Int J Dev Neurosci. 2011;29(4):389-396. doi:10.1016/j.ijdevneu.2011.03.004.

100. Guilarte TR, McGlothan JL, Degaonkar M, et al. Evidence for cortical dysfunction and widespread manganese accumulation in the nonhuman primate brain following chronic manganese exposure: a 1H-MRS and MRI study. Toxicol Sci. 2006;94(2):351-358. doi:10.1093/toxsci/kfl106.

101. Guilarte TR, Chen M-K. Manganese inhibits NMDA receptor channel function: implications to psychiatric and cognitive effects. Neurotoxicology. 2007;28(6):1147-1152. doi:10.1016/j.neuro.2007.06.005.

102. Guilarte TR, Burton NC, McGlothan JL, et al. Impairment of nigrostriatal dopamine neurotransmission by manganese is mediated by pre-synaptic mechanism(s): implications to manganese-induced parkinsonism. $J$ Neurochem. 2008;107(5):1236-1247. doi:10.1111/j.1471-4159.2008.05695.x.

103. Davis AP, Grondin CJ, Lennon-Hopkins K, et al. The Comparative Toxicogenomics Database's 10th year anniversary: Update 2015. Nucleic Acids Res. 2015;43(D1):D914-D920. doi:10.1093/nar/gku935. 


\title{
CHAPTER VII \\ MANUSCRIPT IV
}

\section{EXPOSURE TO ORAL CONTRACEPTIVES AND HORMONE REPLACEMENT THERAPY AND ASSOCIATIONS WITH BRAIN \\ HEALTH: NHANES 2011-2014}

\begin{abstract}
BACKGROUND: The role of estrogenic endocrine disrupting chemicals (EEDCs) and their role in the development of neurodegenerative disease is of great public health concern, due to increasing exposures to these chemicals and increasingly aging population. Evidence suggests EEDCs exposure plays a role in the development of neurodegenerative disease, although epidemiological evidence is lacking in this area. Oral contraceptives (OC) and hormonal replacement therapy (HRT) are known estrogenic endocrine disrupting chemicals (EEDCs), have a strong affinity for estrogen receptors with proven estrogenic activity, and are almost functionally similar to estrogen, which affects estrogen and its protective effects on brain health. OBJECTIVE: The objective of this study is to investigate the relationship between surrogate brain health indicators and to past OC and HRT use among the older individuals of the United States (US) population.

METHODS: In this study, we analyzed participants from the Center for Disease Control and Prevention (CDC) National Health and Nutrition Examination Survey (NHANES) in the survey cycles 2011-2012 and 2013-2014. The participants were 60 years of age and older who had answered medical questions concerning oral contraceptive and hormonal replacement therapy use. Other data pertaining to
\end{abstract}


covariates and demographics were also obtained. The two medical questions analyzed were: 1) "Have you ever taken birth control pills for any reason?" and 2) "Have you ever used female hormones such as estrogen and progesterone?". These responses were analyzed versus various brain health indicators available in the form of test scores and questionnaires, available in the NHANES datasets. The brain health indicators test scores were the following: immediate recall test scores; delayed recall test scores; animal fluency test scores; digit symbol substitution test scores. The brain health indicator questions were as follows: worsening memory over the past 12 months; trouble remembering over the past week; difficulty remembering or because you experience periods of confusion. The following smell and taste questions were also included as brain health indicators due to their potential as pre-clinical indicators of cognitive impairment: phantom odor (phantosmia) and persistent taste in mouth over the past 12 months. Chi-square test of independence was used to test the associations between the medical questions $(\mathrm{Y} / \mathrm{N})$ versus the brain health indicators. Logistic regression was then used to calculate odds ratios (ORs) and 95\% confidence intervals (CIs). Three logistic regression models were presented in our study with all of them stratified by gender: unadjusted; age, race, education; age, race, education, body mass index (BMI), smoking status, blood pressure, diabetes status, alcohol use, coronary heart disease status, heart attack status, stroke status, head injury status, and physical activity status.

RESULTS: We observed a the existence of a relationship between past OC and HRT use and the following surrogates of brain function: immediate recall scores; delayed recall scores; animal flluency scores; DSST scores, and limitations due to difficulty 
remembering or confusion. In our logisitic regression models, when controlling for all known and suspected covariates of cognitive dysfunction and AD in our second adjusted model, we found past OC use to lower the risk of developing cognitive dysfunction and the possible development of AD. Specifically, we found past OC use to be associated with better immediate recall, delayed recall, animal fluency and DSST scores. We also found past HRT use to lower the risk fo developing cognitive dysfunction and the possible development of AD. Specifically, we found past HRT use to be associated with better immediate recall, animal fluency, and DSST scores. Past HRT use was also associated with less occurrences of limitations due to periods of difficulty remembering or periods of confusion.

Conclusion: Our study takes a novel approach to assessing cognitive dysfunction neurodegenerative disease and exposures to past OC and HRT use. It appears there is a link between exposure to past OC and HRT use and adverse brain health. Further research is needed with the use of clinical endpoints to further establish the relationship between neurodegenerative disease and phthalate/BPA exposure. 


\section{Exposure to Oral Contraceptives and Hormone Replacement Therapy and Associations with Brain Health: NHANES 2011-2012 INTRODUCTION}

The role of exposure to EEDCs and neurodegenerative disease development is of great public health concern as stated by the World Health Organization (WHO) ${ }^{1}$, due to cognitive health and neurodegenerative disease emerging as great public health concerns due to an increasingly aging population ${ }^{2}$. Exposures to EEDCs have been linked to neurodegenerative diseases and other adverse brain health conditions, such as Alzheimer's disease (AD). In this study, we examine the associations of past OC and HRT use and their associations with brain health in an older US population.

Pharmaceutical drugs are also considered to be estrogenic endocrine disrupting chemicals ${ }^{3}$. A particularly powerful example are the health effects brought about by the use of diethylstilbestrol a potent estrogen mimic whose use brought about immediate and long-term health effects to the mothers who took the drug ${ }^{4}$. Both had indications as a hormone replacement therapy (HRT) and oral contraceptive (OC).

Oral contraceptives containing specifically containing ethinyl estradiol are considered EEDCs due to its ability to mimic estrogen and influence the reproductive cycle. OC use has been shown to reduce the amount of available endogenous estrogen

in the body ${ }^{5}$. OCs has varying effects brain structure, function, and cognition ${ }^{6}$. Most recent studies have shown varying effects; however, the studies do not differentiate between the various types of contraceptives, making it difficult to find out if the 
contraceptives used contained ethinyl estradiol and inconsistencies are found in the reporting of the type of OC used (Beltz et al, 2015).

A cross-sectional study by Beltz et al. ${ }^{7}$ examined OC effects on spatial and verbal abilities found OC users to perform better on spatial ability and verbal tests. Another cross-sectional study by Egan and Gleason ${ }^{8}$ found OC users to have better performance on cognitive exams compared to non-users. Griksiene and Ruksenas ${ }^{5}$ found OC to negatively affect cognition. A review by Warren et al. ${ }^{9}$ suggest an overall positive effect with OC use and verbal memory.

Hormone replacement therapy, or HRT, has been shown to be associated with the onset of neurodegenerative disease, although evidence points to a timeframe dependent response, depending on when the therapy is initiated. As summarized in Maki and Henderson ${ }^{10}$, initial observation studies and analyses with women indicated the use of HRT's containing estrogen to be associated with a reduced risk of Alzheimer's disease. Studies suggest that the timing of HRT use during parts of the menopausal stage may dictate whether beneficial adverse effects are observed ${ }^{10,11}$. Several analytical studies, however, showed associations between the use of HRT and neurodegenerative disease. In the Women's Health Initiative Memory Study (WHIMS), a randomized, double-blind, placebo-controlled clinical trial, it was found that an HRT treatment of estrogen plus progestin increased the risk for dementia in postmenopausal women and did not prevent cognitive impairment ${ }^{12}$. In another study from the same trial, it was found that estrogen only HRT did not reduce the incidence of dementia, or cognitive impairment, and increased the risk for both ${ }^{13}$. 
An ancillary to the WHIMS study, the Women's Health Initiative Study of Cognitive Aging, or WHISCA, further supports HRT's association with neurodegenerative disease, however, results are varied. One finding from Resnick et al ${ }^{14}$ from the WHISCA study found that a combination of conjugated equine estrogen with medroxyprogesterone acetate (estrogen + progestin) appeared to negatively impact verbal memory, but positively affect figural memory among postmenopausal women, free of probable dementia, and compared to controls. Another finding from Resnick et al ${ }^{15}$ showed estrogen alone, as conjugated equine estrogen, did not improve cognitive functioning and lowered certain cognitive functions in women with prior hysterectomy. Other studies have indicated that no visible improvement has been observed in using estrogen only treatments for cognitive function ${ }^{16-19}$. New studies and reviews give mixed results. A cohort study conducted by Shao et al, ${ }^{20}$ showed increased Alzheimer's disease risk amongst women who used HRT more than five years after menopause, but observed a decreased risk of AD if used within five years of menopause. Another recent meta-analysis showed no observable associated between postmenopausal HRT use and AD and dementia ${ }^{21}$. While another study found that an increased risk in the type of hormonal therapy used and PD risk 22.

\section{OBJECTIVE}

There is limited information regarding exposures to OC and HRT use the development of cognitive dysfunction and neurodegenerative disease in older populations. In this study we examine the relationship between OC and HRT use with surrogate brain health indicators, from the CDC's NHANES 2011-2012 and 2013- 
2014 data cycles. The objectives of the study are as follows: 1) to assess past OC and HRT use and any relationships in older adults in the US, 60 years of age and above with the surrogates of brain health indicators in the US population, 2) assess the association between phthalate and BPA levels and surrogate brain health indicators in older adults in the US, to find the risk of poor cognitive function and development of mild cognitive impairment, dementia, and AD.

\section{METHODS}

Study Design and Population: NHANES is a continuous cross-sectional data collection utilizing a complex multi-stage sampling design that creates a survey representative of the non-institutionalized population of the United States ${ }^{23,24}$. The survey has been conducted since 1999 and consists of an at-home questionnaires followed by a standardized physical examination and specimen collection conducted in mobile examination centers (MEC) ${ }^{23,24}$. Eligibility is determined using preset selection probabilities for the desired demographic subdomains ${ }^{24}$. A household screener is performed before to determine if any household members are eligible for the interview and examination ${ }^{24}$. The interview collects demographic, health, nutrition, and household information, while the physical examination includes physical measurements, dental examination, and the collection of blood and urine specimens for laboratory testing ${ }^{24}$. Prior to any to interviews and examinations, informed consent was obtained and all procedures were approved by the CDC Institutional Review Board ${ }^{25}$

In our study, we merged the NHANES 2011-2012 and 2013-2014 data cycles. All our analyses were limited to individuals 60 years of age and older who have recorded 
responses to cognitive test scores and/or memory and taste/smell questions and have responses to questions concerning oral contraceptive and hormonal therapy use.

\section{Inclusion/Exclusion Criteria}

\section{$\underline{\text { Inclusion criteria: }}$}

13. Females, 60 years of age and older

14. History of OC and/or HRT use.

15. Complete responses to identified outcome variables.

\section{Exclusion criteria:}

13. All males

14. Females, 59 years of age and younger

16. Unavailable History of OC and/or HRT use.

15. Complete responses to identified outcome variables.

\section{Oral Contraceptive Use Assessment and Measurements}

Oral contraceptive use was recorded by the question yes/no question, "Have you ever taken birth control pills for any reason?". Female participants aged 12 years and older were eligible ${ }^{26,27}$. These questions were administered in the mobile examination center (MEC) by trainer interviewers ${ }^{26,27}$. A total of 1670 female subjects over the age of 60 , provided a response, with 952 participants responding "yes" and 718 participants responding "no".

\section{Hormonal Replacement Therapy Use Assessment and Measurements}

Oral contraceptive use was recorded by the question yes/no question, "Have you ever used female hormones such as estrogen and progesterone?”. Female participants aged 12 years and older were eligible ${ }^{26,27}$. These questions were 
administered in the mobile examination center (MEC) by trainer interviewers ${ }^{26,27}$. A total of 1662 female subjects over the age of 60, provided a response, with 628 participants responding "yes" and "1034" participants responding "no".

Assessment of Surrogate Brain Health Indicators - Cognitive Scores CERAD Word Learning Subtest - Immediate Recall and Delayed Recall: The Consortium to Establish a Registry for Alzheimer's Disease (CERAD) Word assesses both immediate and delayed learning ${ }^{28,29}$. The delayed and immediate recall tests available in NHANES assess the ability to process new verbal information ${ }^{28,29}$. The tests are part of the neuropsychological assessment for the entire CERAD testing protocol, which was initially created to standardize AD assessment and diagnosis ${ }^{30}$. The tests in the neuropsychological assessment itself were chose because of their ability to assess cognitive functions inherent in $\mathrm{AD}^{30}$. The assessments have the ability to differentiate those of adequate cognitive status versus those who have mild cognitive impairment or dementia ${ }^{30-33}$. Although developed for use in the assessment of $\mathrm{AD}$, the CERAD assessments have shown utility in use for Parkinson's disease ${ }^{33}$ and frontotemporal lobar degeneration ${ }^{34}$.

Immediate Recall: For immediate recall, , the subjects are asked to read aloud a sequence of 10 unrelated words as they are presented to them and immediately after, they are asked to recall as many words as possible ${ }^{28,29}$. This is done in three trials with the order of the words differing in each trial. ${ }^{28,29}$. Each trial has a maximum score of 10 , with a maximum overall score of $30^{28,29,31}$.

In our study, we included individuals 60 years of age and older who completed all immediate recall word list trials identified as: CFDCST1, CFDCST2, 
and CFDCST3 in the 2011-2012 and 2013-2014 NHANES data cycles. Those who did not have three trials completed were not included in the immediate recall analysis. We summed the total of the three trials and created a new variable with cut-off scores named IMMEDIATERECALL. A cut-off score of $\leq 13$ and $\geq 14$ was used as it is the standard in other assessments ${ }^{31,35}$. A total of 3,149 subjects from the 2011-2012 and 2013-2014 responded with complete immediate recall trials. We then accounted for those who provided responses to OC and HRT use. After, our OC/immediate recall study population consisted of 1576 subjects, 193 subjects $\leq 13$ and 1383 subjects $\geq$ 14. Our HRT/immediate recall study population consisted 1567 subjects, with 189 subjects $\leq 13$ and 1378 subjects $\geq 14$.

Delayed Recall: For delayed recall, the subject is asked to repeat the sequence of 10 unrelated words after the other cognitive tests are completed, which is typical 8 to 10 minutes after the start of the word learning trials ${ }^{28,29}$. The maximum score is 10 for delayed recall ${ }^{28,29}$.

In our study, we included subjects 60 years of age and older who completed the delayed recall trial, identified as CFDCSR, for the 2011-2012 and 2013-2014 NHANES data cycles. After we created a new variable with the cut-off scores named DELAYEDRECALL. A cut-off score of $\leq 13$ and $\geq 14$ was used as it is the standard in other assessments ${ }^{31,35,36}$. A total of 3,126 subjects from the 2011-2012 and 20132014 responded with a complete delayed recall trial. We then accounted for those who provided responses to OC and HRT use. After, our OC/delayed recall study population consisted of 1568 subjects, 218 subjects $\leq 3$ and 1350 subjects $\geq 4$. Our 
HRT/immediate recall study population consisted of 1560 subjects with 213 subjects $\leq 3$ and 1347 subjects $\geq 4$.

Animal Fluency: The animal fluency test is used to determine categorical verbal fluency, which is part of executive function and can differentiate between with normal cognition versus those with MCI and more severe cognitive impairment ${ }^{28,29}$. Since the test uses animal names, it does not require cultural consideration or formal education experience ${ }^{28,29}$. In the test, subjects are asked to name as many animals in a one minute span, with a maximum range of 40 words in the NHANES 2011-2014 data set. ${ }^{28,29}$. A sample test is given to each subject before the actual test ${ }^{28,29}$. In our study, we included subjects 60 years of age and older who completed the animal fluency trial, identified as CFDAST, for the 2011-2012 and 2013-2014 NHANES data cycles. After we created a new variable with cut-off scores named VERBALFLUENCY. cut-off score of $\leq 11$ and $\geq 12$ was used as it is the standard in other assessments ${ }^{31,35-37}$. A total of 3,110 subjects from the 2011-2012 and 20132014 responded with a complete animal fluency trial. We then accounted for those who provided responses to OC and HRT use. After, our OC/Animal Fluency study population consisted of 1565 subjects, 321 subjects $\leq 321$ and 1244 subjects $\geq 12$. Our HRT/Animal Fluency study population consisted of 1557 subjects with 318 subjects $\leq 11$ and 1239 subjects $\geq 12$.

Digit Symbol Substitution Test: The Digit Symbol Substitution Test (DSST) is part of the Wechsler Adult Intelligence Scale (WAIS III) ${ }^{28,29,38}$. The test measures processing speed, sustained attention, and working memory ${ }^{28,29,38}$. The subtests have shown utility in the identification of dementia and other neurodegenerative disorders 
${ }^{39-41}$. The test is given in paper form, with a key that has 9 numbers paired to different symbols. The subject has 2 minutes to match each symbol to 133 boxes with a number associated to it, with the score as the total correct matches with a maximum score of 105 in the 2011-2014 NHANES dataset. ${ }^{28,29}$. A sample test is given to each subject before the actual test ${ }^{28,29}$.

In our study, we included subjects 60 years of age and older who completed the DSST trial, identified as CFDDS, for the 2011-2012 and 2013-2014 NHANES data cycles. After we created a new variable with cut-off scores named DSST, cut-off

score of $\leq 27$ and $\geq 28$ was used as it is the standard in other assessments ${ }^{42-44}$. A total of 3,104 subjects from the 2011-2012 and 2013-2014 responded with a complete delayed recall trial. We then accounted for those who provided responses to OC and HRT use. After, our OC/DSST study population consisted of 1511 subjects, 227 subjects $\leq 27$ and 1284 subjects $\geq 28$. Our HRT/DSST study population consisted of 1505 subjects with 226 subjects $\leq 27$ and 1279 subjects $\geq 28$.

\section{Assessment of Surrogate Brain Health Indicators - Memory Function}

\section{During the past 12 months, have you experienced confusion or memory loss that} is happening more often or getting worse?: In our study we included subjects 60 years of age and older who had phthalate and BPA samples taken and responded to the yes $=0 /$ no=1 question," During the past 12 months, \{have you/has she/has he $\}$ experienced confusion or memory loss that is happening more often or is getting worse?" ${ }^{45}$. Memory issues, such as confusion and memory loss often precede the development of dementia and neurodegeneration ${ }^{2,46}$, which makes this question a potential surrogate for the development of memory issues. 3,628 subjects responded 
to this question in the 2011-2012 and 2013-2014 survey cycle. We then accounted for those who provided responses to OC and HRT use. After, our OC/MCQ084 study population consisted of 1669 subjects, 274 subjects responded "yes" and 1395 subjects responded "no". Our HRT/MCQ084 study population consisted of 1661 subjects with 272 subjects responded "yes" and 1389 subjects responded "no".

\section{During the past 7 days, how often have you had trouble remembering where you}

put things?: In our study we included subjects 60 years of age and older who had phthalate and BPA samples taken and responded to the question," During the past 7 days, how often $\{$ have you/has SP $\}$ had trouble remembering where $\{y o u / h e / s h e\}$ put things, like $\{$ your/his/her $\}$ keys or $\{$ your/his/her $\}$ wallet?" ${ }^{45}$. Memory issues, such as confusion and memory loss often precede the development of dementia and neurodegeneration ${ }^{2,46}$, which makes this question a potential surrogate for the development of memory issues. 3,448 subjects responded to this question in the 20112012 and 2013-2014 survey cycle.

The question is multi-leveled, where 1,993 subjects answered "Never" $=0,809$ subjects answered "About once" =1, 544 subjects answered "Two or three times" =2, 175 subjects answered "Nearly every day" =3, and 102 subjects answered "Several times a day" $=4$. We created a new variable named MCQ380_WK, which combines responses coded as $0 /$ Never and $1 /$ About once into a variable $=2$ coded as "no" and $2 /$ Two or three times, $3 /$ Nearly every day and $4 /$ Several times a day into a variable $=1$ coded as "yes"

We then accounted for those who provided responses to OC and HRT use. After, our OC/MCQ380_WK study population consisted of 1576 subjects, 335 subjects 
responded "yes" and 1241 subjects responded "no". Our HRT/MCQ380_WK study population consisted of 1568 subjects with 332 subjects responded "yes" and 1236 subjects responded "no".

\section{$\underline{\text { Are you limited in any way because of difficulty remembering or because you }}$}

experience periods of confusion?: In our study we included subjects 60 years of age and older who had responses to OC and/or HRT use and responded to the question," $\{$ Are you/Is SP\} limited in any way because of difficulty remembering or because $\{$ you/s/he $\}$ experience $\{s\}$ periods of confusion?" ${ }^{45}$. Limitations in physical movement due to difficulty remembering and confusion can indicate the development of cognition issues ${ }^{47} .3,629$ subjects responded to this question in the 2011-2012 and 2013-2014 survey cycle.

We then accounted for those who provided responses to OC and HRT use. After, our OC/PFQ057 study population consisted of 1670 subjects, 259 subjects responded “yes" and 1411 subjects responded "no". Our HRT/PFQ057 study population consisted of 1662 subjects with 254 subjects responded "yes" and 1408 subjects responded "no".

\section{Assessment of Surrogate Brain Health Indicators - Taste and Smell Function}

It has been observed that neurodegenerative disease has been shown to be preceded by smell and taste disorders ${ }^{48-51}$. The causes of these disorders have been linked to genetic alterations ${ }^{48}$, overexpression of key proteins ${ }^{49}$, and direct effect of some environmental chemicals on the olfactory mucosa ${ }^{52}$, which can have associations with exposure to EEDCs ${ }^{48,49,51,52}$. However, issues with olfaction can 
also be caused by upper respiratory tract infections, sino-nasal disease, head trauma, idiopathic causes, surgery of the nasal area, and congenital loss of smell ${ }^{53}$.

The two most common and prevalent neurodegenerative diseases, $\mathrm{AD}$ and PD have been shown to be preceded by smell disorders ${ }^{54-59}$. These disorders manifest themselves when evidence of pathological changes in the olfactory system are evident 59. These are characterized by the build-up of pathological proteins, which cause the death of olfactory cells ${ }^{59}$. Several human epidemiological studies have also alluded to the utility of using sensory biomarkers as an early detection for neurodegenerative diseases ${ }^{60-63}$

Do you sometimes smell and unpleasant, bad, or burning odor when nothing is

there?: In our study we included subjects 60 years of age and older who had OC and/or HRT responses and responded to the question," $\{$ Do you/Does SP $\}$ sometimes smell an unpleasant, bad or burning odor when nothing is there?" 45 . 3,617 subjects responded to this question in the 2011-2012 and 2013-2014 survey cycle.

We then accounted for those who provided responses to OC and HRT use. After, our OC/CSQ040 study population consisted of 1664 subjects, 123 subjects responded "yes" and 1541 subjects responded "no". Our HRT/CSQ040 study population consisted of 1655 subjects with 123 subjects responded "yes" and 1532 subjects responded "no".

\section{During the past 12 months have you had a taste or other sensation in your}

mouth that does not go away?: In our study we included subjects 60 years of age and older who provided responses to OC/HRT use and responded to the question," During the past 12 months $\{$ have you/has SP $\}$ had a taste or other sensation in 
\{your/his/her\} mouth that does not go away?"45. 3,623 subjects responded to this question in the 2011-2012 and 2013-2014 survey cycle.

We then accounted for those who provided responses to OC and HRT use. After, our OC/CSQ110 study population consisted of 1667 subjects, 134 subjects responded "yes" and 1533 subjects responded "no". Our HRT/CSQ110 study population consisted of 1658 subjects with 134 subjects responded "yes" and 1524 subjects responded "no".

\section{Covariates}

In our study we included a number of covariates, based off a review of literature and well-known risk factors for neurodegenerative diseases, if they were available in the NHANES datasets.

The demographic variables are as follows: gender (male, female), age (60-69, 7-79, 80+), Race/Ethnicity (Hispanic, Non-Hispanic White, Non-Hispanic Black, NonHispanic Asian/Other), Family Income (Under 24k, 25k to 54,999k, 55k to 74,999k, over $75 \mathrm{k})$, Education $\left(<12^{\text {th }} \text { grade, completed high school, }>12^{\text {th }} \text { grade }\right)^{45}$. Modifiable health variables and risk factors are as follows: ever smoked (yes, no), blood pressure (normal/high), diabetes (yes, no, borderline), coronary heart disease (yes, no), stroke (yes, no), heart attack (yes, no), head trauma (yes, no), alcohol use (yes, no), ever use birth control (yes, no), every use hormonal replacement therapy (yes, no $)^{45}$.

\section{Statistical Analysis}

Statistical analysis was performed using SAS software ${ }^{64}$. The 20112012 and 2013-2014 survey cycles were merged and a four-year sampling weight was 
calculated to account for the complex sampling design in order to calculate correct statistical estimates and standard errors when calculating means, geometric means, and other statistics ${ }^{65}$.

For OC AND HRT variables, a value of "1" indicates a "yes" response and "2" indicates a "no" response. We used the SAS Survey procedures to account for the complex sampling design of the NHANES data sets ${ }^{66}$.

We used PROC SURVEYFREQ was used to obtain descriptive statistics for the different populations we were examining in our study which accounts for the complex survey design of the NHANES data sets ${ }^{66}$. Descriptive statistics were organized based on the following categories per variable: gender (male, female), age (60-69, 779, 80+), Race/Ethnicity (Hispanic, Non-Hispanic White, Non-Hispanic Black, NonHispanic Asian/Other), Family Income (Under 24k, 25k to 54,999k, 55k to 74,999k, over $75 \mathrm{k}$ ), Education ( $<12^{\text {th }}$ grade, completed high school, $>12^{\text {th }}$ grade), ever smoked (yes, no), blood pressure (normal/high), diabetes (yes, no, borderline), coronary heart disease (yes, no), stroke (yes, no), heart attack (yes, no), head trauma (yes, no), alcohol use (yes, no), ever use birth control (yes, no), every use hormonal replacement therapy (yes, no) ${ }^{45}$.

We also used PROC SURVEYFREQ to perform chi-square test of independence to examine associations between OC/HRT use and our select surrogate of brain health indicators.

We used PROC SURVEYREG and guidance provided by the SAS institute to directly to determine the geometric mean of the EEDC to test if they were significant between the responses of our outcome variables ${ }^{66,67}$. The standard errors were 
calculated using the Taylor Series linearization method, which is the default method in the survey procedures to calculate standard error ${ }^{66}$. Geometric means (GM), geometric standard errors (GSE), and number of subjects were reported for the results of the outcome variables for all subjects that had EEDCs over the LOD. We looked at geometric means between the outcome variables (yes vs. no, low test score vs. high test score), and also performed age-specific, gender-specific, and race/ethnicityspecific geometric means between the responses to the outcome variable. Due to the smaller range of ages in our dataset, 60 years and older, we calculated age-specific rates in lieu of age-standardized rates.

We used PROC SURVEYLOGISTIC to find the unadjusted and adjusted odds ratios (ORs) and the 95\% confidence intervals (CI) to examine the association between our outcome variables and exposures to phthalates and BPA ${ }^{66}$. Analysis was done per EEDC per outcome variable. We presented three logistic regression models which were stratified by gender and presented the EEDC as a continuous variable as well as a ranked variable $<$ LOD to $50^{\text {th }}$ percentile (reference) and $\geq 50^{\text {th }}$ percentile: unadjusted, adjusted for known risk factors, age, education, race/ethnicity, adjusted for known and suspected risk factors, age, education, race/ethnicity, smoking, blood pressure history, history of coronary heart disease, stroke, heart attack, diabetes status, head trauma, and alcohol use. We did not include income use as variables as they significantly reduced the size of the population. 


\section{RESULTS}

Descriptive statistics for the Surrogates of Brain Health Indicators and Covariates:

Descriptive statistics of the study populations are described in table 1.1 and 1.1a for each of our 9 outcomes with their respective covariates for OC and HRT use respectively. 


\begin{tabular}{|c|c|c|c|c|c|c|c|c|c|c|c|c|c|c|c|c|c|c|c|c|c|c|c|c|c|c|c|c|c|c|c|c|c|c|c|c|}
\hline \multirow{2}{*}{$\begin{array}{c}\text { Table 1.1 - Descriptive Statistics } \\
\text { - Surrogate brain health } \\
\text { indicators and covariates for } \\
\text { OC use }\end{array}$} & \multicolumn{4}{|c|}{ Immediate Recall (n=1576) } & \multicolumn{4}{|c|}{ Delayed Recall (n=1568) } & \multicolumn{4}{|c|}{ Animal Fluency (n=1565) } & Digit S & $\begin{array}{l}\text { Symbol S S } \\
\text { Test }\end{array}$ & $\begin{array}{l}\text { Substitu } \\
=1511)\end{array}$ & itution & & $\begin{array}{l}12 \text { montt } \\
\text { ing worse }\end{array}$ & $\begin{array}{l}\text { ths, mem } \\
\text { se? (n=16 }\end{array}$ & & & $\begin{array}{l}\text { st } 7 \text { days } \\
\text { embering }\end{array}$ & $\begin{array}{l}\text { s, trouble } \\
\text { ? (n=157 }\end{array}$ & & $\begin{array}{l}\text { Limite } \\
\text { rememb }\end{array}$ & $\begin{array}{l}\text { ted due to } \\
\text { bering or } \\
\qquad(\mathrm{n}=16\end{array}$ & $\begin{array}{l}\text { to difficul } \\
\text { Ir confusi } \\
670)\end{array}$ & & \begin{tabular}{|l} 
Do you \\
unpleas \\
odor wh
\end{tabular} & $\begin{array}{l}\text { usometin } \\
\text { sant, bad } \\
\text { hen noth } \\
\text { (n=16) }\end{array}$ & $\begin{array}{l}\text { imes sman } \\
\text { ad or bur } \\
\text { thing it the } \\
664 \text { ) }\end{array}$ & $\begin{array}{l}\text { mell an } \\
\text { uning } \\
\text { there? }\end{array}$ & $\begin{array}{l}\text { Past } 1 \\
\text { had a } \\
\text { your m } \\
\text { a }\end{array}$ & $\begin{array}{l}12 \text { mont } \\
\text { ataste or } \\
\text { mouth tha } \\
\text { away? }\end{array}$ & $\begin{array}{l}\text { ths, hav, hav } \\
\text { of sensat } \\
\text { hat does } \\
\text { (n=1677) }\end{array}$ & $\begin{array}{l}\text { ive you } \\
\text { ation in } \\
\text { es not go } \\
\text { (7) }\end{array}$ \\
\hline & $\mathrm{n}$ & $\%$ & $\mathrm{n}$ & $\%$ & $\mathrm{n}$ & $\%$ & $\mathrm{n}$ & $\%$ & $\mathrm{n}$ & $\%$ & $\mathrm{n}$ & $\%$ & $\mathrm{n}$ & $\%$ & $\mathrm{n}$ & $\%$ & $\mathrm{n}$ & $\%$ & $\mathrm{n}$ & $\%$ & $\mathrm{n}$ & $\%$ & $\mathrm{n}$ & $\%$ & $\mathrm{n}$ & $\%$ & $\mathrm{n}$ & $\%$ & $\mathrm{n}$ & $\%$ & $\mathrm{n}$ & $\%$ & $\mathrm{n}$ & $\%$ & $\mathrm{n}$ & $\%$ \\
\hline & $\leq 1$ & & $\geq 1$ & & $\leq$ & \begin{tabular}{l|l}
3 & \\
\end{tabular} & $\geq 4$ & \begin{tabular}{l|l}
4 \\
\end{tabular} & $\leq 1$ & 11 & $\geq 12$ & 12 & $\leq 2$ & \begin{tabular}{l|l}
27 & \\
\end{tabular} & $\geq 28$ & \begin{tabular}{|l|l|}
28 & \\
\end{tabular} & $\mathrm{Yes}_{\mathrm{S}}$ & \begin{tabular}{l|l} 
es \\
\end{tabular} & $\mathrm{N}_{0}$ & & Yes & \begin{tabular}{l|l} 
es \\
\end{tabular} & No & & \begin{tabular}{|l|l} 
Yes \\
\end{tabular} & & № & & Yes & & No & & $\mathrm{Ye}$ & Yes & & № \\
\hline Total Population & 193 & 9.83 & 1383 & 90.17 & 218 & 11.88 & 1350 & 88.12 & 321 & 13.72 & 1244 & 86.28 & 227 & 9.00 & 1284 & 91.00 & 274 & 14.47 & 1395 & 85.53 & 3352 & 20.76 & 12417 & \begin{tabular}{|c|}
79.24 \\
\end{tabular} & 259 & 11.88 & 14118 & 88.12 & 123 & 6.42 & 1541 & \begin{tabular}{l|l}
93.58 \\
\end{tabular} & & 6.38 & 1533 & 93.62 \\
\hline Age (years) & & & & & & & & & & & & & & & & & & & & & & & & & & & & & & & & & & & & \\
\hline $60-69$ & 58 & 2.31 & 768 & 50.53 & 71 & 3.40 & 753 & 49.62 & 120 & 3.81 & 699 & 48.88 & 79 & 2.31 & 736 & 51.74 & 124 & 6.55 & 736 & 45.37 & 160 & 10.33 & 659 & 42.25 & 115 & 4.70 & $746 \quad 4$ & 47.25 & 77 & 3.91 & 782 & 48.07 & 82 & 3.66 & 778 & 48.30 \\
\hline $70-79$ & 6 & 3.11 & 411 & 26.55 & 66 & 3.69 & 400 & 25.82 & 116 & 5.41 & 352 & 24.37 & 81 & 3.17 & 366 & 26.37 & 65 & 3.29 & 429 & 26.14 & 94 & 5.56 & 3762 & 24.00 & 52 & 2.24 & $442 \quad 2$ & 27.18 & 30 & 1.75 & 462 & 27.64 & 26 & 1.23 & 468 & 28.20 \\
\hline $80+$ & 75 & 4.41 & 204 & 13.09 & 81 & 4.79 & 197 & 12.67 & 85 & 4.50 & 193 & 13.03 & 67 & 3.52 & 182 & 12.90 & 85 & 4.62 & 230 & 14.01 & 81 & 4.87 & 206 & 12.99 & 92 & 4.95 & 2231 & 13.68 & 16 & 0.76 & 297 & 17.87 & 26 & 1.49 & 287 & 17.12 \\
\hline Race/Ethnicity & & & & & & & & & & & & & & & & & & & & & & & & & & & & & & & & & & & & \\
\hline Hispanic & 53 & 1.46 & 254 & 6.12 & 54 & 1.46 & 251 & 6.11 & 77 & 1.92 & 229 & 5.67 & 86 & 2.25 & 195 & 4.84 & 68 & 1.67 & 252 & 5.99 & 64 & 1.57 & 223 & 5.63 & 74 & 1.73 & 246 & 5.92 & 33 & 0.81 & 285 & 6.82 & 53 & 1.20 & 266 & 6.44 \\
\hline $\mathrm{N}$ & 79 & 6.67 & 682 & 71.19 & 97 & 8.46 & 661 & 69.42 & 97 & 7.65 & 658 & 70.19 & 60 & 4.40 & 686 & 74.38 & 126 & 10.63 & 663 & 66.29 & 161 & 16.07 & 591 & 61.25 & 102 & & 6886 & 69.19 & 47 & & 742 & & 47 & & 743 & 72.73 \\
\hline Non-Hispanic & 39 & 1.06 & 319 & 8.29 & 51 & 1.41 & 306 & 7.97 & 108 & 3.00 & 248 & 6.38 & 64 & 1.88 & 279 & 7.25 & 45 & 1.15 & 336 & 8.38 & 73 & 2.00 & 292 & 7.57 & 49 & 1.26 & 332 & 8.27 & 34 & 0.85 & 345 & 8.66 & 25 & 0.64 & 355 & 8.89 \\
\hline Non-Hispanic Asian/Other & 22 & 0.65 & 128 & 4.56 & 16 & 0.56 & 132 & 4.62 & 39 & 1.15 & 109 & 4.04 & 17 & 0.46 & 124 & 4.52 & 35 & 1.02 & 144 & 4.87 & 37 & 1.12 & 135 & 4.79 & 34 & 1.15 & 145 & 4.74 & 9 & 0.28 & 169 & 5.59 & 9 & 0.32 & 169 & 5.55 \\
\hline Education & & & & & & & & & & & & & & & & & & & & & & & & & & & & & & & & & & & & \\
\hline$<12$ th Grade & 88 & 3.24 & 327 & 13.95 & 90 & 3.49 & 322 & 13.63 & 145 & 5.15 & 268 & 12.06 & 144 & 4.70 & 230 & 11.42 & 105 & 3.94 & 366 & 14.73 & 104 & 4.71 & 330 & 13.55 & $\mid 127$ & 4.38 & $344 \quad 1$ & 14.28 & 55 & 2.17 & 413 & 16.46 & 67 & 2.32 & 402 & 16.32 \\
\hline Completed Hig & 43 & 2.52 & 327 & 21.53 & 53 & 2.99 & 316 & 21.15 & 80 & 3.56 & 289 & 20.47 & 44 & 2.15 & 317 & 22.07 & 54 & 2.55 & 335 & 21.37 & 80 & 4.79 & 290 & 19.28 & 49 & 2.23 & $340 \quad 2$ & 21.68 & 25 & 1.38 & 363 & 22.50 & 24 & 1.22 & 365 & 22.69 \\
\hline$>12$ Grade & 61 & 4.08 & 728 & 54.68 & 75 & 5.41 & 711 & 53.34 & 96 & 5.02 & 686 & 53.75 & 39 & 2.15 & 736 & 57.51 & 113 & 7.90 & 693 & 49.52 & 149 & 11.18 & 620 & 46.49 & 81 & 5.18 & $726 \quad 5$ & 52.25 & 43 & 2.87 & 762 & 54.62 & 43 & 2.85 & 763 & 54.59 \\
\hline & & & & & & & & & & & & & & & & & & & & & & & & & & & & & & & & & & & & \\
\hline Underweight & 1. & & 44 & & 18 & & 39 & 2.58 & 19 & 0.94 & 38 & 2.64 & 17 & 0.82 & 34 & 2.55 & 16 & 0.73 & 50 & 3.03 & 11 & 0.77 & 4 & 2.55 & 3 & 0.95 & 4 & 2.82 & & 0.14 & & 3.62 & & 0.38 & 57 & 3.37 \\
\hline Normal W & 58 & 3.09 & 343 & 23.57 & 59 & 3.61 & 338 & 26.55 & 79 & 3.69 & 316 & 22.89 & 47 & 1.91 & 337 & 24.45 & 88 & 4.73 & 346 & 22.25 & 91 & 6.24 & 3112 & 20.46 & 82 & 4.05 & $352 \quad 2$ & 22.92 & 27 & 1.31 & 405 & 25.66 & 30 & 1.13 & 402 & 25.82 \\
\hline & 60 & 3.20 & 400 & 27.67 & 68 & 3.73 & 388 & 27.07 & 96 & 4.26 & 367 & 26.84 & 70 & 3.08 & 375 & 28.25 & 69 & 3.55 & 418 & 27.36 & 95 & 5.80 & 3662 & 25.23 & 65 & 3.02 & $422 \quad 2$ & 27.87 & 30 & 1.39 & 457 & 29.55 & 35 & .93 & 452 & 28.97 \\
\hline $\mathrm{Ob}$ & 62 & 2.75 & 596 & 36.16 & 73 & 3.56 & 585 & 35.53 & 127 & 4.83 & 523 & 33.91 & 93 & 3.19 & 538 & 35.75 & 101 & 5.46 & 581 & 32.89 & 138 & 7.94 & 519 & 31.00 & 89 & 3.87 & 594 & 34.51 & 63 & 3.59 & 617 & 34.75 & 61 & 2.94 & 622 & 35.46 \\
\hline Alcohol Us & & & & & & & & & & & & & & & & & & & & & & & & & & & & & & & & & & & & \\
\hline & 71 & & 753 & 56.85 & 84 & 5.52 & 738 & & \begin{tabular}{|l|l|}
129 \\
\end{tabular} & 5.91 & 692 & & 82 & 3.48 & 725 & & 130 & 7.85 & 713 & & & 12.96 & 623 & & & 5.58 & $736 \quad 5$ & & & .80 & 778 & & & 43 & 782 & 56.26 \\
\hline No & 12 & 5.77 & 630 & 33.32 & 134 & 6.37 & 612 & 32.70 & 192 & 7.81 & 552 & 31.08 & 145 & 5.51 & 559 & 32.58 & 142 & 6.58 & 682 & 33.77 & 160 & 7.81 & 616 & 32.02 & 49 & 6.26 & 67 & 34.06 & 58 & & 761 & 37.64 & 73 & 1.96 & 749 & 37.35 \\
\hline Ever Smoke & & & & & & & & & & & & & & & & & & & & & & & & & & & & & & & & & & & & \\
\hline Yes & 6 & 3.34 & 538 & 37.97 & 65 & 4.00 & 532 & 37.35 & \begin{tabular}{|l|l|}
110 \\
\end{tabular} & 5.16 & 484 & 36.13 & \begin{tabular}{|l|l} 
& 71 \\
\end{tabular} & 3.04 & 505 & 38.24 & 113 & 6.47 & 50 & 34.39 & 129 & 8.52 & 453 & 32.22 & 94 & 4.57 & 527 & 36.27 & 60 & 3.43 & 560 & 37.47 & & .63 & 571 & 38.22 \\
\hline No & 13 & 6.50 & 843 & 52.19 & 153 & 7.89 & 816 & 50.75 & 211 & 8.57 & 758 & 50.14 & 156 & 5.96 & 778 & 52.76 & 161 & 8.01 & 885 & 51.13 & 206 & 12.25 & 786 & 47.01 & 165 & 7.32 & 88 & 51.83 & 63 & 2.99 & 979 & 56.11 & 85 & 3.76 & 960 & 55.40 \\
\hline Physically $\mathrm{Ac}$ & & & & & & & & & & & & & & & & & & & & & & & & & & & & & & & & & & & & \\
\hline $\mathrm{Ye}$ & 54 & 2.98 & 528 & 37.38 & 60 & 3.68 & 519 & 36.67 & \begin{tabular}{|l}
69 \\
\end{tabular} & 2.9 & 509 & 37.54 & \begin{tabular}{|l|l}
59 \\
\end{tabular} & 1.96 & 506 & 38.81 & 6 & 3.42 & 536 & 36.29 & 118 & 7.74 & 461 & 32.69 & 52 & 2.46 & $547 \quad 3$ & 37.23 & 31 & 2.02 & 568 & 37.73 & 33 & 1.84 & 565 & 37.85 \\
\hline $\mathrm{N}$ & 139 & 6.86 & 855 & 52.78 & 158 & 8.20 & 831 & 51.45 & 252 & 10.82 & 735 & 48.74 & 168 & 7.04 & 778 & 52.19 & 211 & 11.05 & 859 & 49.24 & 217 & 13.02 & 780 & 46.55 & \begin{tabular}{|l|l}
207 \\
\end{tabular} & 9.42 & 864 & 50.89 & 92 & 4.40 & 973 & 55.85 & 101 & 4.54 & 968 & 55.77 \\
\hline Diabe & & & & & & & & & & & & & & & & & & & & & & & & & & & & & & & & & & & & \\
\hline $\mathrm{Yc}$ & 46 & 2.0 & 297 & 15.51 & $5:$ & & 287 & 14.85 & \begin{tabular}{|l|l|}
91 \\
\end{tabular} & & 251 & 14.49 & 70 & & 245 & 14.31 & & 3.73 & 287 & 14.07 & & 385 & 265 & 13.42 & & 376 & 1 & 14.03 & & 2.02 & 331 & 15.78 & & 1.54 & 331 & 16.24 \\
\hline & 13 & 7.2 & 1025 & 71.05 & 150 & & 1005 & 69.89 & 215 & & 936 & 68. & 142 & & 975 & 72.77 & 180 & & 1041 & 67.67 & 246 & 6.46 & 914 & 62.23 & 165 & & 10577 & 70.30 & 75 & & 1142 & 74.22 & 88 & & & 73.71 \\
\hline & 12 & 0.49 & 60 & 3.61 & 13 & 0.60 & 57 & 3.37 & 14 & 0.62 & 57 & 3.45 & 6 & 0.17 & 63 & 3.92 & & 0.27 & 67 & 3.81 & & 0.45 & 61 & 3.59 & & 0.28 & 68 & 3.81 & 8 & 0.51 & 67 & 3.58 & 6 & 0.41 & 69 & 3.67 \\
\hline Blood Pry & & & & & & & & & & & & & & & & & & & & & & & & & & & & & & & & & & & & \\
\hline & 10 & 4.7 & 933 & 63.46 & 127 & & 908 & 61.63 & 19 & 7.92 & 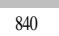 & 60.25 & $\mid 127$ & 4.91 & 869 & 63.51 & 175 & 9.68 & 919 & 58.12 & 219 & 13.91 & 5 & 54.65 & $\mid 161$ & 675 & $934 \quad 6$ & 61.07 & & & 1006 & 63.30 & & 3.57 & 1012 & 64.27 \\
\hline & 87 & 5.04 & 450 & 26.71 & 91 & 5.11 & 442 & 26.48 & 128 & 5.80 & 404 & 26.03 & \begin{tabular}{|l|l}
100 \\
\end{tabular} & 4.09 & 415 & 27.49 & 99 & 4.79 & 476 & 27.41 & 116 & 6.85 & 416 & 24.60 & 98 & 5.13 & $477 \quad 2$ & 27.05 & 39 & 1.94 & 535 & 30.28 & 52 & 2.82 & 521 & 29.35 \\
\hline Myocardial & & & & & & & & & & & & & & & & & & & & & & & & & & & & & & & & & & & & \\
\hline $\mathrm{Ye}$ & 17 & 0. & 83 & & 1 & & 88 & 5.49 & & & 74 & 5.22 & & & 72 & 5.22 & & 1.22 & 82 & 5.12 & & 1.21 & 74 & 5.01 & & & 79 & 4.72 & & 0.84 & o & 5.52 & & 0.47 & 97 & 5.88 \\
\hline № & 176 & 8.97 & 1298 & 84.67 & 206 & 11.00 & 1260 & 82.62 & \begin{tabular}{|l|}
298 \\
\end{tabular} & 12.70 & 1168 & 81.06 & 206 & 8.06 & 1211 & 85.80 & 250 & 13.24 & 1311 & 80.41 & 312 & 19.56 & 11657 & 74.22 & 232 & 10.27 & 1330 & 83.39 & 106 & 5.58 & 1450 & 88.06 & 125 & 5.92 & 1434 & 87.73 \\
\hline Stroke & & & & & & & & & & & & & & & & & & & & & & & & & & & & & & & & & & & & \\
\hline Yes & 2 & 1.1 & 94 & & 23 & 1. & 93 & 5.23 & & & 74 & 4.4 & & 1.53 & 74 & 4.94 & & 1.60 & 99 & 5.43 & & 2.29 & 84 & 4.43 & & 1.92 & 89 & 5.10 & & 0.73 & 117 & 6. & 16 & & 117 & 6.19 \\
\hline $\mathrm{N}$ & 170 & 8.72 & 1285 & 84.75 & 195 & 10.71 & 1253 & 82.86 & 276 & 11.51 & 1169 & 81.96 & 192 & 7.48 & 1207 & 86.05 & 239 & 12.87 & 1293 & 80.11 & 296 & 18.47 & 1154 & 74.81 & 214 & 9.97 & 13198 & 83.01 & 108 & 5.70 & 1420 & 87.29 & 118 & 5.56 & 1412 & 87.42 \\
\hline Coronary $\mathrm{He}$ & & & & & & & & & & & & & & & & & & & & & & & & & & & & & & & & & & & & \\
\hline $\mathrm{Ye}$ & 1 & 0. & 89 & 5.77 & 20 & 1 & 86 & 5.6 & & & 75 & & & & 76 & 5. & & 1.57 & 84 & 5. & & & 78 & 5.19 & & .97 & 80 & 4. & 4 & 0.65 & 103 & 6.26 & 13 & 1.69 & 104 & 6.21 \\
\hline $\mathrm{N}$ & 175 & 8.91 & 1286 & 84.39 & 196 & 10.72 & 1257 & 82.54 & 289 & 12.46 & 1163 & 80.92 & 200 & 7.64 & 1203 & 85.89 & 241 & 12.99 & 1302 & 80.11 & 301 & 19.07 & 11607 & 74.21 & 220 & 9.73 & 13248 & 83.37 & 107 & 5.73 & 1431 & 87.36 & 119 & 5.68 & 1422 & 87.41 \\
\hline Head Tr & & & & & & & & & & & & & & & & & & & & & & & & & & & & & & & & & & & & \\
\hline Yes & 2 & 1. & 139 & 10. & 15 & 0.85 & 143 & 10. & & 0 & 136 & 10 & & 0.72 & 132 & 10.80 & & 53 & 132 & 9. & & .26 & 110 & 8.85 & & 1.70 & 127 & 9.76 & & 1.41 & 143 & & 3 & 1.86 & & 10.60 \\
\hline $\mathrm{N}$ & 173 & 8.82 & 1241 & & 203 & 11.06 & 1204 & & 00 & 12.80 & 1105 & & & 8.30 & 1149 & 80.18 & & 12.97 & 1260 & 55.56 & & 18.46 & 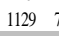 & 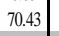 & & & 128 & & & 5.02 & 1395 & & & & & \\
\hline Femalc & & & & & & & & & & & & & & & & & & & & & & & & & & & & & & & & & & & & \\
\hline & 37 & & 582 & 46.48 & & 86 & 566 & 45.22 & 15 & & 541 & 45.35 & & 1.86 & 563 & 47.49 & & 6.20 & 536 & & 120 & 9.81 & 476 & & & 3.42 & 565 & & 40 & 2.66 & 586 & & 44 & 2.85 & 583 & 44.77 \\
\hline No & 151 & 6.90 & 795 & 43.92 & \begin{tabular}{|l|l|}
163 \\
\end{tabular} & 7.82 & 779 & 43.10 & 242 & 9.71 & 697 & 41.13 & 182 & 7.09 & 715 & 43.55 & 180 & 7.98 & 852 & 44.44 & 211 & 10.72 & 758 & 41.34 & \begin{tabular}{|l|l}
191 \\
\end{tabular} & 8.14 & $841 \quad 4$ & 44.26 & \begin{tabular}{|l|}
83 \\
\end{tabular} & 3.80 & 944 & 48.55 & \begin{tabular}{|l|l}
90 \\
\end{tabular} & 3.57 & 939 & 48.81 \\
\hline
\end{tabular}




\begin{tabular}{|c|c|c|c|c|c|c|c|c|c|c|c|c|c|c|c|c|c|c|c|c|c|c|c|c|c|c|c|c|c|c|c|c|c|c|c|c|c|c|c|}
\hline \multirow[t]{2}{*}{$\begin{array}{c}\text { Table 1.la - Descriptive } \\
\text { Statistics --Surrogate brain } \\
\text { health indicators and covariates } \\
\text { for HRT use }\end{array}$} & \multicolumn{4}{|c|}{ Immediate Recall (n=1567) } & \multicolumn{4}{|c|}{ Delayed Recall (n=1560) } & & imal Fluer & ncy $(\mathrm{n}=$ & =1557) & & $\begin{array}{l}\text { tSymbo } \\
\text { Test (I) }\end{array}$ & $\begin{array}{l}\text { S Substit } \\
n=1505)\end{array}$ & & & $\begin{array}{l}\text { ast } 12 \mathrm{n} \\
\text { Setting }\end{array}$ & $\begin{array}{l}\text { month } \\
\text { worse }\end{array}$ & $\begin{array}{l}\mathrm{ns}, \mathrm{mem} \\
\text { ? }(\mathrm{n}=16\end{array}$ & & & $\begin{array}{l}\text { ast } 7 \text { day } \\
\text { embering }\end{array}$ & s, troub $\mathrm{g}$ ? $(\mathrm{n}=1$ & & & $\begin{array}{l}\text { inited } \\
\text { nember }\end{array}$ & $\begin{array}{l}\text { d due to torig } \\
\text { (n=16 }\end{array}$ & $\begin{array}{l}\text { odifficic } \\
\text { ronfu } \\
\text { 602) }\end{array}$ & $\begin{array}{l}\text { culty } \\
\text { usion? }\end{array}$ & & $\begin{array}{l}\text { Ou some } \\
\text { easant, } \\
\text { when no } \\
\text { (n=1 }\end{array}$ & $\begin{array}{l}\text { times sn } \\
\text { ad or bu } \\
\text { thing gis } \\
\text { 6655) }\end{array}$ & $\begin{array}{l}\text { mell an } \\
\text { uning } \\
\text { shere? }\end{array}$ & & $\begin{array}{l}\text { ast } 12 \mathrm{~m} \\
\text { d a taste } \\
\text { ur mouth } \\
\text { awa }\end{array}$ & $\begin{array}{l}\text { te or } \mathrm{s} \\
\text { ht that } \\
\text { yy? }\end{array}$ & $\begin{array}{c}\text { ensatitios } \\
\text { dees n } \\
=1658)\end{array}$ & $\begin{array}{l}\text { e you } \\
\text { tion in } \\
\text { not go } \\
\text { nat }\end{array}$ \\
\hline & $\mathrm{n}$ & $\%$ & $\mathrm{n}$ & $\%$ & $\mathrm{n}$ & $\%$ & $\mathrm{n}$ & $\%$ & $\mathrm{n}$ & $\%$ & $\mathrm{n}$ & $\%$ & $\mathrm{n}$ & $\%$ & $\mathrm{n}$ & $\%$ & $\mathrm{n}$ & $\%$ & $\%$ & $\mathrm{n}$ & $\%$ & $\mathrm{n}$ & $\%$ & $\mathrm{n}$ & $\%$ & $\mathrm{n}$ & & $\%$ & $\mathrm{n}$ & $\%$ & $\mathrm{n}$ & $\%$ & $\mathrm{n}$ & $\%$ & & 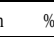 & $\%$ & $\mathrm{n}$ & $\%$ \\
\hline & & 13 & & 14 & & 3 & & 24 & & 811 & & 12 & & 27 & & 28 & & Yes & & $\mathrm{No}$ & & $\mathrm{Y}_{\mathrm{C}}$ & \begin{tabular}{|l|l|l|} 
es & \\
\end{tabular} & & No & & Yes & & $\mathrm{No}$ & & & $\begin{array}{l}\text { Yes } \\
\text {. }\end{array}$ & & No & & Yes & & No & to \\
\hline Total Population & 193 & 9.83 & 1383 & 90.17 & 218 & 11.88 & 1350 & 88.12 & 321 & 13.72 & 1244 & 86.28 & 227 & 9.00 & 1284 & 91.00 & 274 & $\begin{array}{ll}4 & 14.4 \\
4\end{array}$ & 4.471 & 1395 & 85.53 & 335 & 20.76 & 1241 & 79.24 & 259 & $\begin{array}{ll}99 & 11 \\
2\end{array}$ & 11.881 & 1411 & 88.12 & 123 & 6.42 & 1541 & 93.58 & & $34 \quad 6.3$ & & & 93.62 \\
\hline Age (years) & & & & & & & & & & & & & & & & & & & & & & & & & & & & & & & & & & & & & & & \\
\hline $60-69$ & 58 & 2.31 & 768 & 50.53 & 71 & 3.40 & 753 & 49.62 & 120 & 3.81 & 699 & 48.88 & 79 & 2.31 & 736 & 51.74 & 124 & & 6.55 & 736 & 45.37 & 160 & 10.33 & 659 & 42.25 & 115 & & 4.70 & 746 & 47.25 & 77 & 3.91 & 782 & 48.07 & 82 & & 667 & 778 & 48.30 \\
\hline $70-79$ & 60 & 3.11 & 411 & 26.55 & 66 & 3.69 & 400 & 25.82 & 116 & 5.41 & 352 & 24.37 & 81 & 3.17 & 366 & 26.37 & 65 & & 3.29 & 429 & 26.14 & 94 & 5.56 & 376 & 24.00 & 52 & & 2.24 & 442 & 27.18 & 30 & 1.75 & 462 & 27.64 & 26 & 1.2 & .23 & 468 & 28.20 \\
\hline $80+$ & 75 & 4.41 & 204 & 13.09 & 81 & 4.79 & 197 & 12.67 & 85 & 4.50 & 193 & 13.03 & 67 & 3.52 & 182 & 12.90 & 85 & & 1.62 & 230 & 14.01 & 81 & 4.87 & 206 & 12.99 & 92 & & 4.95 & 223 & 13.68 & 16 & 0.76 & 297 & 17.87 & 26 & 6 & 49 & 287 & 17.12 \\
\hline Race/Ethnicity & & & & & & & & & & & & & & & & & & & & & & & & & & & & & & & & & & & & & & & \\
\hline & & 1.46 & 254 & 6.12 & 54 & 1.46 & 251 & 6.11 & 77 & 1.92 & 229 & 5.67 & 86 & 2.25 & 195 & 4.84 & \begin{tabular}{|c|}
68 \\
68
\end{tabular} & & 1.67 & 252 & 5.99 & 64 & 1.57 & 223 & 5.63 & 74 & & 1.73 & 246 & 5.92 & 33 & 0.81 & 285 & 6.82 & 53 & 1.2 & 20 & 266 & 6.44 \\
\hline Non-Hispanic White & 79 & 6.67 & 682 & 71.19 & 97 & 8.46 & 661 & 69.42 & 97 & 7.65 & 658 & 70.19 & 60 & 4.40 & 686 & 74.38 & 126 & & 0.63 & 663 & 66.29 & 161 & 16.07 & 591 & 61.25 & 102 & & 7.74 & 688 & 69.19 & 47 & 4.48 & 742 & 72.51 & 47 & & 23 & 743 & 72.73 \\
\hline Non-Hispanic Bla & 39 & 1.06 & 319 & 8.29 & 51 & 1.41 & 306 & 7.97 & 108 & 3.00 & 248 & 6.38 & 64 & 1.88 & 279 & 7.25 & 45 & & 1.15 & 336 & 8.38 & 73 & 2.00 & 292 & 7.57 & 49 & & 1.26 & 332 & 8.27 & 34 & 0.85 & 345 & 8.66 & 25 & 0.6 & 64 & 355 & 8.89 \\
\hline Non-Hispanic & 22 & 0.65 & 128 & 4.56 & 16 & 0.56 & 132 & 4.62 & 39 & 1.15 & 109 & 4.04 & 17 & 0.46 & 124 & 4.52 & 35 & & 1.02 & 144 & 4.87 & 37 & 1.12 & 135 & 4.79 & 34 & & 1.15 & 145 & 4.74 & & 0.28 & 169 & 5.59 & & & 32 & 169 & 5.55 \\
\hline Education & & & & & & & & & & & & & & & & & & & & & & & & & & & & & & & & & & & & & & & \\
\hline$<12$ th Grac & & 3.24 & 327 & 13.95 & 90 & 3.49 & 322 & 13.63 & $\mid 145$ & 5.15 & 268 & 12.06 & $\mid 144$ & 4.70 & 230 & 11.42 & \begin{tabular}{|l|l} 
\\
\end{tabular} & & 3.94 & 366 & 14.73 & 104 & 4.71 & 330 & 13.55 & 127 & & 4.38 & 344 & 14.28 & 55 & 2.17 & 413 & 16.46 & & & 32 & 402 & 16.32 \\
\hline Completed High Scho & 43 & 2.52 & 327 & 21.53 & 53 & 2.99 & 316 & 21.15 & \begin{tabular}{|l}
80 \\
80
\end{tabular} & 3.56 & 289 & 20.47 & 44 & 2.15 & 317 & 22.07 & 54 & & 2.55 & 335 & 21.37 & 80 & 4.79 & 290 & 19.28 & 49 & & 2.23 & 340 & 21.68 & 25 & 88 & 363 & 22.50 & 24 & 4 & .22 & 365 & 22.69 \\
\hline$>12$ Grade & 61 & 4.08 & 728 & 54.68 & 75 & 5.41 & 711 & 53.34 & 96 & 5.02 & 686 & 53.75 & 39 & 2.15 & 736 & 57.51 & 113 & & 190 & 693 & 49.52 & 149 & 11.18 & 620 & 46.49 & 81 & & 5.18 & 726 & 52.25 & 43 & 2.87 & 762 & 54.62 & & & .85 & 763 & 54.59 \\
\hline BMI $(\mathrm{kg} / \mathrm{m} 2)$ & & & & & & & & & & & & & & & & & & & & & & & & & & & & & & & & & & & & & & & \\
\hline Underweight $(<18.5)$ & & 0.80 & 44 & 2.76 & 18 & 0.99 & 39 & 2.58 & 19 & 0.94 & 38 & 2.64 & 17 & 0.82 & 34 & 2.55 & 16 & & 0.73 & 50 & 3.03 & 11 & 0.77 & 45 & 2.55 & 23 & & 0.95 & 43 & 2.82 & & 0.14 & 62 & 3.62 & & & 38 & 57 & 3.37 \\
\hline Normal W & 58 & 3.09 & 343 & 23.57 & 59 & 3.61 & 338 & 26.55 & 79 & 3.69 & 316 & 22.89 & 47 & 1.91 & 337 & 24.45 & 88 & & 4.73 & 346 & 22.25 & 91 & 6.24 & 311 & 20.46 & 82 & & 4.05 & 352 & 22.92 & 27 & 1.31 & 405 & 25.66 & & 0 & 13 & 402 & 25.82 \\
\hline rweight (25.0 & 60 & 3.20 & 400 & 27.67 & 68 & 3.73 & 388 & 27.07 & 96 & 4.26 & 367 & 26.84 & 70 & 3.08 & 375 & 28.25 & 69 & & 3.55 & 418 & 27.36 & 95 & 5.80 & 366 & 25.23 & 65 & & 3.02 & 422 & 27.87 & 30 & 1.39 & 457 & 29.55 & 35 & & 93 & 452 & 28.97 \\
\hline Obese 300. & 62 & 2.75 & 596 & 36.16 & 73 & 3.56 & 585 & 35.53 & 127 & 4.83 & 523 & 33.91 & 93 & 3.19 & 538 & 35.75 & 101 & & 5.46 & 581 & 32.89 & 138 & 7.94 & 519 & 31.00 & 89 & & 3.87 & 594 & 34.51 & 63 & 3.59 & 617 & 34.75 & 61 & & 94 & 622 & 35.46 \\
\hline & & & & & & & & & & & & & & & & & & & & & & & & & & & & & & & & & & & & & & & \\
\hline Ye. & & & 753 & 56.85 & 8 & 5.52 & 738 & 55.41 & $\mid 129$ & & 692 & 55.20 & & & 72. & 58.42 & 130 & & 7.85 & 713 & 51.81 & 175 & 12.96 & 623 & 47.21 & 108 & & 5.58 & 736 & 54.10 & & & 778 & 55.94 & & & & 782 & 56.26 \\
\hline No & 12 & 5.77 & 630 & 33.32 & 134 & 6.37 & 612 & 32.70 & 192 & 7.81 & 552 & 31.08 & 145 & 5.51 & 559 & 32.58 & $142 \mathrm{z} x$ & & 6.58 & 682 & 33.77 & 160 & 7.81 & 616 & 32.02 & 145 & & 6.26 & 675 & 34.06 & 58 & 2.62 & 761 & 37.64 & & & 96 & 749 & 37.35 \\
\hline Ever Smok & & & & & & & & & & & & & & & & & & & & & & & & & & & & & & & & & & & & & & & \\
\hline Yes & & 3.34 & 538 & 37.97 & 65 & 4.00 & 532 & 37.35 & & & 484 & 36.13 & & & 505 & 38.24 & 113 & & 6.47 & 508 & 34.39 & 12 & .5. & 453 & 32.22 & & & 4.57 & 527 & 36.27 & & 3.43 & 560 & 37.47 & & & & 571 & 38.22 \\
\hline № & & 6.50 & 843 & 52.19 & 153 & 7.89 & 816 & 50.75 & 211 & 8.57 & 758 & 50.14 & 156 & 5.96 & 778 & 52.76 & 16 & & .01 & 885 & 51.13 & 206 & 12.25 & 786 & 47.01 & 16. & & 7.32 & 882 & 51.8 & 63 & 2.99 & 979 & 56.11 & & & 76 & 960 & 55.40 \\
\hline Physically Active? & & & & & & & & & & & & & & & & & & & & & & & & & & & & & & & & & & & & & & & \\
\hline Ye & & & 52 & 37.38 & & & 519 & 36.67 & & & 509 & 37.54 & & & 50 & 38.8 & & & 3.42 & 536 & 36.29 & 18 & 7.74 & 461 & 32.65 & & & 2.46 & 547 & 37.2 & & 2.02 & 568 & 37.73 & & & .84 & 565 & 37.85 \\
\hline No & 13 & 6.86 & 855 & 52.78 & 158 & 8.20 & 831 & 51.45 & 252 & 10.82 & 735 & 48.74 & 168 & 7.04 & 778 & 52.19 & 211 & & 1.05 & 859 & 49.24 & 217 & 13.02 & 780 & 46.5 & 207 & & 9.42 & 864 & & 92 & 4.40 & 973 & 55.85 & 101 & & 54 & 968 & 55.77 \\
\hline Diabetes & & & & & & & & & & & & & & & & & & & & & & & & & & & & & & & & & & & & & & & \\
\hline $\mathrm{Y}$ & & & & & & & & 14.85 & & & & & & & & 14. & & & & & 14.07 & & & & & & & & & & & & & & & & & & 16.24 \\
\hline $\mathrm{N}$ & 13 & & 1025 & 71.05 & 150 & 8.49 & 1005 & 69.89 & 215 & 9.91 & 936 & 68.37 & 142 & 5.75 & 975 & 72.77 & 180 & & 0.441 & 1041 & 67.67 & 246 & 16.46 & 914 & 62.23 & 16. & & 7.82 & 1057 & 70.30 & 75 & 3.89 & 1142 & 74.22 & & & 43 & 1132 & 73.71 \\
\hline & 12 & 0.49 & 60 & 3.61 & 13 & 0.60 & 57 & 3.37 & 14 & 0.62 & 57 & 3.45 & 6 & 0.17 & 63 & 3.92 & & & .27 & 67 & 3.81 & 11 & 0.45 & 61 & 3.59 & & & 0.28 & 68 & 3.81 & 8 & 0.51 & 67 & 3.58 & & 0.4 & 41 & 69 & 3.67 \\
\hline & & & & & & & & & & & & & & & & & & & & & & & & & & & & & & & & & & & & & & & \\
\hline & & & 933 & 63.46 & 12 & 6.7 & 908 & 61.63 & & & 840 & 60.25 & & & 869 & 63.5 & 175 & & .68 & 919 & 58.12 & & 13.91 & 825 & 54.6 & 161 & & 6.75 & 934 & & & 4.48 & 1006 & 63.30 & & & & 1012 & 64.27 \\
\hline & 87 & 5.04 & 450 & 26.71 & 91 & 5.11 & 442 & 26.48 & 128 & 5.80 & 404 & 26.03 & 100 & 4.09 & 415 & 27.4 & \begin{tabular}{|l}
99 \\
99
\end{tabular} & & 4.79 & 476 & 27.41 & 116 & 6.85 & 416 & 24.60 & 98 & & 5.13 & 477 & 27.05 & 39 & 1.94 & 535 & 30.28 & 52 & 2 & 82 & 521 & 29.35 \\
\hline Myocardial & & & & & & & & & & & & & & & & & & & & & & & & & & & & & & & & & & & & & & & \\
\hline $\mathrm{Ye}$ & & & 83 & 5.4 & 12 & & 88 & 5.4 & & & 74 & 5.2 & & & 72 & 5.22 & & & 1.23 & 82 & 5.12 & & & 74 & 5.01 & & & 1.62 & 79 & 4.7 & & 0.84 & 89 & 5.52 & & & .47 & 97 & 5.88 \\
\hline & 176 & 8.97 & 1298 & 84.67 & 206 & 11.00 & 1260 & 82.62 & 298 & 12.70 & 1168 & 81.06 & 206 & 8.06 & 1211 & 85.80 & 250 & & 3.24 & 1311 & 80.41 & 312 & 19.56 & 1165 & 74.22 & 232 & 32 & 10.27 & 1330 & 83.39 & 106 & 5.58 & 1450 & 88.06 & 125. & 25 & 92 & 14348 & 87.73 \\
\hline Stroke & & & & & & & & & & & & & & & & & & & & & & & & & & & & & & & & & & & & & & & \\
\hline Yes & & & 94 & 5.4 & & 1.2 & 93 & 5.2 & & & 74 & & & & 74 & 4.9 & & & & 99 & 5.4 & & & & 4.43 & & & & & & & & & & & & .84 & & 6.19 \\
\hline No & 17 & 8.72 & 1285 & 84.75 & 195 & 10.71 & 1253 & 82.86 & 276 & 11.51 & 1169 & 81.96 & 1 & 7.48 & 1207 & 86.0. & 239 & $\begin{array}{ll}99 & 12.8\end{array}$ & 2.87 & 1293 & 80.11 & 296 & 18.47 & 1154 & 74.8 & 214 & 14 & 9.97 & 1319 & 83.01 & 108 & 5.70 & 142 & 87.29 & 118 & 18 & .56 & 1412 & 87.42 \\
\hline Coronary $\mathrm{He}$ & & & & & & & & & & & & & & & & & & & & & & & & & & & & & & & & & & & & & & & \\
\hline $\mathrm{Ye}$ & & 0.9 & 89 & 5.7 & 20 & 1.1 & 86 & 5.6 & & 1.2 & 75 & & & 1.13 & 76 & & & & & & & & & & 5.19 & & & 1.9 & & 4.94 & & 0.65 & & 6.26 & & & 69 & & 6.21 \\
\hline $\mathrm{N}$ & 17 & 8.91 & 1286 & 84.39 & 196 & 10.72 & 1257 & 82.54 & 289 & 12.46 & 1163 & 80.92 & 200 & 7.64 & 1203 & 85.85 & $\mid 241$ & & 2.991 & 1302 & 80.11 & 301 & 19.07 & 1160 & 74.21 & & & 9.73 & 1324 & 83.37 & 107 & 5.73 & 1431 & 87.36 & 115 & 19 & 68 & 1422 & 87.41 \\
\hline Head Trau & & & & & & & & & & & & & & & & & & & & & & & & & & & & & & & & & & & & & & & \\
\hline $\mathrm{Ye}$ & 2 & 1.0 & 139 & 10.67 & 1. & 0.8 & 143 & 10.85 & 2 & 0.5 & 136 & 10.73 & & 0.72 & 132 & 10.80 & & & 1.53 & 13 & 9.93 & & 2.2 & 110 & 8.85 & & & 1.70 & 127 & 9.76 & & 1.41 & 43 & 10.04 & & 0.8 & 86 & 15 & 10.60 \\
\hline $\mathrm{N}$ & 173 & 8.82 & 1241 & 79.47 & 203 & 11.06 & 1204 & 77.24 & 300 & 12.80 & 1105 & 75.51 & 212 & 8.30 & 1149 & 80.18 & 242 & & 2.971 & 1260 & 75.56 & 295 & 18.46 & 1129 & 70.4 & 222 & 22 & 10.21 & 1281 & 78.33 & 103 & 5.02 & 1395 & 83.52 & 12 & 21 & 54 & 1379 & 83.00 \\
\hline Ever Used Fem & & & & & & & & & & & & & & & & & & & & & & & & & & & & & & & & & & & & & & & \\
\hline $\mathrm{Ye}$ & 37 & 2.6 & 582 & 46.48 & 50 & 3.8 & 566 & 45.22 & 1. & 3. & ${ }^{341}$ & 45.35 & 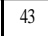 & & 50. & 47.49 & & 0 & & 53 & 41.38 & 20 & & 476 & 38.13 & 62 & 2 & 3.42 & 65 & 44.19 & 40 & 2.66 & 86 & 45.00 & 44 & 4 & 85 & 583 & 44.77 \\
\hline $\mathrm{N}$ & 15 & 6.90 & 795 & 43.92 & 163 & 7.82 & 779 & 4310 & ? & 9. & 697 & & 182 & 7.09 & 715 & 435 & & & & & & & 10.72 & 759 & 413 & & & 14 & & & 83 & 3.80 & 44 & & & 10 & & 39 & 48.81 \\
\hline
\end{tabular}




\section{Associations Between Exposures to Past OC and HRT use and Cognitive Test}

\section{Scores}

Exposures OC and HRT and four cognitive scores (immediate and delayed recall, animal fluency, and DSST score) are summarized in tables 1.2 to 1.13 . The cognitive test scores have been used as a surrogate indicator of brain health to assess cognitive decline and the possible development of mild cognitive impairment, dementia, and/or AD elderly patients as part of neuropsychological testing.

Immediate Recall Scores and Exposure to OC and HRT: Tables 1.2 and 1.3

present chi-square results for immediate recall scores and OC use and HRT use. We observed a significant relationship exists between OC use and HRT use with respect to immediate recall scores.

\begin{tabular}{|c|c|c|}
\hline \multicolumn{3}{|c|}{ Chi Square Results } \\
\hline \multirow{2}{*}{ Table 1.2 Immediate Recall Cut-off Scores } & Yes & OC use? \\
\cline { 2 - 3 } & $63(3.35 \%)$ & $130(6.48 \%)$ \\
\hline 13 & $865(60.83 \%)$ & $518(29.34 \%)$ \\
\hline$\left.\chi^{2}(\mathrm{df}=1, \mathrm{~N}=1576) \mathrm{F}=72.45, \mathrm{p}<0.0001\right)$ \\
\hline \multicolumn{2}{|c|}{ The relationship is significant. } \\
\hline
\end{tabular}

\begin{tabular}{|c|c|c|}
\hline \multicolumn{3}{|c|}{ Chi Square Results } \\
\hline \multirow{2}{*}{ Table 1.3 Immediate Recall Cut-off Scores } & Yes & HRT use? \\
\cline { 2 - 3 } & $37(2.69 \%)$ & $152(6.91 \%)$ \\
\hline 13 & $583(46.60 \%)$ & $795(43.81 \%)$ \\
\hline$\chi 2$ & $(\mathrm{df}=1, \mathrm{~N}=1567) \mathrm{F}=35.55, \mathrm{p}<0.0001)$ \\
\hline \multicolumn{2}{|c|}{ The relationship is significant. } \\
\hline
\end{tabular}

Table 1.4 presents the ORs and 95\% CIs for OC and HRT use and immediate recall scores. In immediate recall scores and OC use, it was observed that OC use was associated with lower odds of a low immediate recall score in the unadjusted model, adjusted model \#1, and adjusted model \#2. 
In immediate recall scores and HRT use it was observed that HRT use was also associated with lower odds of a low immediate recall score in the adjusted model, adjusted model \#1, and adjusted model \#2.

\begin{tabular}{|c|c|c|c|c|c|c|c|c|c|}
\hline EEDC & $\leq 13 / \geq 14$ & $\begin{array}{c}\text { Unadjusted Odds } \\
\text { Ratio }^{1}\end{array}$ & $95 \% \mathrm{CI}$ & $\leq 13 / \geq 14$ & $\begin{array}{c}\text { Adjusted Odds Ratio } \\
\# 1^{2}\end{array}$ & $95 \% \mathrm{CI}$ & $\leq 13 / \geq 14$ & 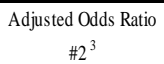 & $95 \% \mathrm{CI}$ \\
\hline OC use & $193 / 1383$ & $0.249^{* * *}$ & $0.175-0.356$ & 193/1382 & $0.487^{* * * *}$ & $0.343-0.692$ & $192 / 1364$ & $0.521^{* *}$ & $0.358-0.760$ \\
\hline HRT use & 189/1378 & 0.366 *** & $0.252-0.530$ & 189/1377 & $0.507 * *$ & $0.332-0.773$ & $187 / 1359$ & $0.517^{* *}$ & $0.340-0.785$ \\
\hline
\end{tabular}

Delayed Recall Scores and Exposure to OC and HRT: Tables 1.5 and 1.6 present

chi-square results for delayed recall scores and OC use and HRT use. We observed a significant relationship exists between OC use and HRT use with respect to delayed recall scores.

\begin{tabular}{|c|c|c|}
\hline \multicolumn{3}{|c|}{ Chi Square Results } \\
\hline \multirow{2}{*}{ Table 1.5 Delayed Recall Cut-off Scores } & \multicolumn{2}{|c|}{ OCuse? } \\
\hline & Yes & No \\
\hline$\leq 3$ & $82(4.71 \%)$ & $136(7.18 \%)$ \\
\hline$\geq 4$ & $842(59.46 \%)$ & $506(28.66 \%)$ \\
\hline \multicolumn{3}{|c|}{$\left.\chi^{2}(\mathrm{df}=1, \mathrm{~N}=1568) \mathrm{F}=30.53, \mathrm{p}<0.0001\right)$} \\
\hline \multicolumn{3}{|c|}{ The relationship is significant. } \\
\hline
\end{tabular}

\begin{tabular}{|c|cc|}
\hline \multicolumn{3}{|c|}{ Chi Square Results } \\
\hline \multirow{2}{*}{ Table 1.6 Delayed Recall Cut-off Scores } & \multicolumn{3}{|c|}{ HRT use? } \\
\cline { 2 - 3 } & Yes & No \\
\hline$\leq 3$ & $50(3.85 \%)$ & $163(7.80 \%)$ \\
$\geq 4$ & $567(45.34 \%)$ & $780(43.01 \%)$ \\
\hline$\left.\chi^{2}(\mathrm{df}=1, \mathrm{~N}=1560) \mathrm{F}=13.56, \mathrm{p}<0.0008\right)$ \\
\hline \multicolumn{2}{|c|}{ The relationship is significant. } \\
\hline
\end{tabular}

Table 1.7 presents the ORs and 95\% CIs for OC and HRT use and delayed recall scores. In delayed recall scores and OC use, it was observed that OC use was associated with lower odds of a low delayed recall score in the unadjusted model, adjusted model \#1, and adjusted model \#2. 
In immediate recall scores and HRT use it was observed that HRT use was also associated with lower odds of a low delayed recall score in the adjusted model and adjusted model \#1.

\begin{tabular}{|c|c|c|c|c|c|c|c|c|c|}
\hline EEDC & $\leq 3 / \geq 4$ & $\begin{array}{l}\text { Unadjusted Odds } \\
\text { Ratio }^{1}\end{array}$ & $95 \% \mathrm{CI}$ & $\leq 3 / \geq 4$ & \begin{tabular}{|c|} 
Adjusted Odds Ratio \\
$\# 1^{2}$ \\
\end{tabular} & $95 \% \mathrm{CI}$ & $\leq 3 / \geq 4$ & \begin{tabular}{|c|} 
Adjusted Odds Ratio \\
$\# 2^{3}$ \\
\end{tabular} & $95 \% \mathrm{CI}$ \\
\hline OCuse & $218 / 1350$ & $0.316 * * *$ & $0.202-0.495$ & $218 / 1349$ & $0.538 *$ & $0.323-0.895$ & $216 / 1332$ & $0.560 *$ & $0.323-0.971$ \\
\hline HRT use & 213/1347 & $0.468 * * *$ & $0.306-0.717$ & $213 / 1346$ & $0.609 *$ & 0.389-0.954 & $211 / 1328$ & 0.621 & $0.383-1.007$ \\
\hline & & $\begin{array}{r}\text { n, race/et } \\
\text { heart }\end{array}$ & $\begin{array}{l}\text { Adjus } \\
\text { tatus, s }\end{array}$ & $\begin{array}{l}\text { e, educatior } \\
\text { s, physical }\end{array}$ & $\begin{array}{l}.01 * * * p<0.001 \\
\text { elethnicity, alcohol use, } \\
\text { ity status, smoking statu }\end{array}$ & $\begin{array}{l}\text { diabetes sta } \\
\text { Is, head trau }\end{array}$ & $\begin{array}{l}\text { h blood pre } \\
\text { ss. }\end{array}$ & us, coronary heart dis & ease status, \\
\hline
\end{tabular}

Animal Fluency Scores and Exposure to OC and HRT: Tables 1.8 and 1.9 present chi-square results for animal fluency scores and OC use and HRT use. We observed a significant relationship exists between OC use and HRT use with respect to animal fluency scores.

\begin{tabular}{|c|cc|}
\hline \multicolumn{3}{|c|}{ Chi Square Results } \\
\hline \multirow{2}{*}{ Table 1.8 Animal Fluency Cut-off Scores } & \multicolumn{2}{c|}{ OCuse? } \\
\cline { 2 - 3 } & \multicolumn{1}{|c|}{ Yes } & $180(8.01 \%)$ \\
\hline 11 & $141(5.71 \%)$ & $464(27.88 \%)$ \\
\hline$\left.\chi^{2}(\mathrm{df}=1, \mathrm{~N}=1565) \mathrm{F}=39.92, \mathrm{p}<0.0001\right)$ \\
\hline \multicolumn{2}{|c|}{ The relations hip is significant. } \\
\hline
\end{tabular}

\begin{tabular}{|c|cc|}
\hline \multicolumn{3}{|c|}{ Chi Square Results } \\
\hline \multirow{2}{*}{ Table 1.9 Animal Fluency Cut-off Scores } & Yes & HRT use? \\
\cline { 2 - 3 } & \multicolumn{1}{|c|}{ No } \\
\hline 11 & $55(3.80 \%)$ & $243(9.71 \%)$ \\
\hline$\chi 2$ & $542(45.46 \%)$ & $697(41.03 \%)$ \\
\hline \multicolumn{2}{|c|}{ The relationship is significant. } \\
\hline
\end{tabular}

Table 1.10 presents the ORs and 95\% CIs for OC and HRT use and animal fluency scores. In animal fluency scores and OC use, it was observed that OC use was associated with lower odds of a low animal fluency score in the unadjusted model, adjusted model \#1, and adjusted model \#2. In animal fluency scores and HRT use it 
was observed that HRT use was also associated with lower odds of a low animal fluency score in the adjusted model, adjusted model \#1, and adjusted model \#2.

\begin{tabular}{|c|c|c|c|c|c|c|c|c|c|}
\hline \multicolumn{10}{|c|}{ Table 1.10 - ORs and 95\% CIfor OC and HRT Use Levels by Animal Fuency Cut-off Scores for Female Subjects >=60 years of age, NHANES 2011-2014 } \\
\hline EEDC & $\leq 11 / \geq 12$ & $\begin{array}{l}\text { Unadjusted Odds } \\
\text { Ratio }^{1}\end{array}$ & $95 \% \mathrm{CI}$ & $\leq 11 / \geq 12$ & $\begin{array}{c}\text { Adjusted Odds Ratio } \\
\# 1^{2}\end{array}$ & $95 \% \mathrm{CI}$ & $\leq 11 / \geq 12$ & $\begin{array}{c}\text { Adjusted Odds Ratio } \\
\# 2^{3}\end{array}$ & $95 \% \mathrm{CI}$ \\
\hline OCuse & $321 / 1244$ & $0.340 * * *$ & $0.238-0.487$ & $321 / 1243$ & $0.606 *$ & $0.385-0.952$ & $314 / 1231$ & $0.595^{*}$ & $0.369-0.958$ \\
\hline HRT use & $318 / 1239$ & $0.353 * * *$ & $0.266-0.468$ & $318 / 1238$ & $0.533^{* * *}$ & $0.375-0.758$ & $310 / 1226$ & $0.523 * *$ & $0.362-0.756$ \\
\hline${ }^{1}$ Unadjuste & djusted & $\begin{array}{r}\text { ation, race/ethr } \\
\text { heart at }\end{array}$ & $\begin{array}{l}{ }^{3} \text { Adjustec } \\
\text { status, strol }\end{array}$ & ${ }^{*} p<0.05,{ }^{*}$ & thnicity, alcohol use, & liabetes stat & blood pres & tus, coronary heart d & ase status, \\
\hline
\end{tabular}

\section{Digit Symbol Substitution Test (DSST) Scores and Exposure to OC and HRT:}

Tables 1.11 and 1.12 present chi-square results for DSST scores and OC use and HRT use. We observed a significant relationship exists between OC use and HRT use with respect to DSST scores.

\begin{tabular}{|c|cc|}
\hline \multicolumn{2}{|c|}{ Chi Square Results } \\
\hline \multirow{2}{*}{ Table 1.11 DSST Cut-off Scores } & Yes & OC use? \\
\cline { 2 - 3 } & $83(2.83 \%)$ & $144(6.17 \%)$ \\
$\geq 28$ & $834(62.57 \%)$ & $450(28.44 \%)$ \\
\hline$\left.\chi^{2}(\mathrm{df}=1, \mathrm{~N}=1511) \mathrm{F}=103.90, \mathrm{p}<0.0001\right)$ \\
\hline \multicolumn{2}{|c|}{ The relationship is significant. } \\
\hline
\end{tabular}

\begin{tabular}{|c|c|c|}
\hline \multicolumn{3}{|c|}{ Chi Square Results } \\
\hline \multirow{2}{*}{ Table 1.12 DSST Cut-off Scores } & \multicolumn{2}{|c|}{ HRT use? } \\
\hline & Yes & No \\
\hline$\leq 27$ & $43(1.86 \%)$ & $183(7.10 \%)$ \\
\hline$\geq 28$ & $564(47.61 \%)$ & $715(43.44 \%)$ \\
\hline \multicolumn{3}{|c|}{$\left.\chi^{2}(\mathrm{df}=1, \mathrm{~N}=1505) \mathrm{F}=32.64, \mathrm{p}<0.0001\right)$} \\
\hline \multicolumn{3}{|c|}{ The relationship is significant. } \\
\hline
\end{tabular}

Table 1.13 presents the ORs and 95\% CIs for OC and HRT use and DSST scores. In DSST scores and OC use, it was observed that OC use was associated with lower odds of a low DSST scores in the unadjusted model, adjusted model \#1, and adjusted model \#2. In DSST scores and HRT use it was observed that HRT use was also associated with lower odds of a low DSST score in the adjusted model, adjusted model \#1, and adjusted model \#2. 


\begin{tabular}{|c|c|c|c|c|c|c|c|c|c|}
\hline \multicolumn{10}{|c|}{ Table 1.13 - 0Rs and 95\% CI for 0C and HRT Use Levels by DSST Cut-off Scores for Female Subjects $>=60$ years of age, NHANES 2011-2014 } \\
\hline EEDC & $\leq 27 / \geq 28$ & $\begin{array}{c}\text { Unadjusted Odds } \\
\text { Ratio }^{1} \\
\end{array}$ & $95 \% \mathrm{Cl}$ & $\leq 27 / \geq 28$ & $\begin{array}{c}\text { Adjusted Odds Ratio } \\
\# 1^{2} \\
\end{array}$ & $95 \% \mathrm{Cl}$ & $\leq 27 / \geq 28$ & $\begin{array}{c}\text { Adjusted Odds Ratio } \\
\# 2^{3} \\
\end{array}$ & $95 \% \mathrm{Cl}$ \\
\hline OCuse & $227 / 1284$ & $0.208 * *$ & $0.150-0.289$ & $227 / 1283$ & $0.401 * * *$ & $0.262-0.614$ & $222 / 1271$ & $0.411 * * *$ & $0.258-0.656$ \\
\hline HRT use & 22611279 & $0.239 * * *$ & $0.143-0.401$ & $226 / 1278$ & 0.419 ** & $0.234-0.749$ & $220 / 1266$ & $0.434 *$ & $0.231-0.813$ \\
\hline \multicolumn{10}{|c|}{${ }^{*} \mathrm{p}<0.05,{ }^{*}{ }^{*} \mathrm{p}<0.01 * * * \mathrm{p}<0.001$} \\
\hline
\end{tabular}

\section{Associations Between Exposures to OC and HRT and Memory Function}

Exposures to OCs and HRTs and three memory function indicators: 1) During

the past 12 months, have you experienced confusion or memory loss that is happening more often or getting worse? 2) During the past 7 days, how often have you had trouble remembering where you put things? 3) Are you limited in any way because of difficulty remembering or because you experience periods of confusion?) are summarized in tables 1.14 to 1.22 . Memory function have been included as a surrogate indicator of brain health as declining memory function can indicate the development of mild cognitive impairment, dementia, $\mathrm{AD}$, or other memory-related neurodegenerative disease.

Worsening memory past 12 months and Exposure to OC and HRT: Tables 1.14 and 1.15 present chi-square results for the $\mathrm{Y} / \mathrm{N}$ question "During the past 12 months, you experienced confusion or memory loss that is happening more often or is getting worse?" and OC use and HRT use. We observed a significant relationship does not exists between OC use and HRT use with respect to experiencing confusion or memory loss in the past 12 months, that is happening more often or getting worse. 


\begin{tabular}{|c|c|c|}
\hline \multicolumn{3}{|c|}{ Chi Square Results } \\
\hline Table 1.14 During the past 12 months, have & \multicolumn{2}{c|}{ OC use? } \\
\cline { 2 - 3 } $\begin{array}{c}\text { you experienced confusion or memory los } \\
\text { that is happening more often or getting } \\
\text { worse? }\end{array}$ & Yes & No \\
\hline Yes & $132(8.03 \%)$ & $142(6.43 \%)$ \\
\hline No & $819(54.71 \%)$ & $576(30.82 \%)$ \\
\hline \multicolumn{2}{|c|}{$\left.\chi^{2}(\mathrm{df}=1, \mathrm{~N}=1669) \mathrm{F}=0.06, \mathrm{p}=\mathrm{n} . \mathrm{s}.\right)$} \\
\hline \multicolumn{2}{|c|}{ The relationship is not significant. } \\
\hline
\end{tabular}

\begin{tabular}{|c|c|c|}
\hline \multicolumn{3}{|c|}{ Chi Square Results } \\
\hline $\begin{array}{c}\text { Table 1.15 During the past 12 months, have } \\
\text { you experienced confusion or memory loss } \\
\text { that is happening more often or getting } \\
\text { worse? }\end{array}$ & Yes & HRT use? \\
\hline Yes & $90(6.18 \%)$ & $182(8.00 \%)$ \\
\hline No & $537(41.50 \%)$ & $852(44.32 \%)$ \\
\hline \multicolumn{2}{|c|}{$\left.\chi^{2}(\mathrm{df}=1, \mathrm{~N}=1661) \mathrm{F}=1.44, \mathrm{p}=\mathrm{n} . \mathrm{s}.\right)$} \\
\hline \\
The relationship is not signfiicant.
\end{tabular}

Table 1.16 presents the ORs and 95\% CIs for OC and HRT use and Y/N question "During the past 12 months, you experienced confusion or memory loss that is happening more often or is getting worse?". No significant associations were observed.

\begin{tabular}{|c|c|c|c|c|c|c|c|c|c|}
\hline \multicolumn{10}{|c|}{$\begin{array}{l}\text { Table 1.16 - 0Rs and 95\% CIfor OC and HRT Use Levels by Y/N "During the past } 12 \text { months, you experienced confusion or memory loss that is happening more often or is getting worse?" for } \\
\qquad \text { Female Subjects }>=60 \text { years of age, NHANES 2011-2014 }\end{array}$} \\
\hline EEDC & Yes/No & $\begin{array}{l}\text { Unadjusted Odds } \\
\text { Ratio }^{1}\end{array}$ & $95 \% \mathrm{CI}$ & Yes/No & $\begin{array}{c}\text { Adjusted Odds Ratio } \\
\# 1^{2}\end{array}$ & $95 \% \mathrm{CI}$ & Yes $/ N_{0}$ & $\begin{array}{c}\text { Adjusted Odds Ratio } \\
\# 2^{3}\end{array}$ & $95 \% \mathrm{CI}$ \\
\hline OCuse & $274 / 1395$ & 0.703 & $0.482-1.026$ & $272 / 1394$ & 0.983 & $0.640-1.511$ & $268 / 1377$ & 0.973 & $0.634-1.493$ \\
\hline HRT use & 272/1389 & 0.825 & $0.594-1.145$ & $270 / 1388$ & 1.002 & $0.715-1.405$ & $265 / 1371$ & 0.994 & $0.671-1.472$ \\
\hline & & heart a & $\begin{array}{l}\text { ity. }{ }^{3} \text { Adjuste } \\
\text { k status, stro }\end{array}$ & ${ }^{*} \mathrm{p}<0.05,{ }^{*}$ & $01 * * * \mathrm{p}<0.001$ & diabetes sta & $\begin{array}{l}\text { h blood pr } \\
\text { as. }\end{array}$ & , coronary heart & ase status, \\
\hline
\end{tabular}

Trouble remembering past 7 days and Exposure to Metalloestrogens: Tables 1.17

and 1.18 present chi-square results for the $\mathrm{Y} / \mathrm{N}$ question "During the past 7 days, how often have you had trouble remembering where you put things, like your keys or your wallet?" and OC use and HRT use. We observed a significant relationship does not exists. 


\begin{tabular}{|c|c|c|}
\hline \multicolumn{3}{|c|}{ Chi Square Results } \\
\hline \multirow{2}{*}{$\begin{array}{c}\text { Table } 1.17 \text { During the past } 7 \text { days, how often } \\
\text { have you dad trouble remembering where you } \\
\text { put things? }\end{array}$} & \multicolumn{2}{|c|}{ OCuse? } \\
\hline & Yes & No \\
\hline Yes & $188(13.02 \%)$ & $147(7.73 \%)$ \\
\hline No & $722(50.66 \%)$ & $519(28.58 \%)$ \\
\hline \multicolumn{3}{|c|}{$\chi^{2}(\mathrm{df}=1, \mathrm{~N}=1576) \mathrm{F}=0.79, \mathrm{p}=$ n.s. $)$} \\
\hline \multicolumn{3}{|c|}{ The relationship is not significant. } \\
\hline
\end{tabular}

\begin{tabular}{|c|c|c|}
\hline \multicolumn{3}{|c|}{ Chi Square Results } \\
\hline \multirow{2}{*}{$\begin{array}{c}\text { Table } 1.18 \text { During the past } 7 \text { days, how often } \\
\text { have you dad trouble remembering where you } \\
\text { put things? }\end{array}$} & \multicolumn{2}{|c|}{ HRT use? } \\
\hline & Yes & No \\
\hline Yes & $120(9.79 \%)$ & $212(10.71 \%)$ \\
\hline No & $477(38.26 \%)$ & $759(41.25 \%)$ \\
\hline \multicolumn{3}{|c|}{$\chi^{2}(\mathrm{df}=1, \mathrm{~N}=1568) \mathrm{F}=0.02, \mathrm{p}=$ n.s. $)$} \\
\hline \multicolumn{3}{|c|}{ The relationship is not significant. } \\
\hline
\end{tabular}

Table 1.19 presents the ORs and 95\% CIs for OC and HRT use and Y/N question "During the past 7 days, how often have you had trouble remembering where you put things, like your keys or your wallet?" No significant associations were observed.

\begin{tabular}{|c|c|c|c|c|c|c|c|c|c|}
\hline \multicolumn{10}{|c|}{$\begin{array}{l}\text { Table 1.19 - 0Rs and 95\% CI for OC and HRT Use Levels by YN "During the past } 7 \text { days, how often hare you had trouble remembering where you put things, like your keys or your wallet?" for } \\
\text { Female Subjects }>=60 \text { years of age, NHANES 2011-2014 }\end{array}$} \\
\hline EEDC & Yes/No & $\begin{array}{l}\text { Unadjusted Odds } \\
\text { Ratio }^{1}\end{array}$ & $95 \% \mathrm{CI}$ & Yes/No & $\begin{array}{c}\text { Adjusted Odds Ratio } \\
\# 1^{2}\end{array}$ & $95 \% \mathrm{CI}$ & Yes/No & $\begin{array}{c}\text { Adjusted Odds Ratio } \\
\#^{3}{ }^{3}\end{array}$ & $95 \% \mathrm{Cl}$ \\
\hline OCuse & $335 / 1241$ & 0.950 & \begin{tabular}{|l|}
$0.651-1.389$ \\
\end{tabular} & $333 / 1240$ & 1.134 & 0.7141 .803 & $326 / 1227$ & 1.151 & $0.737-1.800$ \\
\hline HRT use & $332 / 1236$ & 0.986 & $0.776-1.252$ & $330 / 1235$ & 1.091 & 0.8241 .443 & $323 / 1221$ & 1.097 & 0.830-1.449 \\
\hline
\end{tabular}

\section{Limitations Due to Difficulty Remembering or Confusion and Exposure to OC}

and HRT: Tables 1.20 and 1.21 present chi-square results for the Y/N question "Are you limited in any way because of difficulty remembering or because you experience periods of confusion?" and OC use and HRT use. We observed a significant relationship exists between OC use and HRT use with respect to being limited in any way because of difficulty remembering or because you experience periods of confusion. 


\begin{tabular}{|c|c|c|}
\hline \multicolumn{3}{|c|}{ Chi Square Results } \\
\hline \multirow{2}{*}{$\begin{array}{c}\text { Table 1.20 Are you limited in any way because } \\
\text { of difficulty remembering or because you } \\
\text { experience periods of confusion? }\end{array}$} & \multicolumn{2}{|c|}{ OCuse? } \\
\hline & Yes & No \\
\hline Yes & $115(5.50 \%)$ & $144(6.39 \%)$ \\
\hline No & $837(57.26 \%)$ & $574(30.85 \%)$ \\
\hline \multicolumn{3}{|c|}{$\left.\chi^{2}(\mathrm{df}=1, \mathrm{~N}=1670) \mathrm{F}=13.75, \mathrm{p}=0.0008\right)$} \\
\hline \multicolumn{3}{|c|}{ The relationship is significant. } \\
\hline
\end{tabular}

\begin{tabular}{|c|c|c|}
\hline \multicolumn{3}{|c|}{ Chi Square Results } \\
\hline \multirow{2}{*}{$\begin{array}{l}\text { Table } 1.21 \text { Are you limited in any way } \\
\text { because of difficulty remembering or because } \\
\text { you experience periods of confusion? }\end{array}$} & \multicolumn{2}{|c|}{ HRT use? } \\
\hline & Yes & No \\
\hline Yes & $62(3.41 \%)$ & $192(8.13 \%)$ \\
\hline No & $566(44.29 \%)$ & $842(44.16 \%)$ \\
\hline \multicolumn{3}{|c|}{$\left.\chi^{2}(\mathrm{df}=1, \mathrm{~N}=1662) \mathrm{F}=32.06, \mathrm{p}=0.0001\right)$} \\
\hline \multicolumn{3}{|c|}{ The relationship is significant. } \\
\hline
\end{tabular}

Table 1.22 presents the ORs and 95\% CIs for OC and HRT use and responses to the Y/N question "Are you limited in any way because of difficulty remembering or because you experience periods of confusion?" With regard to OC use, it was observed that OC use was associated with lower odds in the unadjusted model. With regard to HRT use it was observed that HRT use was also associated with lower odds in the adjusted model, adjusted model \#1, and adjusted model \#2.

\begin{tabular}{|c|c|c|c|c|c|c|c|c|c|}
\hline \multicolumn{10}{|c|}{$\begin{array}{l}\text { Table 1.22 - 0Rs and } 95 \% \text { CI for OC and HRT Use Levels by Y/N" "Are you limited in any way because of difficulty remembering or because you experience periods of confusion?" for Female } \\
\text { Subjects }>=60 \text { years of age, NHANES 2011-2014 }\end{array}$} \\
\hline EEDC & Yes/No & $\begin{array}{c}\text { Unadjusted Odds } \\
\text { Ratio }^{1}\end{array}$ & $95 \% \mathrm{CI}$ & Yes/No & $\begin{array}{c}\text { Adjusted Odds Ratio } \\
\# 1^{2}\end{array}$ & $95 \% \mathrm{CI}$ & Yes/No & $\begin{array}{c}\text { Adjusted Odds Ratio } \\
\#^{3}\end{array}$ & $95 \% \mathrm{CI}$ \\
\hline OCuse & $259 / 1411$ & $0.464 *$ & $0.300-0.716$ & $257 / 1410$ & 0.788 & $0.479-1.295$ & $251 / 1395$ & 0.809 & $0.462-1.417$ \\
\hline HRT use & $254 / 1408$ & $0.418^{* * *}$ & $0.304-0.575$ & $252 / 1407$ & $0.555 *$ & $0.396-0.778$ & 246/1391 & $0.574 * *$ & $0.400-0.023$ \\
\hline \multicolumn{10}{|c|}{${ }_{p} \mathrm{p}<0.05,{ }^{*} \mathrm{p}<0.01 * * * \mathrm{p}<0.001$} \\
\hline
\end{tabular}

\section{Associations Between Exposures to Phthalate and BPA and Taste/Smell}

\section{Function}

Exposures to the metalloestrogens $\mathrm{Cd}, \mathrm{Mn}$, and $\mathrm{As}$, and two indicators of taste/smell function: 
1) Do you sometimes smell an unpleasant, bad, or burning odor when nothing is there (phantosmia), and 2) During the past 12 months have you had a taste or other sensation in your mouth that does not go away? Taste and smell indicators have been included as a surrogate indicator of brain health as taste and smell dysfunction is a possible pre-clinical indicator in the development of $\mathrm{AD}$ and other memory-related neurodegenerative diseases.

Smell Dysfunction and Exposure to OC and HRT: Tables 1.23 and 1.24 present chi-square results for the $\mathrm{Y} / \mathrm{N}$ question "Do you sometimes smell an unpleasant, bad or burning odor when nothing is there?" and OC use and HRT use. We observed a significant relationship does not exist.

\begin{tabular}{|c|c|c|}
\hline \multicolumn{3}{|c|}{ Chi Square Results } \\
\hline \multirow{2}{*}{$\begin{array}{l}\text { Table } 1.23 \text { Do you sometimes smell and } \\
\text { unpleasant, bad, or burning odor when } \\
\text { nothing is there? }\end{array}$} & \multicolumn{2}{|c|}{ OCuse? } \\
\hline & Yes & No \\
\hline $\begin{array}{c}\text { Yes } \\
\end{array}$ & $75(4.19 \%)$ & $48(2.23 \%)$ \\
\hline No & $877(58.67 \%)$ & $664(34.91)$ \\
\hline \multicolumn{3}{|c|}{$\chi^{2}(\mathrm{df}=1, \mathrm{~N}=1664) \mathrm{F}=0.23, \mathrm{p}=$ n.s. $)$} \\
\hline \multicolumn{3}{|c|}{ The relationship is not significant. } \\
\hline
\end{tabular}

\begin{tabular}{|c|c|c|}
\hline \multicolumn{3}{|c|}{ Chi Square Results } \\
\hline \multirow{2}{*}{$\begin{array}{l}\text { Table } 1.24 \text { Do you sometimes s mell and } \\
\text { unpleasant, bad, or burning odor when } \\
\text { nothing is there? }\end{array}$} & \multicolumn{2}{|c|}{ HRT use? } \\
\hline & Yes & No \\
\hline Yes & $40(2.65 \%)$ & $83(3.79 \%)$ \\
\hline No & $587(45.11 \%)$ & 945 (48.45\%) \\
\hline \multicolumn{3}{|c|}{$\left.\chi^{2}(\mathrm{df}=1, \mathrm{~N}=1655) \mathrm{F}=1.53, \mathrm{p}=0.22\right)$} \\
\hline \multicolumn{3}{|c|}{ The relationship is not significant. } \\
\hline
\end{tabular}

Table 1.25 presents the ORs and 95\% CIs for OC and HRT use and responses to the Y/N question "Do you sometimes smell an unpleasant, bad or burning odor when nothing is there?". With both OC use and HRT use, we did not observe any significant relationships. 


\begin{tabular}{|c|c|c|c|c|c|c|c|c|c|}
\hline \multicolumn{10}{|c|}{$\begin{array}{l}\text { Table 1.25 - ORs and 95\% CI for OC and HRT Use Levels by Y/N "Do you sometimes smell an unpleasant, bad or burning odor when nothing is there?" for Female Subjects >=60 years of age, } \\
\text { NHANES 2011-2014 }\end{array}$} \\
\hline EEDC & Yes/No & $\begin{array}{l}\text { Unadjusted Odds } \\
\text { Ratio }^{1}\end{array}$ & $95 \% \mathrm{Cl}$ & Yes/No & $\begin{array}{l}\text { Adjusted Odds Ratio } \\
\qquad 1^{2}\end{array}$ & $95 \% \mathrm{CI}$ & Yes/No & $\begin{array}{l}\text { Adjusted Odds Ratio } \\
\# 2^{3}\end{array}$ & $95 \% \mathrm{CI}$ \\
\hline OCuse & $123 / 1541$ & 1.116 & $0.704-1.770$ & $123 / 1538$ & 0.902 & $0.557-1.460$ & $121 / 1519$ & 0.952 & $0.556-1.630$ \\
\hline HRT use & $123 / 1532$ & 0.751 & $0.464-1.216$ & $123 / 1529$ & 0.865 & $0.514-1.455$ & $121 / 1509$ & 0.880 & $0.531-1.457$ \\
\hline
\end{tabular}

Taste Dysfunction and Exposure to OC and HRT: Tables 1.26 and 1.27 present

chi-square results for the $\mathrm{Y} / \mathrm{N}$ question "During the past 12 months have you had a taste or other sensation in your mouth that does not go away?" and OC use and HRT

use. We observed a significant relationship does not exist.

\begin{tabular}{|c|c|c|}
\hline \multicolumn{3}{|c|}{ Chi Square Results } \\
\hline \multirow{2}{*}{$\begin{array}{c}\text { Table } 1.27 \text { During the past } 12 \text { months have } \\
\text { you had a taste or other sensation in your } \\
\text { mouth that does not go away? }\end{array}$} & \multicolumn{2}{|c|}{ HRT use? } \\
\hline & Yes & No \\
\hline Yes & $44(2.85 \%)$ & $90(3.56 \%)$ \\
\hline No & $584(44.89 \%)$ & $940(48.71 \%)$ \\
\hline \multicolumn{3}{|c|}{$\left.\chi^{2}(\mathrm{df}=1, \mathrm{~N}=1658) \mathrm{F}=0.35, \mathrm{p}=0.55\right)$} \\
\hline \multicolumn{3}{|c|}{ The relationship is not significant. } \\
\hline
\end{tabular}

\begin{tabular}{|c|c|c|}
\hline \multicolumn{3}{|l|}{ Chi Square Results } \\
\hline \multirow{2}{*}{$\begin{array}{c}\text { Table } 1.26 \text { During the past } 12 \text { months have } \\
\text { you had a taste or other sensation in your } \\
\text { mouth that does not go away? }\end{array}$} & \multicolumn{2}{|c|}{ OC use? } \\
\hline & Yes & No \\
\hline Yes & $75(3.79 \%)$ & $59(2.59 \%)$ \\
\hline No & $876(58.97 \%)$ & $657(34.64 \%)$ \\
\hline \multicolumn{3}{|c|}{ 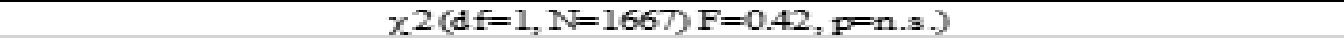 } \\
\hline \multicolumn{3}{|c|}{ The relations hip is not significant. } \\
\hline
\end{tabular}

Table 1.28 presents the ORs and 95\% CIs for OC and HRT use and responses to the Y/N question "During the past 12 months have you had a taste or other sensation in your mouth that does not go away?". With both OC use and HRT use, we did not observe any significant relationships 


\begin{tabular}{|c|c|c|c|c|c|c|c|c|c|}
\hline \multicolumn{10}{|c|}{$\begin{array}{c}\text { Table 1.28.0Rs and 95\% Clfor OC and HRT Lse Lerels by YN "During the past } 12 \text { months hare you had ataste or other sensation in your mouth that does not go aray?" for Female Subjects >=60 } \\
\text { years of age, NHANES 2011-2014 }\end{array}$} \\
\hline EEDC & Yes/No & $\begin{array}{c}\text { Unadjusted Odds } \\
\text { Ratio }^{1}\end{array}$ & $95 \% \mathrm{Cl}$ & Yes/No & $\begin{array}{c}\text { Adjusted Odds Ratio } \\
\# 1^{2}\end{array}$ & $95 \% \mathrm{Cl}$ & Yes/No & $\begin{array}{c}\text { Adjusted Odds Ratio } \\
\# 2^{3}\end{array}$ & $95 \% \mathrm{Cl}$ \\
\hline OCuse & $134 / 1533$ & 0.861 & $0.5388-1.378$ & 13411530 & 0.921 & $0.578-1.468$ & $132 / 1511$ & 0.947 & $0.587-1.528$ \\
\hline HRT use & 13441524 & 0.867 & $0.531-1.417$ & 1341521 & 1.081 & $0.685-1.706$ & $132 / 1501$ & 1.180 & $0.748-1.859$ \\
\hline${ }^{1}$ Unadjust & usted f & heart? & status, $s$ & ${ }^{*} p<0.05, *$ & $01 * * * * 0 \times 0.001$ & s, head tr & loodp & tusc coronary heart di & asestatus, \\
\hline
\end{tabular}

\section{DISCUSSION}

This study takes a novel approach in the assessment of exposure to OC and HRT in the development of cognitive dysfunction and possible neurodegenerative disease using surrogate indicators of brain health. The study examined an older geriatric-aged population of US adults 60 years of age and older. We first assessed the existence of a relationship between past OC and HRT use by calculating and chisquare test of independence versus each surrogate indicator of brain health, and subsequently used ORs and $95 \% \mathrm{CI}$ to determine the risk of developing cognitive dysfunction.

Major Findings: Overall, we observed a the existence of a relationship between past OC and HRT use and the following surrogates of brain function: immediate recall scores; delayed recall scores; animal flluency scores; DSST scores, and limitations due to difficulty remembering or confusion (Tables 1.2 to 1.28). In our logisitic regression models, when controlling for all known and suspected covariates of cognitive dysfunction and $\mathrm{AD}$ in our second adjusted model, we found past $\mathrm{OC}$ use to lower the risk of developing cognitive dysfunction and the possible development of 
AD. Specifically, we found past OC use to be associated with better immediate recall, delayed recall, animal fluency and DSST scores.

We also found past HRT use to lower the risk fo developing cognitive dysfunction and the possible development of AD. Specifically, we found past HRT use to be associated with better immediate recall, animal fluency, and DSST scores. Past HRT use was also associated with less occurrences of limitations due to periods of difficulty remembering or periods of confusion. Tables (1.2 to 1.28).

With regards to past OC use, it was not possible to differentiate between OCs containing estrogen and those not containing it, which is an issue with past studies ${ }^{68}$. Past OC use with other studies that suggest its' use can improve cognitive function with regards to cognitive tests ${ }^{8}$ and better performance of spatial ability and cognitive tests ${ }^{68}$. The majority of evidence with regard to past HRT use suggests that HRT use may be harmful to brain health. Increased risk of dementia ${ }^{12,13,16-19}$.

One of the prevailing hypothesis states the timing of estrogen therapies may dictate its' effectiveness in protecting cognition and function. An increase in estrogen in early to mid-adulthood and early on in menopause suggests a possible protective benefit from the use of OC and HRT ${ }^{69}$, which was shown in one study, which showed increased AD risk if HRTs were used more than five years after menopause and a decreased risk if HRTs were used within five years after menopause ${ }^{20}$. From our study, we cannot fully determine the composition of the OC and HRT therapies. It is suggested that the composition, and hence, the estrogen amount and dosage may also dictate the effectiveness, or adversness of the OC and/or HRT therapy. 
Biological Mechanisms and Brain Health: OCs and HRTs, although considered to EEDCs because of their ability to mimic estrogen are unique since their main active ingredients may contain derivatives of estrogen, making them highly estrogenresponsive with their high affinity for estrogen receptors ${ }^{70}$ which is very similar to biologically available estrogen, with its effects mediated through the known estrogen receptor pathways, ERa, ERß, GPER/GPR30, which are widely expressed in the brain ${ }^{71}$. Estrogen itself has been demonstrated to have roles in neuroprotection as well as exert anti-inflammatory effects on the brain ${ }^{72-77}$ and with its' similar bioactivity to biological estrogen, may provide a protective effect to the aging brain.

Although a number genes that are implicated in $\mathrm{AD}$ and other neurodegenerative diseases are estrogen-sensitive ${ }^{71}$, it is possible that a deficit in naturally circulating estrogen may be one of culprits of neurodegeneration which supports the critical window hypothesis when estrogen production is lowest around menopause ${ }^{69}$.

Strengths and Limitations: There are strengths and limitations to our study. The largest limitations the cross-sectional design of our study. The data is self-reported which makes inference difficult and prone to misclassification bias. Our OC and HRT variable does not give us a dosage, or amount. It also does not indicate the length of time, or when the OC/HRT therapy was stated and finished. Our outcome variables are surrogates rather than actual clinical endpoints, which limits our ability to say that our outcome will lead to neurodegenerative disease. The cut-off scores of our cognitive exams were based on previous studies, however, performance on those exams are heavily dependent on education, so there is a possibility that poor 
performance might be a function of lack of education. For our taste/smell indicators, the senses heavily affected by numerous confounders, some of which were not feasible in study due to making the sample smaller, or the confounder not being available. Strengths of our study include the novelty of such an analysis to be conducted on an older population. We had relatively large sample sizes available for our analyzes. The generalizability of our study to the US population and the large amount of chemical measurements available gives the study the ability to examine different aspects of chemical exposure.

\section{Conclusion}

Based on our findings, OC and HRT use has a beneficial and possible protective effect on cognitive function and might possibly be protective against neurodegenerative disease. Further study is needed, especially to examine time frames and extent of OC and HRT use to establish the most effective points to take it in a female's life. This may add to the therapeutic benefits of these therapies.

\section{References}

1. Bergman A, Heindel JJ, Jobling S, Kidd KA, Zoeller TR. State of the science of endocrine disrupting chemicals. World Heal Organ United Nations Environ Program. 2012:1-38. http://apps.who.int/iris/handle/10665/78102.

2. Alzheimer's Association. 2017 Alzheimer's Disease Facts and Figures. Alzheimers Dement . 2017;13:325-373. doi:10.1016/j.jalz.2017.02.001. 
3. Diamanti-Kandarakis E, Bourguignon J-P, Giudice LC, et al. Endocrinedisrupting chemicals: an Endocrine Society scientific statement. Endocr Rev. 2009;30(4):293-342. doi:10.1210/er.2009-0002.

4. Giusti RM, Iwamoto K, Hatch EE. Diethylstilbestrol revisited: a review of the long-term health effects. Ann Intern Med. 1995;122(10):778-788. http://www.ncbi.nlm.nih.gov/pubmed/7717601. Accessed February 4, 2018.

5. Griksiene R, Ruksenas O. Effects of hormonal contraceptives on mental rotation and verbal fluency. Psychoneuroendocrinology. 2011;36(8):1239-1248. doi:10.1016/j.psyneuen.2011.03.001.

6. Pletzer BA, Kerschbaum HH. 50 years of hormonal contraception - time to find out, what it does to our brain. Front Neurosci. 2014. doi:10.3389/fnins.2014.00256.

7. Beltz AM, Hampson E, Berenbaum SA. Oral contraceptives and cognition: A role for ethinyl estradiol. Horm Behav. 2015;74:209-217.

doi:10.1016/j.yhbeh.2015.06.012.

8. Egan KR, Gleason CE. Longer Duration of Hormonal Contraceptive Use Predicts Better Cognitive Outcomes Later in Life. J Women's Heal. 2012;21(12):120920061605006. doi:10.1089/jwh.2012.3522.

9. Warren AM, Gurvich C, Worsley R, Kulkarni J. A systematic review of the impact of oral contraceptives on cognition. Contraception. 2014;90(2):111-116. doi:10.1016/j.contraception.2014.03.015.

10. Maki PM, Henderson VW. Hormone therapy, dementia, and cognition: the Women's Health Initiative ten years on. Climacteric. 2012;15(3):256-262. doi:10.1016/j.biotechadv.2011.08.021.Secreted.

11. Xiao M, Cao GL, Marshall C, Hu G. Hypothesis: Multiple factors are associated with the lack of any beneficial effects of oestrogen-replacement therapy in the late postmenopausal stage. Clin Exp Pharmacol Physiol. 2010;37(8):873-876.

doi:10.1111/j.1440-1681.2010.05400.x. 
12. Shumaker S a, Legault C, Rapp SR, et al. Estrogen Plus Progestin and the Incidence of Dementia and Mild Cognitive Impairment in Postmenopausal Women. JAMA. 2003;289(20):2651-2662.

13. Shumaker S a, Legault C, Kuller L, et al. Conjugated equine estrogens and incidence of probable dementia and mild cognitive impairment in postmenopausal women: Women's Health Initiative Memory Study. JAMA. 2004;291(24):29472958. doi:10.1097/01.ogx.0000140470.91959.32.

14. Resnick SM, Maki PM, Rapp SR, et al. Effects of combination estrogen plus progestin hormone treatment on cognition and affect. J Clin Endocrinol Metab. 2006;91(5):1802-1810. doi:10.1210/jc.2005-2097.

15. Resnick SM, Espeland M a., An Y, et al. Effects of conjugated equine estrogens on cognition and affect in postmenopausal women with prior hysterectomy. J Clin Endocrinol Metab. 2009;94(11):4152-4161. doi:10.1210/jc.2009-1340.

16. Yaffe K, Vittinghoff E, Ensrud KE, et al. Effects of ultra-low-dose transdermal estradiol on cognition and health-related quality of life. Arch Neurol. 2006;63(7):945-950. doi:10.1001/archneur.63.7.945.

17. Viscoli CM, Brass LM, Kernan WN, Sarrel PM, Suissa S, Horwitz RI. Estrogen therapy and risk of cognitive decline: Results from the Women's Estrogen for Stroke Trial (WEST). Am J Obstet Gynecol. 2005;192(2):387-393. doi:10.1016/j.ajog.2004.08.017.

18. Almeida OP, Lautenschlager NT, Vasikaran S, Leedman P, Gelavis A, Flicker L. A 20-week randomized controlled trial of estradiol replacement therapy for women aged 70 years and older: Effect on mood, cognition and quality of life. Neurobiology. 2006;27:141-149. doi:10.1016/j.neurobiolaging.2004.12.012.

19. Pefanco MA, Kenny AM, Kaplan RF, et al. The effect of 3-year treatment with $0.25 \mathrm{mg} /$ day of micronized 17??-estradiol on cognitive function in older postmenopausal women. J Am Geriatr Soc. 2007;55(3):426-431. doi:10.1111/j.1532-5415.2007.01085.x.

20. Shao H, Breitner JCS, Whitmer R a., et al. Hormone therapy and Alzheimer disease dementia: New findings from the Cache County Study. Neurology. 2012;79(18):1846-1852. doi:10.1212/WNL.0b013e318271f823. 
21. O'Brien J, Jackson JW, Grodstein F, Blacker D, Weuve J. Postmenopausal hormone therapy is not associated with risk of all-cause dementia and alzheimer's disease. Epidemiol Rev. 2014. doi:10.1093/epirev/mxt008.

22. Lundin JI, Ton TG, LaCroix AZ, et al. Formulations of Hormone Therapy and Risk of Parkinson's Disease. Mov Disord. 2014;29(13):1631-. doi:10.1002/mds.26037.

23. Johnson CL, Paulose-Ram R, Ogden CL, et al. National health and nutrition examination survey: analytic guidelines, 1999-2010. Vital Health Stat 2. 2013;(161):1-24. http://www.ncbi.nlm.nih.gov/pubmed/25090154.

24. Johnson CL, Dohrmann SM, Burt VL, Mohadjer LK. National Health and Nutrition Examination Survey: Sample Design , 2011 - 2014. Vital Heal Stat. 2014;2(162):1-33. http://www.cdc.gov/nchs/data/series/sr_02/sr02_162.pdf.

25. Centers for Disease Control and Prevention (CDC). National Center for Health Statistics (NCHS). National Health and Nutrition Examination Survey NCHS Research Ethics Review Board (ERB) Approval. Hyattsville, MD: U.S. Department of Health and Human Services, Centers for Disease Control and Prevention. https://www.cdc.gov/nchs/nhanes/irba98.htm. Published 2016.

26. Centers for Disease Control and Prevention (CDC). National Center for Health Statistics (NCHS). No TitleNational Health and Nutrition Examination Survey 2011-2012 Data Documentation, Codebook, and Frequencies Reproductive Health (RHQ_G). Hyattsville, MD: U.S. Department of Health and Human Services, Centers for Disease Control and Prevention. https://wwwn.cdc.gov/nchs/nhanes/2011-2012/RHQ_G.htm\#RHQ420. Published 2017.

27. Centers for Disease Control and Prevention (CDC). National Center for Health Statistics (NCHS). National Health and Nutrition Examination Survey 2013-2014 Data Documentation, Codebook, and Frequencies Reproductive Health (RHQ_H). https://wwwn.cdc.gov/Nchs/Nhanes/2013-2014/RHQ_H.htm. Published 2017. 
28. Centers for Disease Control and Prevention (CDC). National Center for Health Statistics (NCHS). National Health and Nutrition Examination Survey 2013-2014 Data Documentation, Codebook, and Frequencies Cognitive Functioning (CFQ_H). Hyattsville, MD: U.S. Department of Health and Human Services, Centers for Disease Control and Prevention. https://wwwn.cdc.gov/Nchs/Nhanes/2013-2014/CFQ_H.htm. Published 2017.

29. Centers for Disease Control and Prevention (CDC). National Center for Health Statistics (NCHS). National Health and Nutrition Examination Survey 2011-2012 Data Documentation, Codebook, and Frequencies Cognitive Functioning (CFQ_G). Hyattsville, MD: U.S. Department of Health and Human Services, Centers for Disease Control and Prevention. https://wwwn.cdc.gov/Nchs/Nhanes/2011-2012/CFQ_G.htm\#CFDCST3. Published 2017.

30. Fillenbaum GG, van Belle G, Morris JC, et al. Consortium to Establish a Registry for Alzheimer's Disease (CERAD): The first twenty years. Alzheimer's Dement. 2008;4(2):96-109. doi:10.1016/j.jalz.2007.08.005.

31. Bertolucci PHF, Okamoto IH, Brucki SMD, Siviero MO, Neto JT, Ramos LR. Applicability of the CERAD neuropsychological battery to Brazilian elderly. Arq Neuropsiquiatr. 2001;59(3 A):532-536. doi:10.1590/S0004282 X2001000400009.

32. Karrasch M, Sinervä E, Grönholm P, Rinne J, Laine M. CERAD test performances in amnestic mild cognitive impairment and Alzheimer's disease. Acta Neurol Scand. 2005;111(3):172-179. doi:10.1111/j.16000404.2005.00380.x.

33. Karrasch M, Laatu S, Martikainen K, Marttila R. CERAD test performance and cognitive impairment in Parkinson's disease. Acta Neurol Scand. 2013;128(6):409-413. doi:10.1111/ane.12138.

34. R.M. H, N.-M. S, P. H, et al. The CERAD neuropsychological battery in patients with frontotemporal lobar degeneration. Dement Geriatr Cogn Dis Extra. 2015;5(1):147-154. doi:10.1159/000380815. 
35. Berres M, Monsch AU, Bernasconi F, Thalmann B, Stähelin HB. Normal ranges of neuropsychological tests for the diagnosis of Alzheimer's disease. Stud Health Technol Inform. 2000;77:195-199.

http://www.ncbi.nlm.nih.gov/pubmed/11187541. Accessed January 21, 2018.

36. Alegret M, Espinosa A, Valero S, et al. Cut-off Scores of a Brief Neuropsychological Battery (NBACE) for Spanish Individual Adults Older than 44 Years Old. Brucki S, ed. PLoS One. 2013;8(10):e76436. doi:10.1371/journal.pone.0076436.

37. American Academy of Family Physicians. BB, Hartmann B, DeJoseph D. American Family Physician. Vol 84. American Academy of Family Physicians; 1970. https://www.aafp.org/afp/2011/1015/p895.html. Accessed January 21, 2018.

38. Axelrod BN. Administration duration for the Wechsler Adult Intelligence ScaleIII and Wechsler Memory Scale-III. Arch Clin Neuropsychol. 2001;16(3):293301. doi:10.1016/S0887-6177(00)00048-2.

39. Matsuda O, Saito M, Kato M, Azami H, Shido E. Wechsler Adult Intelligence Scale-III profile in the early stages of Alzheimer's disease: Performance in subtests sensitive to and resistant to normal decline with ageing. Psychogeriatrics. 2015;15(1):1-6. doi:10.1111/psyg.12066.

40. Donnell AJ, Pliskin N, Holdnack J, Axelrod B, Randolph C. Rapidlyadministered short forms of the Wechsler Adult Intelligence Scale-3rd edition. Arch Clin Neuropsychol. 2007;22(8):917-924. doi:10.1016/j.acn.2007.06.007.

41. Nielsen NP, Ringström R, Wiig EH, Minthon L. Associations between AQT processing speed and neuropsychological tests in neuropsychiatric patients. Am $J$ Alzheimers Dis Other Demen. 2011;22(3):202-210. doi: $10.1177 / 1089253206300417$.

42. Proust-Lima C, Amieva H, Dartigues JF, Jacqmin-Gadda H. Sensitivity of four psychometric tests to measure cognitive changes in brain aging-population-based studies. Am J Epidemiol. 2007;165(3):344-350. doi:10.1093/aje/kwk017. 
43. Rosano C, Perera S, Inzitari M, Newman AB, Longstreth WT, Studenski S. Digit symbol substitution test and future clinical and subclinical disorders of cognition, mobility and mood in older adults. Age Ageing. 2016;45(5):687-694. doi:10.1093/ageing/afw116.

44. Saxton J, Lopez OL, Ratcliff G, et al. Preclinical Alzheimer disease: neuropsychological test performance 1.5 to 8 years prior to onset. Neurology. 2004;63(Mh 46643):2341-2347. doi:10.1212/01.WNL.0000147470.58328.50.

45. Centers for Disease Control and Prevention (CDC). National Center for Health Statistics (NCHS). National Health and Nutrition Examination Survey Questionnaires, Datasets, and Related Documentation. Hyattsville, MD: U.S. Department of Health and Human Services, Centers for Disease Control and Prevention. https://www.cdc.gov/nchs/nhanes/nhanes_questionnaires.htm. Published 2015.

46. Brooks LG, Loewenstein DA. Assessing the progression of mild cognitive impairment to Alzheimer's disease: current trends and future directions. Alzheimers Res Ther. 2010;2(5):28. doi:10.1186/alzrt52.

47. Tolea MI, Morris JC, Galvin JE. Longitudinal associations between physical and cognitive performance among community-dwelling older adults. PLoS One. 2015;10(4):1-12. doi:10.1371/journal.pone.0122878.

48. Ansoleaga B, Garcia-Esparcia P, Llorens F, Moreno J, Aso E, Ferrer I. Dysregulation of brain olfactory and taste receptors in AD, PSP and CJD, and AD-related model. Neuroscience. 2013;248:369-382. doi:10.1016/j.neuroscience.2013.06.034.

49. Macknin JB, Higuchi M, Lee VM-Y, Trojanowski JQ, Doty RL. Olfactory dysfunction occurs in transgenic mice overexpressing human $\tau$ protein. Brain Res. 2004;1000(1-2):174-178. doi:10.1016/j.brainres.2004.01.047.

50. Lee S, Manly J, Andrews H, et al. Olfactory deficits predict cognitive decline and Alzheimer dementia in an urban community. 2014.

51. Hummel T, Landis B, Huttenbrink K. Smell and taste disorders. GMS Curr Tpoics Otorhinolaryngol Head Neck Surg. 2011;10:1-15. doi:10.3205/cto000077. 
52. Doty RL. The olfactory vector hypothesis of neurodegenerative disease: Is it viable? Ann Neurol. 2008;63(1):7-15. doi:10.1002/ana.21327.

53. Liu J. Clinical features of olfactory disorders in patients seeking medical consultation. Med Sci Monit. 2013;19:444-450. doi:10.12659/MSM.883938.

54. Hüttenbrink K-B, Hummel T, Berg D, Gasser T, Hähner A. Olfactory dysfunction: common in later life and early warning of neurodegenerative disease. Dtsch Arztebl Int. 2013;110(1-2):1-7, e1. doi:10.3238/arztebl.2013.0001.

55. Barresi M, Ciurleo R, Giacoppo S, et al. Evaluation of olfactory dysfunction in neurodegenerative diseases. J Neurol Sci. 2012;323(1-2):16-24. doi:10.1016/j.jns.2012.08.028.

56. Ruan Y, Zheng XY, Zhang HL, Zhu W, Zhu J. Olfactory dysfunctions in neurodegenerative disorders. J Neurosci Res. 2012;90(9):1693-1700. doi:10.1002/jnr.23054.

57. Albers MW, Tabert MH, Devanand DP. Olfactory dysfunction as a predictor of neurodegenerative disease. Curr Neurol Neurosci Rep. 2006;6(5):379-386. doi:10.1007/s11910-996-0018-7.

58. Winner B, Kohl Z, Gage FH. Neurodegenerative disease and adult neurogenesis. Eur J Neurosci. 2011;33(6):1139-1151. doi:10.1111/j.1460-9568.2011.07613.x.

59. Attems J, Walker L, Jellinger KA. Olfactory bulb involvement in neurodegenerative diseases. Acta Neuropathol. 2014;127(4):459-475. doi:10.1007/s00401-014-1261-7.

60. Makowska I, Kloszewska I, Grabowska A, Szatkowska I, Rymarczyk K. Olfactory deficits in normal aging and Alzheimer's disease in the polish elderly population. Arch Clin Neuropsychol. 2011;26(3):270-279. doi:10.1093/arclin/acr011.

61. Sohrabi HR, Bates K a, Weinborn MG, et al. Olfactory discrimination predicts cognitive decline among community-dwelling older adults. Transl Psychiatry. 2012;2(5):e118. doi:10.1038/tp.2012.43. 
62. Driver-Dunckley E, Adler CH, Hentz JG, et al. Olfactory dysfunction in incidental Lewy body disease and Parkinson's disease. Parkinsonism Relat Disord. 2014;20(11):1260-1262. doi:10.1016/j.parkreldis.2014.08.006.

63. Ward AM, Calamia M, Thiemann E, Dunlap J, Tranel D. Association between olfaction and higher cortical functions in Alzheimer's disease, mild cognitive impairment, and healthy older adults. J Clin Exp Neuropsychol. November 2016:1-15. doi:10.1080/13803395.2016.1253667.

64. SAS 9.4. 2017.

65. Centers for Disease Control and Prevention (CDC). National Center for Health Statistics (NCHS). National Health and Nutrition Examination Survey - Overview of NHANES Survey Design and Weights. Hyattsville, MD: U.S. Department of Health and Human Services, Centers for Disease Control and Prevention. https://www.cdc.gov/nchs/tutorials/environmental/orientation/sample_design/. Published 2013.

66. SAS Institute Inc. Sas/Stat ${ }^{\circledR}$ 9.3. 2011:User's Guide: The GLIMMIX Procedure (Chapter).

67. SAS Institute Inc. Estimating Geometric Means Using Data from a Complex Survey Sampling Design. http://citeseerx.ist.psu.edu/viewdoc/download?rep=rep1\&type=pdf\&doi=10.1.1.1 92.593. Published 2017.

68. Beltz AM, Hampson E, Berenbaum SA. Oral Contraceptives and Cognition: A Role for Ethinyl Estradiol. Horm Behav. 2015. doi:10.1016/j.yhbeh.2015.06.012.

69. Maki PM. Critical window hypothesis of hormone therapy and cognition: a scientific update on clinical studies. Menopause. 2013;20(6):695-709. doi:10.1097/GME.0b013e3182960cf8.

70. Blair RM, Fang H, Branham WS, et al. The estrogen receptor relative binding affinities of 188 natural and xenochemicals: structural diversity of ligands. Toxicol Sci. 2000;54(1):138-153. doi:10.1093/toxsci/54.1.138. 
71. Preciados M, Yoo C, Roy D. Estrogenic Endocrine Disrupting Chemicals Influencing NRF1 Regulated Gene Networks in the Development of Complex Human Brain Diseases. Int J Mol Sci. 2016;17(12):2086. doi:10.3390/ijms 17122086 .

72. Arevalo M-A, Azcoitia I, Garcia-Segura LM. The neuroprotective actions of oestradiol and oestrogen receptors. Neuroscience. 2015;16(1):17-29. doi: $10.1038 / \mathrm{nrn} 3856$.

73. Chakrabarti M, Haque A, Banik NL, Nagarkatti P, Nagarkatti M, Ray SK. Estrogen receptor agonists for attenuation of neuroinflammation and neurodegeneration. Brain Res Bull. 2014;109:22-31. doi:10.1016/j.brainresbull.2014.09.004.

74. Green PS, Simpkins JW. Neuroprotective effects of estrogens: Potential mechanisms of action. Int J Dev Neurosci. 2000;18(4-5):347-358. doi:10.1016/S0736-5748(00)00017-4.

75. Hristian C, Ruber JG, Alter W, et al. Production and actions of estrogens. $N$ Engl J Med. 2002;346(5). doi:10.1016/S0960-0760(01)00184-4.

76. Pozzi S, Benedusi V, Maggi A, Vegeto E. Estrogen action in neuroprotection and brain inflammation. Ann N Y Acad Sci. 2006;1089(2006):302-323. doi:10.1196/annals.1386.035.

77. Wise PM, Dubal DB, Wilson ME, Rau SW, Liu Y. Estrogens: trophic and protective factors in the adult brain. Front Neuroendocrinol. 2001;22(1):33-66. doi:10.1006/frne.2000.0207. 


\section{CHAPTER VIII}

\section{OVERALL CONCLUSIONS}

The goal of this research was to find out if exposures to EEDCs had an adverse effect on brain health, using our surrogates of brain health indicators found in the CDC's NHANES datasets. Our surrogate indicators, cognitive scores that include immediate recall, delayed, recall, animal fluency, and digit symbol substitution scores, have been proven to differentiate between normal cognitive function, cognitive decline, and the development of dementia and AD. They are further supported by their use in a clinical setting. The memory questions that indicated if memory issues were present in a year's time and a week's time have utility in the fact that the first symptom of cognitive decline is memory issues. Taste and smell questions have their utility in taste and smell dysfunction can be an early sub-clinical indicator of cognitive dysfunction.

The EEDCs considered in this study, phthalates, BPA, PCBs, Cd, As, and Mn, all have proven estrogenic activity and can interfere with the protective and therapeutic effect that estrogen has on brain health and function. The epidemiologic evidence, however, is lacking, as most exposure studies have been performed at the cellular, animal, or before, during, and after birth in humans. Our study addresses the gaps present in this study area.

Our first specific aim, assessing exposure to phthalates and bisphenol-A (BPA) using urinary biomarkers from the CDC NHANES 2011-2014 datasets to find associations between phthalate and BPA bioburden and adverse brain health using surrogate indicators of brain health was addressed in manuscript II. 
Increased levels of phthalate metabolites were observed in those who had lower cognitive test scores, reported having memory issues, and reported taste and smell deficits compared to those who did not have any observed brain health issues. Females have a greater bioburden of phthalates compared to males. In females, the phthalates ECP, MBP, MOH, MZP, and MIB were observed to have significantly higher bioburdens among two or more of the surrogate brain health indicators. In males, the phthalates $\mathrm{ECP}, \mathrm{MHH}, \mathrm{MOH}$, and MIB were observed to have significantly higher bioburdens among two or more of the surrogate brain health indicators. BPA did not have any significant results in any of our tests. When controlling for known and suspected covariates of $\mathrm{AD}$ in males in our final logistic regression model, $\mathrm{COP}, \mathrm{ECP}, \mathrm{MBP}, \mathrm{MC1}$, MEP, $\mathrm{MHH}, \mathrm{MOH}$, and MIB were associated with one or more of the surrogate brain health indicators. When controlling for known and suspected covariates of $\mathrm{AD}$ in females in our final logistic regression model, ECP, MBP, MHH, MOH, MZP, and MIB were associated with one or more of the surrogate brain health indicators. BPA did not have any significant results in any of our tests.

Our second specific aim, assessing exposure to the metalloestrogens, cadmium $(\mathrm{Cd})$, arsenic (As), and manganese $(\mathrm{Mn})$ using urinary biomarkers from the CDC NHANES 2011-2014 datasets to find associations between phthalate and BPA bioburden and adverse brain health using surrogate indicators of brain health was addressed in manuscript III. $\mathrm{Cd}$ and Mn bioburdens were found to be more evident in individuals who have lower cognitive scores and/or have memory cognition issues. In our logistic regression models, $\mathrm{Cd}$ stood out as the 
metalloestrogen that plays a larger role in the development of neurodegenerative disease and adverse brain health in an older population. No significant results were observed in our full logistic regression models.

Our third specific aim, assessing exposure to oral contraceptives (OC) and hormonal replacement therapy (HRT) using urinary biomarkers from the CDC NHANES 2011-2014 datasets to find associations between phthalate and BPA bioburden and adverse brain health using surrogate indicators of brain health, was addressed in manuscript IV. We observed the existence of a relationship between past OC and HRT use and the following surrogates of brain function: immediate recall scores; delayed recall scores; animal flluency scores; DSST scores, and limitations due to difficulty remembering or confusion. In our logisitic regression models, when controlling for all known and suspected covariates of cognitive dysfunction and $\mathrm{AD}$ in our second adjusted model, we found past $\mathrm{OC}$ use to lower the risk of developing cognitive dysfunction and the possible development of AD. Specifically, we found past OC use to be associated with better immediate recall, delayed recall, animal fluency and DSST scores. We also found past HRT use to lower the risk fo developing cognitive dysfunction and the possible development of AD. Specifically, we found past HRT use to be associated with better immediate recall, animal fluency, and DSST scores. Past HRT use was also associated with less occurrences of limitations due to periods of difficulty remembering or periods of confusion. 
Our fourth specific aim, to Assess estrogen-responsive genes networks common to the EEDCs, phthalates, BPA, Cd, As, and Mn, NRF1, and neurodegenerative disease using bioinformatics methods, was addressed in manuscript I. From our bioinformatics approach, we identified several novel gene NRF1 gene targets that are both estrogen-responsive and responsive to our selection of EEDCs which are also implicated in the gene networks of several major neurodegenerative diseases.

Our study has several strengths. We had sufficient sample sizes to examine our variables, and our covariates had a large response rate. The generalizability of our study to the US population and the large amount of chemical measurements available gives the study the ability to examine different aspects of chemical exposure.

Our overall study has several limitations. Most of the data is self-reported, which lends itself to misclassification bias, with the chance of incorrectly reported endpoints. Also, the lack of true clinical endpoints allows us to make limited inference pertaining to disease diagnosis. Our OC and HRT variables do not give information on the type of treatment given, so we could not assess if synthetic estrogens were part of the drug formulation. Our cut-off scores were based on previous studies, however, performance on these test is also influenced by education. For our taste/smell indicators, the senses heavily affected by numerous confounders, some of which were not feasible in study due to making the sample smaller, or the confounder not being available. 
In summary, our findings suggest that EEDCs do play a role in the development of cognitive dysfunction and the development of neurodegenerative disease in older people. These findings are further supported by our finding of gene targets which play a role in the development of various neurodegenerative diseases. 


\section{VITA}

\section{MARK VICERA PRECIADOS}

$2003-2007$

B.S., Microbiology and Immunology

University of Miami

Miami, FL

$2008-2013$

M.P.H., Public Health

Florida International University

Miami, FL

2013 - present

Doctoral Candidate, Public Health

Florida International University

Miami, FL

$2004-2007$

Pharmacy Technician

Miami, FL

2007-2008

Quality Control Analyst

Bioheart, Inc

Sunrise, FL

2008-2009

Microbiologist

Bionucleonics, Inc

Doral, FL

2009 - present

Consumer Safety Officer

US Food and Drug Administration

\section{PUBLICATIONS AND PRESENTATIONS}

Preciados M, Yoo C, Roy D. Estrogenic Endocrine Disrupting Chemicals Influencing NRF1 Regulated Gene Networks in the Development of Complex Human Brain Diseases. Int J Mol Sci. 2016;17(12):2086.

doi:10.3390/ijms 17122086.

Preciados, Mark, (March, 2017). Role of Estrogenic Endocrine Disruptors (EEDs)-Genes interactions associated with both estrogen signaling and NRF1 signaling pathways in Alzheimer's Disease (AD). Poster presented at the Society of Toxicology Meeting, Baltimore, MD, March 2017. 